

\section{MHC IAIMt:R}

This document was prepared an an uccount of work sponored by an agency of the I nited Statew cinsernment. Seither the I nited States Covernment nor the I niversity of California nor any of their emplosecs. makes an! warranty. enpress or implicd. nr ussumes any legal liahility or responsibility ar the accuracs, compluteness, or usefulness of any information, apparatus, product, or procens disclosed, or represents that its use nould not infringe pris ately on ned rights. Keference herein to any specific comnercial products, process. or service by trade name, trademark, manufacturer. or atherh ise, does not necessarily constitute ur imply it: endorsement, recommendation, or favoring hy the I nited Silales Giovernment or the I niversity of California. The views and opinions of authors supressed herein do not necessarily state or reflect those of the I nited states Covernment thereof, and shall not be used for advertising or product indersienent purposes. 


\title{
Energetic Particle Drift Motions in the Outer Dayside Magnetosphere
}

\author{
Richard McCullough Buck \\ (Ph.D. Thesis)
}

\section{Manuscript date: December 1987}

\author{
DISCLAIMER
}

\begin{abstract}
This report was prepared as an scoount of work sponsoced by an atency of the United States Government. Neither be United Stutes Goverument nor any acency thereof, nor any of beir employees, makes uny warranly, expreas or implied, or annmes any legal liability of reaponibility for the accuracy, completenese, or usefulnes of any information, apparatur, product, or process discloed, or represents thet its une would not infringe privately owned rights. Reforenoe herein to any specific commercial product, proceat, or service by trade name, trademart. manufacturer, or otherwive doen not necesasrily constitute or imply its endorsement, recommendation, of favoring by the Unitod States Government or any agency thereof. The views and opinicas of authors expreand herein do not necenarily state or reflect those of the United States Government or any abeacy thereof.
\end{abstract}

\section{LAWRENCE LIVERMORE NATIONAL LABORATORY University of California - Livermore, California - 94550}

Available from: National Technical information Service - U.S. Department of Commerre 5285 Port Royal Road - Springfield, VA

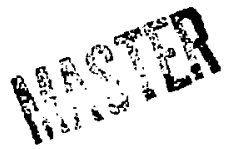




\section{Dedication}

To my wife Viola, whose understanding and support made this work possible, and to the memory of Dr. Abraham Goldberg, Graduate Adviser, whose personal interest and encouragement were essential to the completion of this dissertation. 


\section{Contents}

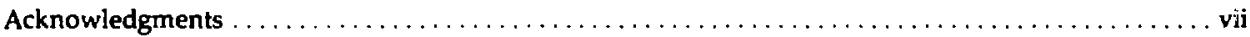

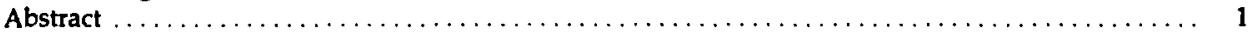

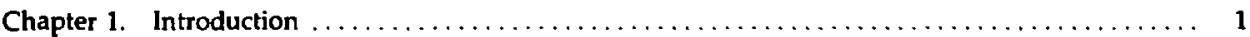

Overview of the Magnetosphere $\ldots \ldots \ldots \ldots \ldots \ldots \ldots \ldots \ldots \ldots \ldots \ldots \ldots \ldots \ldots \ldots \ldots \ldots$

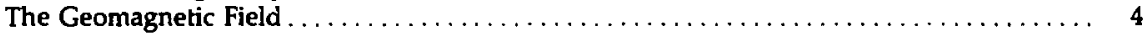

Magnetic Activity . . . . . . . . . . . . . .

Energetic Particles in the Magnetosphere $\ldots \ldots \ldots \ldots \ldots \ldots \ldots \ldots \ldots \ldots \ldots \ldots \ldots, 9$

Particle Flux Measurements in the Magnetosphere $\ldots \ldots \ldots \ldots \ldots \ldots \ldots \ldots \ldots \ldots \ldots$

Adiabatic Invariants of the Motion $\ldots \ldots \ldots \ldots \ldots \ldots \ldots \ldots \ldots \ldots \ldots \ldots \ldots \ldots \ldots$

Phase-Space Density and the Directional Flux $\ldots \ldots \ldots \ldots \ldots \ldots \ldots \ldots \ldots \ldots \ldots \ldots$

Organizing Directional Fluxes on Drift Shells $\ldots \ldots \ldots \ldots \ldots \ldots \ldots \ldots \ldots \ldots \ldots \ldots, 18$

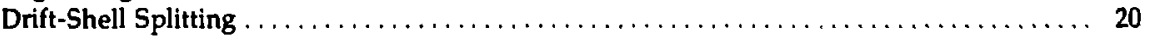

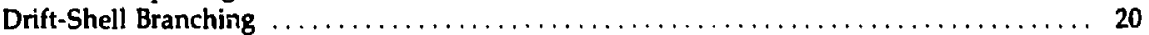

Energetic Particles as Tracers of Magnetic Field Configuration $\ldots \ldots \ldots \ldots \ldots \ldots \ldots \ldots, 21$

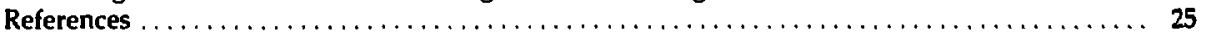

Chapter 2. The OGO-5 Spacecraft and the LLNL Electron and Proton Spectrometer $\ldots \ldots \ldots \ldots 28$

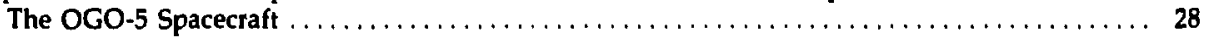

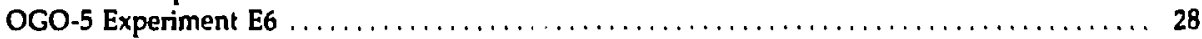

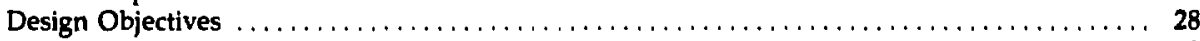

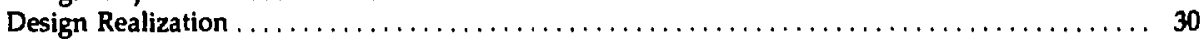

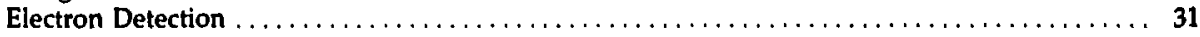

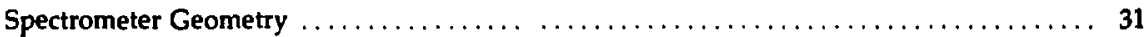

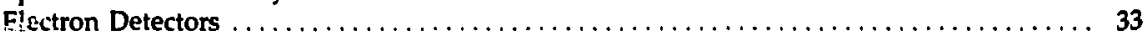

Calibration, Backgrounds, and Stability $\ldots \ldots \ldots \ldots \ldots \ldots \ldots \ldots \ldots \ldots \ldots \ldots \ldots \ldots \ldots \ldots \ldots$

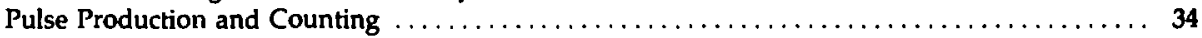

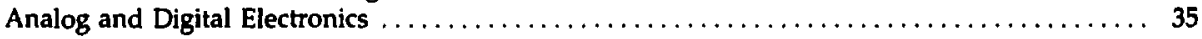

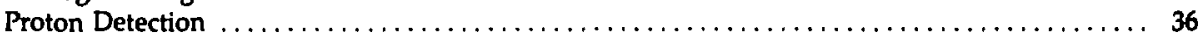

Proton Detector Calibration and Backgrounds $\ldots \ldots \ldots \ldots \ldots \ldots \ldots \ldots \ldots \ldots \ldots \ldots \ldots \ldots$

Proton Detector Electronics $\ldots \ldots \ldots \ldots \ldots \ldots \ldots \ldots \ldots \ldots \ldots \ldots \ldots \ldots \ldots \ldots \ldots \ldots \ldots \ldots$

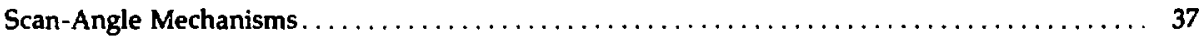

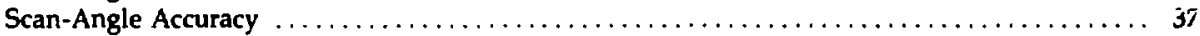

Magnetic Field Measurement $\ldots \ldots \ldots \ldots \ldots \ldots \ldots \ldots \ldots \ldots \ldots \ldots \ldots \ldots \ldots \ldots \ldots \ldots, 38$

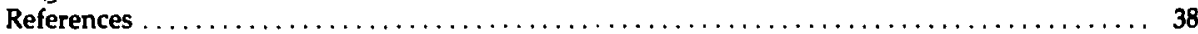

Chapter 3. Theory of Charged Particle Motion in a Magnetic Field $\ldots \ldots \ldots \ldots \ldots \ldots \ldots \ldots \ldots \ldots 39$

Equations of Motion of a Charged Particle in Static Electric and Magnetic Fields .......... 39

Static Magnetic Field $\ldots \ldots \ldots \ldots \ldots \ldots \ldots \ldots \ldots \ldots \ldots \ldots \ldots \ldots \ldots \ldots \ldots \ldots \ldots, 40$

Homogeneous Magnetic Field $\ldots \ldots \ldots \ldots \ldots \ldots \ldots \ldots \ldots \ldots \ldots \ldots \ldots \ldots \ldots \ldots, 40$

Particle Mation in General Fields $\ldots \ldots \ldots \ldots \ldots \ldots \ldots \ldots \ldots \ldots \ldots \ldots \ldots \ldots \ldots, 41$

Constant Electric and Magnetic Fields $\ldots \ldots \ldots \ldots \ldots \ldots \ldots \ldots \ldots \ldots \ldots \ldots \ldots \ldots \ldots \ldots \ldots$

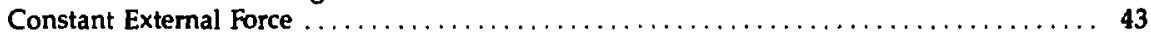

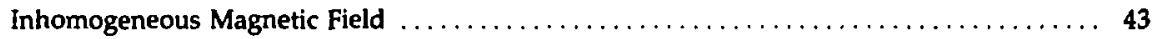

The Guiding-Center Approximation $\ldots \ldots \ldots \ldots \ldots \ldots \ldots \ldots \ldots \ldots \ldots \ldots \ldots \ldots \ldots \ldots \ldots$

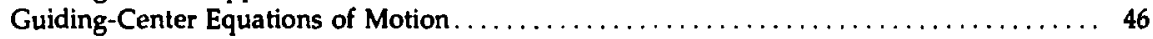

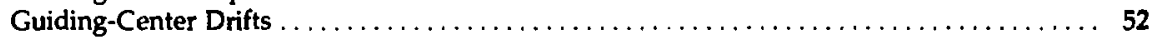

Hamiltonian Dynamics of Charged Particle Motion in a Magnetic Field $\ldots \ldots \ldots \ldots \ldots \ldots \ldots, 55$

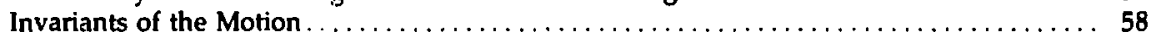

Adiabatic Invariants of Charged Particle Motion in a Magnetic Field $\ldots \ldots \ldots \ldots \ldots \ldots 67$

References $\ldots \ldots \ldots \ldots \ldots \ldots \ldots \ldots \ldots \ldots \ldots \ldots \ldots \ldots \ldots \ldots \ldots \ldots \ldots \ldots \ldots \ldots \ldots$

Chapter 4. Energetic Electron Trajectories in the Outer Dayside Magnetosphere $\ldots \ldots \ldots \ldots \ldots \ldots$

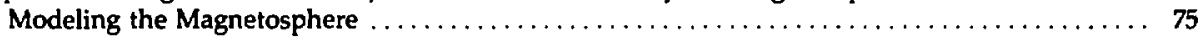

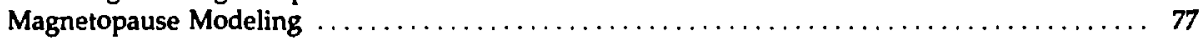

Self-Consistent Calculation of the Magnetopause $\ldots \ldots \ldots \ldots \ldots \ldots \ldots \ldots \ldots \ldots \ldots \ldots \ldots$ 
The Choe-Beard Model as a Representative Magnetospheric Magnetic Field Model........ 90

Definition of the Nominal Choe-Beard Field $\ldots \ldots \ldots \ldots \ldots \ldots \ldots \ldots \ldots \ldots \ldots \ldots, 90$

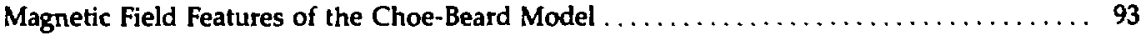

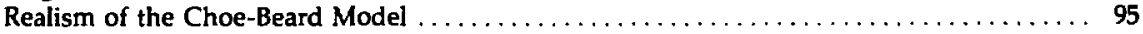

Particle Drift Shells in the Outer Magnetosphere $\ldots \ldots \ldots \ldots \ldots \ldots \ldots \ldots \ldots \ldots \ldots \ldots, 97$

Drift-Shell Branching $\ldots \ldots \ldots \ldots \ldots \ldots \ldots \ldots \ldots \ldots \ldots \ldots \ldots \ldots \ldots \ldots \ldots$

Effects of Shell Branching on Adiabatic Invariants $B_{M}$ and $I \ldots \ldots \ldots \ldots \ldots \ldots \ldots \ldots$

Trajectory Calculations ................................. 103

Effects of Shell Branching on the Directional Flux $\ldots \ldots \ldots \ldots \ldots \ldots \ldots \ldots \ldots \ldots \ldots \ldots$

Drift Shell Tracing in the Outer Magnetosphere; the Choe-Beard Model . . . . . . . . . . . 107

Mapping Drift Shells by Means of Adiabatic Invariants $\ldots \ldots \ldots \ldots \ldots \ldots \ldots \ldots \ldots \ldots$

The Drift Loss-Cone and Regions of Stable and Pseudo-trapping $\ldots \ldots \ldots \ldots \ldots \ldots \ldots \ldots$

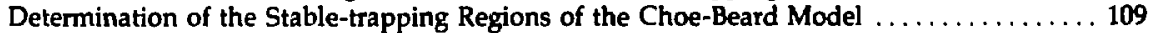

Effects of Shell Branching vs Shell Splitting on PADs $\ldots \ldots \ldots \ldots \ldots \ldots \ldots \ldots \ldots \ldots, 122$

Energetic Electron Fluxes in the Oi iter Dayside Magnetosphere:

Experimental Measurements and Adiabatic Theory Interpretation $\ldots \ldots \ldots \ldots \ldots \ldots \ldots \ldots 128$

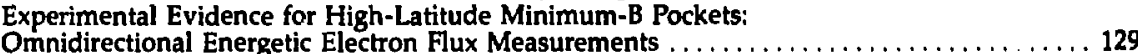

Experimental Evidence for High-Latitude Minimum-B Pockets:

Directional Flux Measurements of Energetic Electrons $\ldots \ldots \ldots \ldots \ldots \ldots \ldots \ldots \ldots \ldots, 130$

OGO-5 Observations on Day 66 (March 6), $1968 \ldots \ldots \ldots \ldots \ldots \ldots \ldots \ldots \ldots \ldots \ldots \ldots$

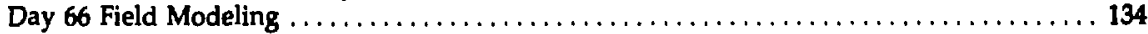

Day 66 Synthetic Pitch Angle Distributions $\ldots \ldots \ldots \ldots \ldots \ldots \ldots \ldots \ldots \ldots \ldots \ldots \ldots \ldots \ldots$

Day 66 Energetic Electron Pitch Angle Distributions $\ldots \ldots \ldots \ldots \ldots \ldots \ldots \ldots \ldots \ldots \ldots$

OGO-5 Observations on Day 41 (February 10), $1969 \ldots \ldots \ldots \ldots \ldots \ldots \ldots \ldots \ldots \ldots$

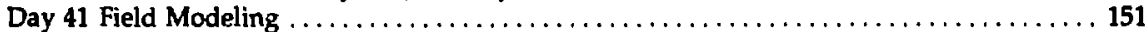

Day 41 Energetic Electron Pitch Angle Distributions $\ldots \ldots \ldots \ldots \ldots \ldots \ldots \ldots \ldots \ldots \ldots$

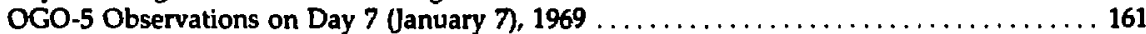

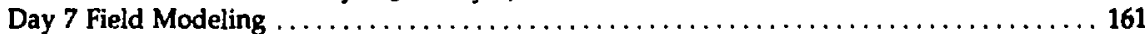

Day 7 Energetic Electron Pitch Angle Distributions $\ldots \ldots \ldots \ldots \ldots \ldots \ldots \ldots \ldots \ldots \ldots$

OGO-5 Observations on Day 5 (January 5), $1969 \ldots \ldots \ldots \ldots \ldots \ldots \ldots \ldots \ldots \ldots \ldots$

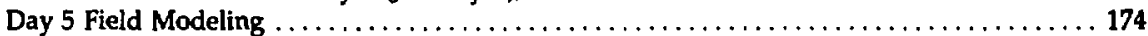

Day 5 Energetic Electron Pitch Angle Distributions $\ldots \ldots \ldots \ldots \ldots \ldots \ldots \ldots \ldots \ldots \ldots \ldots$

OGO-5 Observations on Day 10 (January 10), $1969 \ldots \ldots \ldots \ldots \ldots \ldots \ldots \ldots \ldots \ldots$

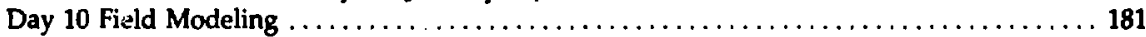

Day 10 Energetic Electron Pitch Angle Distributions $\ldots \ldots \ldots \ldots \ldots \ldots \ldots \ldots \ldots \ldots$

Discussion of Experimental Results $\ldots \ldots \ldots \ldots \ldots \ldots \ldots \ldots \ldots \ldots \ldots \ldots \ldots \ldots \ldots$

Shell-branching Studies Using Other Magnetic Field Models $\ldots \ldots \ldots \ldots \ldots \ldots \ldots \ldots \ldots$

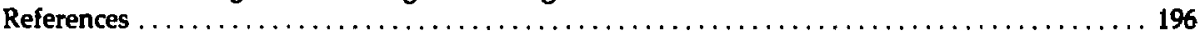

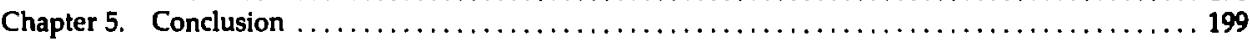

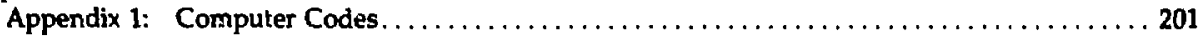

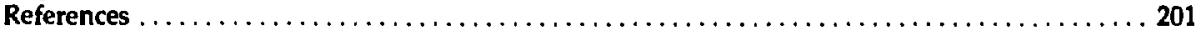




\section{Acknowledgments}

I would like to thank Dr. Har-y I. West, Jr., Principal Investigator for the E6 experiment on the OGO-5 satellite. for providing the energetic particle data which formed the basis of my research. I am truly indebted to him for introducing me to space sciences and allowing me the opportunity to learn magnetospheric physics through participation in his research studies. I have greatly benefited from his keen insights into the physical processes underlying the data and his willingness to patiently explain them in numerous amiable and stimulating discussions. I am especially grateful for his contagious scientific enthusiasm, and for his personal interest and persistent encouragement that were so instrumental in motivating my studies. I will always greatly value our association and friendship.

I thank Drs. P. J. Coleman and C. T. Russell of UCLA for providing the fluxgate magnetometer data used in this work. I thank Dr. J. R. Walton of LLNL for providing the computer codes which produced the experimental data plots. I thank my chairman Dr. John Killeen for his encouragement of my candidacy and for his efforts to help steer me around the various obstacles encountered along the road to graduation.

I am especially grateful for the substantial Educational Aid support provided by the Laboratory, which truly made this work possible. 


\title{
Energetic Particle Drift \\ Motions in the Outer \\ Dayside Magnetosphere
}

\begin{abstract}
Models of the geomagnetic field predict that within a distance of approximately one earth radius inside the dayside magnetopause, magnetic fields produced by the ChapmanFerraro magnetopause currents create high-latitude minimum-B "pockets" in the geomagnetic field. These pockets are theoretically capable of temporarily trapping azimuthallydrifting electrons and modifying electron directional distributions. The Lawrence Livermore National Laboratory's scanning electron spectrometer aboard the OGO-5 satellite provided detailed energetic ( $E>70 \mathrm{keV}$ ) electron pitch angle distributions throughout the magnetosphere. Distributions obtained in the outer dayside magnetosphere over a wide range of longitudes show unusual flux features. In this dissertation, we analyze drift-shell branching caused by the minimum-B pockets, and interpret the observed flux features in terms of an adiabatic shell branching and rejoining process.

'Yie examine $i_{1}$ ? shell-branching process for a static field in detail, using the Choe-Beard 1974 magnetospheric magnetic field model. We find that shell branching and rejoining conserves the particle mirror field $B_{M}$, the fieldline integral invariant $I$, and the directional electron flux $\mathrm{j}$. We determine the spatial extent of the stable trapping regions for the ChoeBeard model. We develop an adiabatic branching map methodology which completely identifies and describes the location of shell-branching points and the adiabatic trajectories of particles on branched shells, for any model field. We employ the map to develop synthetic pitch angle distributions near the dayside magnetopause by adiabatically transforming observed midnight distributions to the dayside. We find that outer dayside lines contain particles moving on branched and unbranched shells, giving rise to distinctive pitch angle distribution features.

We find a good correlation between the pitch angles which mark the transition from branched to unbranched shells in the model, and the distinctive features of the OGO-5 distributions. In the morning sector, we observe large flux changes at critical pitch angles which correspond to B-pocket edges in the model. Measurements on inbound passes in the afternoon sector show first the adiabatic particle shadow, then the arrival of fluxes on rejoined shells, then fluxes on unbranched shells-in accord with model predictions. The B-pockets have interesting implications for the entry and transport of solar-wind particles within the magnetosphere.
\end{abstract}

\section{Chapter 1. Introduction}

The science of magnetospheric physics was born when the first instrumented satellites were launched in the late 1950's, beginning the so-called "Sputnik era" of space exploration. An early discovery of major proportions was the detection of large fluxes of energetic particles circulating in broad belts about the earth. In March of 1958, the Explorer III Geiger counter experiment designed by the Van Allen group at Jowa returned data which showed an unusual effect. As the spacecraft neared apogee, the Geiger counting 
rate dropped abruptly to nearly zero. After some analysis, it dawned on the investigators that the countingrate dropout could be caused by the passage of the satellite through a region of intense corpuscular radiation which saturated the counter, increasing counter dead-time and causing the anomalously low readings. Owing to the $1 \mathrm{gm} / \mathrm{cm}^{2}$ of shielding material around the counters, only electrons of energy $E_{\mathrm{e}}>1.6 \mathrm{MeV}$ and protons of energy $E_{\mathrm{p}}>30 \mathrm{MeV}$ could penetrate and be counted. This radiation belt discovery, termed by Van Allen "the most interesting and least expected result" of early space researches, was confirmed by the space probes which followed. These counting experiments in fact revealed the presence of two concentric belts of energetic particles surrounding the earth (Fig. 1-1).

Subsequent satellite-borne experiments sensitive to a wider energy spectrum established that energetic electrons and protons were distributed throughout near-earth space, extending out to the limits of the earth's field. Magnetic field rieasurements by Cahill and Amazeen on Explorer XII in 1961 first sensed the magnetopause or boundary which separates the terrestrial field from the plasma and fields of interplanetary space. Created by the action of the flowing solar wind plasma on the earth's field, this boundary completely encloses the earth, forming the volume of the magnetosphere (Fig. 1-2). This is the province of magnetospheric physics, a major section of which is devc sed to the study of the magnetosphere's charged particles: their sources, sinks, and distributions, and the mechanisms of particle energization and transport.

The motion of a charged particle in a magnetic field is governed by the Lorentz force, which is written in rationalized MKS units as:

$\mathbf{F}=\mathbf{q} \mathbf{v} \times \mathbf{B}$,

where

$\mathrm{q}$ is the particle charge (coulombs),

$v$ is particle velocity (meters/second),

$B$ the magnetic induction (teslas).

This force acts on a radiation belt particle to produce a trajectory which consists of a spiralling motion along the field lines, coupled with a slow azimuthal drift about the earth (Fig. 1-3). The earth's basically dipolar field forms a gigantic natural "mirror machine" which arrests the particle's poleward motion and reflects it equatorward, while conserving particle energy. The radial gradients in the field arid the curvature of the field lines combine to generate the azimuthal drift. The evolution of the orbit produces a drift shell about the earth over which the particle perpetually moves (in the limit of no scattering). In the inner

Figure 1-1. Inner and outer radiation belts as determined by Geiger counter measurements on Explorer IV and Pioneer III. Contours are counting rates of protons $>30 \mathrm{MeV}$ and electrons $>$ 1.6 MeV. Satellite trajectories are shown (Van Allen and Frank, 1959a,b).

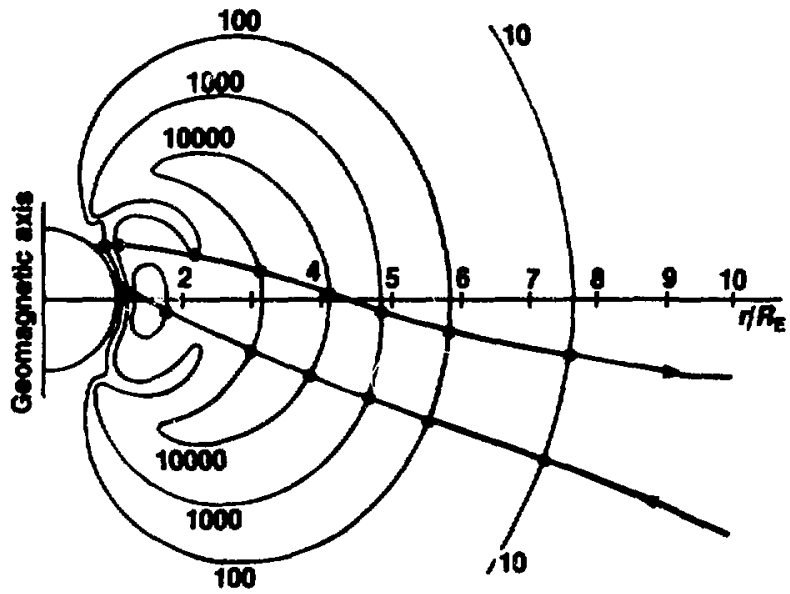



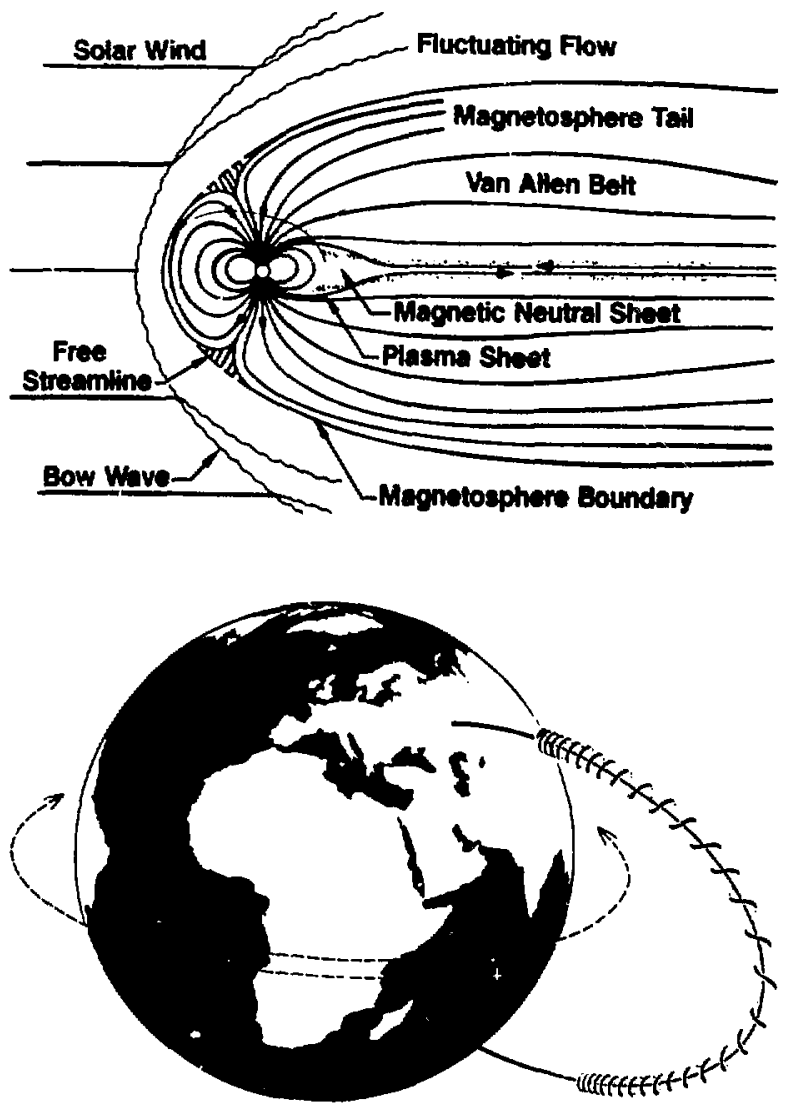

Figure 1-2. Noon-midnight crosssection of the magnetosphere and bow shock. Nominal geocentric distance of the magnetospheric sub-solar point is $\approx 10 \mathrm{R}_{\mathrm{E}}$ (earth radii) (Wolfe and Intriligator, 1970).

Figure 1-3. Motion of a charged particle trapped in the geomagnetic field. The motion has three components: a rapid nearly-cir. cular motion in a plane perpendicular to the local magnetic field; a slower bounce motion along the guiding field line between reflection (mirror) points in the northern and southern hemispheres; and an even olower drift in azimuth about the earth. Electrons (negative) and protons (positive) drift in opponite directions. (Van Allen, 1959)

magnetosphere the field is nearly difolar and azimuthally symmetric, and has very little time-variation. The particle distributions in this stable core show no significant azimuthal dependence.

Very different conditions prevail in the outer regions of the magnetosphere near the magnetopause, the surface that bounds the earth's field. Field lines are highly distorted from the dipolar configuration by the large electrical currents that flow on the magnetopausn. and by the distributed currents that flow in the magrietosnheric plasma. These magnetic field distortions perturb the distributions of energetic particles that drift azimuthally through the outer magnetosphere, producing characteristic distribution features. By comparing distributions measured at various azimuths, one can identify the integral effect of the perturbations encolintered along the drift path, which can in turn be analyzed in terms of field distortions.

In this study we foc'ss on the characteristics of energetic electron distributions in the outer dayside magnetosphere, analyzing viata measured by the Lawrence Livermore National Laboratory's spectrometer on the OGO-5 satellite. We find features which indicate that particles near the magnetopause depart from their normal equator-crossing bounce trajectories anc become temporarily trapped in lonalized highlatitude minimum-B pockets near the noon magnetopause. These unusual trajectories may offer a route for the injection of solar-wind particles into the trapping regions of the magnetosphere.

We begin with a brief exposition of magnetospheric physics and phenomena, with an emphasis on energetic particles and magnetic fields, in order to provide an appropriate context for the subsequent studies. In Chapter 2 we review the OGO-5 E6 spectrometer experiment which provided most of our experimental data. Chapter 3 treats the theory of charged particle motion in magnetic fields. We review 
guiding center theory, and develop the highly-useful adiabatic invariants of particle motion via classical Hamilton-Jacobi theory. In Chapter 4 we treat in more detail the use of energetic particles to trace magnetic fieldline topology and reveal field distortions. We examine the effects on particle distributions of drift-shell splitting and branching induced by field asymmetries. We describe various standard models of the geomagnetic field, and evaluate their capabilities to accurately represent the outer field regions. Using the best of these models, the Choe-Beard 1974 field model, we compute theoretical trajectories and establish the limits of stable trapping. The model predicts that particle populations on outer magnetospheric field lines should contain distinct classes of particles that follow very different drift trajectories, giving rise to distinctive pitch angle distribution features. We compare these predictions to detailed observations in the outer dayside magnetosphere. We discuss our findings and their implirations for particle entry and transport.

\section{Overview of the Magnetosphere}

During nearly three decades of ongoing space exploration, the full complexity of particles and fields in the magnetosphere has become evident. Nearly all phenomena of interest in this domain have a single fundamental source - the interaction of the continuously-flowing hot magnetized solar-wind plasma with the nearly dipolar magnetic field of the earth. Yet this seemingly simple interaction (which has been very successfully modeled from a magnetopause shape standpoint by considering the magnetosphere to be a blunt obstacle in the flow of a supersonic gas) is respunsible for a rich diversity of effects which are even today incompletely understood.

\section{The Geomagnetic Field}

A fundamental aspect of solar-terrestrial relations is the confinement of the earth's dipolar magnetic field to a finite volume - the magnetosphere-by the action of the flowing solar wind (see, for example, Fig. 1-2). Many of the most interesting problems in magnetospheric physics-for example, whether magnetic flux tubes of solar origin can connect to field lires of earth origin, and how solar wind particles gain access to the trapping regions of the magnetosphere--depend on the mechanism of the solar windmagnetosphere interaction.

Prior to the advent of space probes in the 1950's, the earth's magnetic field was imagined to extend indefinitely into space, weakening "uith distance (according to the dipole formula) as $1 / \mathrm{r}^{3}$. The sun was not thought to be a source of a continuous wind of ionized subatomic particles but rather an intermittent emitter of plasma via solar flares. The magnetic "storms"- world-wide large-scale variations in the trieasured field-which followed certain types of flares were studied in the 1930's by Chapman and Ferraro (Chapman and Ferraro, 1931; 1932; 1940). They proposed that a flare-emitted cloud of neutral plasma, arriving at earth a day or two later, would compress the earth's field and give rise to the abrupt field increase (sudden commencement) associated with storm onset. In this theory the earth's field reflects the solar charged particles but in doing so is compressed by the particle pressure produced by the momentum change; the interface between solar plasma and geomagnetic field moves inward until the particle pressure inwards is balanced by the outward magnetic pressure (Fig. 1-4). The iolar electrons and protons impinging on the field are turned in opposite directions, setting up a boundary layer of about one proton gyroradius in thickness in which electrical currents flow. These Chapman-Ferraro currents produce a magnetic field which cancels the earth's field exterior to the boundary, and approximately doubles the earth's field just inside the boundary.

This theory not only presented the correct explanation for the sudden commencement of magnetic storms, but also provided the key elements needed to determine the general features of the magnetospheric boundary shape and its location. Figure 1-5 depicts the magnetic induction measured along the earth-sun line vs distance from earth in $R_{E}\left(1 R_{E}=\right.$ nominal earth radius $=6370 \mathrm{~km}$.). As the ChapmanFerraro theory predicts, the measured field just inside the magnetopause at $10 R_{E}$ is roughly iwice that of the field due to the earth's dipole moment.

We now know that the solar wind is a permanent feature of the interplanetary environment [as proposed by Johnson (1960)]. Observations of the tails of comets (Biermann 1951) showed that a corpuscular radiation emitted from the sun was responsible for the gaseous, anti-solar-directed cometary tail. Since certain types of comets always produced these tails irrespective of position around the sun, he 


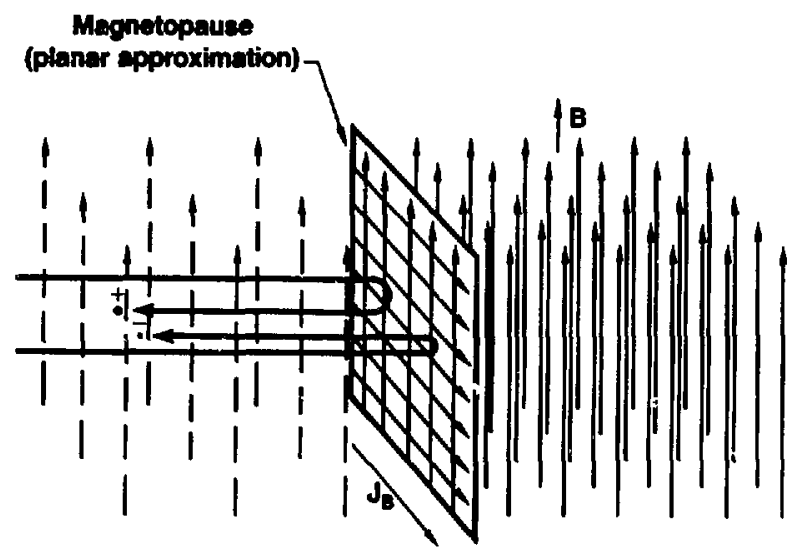

Figure 1-4. The Chapman-Ferraro theory of magnetic storms proposed that a plasms sioud ejected from the sun compresses the earth's magnetic field untll the cloud's forward momentum is arreated by the magnetic pressure. The incident solar nrotons and electrons are turned in opposile directions and reflected by the field at the boundary, eetting up the boundary currents $\mathrm{J}_{\mathrm{s}}$. The currents in turn give rise to a field which reinforces the earth't field juat earthward of the boundary (producing a net field approximately twice the earth's field), and cancelo the earth's field in the interplanetary region. (Cahill, 1965)

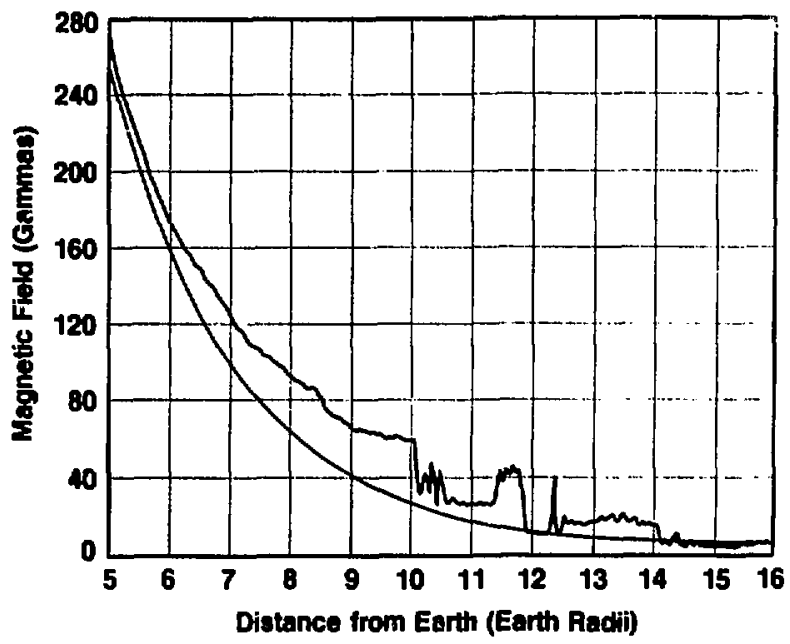

Figure 1-5. The jagged trace is the total magnetospheric magnetic field strength measured by a spacecraft magnetometer in the noon meridian, along the earth-sun line. The smooth trace is the field due to the earth's internal dipole. These fields are nearly identical within about $5 \mathbf{R}_{\mathrm{E}}$ of the earth. Beyond $5 \mathbf{R}_{E}$ the contributions from the magnetopause currents begin to have a significant effect on total field magnitude. Just inside the magnetopause (at about $10 R_{E}$ for this pass), the total field strength is nearly twice the dipole contribution. Between 10 and $15 R_{E}$ the irregular, fluctuating field downstream of the bow shock is seen. A very weak interplanetary field is observed beyond the shock (Cahill, 1965). 
concluded that a radially directed particulate wind must always be flowing outwards from the sun The solar wind prope:ties were subsequently measured by satellites, first by the Lunik II and III spacecrafts (Gritıgauz et al., 1960), then by Mariner 2 (Neugebauer and Snyder, 1962) and Explorer 10 (Bonetti et al., 1963) and Explorer 12 (Freeman et al., 1963). Typically the quiet-time solar wind at the earth's orbit is composed mainly of hydrogen ions with a few percent helium, with a density of 3-10 ion pairs $/ \mathrm{cm}^{3}$, a flow velocity of $300-400 \mathrm{~km} / \mathrm{s}$, and an ion temperature of $7 \mathrm{eV}$ (Anderson, 1966). During disturbed times the density can increase to $20-40$ ion pairs $/ \mathrm{cm}^{3}$, the flow velocity to $800 \mathrm{~km} / \mathrm{s}$, and the temperature to $25 \mathrm{eV}$. Embedded in the plasma and carried with it are filaments of the solar magnetic field with an induction of 5-30 gamma (one gamma $=10^{-9}$ tesla). Because of the highly-conductive nature of the plasma, the field is considered to be "frozen-in" to the plasma. The energy density of the magnetic field $\left(\mathrm{B}^{2} / 2 \mu_{0}\right)$ is comparable to the thermal energy density $(3 / 2 \mathrm{nkT})$ but a thousand times less than the energy density associated with flow; consequently the flow dynamics is dominated by the solar wind bulk flow momentum and the frozen-in field is transported by the particles.

Figure 1-6 presents a schematic view of the magnelosphere in the plane containing the sun and the geomagnetic dipole axis. The presence of the magnetic field in the solar wind endows it with fluid properties (Piddington, 1969). At the earth's orbit, the supersonic (Mach number 3-5) solar wind encounters the obstacle of the earth's field, and a bow shock is formed. The plasma flowing through the shock is slowed to subsonic speeds and heated. In the magnetosheath region between the shock and the magnetosphere the plasma expands around the flanks and becomes supersonic again. A boundary or magnetopause separates the solar wind particles and fields from the interior of the magnetosphere where all field lines have terrestrial origins. The magnetopause in the dipole equator is typically at a geocentric distance of 10-12 $\mathrm{R}_{\mathrm{E}}$, althougr. during periods of peak solar wind pressure the magnetopause has been detected at 6-7 $\mathrm{R}_{\mathrm{E}}$. High-latitude field lines on the dayside are pulled back by the flowing plasma fo form an elongated tail in the anti-solar direction, which by satellite measurement (Pioneer 7 ) extends to at least $1000 R_{E}$ (Behannon and Ness, 1968). The sunward surface of the magnetopause is roughly a hemisphere of radius $15 R_{E}$ (Voigt, 1972) joined to a flaring cylindrical tail on the nightside of radius $20-30 R_{E}$. Beyond about $10 R_{E}$ in the midnight meridian the magnetic field becomes increasingly tail-like (Fig. 1-7), evolving into a roughly cylindrical configuration with solar-directed fields in the northern half of the cylinder, and antisolar-directed field in the southern half (Ness, 1965). A quasi-neutral sheet separates the nearly antiparallel fields at the cylinder midplane. It is embedded in a sheet of plasma ( $\approx 6 R_{E}$ thick at a distance of $17 R_{E}$ (Bame et al., 1966) which balances the magnetic pressure and stabilizes the tail structure (Fig. 1-8).

Figure 1-6. Noon-midnight crooesection of the magnetosphere, showing the major magnetic field features and particle populations (Rosenbauer et al., 1975). See text for explanation.

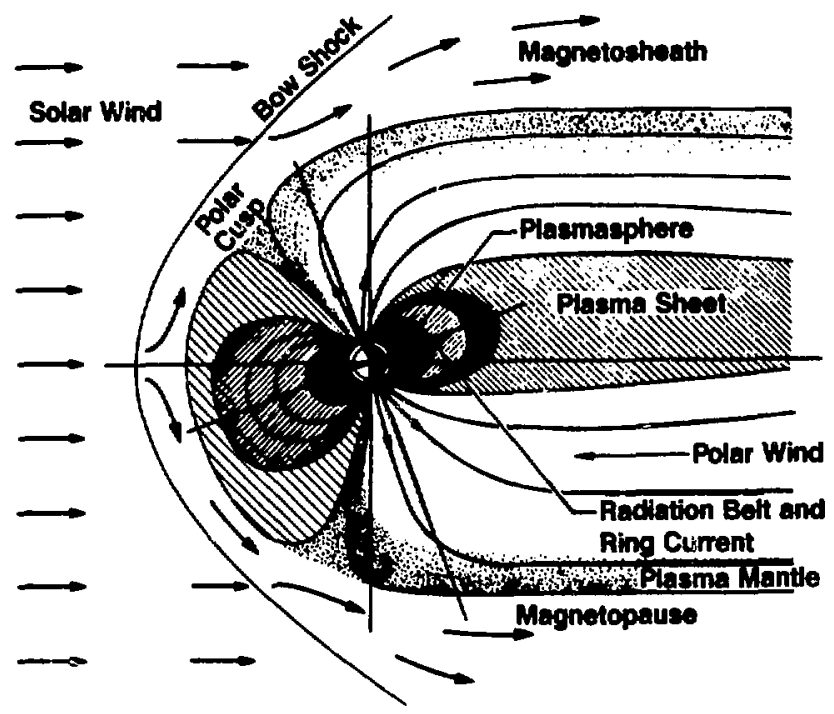




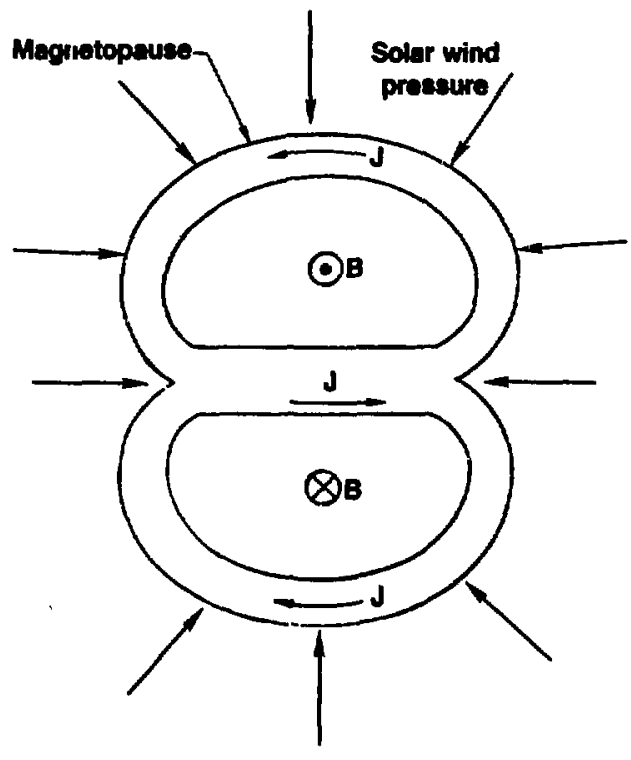

Figure 1-7. Schematic cross-section of the geomagnetic tail as viewed from earth. The elongated tail appears as a two-lobed bundle of flux tubes with a magnetic quasi-neutral sheet in the midplane. The tail field is sustained by a tail current system (U) comprising cross-tail currents flowing in the dawn-todusk direction near the neutral sheet, and magnetopause return currents which close the system (Axford et al., 1965).

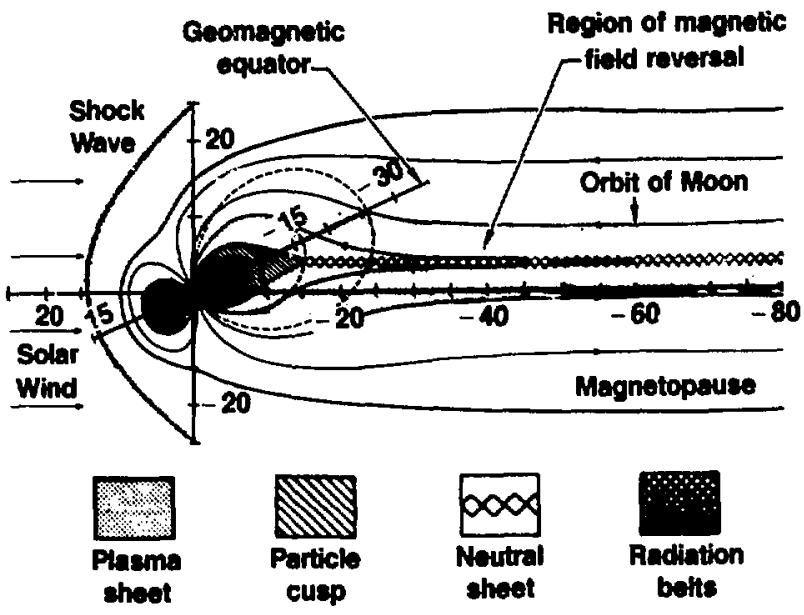

Figure 1-8. Noon-midnight crosssection of the magnetosphere, showing the tail structure (Ness, 1965).

The plasma sheet may be maintained by the influx of magnetosheath plasma through the weak-field regions of the polar cusp (Heikkila and Winningham, 1971), forming the plasma mantle just inside the tailward magnetopause (Rosenbauer et al., 1975). Under the influence of convection electric fields, the mantle plasma flows towards the distant tail plasma sheet (Mencke Hansen et al., 1976).

\section{Magnetic Activity}

The geomagnetic field is subject io constant transient variations, a fact noted as early as 1722 . There are two principal types of impulsive magnetic activity that influence the distributions of energetic particles 
in the magnetosphere: magnetic storms and polar magnetic substorms. Both have characteristic signatures in earth-station magnetograms.

The magnetic storm has a world-wide effect, often beginning with a sudden commencement (SC) seen as a rapid rise in the horizontal component of the field at equatorial stations. This is attributable to a sudden large increase in the solar wind pressure which drives the magnetopause inwards, compressing the field until a new equilibrium is achieved between external plasma pressure and internal magnetic pressure. A good example of the SC is shown in the magnetograms of Fig. 1-9. This event was initiated by a strong shock wave that pushed the magnetopause inwards from its nominal $10 R_{E}$ distance to a position within the orbit of satellite GEOS-1 $\left(\approx 7 R_{E}\right)$. The main phase of a storm, also seen in Fig. 1-9, is characterized by a large decrease in the horizontal component following the SC. This phase typically develops in a day and decays away over a period of several days. This signature is caused by the development of a diamagnetic ring current about the earth, at a distance of a few $R_{E}$. The ring current generates a field which opposes the earth's field in the earthward region, as first postulated by Singer (1957). The SC abruptly displaces particle drift shells in an irreversible manner, leading to radial transport of radiationbelt particles.

The polar substorm is a complex, localized event driven by the sudden conversion of magnetic energy stored in the magnetotail into plasma kinetic energy. The normally stretched-out lines of the tail an maintained by cross-tail currents fowing in the plasma sheet. Some initiating event causes the plasma sheet to thin down (as observed by Buck et al., 1973) until the nearly anti-parallel field lines north and south of the neutral sheet begin to merge. An X-type neutral line is formed (Fig. 1-10). The cross-tail current is disrupted, allowing the lines earthward of the neutral line to relax into a more dipolar configuration. The near-earth tail collapses earthward, generating large flows of energized plasma along the field lines. This process precipitates particles into the upper atmosphere in the polar regions, producing bright auroral displays. In addition, some fraction of the plasma sheet particles can be accelerated to hundreds of $\mathrm{keV}$ and injected into the radiation-belt regions (Young, 1983).

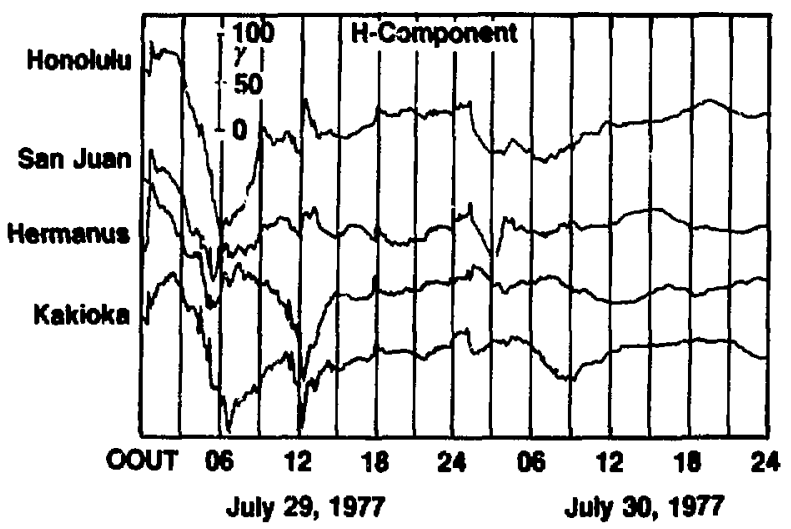

Figure 1-9. Horizontal-component magnetograms from near-equatorial stations during the magnetic storm of July 29, 1977 (Manka, 1979). The storm began with a strong shock wave in the solar wind which pushed the magnetopause inward from $\approx 10 R_{E}$ to $\approx 7 R_{E}$ producing the sudden commencement (SC) observed at 0027 UT. The H-component then developed a large negative decrease, reaching an extremum at about 0600 UT. This began the main phase of the storm, produced by the intensification of the diamagnetic ring current about the earth by injected and accelerated solarwind particles (principally protons). Recovery to pre-storm conditions takes several days. Such events significantly modify trapped particle populations by enhancing rates of injection, diffusion, and loss. 

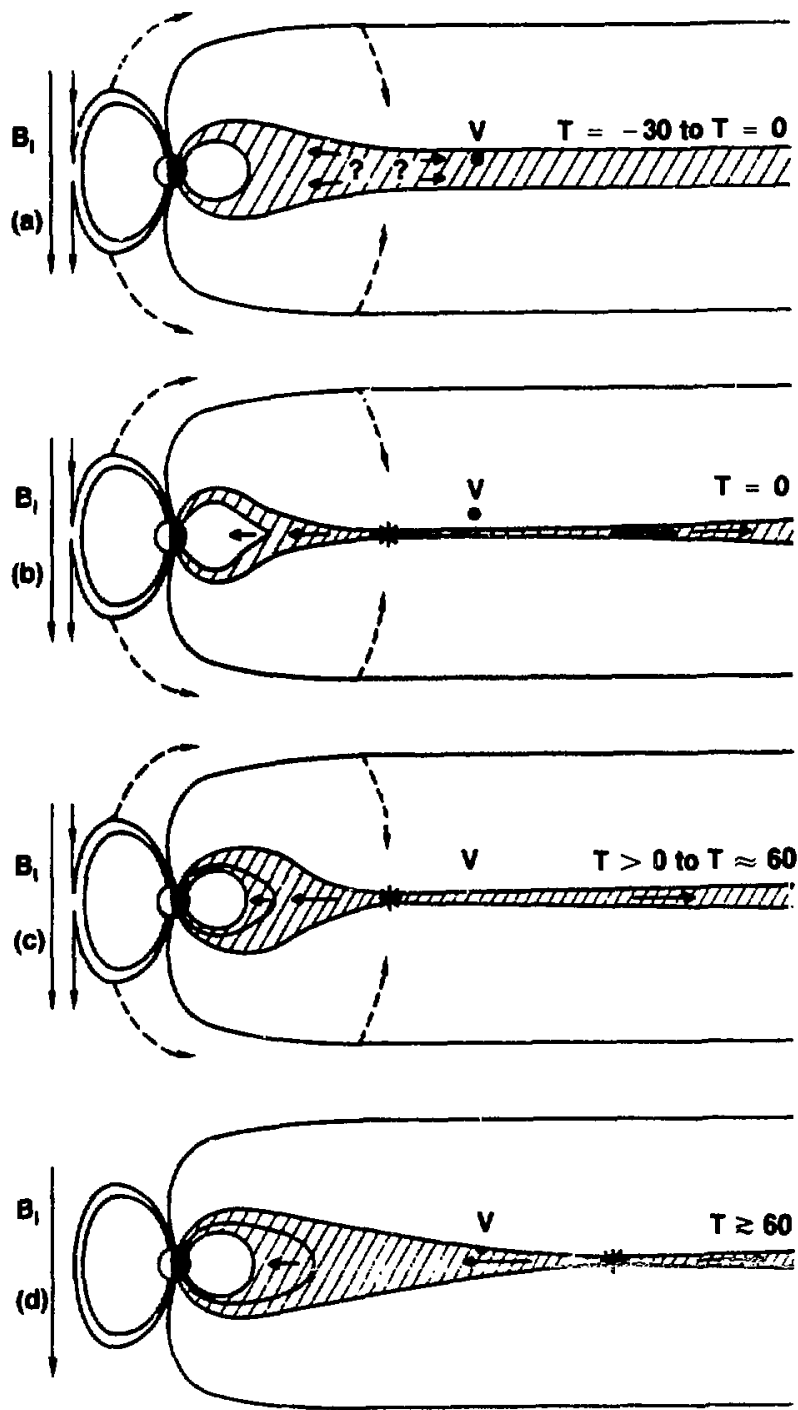

Figure 1-10. Sequence of magnetic substorm events in the tail. Prior to substorm onset the plasma sheet begins to thin down (a). A satellite (V) observes the drop-out of particles. At onset (b) an X-type neutral line is formed, transforming magnetic energy into plasma kinetic energy. Heated plasma flows earthward, leading to enhanced auroral precipitation and injection of particles into the radiation belt regions. With the disruption of the cross-tail current system, the field lines relax earthward into a more dipolar configuration (c). The neutral line moves tailward, and the satellite observes the expanding plasma (d) (Hones, 1973).

\section{Energetic Particles in the Magnetosphere}

The regime of energetic particles has historically been taken to include particles of $\mathrm{E}>45 \mathrm{keV}$, which could penetrate the shielding of a Geiger tube. The pioneering particle observations by Van Allen and Frank (1959a, 1959b) on Explorer IV and Pioneer III and IV discerned an inner and outer rarliation belt with about a $1 \mathrm{R}_{E}$ radial interbelt separation. The inner belt peak counting rate (at $1.5 R_{E}$ ) was later attributed to energetic protons $\left(E_{\mathrm{T}}>30 \mathrm{MeV}\right.$ ) while the peak rate in the outer belt (at $3.5 R_{E}$ ) was due to electrons of $E_{e}>1.6 \mathrm{MeV}$ (Poeverlein, 1972). As more refined instrumentation extended the detected spectrum, the two belts were seen to nearly merge into a single belt at low energies (Fig. 1-11) although the regions have distinctive particle spectra and flux dynamics. 


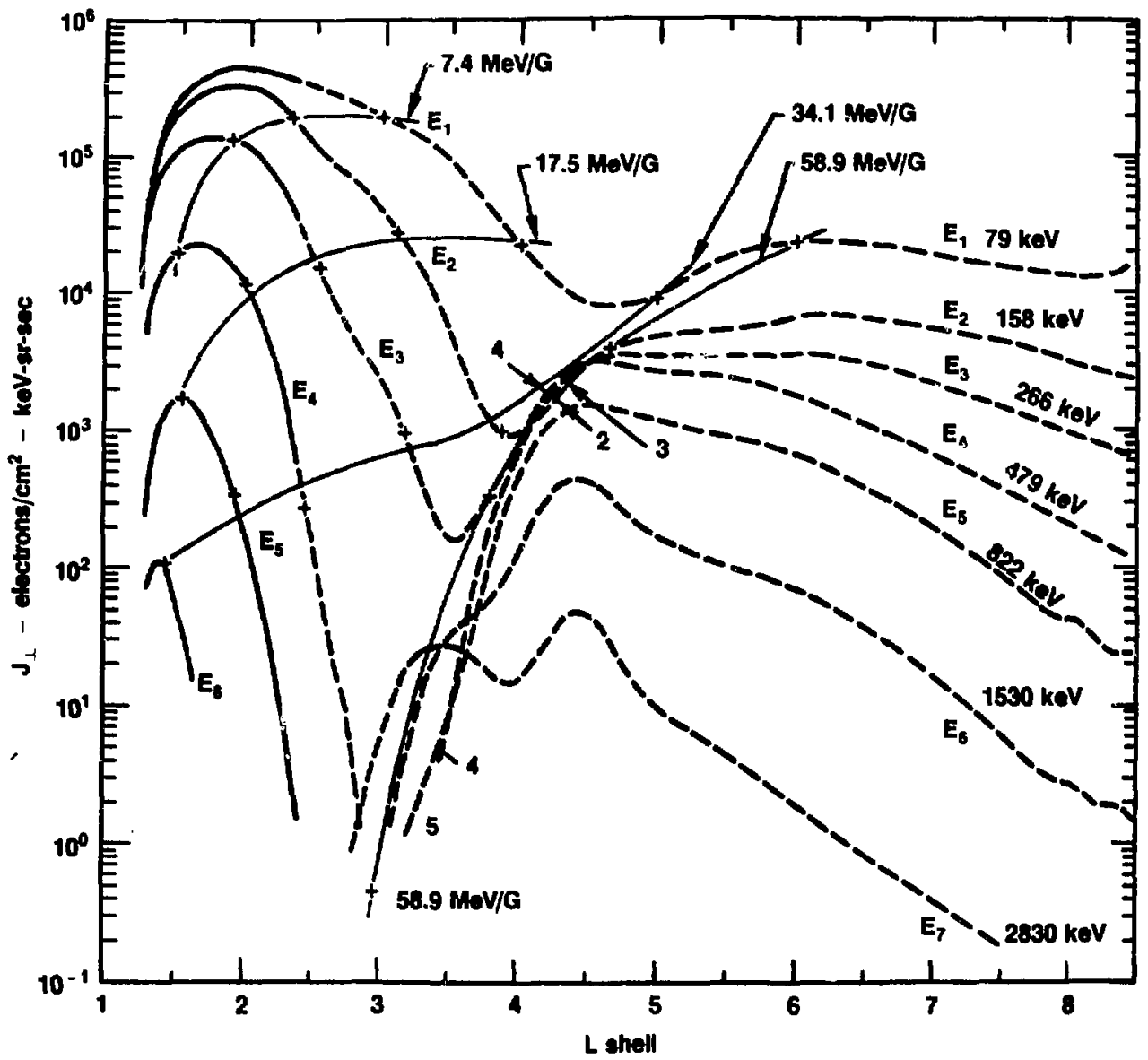

Figure 1-11. Energetic electrons in the equatorial regions of the inner and outer belts on Day 69, 1968 , for the seven spectrometer channels $\mathrm{E}_{1}-\mathrm{E}_{7}$. These OGO-5 observations show clearly the energy daFsenderice of the inter-beit "slứt" in the range $\bar{z} \leq \bar{L} \leq 5$ (west and Buck, 1976).

The shell on which a particle drifts is conveniently labeled by its $L$-value, which is computed as a function of the adiabatic invariants $\left(B_{M}\right.$ and I) that describe the particle's orbit. In a dipole field (axial symmetry), $L$ is equal to the equatorial distance $R_{E Q}$ of the shell. In the actual geomagnetic field the $R_{E O}$ of a shell is longitude dependent due to the presence of non-dipolar field components. The L-value calculation for a shell in the actual field serves to map that shell into a dipole field, where it has a single value of $R_{E Q} . L$ is conventionally measured in units of $R_{E}$. The organizational value of $L$ is that for shells in the inner magnetosphere $(L<\approx 5)$, particles initially mirroring on the same field line will drift on the same shell (i.e., have the same L-value).

The inner belt ( $L<\approx 2.5-4.0$ depending on energy) is populated by energetic protons with a spectrum which extends to several hundred $\mathrm{MeV}$ (Fig. 1-12). The fluxes are very stable with a lifetime of the order of a year (Freden and White, 1960). Losses of the very energetic component occur due to Coulomb drag (ionization loss) and nuclear collisions in the upper atmosphere; for energies below an 


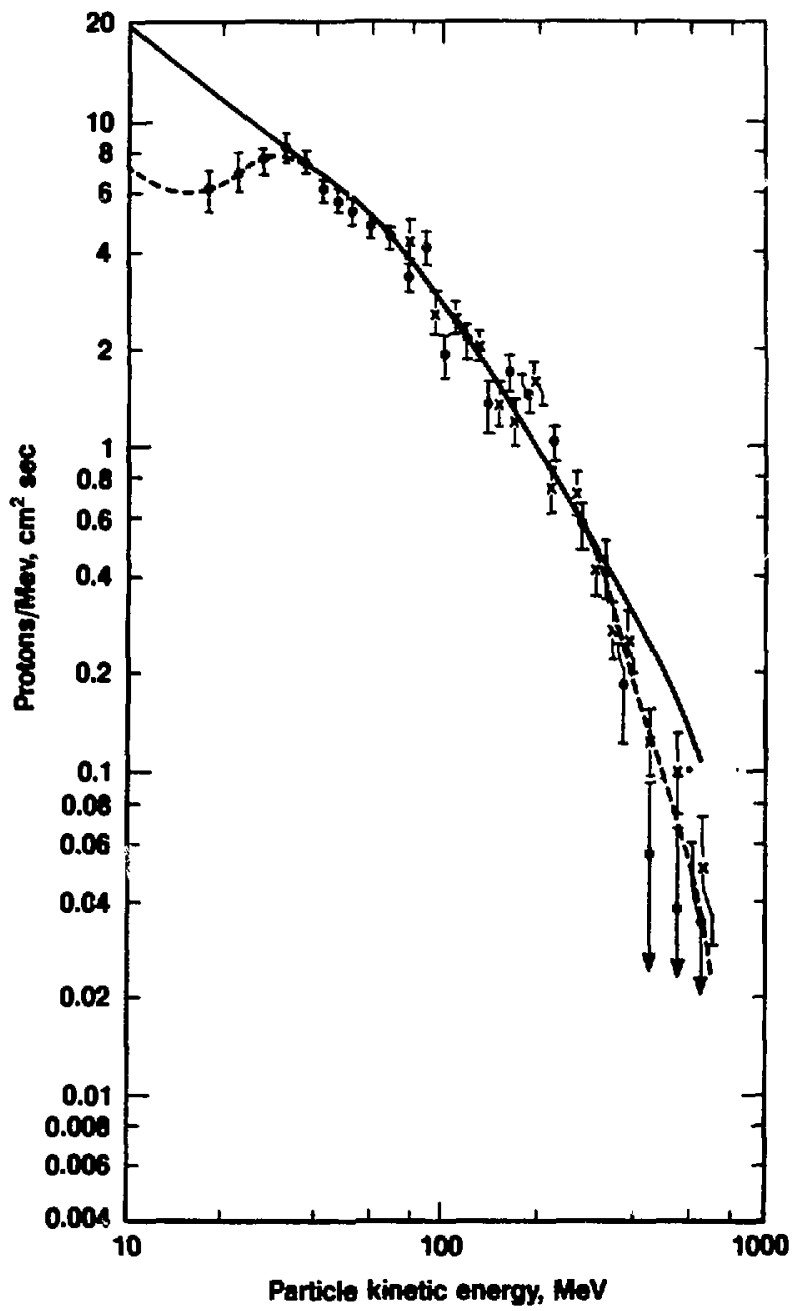

Figure 1-12. Energetic proton spectrum in the inner belt. Solid curve is the theoretical spectrum derived from the cosmic-ray neutron albedo theory (Freden and White, 1360).

Particlo kinotic energy, mov

$\mathrm{MeV}$ charge exchange with neutrals in the upper atmosphere is an effective loss mechanism. Cosmic ray albedo neutron decay (CRAND) was soon proposed as the major source of the energetic protons (Lingenfelter, 1963); (Hess and Killeen, 1966). As Fig. 1-12 shows, agreement of CRAND theory with observation was very good except for $E_{P}<30 \mathrm{Mev}$. These discrepancies at low energies and the differences between the observed spatial distribution of energetic protons and CRAND predictions were significantly reduced by the theoretical efforts of Farley and Walt (1971), who analyzed the belt structure in terms of diffusive transport.

The energetic electron component of the inner belt is also highly stable, with a lifetime of the order of a year (Pfitzer et al., 1966), (West and Buck, 1976). Only the largest of the major magnetic storms (which occur about once a year) can cause injection of electrons into the inner belt region. At other times inward diffusion of particles from the outer belt region appears to balance the belt losses. The upper atmosphere is a sink for energetic electrons, causing loss by Coulomb scattering near the mirror points. 
The outer belt (beyond $L \approx 4$ ) contains a much softer proton spectrum than the inner belt (Fig. 1-13), which is quite stable over time (Hess, 1968). By contrast, the energetic electrons in the region are highly variable and significantly affected by magnetic disturbances. A careful study of decay rates (West et al., 1979) following major injection episodes determined decay times that ranged from 1-50 days, depending on electron energy and L-shell (Fig. 1-14).

It has been established that outer-belt electrons are transported radially by the combined effects of pitch-angle scattering and magnetic field perturbations (Walt, 1971; Walt and Newkirk, 1971; Falthammer, 1972). Within $L=4$, the interaction of whistler-mode electromagnetic radiation with energetic electrons causes pitch-angle scattering. Theoretical calculations of these wave-particle interactions (Lyons et al., 1971; Lyons et al., 1972) have produced characteristic pitch angle distribution shapes that agree with OGO-5 observations of West et al., (1973a,b) (Fig. 1-15). The static flux profiles observed during quiet times are the result of an equilibrium between the effects of pitch-angle diffusion and radial diffusion (Schultz, 1974).

The sources of the energetic belt particles (aside from the CRAND source mentioned above) has been a long-standing question of magnetospheric physics. Diffusion analysis sheds some light on particle origins. The radial flow of particles is found to be proportional to a quantity which has a negative sign for distances less than about $L=10$, suggesting inward diffusion from a boundary at this distance (Schultz, 1982). In addition, a great deal of data indicates that particles from solar flares have rapid access to the magnetosphere's psuedo-trapping regions (Vampola, 1971; Scholer et al., 1974) and even well into the stable trapping (radiation belt) regions (Paulikas and Blake, 1979; Lanzerotti, 1971). Entry of these particles into the magnetosphere appears to occur in the distant $\left(R \approx 1000 R_{E}\right)$ tail (Lanzerotti, 1972). Measurements made on the HEOS 1 and 2 spacecraft indicated that magnetosheath plasma enters the magnetosphere near the dayside neutral points (Paschmann, et al., 1976; Fairfield, 1977, 1979), and is likely a source of plasma sheet particles. Some sheet particles can diffuse inwards and gain energy through both

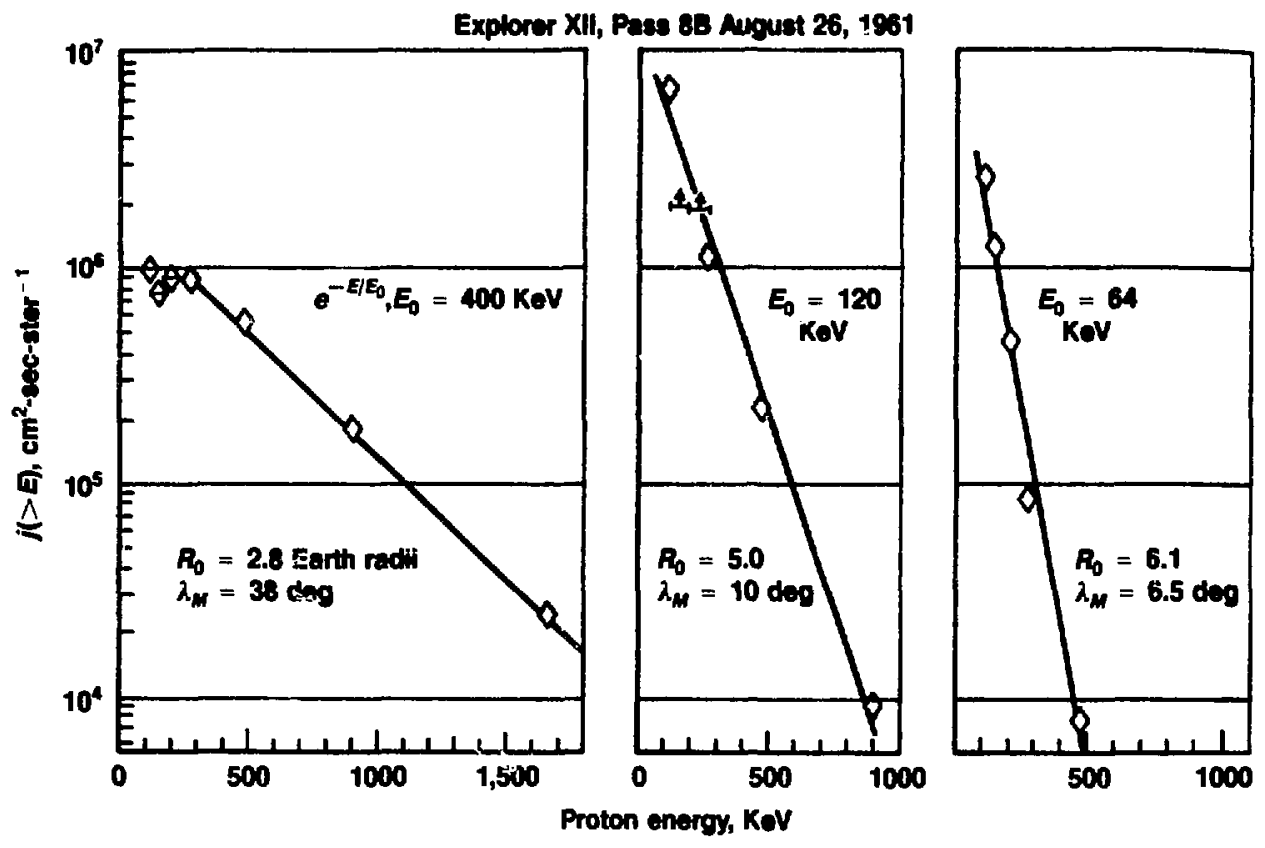

Figure 1-13. Proton directional-flux spectra at three locations in the outer belt. Solid line is a fit of the form, $i(<E)=k \cdot \exp \left(-E / E_{0}\right)$ (Davis and Willlamson, 1963). 


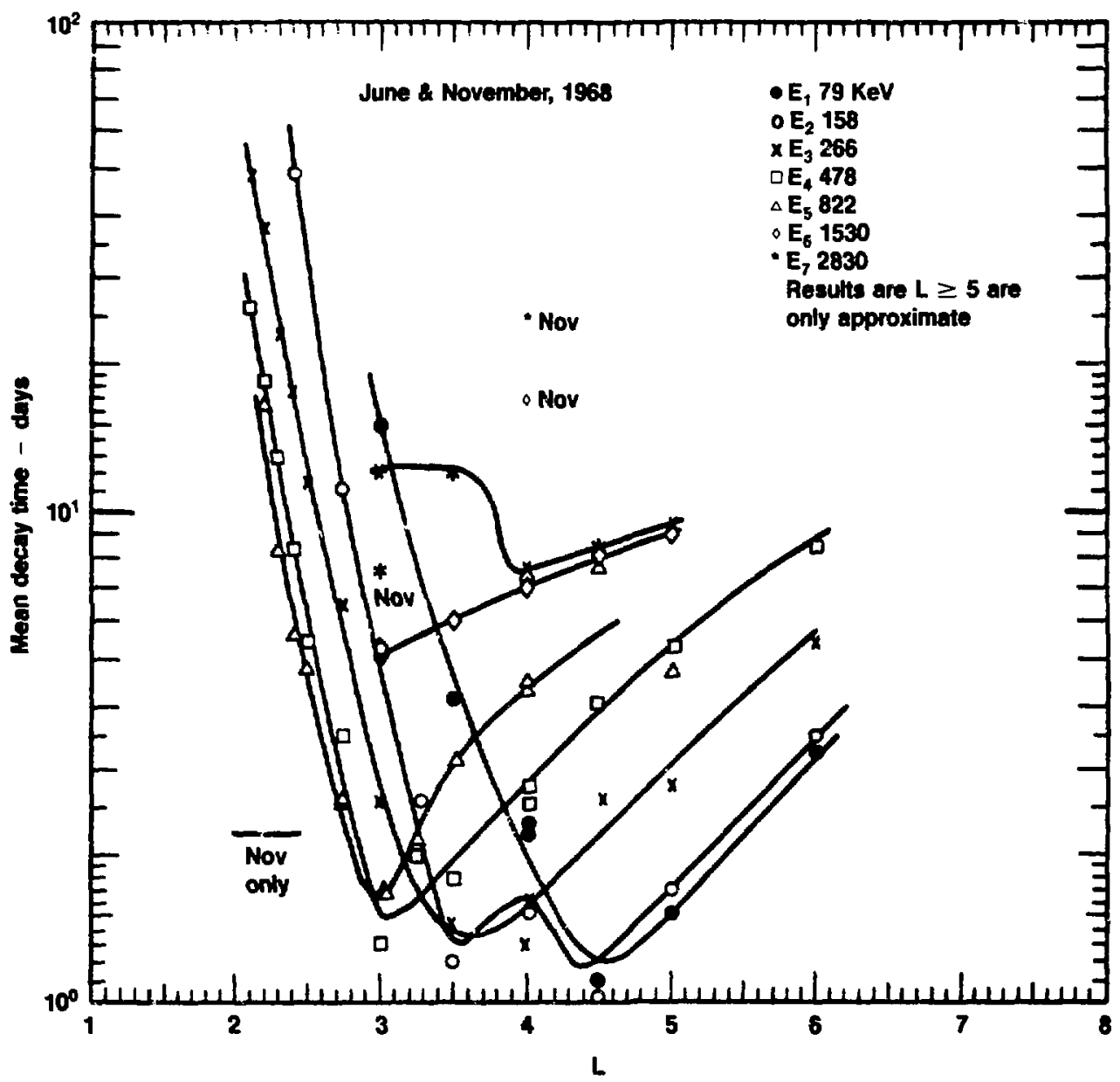

Figure 1-14. Decay rates of energetic electrons following injection by major storms in June and November, 1968 (West et al., 1979).

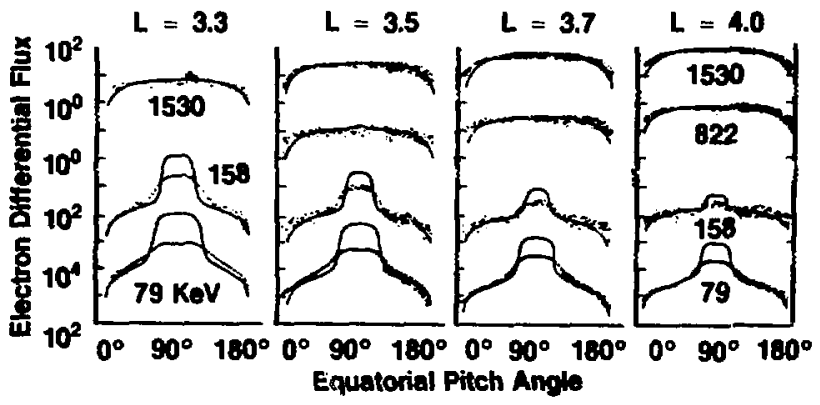

Figure 1-15. Comparison of theoretical equatorial pitch-angle distributions of diffusing energetic electrons (solid curves) with OGO-5 observations (dots), following an injection event (Lyons et al., 1972) 
adiabatic and non-adiabatic processes (Lyons and Speiser, 1982) until they enter the stable-trapping regions of the outer belt. The high-latitude B-pockets are found adjacent to the cusp region (Fig. 1-16), and it is possible that scattering, energization, and diffusion of magnetosheath plasma onto B-pocket lines leads to injection of particles into the stable-trapping regions.

\section{Particle Flux Measurements in the Magnetosphere}

Except for the role played by neutrals in the upper atmosphere, the whole of magnetospheric physics is concerned with the interaction of charged particles with the electric and magnetic fields of the magnetosphere. Magnetospheric physics is a specialized form of plasma physics, featuring generally collisionless, low-temperature, low-beta plasmas. Despite these simplifications, these plasmas are responsible for a diverse phenomenology. Satisfactory theoretical models of such phenomena-including auroral displays, magnetic substorms, ring currents, and raciation belt formation, transport, and depletion-must be based on detailed knowledge of the particle populations and their interactions with external and self-generated fields. Accordingly, a major goal of magnetospheric physics has been the description of the magnetosphere's particles and fields and the elucidation of particle orbits and transport mechanisms. To this end, the typical scientific earth satellite is equipped with numerous experiments to measure particles and fields encountered along the spacecraft trajectory.

Broadly speaking, the purpose of charged particle measurement in the magnetosphere is to characterize particle distributions in terms of particle species, energy, and the spatial and temporal varlations in number density. Thoroughly charting the magnetosphere's particles in this way would how:ver be nearly a hopeless task if the distributions could vary arbitrarily in three dimensions. Fortunately for the advancement of space science, the spatial variations of particle distributions are severely constrained by two fundamental principles which greatly simplify the organization of particle measurements: the adiabatic invariants of charged-particle motion, and the Liouville theorem of particle conservation.

\section{Adiabatic Invariants of the Motion}

The adiabatic invariants are integrals of the equation of motion, associated with the Hamiltonian of charged-particle motion in a magnetic field. These invariants are conserved even under substantial changes in the magnetic field, provided that the changes are slow enough. There is an integral for each of the three periodicities in the motion of a magnetospheric charged particle, which as we have seen are the rapid gyro-motion about a ield line, the slower bounce motion along the field line, and the even slower azimithal drift about the earth. The adiabatic invariants associated with these motions are termed $\mu, \mathrm{J}$, and $\phi$ sspectively. Typical periods for an energetic $\left(E_{e}>45 \mathrm{keV}\right)$ electron are approximately milliseconds for the gyroperiod, seconds for the bounce period, and hours for the drift period.

The formulation of these quantities is discussed in detail in Chapter 2. For the moment, we just want to point out that these adiabatic invariants are highly useful in determining points along the narticle's trajectory. By use of the invariants one can determine the set of field lines comprising the drift shell along which a particle moves in the course of its azimuthal drift about the earth, without having to solve the complex equations of motion. Each adiabatic invariant is formally expressed in terms of an asymptotic series, whose coefficients beyond first order are very difficult to derive. It is possible to avoid computing terms of order $>0$ however if the adiabatic invariants are evaluated at a particle bounce-mirror position. At this position we may write for the first two adiabatic invariants:

$\mu=\frac{p^{2}}{2 m_{0} B_{m}}$;

where

$\mathrm{P}$ = particle momentum,

$\mathbf{B}_{\mathrm{M}}=$ magnetic induction evaluated at the mirror-point,

$m_{0}=$ particle rest mass;

$J=\oint p \cdot d s=2 p \int_{m_{1}}^{m_{2}}\left[1-\frac{B(s)}{B_{M}}\right]^{1 / 2} d s$ 


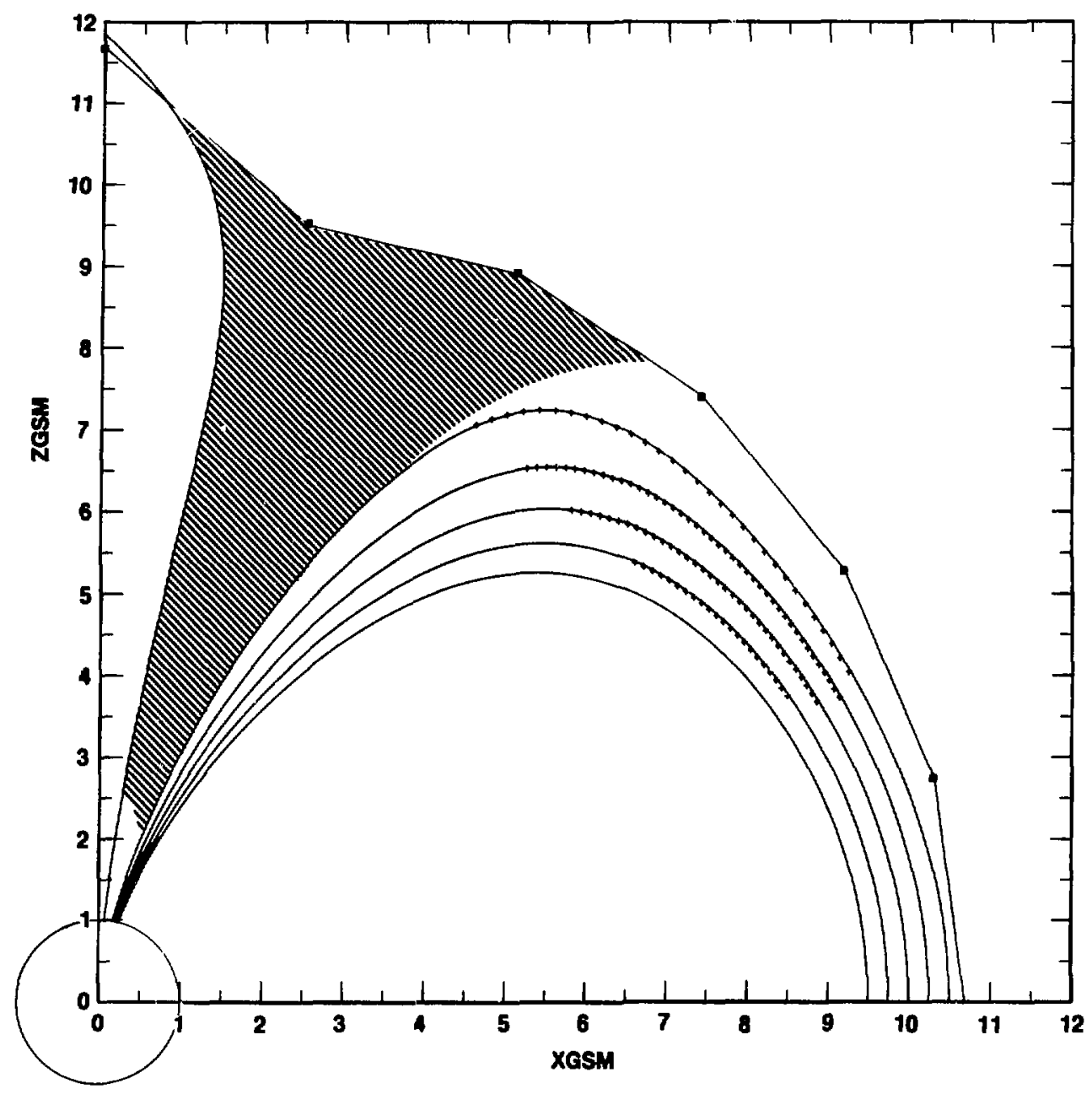

Figure 1-16. Outer magnetospheric field lines in the noon meridian. Line sections designated by + are B-pocket regions of depressed field strength, formed by the Chapman-Ferraro currents of the nearby magnetopause. Drift shells in the outer magnetosphere anch into segments which pass through these high-latitude B-pockets. Magnetosheath plasma penetrating the dayside cusp region (shaded) may diffuse into the B-pocket regions and be subsequently tranoported into the trapping regions of the magnetosphere. 
where the integral is evaluated along the field line from one mirror point $m_{1}$ to its conjugate point $m_{2}$, $B\left(m_{1}\right)=B\left(m_{2}\right)=B_{M}$ (Fig. 1-17).

An immediate consequence of Eq. (1.1) is that the magnetic aspect angle $\alpha$ of a particle at point $P$ in space, defined as:

$\cos \alpha=\frac{\mathbf{P} \cdot \mathrm{B}(\mathrm{P})}{\mathrm{PB}(\mathrm{P})}$,

is a function only of the ratio $B(P) / B_{M}$ :

$\cos \alpha=\left[1-\frac{B(P)}{B_{M}}\right]^{1 / 2}$.

A limiting case of practical importance occurs when the particle kinetic energy is conserved. In the absence of electric fields (whether arising from polarization, plasma convection, or time-varying B), the particle energy will be constant. (For energetic particles this case frequently pertains, since the kinetic energy perturbations due to polarization and convection electric field's are small. The magnitude of timevarying B-fields varies with location, producing substantial effects in the outer regions but only minor effects in the inner belt). In this case we can take $B_{M}$ and I to be the first two invariants, where $B_{M}$ is the particle's mirror field and $I=J / 2 p$. Note that $B_{M}$ and $I$ are properties only of the geomagnetic field, independent of charged particle properties.

Gradients in the magnetic field act on the bouncing particle to effect a slow azimuthal drift with each bounce. Under the assumption of adiabatic motion, the particle mirrors at points in the field such that the initial invariant values $B_{M 0}, I_{0}$ are conserved. Given an initial mirror point position in a model field, the entire drift shell may be traced out by locating at all azimuths the set of field points satisfying $B=B_{M 0}$. $I=I_{0}$. The drift shell consists of all such points and the field lines connecting them. The distorted dipole field of the magnetosphere exhibits sufficient regularity (except perhaps for regions adjacent to strong local current systems) that the drift shell trace in any azimuth is just a single field-line segment. In the inner magnetosphere the field possesses such azimuthal symmetry that a drift shell is nearly a surface of revolution (Fig. 1-18).

In effect, the adiabatic invariants constrain the possible variations of a charged-particle trajectory. The Liouville theorem, as we will see now, constrains the possible variations of particie flux along a trajectory.

Figure 1-17. Parameters of the bounce motion. Particle mirrors at points $\mathrm{M}_{1}$ and $\mathrm{M}_{2}$, where the magnetic aspect angle $a=90^{\circ}$. The aspect angle at any trajectory point $P$ is given by:

$\cos \alpha=\left[1-\frac{B(P)}{B_{M}}\right]^{1 / 2}$

where $B_{M}=B\left(M_{1}\right)=B\left(M_{2}\right)$.

(Rossi and Olbert, 1970).

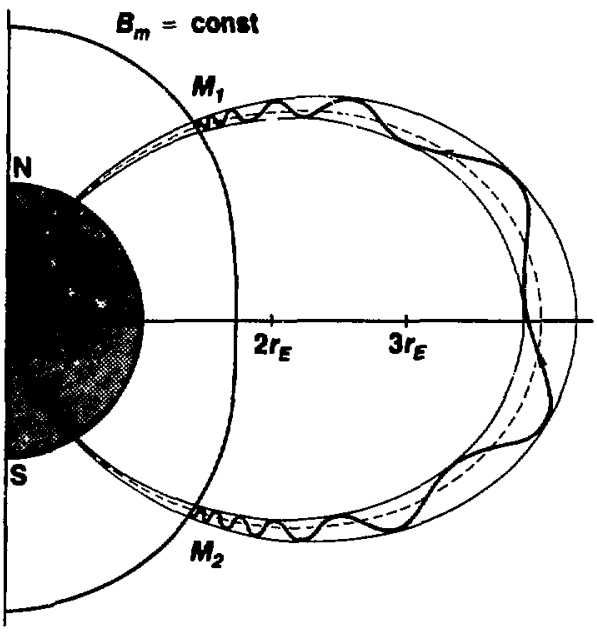




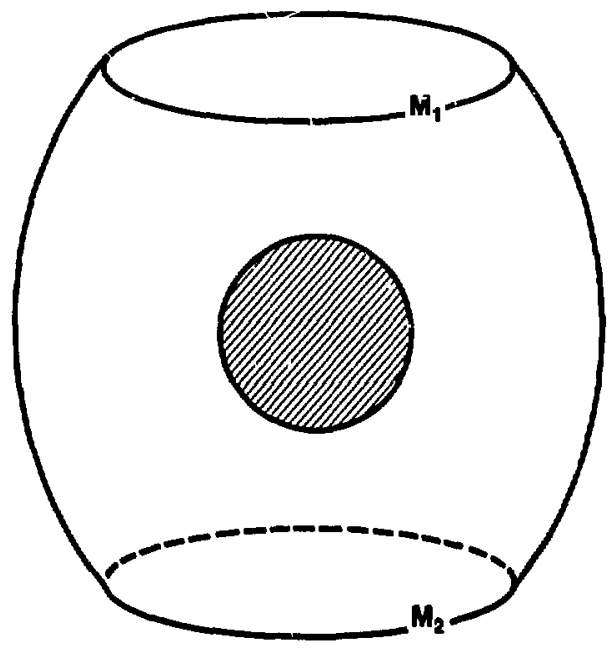

Figure 1-18. Drift surface or shell for a particle of specified adiabatic invariants $\mathrm{B}_{\mathrm{M} 0}$ and $\mathrm{I}_{0}$, and constant energy. The drift shell consists of all field points satisfying the conditions $B_{M}=$ $B_{M O}, I=I_{0}$, and the field lines connecting these points. $M_{1}$ and $M_{2}$ are the mirror-point traces.

\section{Phase-space Denoity and the Directional Flux}

The Liouville theorem states that the density of particles $f(x, p)$ in the six-dimensional phase space $(x, p)$ is constant along the dynamical path of a particle, provided there is no scattering, or particle sources or sinks (Spitzer, 1962). It is readily shown (e.g., Hess, 1968) that the phase space density $f$ can be expressed as:

$f=j / p^{2}$

where $j$ is the directional (or differertial) flux, an observable quantity.

The directional flux $j(\alpha, E)$ at a point in space is defined as the number of particles $d N$ of energy $E$ crossing a differential area $\mathrm{dA}_{\perp}$ oriented normal to the flux, whose velocities lie in the solid angle $\mathrm{d} \Omega=2 \pi \sin \alpha \mathrm{d} \alpha$, per unit area, per unit time, per unit solid angle, per unit energy:

$\mathrm{j}(\boldsymbol{\alpha}, \mathrm{E})=\frac{\mathrm{dN}(\boldsymbol{\alpha}, \mathrm{E})}{\mathrm{dA} \mathrm{A}_{\perp} \mathrm{d} \Omega \mathrm{dtd} \mathrm{E}}$

The polar coordinate system defining the flux direction is oriented along $B$, so that $\alpha \mathrm{i}$ the magnetic aspect angle given in Eq. (1.3). Figure 1-19 illustrates the geometric properties of the directional flux. Common units of $\mathrm{j}$ are:

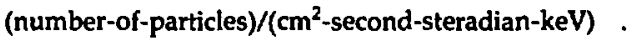

The directional flux can be directly measured by a particle-sounting detector that has high resolution in time, energy, and angle, such as the OGO-5 E6 scanning electron spectrometer experiment of West (West et al., 1969). The directional flux is the most fundamental flux measurement that can be made, because it is equivalent to directly sampling the particle phase space density $f$. The conversation of $f$ along a dynamical trajectory in phase space implies the conservation of $j / p^{2}$ along a drift shell in real space. For a static magnetic field the Liouville theorem implies the directional flux conservation law

$\mathrm{j}\left[\mathrm{a}\left(\mathrm{B}_{\mathrm{M}}\right), \mathrm{E}\right]=$ constant

along the shell designated by $B_{M}, I$. 


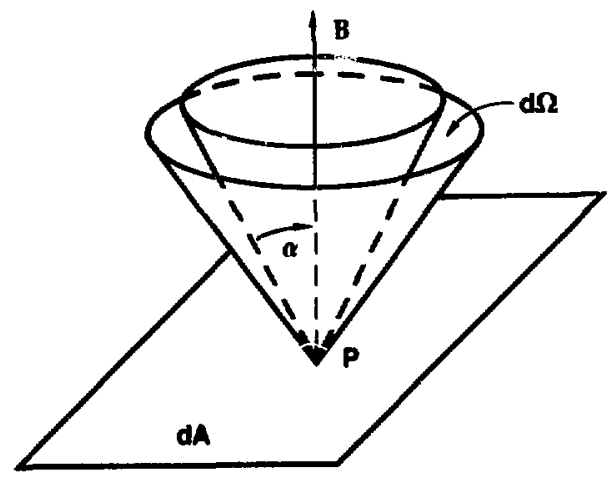

Figure 1-19. Geometry of the directional fiux j(a,E). The particles contributing to the directional flux $j(\alpha, E)$ at point $P$ have velocity vectors which lie in the differential solid angle $d \Omega$ $=2 \pi s i n a \mathrm{~d} a$ about $\mathrm{B} . \mathbf{j}(\alpha, E)$ is itien the number of particles dN with energies in the interval dE about $E$, and with velocity vectors in $\mathbf{l} \Omega$, which pass through 2 differential area $\mathrm{dA}_{\perp}$ oriented normal to the flux, per unit time-solid angleenergy-area.

\section{Organizing Direct" isal Fluxes on Drift Shells}

This directionai flux conservation law provides a powerful means for organizing particle flux measurements. Where this law holds, we are able to cilaracterize the directional fluxes on an entire drift shell from measurements made on a single line of the shell. If for the shell designated by $B_{M}$ I we measure the directional flux in the meridian $\phi_{0}$ and obtain at point $P$ :

id $[0(P), E]$;

where

$\alpha(P)=\cos ^{-1}\left[1-\frac{\dot{L}(\mathrm{P})}{\mathrm{B}_{\mathrm{M}}}\right]^{1 / 2}$ (by Eq. 1.4) ;

then go to another meridian $\phi_{1}$ on the same shell and make a measurement at an arbitrary point $Q$ :

$\mathrm{j}_{1}\{a(\mathrm{Q}), \mathrm{E}] \quad$;

where

$\alpha(Q)=\cos ^{-1}\left[1-\frac{B(Q)}{B_{M}}\right]^{1 / 2} ;$

we find that $j_{0}[\alpha(P), E]=j_{1}[\alpha(Q), E]$.

A pitch-angle distribution (PAD) is obtained at a point in spare by measuring the directional fiux over all pitch (magnetic aspect) angles, $-180^{\circ} \leq \alpha \leq 180^{\circ}$. $A$ tyfical PAD is illustrated in Fig. 1-20. This one is tern id a normal or loss-cone type of distributicn because the flux peaks at $a=90^{\circ}$. Particles with pitch angles near $\pm 180^{\circ}$ have mirror points in the upper atmosphere where they are likely to be lost from the distribution by scattering with neutrals; these particles are said to lie in the atmospheric loss cone. An initially isotropic distribution will relax to the loss-cone distribution after many bounce periods.

A typical magnetospheric field line has a single minimum in field strength, which occurs in the magnetic equatorial plane if the field is strictly dipolar. The set of all such loci in the real field forms a - on-planar surface Since all particles on a field line must pass through this surface, a PAD obtained at the intersection of a ficld line with this surface completely describes the dirertional fluxes of all particles moving on the line. A satellite passing through the magnetosphere alor" se magnetic equatorial surface can collec: a complete set of PAD measurements over an extended radi.. range. These measurements will completely characterize the directional Gitixes for all ärift shells that intersect the satellite rajectory (Fig. 1-2l), thereby gathering the sanie information that would be provided by sarapling the diztribution funcion f over a vast volume of space. 


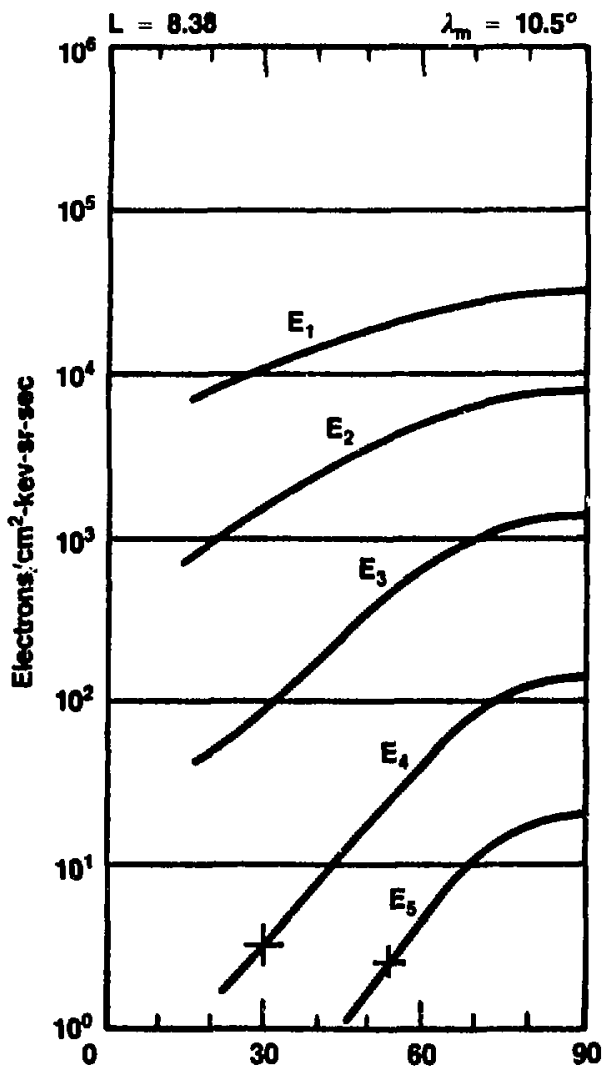

Pitch Angle a

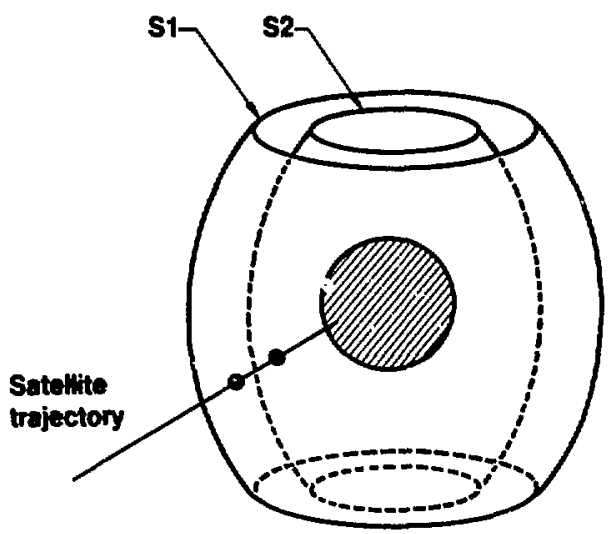

Figure 1-20. Typical OGO-5 electron pitch-angle distribution (PAD) illustrating the frequently-observed "normal" or loss-cone distribution which is characteristic of bouncetrapped radiation. This is a unimodal distribution with a peak at $\alpha=90^{\circ}$ (West et al., 1973a).
Figure 1-21. Equivalence of equatorial pitchangle distributions (PAD) and the phase space density. A spacecraft trajectory is shown along the magnetic equator through a region of space bounded by the drift shells $S_{1}$ and $S_{2}$. PAD measurements made along this trajectory will completely characterize the particle distributions in the volume $\mathbf{V}$ between the shells, acquiring information equivalent to sampling the phase-space density f over the entire volume. 
We have seen that the invariant pair $B_{M}, I$ is sufficient (in the absence of electric fields) to identify a particle trajectory, along which the directional flux $j$ is conserved. In the inner magnetosphere a further simplification of drift shell identification is made possible by the azimuthal symmetry of the geomagnetic field. In the inner belt region, drift shells are nearly degenerate in the sense that the shells for all particles initially on the same field line, nearly coincide at all azimuths. One would therefore desire a single parameter which could chàcterize such a degenerate shell. The Mcllwain L-value was introduced to provide such a drift shell categorization (Mcllwain, 1961). $\mathrm{L}$ is computed from a model of the geomagnetic field. $L$ is a function of $B_{M}, I$ which assigns to every drift shell a number which is equal to the equatorial radius of a dipole field line possessing the same $B_{M}, I$ value. (In a dipole field, a pair of $B_{M}, I$ values specifies a unique field line at a specified azimuth). The L-calculation serves to map the model field into a dipole field, roughly speaking. The condition of nearly-degenerate drift shells is equivalent to having nearly constant $\mathrm{L}$-values along a field line. Thus $\mathrm{L}$ serves as a more useful coordinate for labelling drift shells in the inner magnetosphere than does the pair of invariants $B_{M}, I$, since both $B_{M}$ and $I$ vary greatly along a field line. We can now write

$j\left[\alpha\left(B_{M}\right), E\right]=$ constant

along the (degenerate) drift shell designated by $L=$ constant. $L$ is a convenient and accurate parameter for characterizing drift shells within about $5 R_{E}$ of earth. $A$ plot of $j\left[\alpha\left(B_{M}\right), E\right]$ vs $B_{M}$ for $L=$ constant will be independent of azimuth, and can be determined from a single equatorial PAD measurement on the shell at any azimuth.

\section{Drift-Shell Splitting}

Beyond this distance, the field becomes increasingly aximuthally-asymmetric due to contributions from the Chapman-Ferraro magnetopause currents and from distributed plasma currents (e.g. the crosstail current system). Shell degeneracy is brcken, and $L$ is not close to constant along a field line. $j\left[a\left(B_{M}\right), E\right]$ vs $B_{M}$ for $L=$ constant will now depend on the azimuth of observation. This breakdown of degeneracy is termed shell-splitting, since the family of shells generated by particles distributed initially along a single field line are now tangent only on the original line. At other azimuths the shells split in the sense that the radial distance $R_{E Q}$ to the equatorial crossing of these shells is not a constant, but varies with $B_{M}, 1$. The radial range spanned by such a family of shells is usually most extreme at noon or midnight. This is illustrated by Fig. 1-22, which shows shell-splitting as computed by Roederer (1967) for the MeadWilliams magnetic field model.

Particles mirroring on a common field line in the noon meridian are distributed over a wide range of field lines at midnight. Particles of smaller equatorial pitch angle $\boldsymbol{\alpha}_{0}$ move to more distant lines than do particles of larger $\alpha_{0}$. A PAD measurement on a distant line at midnight therefore detects at small pitch angles, particles which at noon mirrored closer to the earth than the particles detected at large pitch angles. Since the particle flux gradient is normally directed earthward, this implies enhanced fluxes at small pich angles compared to angles near $90^{\circ}$, leading to a nearly field-aligned type of PAD. This type of distribution is also known as a "butterfly" distribution because of its appearance on a polar plot. The butterfly PAD is commonly observed on the nightside as a consequence of drift shell splitting (Fig. 1-23).

Figure 1-24 illustrates the shell-splitting of a family of drift shells emanating from an initial field line at midnight. At noon, the shells have split radially such that particles of large initial $\alpha_{0}$ have inoved to more distant lines than have particles of small initial $\alpha_{0}$ just reversing the shell-splitting encountered previously in going from noon to midnight. The PADs in this region are of the normal type, peaking at $\alpha=90^{\circ}$ (Fig. 1-20).

\section{Drift-Shell Branching}

An even more dramatic distortion of drift shells occurs near the dayside magnetopause. The magnetic field generated by the Chapman-Ferraro currents flowing on the nearby magnetopause creates minimum$B$ "pockets" in the field at latitudes of about $\pm 50^{\circ}$ (Fig. 1-16). Particles in a certain range of $B_{M}, I$ values can become temporarily trapped in these pockets in the course of their azimuthal drift. The drift shell branches into disjoint northern and southern segments, and the particles pass around the noon meridian on trajectories which do not cross the equator (Fig. 1-25). Post-noon the segments rejoin into a single, equator-crossing shell. 


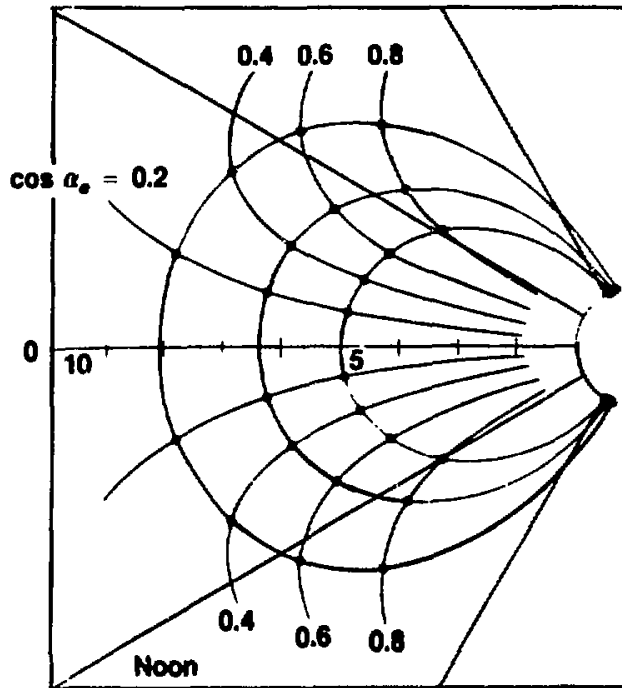

30

60

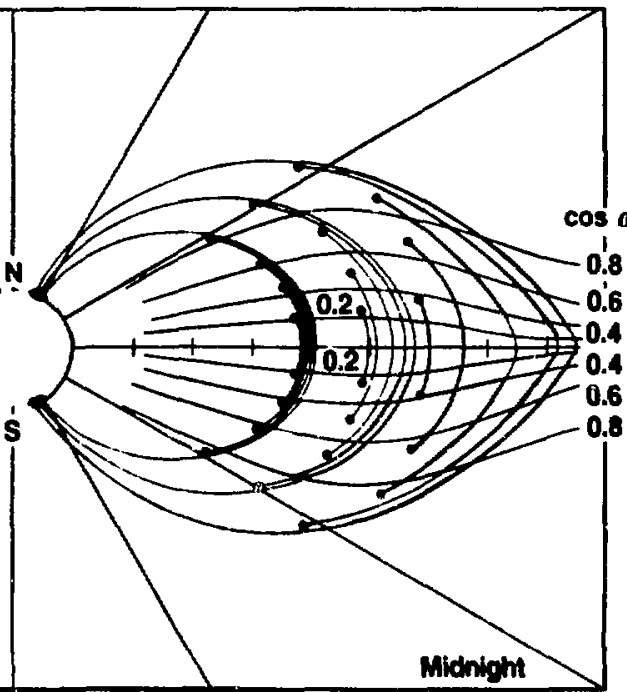

90

60

Figure 1-22. Shell-splitting in the Mead-Williams model for particles initially on a common noon field line (Roederer, 1967). Contour of contant-conine of the equatorial pitch angle are shown. Particles from a given noon field line are distributed over a range of field lines at midnight. Particles are assumed to move adiabatically such that their initial values of $B_{M} I$ are conserved. For any line at noon, particles of large I (small equatorial pitch angle) are found farthest from the earth on the nightaide. This splitting, coupled with the negative radial gradient in $j$, produces night-side "butterfly distributions"-PAD, which peak at mall pitch angles.

\section{Energetic Particles as Tracers of Magnetic Field Configuration}

All non-symmetric field distortions (such as those causing drift-shell splitting and drift-shell branching) introduce characteristic features into the PADs of drifting energetic particles. With the aid of geomagnetic field models, these features can be analyzed to infer the distortions or topological changes in the real field. (Energetic particles are preferred as magnetic-field distortion sensors because their trajeciories are not affected by the electric fields associated with plasma convective flows in the magnetosphere, which do strongly influence the drift of low-energy particles).

The ability of a drifting particle distribution to "encode" field distortions makes the PAD a highly useful tool for studying magnetic field line configuration in the outer dayside magnetosphere, where temporal changes due to the fluctuating properties of the solar wind are very significant. Satellite magnetometers, which provide detailed but highly-localized field information, cannot determine the instantaneous configuration of the field along a drift path. Even when multiple satellites are used, the spatial and temporal intervals between samples of a common particle drift shell are usually too coarse to produce more than an average picture of fieldline configuration. As we shall see, field models based on average configuration data fail to produce physically realistic gradients and fieldline configurations near the magnetopause. On the other hand, energetic electrons drift so rapidly around the earth (having a drift period of about 1 hour) that their distributions provide practically a snapshot of the field distortion effects encountered by the drifting particles.

Perhaps the earliest use of energetic particle distributions to detect topological changes was that of McDiarmid and Burrows (1968). They used the observed drop-out in energetic electron fluxes at high latitudes in the dayside magnetosphere to indicate the transition of the satellite from magnetic field lines 
Figure 1-23. Experimentally-observed energetic electron PADs in the midnight sector of the outer magnetosphere (West, 1979). Channels range in energy from E1 (79 keV) to E6 (1530 keV). All show the "butterfly distribution," peaking at small values of the pitch angle $\alpha$.
Sept 18, 1968

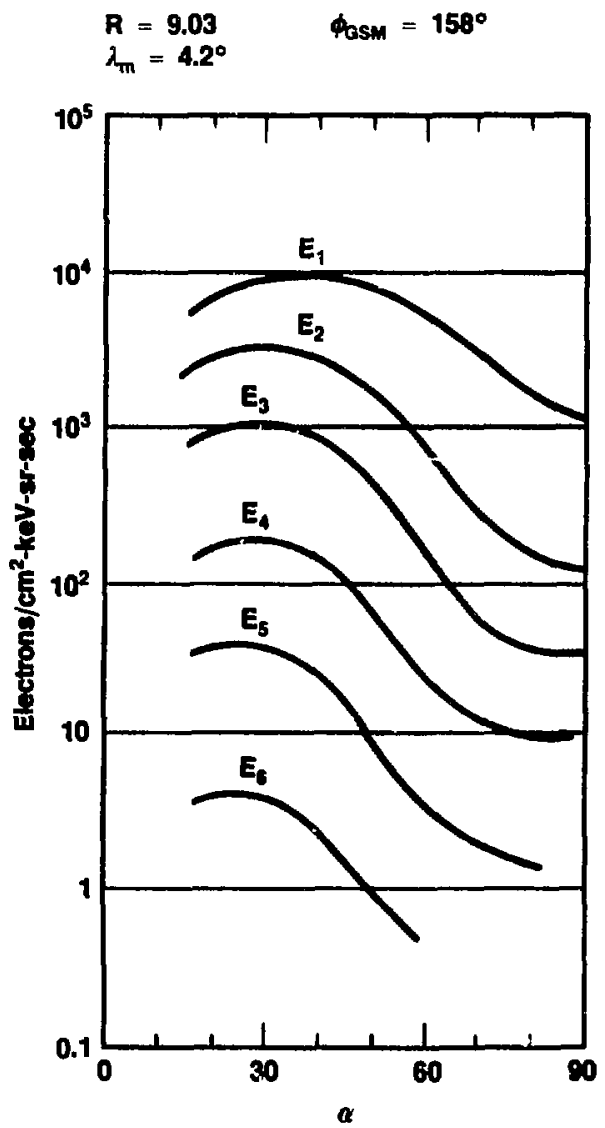




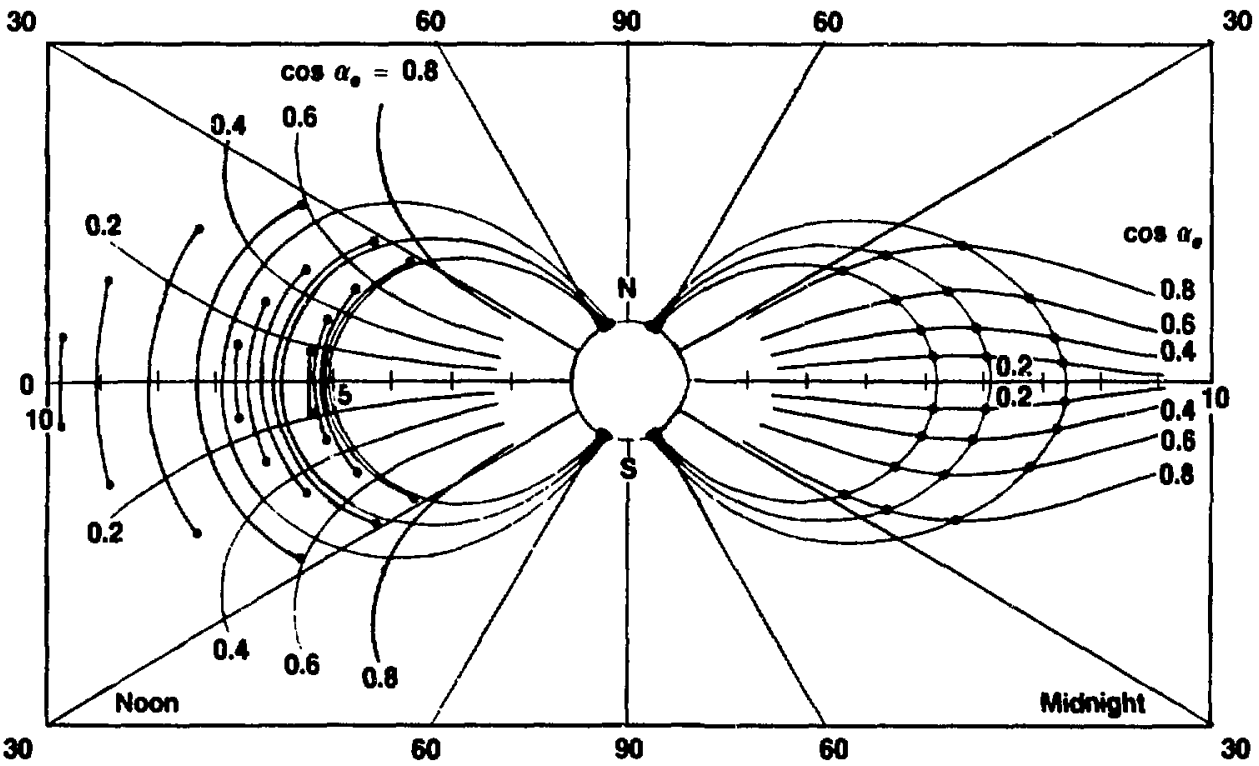

Figure 1-24. Shell-splitting in the Mead-Williams model for particles initially on a common midnight field line (Roederer, 1967). Contours of constant-cosine of the equatorial pitch angle are shown. Particles from a given midnight field line are distributed over a range of field lines at noon. Particles are assumed to move adiabatically such that their initial values of $B_{M}, I$ are conserved. For any line at midnight, particles of small I (large equatorial pitch angle) are found farthest from the earth on the dayside. This splitting reverses the effects of the drift from noon to midnight (illustrated in Fig. 1-22) and restores the loss-cone type of PAD found in the noon sector (e.g., Fig. 1-20). 


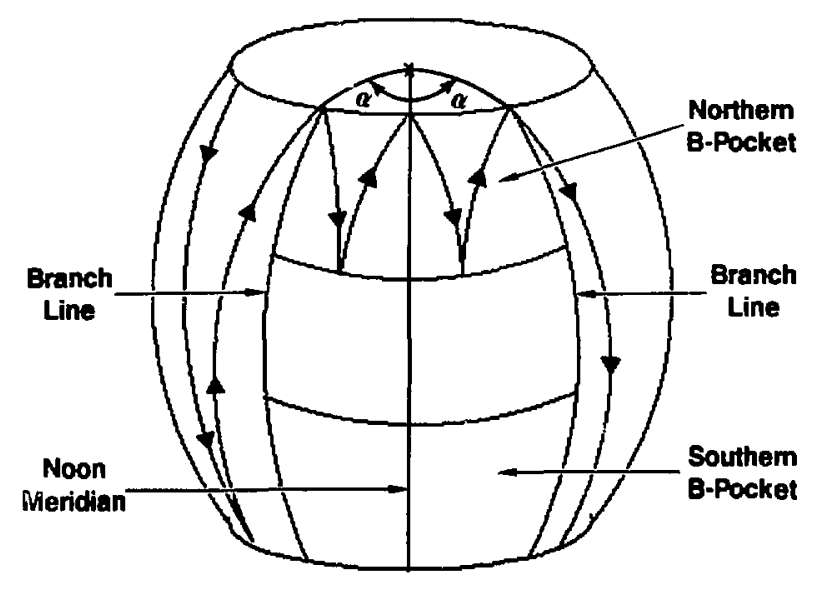

Figure 1-25. Particle motion on a branched drift shell. The arrowed line traces the bounce trajectory of an energetic electron as it drifts eastward through the outer dayoide magnetosphere. It first exhibits normal equator-crossing bounce motion, its poleward motion limited by high-latitude mirror points where $B=B_{M}$. After crossing the branch line at azimuthal angle $\alpha$ pre-noon, its bounce motion is confined to the northern high-latitude B-pocket by the presence of an off-equator local $B$-maximum of strength $B_{M A x}>B_{M}$. The electron passes around the noon meridian without ever crossing the equator. At an azimuthal angle $\alpha$ post-noon, the local B-maximum strength drops below $B_{M}$ and the particle returns to normal equator-crossing bounce motion. Depending on bounce phase, all particles on this drift shell will pass into either the northern B-pocket, the southern B-pocket, or the equatorial segment as they cross the branch line towards noon. The drift shell is said to branch along the branch line into these three segmenta. Post-noon these segmento are rejoined into one unified shell. These high-latitude minimum-B pockets may offer a route for the injection of solarwind particles into the trapping regions of the magnetosphere.

which closed on the dayside to lines which were swept back into the tail (and which could not trap energetic particles). The PAD features arising from the splitting of electron drift shells in the outer magnetosphere have been frequently observed, for example by Bogott and Mozer (1971) and West et al., (1972). Shell-splitting effects were explained by tracing the adiabatic drift paths of particles in model magnetic fields (Roederer 1967, 1972; Shabansky 1971, 1972).

Energetic protons $\left(E_{p}>100 \mathrm{keV}\right)$ have also been employed to detect spatial boundaries. The considerable rigidity of energetic protons means that a proton flux measurement actually samples a population whose guiding centers lie a fraction of an $R_{E}$ distant from the satellite. By analyzing directional energetic proton fluxes arriving from various angles at the satellite, one can derive the local spatial gradient of the proton flux. This so-called "East-West" effect was employed by Buck et al. (1973) to measure the dynamic changes in the tail plasma sheet during a substorm. The same effect was utilized by Kaufman and Konradi (1973), and Williams et al. (1979) to study magnetopause boundary motion, and also by Palmer et al. (1976) to investigate field line dynamics in the distant tail.

In this dissertation we utilize energetic electron distributions to probe field distortions and changes in fieldline topology near the dayside magnetopause. For this study, we analyze detailed energetic electron pitch angle distribution measurements acquired by the ILNL spectrometer experiment aboard the OGO-5 spacecraft in 1968-1969. This experiment provided a wealth of detailed distribution information which has not been matched by subsequent experiments. We evaluate distinctive PAD features by tracing the trajectories of charged particles in model magnetospheric fields, using both adiabatic conservation methods and numerical integrations of the equations of motion. We find features in the spectrometer data which are most readily interpreted in terms of particle motion on B-pocket fieldilines. We explore the consequences of these pockets for solar particle entry, trapping, and transport. 


\section{References}

Anderson, K., Annual Review of Nuclear Science, 16, 291, 1966.

Axford, W. I., H. E. Petschek, and G. L. Siscoe, Tail of the Magnetosphere, J. Geophys. Res., 70, 1231, 1965.

Bame, S. J., J. R. Asbridge, H. E. Felthauser, R. A. Olson, and I. B. Strong, Electrons in the plasma sheet of the earth's magnetic tail, Phys. Rev. Letters, 16, 138, 1966.

Behannon, K. W., and N. F. Ness, Satellite studies of the earth's magnetic tail, in Physics of the Magnetosphere, edited by R. L. Carovillano, J. F. McClay, and H. R. Radoski, p409, D. Reidel, Dordrecht, Holland, 1968.

Biermann, L., Kometenschweife und solare korpuskularstrahlung, Z. Astrophys., 29, 274, 1951.

Bogott, F. H., and F. S. Mozer, Equatorial electron angular distributions in the loss-cone and at large angles, J. Geophys. Res., 76, 6790, 1971.

Bonetti, A, H. S. Bridge, A. J. Lazarus, B. Rossi, and F. Scherb, Explorer 10 plasma measurements, J. Geophys. Res., 68, 4017, 1963.

Buck, R. M., H. l. West, Jr., and R. G. D'Arcy, Jr., Satellite studies of magnetospheric substorms on August 15, 1968: 7. OGO-5 energetic proton observations-spatial boundaries, J. Geophys. Res., 78, 3101, 1973.

Cahill, L. J., and P. G. Amazeen, The boundary of the geomagnetir field, J. Geophys. Res., 68, 1835, 1963.

Cahill, L. J., Jr., The magnetosphere, Sci. Am., 212, 1965.

Chapman, W., and V. C. A. Ferraro, A new theory of magnetic storms, Terrestrial Magnetism and Atmospheric Electricity, 36, 77, 1931.

Chapman, W., and V. C. A. Ferraro, A new theory of magnetic storms (continued), Terrestrial Magnetism and Atmospheric Electricity, 37, 147, 1932.

Chapman, W., and V. C. A. Ferraro, The theory of the first phase of a geomagnetic storm, Terrestrial Magnetism and Atmospheric Electricity, 45, 245, 1940.

Davis, L. R., and J. M. Williamson, Low-energy trapped protons, Space Res., 3, 365, 1963.

Fairfield, D. H., Electric and magnetic fields in the high-latitude magnetosphere, Rev. Geophys. Space Phys., 15, 285, 1977.

Fairfield, D. H., Structure of the magnetopause: observations and implications for reconnection, Space Sci. Rev., 3, 427, 1979.

Falthammer, C. G., Diffusion and acceleration in the earth's radiation belts, in Solar Terrestrial Physics $/ 1970$, edited by E. R. Dyer, P270, D. Reidel, Dordrecht, Holland, 1972.

Farley, T. A., and M. Walt, The source and loss processes of inner radiation belt protons, J. Geophys, Res., $76,8223,1971$.

Freden, S. C., and R. S. White, Particle fluxes in the inner radiation belt, J. Geophys. Res., 65, 1377, 1960.

Freeman, J. W., J. A. Van Allen, and L. J. Cahill, Explorer 12 observations of the magnetospheric boundary and the associated solar plasma on September 13, 1961, J. Geophys. Res., 68, 2121, 1963.

Gringauz, K. I., V. V. Bezrukikh, V. D. Ozerov, and R. E. Rybchinskii, A study of the interplanetary ionized gas, high-energy electrons, and corpuscular radiation from the sun by means of the three electrode trap for charged particles on the second Soviet cosmic rocket, Dakl. Adak. Nauk. SSSR, 131, 1301, Soviet Physics Dokl., 5, 361, 1960.

Heikkila, W. J., and J. D. Winningham, Pene'ration of magnetosheath plasma to low altitudes through the dayside magnetospheric cusps, J. Geophys. Res., 76, 883, 1971.

Hess, W. N., The Radiation Belt and Magnetosphere, Blaisdell, Waltham, Mass, 1968.

Hess, W. N., and J. Killeen, Spatial distribution of protons from neutron decay trapped by the geomagnetic field, J. Geophys. Res., 71, 2799, 1966.

Hones, E. W., Jr., Plasma flow in the plasma sheet and its relation to substorms, Radio Sci., 8, $979,1973$.

Johnson, F. S., The gross character of the geomagnetic field in the solar wind, J. Geophys. Res. 65, 3049, 1960.

Kaufman, R. L., and A. Konradi, Speed and thickness of the magnetopause, J. Geophys, Res., 78, 6549, 1973.

Lanzerotti, L. J., Equatorial and precipitating solar protons in the magnetosphere: 1. Low-energy diurnal variations, J. Geophys. Res., 76, 5235, 1971.

Lanzerotti, L. J., Solar energetic particles and the configuration of the magnetosphere, Rev. Geophys. Space Phys., 10, 379, 1972.

Lingenfelter, R. E., The cosmic ray neutron leakage flux, J. Geophys. Res., 68, 5633, 1963. 
Lyons, L. R., R. M. Thorne, and C. F. Kennel, Electron pitch-angle diffusion driven by oblique whistler-mode turbulence, J. Plasma Phys., 6, 589, 1971.

Lyons, L. R., R. M. Thome, and C. F. Kennel, Pitch-angle diffusion of radiation belt electrons within the plasmasphere, J. Geophys. Res., 77, 3455, 1972.

Lyons, L. R., and T. W. Speiser, Evidence for current sheet acceleration in the geomagnetic tail, J. Geophys. Res., 87, 2276, 1982.

Manka, R. H., Review of the July 29, 1977 substorm, in Quantitative Modeling of Magnetospheric Processes, Geophys. Monogr. Ser., Vol. 21, edited by W. P. Olson, p644, AGU, Washington, D. C., 1979.

McDiarmid, I. B., and J. R. Burrows, Local time asymmetries in the high-latitude boundary of the outer radiation zone for the different electron energies, Can. J. Phys., 46, 49, 1968.

Mcllwain, C. E., Coordinates for mapping the distribution of magneticali.y trapped particles, J. Geophys. Res., 66, 3681, 1961.

Mencke Hansen, A., A. Bahnsen, and N. D'Angelo, The cusp-magnetosheath interface, J. Geophys. Res., 81, $556,1976$.

Ness, N. F., The earth's magnetic tail, J. Geophys. Res., 70, 2989, 1965.

Neugebauer, M., and C. W. Snyder, Solar plasma experiment: preliminary mariner 2 observations, Science, 138, $1095,1962$.

Palmer, I. D., P. R. Higbie, and E. W. Hones, Jr., Gradients of solar protons in the high-latitude magnetotail and the magnetospheric electron field, I. Geophys. Res., 81, 562, 1976.

Paschmann, G., G. Haerendel, N. Sckopke, H. Rosenbauer, and P. C. Hedgecock, Plasma and magnetic field characteristics of the distant polar cusp near local noon: The entry layer, /. Geophys. Res., 81, 2883, 1976.

Paulikas, G. A., and I. B. Blake, Effects of the solar wind on magnetosphertc dynamics: energetic electrons at the synchronous orbit, in Quantitative Modeling of Magnetospheric Processes, Geophys. Monogr. Ser., Vol. 21, edited by W. P. Olson, p180, AGU, Washington, D. C., 1979.

Pfitzer, K. A., S. Kane, and J. R. Winkler, The spectra and intensity of electrons in the radiation belts, Space Res. 6, 702, 1966.

Piddington, J. H., Cosmic Electrodynamics, John Wiley and Sons, New York, 1969.

Poeverlein, H., The earth's magnetosphere, in Handbuch der Physik, Vol. 49-4, 7, 1972.

Roederer, J. G., On the adiabatic motion of energetic particles in a model magnetosphere, J. Geophys. Res., $72,981,1967$.

Roederer, J. G., Geomagnetic field distortions and their effects on radiation belt particles, Rev. Geophys. Space Phys., 10, 599, 1972.

Rosenbauer, H., H. Grunwaldt, M. D. Montgomery, G. Paschmann, and N. Sckopke, Heos 2 plasma observations in the distant polar magnetosphere: The plasma mantle, J. Geophys. Res., 80, 2723, 1975.

Scholer, M., G. Morfill, and D. Hovestadt, Statistical analysis of solar proton pitch angle distributions over the polar cap during disturbed geomagnetic times, J. Geophys. Res., 40, 37, 1974.

Schultz, M., Geomagnetically trapped radiation, Space Sci. Rev., 17, 481, 1974.

Schultz, M., Earth's radiation belts, Rev. Geophys. Space Phys., 20, 613, 1982.

Shabansky, V. P., Some processes in the magnetosphere, Space Sci. Rev., 12, 299, 1971.

Shabansky, V. P., Particle motion in the earth's magnetosphere, in Solar Terrestrial Physics/1970: Part III, edited by E. R. Dyer, p25, D. Reidel, Dordrecht, Holliand, 1972.

Singer, S. F., A new model of magnetic storms and auror:., Trans. Am. Geophys. Union, 38, 175, 1957.

Spitzer, L., Jr., Physics of Fully lonized Gases, second edition, Interscience, New York, 1962.

Vampola, A. L., Access of solar electrons to closed field lines, J. Geophys. Res., 77, 36, 1971.

Van Allen, J. A., Radiation belts around the earth, Sci. Am., 200, 39, 1959.

Van Allen, J. A., and L. A. Frank, Radiation around the earth to a radial distance of $107,400 \mathrm{~km}$, Nature, $183,430,1959$ a.

Van Allen, J. A., and L. A. Frank, Radiation measurements to $658,300 \mathrm{~km}$ with Pioneer IV, Nature, 184, 219, 1959b.

Voigt, G. H., A three dimensional analytical magnetospheric model with defined magnetopause, $J$. Geophys, 38, 319, 1972.

Walt, M., Radial diffusion of trapped particles and some of its consequences, Rev. Geophys. Space Sci., 9, 11, 1971. 
Walt, M., and L. L. Newkirk, Comments on radial diffusion of outer-zone electrons, /. Geophys. Res., 76, $5368,1971$.

West, H. I., Jr., J. H. Wujeck, J. H. Mcquaid, J. C. Jenson, R. G. D'Arcy, Jr., R. W. Hill, and R. M. Bogandowicz, The LRL electron and proton spectrometer on NASA's Orbiting Geophysical Observatory V(E), Lawrence Livermore National Laboratory, Livermore, CA., UCRL-50572, 1969.

West, H. I., Jr., R. M. Buck, and J. R. Walton, Shadowing of electron azimuthal-drift motions near the noon magnetopause, Nature Physical Sci., 240, 6, 1972.

West, H. I., Jr., R. M. Buck, and J. R. Walton, Electron pitch angle distributions throughout the magnetosphere as observed on OGO-5, J. Geophys. Res., 78, 1064, 1973a.

West, H. I., Jr., R. M. Buck, and J. R. Walton, Satellite studies of magnetospheric substorms on August 15, 1968: 6. OGO-5 energetic electron observations-pitch angle distributions in the nighttime magnetosphere, J. Geophys. Res., 78, 3093, 1973 b.

West, H. I., Jr., and R. M. Buck, Energetic electrons in the inner belt in 1968, Planet. Space Sci, 24, 643, 1976.

West, H. 1., Jr., The signatures of the various regions of the outer magnetosphere in the pitch angle distributions of energetic particles, in Quantitative Modeling of Magnetospheric Processes, Geophys. Monogr. Ser., Vol. 21, edited by W. P. Olson, p150, AGU, Washington, D. C., 1979.

West, H. I., Jr., R. M. Buck, and G. Davidson, Study of energetic electrons in the outer radiation-belt regions using data obtained by the LLL spectrometer on OGO-5 in 1968, Lawrence Livermore National Laboratory, Livermore, CA., UCRL-52807, 1979.

Williams, D. J., T. A. Fritz, B. Wilken, and E. Keppler, An energetic particle perspective of the magnetopause, J. Geophys. Res., 84, 6385, 1979.

Wolfe, J. H., and D. S. Intriligator, The solar wind interaction with the geomagnetic field, Space Sci. Rev., $10,511,1970$.

Young, D. T., Near-equatorial magnetospheric particles from $\approx 1 \mathrm{eV}$ to $\approx 1 \mathrm{MeV}$, Rev. Geophys. Space Phys., 21, 402, 1983. 


\section{Chapter 2. The OGO-5 Spacecraft and the LLNL Electron and Proton Spectrometer}

\section{The OGO-5 Spacecraft}

The OGO-5 spacecraft was the fifth of six satellites to be launched by the National Aeronautics and Space Administration as part of the Orbiting Geophysical Observatory program. Three of the spacecraft were placed in polar orbit, and three in highly elliptical orbits which were inclined about 30 degrees to the equator. Launched on March 4, 1968, OGO-5 became the last elliptic-orbit spacecraft in the program. The satellite carried a payload of 22 scientific experiments designed to measure charged particles (over a range that spanned plasma to cosmic-ray energies) and magnetic fields and waves. The orbit achieved by the spacecraft permitted the sampling of particle populations from the inner radiation belt, through the outer regions of the magnetosphere, and in the interplanetary medium. Among the experiments were the

Lawrence Livermore National Laboratory's Electron and Proton Measurement Experiment E6, which supplied the differential particle flux data used in this dissertation, and the UCLA Magnetic Field Experiment E14, which provided values of ambient magnetic field needed in conjunction with the particle data to construct directional particle flux distributions.

A diagram of the OGO-5 spacecraft is shown in Fig, 2-1 [Linder, 1968]. The large rectangular solar paddle arrays provided electric power. The two Orbital Plane Experimental Packages (OPEP-1 and OPEP-2) were located at opposite ends of a rotatable shaft. The LLNL electron/proton spectrometer experiment was housed in the OPEP-2 enclosure. The UCLA E14 fluxgate magnetometer was carried at the end of a $22 \mathrm{ft}$ boom to reduce magnetic interference from the spacecraft's main body.

The spacecraft was stabilized in an attitude such that the solar paddles were normal to the incident sunlight, and the OPEP shaft pointed at the center of the earth. Attitude control was maintained by gas reaction thrusters and reaction wheels. Although the nitrogen bottle supplying the reaction thrusters was expected to become exhausted after about one year of flight, causing the spacecraft to tumble and terminate its useful life, so little reaction gas was expended in placing the spacecraft in orbit that normal stabilized operations continued for more than 3 years.

The initial orbital parameters for OGO-5 were an orbital inclination of $31.3^{\circ}$, apogee at $22.9 \mathbf{R}_{E}$ $\left(1 R_{E}=6400 \mathrm{~km}=\right.$ mean Earth equatorial radius), perigee at $150 \mathrm{~km}$, and an orbital period of 62.4 hours. Figure 2-2 depicts the satellite orbit. Due to the revolution of the earth about the sun, apogee moved westward in geographical coordinates about one degree/day. Over the course of a year the orbit swept out a large volume of the magnetosphere and allowed the experiments to sample near-equatorial space at all local times.

\section{OGO-5 Experiment E6}

The OGO-5 E6 experiment package was designed and developed by Harry I. West, Jr., Principal Investigator, under NASA Order No. S-70014-G. It consisted of two magnetic electron spectrometers and a proton range-energy telescope. The experiment was designed to provide energy- and time-resolved measurements of fluxes of energetic electrons $(80 \mathrm{keV}-2.8 \mathrm{MeV})$ and protons $(0.1-100 \mathrm{MeV})$ throughout the magnetosphere and in interplanetary space. The following sections provide an overview of the experimental design and principles of operation. Details of the instrumentation are given in West et al., 1969.

\section{Design Objectives}

The energetic electrons and protons in the magnetosphere represent the high-energy tails of the particle populations. Studies of their distributions in magnetically-quiet times and during periods of magnetic activity help elucidate the processes by which particles are injected into the magnetosphere, accelerated, transported, and lost. The understanding of these processes is central to much of the ongoing work in magnetospheric physics. 


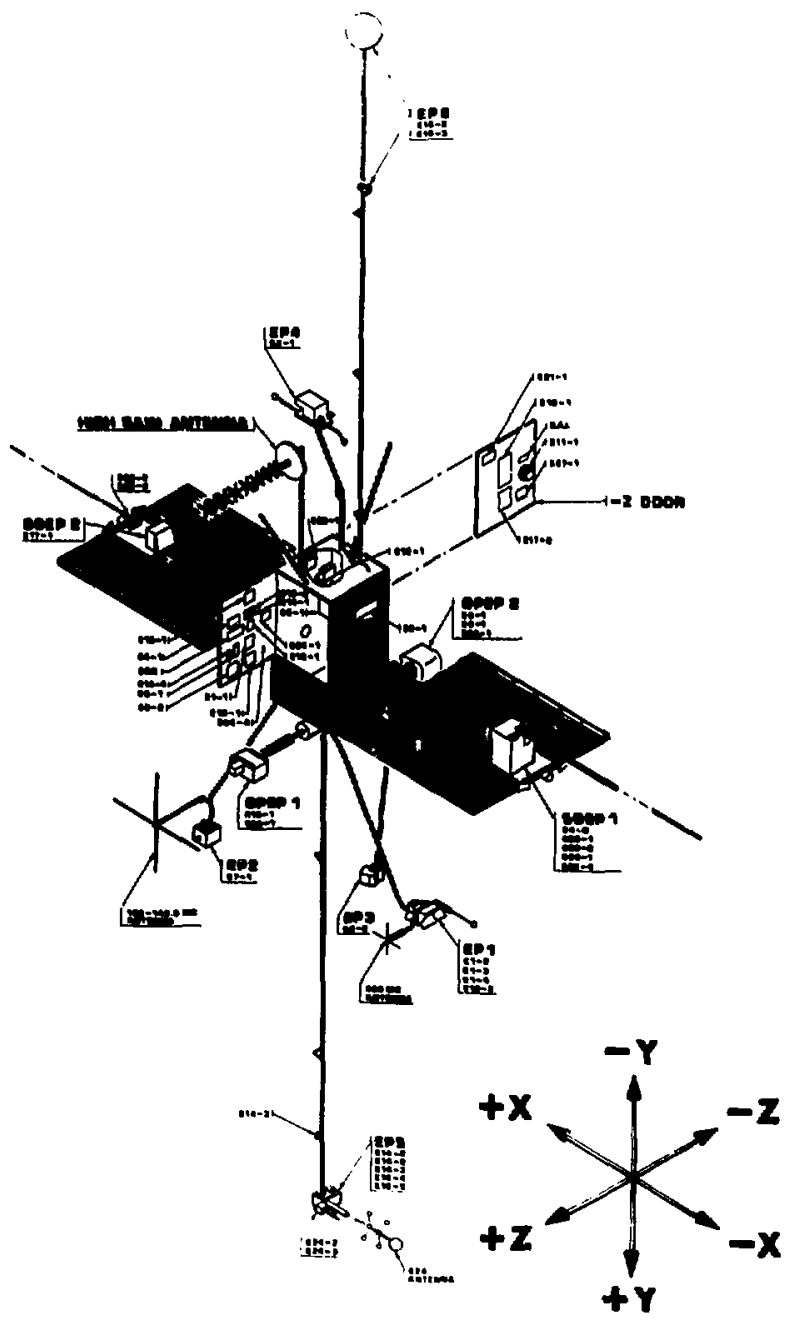

Figure 2-1. OGO-5 Spacecraft with experiment locations [Linder, 1968).

The most critical particle meastrement that can be made from the standpoint of theoretical importance, is the directional flux $j$. This quantity is proportional to the number of particles of energy $E$, coming from a specified direction with respect to the local magnetic field, which cross a unit area oriented normal to the incident flux. The quantity $j / p^{2}$, where $p$ is the particle momentum, is conserved along a particle trajectory in space (in the absence of collisions). A set of $j$-measurements made over a range of energies and trajectories can provide evidence for particle scattering and the action of injection, diffusion, and loss processes.

It was the principal design goal of the E6 experiment to provide accurate measurements of directional fluxes of energetic electrons $\left(E_{e}>70 \mathrm{keV}\right)$ and protons $\left(E_{\mathrm{p}}>0.1 \mathrm{MeV}\right)$ encountered along the orbit of the OGO-5 spacecraft. This goal led to the following design requirements:

- A dynamic range of about $10^{8}$ to cover both the low-level interplanetary fluxes and the high-level radiation-belt fluxes; 


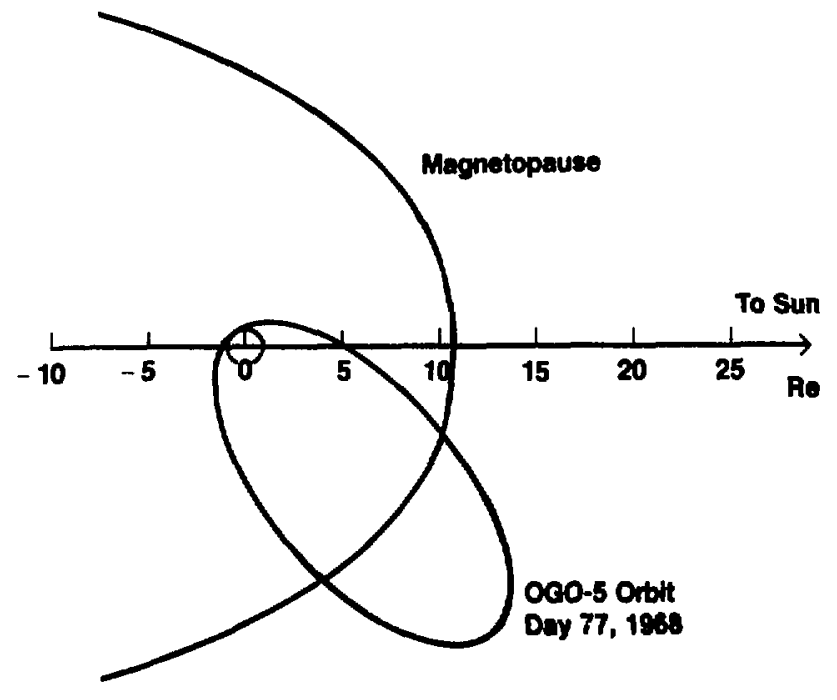

Figure 2-2. OGO-5 Spacecraft orbit, projected on ecliptic plane.

- A mechanism for separating (or uniquely identifying) protons from elsctrons;

- High directional pointing accuracy;

- Spectral coverage from $\sim 70 \mathrm{keV}$ to several $\mathrm{MeV}$ for electrons and from 0.1 to tens of $\mathrm{MeV}$ for protons;

- Many channels for good energy resolution;

- Suppression of backgrounds as far as feasible, and accurate determination of residual backgrounds;

- A mechanism for scanning the instrument with respect to the local magnetic field, in order to measure fluxes over a wide range of magnetic pitch angles;

- An onboard vector magnetometer to provide accurate measures of the strength and direction of the ambient magnetic field.

\section{Design Realization}

These requirements were met by a design based on the single-focussing semi-circular beta-ray spectrometer (for electrons) and on the range-energy telescope (for protons). In the West design, the total desired energy range for electrons was subdivided into a low and a high energy range. Each range was covered by a constant-field magnetic spectrometer which energy-analyzed the electron fluxes (Figs. 2-3 and 2-4). Electrons incident on the entrance aperture were bent through $180^{\circ}$ by the permanent magnet field and focussed (in first order) on as row of solid-state detectors. The field also served as a "broom" to sweep incident protons away from the detectors. $"$ " shielding provided by the iron spectrometer case, ferroceramic magnet poles, and gold detector envelopes served to block out penetrating protons (of $\mathrm{E}_{\mathrm{p}}<$ $80 \mathrm{MeV}$ ) and bremsstrahlung radiation. A stack of solid-state detectors and absorbers (the proton telescope) was located in the high-field spectrometer to count energetic protons. By placing the two spectrometers with poles antiparallel, and surrounding the spectrometers with a magnetic shield, the residual leakage field from the magnets was kept within the limits required to avoid interference with other spacecraft experiments (such as the magnetometer). 


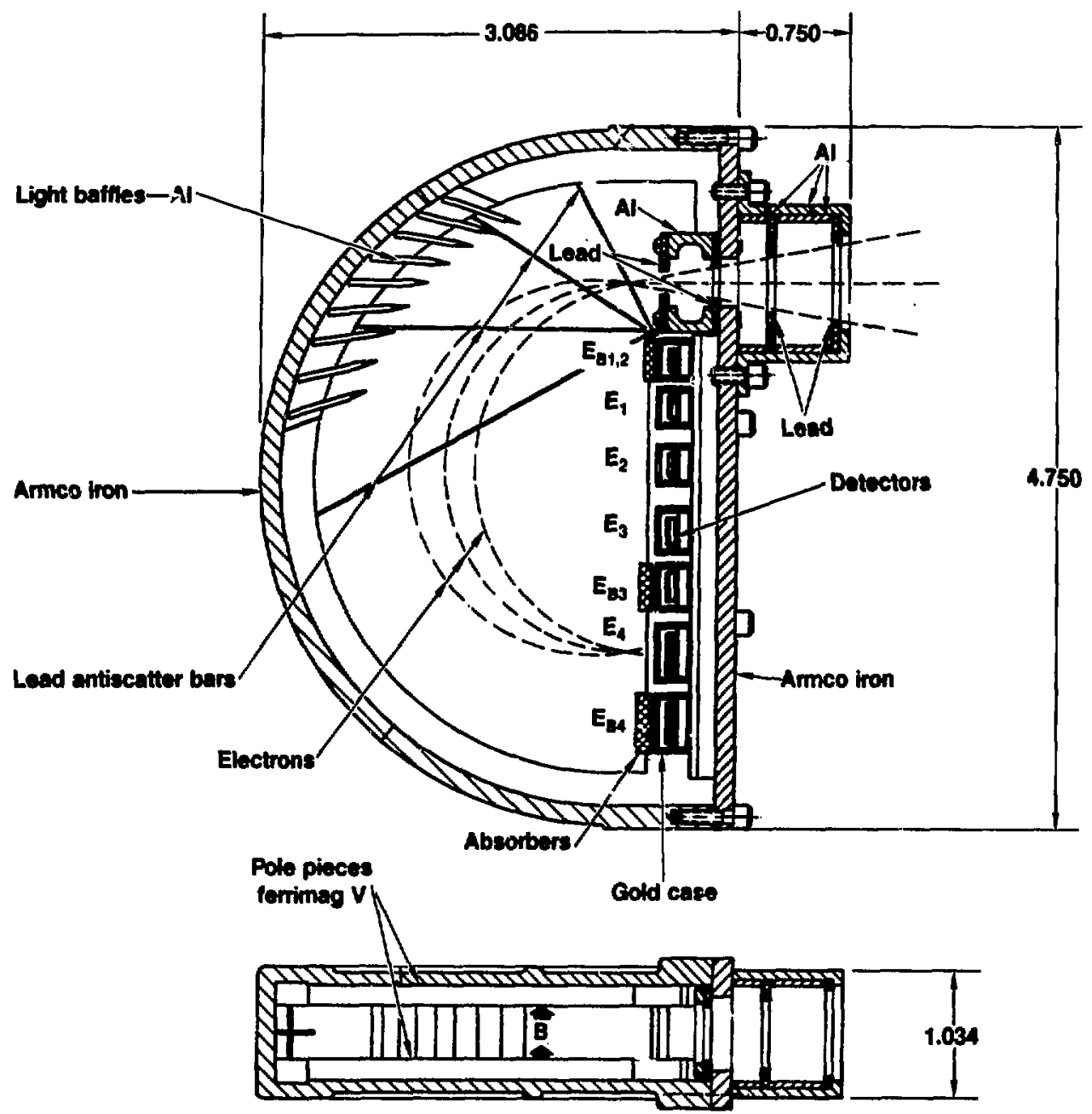

Figure 2-3. Low-field $(B=.0863$ tesla) magnetic spectrometer for electron detection. Energy range: $79-479 \mathrm{keV}$. (Dimensions in inches). [West et al., 1969]

\section{Electron Detection}

\section{Spectrometer Geometry}

The entrance to each spectrometer was defined by a lead disk collimator which limited the incident flux to $\sim 30^{\circ}$ in the plane of focussing, but only $\sim 5^{\circ}$ in the perpendicular plane (see Table 1). For most of the dayside magnetosphere, it was this narrow dimension of the collimator which fixed the effective angular resolution. Solid-state detectors were located in a linear array to intercept the electron trajectories after they were bent by the spectrometer magnetic field through $180^{\circ}$. The theory of the semicircular 


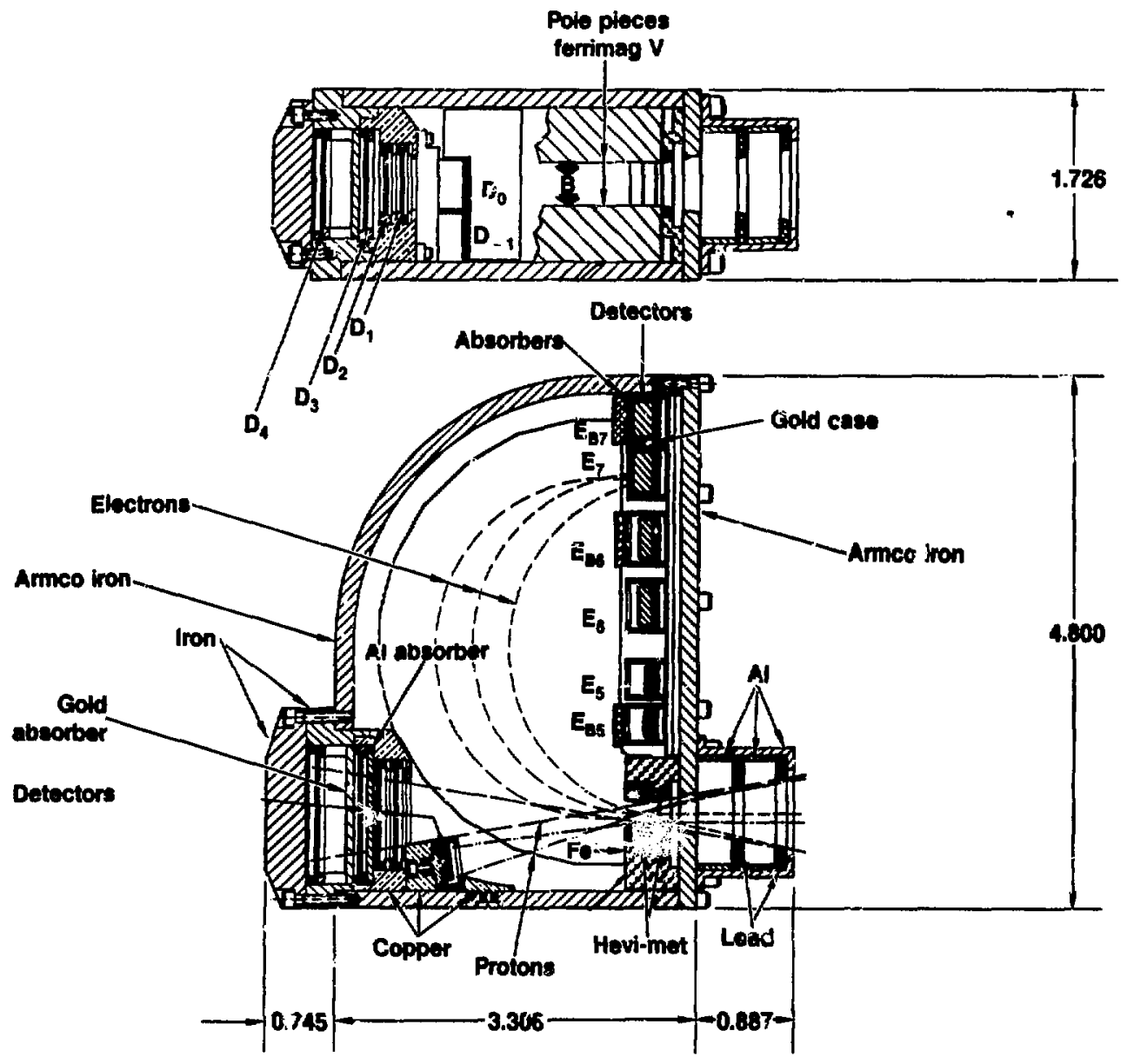

Figure 2-4. High-field $(B=.269$ tesla) electron spechometer and proton telescope. Election ewergy range: 822-2820 keV. Proton energy range: 0.1-94 MeV. (Dimensions in inches). [Weat et al., 1969]

Table 1. Electron channel sharacteristics, after [West et al., 1969].

\begin{tabular}{|c|c|c|c|c|}
\hline $\begin{array}{c}\text { Channel } \\
\text { No. }\end{array}$ & $\begin{array}{c}\text { Center Energy } \\
\text { (keV at } 0^{\circ} \mathrm{C} \text { ) }\end{array}$ & $\begin{array}{l}\text { Channel Width } \\
\text { (FWHM at } 0^{\circ} \mathrm{C} \text { ) }\end{array}$ & $\begin{array}{l}\text { Acceptance } \\
\text { angle (deg.) }\end{array}$ & $\begin{array}{l}\text { Geometrical Factor } \\
\mathrm{cm}^{2}-\mathrm{keV}_{-1} \text { itr } \\
\text { (at } 0^{\circ} \mathrm{C} \text { ) }\end{array}$ \\
\hline 1 & 39 & 22 & 7.6 & 0.180 \\
\hline 2 & 158 & 27 & 5.4 & 0.277 \\
\hline 3 & 266 & 21 & 4.7 & 0.590 \\
\hline 4 & 479 & 66 & 3.5 & 0.605 \\
\hline 5 & 822 & 250 & 5.3 & 4.43 \\
\hline 6 & 1530 & 352 & 4.1 & 8.57 \\
\hline 7 & 2820 & - & 2.5 & s.80 \\
\hline
\end{tabular}


magnetic spectrometer is well known (e.g., Siegbahn, 1955). Such a spectrometer sorts electrons by momentum, bringiris an initially-divergent pencil of monoenergetic electrons to an approximate focus in the plane of the spectrometer, after half a revolution in the field. The radius $r$ of the half-circular trajectory (ignoring variations in field strength) is given in MKS units by:

$r=p / e B$

where

$\mathrm{p}=$ electron momentum

$B=$ magnetic field strength

e = electronic charge.

In terms of the electron's kinetic energy $T$, the orbital radius for a central-ray electron is:

$r=\frac{1}{\mathrm{eBc}}\left[\left(E_{0}+T\right)^{2}-E_{0}^{2}\right]^{1 / 2}$

where $E_{0}=$ the electron rest energy, so that, for example, the center of detector $E 4(r=3.3 \mathrm{~cm})$ intercepts electrons of energy $\sim 480 \mathrm{keV}$.

\section{Electron Detectors}

Electrons incident on the detector array were counted by silicon solid-state detectors. For all but the two lowest energy channels, the depletion layer depth of each detector was matched to the range of the magnetically-selected electrons incident on the detector. The range-matching procedure minimized the detector response to background particles (electrons scattered inside the spectrometer and penetrating protons) which had energies different from the design energy. Background particles which had ranges significantly different from the depletion layer thickness produced pulses of lower amplitude; these small pulses were rejected by the thresholding discriminator in the pulse-handling electronics. To achieve the thick depletion depths required to match the ranges of the more energetic electrons, lithium drift detectors were required.

The depletion-layer thicknesses for the two lowest-energy channels were determined by signal/noise considerations. (Range-matching in this case would have resulted in thinner junctions with an undesirable increase in capacitance and noise.) These detectors were fabricated with diffused junctions, which offered greater resistance to radiation damage than those of the lithium-drift type. Because the typical radiation belt electron spectra decreases with energy, the fluxes incident on the lowest energy channel were often orders of magnitude larger than for the high-energy detectors, thus raising the possibility of radiation damage.

The effects of radiation damage appear as increased detector leakage current and decreased resolution. An increased leakage current decreases the detector bias voltage, leading to lower pulse amplitude and lower detector efficiency. The design included overbiasing the detectors by as much as a factor of two, to prevent this pulse amplitude degradation. Additionally, the radiation damage sites tend to recover over time, and since the satellite spent most of its time near apogee outside the radiation belts, accumulated radiation-induced damage was not thought to be a problem over the lifetime of the satellite.

\section{Calibration, Backgrounds, and Stability}

A considerable effort in the experimental design went towards ensuring that the flux measurements were accurate, that backgrounds were reduced as far as practicable and residual backgrounds were accurately measured, and that the electronic circuits which conditioned and counted the pulses from the detectors were stable.

The range of electron energies "seen" by each detector was determined by placing calibrai ed detectors of known energy response into the spectrometer detection plane. The spectrometer was then exposed 
to a broad-spectrum source, and the full-height pulses from the detectors were analyzed for the energy of the incident electron. This procedure mapped the slit-detector distance into electron energy.

The conversion from measured counting rates to directional particle fluxes (particles $/ \mathrm{cm}^{2}-\mathrm{sec}$ steradian-keV) incident on the spectrometer aperture required determination of the geometrical factor for each detector. In this case it can be expressed as:

\section{$A \Omega \delta$ in units of $\mathrm{cm}^{2}$-steradian-keV}

where
$A=$ effective area of the apertire;
$\boldsymbol{\Omega}=$ solid angle witich the detector subtends, viewed from a point in the aperture;
$\delta \mathrm{E}=$ effective energy range detected.

These quantities can be calculated from the spectrometer-detector geometry and the magnetic field. However, a more accurate procedure (which does not involve modeling trajectories in the detalled field) is to determine the geometric factor by in-situ measurement. A set of extended uniform sources of beta-ray emitters were placed over the aperture, and the geometric factor was straightforwardly obtained. This provided absolute geometric factors accurate to $\pm 15 \%$ for channel E1, and to $\pm 10 \%$ for channels E2-E7. The relative error between channels was a factor of two smaller.

Backgrounds were minimized by two levels of shielding. The iron spectrometer case and magnet assembly prevented electrons and bremsstrahlung from penetrating to the detectors, and stopped penetrating protons with $\mathrm{E}_{\mathrm{p}}<80 \mathrm{MeV}$. Close-in shielding was provided by a thin gold case around the detector. The gold case also tended to elastically scatter back into the detector electrons exiting the sides and front surface of the detector, thus increasing the counting efficiency of the detector and permitting better rejection of background signals.

These measures were effective in providing a large signal/background ratio through most of the spacecraft orbit. However, to ensure the highest quality data, residual backgrounds were accurately determined. Each detector was matched with an adjacent shielded detector of identical sensitive volume and construction, except that a lead absorber was placed over the background detector window to block signal electrons. Pulses from the background detectors were routed through the same amplification and counting stages as the signal channels to assure uniform handling.

Provisions were made to monitor the status of critical parameters of the detection systems while in fight, to check for system drift or malfunction. An inflight pulser was triggered to insert a train of pulses of increasing amplitude into each detector preamplifier. This allowed the checking of discriminator thresholds and the operation of the counting logic. Hall probes in each spectrometer returned measurements of the magnetic field strength, which could change due to temperature variation and aging. Detector leakage current was measured to assess cumulative radiation damage to the detectors. Temperatures of the electronics package and both spectrometer magnets were reported. Voltages were sampled at several places in the electronics package to indicate abnormalities.

\section{Pulse Production and Counting}

Charged particles incident on the detectors lost energy through inelastic Coulomb scattering in the semiconductor material, creating electron-hole pairs. Creation of each pair required about $1.1 \mathrm{eV}$ of incident particle energy. The pairs produced in the junction layer were collected by the detector, and produced a current pulse whose integral was proportional to the number of electron-hole pairs collected, and thus proportional to the energy lost by the incident particle in the sensitive volume. 


\section{Analog and Digital Electronics}

Detector output pulses were amplified by low-noise circuits and routed to a differential discriminator, which passed only puises with amplitudes in a specified range (coinciding with the peak of the pulsenumber vs amplitude distribution). This pulse thresholding reduced the probability of counting scattered electrons, bremsstrahlung, and penetrating protons (Fig. 2-5).

Pulses passed by each discriminator were routed to an accumulator, which accumulated (counted) pulses for a prescribed period. On command from the main spacecraft electronics, the accumulated counts for each detector were formatted into a floating-point digital word and loaded into a word of the telemetry frame (Fig. 2-6).

The frame was then either transmitted to earth ground stations in real time or stored on the spacecraft tape recorder for later transmission. Data were read out at one of three rates: 64, 8, or 1 kilobits/second. The $64 \mathrm{~Kb}$ rate offered the highest time resolution and was used for real-time transmission when the spacecraft was sufficiently close to an earth station that the signal strength permitted high-bandwidth transmission. At greater distances, the $\mathrm{B} \mathrm{Kb}$ or $1 \mathrm{~Kb}$ rate was used. In the record-to-tape mode, only the $1 \mathrm{~Kb}$ rate could be used.

The digital word used 9 bits to represent the number of accumulated counts, of which 5 bits were the mantissa and 4 bits the exponent. This yielded a dynamic range of $10^{6}$ counts.

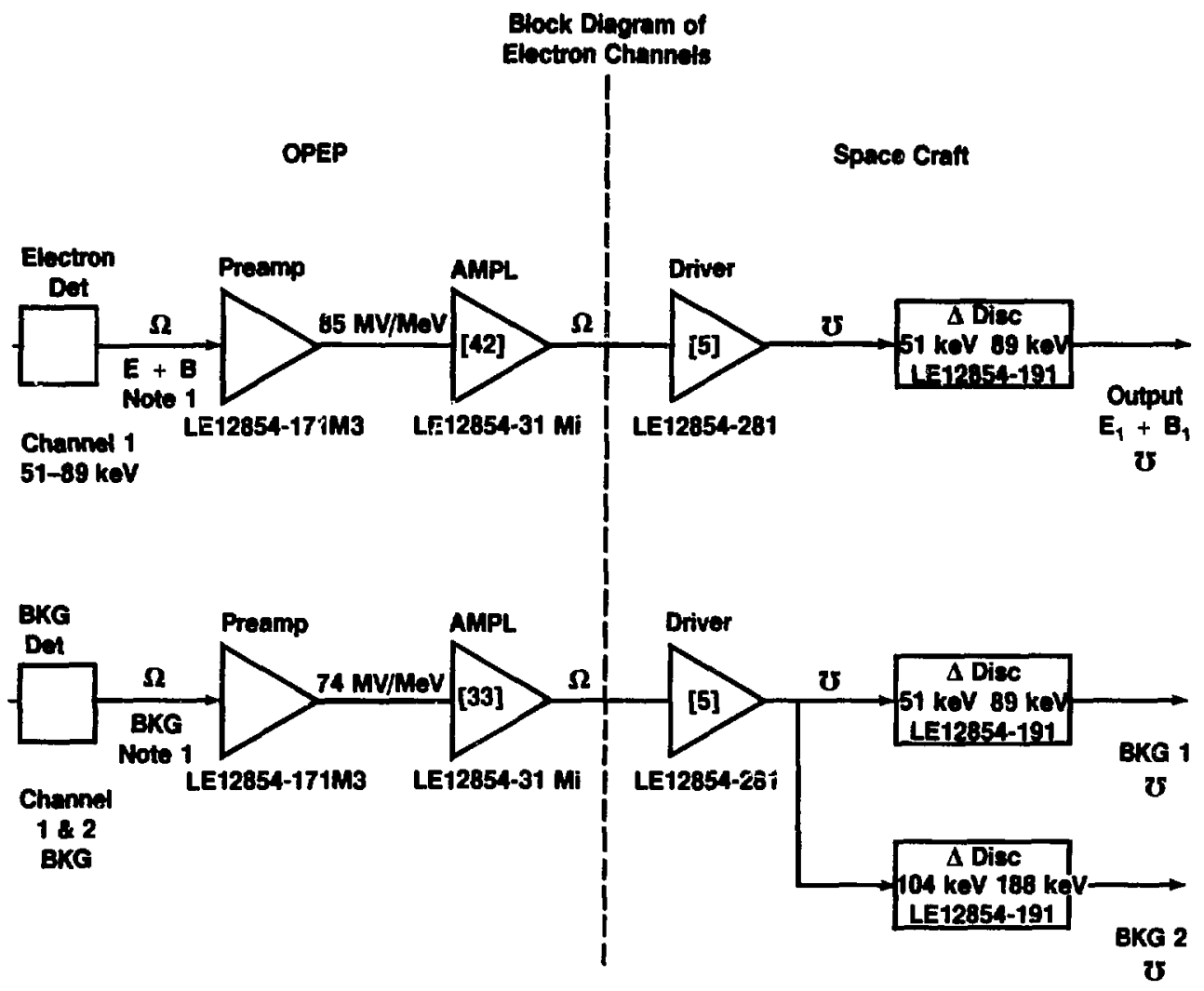

Figure 2-5. Block diagram of analog electronics for typical electron primary and background channel [West et al., 1969]. 


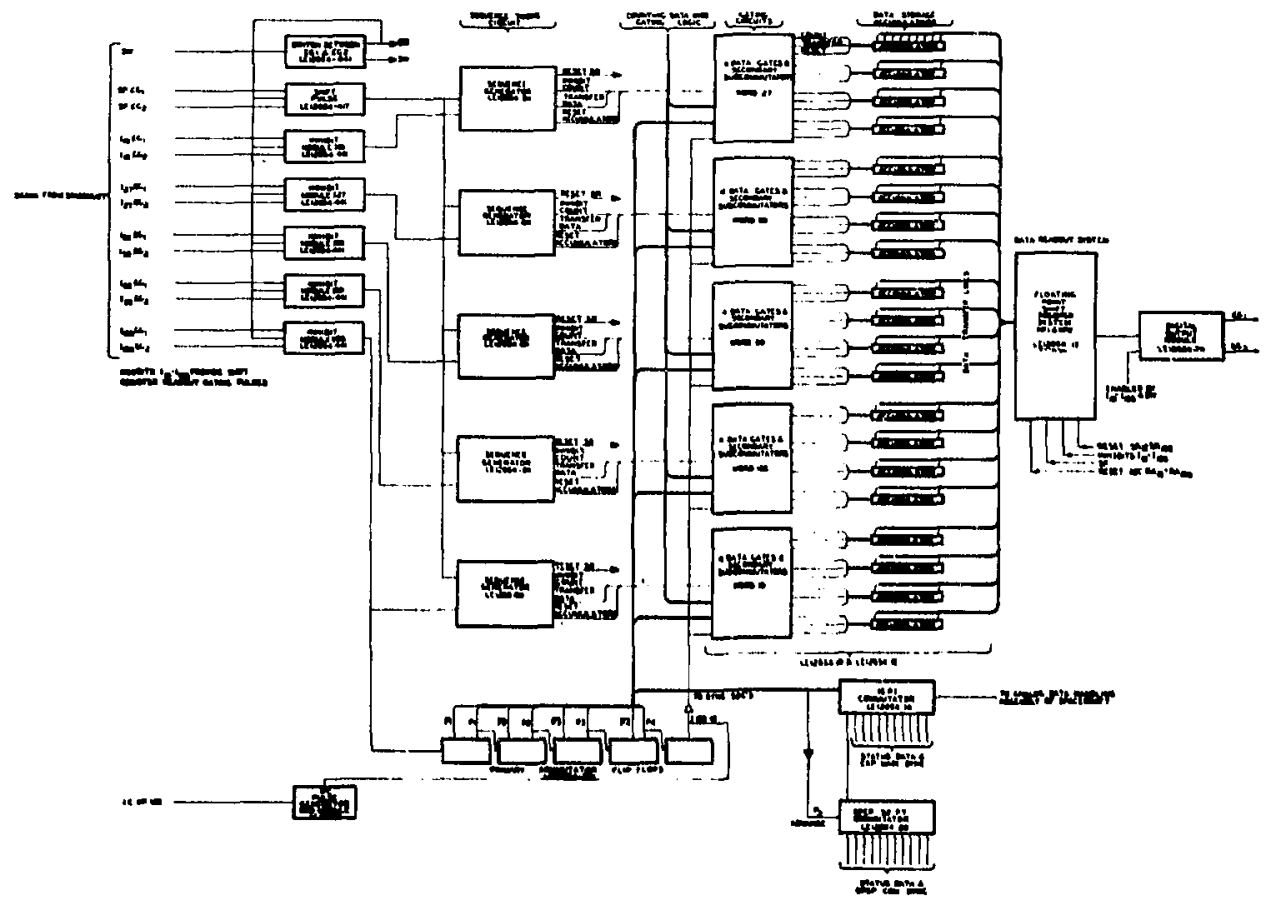

Figure 2-6. Block diagram of digital data-handling system for detector data. After [Weat et al., 1969].

\section{Proton Detection}

The proton detection system (Fig. 2-4) consisted of a proton telescope (a stack of 4 detectors and 2 absorbers) which spanned the energy range $0.23<\mathrm{E}_{\mathrm{p}}<94 \mathrm{MeV}$, and a separate detector for low-energy protons in the range $0.10<\mathrm{E}_{\mathrm{p}}<0.15 \mathrm{MeV}$. The proton detectors were placed in the high-field spectrometer so the field would divert the large fluxes of electrons of $E_{e}<4 \mathrm{MeV}$ from impinging on the proton system detectors. The spectrometer iron case shielded the detection system from penetrating eleclrons and from penetrating protons of $\mathrm{E}_{\mathrm{p}}<80 \mathrm{MeV}$.

The energy of a proton incident on the telescope was determined by analyzing the energy deposited in each of the 4 detector elements as the particle passed through the stack. Each detector put out a current pulse whose integral was proportional to the energy lost by the proton in transiting the detector's sensitive volume. Threshold discriminators sorted the detector pulses by energy into a set of pre-defined energy bands. Subsequent logic circuits combined the discriminator outputs in appropriate coincidence and anticoincidence arrangements to deduce the particle incident energy. Table 2 indicates the characteristics of each energy channel. The total telescope energy range $\left(0.23<\mathrm{E}_{\mathrm{p}}<94 \mathrm{MeV}\right)$ was thereby divided into 6 channels, approximately logarithmically spaced. The detectors were silicon diffused-junction solid-state detectors, which offered good energy resolution and resistance to radiation-induced damage.

\section{Proton Detector Calibration and Backgrounds}

The geometrical factor for each channel (required to convert counting rates into directional fluxes) were derived from measurements of detector areas and channel energy widths, and calculations of the 
Table 2. Proton channel characteriatics, after [Weat et al., 1969].

\begin{tabular}{ccc}
$\begin{array}{c}\text { Channel } \\
\text { No. }\end{array}$ & $\begin{array}{c}\text { Energy range } \\
\text { (MeV) }\end{array}$ & $\begin{array}{c}\text { Geometrical factor } \\
\text { (cm }\end{array}$ \\
\hline 1 & $0.10-0.15$ & $2.06 \times 10^{-3}$ \\
2 & $0.23-0.57$ & $1.30 \times 10^{-2}$ \\
3 & $0.57-1.35$ & $1.30 \times 10^{-2}$ \\
4 & $1.35-5.40$ & $1.30 \times 10^{-2}$ \\
5 & $5.60-13.3$ & $1.25 \times 10^{-2}$ \\
6 & $14.0-46.0$ & $1.72 \times 10^{-2}$ \\
7 & $43 .-94$. & $1.98 \times 10^{-2}$ \\
\hline
\end{tabular}

effective solid angle. For the latter, a computer code averaged the solid angle seen by differential areas of each detector over the detector area. Errors in geometrical factor determination appeared to be better than $10 \%$. The detection system responded also to protons and bremsstrahlung that penetrated the spectrometer case. To monitor these background fluxes, channels were designed to measure backgrounds in every energy range.

\section{Proton Detector Electronics}

Pulses from the proton detectors were amplified by multiple stages, then passed to integral discriminators which provided an output pulse for each discriminator threshold level exceeded. The logic circuits then combined the pulses with logical operations to produce counts in the appropriate energy channels. The counts were accumulated in registers as for the electron detection system, then periodically read out into the spacecraft telemetry frame.

\section{Scan-Angle Mechanisms}

The experiment required a scanning mechanism to sweep the spectrometer aperture over a range of angles with respect to the local magnetic field direction, thus acquiring pitch-angle distributions (PADs). Two mechanisms were provided. The OPEP shaft drive could operate in two modes: the scan mode, in which the shaft was slowly oscillated through $230^{\circ}$, at $1.7^{\circ} / \mathrm{s}$, and the "gyro" mode in which the shaft motor was occasionally stepped in order to keep the OPEP $x$-axis in the plane of the orbit, looking forward.

In addition, the OPEP-2 package had its own scan mechanism which could scan about the same axis as the OPEP shaft at a rate of $3 \% / \mathrm{s}$, through a range of $230^{\circ}$. During typical operations, this latter scan mechanism was the ore utilized.

\section{Scan-Angle Accuracy}

The shaft scan was not very accurately calibrated-the calibration table used to convert shaft-angle sensor voltage to degrees had errors as large as $\pm 5^{\circ}$. The OPEP-2 positioning error was much betterno larger than $0.5^{\circ}$. In the usual mode of operation (with the shaft in "gyro" mode and the OPEP-2 scan operating), the angular error was constant over extended periods of data acquisition. The error could usually be readily determined from the natural symmetry of the observed PADs about the $90^{\circ}$ pitch-angle position. The correction process-shifting the observed PADs so that the point of distribution symmetry fell at $90^{\circ}$-also served to correct for the errors in the determination of the magnetic field direction (by the UCLA magnetomete: experiment). Residual pitch-angle errors following the correction process are expected to be less than $2^{\circ}$. The studies reported herein require accuracies of the order of $5^{\circ}$. 


\section{Magnetic Field Measurement}

In order to determine the particle flux distribution as a function of local magnetic pitch-angle, vector B-field measurements were required. The UCLA magnetometer experiment provided accurate values of ambient B-field magnitude and direction throughout the orbit. The instrument design [Snare and Benjamin, 1966] was based on a three-axis fluxgate magnetometer. The instrument had sufficient dynamic range to measure fields from the surface of the earth $(\sim 30,000 \mathrm{nT})$ to interplanetary space $(\sim 1 \mathrm{nT})$, with a resolution of $1 / 16 \mathrm{nT}$.

\section{References}

Linder, H. G., Data Processing Plan for Eccentric Orbiting Geophysical Observatory (OGO-E), Publication X-565-68-19, Goddard Space Flight Center, Greenbelt, MD, 1968.

Siegbahn, K., Beta- and gamma-ray spectroscopy, North Holland Publishing Co., Amsterdam, 1955.

Snare, R. C. and C. R. Benjamin, A magnetic field instrument for the OGO-E spacecraft, IEEE Trans. Nuclear Science NS-13, 6, 333, 1966.

West, H. I. Jr., J. H. Wujeck, J. H. McQuaid, N. C. Jensen, R. G. D'Arcy, Jr, R. W. Hill, and R. M. Bogdanowicz, The LRL electron and proton spectrometer on NASA's Orbiting Geophysical Observatory V(E) (Instrumentation and Calibration), Lawrence Livermore National Laboratory, Livermore, CA, UCRL-50572, 1969. 


\section{Chapter 3. Theory of Charged Particle Motion in a Magnetic Field}

This chapter reviews the theory of charged particle trajectories in a magnetic field. In principle, knowledge of the electromagnetic field and the particle initial conditions coupled with the Lorentz force equation, provides all the information needed to calculutc the particle position at any subsequent time. However, the difficulty encountered in analytically solving the Lorentz equation for all but the simplest magnetic field configurations motivates the development of adiabatic invariants of the motion, via the Hamilton-Jacobi equation. These adiabatic integral invariants are seen to parameterize the particle orbit, allowing one to easily extrapolate a trajectory in a known field without first solving the equations of motion. In the magnetosphere, the adiabatic invariants form a simple basis for organizing particle flux measurements made over extended spatial regions.

\section{Equations of Motion of a Charged Particle in Static Electric and Magnetic Fields}

The motion of a charged particle in the presence of electric and magnetic fields is governed by the Lorentz force (Panofsky and Phillips, 1962):

$F_{L}=e(E+v \times B)$

where

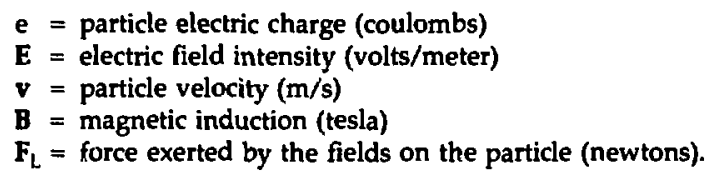

The instantaneous rate of change of relativistic momentum is then:

$\frac{d \mathbf{P}}{d t}=F_{L} \quad ; \quad P=\gamma m_{0} \frac{d r}{d t}$

where

$\mathbf{m}_{0}$ is the particle rest mass $(\mathrm{kg})$;

$\gamma$ is the relativistic factor $\gamma=1 /\left[1-(v / c)^{2}\right]^{1 / 2}$;

$c$ is the speed of light $(\mathrm{m} / \mathrm{s})$;

$r$ is the position vector from the origin to the particle $(m)$.

When Eq. (3.2) is expanded in Cartesian components, a set of three coupled second-order non-linear differential equations of motion is formed:

$$
\begin{aligned}
& \frac{d(\gamma \dot{x})}{d t}=\frac{e}{m_{0}}\left[E_{x}+\left(\dot{y} B_{z}-\dot{z} B_{y}\right)\right] \\
& \frac{d(\gamma \dot{y})}{d t}=\frac{e}{m_{0}}\left[E_{y}+\left(\dot{z} B_{x}-\dot{x} B_{z}\right)\right] \\
& \frac{d(\gamma \dot{z})}{d t}=\frac{e}{m_{0}}\left[E_{z}+\left(\dot{x} B_{y}-\dot{y} B_{x}\right)\right] .
\end{aligned}
$$


Because of this complexity, there exists no simple analytical solution for general B and E (Dragt, 1965). But for various special cases, the equations of motion can be readily solved.

\section{Static Magnetic Field}

In a static magnetic field and with $\mathrm{E}=0$, the equation of motion becomes:

$\frac{d P}{d t}=F_{L}=e(v \times B)$.

The kinetic energy of the particle is given by:

$\mathrm{T}=m c^{2}-\mathrm{m}_{0} \mathrm{c}^{2}=(\gamma-1) \mathrm{m}_{0} \mathrm{c}^{2}$.

The rate at which work is done by the field on the particle is:

$$
\begin{aligned}
\frac{d T}{d t} & =F_{L} \cdot v=e v \cdot(v \times B)=e(v \times v) \cdot B \\
& =0 \text { at all times. }
\end{aligned}
$$

Therefore a static field does not change the particle kinetic energy, and so $|v|$ and $\gamma$ are constants of the motion. The particle acceleration is:

$$
a=\frac{d v}{d t}=\frac{1}{\gamma m_{0}} \frac{d P}{d t}=\frac{e(v \times B)}{\gamma m_{0}}
$$

and $\mathbf{a}$ is perpendicular to both $\mathbf{v}$ and $B$.

\section{Homogeneous Magnetic Field}

If the magnetic field is static and also homogeneous, then the magnitude of the acceleration is a constant of the motion:

$$
\begin{aligned}
|a| & =\text { constant } \\
& =\frac{e v_{\perp} B}{\gamma m_{0}}
\end{aligned}
$$

where

$v_{\perp}$ is the component of $\mathbf{v}$ in the plane perpendicular to $\mathbf{B}$.

(Because the Lorentz force involves only the velocity component perpendicular to $B$, it is convenient from an analysis standpoint to decompose $\mathbf{v}$ into components parallel and perpendicular to $\mathbf{B}$. We can write $\mathbf{v}$ as:

$\mathbf{v}=\mathbf{v}_{\perp}+\mathbf{v}_{\boldsymbol{i}}$

where

$$
\mathbf{v}_{\|}=\text {parallel component }=\frac{(v \cdot B) B}{B^{2}}
$$

and

$$
\mathbf{v}_{\perp}=\text { perpendicular component }=\mathbf{v}-\mathbf{v}_{\mathrm{h}} \text { ) }
$$


The solution for motion in a plane at constant speed, with constant acceleration perpendicular to the velocity, is a circular trajectory with:

radius $\sigma=v_{\perp}^{2} / a ;$

angular frequency $\Omega=a / v_{\perp}$.

Therefore ir, the plane normal to B, the trajectory is a circle with gyroradius:

$\sigma=\frac{\gamma \mathbf{m}_{0} \mathbf{v}_{\perp}}{\mathbf{e B}}$

and gyrofrequency:

$\Omega=\frac{\mathrm{eB}}{\gamma \mathrm{m}_{0}}$

plus a rectilinear motion along $B$ with speed $\mathbf{v}_{\|}$.

\section{Particle Motion in General Fielda}

For the cases of external conservative forces, inhomogeneous static fields, and time-varying fields, the particle trajectory becomes increasingly complicated to treat exactly. However very useful results can be derived by the application of perturbation theory to the particle motion. In this theory, the effect of inhomogeneities and varying external forces are viewed as perturbations to the solution of the homogeneous-field case.

The motion is decomposed into two components:

1. A gyromotion of the particle in a (nearly) circular orbit in the plane perpendicular to $B$, about the guiding center point.

2. The motion of the guiding center itself.

This guiding-center theory was pioneered by Alfvén in studies of cosmic plasmas (Alfvén and Falthammar, 1963).

The guiding-center method produces a trajectory for the guiding-center and not for the particle. In many cases of interest in the magnetosphere, one is interested in trajertories which span distances large compared to a gyro-radius and hence the error in neglecting the displacement of the particle from its guiding center is negligible. The guiding-center approximation will be valid if a frame of reference can be found in which the particle motion is periodic in the plane perpendicular to $B$ for at least a full cycle (Roederer, 1970). Periodic in this sense means that in one period $\tau$, the particle orbit in the guiding-center coordinate system nearly closes on itself. The guiding-center geometry is illustrated in Fig. 3-1.

The position of the guiding center is:

$\mathbf{r}_{\mathrm{c}}=\mathbf{r}-\boldsymbol{\sigma}_{\mathrm{c}}$

where $\mathbf{r}$ is the instantaneous position vector of the particle and $\sigma_{c}=$ vector from the guiding center to $\mathbf{r}$.

In the guiding-center frame, the motion is nearly circular, so that

$\sigma_{c}=-\frac{m(v-u) \times B}{e B^{2}}$

where $\mathbf{u}$ is the velocity of the guiding-center frame (drift velocity).

In this frame, the particle has a gyration velocity:

$\mathbf{v}_{\mathrm{gy}}=\mathbf{v}-\mathbf{u}$. 


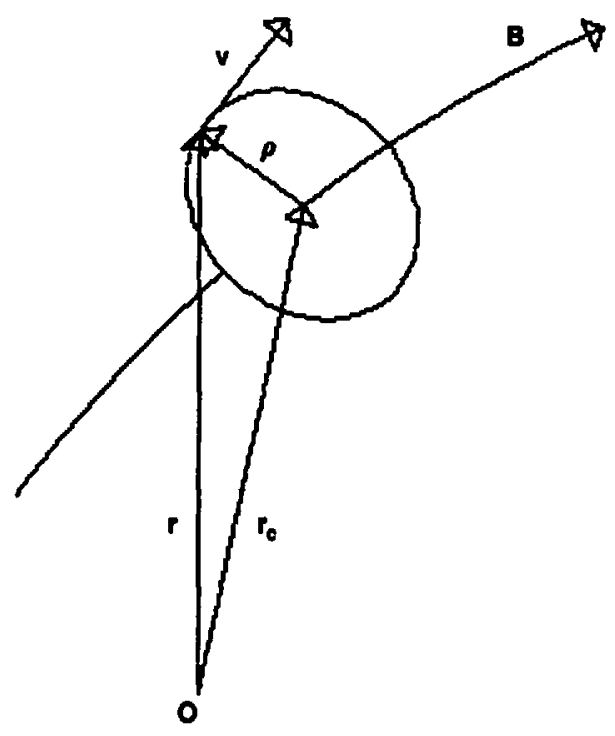

Figure 3-1. Guiding-center geometry for positively-charged particle.

\section{Constant Electric and Magnetic Fields}

If a constant electric field $\mathbf{E}$ is applied perpendicular to a constant magnetic field $\mathrm{B}$, the drift velocity $\mathbf{u}$ which results can be derived from the transformation properties of the electromagnetic field.

A Lorentz transformation applied to the covariant electromagnetic field tensor yields, for a coordinate system moving at uniform velocity $U$ with respect to a reference system, these expressions for the electric and magnetic fields in the moving frame (Panofsky and Phillips, 1962):

$$
\begin{aligned}
& \mathbf{E}_{\|}^{\prime}=\mathbf{E}_{\|} \\
& \mathbf{E}_{\perp}^{\prime}=\gamma\left(\mathbf{E}_{\perp}+\mathbf{U} \times \mathbf{B}\right) \\
& \mathbf{B}_{\|}^{\prime}=\mathbf{B}_{\|} \\
& \mathbf{B}_{\perp}^{\prime}=\gamma\left(\mathbf{B}_{\perp}-\frac{\mathrm{U} \times \mathbf{E}}{c^{2}}\right) \approx B_{\perp} \text { for }|\mathbf{U}| \ll c .
\end{aligned}
$$

Here E, B are the fields in the reference frame; E', B' are the fields in the moving frame. The subscripts \| and $\perp$ designate field components parallel and perpendicular to $U$, respectively.

If the moving-frame velocity $U$ is chosen to be:

$\mathbf{U}_{\mathrm{E}}=\frac{\mathbf{E} \times \mathbf{B}}{\mathrm{B}^{2}}$

then the electric field $\mathbf{E}^{\prime}$ vanishes identically (for $\mathbf{E} \cdot \mathbf{B}=0$ ), and there are only magnetic forces exerted on the particle. In this frame the motion of the particle is circular, which is the definition of the guiding center frame. Therefore the drift velocity $u$ must be $U_{E}$. 
The instantaneous non-relativistic kinetic energy of the particle is given by:

$$
\begin{aligned}
T & =\frac{1}{2} m v \cdot v=\frac{1}{2} m\left(u+v_{g y}\right) \cdot\left(u+v_{g y}\right) \\
& =\frac{1}{2} m\left(u^{2}+v_{g y}^{2}+2 u \cdot v_{g y}\right) .
\end{aligned}
$$

When $T$ is averaged over one gyrocycle, the $\mathbf{u} \cdot \mathbf{v}_{\mathrm{gy}}$ term vanishes, so that the mean total kinetic energy is just the sum of the drift energy of the guiding center ( as measured in the reference frame) plus the kinetic energy of gyration in the guiding-center frame.

The drift motion is normal to the electric field:

$\mathbf{E} \cdot \mathbf{u}=\mathbf{E} \cdot(\mathbf{E} \times \mathbf{B}) / \mathrm{B}_{2}=0$

therefore the drift trajectory is along an equipotential surface.

\section{Constant External Force}

The above results for an electrostatic field can be extended to the case of a general external force. Let $F=F_{\|}+F_{\perp}$ be a constant external force exerted on the particle.

The parallel and perpendicular equations of motions are:

$$
\begin{aligned}
& \frac{d P_{\|}}{d t}=F_{\|} \\
& \frac{d P_{\perp}}{d t}=F_{\perp}+e v \times B
\end{aligned}
$$

As in the case of an electric field, the perpendicular force $\mathbf{F}_{\perp}$ results in a drift of the gyrocenter in a direction perpendicular to $F$ and to $B$.

The guiding-center frame of reference will move at a velocity such that the transformation E-field $\mathbf{E}^{\prime}$ cancels the force $F_{\perp}$, so that the motion in the plane perpendicular to $B$ is again circular.

This condition $E^{\prime}=\frac{-F_{\perp}}{e}=\mathbf{u} \times \mathbf{B}$ yields the drift velocity:

$\mathbf{u}=\frac{\mathbf{F}_{\perp} \times \mathbf{B}}{\mathbf{e}^{B^{2}}} ; \quad$ limited to $\mathbf{u} \ll c$

If the generalized force is nut constant in space but is derivable from a potential function $\Phi$, the equations of motion (3.19a) and (3.19b) and the drift velocity Eq. (3.20) are still valid, provided that the change in particle energy over a cycle is small compared to the initial kinetic energy, i.e., if:

$\sigma \nabla \Phi \ll T$

\section{Inhomogeneous Magnetic Field}

If the magnetic field is inhomogeneous, the magnetic force on the particle will vary duing a gyrocycle. If the force is averaged over one cycle a net force results, which can play the role of the generalized external force in Eq. (3.19b). This allows the drift equation for a general force Eq. (3.20) to be used to evaluate the drift due to magnetic field gradients.

Let the gradient of the scalar field be written in terms of components parallel and perpendicular to the magnetic field at the guiding center:

$\nabla B=\nabla_{\perp} B+\nabla_{\|} B$ 
The field at the particle is then approximately given by:

$\mathbf{B}(\sigma)=\mathbf{B}_{0}+\left(\sigma \cdot \nabla_{\perp}\right) \mathbf{B}+$ terms of $\mathrm{O}\left[(\sigma \nabla \mathrm{B})^{2}\right]$.

The perpendicular force experienced by the particle can be written:

$F_{\perp}=e v \times B \approx e v \times\left[B_{0}+\left(\sigma \cdot \nabla_{\perp}\right) B\right]$

which can be decomposed into:

$F_{\perp}=F_{\perp 0}+F_{\text {GRAD }}=\left[\mathrm{ev} \times B_{0}\right]+\left[\mathrm{ev} \times\left(\sigma \cdot \nabla_{\perp}\right) B\right]$.

If Eq. (3.25) is averaged over a gyrocycle, then $\left\langle F_{\perp 0}\right\rangle=\left\langle e v \times B_{0}\right\rangle=0$.

The remaining force term is:

$\left\langle\mathbf{F}_{\mathrm{GRAD}}\right\rangle=\left\langle\mathrm{eV} \times\left(\sigma \cdot \nabla_{\perp}\right) \mathrm{B}\right\rangle$.

To evaluate this average, consider the magnetic field geometry of Fig. 3-2. From the figure, we have:

$B_{0}=-B_{0} \mathbf{k}$

$v_{\perp}=v_{\perp}[-\sin (\omega t) \hat{\mathbf{i}}+\cos (\omega t) \hat{f}]$

$\sigma=\sigma_{0}[\cos (\omega t) \hat{\imath}+\sin (\omega t) \hat{\jmath}] ;$ where $\sigma_{0}=\mathrm{mv}_{\perp} / \mathrm{eB}$

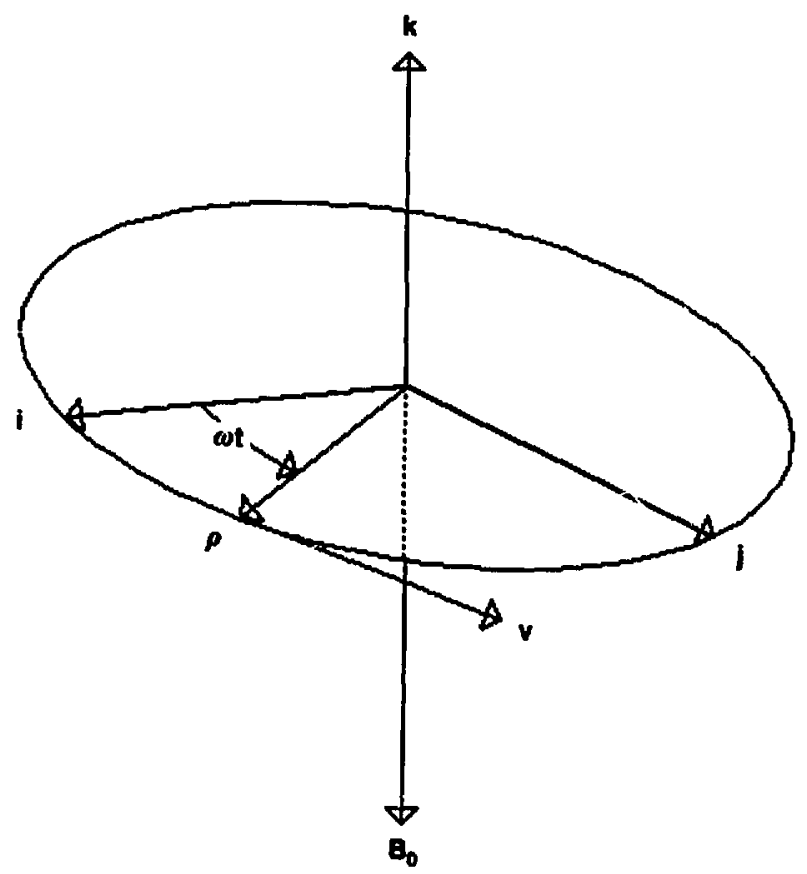

Figure 3-2. Magnetic field geometry for gradient force evaluation. Positive particle charge. 
This expression for $\sigma$ is an approximation valid when the change in $B$ over a gyrocycle is small, i.e., when $\sigma_{0} \nabla_{\perp} B / B \ll 1$.

Expanding the right-hand side of Eq. (3.26) in component form,

$\left(\sigma \cdot \nabla_{\perp}\right) B=-\sigma_{0}\left[\cos (\omega t) \frac{\partial}{\partial x}+\sin (\omega t) \frac{\partial}{\partial y}\right] B \hat{k} ;$

and

$v \times\left(\sigma \cdot \nabla_{\perp}\right) B=-v_{\perp} \sigma_{0}\left\{\left[\cos ^{2}(\omega t) \frac{\partial B}{\partial x}+\sin (\omega t) \cos (\omega t) \frac{\partial E}{\partial y}\right] \hat{\imath}+\left[\sin (\omega t) \frac{\cos (\omega t) \partial B}{\partial x}+\sin ^{2}(\omega t) \frac{\partial B}{\partial y}\right] \hat{i}\right\}$

Averaged over one gyrocycle, the mean values of the sinusoidal terms are:

$(\sin (\omega t) \operatorname{co}(\omega t))=0$;

$\left\langle\sin ^{2}(\omega t)\right\rangle=\left\langle\cos ^{2}(\omega t)\right\rangle=1 / 2$.

Then Eq. (3..6) becomes:

$\left\langle\mathbf{F}_{\text {GRAD }}\right)=-\frac{e v_{\perp} \sigma_{0}}{2}\left[\frac{\partial \mathrm{B}}{\partial \mathrm{x}} \hat{\imath}+\frac{\partial \mathrm{B}}{\partial \mathrm{y}} \hat{\mathrm{j}}\right]=-\frac{\mathrm{ev} \mathrm{v}_{\perp} \sigma_{0} \nabla_{\perp} \mathrm{B}}{2}$.

Substituting the average gradient-B force $\left\langle F_{C R A D}\right\rangle$ for the external force $F_{\perp}$ in Eq. (3.20). the gradient drift velocity $\mathbf{U}_{\mathrm{C}}$ tecomes:

$\mathbf{U}_{\mathrm{G}}=\frac{\left\langle\mathbf{F}_{\mathrm{GRAD}} \times \mathbf{B}\right.}{\mathrm{eB}^{2}}=\frac{\mathbf{m v}_{\perp}^{2} \mathbf{B} \times \nabla_{\perp} \mathrm{B}}{2 \mathrm{eB}^{3}}$

To this point, the results of guiding-center theory have been displayed for the drift forces which are most important in determining the trajectory of energetic charged particles in the static magnetosplere; i.e., electric field and gradient-B drifts. In the next section, the guiding-center theory is formulated in a more rigorous context in which the order of each force ter. $n$ is made explicit. forces.

First, it will be useful to compare the relative $n:-$ gnitudes of the drifts due to electric and magnetic

The ratio of electric-field force to gradient-B force in the plane perpendicular to $B$ is (nonrelativistically):

$\mathbf{r}_{\mathrm{EB}}=\frac{e E_{\perp}}{T_{\perp} \frac{\nabla_{\perp} B}{B}}$

where

$$
\mathrm{T}_{\perp}=\frac{1}{2} m v_{\perp}^{2}=\mathrm{eV}
$$

electron-volis, the particle's perpendicular kinetic energy.

For the electric-field drift to be ignorable, $r_{E B}$ must be $\ll 1$, or equivalently:

$E_{\perp} \ll v \frac{\left|\nabla_{\perp} B\right|}{B}$ 
Now $\mathrm{B} / \nabla_{\perp} \mathrm{B}$ is a measure of the length over which the magnetic field changes significantly, a distance we will take as the magnetic field scale length $L$.

Thus for sufficiently energetic particles, $V$ will satisfy the condition:

$V \gg L_{\perp}$

In a dipole magnetic field, the equatorial field strength is

$\mathrm{B}_{\mathrm{EQ}}=\frac{\mathrm{B}_{0}}{\mathbf{R}_{\mathrm{EQ}}^{3}}$

and

$L=\left|\frac{B}{\nabla_{\perp} B}\right|=\frac{R_{E Q}}{3}$.

The condition on V [Eq. (3.37)] becomes:

$V \gg \frac{E_{\perp} R_{E Q}}{3}$

The typical qua: $i$-steady state electric field in the outer dayside magnetosphere at $\mathbf{R}_{\mathfrak{E Q}}=9$ is usually less than $1 \mathrm{mv} / \mathrm{m}\left(6.4 \mathrm{kV} / \mathrm{R}_{\mathrm{E}}\right)$ [Smith et al.,1979]. For this case the condition on $\mathrm{V}$ is:

$\mathrm{V} \gg 19 \mathrm{kV}$.

For the particles measured by the OGO-5 E6 experiment ( $E_{E}>79 \mathrm{keV}, E_{P}>100 \mathrm{keV}$ ), this inequality is satisfied (if not by a wide margin) and field effects will be onitted in subsequent developments.

\section{The Guiding-Center Approximation}

\section{Guiding-Center Equations of Motion}

In this section we derive the charged-particle equations of motion in the guiding-center approximation. We begin with the Lorentz force equation (3.1). We first convert the variabies into a normalized form, so that the resulting equation is dimensionless. Then we expand the fields about the gyrocenter position in the normalized small paramp ${ }^{r} \rho / L$, where $\rho$ is the gyroradius vector and $L$ is the scale length of the fields. For the guiding-center approximation to hold, $\rho / L$ must be $\ll 1$, and both spatial and temporal changes to $B$ and $E$ must be small during one gyrocycle; i.e., we must have:

$\frac{\delta B_{t}}{B}=\frac{1}{B} \frac{\partial B}{\partial t} \tau=O(\epsilon) ;$

$\frac{\delta B_{x}}{B}=\rho \cdot \frac{\nabla B}{B}=O(\epsilon)$

$\frac{\delta \mathrm{E}_{\mathrm{t}}}{\mathrm{E}}=\frac{1}{\mathrm{E}} \frac{\partial \mathrm{E}}{\partial \mathrm{t}} \tau=\mathrm{O}(\epsilon) ;$

$\frac{\delta E_{x}}{E}=p \cdot \frac{\nabla E}{E}=O(\epsilon) ;$ 
where

$\epsilon=\rho / L \ll 1 ;$

Let us define the following normalized quantities:

$\mathbf{R}=\mathbf{r} / \mathrm{L} ;$

$\tilde{B}=\mathbf{B}(\mathbf{r}, t) / \mathrm{B}_{0}(t)$, where $\mathrm{B}_{0}(t)$ is the magnetic field at the initial point, evaluated at time $t$;

$T=v_{0} t / L$, where $v_{0}$ is the initial particle velocity;

$\tilde{E}=E(r, t) / v_{0} B_{0}$.

With these definitions, the normalized velocity $\mathrm{V}$ is:

$\mathbf{V}=\frac{\mathrm{dR}}{\mathrm{dT}}=\frac{1 / \mathrm{L}}{\mathrm{v}_{0} / \mathrm{L}} \frac{\mathrm{d} \mathbf{r}}{\mathrm{dt}}=\frac{\mathbf{v}}{\mathrm{v}_{0}}$

and the derivative operators are:

$\frac{d}{d T}=\frac{1}{v_{0} / L} \frac{d}{d t}$

$\frac{d}{d x}=\frac{d x}{d x} \frac{d}{d x}=L \frac{d}{d x}$.

Substituting these normalized variables, the Lorentz equation can be expressed in the dimensionless form:

$\frac{\rho_{0}}{\mathrm{~L}} \frac{\mathrm{d}^{2} \mathbf{R}}{\mathrm{dT}^{2}}=\tilde{\mathrm{E}}+\frac{\mathrm{dR}}{\mathrm{dT}} \times \tilde{\mathrm{B}}$

where $\rho_{0}=\frac{\mathrm{mv}_{0}}{\mathrm{eB}_{0}(\mathrm{t})}$, the gyro-radius corresponding to the normalizing values of $\mathrm{B}$ and $v$.

We now expand this equationi about the guiding-center position, retaining terms up through first order in $\epsilon$.

The expanded electric and magnetic fields are:

$B(\mathbf{r})=B\left(\mathbf{r}_{c}\right)+(\boldsymbol{\rho} \cdot \nabla) B\left(\mathbf{r}_{c}\right)+\frac{1}{2}(\boldsymbol{\rho} \rho: \nabla \nabla) B\left(\mathbf{r}_{c}\right)+\ldots$

$\mathbf{E}(\mathbf{r})=\mathbf{E}\left(\mathbf{r}_{c}\right)+(\boldsymbol{\rho} \cdot \nabla) \mathbf{E}\left(\mathbf{r}_{c}\right)+\frac{1}{2}(\rho \rho: \nabla \nabla) \mathbf{E}\left(\mathbf{r}_{c}\right)+\ldots$

where $\boldsymbol{p}$ is the gyroradius vector from the gyrocenter to the particle position $\mathbf{r}$ (Fig. 3-1).

To express these expansions in the norrialized variables, we note that

$\ddot{\nabla}=L \nabla$.

We have for $B$ in dimensionless form:

$\tilde{\mathbf{B}}(\mathbf{r})=\tilde{\mathbf{B}}\left(\mathbf{r}_{c}\right)+\frac{(\boldsymbol{\rho} \cdot \bar{\nabla}) \overline{\mathbf{B}}\left(\mathbf{r}_{c}\right)}{\mathbf{L}}+\frac{1}{2 \dot{L}^{2}}(\boldsymbol{\rho} \boldsymbol{\rho}: \bar{\nabla} \bar{\nabla}) \tilde{\mathbf{B}}\left(\mathbf{r}_{c}\right)$. 
If $\frac{\rho}{\mathrm{L}} \ll 1$ as required for the guiding-center theory to be valid, then the term:

$\frac{(\boldsymbol{\rho} \cdot \tilde{\nabla}) \overline{\mathbf{B}}}{\mathrm{L}}$

is of $O(\epsilon)$ while the term:

$\frac{(\boldsymbol{\rho} \boldsymbol{\rho}: \tilde{\nabla} \tilde{\nabla}) \tilde{B}}{2 L^{2}}$

is of $O\left(\epsilon^{2}\right)$. So to first order in the small expansion parameter $\epsilon$, we have:

$\tilde{\mathbf{B}}(\mathbf{r}) \approx \tilde{\mathbf{B}}\left(\mathbf{r}_{\mathbf{c}}\right)+\frac{(\boldsymbol{\rho} \cdot \tilde{\nabla}) \tilde{\mathbf{B}}\left(\mathbf{r}_{c}\right)}{\mathrm{L}}+O\left(\boldsymbol{\epsilon}^{2}\right)$

The expansion for $\mathbf{E}$ can be treated in a similar way to obtain:

$\tilde{\mathbf{E}}(\mathbf{r}) \approx \tilde{\mathbf{E}}\left(\mathbf{r}_{\mathrm{c}}\right)+\frac{(\boldsymbol{p} \cdot \tilde{\nabla}) \tilde{\mathbf{E}}\left(\mathbf{r}_{\mathrm{c}}\right)}{\mathrm{L}}+O\left(\boldsymbol{\epsilon}^{2}\right)$

Substituting these expressions for $\vec{B}$ and $\mathbf{E}$ into the Lorentz equation (3.47) forms:

$\frac{\left(\rho_{0}\right)}{\mathrm{L}} \frac{\mathrm{d}^{2} \mathbf{R}}{\mathrm{dT}^{2}}=\tilde{\mathbf{E}}\left(\mathbf{r}_{\mathrm{c}}\right)+\frac{(\boldsymbol{\rho} \cdot \tilde{\nabla}) \tilde{\mathbf{E}}\left(\mathbf{r}_{\mathrm{c}}\right)}{\mathrm{L}}+\frac{\mathrm{dR}}{\mathrm{dT}} \times \frac{\left[\tilde{\mathbf{B}}\left(\mathbf{r}_{\mathrm{c}}\right)+(\boldsymbol{\rho} \cdot \tilde{\nabla}) \tilde{\mathbf{B}}\left(\mathbf{r}_{\mathrm{c}}\right)\right]}{\mathrm{L}}+O\left(\epsilon^{2}\right)$

If we now substitute $\frac{r_{c}}{L}+\frac{\rho}{L}$ for $R$, we have the dimensionless expanded Lorentz equation:

$\frac{\left(\rho_{0}\right)}{L} \frac{\mathrm{d}^{2}\left(\mathbf{r}_{c}+\rho\right)}{\mathrm{dT}^{2} \mathrm{~L}}=\tilde{\mathbf{E}}\left(\mathbf{r}_{c}\right)+\frac{(\boldsymbol{\rho} \cdot \tilde{\nabla}) \tilde{\mathbf{E}}\left(\mathbf{r}_{c}\right)}{\mathrm{L}}+\frac{\mathrm{d}\left(\mathbf{r}_{\mathrm{c}}+\boldsymbol{\rho}\right)}{\mathrm{dT} \mathrm{L}} \times \frac{\left[\tilde{\mathbf{B}}\left(\mathbf{r}_{c}\right)+(\boldsymbol{p} \cdot \tilde{\nabla}) \tilde{\mathbf{B}}\left(\mathbf{r}_{c}\right)\right]}{\mathrm{L}}+O\left(\epsilon^{2}\right)$.

The guiding-center equation of motion which we seek is found by averaging Eq. (3.53) over one gyroperiod and discarding terms which are higher than first order in $\epsilon$. For this averaging process we construct a local coordinate system (Fig. 3-3) with origin at the gyrocenter $\mathbf{r}_{c}$, the $\mathbf{k}$-axis aligned parallel to $B\left(r_{c}\right)$, the $\hat{j}$-axis parallel to the initial gyroradius vector $\rho(t=0)$, and $\hat{i}$-axis defined to form a right-handed coordinate system. In this system the gyroradius vector and its time derivatives may be written:

$\rho(t)=\rho[\sin (\omega t) \hat{i}+\cos (\omega t) \hat{\jmath}]$

$\frac{d}{d T} \frac{(\rho)}{!}=\frac{1}{v_{0}}\{\rho[\sin (\omega t) \hat{i}+\cos (\omega t \hat{j}]+\rho \omega[\cos (\omega t) \hat{\imath}-\sin (\omega t) \hat{j}]]$

$\frac{\mathrm{d}^{2}}{\mathrm{dT}^{2}} \frac{(\boldsymbol{\mu})}{\mathrm{L}}=\frac{\mathrm{L}}{\mathrm{v}_{0}^{2}}\left[\left(\not \rho-\rho \omega^{2}\right)[\sin (\omega t) \hat{\mathrm{i}}+\cos (\omega t) \hat{\mathrm{j}}]+(2 \rho \omega+\rho \omega)[\cos (\omega t) \hat{\mathrm{i}}-\sin (\omega t) \hat{\mathrm{j}}]\right]$

In averaging these expressions over a gyrocycle, we treat coefficients like $\rho$ and $\phi$ as constants. The results for $\rho$ and its derivatives are:

$\langle\boldsymbol{p}\rangle=\langle\boldsymbol{\rho}\rangle=\langle\boldsymbol{\rho}\rangle=\mathbf{0}$.

Thus the left-hand side of Eq. (3.53) averages to: 


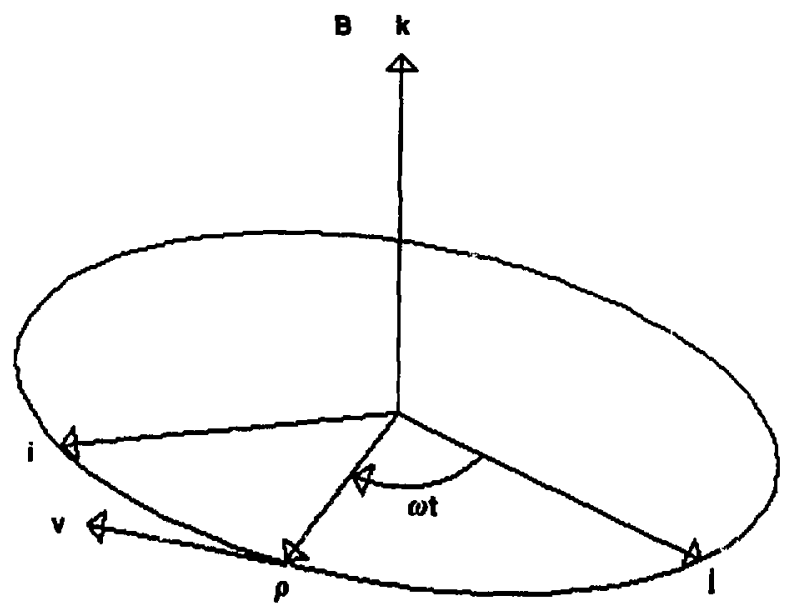

Figure 3-3. Local gyro-center coordinate aystem.

$\frac{\rho_{0}}{\mathrm{~L}} \frac{\mathrm{d}^{2}}{\mathrm{dT}^{2}} \frac{\left(\mathbf{r}_{\mathrm{c}}\right)}{\mathrm{L}}$

For the right-hand side we must avaluate these averages:

$\left\langle\tilde{\mathbf{E}}\left(\mathbf{r}_{\mathbf{c}}\right)\right\rangle$

$\left\langle\left(\frac{\boldsymbol{p}}{\mathbf{L}} \cdot \tilde{\nabla}\right) \tilde{\mathbf{E}}\left(\mathbf{r}_{\mathrm{c}}\right)\right)$

$\left\langle\frac{\mathrm{d}}{\mathrm{dT}} \frac{\left(\mathbf{r}_{\mathrm{c}}\right)}{\mathrm{L}} \times \tilde{\mathrm{B}}\left(\mathbf{r}_{\mathrm{c}}\right)\right)$

$\left\langle\frac{\mathrm{d}}{\mathrm{dT}} \frac{\left(\mathbf{r}_{\mathrm{c}}\right)}{\mathrm{L}} \times \frac{(\boldsymbol{\rho} \cdot \tilde{\nabla})}{\mathrm{L}} \tilde{\mathbf{B}}\left(\mathbf{r}_{\mathrm{c}}\right)\right\rangle$

$\left\langle\frac{\mathrm{d}}{\mathrm{dT}} \frac{(\boldsymbol{\rho})}{\mathrm{L}} \times \tilde{\mathrm{B}}\left(\mathbf{r}_{\mathrm{c}}\right)\right\}$

$\left\langle\frac{\mathrm{d}}{\mathrm{dT}} \frac{(\boldsymbol{\rho})}{\mathrm{L}} \times \frac{(\boldsymbol{\rho} \cdot \bar{\nabla})}{\mathrm{L}} \overline{\mathrm{B}}\left(\mathbf{r}_{\mathrm{c}}\right)\right\rangle$

These are evaluated as follows:

$\left\langle\tilde{\mathbf{E}}\left(\mathbf{r}_{\mathrm{c}}\right)\right\rangle=\tilde{\mathbf{E}}\left(\mathbf{r}_{\mathrm{c}}\right)$

$\left\langle\frac{(\boldsymbol{\rho} \cdot \dot{\nabla}) \hat{\mathbf{E}}\left(\mathbf{r}_{\mathrm{c}}\right)}{\mathrm{L}}\right)=\left\langle\frac{\rho}{\mathrm{L}}\left[\sin (\omega t) \dot{\nabla}_{x} \tilde{\mathbf{E}}=\cos (\omega t) \tilde{\nabla}_{y} \tilde{\mathbf{E}}\right]\right\rangle=0$ to $O(\theta)$.

$\left\langle\frac{\mathrm{d}}{\mathrm{dT}} \frac{\left(\mathbf{r}_{\mathrm{c}}\right)}{\mathrm{L}} \times \tilde{\mathrm{B}}\left(\mathbf{r}_{\mathrm{c}}\right)\right)=\frac{\mathrm{d}}{\mathrm{dt}} \frac{\left(\mathbf{r}_{\mathrm{c}}\right)}{\mathrm{L}} \times \tilde{\mathbf{B}}\left(\mathbf{r}_{\mathrm{c}}\right)$ 


$$
\begin{aligned}
\left\langle\frac{\mathrm{d}}{\mathrm{dT}} \frac{\left(\mathbf{r}_{\mathrm{c}}\right)}{\mathrm{L}} \times \frac{(\boldsymbol{\rho} \cdot \tilde{\nabla})}{\mathrm{L}} \tilde{\mathbf{B}}\left(\mathbf{r}_{\mathrm{c}}\right)\right\rangle & =\left\langle\frac{\mathrm{d}}{\mathrm{dT}} \frac{\left(\mathbf{r}_{\mathrm{c}}\right)}{\mathrm{L}} \times \frac{\rho}{\mathrm{L}}\left[\sin (\omega t) \tilde{\nabla}_{\mathrm{x}} \tilde{\mathrm{B}}+\cos (\omega t) \tilde{\nabla}_{\mathrm{y}} \tilde{\mathrm{B}}\right]\right) \\
& =0 \text { to } \mathrm{O}(\epsilon)
\end{aligned}
$$

since $\frac{d}{d T} \frac{\left(\mathbf{t}_{c}\right)}{L}, \tilde{\nabla}_{x} \tilde{\mathbf{B}}, \tilde{\nabla}_{\mathbf{y}} \tilde{\mathbf{B}}$ are constant vectors to order zero over a cycle.

$\left\langle\frac{\mathrm{d}}{\mathrm{dT}} \frac{(\rho)}{\mathrm{L}} \times \tilde{\mathrm{B}}\left(\mathbf{r}_{\mathrm{c}}\right)\right\rangle=\left\langle\frac{\rho \omega}{\mathrm{v}_{0}}[-\tilde{\mathrm{B}} \sin (\omega t) \hat{\mathbf{i}}-\tilde{\mathrm{B}} \cos (\omega t) \hat{i}]\right\rangle+\left\langle\frac{\rho}{v_{0}}[\tilde{\mathrm{B}} \cos (\omega t) \hat{\mathbf{i}}-\tilde{\mathrm{B}} \sin (\omega t) \hat{\jmath}]\right\rangle=0$.

$\left\langle\frac{\mathrm{d}}{\mathrm{dT}} \frac{(\rho)}{\mathrm{L}} \times \frac{(\rho \cdot \tilde{\nabla})}{\mathrm{L}} \tilde{\mathbf{B}}\left(\mathrm{r}_{\mathrm{c}}\right)\right\rangle=\left\langle\frac{\rho^{2} \omega}{\mathrm{v}_{0} \mathrm{~L}}[\cos (\omega t) \hat{\mathrm{i}}-\sin (\omega t) \hat{]}] \times\left[\sin (\omega t) \tilde{\nabla}_{\mathrm{x}}+\cos (\omega t) \tilde{\nabla}_{y}\right] \dot{\mathbf{B}}\right\rangle$

$$
+\left\langle\frac{\rho \rho}{v_{0} L}[\sin (\omega t) \hat{\mathbf{i}}+\cos (\omega t) \hat{\jmath}] \times\left[\sin (\omega t) \dot{\nabla}_{x}+\cos (\omega t) \dot{\nabla}_{y}\right] \tilde{\hat{s}}\right\rangle .
$$

We can reduce our effort in evaluating these averages by first examining the order of each term. By definition, $\rho / L=\epsilon$, and $\omega=v / \rho=v / L[1 / \epsilon]$.

The coefficient of the first term on the right side of Eq. (3.57) is $\frac{\rho^{2} \omega}{v_{0} L}$, which can be written as:

$\left[\frac{\rho}{L}\right]^{2}\left[\frac{L \omega}{v_{0}}\right]=O\left(\epsilon^{2}\right) \cdot O\left[\frac{1}{\epsilon}\right]=O(\epsilon)$

The second term on the right side is of $O\left[\frac{\rho \rho}{v_{0} L}\right]$.

To evaluate this, note that the change in $\rho$ over one gyrocycle is:

$\delta \rho=\rho \tau=\delta(\mathrm{mv} / \mathrm{eB})=\rho \delta \mathrm{v} / \mathrm{v}-\rho \delta \mathrm{B} / \mathrm{B}=\rho \cdot[$ term of $\mathrm{O}(\epsilon)]$

Hence $\rho \tau / \rho=O(\epsilon)$.

We can now write

$$
\begin{aligned}
\frac{\rho \tau}{\rho} & =\left[\frac{\rho}{\mathrm{v}_{0}}\right]\left[\frac{1}{\rho / \mathrm{L}}\right]\left[\frac{2 \pi}{\omega \mathrm{L} / \mathrm{v}_{0}}\right] \\
& =\left[\frac{\rho}{\mathrm{v}_{0}}\right]\left[\frac{1}{\epsilon}\right][2 \pi \epsilon]
\end{aligned}
$$

so $\rho / v_{0}=O(\rho \tau / \rho)=O(\epsilon)$.

Now the coefficient of the second term on the right side of Eq. (3.57f) can be written:

$\frac{\rho \rho}{\mathrm{L} \mathrm{v}_{0}}=(\rho / \mathrm{L})\left(\rho / \mathrm{v}_{0}\right)=\mathrm{O}(\epsilon) \cdot \mathrm{O}(\epsilon)$,

$$
=O\left(\epsilon^{2}\right) ;
$$


therefore the second term of Eq. (3.57f) can be neglected compared to the $O(f)$ first term.

Expanding the cross-product in the first term of Eq. (3.57), we have:

$$
\begin{aligned}
\frac{\rho^{2} \omega}{v_{0} L}\langle & -\hat{\imath}\left[\sin ^{2}(\omega t) \dot{\nabla}_{x} \bar{B}_{z}+\sin (\omega t) \cos (\omega t) \tilde{\nabla}_{y} \tilde{B}_{z}\right]-\hat{j}\left[\sin (\omega t) \cos (\omega t) \tilde{\nabla}_{x} \tilde{B}_{z}+\cos ^{2}(\omega t) \tilde{\nabla}_{y} \tilde{B}_{z}\right] \\
& \left.+\hat{k}\left[\sin (\omega t) \cos (\omega t) \tilde{\nabla}_{x} \tilde{B}_{y}+\cos ^{2}(\omega t) \tilde{\nabla}_{y} \tilde{B}_{y}+\sin ^{2}(\omega t) \dot{\nabla}_{x} \tilde{B}_{x}+\sin (\omega t) \cos (\omega t) \tilde{\nabla}_{y} \tilde{B}_{x}\right]\right\rangle .
\end{aligned}
$$

On taking the time-averages indicated, this becomes:

$\frac{\rho^{2} \omega}{2 v_{0} L}\left[-\hat{\mathrm{i}} \tilde{\nabla}_{x} \tilde{B}_{z}-\hat{j} \dot{\nabla}_{y} \tilde{B}_{z}+\hat{k}\left(\bar{\nabla}_{x} \tilde{B}_{x}+\tilde{\nabla}_{y} \tilde{B}_{y}\right)\right]$.

We note that the field is divergence-free,

$\dot{\nabla} \cdot \tilde{\mathbf{B}}=\left(\tilde{\nabla}_{x} \tilde{B}_{x}+\tilde{\nabla}_{y} \tilde{B}_{y}+\dot{\nabla}_{z} \tilde{B}_{z}\right)=0$,

so that the $k$ component of the above expression $=-\tilde{\nabla}_{z} \tilde{B}$.

With this substitution, the bracketed expression is seen to be just $-\tilde{\nabla} \tilde{B}$, and we have finally for the average of Eq. (3.57):

$\frac{-\rho^{2} \omega}{2 v_{0} L} \tilde{\nabla} \tilde{B}$

Collecting together all the averaged terms, we have for the dimensionless guiding-center equation of motion:

$\frac{\rho_{0}}{\mathrm{~L}} \frac{\mathrm{d}^{2}}{\mathrm{dT}^{2}} \frac{\left(\mathbf{r}_{\mathrm{c}}\right)}{\mathrm{L}}=\tilde{\mathbf{E}}\left(\mathbf{r}_{\mathrm{c}}\right)+\frac{\mathrm{d}}{\mathrm{dT}} \frac{\left(\mathbf{r}_{\mathrm{c}}\right)}{\mathrm{L}} \times \tilde{\mathrm{B}}\left(\mathbf{r}_{\mathrm{c}}\right)-\frac{\rho^{2} \omega}{2 \mathrm{v}_{0} \mathrm{~L}} \tilde{\nabla} \tilde{\mathrm{B}}\left(\mathbf{r}_{\mathrm{c}}\right)+\mathrm{O}\left(\boldsymbol{\epsilon}^{2}\right)$.

From Eq. (3.64) we can derive equations for the parallel and perpendicular motion of the guiding-center.

The parallel equation is formed by taking the inner product of $\mathrm{Eq}$. (3.64) with $\hat{k}$, the unit vector parallel to $\mathbf{B}\left(\mathbf{r}_{\mathrm{c}}\right)$.

$\hat{k} \cdot E_{q} \cdot(3.64)=\frac{\rho_{0}}{L}\left[\frac{d^{2}}{d T^{2}} \frac{\left(\mathbf{r}_{c}\right)}{L}\right]_{\|}=\tilde{E}_{\|}-\frac{\rho^{2} \omega}{2 v_{0} L} \tilde{\nabla}_{\|} \tilde{B}+O\left(\epsilon^{2}\right)$.

or

$\left[\frac{\mathrm{d}^{2}}{\mathrm{dT}^{2}} \frac{\left(\mathbf{r}_{c}\right)}{\mathrm{L}}\right]_{\|}=\left(\frac{1}{\rho_{0} / L}\right) \tilde{\mathrm{E}}_{\|}-\left(\frac{1}{\rho_{0} / L}\right) \frac{\rho^{2} \omega}{2 v_{0} L} \tilde{\nabla}_{\|} \dot{B}+O(\epsilon)$.

where the subscript $\|$ designates a component parallel to $\mathbf{B}$, i.e., $\nabla_{\|}=\hat{k} \cdot \tilde{\nabla}$.

In this form it is evident that the parallel electric field force is of $O(1 / \epsilon)$ while the parallel magnetic force is $\mathrm{O}(0)$. A more understandable physical form of the parallel equation of motion is produced by expressing Eq. (3.65) in the original (unscaled) variables, yielding:

$\mathbf{y}_{\mathrm{c}} \cdot \hat{\mathrm{k}}=\frac{\mathrm{e}}{\mathrm{m}}\left[\mathrm{E}_{\|}-\frac{\rho^{2} \omega}{2} \nabla_{\|} B\right]$.

The parallel magnetic force can be expanded in terms of the particle's magnetic moment $\mu$, defined as the product of the equivalent current of the circulating particle and the area of the "current loop" or gyrocircle, 
$\mu=\mathrm{I}\left(\pi \rho^{2}\right)=\left(\frac{e \omega}{2 \pi}\right)\left(\pi \rho^{2}\right)=\frac{e \rho^{2} \omega}{2} ;$

and Eq. (3.66) becomes the Guiding-Center Parallel Equation of Motion:

$\mathbf{r}_{\mathbf{c}} \cdot \hat{\mathbf{k}}=\frac{\mathbf{e}}{\mathrm{m}} \mathbf{E}_{\|}-\frac{\mu}{\mathrm{m}} \nabla_{\hat{k}} B$

The magnetic field gradients along a field line provide a restoring force that can eventually arrest and reverse the motion of the guiding center, thus leading to the well-known oscillation of a charged particle trapped in a region between two high-field magnetic "mirrors". The magnetospheric magnetic field is one example of a naturally occurring mirror field that effectively traps charged particles over a wide range of energies.

In models of magnetospheric electric fields, quasi-static parallel electric fields are assumed not to exist (e.g., Pedersen and Grard, 1979) because of the extremely large conductivity along field lines. Such fields would produce large persistent field-aligned currents of accelerated electrons and protons, an effect not observed by in-situ measurements.

The guiding-center motion in the plane perpendicular to $\mathbf{B}$ is found by forming the vector product of $\hat{k}$ and Eq. (3.64).

$\hat{k} \times E_{q} \cdot(3.64)=\hat{k} \times \frac{\rho_{0}}{L} \frac{d^{2}}{d^{2} T^{2}} \frac{\left(\mathbf{r}_{c}\right)}{L}=\hat{k} \times \hat{E}\left(\mathbf{r}_{c}\right)+\hat{k} \times\left[\frac{d}{d T} \frac{\left(\mathbf{r}_{c}\right)}{L} \times \hat{B}\left(\mathbf{r}_{c}\right)\right]-\hat{k}$

$$
\times \frac{\rho^{2} \omega}{2 v_{0} L} \tilde{\nabla} \tilde{B}\left(\mathbf{r}_{c}\right)+O\left(\epsilon^{2}\right)
$$

Now

$\hat{k} \times\left[\frac{d}{d T} \frac{\left(r_{c}\right)}{L} \times \tilde{B}\right]=\frac{d}{d T} \frac{\left(r_{c}\right)}{L} \bar{B}-\tilde{B}\left[\frac{d}{d T} \frac{\left(r_{c}\right)}{L}\right]_{l}=\tilde{B}\left[\frac{d}{d T} \frac{\left(r_{c}\right)}{L}-\left[\frac{d}{d T} \frac{\left(r_{c}\right)}{L}\right]_{\|} \hat{k}\right]=\tilde{B}\left[\frac{d}{d T} \frac{\left(r_{c}\right)}{L}\right]_{\perp}$.

With this substitution Eq. (3.69) becomes the Guiding-Center Perpendicular Equation of Motion:

$\hat{k} \times \frac{\rho_{0}}{\mathrm{~L}} \frac{\mathrm{d}^{2}}{\mathrm{dT}^{2}} \frac{\left(\mathbf{r}_{\mathrm{c}}\right)}{\mathrm{L}}=\hat{\mathrm{k}} \times \tilde{\mathbf{E}}\left(\mathbf{r}_{\mathrm{c}}\right)+\tilde{\mathbf{B}}\left(\mathbf{r}_{\mathrm{c}}\right)\left[\frac{\mathrm{d}}{\mathrm{dT}} \frac{\left(\mathbf{r}_{\mathrm{c}}\right)}{\mathrm{L}}\right]_{\perp}-\frac{\rho^{2} \omega}{2 \mathrm{v}_{0} \mathrm{~L}} \hat{\mathrm{k}} \times \tilde{\nabla} \tilde{\mathrm{B}}\left(\mathbf{r}_{\mathrm{c}}\right)+O\left(\boldsymbol{\epsilon}^{2}\right)$.

\section{Guiding-Center Drifts}

In this section we use the parallel Eq. (3.68) and perpendicular Eq. (3.70) guiding-center equations of motion to derive the guiding-center drift velocity $U_{\perp}$. Knowledge of $U_{\perp}$ will allow us to determine the average motion of a test particle in a model magnetospheric field from local field properties (gradient and field-line curvature). For simple dipolar fields, drift velocities have been extensively tabulated by analytically evaluating $U_{\perp}$ (Hamlin, et. al. 1961). For the more complex fields of the outer magnetosphere, recourse must be had to numerical evaluations of model fields.

We define $\tilde{\mathrm{U}}_{\perp}$ to be $\left[\frac{\mathrm{d}}{\mathrm{dT}} \frac{\left(\boldsymbol{r}_{\mathrm{c}}\right)}{\mathrm{L}}\right]_{\perp}$ the dimensionless perpendicular drift velocity. By rearranging Eq. (3.70), we can write for the drift velocity:

$\tilde{\mathbf{U}}_{\perp}=-\frac{\hat{\mathbf{k}} \times \hat{\mathbf{E}}}{\hat{\mathrm{B}}}+\frac{\rho^{2} \omega}{2 \mathrm{v}_{0} \mathrm{~L}} \hat{\mathrm{K}} \times \frac{\hat{\nabla} \tilde{\mathrm{B}}}{\hat{\mathrm{B}}}+\frac{\rho_{0}}{\mathrm{~L} \tilde{\mathrm{B}}} \hat{\mathrm{k}} \times \frac{\mathrm{d}^{2}}{\mathrm{dT}^{2}} \frac{\left(\mathbf{r}_{\mathrm{c}}\right)}{\mathrm{L}}+\mathrm{O}\left(\epsilon^{2}\right)$.

The first term on the right side is the electric field drift velocity:

$\tilde{\mathbf{U}}_{\mathrm{E}}=\frac{\overline{\mathbf{E}} \times \tilde{\mathbf{B}}}{\tilde{\mathbf{B}}^{2}}$; 
which we derived earlier from considerations of the transformation properties of the electromagnetic field. In terms of the expansion parameter $\epsilon$, it is of $O(0)$.

The second term in Eq. (3.71) is the gradient-B drift velocity:

$\tilde{\mathbf{U}}_{\mathrm{G}}=\frac{\rho^{2} \omega}{2 \mathrm{v}_{0} \mathrm{~L}} \frac{\tilde{\mathbf{B}} \times \tilde{\nabla} \tilde{B}}{\tilde{\mathrm{B}}^{2}}$.

This term is of $O(\epsilon n)$, since we have shown above that $\frac{\rho^{2} \omega}{v_{0} L}$ is of $O(\epsilon)$.

In dimensional form, Eq. (3.72) is

$\mathbf{U}_{\mathrm{G}}=\frac{\mathbf{m v _ { \perp } ^ { 2 }} \mathbf{B} \times \nabla \mathbf{B}}{2 \mathrm{eB}^{3}}$.

The final term in Eq. (3.71) is the acceleration drift:

$\tilde{U}_{A}=\frac{P_{0} \tilde{B}}{L \tilde{B}^{2}} \times \frac{d^{2}}{\mathrm{dT}^{2}} \frac{\left(\boldsymbol{r}_{\mathrm{c}}\right)}{\mathrm{L}}=\mathrm{O}(\boldsymbol{\epsilon}) \cdot \mathrm{O}\left[\frac{\mathrm{d}^{2}}{\mathrm{dT}^{2}} \frac{\left(\mathrm{r}_{\mathrm{c}}\right)}{\mathrm{L}}\right]$.

Since we are interested only in drifts through first order in $\epsilon$, we need only keep the order zero terms in $\frac{\mathrm{d}^{2}}{\mathrm{dT}^{2}} \frac{\left(\mathbf{r}_{\mathrm{c}}\right)}{\mathrm{L}}$ for $\tilde{U}_{\mathrm{A}}$ to be of $\mathrm{O}(\boldsymbol{\epsilon})$.

We will therefore write the gyrocenter acceleration in terms of the rate of change of the zeroth-order velocities:

$$
\begin{aligned}
& \frac{\mathrm{d}^{2}}{\mathrm{dT}^{2}} \frac{\left(\mathbf{r}_{\mathrm{c}}\right)}{\mathrm{L}}=\frac{\mathrm{d}}{\mathrm{dT}}\left(\tilde{\mathbf{v}}_{\|} \hat{\mathbf{k}}+\tilde{\mathrm{U}}_{\mathrm{E}}\right)+\mathrm{O}(\boldsymbol{\epsilon}) \\
& =\frac{\hat{k}_{\mathrm{d}} \tilde{\mathbf{v}}_{\mathbb{B}}}{\mathrm{dT}}+\tilde{\mathrm{v}}_{\mathbb{H}} \frac{\mathrm{d} \hat{\mathrm{k}}}{\mathrm{dT}}+\frac{\mathrm{d} \tilde{\mathrm{U}}_{\mathrm{E}}}{\mathrm{dT}}
\end{aligned}
$$

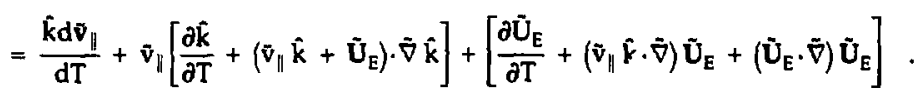

which expands to:

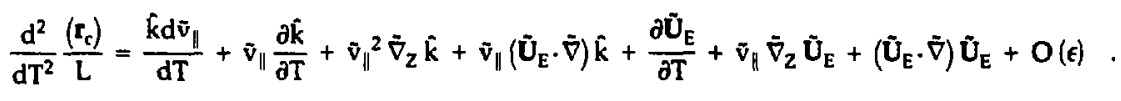

When we substitute this expansion of the gyrocenter acceleration into the normalized drift velocity Eq. (3.71) we obtain:

$$
\begin{aligned}
& \tilde{\mathbf{U}}_{\perp}=\frac{\tilde{\mathbf{E}} \times \tilde{\mathbf{B}}}{\tilde{\mathrm{B}}^{2}}+\frac{\rho^{2} \omega}{2 \mathbf{v}_{0} \mathrm{~L}} \frac{\tilde{\mathbf{B}} \times \tilde{\nabla} \tilde{\mathbf{B}}}{\tilde{\mathrm{B}}^{2}}+\frac{\rho_{0}}{\mathrm{LB}^{2}} \tilde{\mathbf{B}} \times\left[\mathrm{v}_{\|} \frac{\partial \hat{\mathrm{k}}}{\partial \mathrm{T}}+\tilde{\mathbf{v}}_{\|}{ }^{2} \tilde{\nabla}_{Z} \hat{\mathrm{k}}+\tilde{\mathrm{v}}_{\|}\left(\tilde{\mathrm{U}}_{\mathrm{E}} \cdot \tilde{\bar{\nabla}}\right) \hat{\mathbf{k}}\right. \\
& \left.+\frac{\partial \tilde{U}_{E}}{\partial T}+\tilde{v}_{\|} \tilde{\nabla}_{Z} \tilde{U}_{E}+\left(\tilde{U}_{E} \cdot \tilde{\nabla}\right) \tilde{\mathbf{U}}_{\mathrm{E}}\right]+O\left(\epsilon^{2}\right)
\end{aligned}
$$

For the typical magnetospheric case where the fields are quasi-static and $E$ is of $O(\epsilon)$, the guidingcenter drift velocity approximation becomes:

$$
\tilde{\mathbf{U}}_{\perp}=\frac{\tilde{\mathrm{E}} \times \tilde{\mathrm{B}}}{\tilde{\mathrm{B}}^{2}}+\frac{\rho^{2} \omega}{2 \mathbf{v}_{0} \mathrm{~L}} \frac{\tilde{\mathrm{B}} \times \tilde{\nabla} \tilde{\mathrm{B}}}{\tilde{\mathrm{B}}^{2}}+\frac{\rho_{\mathrm{J}}}{\mathrm{LB}^{2}} \tilde{\mathrm{B}} \times \tilde{\mathbf{v}}_{\mathrm{l}}^{2} \tilde{\nabla}_{\mathrm{Z}} \hat{\mathrm{k}}+\mathrm{O}\left(\epsilon^{2}\right)
$$


The surviving acceleration term in the drift-velocity expression represents the centrifugal acceleration experienced by the gyrocenter by virtue of its parallel motion along a curved field line. The drift velocity associated with this term is denoted the "curvature drift." This drift velocity can be expressed in terms of the perpendicular gradient of $B$, and then combined with the other $\bar{\nabla} \bar{B}$ ierm to yield a cr npact expression for the net magnetic-field-induced drifts (to first order in $\epsilon$ ).

We first establish a very useful relation between the local magnetic field-line curvature and the perpendicular gradient of B. [This derivation follows that of Roederer (1970)].

Let a local Cartesian coordinate system be set up as in Fig. 3-4, so that the magnetic field line $P_{1}-P_{2}$ lies entirely in the $y z$ plane, and $B\left(P_{1}\right)$ is parallel to $z$. Let $R_{c}$ be the local radius of curvature of the field line, extending from the center of curvature $C$ to a field point. $\hat{k}$ is a unit vector parallel to $B$.

Evaluated at $P_{1}$,

$$
\begin{aligned}
\nabla B & =\left[\frac{\hat{x} \partial}{\partial x}+\frac{\hat{y} \partial}{\partial y}+\frac{\hat{z} \partial}{\partial z}\right] B_{z} ; \\
& =\frac{\partial B_{z}}{\partial x} \hat{x}+\frac{\partial B_{z}}{\partial y} \hat{y}+\frac{\partial B_{z}}{\partial z} \hat{z} .
\end{aligned}
$$

In the absence of local currents, $\nabla \times B=0$, which implies that:

$$
\begin{aligned}
& \frac{\partial B_{z}}{\partial y}=\frac{\partial B_{y}}{\partial z} ; \\
& \frac{\partial B_{y}}{\partial x}=\frac{\partial B_{z}}{\partial x}=0 ;
\end{aligned}
$$

since by assumption $B$ is independent of $x$, and Eq. (3.79) becomes:

$\nabla B=\nabla_{\perp} B+\nabla_{\|} B=\frac{\partial B_{y}}{\partial z} \hat{y}+\frac{\partial B_{z}}{\partial z} \hat{z}$.

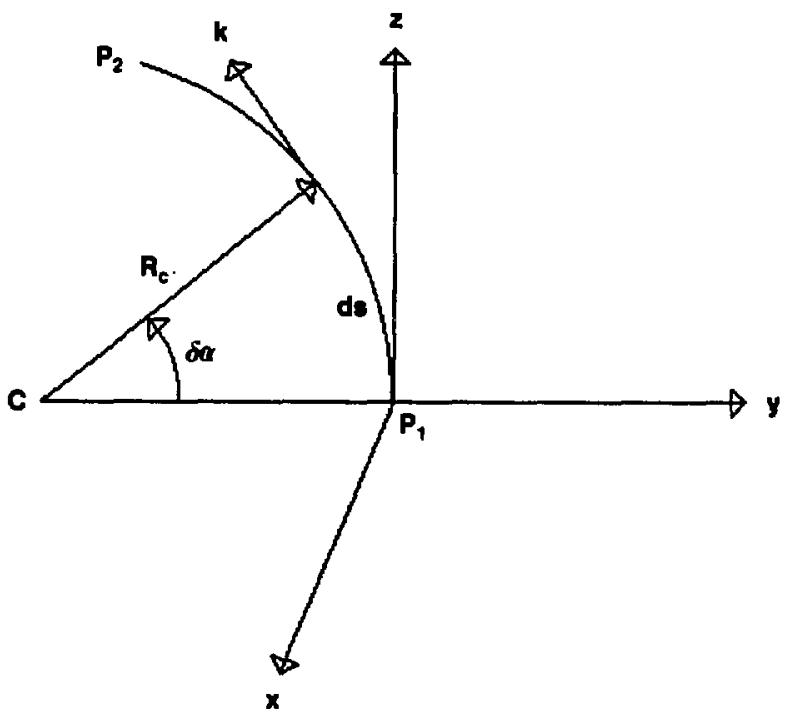

Figure 3-4. Local coordinate system for evaluating magnetic field-line coordinates. Field line $\mathbf{P}_{1}-\mathbf{P}_{\mathbf{2}}$ lies in the yz plane. $\mathbf{L}\left(\mathbf{P}_{\mathbf{1}}\right)=$ B z. $\mathbf{k}\left(\mathbf{R}_{\mathrm{C}}\right)=\boldsymbol{A}\left(\mathbf{R}_{\mathrm{C}}\right) / \mathrm{B}$. $\mathrm{C}$ is local center of curvature. 
In the $\mathbf{R}_{c}, \hat{\mathbf{k}}$ coordinate system,

$\mathbf{R}_{\mathrm{c}}=\mathbf{R}_{\mathrm{c}}[\cos \delta \boldsymbol{\alpha} \hat{\mathbf{y}}+\sin \delta \alpha \hat{\mathbf{z}}]$

$\hat{\mathrm{k}}=[-\sin \delta \alpha \hat{\mathrm{y}}+\cos \delta \alpha \hat{\mathrm{z}}]$

and we can write for the perpendicular gradient magnitude

$\left|\nabla_{\perp} \mathrm{B}\right|=\frac{\partial \mathrm{B}_{\mathrm{y}}}{\partial \mathrm{z}} \approx \frac{\partial \mathrm{B}}{\partial \mathrm{s}} \cdot \frac{\mathrm{R}_{\mathrm{c}}}{\mathrm{R}_{\mathrm{c}}}$.

where $d s$ is a differential step along the field line $d s=R_{r} \delta \alpha$.

$\frac{\partial B}{\partial s} \cdot \frac{R_{c}}{R_{c}}=\left[B \frac{\partial \hat{k}}{\partial s}+\hat{k} \frac{\partial B}{\partial s}\right] \cdot \frac{R_{c}}{R_{c}}=B \frac{\partial \hat{k}}{\partial s} \cdot \frac{R_{c}}{R_{c}}$.

so

$\nabla_{\perp} B=B \frac{\partial \hat{k}}{\partial s}$

The $\nabla_{\mathrm{z}} \mathrm{k}$ factor in the third term of Eq. (3.78) can therefore be written:

$\nabla_{\mathbf{z}} \hat{\mathrm{k}}=\frac{\partial \hat{\mathrm{k}}}{\partial \mathrm{z}}=\frac{\nabla_{1} \mathrm{~B}}{\mathrm{~B}}$

We can now write the curvature drift velocity as

$\tilde{\mathbf{U}}_{\mathrm{C}}=\frac{\rho_{0} \tilde{\mathbf{v}}_{\|}{ }^{2} \tilde{\mathbf{B}}}{\mathrm{L} \tilde{\mathrm{B}}^{2}} \times \frac{\bar{\nabla}_{\perp} \tilde{\mathrm{B}}}{\overline{\mathrm{B}}}=\frac{\rho_{0} \tilde{\mathbf{v}}_{\|}{ }^{2}}{L} \frac{\tilde{\mathrm{B}} \times \tilde{\nabla} \tilde{\mathrm{B}}}{\tilde{\mathrm{B}}^{3}}$.

In dimensional form, Eq. (3.88a) is

$\mathbf{U}_{C}=\frac{m v_{\|}^{2}}{\mathbf{e}} \frac{\mathbf{B} \times \nabla \mathbf{B}}{\mathbf{B}^{3}}$

We combine Eqs. (3.73) and (3.88b) to obtain the total guiding-center drift due to first-order magnetic forces:

$\mathrm{U}_{\mathrm{B}}=\mathrm{U}_{\mathrm{G}}+\mathrm{U}_{\mathrm{C}}=\frac{\mathrm{m}}{2 \mathrm{e}}\left[\mathrm{v}_{\perp}^{2}+2 \mathrm{v}_{\|}^{2}\right] \frac{\mathrm{B} \times \nabla \mathrm{B}}{\mathrm{B}^{3}}$.

\section{Hamiltonian Dynamics of Charged Particle Motion in a Magnetic Field}

In the preceding sections the motion of a charged particle in a magnetic field was analyzed by guiding-center theory-a perturbation technique which treated the effect of a non-magnetic or gradient-B force as a small perturbation of the cyclotron motion in a homogenous B-field. This enabled interesting results to be derived (e.g., drift velocities) without formally solving the equations of motion.

If we now apply the very powerful and well-developed analytical machinery of classical mechanics (and especially Hamiltonian dynamics) to these equations, we can derive additional information in the form of integral invariants and adiabatic invariants. These are conservation laws for the system, and play a primary role in classifying particle trajectories in the geomagnetic field. 
A starting point for this analysis is the Lagrangian function for a particle in an electromagnetic field (Jacksu.:, 1962):

$\mathbf{L}=\mathbf{T}-\mathbf{U}$

where

$$
\begin{aligned}
\mathbf{L} & =\text { system Lagrangian function } \\
\mathrm{T} & =\text { particle kinetic energy ; } \\
& =\frac{1}{2} \mathrm{mv}^{2} \quad \text { (non-relativistic) } \\
\mathrm{U} & =\text { generalized potential ene:gy. }
\end{aligned}
$$

$U$ for the electromagnetic field contains a velocity-dependent magnetic potential term in addition to the conservative electrostatic potential $\Phi$.

$L=L\left(q_{1}, \dot{q}_{1}, t\right), i=1,2,3$

where the $q_{i}$ are position coordinates and the $q_{1}$ are the corresponding velocity components.

$\mathbf{r}=\mathbf{r}\left(q_{1}, q_{2}, q_{3}, t\right)$

$\mathbf{v}=\mathbf{v}\left(\dot{q}_{1}, \dot{q}_{2}, \dot{q}_{3}, t\right)$

The force on the particle is derived from the generalized potential $U$ :

$$
F_{i}=-\frac{\partial U}{\partial q_{i}}+\frac{d}{d t} \frac{(\partial U)}{\left(\partial \dot{q}_{i j}\right)}
$$

or

$F=-\nabla U+\frac{d}{d t}\left[\nabla_{V} U\right]$

where $\nabla_{V}$ is the velocity-space gradient operator.

This force must be equal to the Lorentz force:

$\mathbf{F}_{\mathrm{L}}=\mathbf{e}(\mathbf{E}+\mathbf{v} \times \mathbf{B})$

The form of $U$ can now be obtained by equating the right-hand sides of Eqs. (3.93b) and (3.94).

First we will rewrite the Lorentz equation in terms of the scalar and vector potentials $\Phi$ and $A$.

$B=\nabla \times A\left(q_{i}, t\right)$

From: Faraday's law,

$\nabla \times \mathbf{E}=-\frac{\partial \mathbf{B}}{\partial \mathrm{t}}=-\frac{\partial}{\partial \mathrm{t}}(\nabla \times \mathbf{A})$, so that

$\nabla \times\left(\mathbf{E}+\frac{\partial \mathbf{A}}{\partial t}\right)=0$. 
This implies that the bracketed quantity can be written as the gradient of a scalar function $\Phi$ whose curl vanishes automatically:

$E+\frac{\partial A}{\partial t}=-\nabla \Phi\left(q_{i}, t\right)$

With these substitutions for $E$ and $B$, the Lorentz force Eq. (3.94) becomes:

$\mathbf{F}_{\mathrm{L}}=\mathrm{e}\left[-\nabla \boldsymbol{\Phi}-\frac{\partial \mathbf{A}}{\partial \mathrm{t}}+\mathbf{v} \times(\nabla \times \mathbf{A})\right]$.

The triple cross product can be written

$\mathbf{v} \times(\nabla \times \mathbf{A})=\nabla(\mathbf{A} \cdot \mathbf{v})-(\mathbf{v} \cdot \nabla) \mathbf{A}$

where the $\nabla$ operator in the first term does not operate on $\mathbf{v}$ since $\mathbf{v}$ is a function of the velocities $\phi_{i}$ only. The total derivative of $A$ along the particle trajectory, $\frac{\mathrm{dA}}{\mathrm{dt}}$ can be expressed in terms of the convective
derivative:

$\frac{\mathrm{dA}}{\mathrm{dt}}=\frac{\partial \mathbf{A}}{\partial \mathrm{t}}+(\mathbf{v} \cdot \nabla) \mathbf{A}$

where

$\frac{\partial \mathbf{A}}{\partial \mathrm{t}}$

is the explicit variation of the vector potential with time and $(\mathbf{v} \cdot \nabla) A$ is the change in $A$ experienced by the particle due to its motion through gradients in A. Substituting Eqs. (3.100) and (3.101) in Eq. (3.99), we obtain:

$$
\begin{aligned}
\mathbf{F}_{\mathrm{L}} & =\mathrm{e}\left\{-\nabla \boldsymbol{\Phi}-\left[\frac{\mathrm{d} \mathbf{A}}{\mathrm{dt}}-(\mathbf{v} \cdot \boldsymbol{\nabla}) \mathbf{A}\right]+[\boldsymbol{\nabla}(\mathbf{A} \cdot \mathbf{v})-(\mathbf{v} \cdot \boldsymbol{\nabla}) \mathbf{A}]\right\} \\
& =\mathrm{e}\left\{-\nabla \boldsymbol{\Phi}-\frac{\mathrm{d} \mathbf{A}}{\mathrm{dt}}+\nabla(\mathbf{A} \cdot \mathbf{v})\right\} .
\end{aligned}
$$

Equating this to the Lagrangian force in Eq. (3.93b) gives

$$
-\nabla \mathrm{U}+\frac{\mathrm{d}}{\mathrm{dt}}[\nabla \vee \mathrm{U}]=\mathrm{e}\left[-\nabla \boldsymbol{\Phi}-\frac{\mathrm{d} \mathbf{A}}{\mathrm{dt}}+\nabla(\mathbf{A} \cdot \mathbf{v})\right] \text {. }
$$

We can derive two relations for $U$ by equating first the gradient expressions on both sides of Eq. (3.103):

$$
-\nabla \mathbf{U}=-\mathbf{e}[\nabla \boldsymbol{\phi}-\nabla(\mathbf{A} \cdot \mathbf{v})]
$$

and then equating the remaining terms:

$\frac{\mathrm{d}}{\mathrm{dt}}[\nabla \vee U]=\frac{\mathrm{d}}{\mathrm{dt}}[-\mathrm{eA}]$

This suggests an expression fo: $U$ of the form:

$U=e(\Phi-A \cdot v)+$ constant

which upon substitution into Eq. (3.103) is indeed seen to be a solution. 
Thus the Langrangian function for the motion of a charged particle in electric and magnetic fields is: (Panofsky and Phillips, 1962)

$L=T-e \Phi+e \mathbf{A} \cdot \mathbf{V}$.

The Hamiltonian function $\mathrm{H}$ for any system is defined by:

$H=\sum_{i} p_{i} v_{i}-l$

where

$$
\begin{aligned}
P_{i} & =\text { generalized momentum conjugate to } q_{i} ; \\
& =\frac{\partial L}{\partial v_{1}} \\
& =m v_{i}+e A_{1} \text { for the electromagnetic field. }
\end{aligned}
$$

or

$$
\mathbf{p}=\mathbf{m v}+\mathbf{e A} \text {. }
$$

This generalized momentum for the system includes not only the particle momentum mv but also contains a term proportionai to the vector potential $\mathbf{A}$. The Hamiltonian is then:

$H=\left[e \Phi+\frac{1}{2 m}|\mathbf{p}-\mathrm{eA}|^{2}\right]$

Since the Lagrangian $\mathrm{L}$. is not explicitly time-dependent, $\mathrm{H}$ is a constant of the motion (Goldstein, 1950), And even though the momentum term includes a contribution from the velocity-dependent potential, the Hamiltonian is the total particle energy:

$H=e \Phi+\frac{1}{2} m v^{2}$

\section{Invariants of the Motion}

For every Hamiltonian system in general there exists an integral invariant of the form:

$\oint_{C} \mathbf{p} \cdot d \mathbf{q}=$ constant

where the contour $C$ is a closed curve in phase space whose points are solutions to the equations of motion at a fixed time (Kruskal, 1962). As the system evolves, the curve $C$ in general does not remain constant but changes shape as each point moves along a phase-space trajectory. The existence of this integral invariant [Eq. (3.112)] can in fact be taken as the definition of a Hamiltonian system (Kilmister, 1964).

To make the physical content of the integral invariant more concrete, consider the following oneparticle system of one physical dimension. Let the Hamiltonian be $H=H(q, p)$. Choose $C_{0}(t=0)$ be a closed curve in the p,q plane which encloses the area $A_{0}$ (Fig. 3-5). Each point on the curve represents a possible initial value for the system, consistent with system constraints and conservation of $H$. As the system evolves, each point on $C_{0}$ traces out a trajectory in phase space such that each trajectory point is a solution to the equations of motion. At a subsequent time $t$, these trajectories form another curve $C(t)$. 


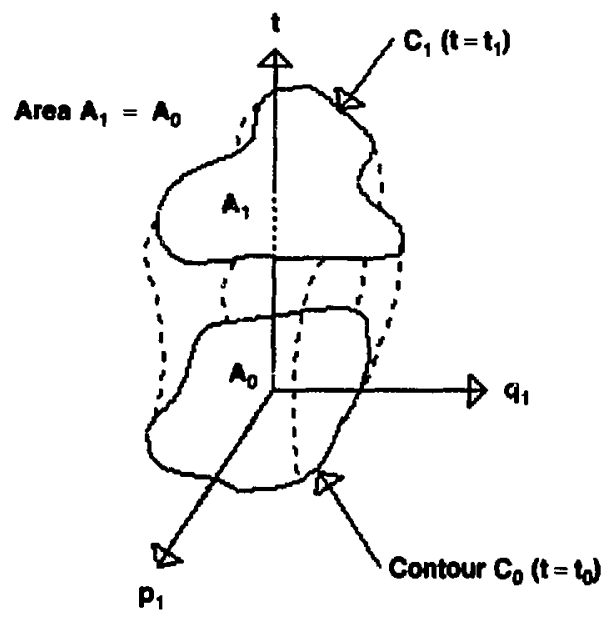

Figure 3-5. Evolution of an integration contour in the $p_{1}, q_{1}$ plane.

Althcugh tise shaps of $C(t)$ in general differs from $C_{0}$, the area $A(t)$ bounded by $C(t)$ equals $A_{0}$, regardless of the nature of te iorces on the particle. That is,

$$
\iint_{A_{0}} d p d q=\iint_{A(t)} d p d q .
$$

But since

$$
\iint_{A_{0}} \mathrm{dpdq}=\oint_{\mathrm{C}_{0}} \mathrm{pdq} \text {, }
$$

we have

$\oint_{C_{0}} p d q=\oint_{C(t)} p d q$

Higher-order integral invariants of the form:

$$
\iiint \int p_{1} d q_{1} d p_{2} d q_{2}
$$

also exist, and in fact any Hamiltonian system possesses an unlimited number of invariants (Kilmister, 1964).

Because the invariance (either absolute or adiabatic) of the integral [Eq. (3.112)] plays a fundamental role in the classification and analysis of charged-particle orbits in the magnetosphere, a general proof of the invariance will be given. This proof is based on notions of Kilmister and Goldstein.

We first -tablish the result ihat if $p_{i}, q_{i}$ are canorical Hamiltonian variables, i.e., if

$\dot{q}_{i}=\frac{\partial H}{\partial p_{i}} ;$ and $\dot{p}_{i}=-\frac{\partial H}{\partial q_{i}} ;$ 
and if $p_{i}, q_{i}$ are subsequently transformed via a canonical transformation to new canonical variables $P_{j}, Q_{i}$, then

$\oint_{C} \sum_{i} p_{i} d q_{i}=\oint_{C} \sum_{i} P_{i} d Q_{i}$

where the integral is taken at fixed time over an arbitrarily-chosen simple closed curve $C$.

We start with a system Lagrangian:

$L=L(\mathbf{q}, \mathbf{q}, \mathbf{t})$

from which we construct the Hamiltonian

$H=H(\mathbf{p}, \mathbf{q}, \mathbf{t})=\sum_{i} p_{i} \mathbf{q}_{i}-\mathbf{L}(\mathbf{q}, \mathbf{q}, \mathbf{t})$

where $p_{i}=$ the generalized momentum associated with coordinate $q_{i}$

$$
=\frac{\partial L}{\partial \dot{q}_{i}} \text {. }
$$

The $p_{i}$ and $q_{i}$ as defined wili satisfy Hainilton's canonical equations (3.116). The integral of interest is:

$\oint_{C} \sum_{i} p_{i} d q_{i}$

We wish to demonstrate the invariance of Eq. (3.120) under a so-called canonical transformation (alternatively termed a contact transformation) which transforms the $q_{i}, p_{i}$ to a new set of coordinates and momenta $Q_{i}, P_{j}$. The $Q_{i}, P_{i}$ will be functions of the old coordinates and time:

$Q_{i}=Q_{i}(p, q, t) ; P_{i}=P_{i}(p, q, t)$

The $Q_{i}, P_{i}$ must also be canonical variables, in that they satisfy a Hamiltonian relationship:

$\dot{Q}_{i}=\frac{\partial K}{\partial P_{i}} ; P_{i}=-\frac{\partial K}{\partial Q_{i}} ;$

where $K=K(\mathbf{Q}, \mathbf{P}, t)$ is the Hamiltonian expressed in the new variables. Now we employ the fact that if $Q_{i}, P_{i}$ are canonical variables, then they must also satisty Hamilton's principle:

$\delta \int_{t_{1}}^{t_{2}}\left[\sum_{i} P_{i} \dot{Q}_{i}-K(\mathbf{Q}, P, t)\right] d t=0$.

This principle imposes a constraint on the motion of a Hamiltonian system in phase space.

Let us take phase space to be a $2 \mathrm{~N}+1$ dimensional space with the $\mathrm{N}$ independent $\mathrm{q}_{\mathrm{i}}$ (position coordinates), $\mathrm{N}$ independent momenta $\mathrm{p}_{\mathrm{i}}$ (momentum coordinates), and time as the basis. $\therefore$ single point in this space then specifies the exact state of the system at an instant of time. As the system evolves from an initial state corresponding to an initial point $\left(q_{1}, p_{1}, t_{1}\right)$, it generates a curve in phase space which terminates at time $t_{2}$ on the point $\left(q_{2}, p_{2}, t_{2}\right)$.

Hamilton's principle states that the motion of the system from time $t_{1}$ to $t_{2}$ is along the one trajectory in phase-space for which the integral in Eq. (3.123) is an extremum, compared to its value on all other 
possible trajectories the system could take. Therefore the variation of this integral will vanish. (In evaluating alternate paths, the end points at $t_{1}$ and $t_{2}$ are considered fixed).

The original canonical variables must satisfy Hamilton's principle as well:

$\delta \int_{t_{1}}^{t_{2}}\left[\sum_{i} p_{i} q_{i}-H(q, p, t)\right] d t=0$.

Comparitig Eqs. (3.123) and (3.124), we see that the integrands must be identical, except for an arbitrary function whose integral variation vanishes.

One such function is $\frac{\mathrm{dF}}{\mathrm{dt}}$, a total time derivative of an arbitrary function $\mathrm{F}$.

lts integral variation will be:

$\delta \int_{t_{1}}^{t_{2}} \frac{\mathrm{dF}(t)}{\mathrm{dt}} \mathrm{dt}=\delta\left[\mathrm{F}\left(\mathrm{t}_{2}\right)-\mathrm{F}\left(\mathrm{t}_{1}\right)\right]=0$

because the variation of the integral at the end-points is zero by definition.

Since the variations of $p(t), q(t), P(t), Q(t)$ vanish at the rixed end-points, $F$ may be written as a function of these variables (and time). But by Eq. (3.121), $Q_{1}$ and $P_{1}$ are functions of the original set of variables $p_{i}, q_{1}$ so that only two of the set $p, q, P, Q$ may be chosen as independent variables for $F$. Here we will choose to write $F$ as:

$\mathbf{F}=\mathbf{F}(\mathbf{q}, \mathbf{Q}, \mathbf{t})$

The difference between the integrands in Eqs. (3.123) and (3.124) is then:

$\sum_{i} p_{i} \dot{q}_{i}-H(\mathbf{p}, \mathbf{q}, t)-\sum_{i} P_{i} \dot{Q}_{i}+K(\mathbf{P}, \mathbf{Q}, t)=\frac{d F(q, Q, t)}{d t}$

The total derivative $\frac{\mathrm{dF}}{\mathrm{dt}}$ can be expanded as:

$\frac{d F}{d t}=\sum_{i} \frac{\partial F}{\partial q_{i}} \dot{q}_{i}+\sum_{i} \frac{\partial F}{\partial Q_{i}} \dot{Q}_{i}+\frac{\partial F}{\partial t}$

and Eq. (3.127) becomes:

$\sum_{i} p_{i} \dot{q}_{i}-\sum_{i} P_{i} \dot{Q}_{i}+K-H=\sum_{i} \frac{\partial F}{\partial q_{i}} q_{i}+\sum_{i} \frac{\partial F}{\partial Q_{i}} \dot{Q}_{i}+\frac{\partial F}{\partial t}$

The $q_{i}, Q_{i}$ are all independent, so that the equality can only hold if:

$p_{i}=\frac{\partial F}{\partial q_{i}}$,

$P_{i}=-\frac{\partial F}{\partial Q_{i}}$,

$K=\mathbf{H}+\frac{\partial F}{\partial t}$. 
The total differential of $\mathrm{F}$ is:

$d F=\sum \frac{\partial F}{\partial q_{i}} d_{i}+\sum \frac{\partial F}{\partial Q_{i}} d Q_{i}+\frac{\partial F}{\partial t} d t$

which with substitutions from Eq. (3.130) becomes:

$\mathrm{dF}=\sum \mathrm{p}_{\mathrm{i}} \mathrm{dq}_{\mathrm{i}}-\sum \mathrm{P}_{\mathrm{i}} \mathrm{dQ} \mathbf{Q}_{\mathrm{i}}+(\mathbf{K}-\mathbf{H}) \mathrm{dt}$

If we evaluate the integral of $\mathrm{dF}$ around a closed contour at a fixed time, it will vanish:

$\oint_{c} d F=\oint_{(T, q)} \sum P_{i} d q_{i}-\oint_{C(P, Q)} \sum P_{i} d Q_{i}=0$

where $C(P, Q)$ is the contour $c(p, q)$ transformed to $P, Q$ space. Therefore, the contour integral:

$\oint_{c} \sum_{i} p_{i} d q_{1}$ is invariant under a canonical transformation.

This completes the first part of our prouf.

We next seek to show that the evolution of a Hamiltonian system can be regarded as a series of cascaded infinitesimal canonical transformations operating on the initial conditions $\left(p_{0}, q_{0}, t_{0}\right)$ to produce every subsequent point along the system phase-space trajectory. Thus if an integral quantity is invariant with respect to a canonical transformation, then it is also invariant with respect to a translation of the system along its natural trajectory in phase-space.

The function $F$ in Eq. (3.126) which relates the two sets of canonical variables can be regarded as the generator of the canonical transformation, since once $F$ is specified the dependence of $Q, P$ on $P, q$ is defined by Eqs. $(3.130 a-c)$. One special form of $F$ is the idendity transformation $F_{1}$, which leaves $p, q$ unchanged.

$F_{l}=\sum_{i} q_{i} P_{i}, \quad$ where we have chosen $q$ and $P$ as the independent variables.

In this case the transformation equations are:

$$
\begin{aligned}
& P_{i}=\frac{\partial F_{l}}{\partial q_{i}}=P_{i}, \\
& Q_{i}=\frac{\partial F_{I}}{\partial P_{i}}=q_{i}, \\
& K=H .
\end{aligned}
$$

The identity transformation serves as the basis for the infinitesimal canonical transformation which moves the system along its path from time $t$ to $t+\delta t$.

Let us define an infinitesimal canonical transformation to be:

$$
S=F_{1}(\mathbf{q}, \mathbf{P}, \mathbf{t})+\epsilon G(\mathbf{q}, \mathbf{P})
$$


where $\epsilon$ is an infinitesimal parameter of the transformation. The transformation equations are:

$$
\begin{aligned}
& p_{i}=\frac{\partial S}{\partial q_{i}}=P_{i}+\epsilon \frac{\partial G}{\partial q_{i}} . \\
& Q_{i}=\frac{\partial S}{\partial P_{i}}=q_{i}+\epsilon \frac{\partial G}{\partial P_{i}} .
\end{aligned}
$$

The change in $p, q$ under this transformation is:

$$
\begin{aligned}
& \delta \mathrm{p}_{i}=\mathrm{P}_{\mathrm{i}}-\mathrm{p}_{\mathrm{i}}=-\epsilon \frac{\partial \mathrm{G}(\mathrm{P}, \mathrm{q})}{\partial \mathrm{q}_{\mathrm{i}}} . \\
& \delta \mathrm{q}_{\mathrm{i}}=\mathrm{Q}_{\mathrm{i}}-\mathrm{q}_{\mathrm{i}}=\epsilon \frac{\partial \mathrm{G}(\mathrm{P}, \mathrm{q})}{\partial \mathrm{P}_{\mathrm{i}}} .
\end{aligned}
$$

By Eq. (3.137a), $P_{1}$ differs from $p_{1}$ only by the infinitesimal 6 , so that to first order in 6 we can write the right-hand side of Eq. (3.137b) as:

$$
\epsilon \frac{\partial G}{\partial P_{i}}=\epsilon \frac{\partial G}{\partial p_{i}} \frac{\partial p_{i}}{\partial P_{i}}=\epsilon \frac{\partial G}{\partial p_{i}}\left[1+0(\theta] \approx \epsilon \frac{\partial G(p, q)}{\partial p_{i}}\right. \text {. }
$$

We can therefore regard $G$ as a function of $p$ and $q$ to first order in $\epsilon$, so that the transformation equations become:

$$
\begin{aligned}
& \delta p_{i}=-\epsilon \frac{\partial G(\mathbf{q}, \mathbf{p})}{\partial q_{i}} . \\
& \delta q_{i}=\epsilon \frac{\partial G(\mathbf{q}, \mathbf{p})}{\partial p_{i}} .
\end{aligned}
$$

Let us now choose $\epsilon$ to be $\delta \mathrm{t}$, so that the transformation represents the incremental motion of the system along its phase-space trajectory. Equations (3.139) become:

$$
\begin{aligned}
& \delta \mathrm{p}_{\mathrm{i}}=-\delta \mathrm{t} \frac{\partial \mathrm{G}(\mathrm{q}, \mathrm{p})}{\partial \mathrm{q}_{\mathrm{i}}}, \\
& \delta \mathrm{q}_{\mathrm{i}}=\delta \mathrm{t} \frac{\partial \mathrm{G}(\mathrm{q}, \mathrm{p})}{\partial \mathrm{p}_{\mathrm{i}}} .
\end{aligned}
$$

But in the limit as $\delta \mathrm{t}=>0$, these become identical with Hamilton's canonical equations, if we identify $G(q, p)$ with the Hamiltonian $H(q, p)$.

So we find that the Hamiltonian function itself is the generator of the infinitesimal carcenical transformation which carries the system along in time, a property termed the unfolding theorem (Kilmister, 1964). The motion of the system can be viewed as a continuous unfolding of a canonical transformation which transforms the coordinates $q(t), p(t)$ to $q(t+\delta t), p(t+\delta t)$ and thus advances the point representing the instantaneous system coordinates along the system trajectory. A point on the trajectory is reached by a succession (a cascade) of such infinitesimal canonical transformations, beginning with an initial point at time $t_{0}$. Since the result of two or more cascaded transformations is equivalent to a single canonical transformation, each point on the trajectory is related to every other point by a single canonical transformation. 
We have now arrived at the final step of our proof. If an integral quantity (computed at a fixed time $t_{0}$ ) is invariant under a canonical transformation, it must be invariant under that particular transformation which advances each point in the integrand along the system phase-space trajectory to a fixed subsequent time $t_{1}$.

From Eq. (3.132), if $F$ is chosen to be the generating function of the canonical transformation which takes $\mathbf{p}\left(t_{0}\right), q\left(t_{0}\right)$ into $p\left(t_{1}\right), q\left(t_{1}\right)$, then it follows that

$$
\oint_{\substack{c_{0} \\ t=t_{0}}} \sum_{i} p_{i} d q_{i}=\oint_{\substack{c_{1} \\ i=t_{1}}} \sum_{i} p_{i} d q_{i} ;
$$

where the contour $C_{0}$ is a simple closed curve, every point of which represents a possible initial value of the system at $t=t_{0}$ (i.e., is a solution to the equations of motion and is consistent with system constraints and conservation of $H$ ), and the contour $C_{1}$ is the contour $C_{0}$ evolved via the system Hamiltonian from time $t_{0}$ to $t_{1}$ (i.e., each point on $C_{0}$ is translated along the trajectory dictated by Hamilton's principle, and at time $t_{1}$ the set of such translated points forms $C_{1}$ ).

For such an integral,

$\oint \sum_{i} p_{i} d q_{i}$ is a constant of the motion.

The above proof can be readily generalized to show that higher-order integral invariants such as:

$\iint::: \iint d p_{1} d q_{1} \ldots d p_{n} d q_{n}$

also exist for every Hamiltonian system. In particular, the integral over a volume in phase space is constant with time, if the surface bounding the volume evolves according to the equations of motion and the system constraints. This is a form of Liouville's theorem (Landau and Lifshitz, 1976) which states that the density in phase space is constant along a system trajectory, a result that leads to useful particle-flux conservation theorems in magnetospheric physics.

The utility of an integral invariant is that it provides a constant of the motion which can yield useful information about the system (for instance, the frequencies of periodic motion) without first finding a complete solution to the equations of motion (Goldstein, 1950).

However, if the contour on which

$\oint$ pdq

is evaluated c-rnlves in a complex way, then the labor involved in determiring the subsequent contour many be no less than that involved in fully solving the equations of motion (Kruskal, 1962). Thus an integral invariant to be generally useful must involve a contour which is either constant over time or evolves in a simply-determined manner.

If the Hamiltonian is independent of a canonical coordinate $q_{k}$, then the conjugate momentum is a constant of the motion and is termed "cyclic" (because in many instances the corresponding motion is a rotation about an axis). That is, if

$\dot{\mathrm{p}}_{\mathrm{k}}=-\frac{\partial \mathrm{H}}{\partial \mathrm{q}_{\mathrm{k}}}=\mathbf{0}$

then $p_{k}=$ constant. In this case the integral invariant contour is a rectangle in the $p_{k}, q_{k}$ plane, whose shape is independent of time. If $q$ is an angle variable, then 
$J=\oint p d q=2 \pi p=$ constant

and $\mathrm{J}$ is termed an "action" variable.

Action-angle variables play a major role in analyzing the equations of motion of periodic mechanical systems (e.g., planetary motion) and were historically important in the Bohr quantum theory of the atom where action variables of atomic systems were expressed as integral multiples of nh (Born, 1960), leading to the quantization of angular momentum and energy.

We now indicate how the solution of a periodic system can be linked to these integral invariants. We showed above that the coordinates $\mathbf{p}(t), q(t)$ of a system at any time $t$, are transformable via a canonical transformation to their initial values. This effects a solution to the equations of motion by expressing the 2n position and momentum coordinates of the problem in terms of their $2 \mathrm{n}$ initial (constant) values,

Alternatively the equations of motion can be couched in Hamilton-Jacobi form, replacing the $2 \mathrm{n}$ Hamilton's equations with one first-order differential equation. The Hamilton-Jacobi equation (for the case of $\mathrm{H}$ conserved) is:

$H\left(q, \nabla_{q} S\right)=E$;

which is a first-order partial differential equation for $S$, having $n$ constants of integration (arising from the $\nabla_{q} S$ term) plus one for the constant energy E. S(q,P) is known as Hamilton's characteristic function (Park, 1979 ), and is the generator of a canonical transformation that relates the $n$ original space variables $q$ to $n$ new canonical momenta $\mathbf{P}$. One choice for the $\mathbf{P}$ which greatly simplifies the solution of the HamiltonJacobi equation is:

$P_{i}=$ constant $=a_{i} ; \quad i=1, \ldots n$.

For this case, Hamilton's equations in the new canonical variables $\mathbf{P}, \mathbf{Q}$ and the new Hamiltonian $K=K(P)$ are:

$\dot{\mathrm{P}}_{\mathrm{K}}=-\frac{\partial \mathrm{K}}{\partial \mathrm{Q}_{\mathrm{K}}}=0$

from which it immediately follows that $K$ is a function of $P$ only;

$\dot{\mathrm{Q}}_{\mathrm{K}}=\frac{\partial \mathrm{K}}{\partial \mathrm{F}_{\mathrm{K}}}=\mathbf{g}_{\mathrm{K}}(\mathrm{P})=\mathbf{g}_{\mathrm{K}}(\boldsymbol{\alpha})$;

where $g_{k}(P)$ is a function of the constant momenta only, and is therefore itself a constant.

These Hamilton's equations can be immediately integrated to obtain:

$P_{K}=\alpha_{K} ;$ constant generalized momenturn ,

$\mathrm{Q}_{\mathrm{K}}=\mathbf{g}_{\mathrm{K}}(\boldsymbol{\alpha}) \mathrm{t}+\mathrm{C}_{\mathrm{K}} ;$ cyclic conjugate coordinate.

A complete solution contains the $n \alpha_{K}$ and the $n C_{K}$ constants, which are to be evaluated in terms of the $2 n$ initial conditions.

The transformation-generator $S$ can be calculated from the transformation-defining equations:

$$
\begin{aligned}
& P_{K}=\frac{\partial S}{\partial q_{k}}(\mathbf{q}, \alpha) ; \\
& Q_{K}=\frac{\partial S}{\partial P_{K}}(\mathbf{q}, \alpha) .
\end{aligned}
$$


The $\alpha$ can be chosen from the $n$ initial momenta, or any $\mathbf{n}$ independent combinations of constants of the motion-initial values, conserved quantities, or integral invariants such as the action integrals.

For the case of a system which exhibits periodic motion, the constants of the motion $a_{i}$ can be chosen to be action variables $\mathrm{j}_{\mathrm{i}}$;

$\mathrm{J}_{i}=\oint \mathrm{p}_{\mathrm{i}} \mathrm{dq} \mathrm{q}_{\mathrm{i}}=2 \pi \mathrm{p}_{\mathrm{i}}=a_{\mathrm{i}}$

where $q_{i}$ is a cyclic angle variable.

It can be readily shown (Goldstein, 1950) that the transformed variable $Q_{i}$ conjugate to the constant generalized momentum $\mathrm{J}_{\mathrm{i}}$ is:

$Q_{i}=f_{i} t+\beta_{i}$

where $f_{i}=1 / \tau_{i}$, the frequency of the periodic motion corresponding to $I_{i}$, and

$\mathbf{f}_{\mathrm{i}}=\dot{\mathrm{Q}}_{\mathrm{i}}=\frac{\partial \mathrm{K}(\mathrm{U})}{\partial \mathrm{J}_{1}}$.

Thus by using action variables as the generalized constant momenta, the frequency of a periodic motion may be obtained from the transformed Hamiltonian $\mathrm{K}$ without recourse to solving the equations of motion, a considerable benefit.

We now apply the action-angle method to the motion of a charged particle in a homogeneous magnetic field. Referring to the earlier section on Hamiltonian dynamics, the generalized momentum for a charged particle in a magnetic field is:

$\mathbf{p}=\mathbf{m v}+\mathbf{e A}$.

In cylindrical coordinates, taking $\mathbf{B}$ in the $\hat{z}$ direction, the canonical momentum in the $r, \theta$ plane is:

$\mathrm{P}_{\theta}=\mathrm{mr}^{2} \dot{\theta}+\mathrm{erA}_{\theta}=$ constant.

To evaluate $\mathrm{J}=\oint \mathrm{P}_{\theta} \mathrm{d} \theta$,

we choose a clockwise sense of integration around the circular contour such that ds. $v_{\theta}>0$, and ds. $\mathbf{A}_{\theta}<0$ (see Fig. 3-3). Then

$J=2 \pi\left[\mathrm{mr}^{2} \dot{\theta}-\mathrm{er} \hat{A}_{\theta}\right]=2 \pi m r^{2}[\dot{\theta}-\mathrm{eB} / 2 \mathrm{~m}]$

is the action variable.

The Hamiltonian $K$ is the particle kinetic energy

$K=\frac{1}{2} m r^{2} \dot{\theta}^{2}$

from which we obtain the frequency of the orbital motion:

$\left.f=\frac{\partial K(J)}{\partial]}-K=f\right]+$ constant

where we may take the arbitrary constant (the system energy when $J=0$ ) to be 0 . Substituting for $K$ and $J$ we obtain: 
$\frac{1}{2} m r^{2} \dot{\theta}^{2}=2 \pi f m r^{2}[\dot{\theta}-e B / 2 m]=\dot{\theta} m r^{2}[\dot{\theta}-e B / 2 m]$

or

$\dot{\theta}=2 \dot{\theta}-\mathrm{eB} / \mathrm{m} ;$ which yields the well-known result for the gyro-frequency:

$\dot{\theta}=\mathrm{eB} / \mathrm{m}$.

We now substitute this expression into Eq. (3.154) to obtain:

$\mathrm{J}=\pi \mathrm{mr} \mathbf{r}^{2} \dot{\theta}$

an integral invariant of the motion. We have previously defined a particle's magnetic moment $\mu$ as

$\mu=\operatorname{er}^{2} \dot{\theta} / 2$,

which can now be expressed in terms of J as:

$\mu=\frac{e)}{2 \pi \cdot n}$.

Since $\mathrm{J}$ is an integral invariant of the motion, then so is $\mu$.

As we will see in the next section, $J$ and $\mu$ are not only integral invariants but also have the property of being adiabatic invariants. That is to say, under changes in the parameters of a periodic mechanical system which are slow compared to the characteristic periods of oscillation, the integral invariants are conserved.

\section{Adiabatic Invariants of Charged Particle Motion in a Magnetic Field}

For a particle with $n$ degrees of freedom, the maximum number of periodicities and therefore action integrals is $\mathbf{n}$. The trajectory of a charged particle in a magnetic field which has a mirror-type geometry (such as the geomagnetic field) is characterized by up to three periodicities-a rapid oscillation of the particle about its guiding center in the plane perpendicular to $B$ (the cyclotron motion), a slower oscillation of the guiding center along the field lines between mirror points (the bounce motion), and a still slower perpendicular motion of the guiding center (the drift motion) which is periodic in fields that possess sufficient azimuthal symmetry.

Associated with each periodicity is an action-variable integral quantity which can be shown to be an adiabatic invariant of the motion. These adiabatic invariants form an important basis for organizing particle flux observations in the magnetosphere. In regions where all three adiabatic invariants are conserved, a single-point particle flux measurement can serve to determine the fluxes everywhere on a magnetic drift shell (for static fields). Observations made during a single satellite pass radially along the magnetic equator will characterize the fluxes for large volumes of the magnetosphere. From the standpoint of magnetospheric physics, it is the general conservation of invariants that produces persistent particle-flux features such as the radiation belts and characteristic particle pitch-angle distributions, whereas it is the violation of the adiabatic invariants (by stochastic or resonant magnetic field perturbations) which leads both to the radial transport of particles into the belts and their subsequent loss by atmospheric scattering. In this section we will establish the forms of these adiabatic invariants.

The three adiabatic invariants associated with charged-particle motion in the magnetospheric magnetic field are conventionally termed $\mu, \mathrm{J}$, and $\Phi, \mu$ is associated with the particle gyromotion, and has a period of the order of milliseconds for an energetic electron $\left(E_{E}>70 \mathrm{keV}\right.$ ) in the outer magnetosphere (magnetic shell parameter $L>6$ ). J is associated with the oscillation along field lines between mirror points, and has a period of about a second. $\Phi$ is associated with the complete azimuthal drift of the particle around the earth, and has a period of the order of an hour (Hamlin et ai., 1961). 
Formally each one is an action-variable:

$A=\oint p d q$

A theorem due to Boltzmann, termed the adiabatic theorem by Ehrenfest (1916), states that the action integral for any cyclic system is an adiabatic invariant, in that slow changes to the parameters of the system produce changes in A which are very slow. This permits the system parameters to vary hy an arbitrarily large amount, without changing $\mathrm{A}$ by more than a specified incremental amount, if the variation is slow enough (Park, 1979).

Adiabatic invariants can be expressed as asymptotic expansions in a smallness parameter $\epsilon$, which embodies the constraint that changes in the parameters which affect the periodic motion be slow. For the three adiabatic invariants associated with charged particle motion, we can write (Northrup et al., 1966):

$$
\begin{aligned}
& \mu=\mu_{0}+\epsilon \mu_{1}+\epsilon^{2} \mu_{2}+\ldots \\
& \mathrm{J}=\mathrm{J}_{0}+\epsilon \mathrm{J}_{1}+\epsilon^{2} \mathrm{~J}_{2}+\ldots \\
& \Phi=\Phi_{0}+\epsilon \Phi_{1}+\epsilon^{2} \Phi_{2}+\ldots
\end{aligned}
$$

These series are divergent, because applying the Cauchy convergence test to the $\mu$ expansion, for example, yields

$$
\lim _{n \rightarrow \infty}\left[\frac{\mu_{n+1}}{\mu_{n}}\right] \rightarrow \infty \text {. }
$$

Each series is however asymptotically convergent in the sense that the error

$\mu-\sum_{k=0}^{n} \mu_{k} \epsilon^{k}$

can be made as small as desired for fixed $n$, by making $\epsilon$ small enough. Formally, the expansion for $\mu$ is said to be asymptotically convergent if for ali $n$,

$\lim _{n \rightarrow 0}\left[\frac{\mu-\sum_{k=0}^{n} \mu_{k} \epsilon^{k}}{\epsilon^{n}}\right]-0$.

(Morse and Feshbach, 1953).

The adiabatic invariance of $\mu$ to order zero was first demonstrated by Alfvén (1950). Hellwig proved the adiabatic invariance of $\mu$ to order $n=1$ (Hellwig, 1955). Subsequently it was shown (Berkowitz and Gardner, 1959) that $\mu$ was an adiabatic invariant to all orders. The second adiabatic invariant J was also shown (Gardner, 1959) to be constant to all orders.

A general proof of the adiabaticity of the action integral for any cyclic system was given by ter Haar (1961), and by Kruskal (1962). The latter proved that Hamiltonian systems whose solutions are all nearly periodic have adiabatic invariants which when expressed in asymptotic series, are constant to all orders of the expansion parameter $\epsilon$.

The asymptotic series expression for $\mu$ was derived through order $\epsilon^{2}$ by Northrup (1963). In dimensionless form it is:

$A=\frac{\tilde{\mathbf{v}}_{\perp}^{2}}{\tilde{\mathrm{B}}}+\frac{2 \epsilon \tilde{\mathbf{v}}_{\|}^{2}}{\tilde{\mathrm{B}}^{3}}\left[\hat{\mathbf{e}}_{1} \times \tilde{\mathbf{v}}_{\perp}\right] \cdot \tilde{\nabla} \tilde{\mathrm{B}}-\frac{\epsilon \tilde{\mathbf{v}}_{\|} \hat{\nu}_{\perp}}{\tilde{\mathrm{B}}^{2}} \cdot\left[\hat{\mathbf{e}}_{1} \times \tilde{\mathbf{v}}_{\perp}\right] \cdot \tilde{\nabla} \hat{\mathbf{e}}_{1}+O\left(\epsilon^{2}\right)$ 
when the electric field $\mathbf{E}$ and current density $\mathbf{J}=\mathbf{0}$.

The dimensionless variables are:

$\nabla=\mathrm{v} / \mathrm{v}_{0}$

$\tilde{\mathbf{B}}=\mathbf{B} / \mathrm{B}_{0}$

$\hat{e}_{1}=$ unit vector in the direction of $B$.

$\bar{\nabla}=\mathrm{L} \nabla$.

$\epsilon=\frac{m v_{0}}{e B_{0} L}=\frac{P_{0}}{L}$; the expansion parameter.

$A=\frac{\mu}{m v_{0}^{2} / 2 B_{0}}$.

where $v_{0}, B_{0}$ and $\rho_{0}$ are the initial particle speed, magnetic induction, and gyro-radius respectively. $B$ and $v$ are evaluated at the guiding center. $L$ is the characteristic B-field scale-length, i.e., the distance over which the field changes significantly.

This expression (3.167) for $\boldsymbol{A}$ can be simplified and put into a more useful form. We first write

$\mu=\mu_{0}+\mu_{11}+\mu_{12}+O\left(\epsilon^{2}\right)$

where

$$
\mu_{0}=\frac{\hat{v}_{\perp}^{2}}{\tilde{\mathbf{B}}}
$$

is the zeroth-order term, and $\mu_{11}$ and $\mu_{12}$ are the two $O(\epsilon)$ terms in Eq. (3.167).

In dimensional form, the zeroth-order term is

$\mu_{0}=\frac{m v_{\perp}^{2}}{2 B}=\frac{P_{\perp}^{2}}{2 m_{0} B}$

which is just the non-relativistic magnetic moment. $\mu_{0}$ has been shown to be also the leading term in the relativistically-correct expansion of the integral invariant (Northrup and Teller, 1960).

The first-order term $\mu_{11}$

$\mu_{11}=\frac{2 \epsilon \bar{v}_{1}^{2}}{\tilde{\mathrm{B}}^{3}}\left[\hat{\mathrm{e}}_{1} \times \tilde{\mathbf{v}}_{\perp}\right] \cdot \bar{\nabla} \tilde{\mathrm{B}} ;$

can be given a greater physical content by expressing it in terms of the dimensionless drift velocity due to field line curvature, $\tilde{\mathbf{U}}_{\mathrm{c}}$ :

$\tilde{U}_{c}=\epsilon \frac{\left[v_{\|}^{2} \hat{e}_{1} \times \tilde{\nabla} \tilde{B}\right]}{\tilde{B}^{2}}$

[cf. Eq. (3.88a)], yielding:

$\mu_{11}=-\frac{2}{\overline{\mathbf{B}}} \overline{\mathbf{v}}_{\perp} \cdot \tilde{\mathbf{U}}_{\mathrm{c}} ;$ 
or in dimensional form:

$\mu_{11}=-\frac{\mathbf{m}}{\mathrm{B}} \mathbf{v}_{\perp} \cdot \mathbf{U}_{\mathrm{c}}$

The other first order term

$\mu_{12}=-\frac{\epsilon \tilde{v}_{\|}}{\tilde{B}^{2}} \nabla_{\perp} \cdot\left[\hat{\mathrm{e}}_{1} \times \nabla_{\perp}\right] \cdot \dot{\nabla}_{\hat{\mathbf{e}}}$

can be simplified by expressing it in terms of the dimensionless gyro-radius

$\rho=\frac{\rho}{\mathrm{L}}$.

In the guiding-center approximation the particle velocity ${ }_{\perp}$ in the plane normal to B can be decomposed as

$\nabla_{\perp}=\nabla_{g}+\nabla_{d}$

where $\nabla_{8}$ is the circular velocity in the guiding-center frame of reference and $\nabla_{d}$ is the drift of the guidingcenter in the pliane normal to $B$. In the absence of electric fields we can take $\nabla_{d}=\tilde{U}_{B}$, the dimensionless total guiding-center drift due to first-order magnetic forces [cf. Eq. (3.89)]:

$\tilde{U}_{B}=\frac{\epsilon}{2}\left[\tilde{v}_{\perp}^{2}+2 \tilde{v}_{\Uparrow}^{2}\right] \frac{\hat{e}_{I} \times \tilde{\nabla} \dot{B}}{\tilde{B}^{2}}$.

In the guiding-center frame,

$\rho=\epsilon \frac{\hat{e}_{1} \times \hat{v}_{B}}{\tilde{B}}$

so we see that $\rho$ is $O(\epsilon)$. Substituting for $\nabla_{g}$, we have

$\boldsymbol{\rho}=\epsilon\left[\frac{\hat{e}_{1} \times \tilde{\mathbf{v}}_{\perp}}{\tilde{B}}-\frac{\hat{e}_{1} \times \tilde{U}_{B}}{\tilde{B}}\right]$

but since $\tilde{\mathbf{U}}_{B}$ is $O(\epsilon)$ [from Eq. (3.177)], we can write

$\boldsymbol{\rho}=\epsilon \frac{\hat{\mathrm{e}}_{1} \times \hat{\nabla}_{\perp}}{\hat{\mathrm{B}}}+O\left(\epsilon^{2}\right)$.

$\mu_{12}$ can now be written:

$$
\begin{aligned}
\tilde{\mu}_{12} & =-\frac{\tilde{\mathbf{v}}_{\|}}{\tilde{\overline{\mathrm{B}}}} \tilde{\mathbf{v}}_{\perp} \cdot(\boldsymbol{\rho} \cdot \tilde{\nabla}) \hat{\mathrm{e}}_{1}+\mathbf{O}\left(\epsilon^{2}\right) \\
& =-\frac{\tilde{\mathrm{v}}_{\|}}{\tilde{\mathrm{B}}}\left(\tilde{\mathbf{v}}_{\perp} \cdot \delta \mathrm{e}_{1}\right) ;
\end{aligned}
$$

where $\delta e_{1}=(\rho \cdot \tilde{\nabla}) \hat{e}_{1}$ is the change in direction of $B$ over a gyro-radius, to first order.

Thus to first order in $\epsilon$, Eq. (3.169) can be written as:

$\tilde{\mu}=\frac{\overline{\mathrm{v}}_{\perp}^{2}}{\tilde{\mathrm{B}}}-\frac{2 \tilde{\mathbf{v}}_{\perp}}{\tilde{\mathrm{B}}} \cdot \tilde{\mathbf{U}}_{\mathrm{c}}-\frac{\tilde{\mathrm{v}}_{\underline{L}}}{\tilde{\mathrm{B}}}\left(\tilde{\mathrm{v}}_{\perp} \cdot \delta \mathrm{e}_{1}\right)+\mathrm{O}\left(\epsilon^{2}\right) ;$ 
or in dimensional form

$\mu=\mu_{0}\left[1-\frac{2 v_{\perp} \cdot U_{c}}{v_{\perp}^{2}}-\frac{v_{\|}}{v_{\perp}^{2}}\left(v_{\perp} \cdot \delta e_{1}\right)\right]+O\left(\epsilon^{2}\right) ;$

where $\mu_{0}=$ non-relativistic magnetic moment $=\mathrm{mv}_{\perp}^{2} / 2 \mathrm{~B}$.

Evidently it is not $\mu_{0}$ which is an adiabatic invariant and therefore conserved under slow changes in the system parameters, but the entire asymptotic series of which $\mu_{0}$ is the leading (and only zerothorder) term.

As a practical matter, one wants to know when a change in system parameters is 'slow enough' so that an adiabatic quantity is conserved. A set of criteria owing to Alfvén (Alfvén and Falthammar, 1963) is commonly used in magnetospheric particle studies to separate adiabatic and non-adiabatic regions in the geomagnetic field (Morfill, 1973). The basic consideration for the conservation of $\mu$ is that the change in $B$ during one gyration be small. This can be expressed in the following requirements:

$\tau_{8}\left|\frac{1}{B} \frac{\partial B}{\partial t}\right| \ll 1 ;$

$\rho\left|\frac{\nabla_{\perp} \mathbf{B}}{\mathbf{B}}\right| \ll 1 ;$

$\tau_{\mathrm{g}} v_{\|}\left|\frac{\nabla_{\|} \mathrm{B}}{\mathrm{B}}\right| \ll 1 ;$

where $\tau_{\mathrm{g}}$ is the gyroperiod. The criterion for conservation of $\mathrm{J}$ is similarly that B should change very little over one bounce period:

$\tau_{\mathrm{b}}\left|\frac{1}{\mathrm{~B}} \frac{\partial \mathrm{B}}{\partial \mathrm{t}}\right| \ll 1 ; \tau_{\mathrm{b}}\left|\frac{v_{\mathrm{d}} \cdot \nabla \mathrm{B}}{\mathrm{B}}\right| \ll 1 ;$

where $\tau_{b}$ is the bounce period and $v_{d}$ is the azimuthal drift velocity. Since $\tau_{b} \gg \tau_{g^{\prime}}$ conservation of $\mathrm{J}$ is much more readily violated by fluctuations in $B$. Conservation of the third or flux invariant $\Phi$ requires the field changes to be small for an even longer period, i.e.,

$\tau_{\mathrm{d}}\left|\frac{1}{\mathrm{~B}} \frac{\partial \mathrm{B}}{\partial \mathrm{t}}\right| \ll 1 ;$

where $\tau_{\mathrm{d}}$ is the drift period-the time required for a complete azimuthal drift about the earth (typically measured in hours).

Returning to our expansion for $\mu$ [Eq. (3.183)], under what conditions do the first-order terms contribute significantly to $\mu$ ? The magnitude of the second term in Eq. (3.183) is

$$
\begin{aligned}
& \left|\frac{2 v_{\perp} \cdot U_{c}}{v_{\perp}^{2}}\right| \approx\left|\frac{2 U_{c}}{v_{\perp}}\right| \text { at maximum. } \\
& \approx\left|\frac{2 m v_{\|}^{2}\left(\hat{e}_{1} \times \nabla B\right)}{e B^{2} v_{\perp}}\right|=\left|\frac{2 m v_{\|}^{2} \nabla_{\perp} B}{e B v_{\perp} B}\right| \\
& =\left|\frac{2 m v_{\|}^{2} \rho \nabla_{\perp} B}{v_{\perp}^{2} B}\right|
\end{aligned}
$$


where we have made use of $\rho=\frac{\mathrm{mv}}{\mathrm{eB}}$.

Since $\left|\rho \nabla_{\perp} \mathrm{B}\right|=\delta \mathrm{B}$, the change in $\mathrm{B}$ over a gyro-radius, we have

$$
\left|\frac{2 v_{\perp} \cdot U_{c}}{v_{\perp}^{2}}\right| \approx \frac{2 v_{\|}^{2} \delta \mathrm{B}}{v_{\perp}^{2} \mathrm{~B}}=2\left[\frac{\cos ^{2} \alpha}{\sin ^{2} \alpha}\right] \frac{\delta \mathrm{B}}{\mathrm{B}} ;
$$

where $\alpha=$ particle magnetic pitch angle $=\cos ^{-1}\left|\frac{\mathbf{v} \cdot \mathrm{B}}{\mathrm{VB}}\right|$.

Similarly we can write for the magnitude of the third term in Eq. (3.183):

$$
\begin{aligned}
\left|\frac{v_{\mathrm{A}}}{v_{\perp}^{2}} v_{\perp} \cdot \delta \mathrm{e}_{1}\right| & \approx\left|\frac{v_{\mathrm{H}} \delta \mathrm{B}}{\mathrm{v}_{\perp} \mathrm{B}}\right| \text { at maximum, }=\left|\frac{\cos \alpha}{\sin \alpha}\right| \frac{\delta \mathrm{B}}{\mathrm{B}} ; \\
\text { using } \delta \mathrm{e}_{1} & \approx \frac{\left(\rho \cdot \nabla_{\perp}\right) \mathrm{B}}{\mathrm{B}}=\frac{\delta \mathrm{B}}{\mathrm{B}},
\end{aligned}
$$

We see from Eqs. (3.186) and (3.187) that the contribution of the first-order term: car become large, even exceeding the zeroth-order term $\mu_{0}$, as the pitch-angle $\alpha \rightarrow 0$. On the other hand, the particle mirror point (where $\alpha-\pi / 2$ ) the tirst-order terms vanish.

For small $\alpha$, the magnitude ratio of the first-order terms is:

$$
\left|\frac{\frac{2 v_{\perp} \cdot u_{c}}{v_{\perp}^{2}}}{\frac{v_{1} v_{\perp} \cdot \delta \mathrm{e}_{1}}{v_{\perp}^{2}}}\right| \approx\left|\frac{2 \cos \alpha}{\sin \alpha}\right| \approx\left|\frac{2}{\alpha}\right| \gg 1 .
$$

The importance of the first-order terms in calculating $\mu$ for particles of very small $\alpha$ was noted by Mozer (1966). He presented an iutuitive derivation of the $v_{\perp} \cdot U_{c}$ term based on the notion that the magnetic moment should be evaluated in a coordinate system which mc es at the guiding-center drift velocity $\mathrm{U}_{\mathrm{B}}$.

In this system the magnetic moment $\mu_{0}^{\prime}$ is:

$\mu_{0}^{\prime}=\mathrm{m} \frac{\left|\mathbf{v}_{\perp}-\mathrm{U}_{\mathrm{B}}\right|}{2 \mathrm{~B}}=\mu_{0}\left|1-\frac{2 \mathbf{v}_{\perp} \cdot \mathrm{U}_{\mathrm{B}}}{\mathrm{v}_{\perp}^{2}}+\frac{\mathrm{U}_{\mathrm{B}}^{2}}{\mathrm{v}_{\perp}^{2}}\right|$.

If $U_{B}$ is taken to be just $U_{c}$, the guiding-center drift due to the parallel component of motion along curved field lines, expression (3.189) correctly yields the zeroth-ordf: term and the larger of the two firstorder terms in the asymptotic series for $\mu$ [Eq. (3.183)]. [This approximation of $U_{B}$ by $U_{c}$ is justified since by Eq. (3.89), $U_{c} / U_{G}=2 v_{h}^{2} / v_{\perp}^{2}=2\left(\cos ^{2} \alpha / \sin ^{2} \alpha\right) \gg 1$ for small $c$ ).

In tracing the trajectories of particles in the magnetosphere, the first-order terms of $\mu$ are important for particles which have pitch angles near zero at the minimum-B point of their bounce motion (Taylor and Hastie, 1971). The values of $\mu$ calculated along a bounce trajectory will fluctuate if only the $\mu_{0}$ term is included; i.e., the motion will appear "non-adiabatic." It is important when calculating trajectories to distinguish between orbits which are genuinely non-adiabatic (because of a violation of the Alfvén criteria) and those which merely do not conserve $\mu_{0}$. Note however that the first-order expansion collapses to $\mu_{0}$ at the mirror point $\left(v_{\|}=0\right)$, so $\mu_{0}$ is an adiabatic invariant at the mirror location.

The second or longitudinal invariant $J_{0}$ exists for a particle trapped in a mirror field configuration, and is defined by:

$\mathrm{J}_{0}=\oint \mathrm{p}_{\|} \mathrm{ds}$, 
where the integral is taken along a fixed guiding field line between mirror points, over a complete cycle. The corresponding adiabatically invariant quantity is the asymptotic series:

$\mathrm{J}=\mathrm{J}_{\mathrm{g}}+\epsilon \mathrm{J}_{1}+\ldots$

(Northrup et al., 1966). At the particle's mirror point, the $\mathrm{J}_{1}$ term vanishes and $\mathrm{J}=\mathrm{J}_{0}$. As the particle moves away from this point, the instantaneous position of the guiding-center moves to a field-line such that the higher-order terms must be evaluated to retain an adiabatically-invariant quantity. In the geomagnetic field a particle drifts azimuthally as it bounces. The loci of the mirror points will lie on the parametric shell $\mathrm{J}_{0}=$ constant if the changes in $B$ experienced by the particle per bounce are small-i.e., if the appropriate Alfvén criteria are satisfied:

$$
\begin{aligned}
& \tau_{\mathrm{b}}\left|\frac{1}{\mathrm{~B}} \frac{\partial \mathrm{B}}{\partial \mathrm{t}}\right| \ll 1 ; \\
& \tau_{\mathrm{b}} \frac{\left|\mathbf{v}_{\mathrm{d}} \cdot \nabla \mathrm{B}\right|}{\mathrm{B}} \ll 1 .
\end{aligned}
$$

In this case $\mathrm{J}_{0}$ will be constant when evaluated at successive mirror points along the guiding-center trajectory, although it will fluctuate if evaluated elsewhere.

\section{References}

Alfvén, H., Cosmical Electrodynamics, Clarendon Press, Oxiord, 1950.

Alfvén, H., and C-G Folthammar, Cosmical Electrodynamics, second ed., Clarendon Press, Oxford 1963.

Berkowitz, J., and C. S. Gardner, On the asymptotic series expansion of the motion of a charged particle in slowly varying fields, Comm. Pure Appl. Math., XII, 501, 1959.

Born, M., The Mechanics of the Atom, F. Ungar Co., New York, 1960.

Dragt, A. J., Trapped orbits in a magnetic dipole field, Rev. Geophys., 3, 255, 1965.

Ehrenfest, P., Annaler. der Physik, vol. li, 327, 1916.

Gardner, C. S., Adiabatic invariants of periodic classical systems, Phys. Rev., 115, 791, 1959.

Goldstein, H., Classical Mechanics, Addison-Wesley, Palo Alto, 1950.

Hamlin, D. A., R. Karplus, R. C. Vik, and K. M. Watson, Mirror and azimuthal drift frequencies for geomagnetically trapper particles, $]$. Geophys. Res.. 66, 1, 1961.

Hellwig, G., Uber die Beweg', 'ng geladener Teilchen iri schwach veranderlichen Magnetfeldern, Zeitschrift fur Naturforschung 10a, 508, 1955.

Jackson, J. D., Classical Electrodynamics, Johni Wiiey and Sons, New York, 1962.

Kilmister, C. W., Hamiltonian Dynamics, American Elievier, New York, 1964.

Kruskal, M., Asymptotic theory of Hamiltonian and other systems with all solutions nearly periodic, J. Math. Phys., 3, 806, 1962.

Landau, L. D, and E. M. Lifshitz, Mechanics, third sd., Pergamon Press, Oxford, 1976.

Morfill, G., Nonadiabatic particle motion in the magnetosphere, J. Geophys. Res., 78, 588, 1973.

Morse, P. M., and H. Feshbach, Methods of Theoretical Physics: Part I, McGraw-Hill, New York, 1953.

Mozer, F. S., Proton trajectories in the radiation belts, J. Geophys. Res., 71, 2701, 1966.

Northrup, T. G., and E. Teller, Stability of the adiabatic motion of charged particles in the earth's field, Phys. Rev., 117, 215, 1960.

Northrup, T. G., The Adiabatic Motion of Charged Particles, Interscience Publishers, New York, 1963.

Northrup, T. G., C. S. Liu, and M. D. Kruskal, First correction to the second adiabatic invariant of chargedparticle motion, Phys. Fluids, 9, 1503, 1966.

Panofsky, W. K. "'- and M. Phillips, Classical Electricity and Magnetism, Addison-Wesley, London, 1962.

Park, D., Lecture Notes in Physics \#110, Classical Dynamics and its Quantum Analogues, Springer-Verlag, Niew York, 1979. 
Pedersen, A., and R. Grard, Quasistatic electric field measurements on the GEOS-1 and GEOS-2 satellites, in Quantitative Modeling of Magnetospheric Processes, Geophys. Monogr. Ser., Vol. 21, edited bv W. P. Olson, American Geophysical Union, Washington, D.C., 1979.

Roederer, J. G., Dynamics of Geomagneticali,' Trapped Radiation, Springer-Verlag, New York, 1970.

Smith, P. H., N. K. Bewtra, and R. A. Hoffman, Motions of charged particles in the magnetosphere under the influence of a time-varying large scale convection electric field, in Quantitative Magnetospheric Processes, Geophys. Monogr. Ser., Vol. 21, edited by W. P. Olson, American Geophysical Union, Washington, D.C., 1979.

Taylor, H. E., and R. J. Hastie, Nonadiabatic behavior of radiation-belt particles, Cosmic Electrodynamics, 2, 211, 1971.

ter Haar, D., Elements of Hamiltonian Mechanics, North-Holland, Amsterdam, 1961. 


\section{Chapter 4. Energetic Electron Trajeciuries in the Outer Dayside Magnetosphere}

In this chapter we correlate the results of numerically tracing drift shells in a model magnetic field with energetic electron pitch angle distributions measured by the LLNL spectrometer experiment on the OGO-5 satellite. We first develop the basis for the particular magnetic field model used in this study: the Choe-Beard self-consistent magnetopause model. We then employ it to evaluate energetic particle drift motions in the outer dayside magnetosphere, using the results of adiabatic theory. We find that certain outer shells branch into disjoint segments near local noon, leading to bounce trajectories which do not sross the magnetic equator. The regions of psuedo-trapping, stable trapping, and branching shells are mapped out for the rep esentative Choe-Beard model. The model predicts that pitch angle distributions in the outer dayside ma letosphere should contain classes of paricles that have traversed distinctly separated trajectories, giving rise to sharacteristic signatures in the distribution. This model-based prediction is then compared to detailed OGO-5 measurements obtained in the outer magnetosphere. We discover faatures which are most readily explained in terms of shell branching.

\section{Modeling the Magnetosphere}

Many numarical models of the magnetospheric magnetic field have been developecil to aid in the investigation and understanding of physical processes in the magne:osphere. This secticn treats those models of greatest utility to detailed studies of energetic particie muxion in the outer dayside magnetosphere. For a more comprehensive model survey and general comparisons of model features, the reader is referred to several excellent reviews of magnetospheric magnetic field models (Roederer, 1969; Walker, 1976; 1979; 1983).

Figure 4-1 illustrales the geometry of the magnetosphere. In the most general orientation shown here, the earth-sun line does not lie in the dipole equator. A commonly-used coordinate system, useful for studies in the outer magnetosphere, is the so-called Geocentric Solar Magnetospheric coordinate system, or GSM. The GSM cartesian coordinate system has its origin at the crnter of the erth. The $X_{G 5 M}$-axis points sunward. The $\mathbf{Y}_{\mathrm{CSM}}$-axis is taken in the direction $\mathbf{N M} \times \mathbf{X}_{\mathrm{CSM}}$, where $\mathbf{N M}$ is a unit vector in the direction of the north magnetic dipole axis. $Z_{C S M}$ is definet so as to complete a right-handed system. The dipole tilt angle $i$ is defined by:

$\tau=\cos ^{-1}\left[\mathbf{N M} \cdot \mathbf{Z}_{\mathrm{GSM}}\right]$.

$\tau$ is positive for $N M$ oriented sunward. The dipole has a diurnal nutation of $\approx 12^{\circ}$ and an annual precession of $\approx 23^{\circ}$, producing a maximum dipole tilt of $\approx \pm 35^{\circ}$ in the summer and winter seasons.

The earliest field models (prior to the discovery of the extended anti-solar "tail" of the magnetosphere) were able to reproduce rather well the gross featuses of the dayside magnetospheric cavity seen here-principally the average loration and shape of the magnetopause formed by the action of the flowing solar wind on the earth's field. The model of Hones (1963) employed a large image dipole to approximate the effects of the currents which flow on the magrietopause and shield the exterior volume from the carth's field (taken to be a geocentric dipole). In the boundary surface model of Mead (Mead, 1964; Meac and Beard, 1964), the magnetopause was determined by seeking a surface on which the dynamic pressure of the solar wind was balanced by the magnetic pressure of the confined field. (The internal source was a geocentric dipole). Both models produced a self-consistent boundary, in that the normal component of B at the surface was zero (i.e., no field lines crossed the boundary). Since the "standoff" distance (the radial distance from the earth's center to the equatorial magnetopause at noon) varies considerably with solar wind pressure, a useful numerical model must have inputs to set the standoff distance to match specified solar wind conditions. This is readily done in the Mead model, which has a standoff distance parameter, but for the Hones model one must adjust the image dipole moment and location (both non-physical parameters) to achieve the desired standoff distance.

Subsequent space probes provided a large body of vector field measurements throughout the magnetosphere, and a picture emerged of magnetospheric internal current systems which made significant contributions to the total field. Whereas the dayside magnetopause could be accurately modeled in terms 


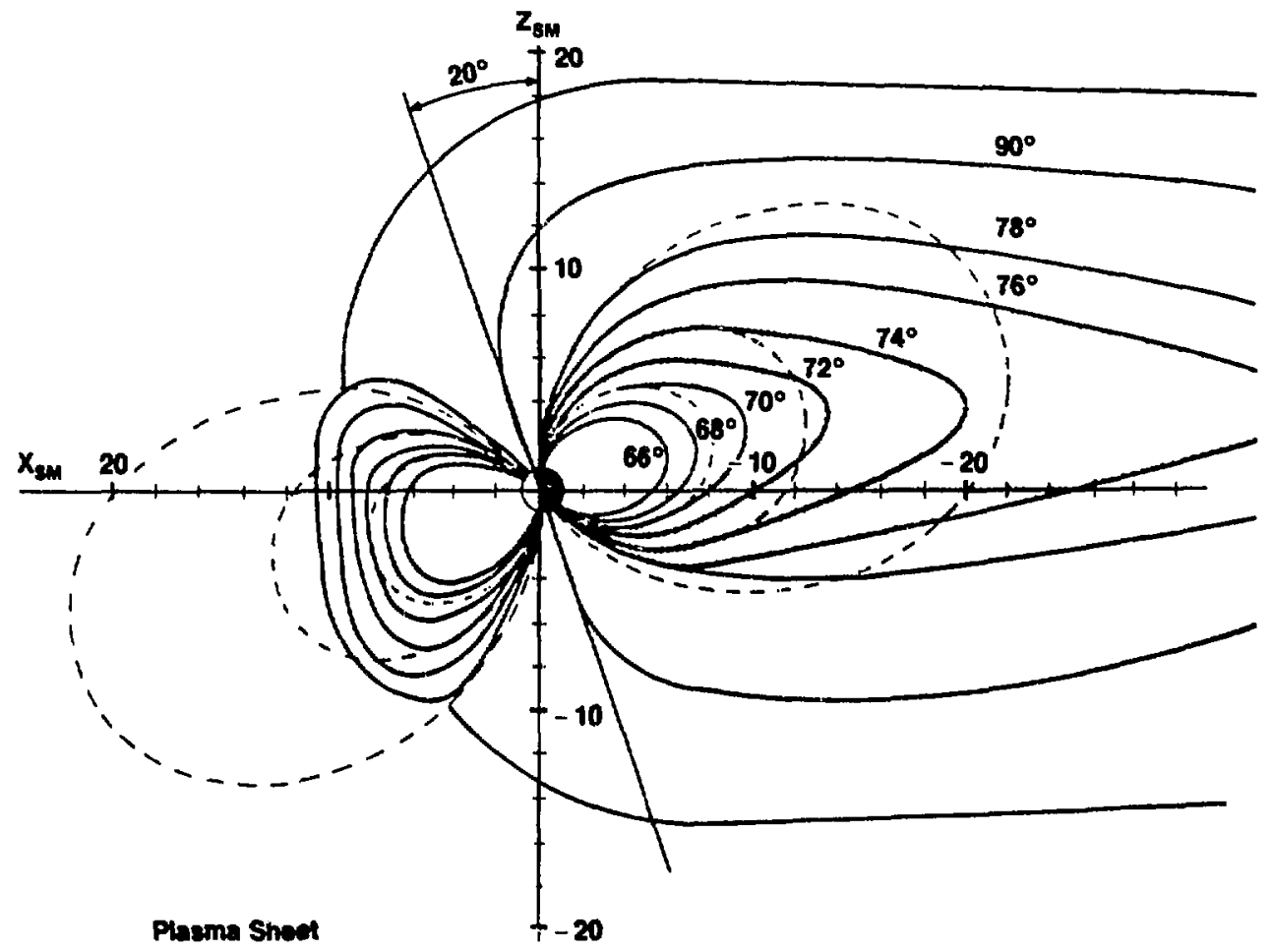

Figure 4-1. Noon-midnight cros-section of the magnetosphere, showing a dipole tilt angle of $20^{\circ}$ in the GSM coordinate aystem. The $X_{\text {GSM }}$ axis points sunward. The $Y_{G S M}$ axis is taken in the direction $N M \times X_{G S M}$ where $N M$ is a unit vector along the north magnetic dipole axis. The $Z_{G S M}$ axis completes a right-handed coordinate system. Field lines are labelled with the dipole latitude of their earth intercept. Dotted dipolar field lines are shown to illustrate the dayside compreasion and the nightoide distension of the magnetospheric field. Dipole tilt varies diumally and seasonally, with a maximum amplitude of $\pm 35^{\circ}$ (Fairfield and News, 1970).

of a dipole interaction with the solar wind, the downstream closure of the magnetosphere in the tail region behind the earth depended strongly on the strength and location of distributed current systems in the tail. The early field models were extended to include simple current sheets in the tail in order to reproduce ".e satellite-measured average tail field properties. The Hones model was modified (Taylor and Hones, 196; Taylor, 1966) by adding a tail current sheet of finite thickness on the night side. The Mead model was augmented with a tail current sheet of zero thickness (Williams and Mead, 1965).

Other models followed $i$ : an attempt to achieve greater realism and more quantitative agreement with satellite observations. The boundary surface model of Choe and Beard (Chue and Beard, 1974; Halderson, et al., 1975) included a tail current system which closed on the nightside magnetopause, and a representation of the magnetopause that was valid for all angles of dipole tilt. The Olson-Pfitzer boundary surface model (Olson, 1969; Olson, 1974; Olson and Pfitzer, 1974) included a dipole-tilt dependent magnetopause, a tail current system, and a current system flowing in an elliptical pattern near the equatorial plane to simulate the quiet-time ring currents of the inner magnetosphere. It should be carefully noted that the magnetopause calculations in tnese latter boundary-surface models were not completely selfconsistent; the pressure-balance equation which deternined the magnetopause location included only 
the earth's dipolar field and not any contributions from interior distributed currents. This lack of selfconsistency can be an issue when attempting to calculate particle orbits near the magnetopause. As we shall see, models which do not determine the magnetopause in at least a quasi-self-consistent manner exhibit anomalous field artifacts near the magnetopause, which render them useless for outer-magnetosphere orbit calculations.

Refinements to the image-dipole model were made by Antonova and Shabansky (1968) and more recently by Willis and Pratt (1972). This latter model added a graduated tail current system which provided a better match to field measurements made by the IMP-1, Explorer-33, and Explorer- 35 satellites in the distant tail. The great virtue of the image-dipole models-apart from their analytical formulation which lends itself to rapid evaluation of the total field-was that the magnetopause was necessarily selfconsistent with the total interior field from all sources (Willis and Pratt, 1972). Even in these improved models, matching the size and shape of the model magnetopause to observational data required rather arbitrary and iterative adjustment of the location and moment of the image dipole.

A variant model type was developed which retained the self-consistency of the image-dipole models but allowed for more realistic magnetopause shape, and could be much more readily adjusted in standoff distance to simulate various solar-wind conditions. Known as defined magnetopause models, they were developed in various forms by Alekseev and Shabansky (1972), Tsyganenko (1976), and Voigt and Fuchs (1979). The magnetopause surface is defined at the outset to have a particular shape. For simplicity a surface of revolution about the earth-sun axis was usually chosen. A current system was assumed to flow on the magnetopause which completely shielded the exterior volume from interior fields, a requirement that translates to the condition that the normal component of $B$ at the boundary must vanish. The magnetic induction from the surface currents is curl-free at any interior point, so we have:

$\nabla \times B_{5 C}(\mathbf{r})=0-B_{5 C}=-\nabla \phi_{5 C}(\mathbf{r})$,

where $\phi_{\mathrm{SC}}$ is the magnetic scalar potential of the field due to the surface currents, and

$\nabla \cdot \mathbf{B}_{\mathrm{SC}}(\mathbf{r})=-\nabla \cdot \nabla \phi_{\mathrm{SC}}(\mathbf{r})=-\nabla^{2} \phi_{\mathrm{SC}}(\mathbf{r})=0$.

This is Laplace's equation for the scalar potential, and it can be solved uniquely for $\phi_{S C}(r)$ if the normal derivative is known on all bounding surfaces (Neumann boundary condition). In this case

$\frac{\partial \phi}{\partial n}=-B_{5 C n}=+B_{l n}$

on the magnetopause, where $B_{I n}$ is the normal component of the field arising from all internal sources (geocentric dipole + distributed currents). This guarantees that the normal component of the total field $\left(B_{S C}+B_{l}\right)$ vanishes. The magnetopause for these models is then consistent with all internal field sources used in the calculation. It does not however represent a surface in equilibrium with the solar wind pressure, although model parameters can be easily adjusted to get a generally good agreement with satellite-determined average boundaries.

Another type of model is the empirical model derived by Mead and Fairfield (1975). This is a leastsquares fit of satellite magnetometer data to a polynomial of degree two in the spatial $x, y, z$ coordinates of the magnetosphere. The magnetopause surface was taken as the average satellite-determined boundary.

In this study of energetic particle drift motions in the outer dayside magnetosphere, we employ models representative of major model types to generate theoretical drift trajectories. These models are the boundary surface models of Choe-Beard and Olson-Pfitzer, and the empirical Mead-Fairfield model. Results of adiabatic drift studies previously reported (Shabansky and Antonova, 1968; Kasimov and Shabansky, 1979) using an image-dipole model will be reviewed. Each model will be discussed in somewhat greater detail to illuminate model features and limitations which are significant to this study.

\section{Magnetopause Modeling}

The boundary surface models determine the magnetopause by seeking a surface which is in equilibrium between the dynamic pressure of the solar wind on the sunward side and the magnetic pressure of 
the confined magnetic field on the other. Pioneering stidies of the interaction between the solar wind plasma and the earth's field were carried out by Chapman and Ferraro $(1931 ; 1932 ; 1933 ; 1940)$ and Ferraro (1952). They reasoned that the diamagnetic plasma would exclude the earth's field, and assumed for simplicity a planar boundary geometry (Fig. 4-2). For a more realistic model, the surface must be allowed to curve three-dimensionally, but much of the boundary physics can be understood from the planar approximation. The following discussion of this simplified magnetopause model is intended to identify the important boundary-formation mechanisms. Comprehensive reviews of the magnetopause structure and fields have been presented by Beard (1964) and Willis (1971).

We assume a cold, neutral, field-free stream of ions and electrons incident from the $-x$ direction upon the flanar magnetopause at $x=0$. The uniform geomagnetic field lies in the $+z$ direction. The charged particles are rotated through $180^{\circ}$ by the field and ejected back across the boundary. The net effect is a specular reflection of the incident solar wind particles. This exerts a dynamic pressure on the confined field which in the steady-state must be balanced by the magnetic pressure $B^{2} / 8 \pi$ (Gaussian units). The reflection of the particles sets up a boundary-layer electrical current flowing in the $-y$ direction. $A$ boundary condition of this problem is that the net magnetic field sunward of the magnetopause must be zero (by assumption, the solar wind is field-free). The effect of the current is to produce a magnetic field which completely nulls the terrestrial field at all points in the sunward half-space. As a consequence (for a boundary layer thin compared to its distance from earth), the magnetic field just earthward of the boundary will be twice the terrestrial contribution. If the incident solar wind speed is $v_{0}$ (for both ions and electrons), the incident ions will penetrate into a uniform magnetic field a factor of $m_{1} / m_{E}$ farther than the electrons. This in turn would create a very large polarization field in the $-x$ direction, slowing the ions and accelerating the electrons, leading to a reduction of the difference in ion/electron ranges in the boundary layer and thus reducing the polarization field. In the steady state, the potential energy of a particle in the E-field must be no greater than the kinetic energy of the solar wind

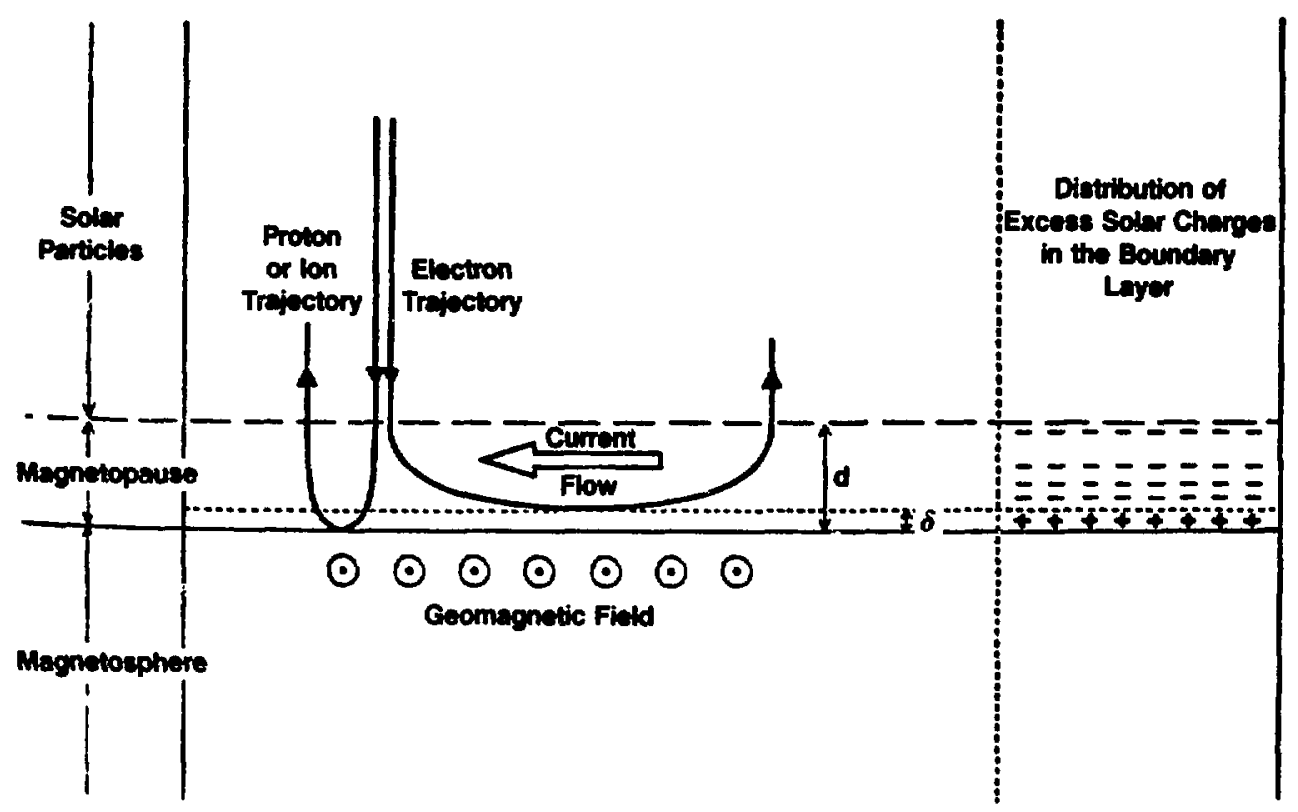

Figure 4-2. Schematic illustration of the trajectories of ions and electrons incident normally on a plane boundary when the polarization electric field due to charge separation is present; $d \approx 1 \mathrm{~km}, \delta$ $\approx I$ meter (Willis, 1971). 
particles which are creating the field, if energy is to be conserved. This requirement leads to approximate charge neutrality over nearly the entire depth of the boundary layer.

The equations of motion of a solar wind particle are (Dungey, 1958):

$F_{X}=m \ddot{x}=e E(x)+\frac{[e v \times B(x)] x}{c}$,

$F_{Y}=m \ddot{y}=\frac{[e v \times B(x)] y}{c}$.

The solutions have been discussed by Beard $(1960,1964)$. The latter equation can be written as:

$m \frac{d y}{d t}=-\frac{e \dot{x} B(x)}{c}$;

which can be integrated once to obtain:

$\left.m \dot{y}\right|_{-\infty} ^{x}=-\frac{e}{c} \int_{-\infty}^{x} B\left(x^{\prime}\right) d x^{\prime}$

or

$v_{Y}(x)=\frac{-e}{m c} \int_{-\infty}^{x} B\left(x^{\prime}\right) d x^{\prime}$

(at the source, $v_{Y}(-\infty)=v_{Y 0}=0$ ).

If Eq. (4.8) is substituted into Eq. (4.5a), an equation for the $x$-velocity results:

$m \ddot{x}=e E(x)-\frac{e^{2} 3(x)}{m c^{2}} \int_{-\infty}^{x} B\left(x^{\prime}\right) d x^{\prime}$.

To produce an easily integrable form, Eq. (4.9) is multiplied by $\dot{x}$ to produce:

$m \dot{x} \ddot{x}=e E(x) \dot{x}-\frac{e^{2} \dot{x}}{m c^{2}} B(x) \int_{-\infty}^{x} B\left(x^{\prime}\right) d x^{\prime}$

which can be written

$\frac{m}{2} \frac{d(\dot{x})^{2}}{d t}=e E(x) \dot{x}-\frac{e^{2} x}{2 m c^{2}} \frac{d}{d x}\left[\int_{-\infty}^{x} B\left(x^{\prime}\right) d x^{\prime}\right]^{2}$.

This equation can be integrated over $\mathrm{dt}$ to obtain:

$\left.\frac{m}{2} \dot{x}^{2}\right|_{-\infty} ^{x}=e \int_{-\infty}^{x} E\left(x^{\prime}\right) d x^{\prime}-\frac{e}{2 m c^{2}}\left[\int_{-\infty}^{x} B\left(x^{\prime}\right) d x^{\prime}\right]^{2}$

yielding:

$\frac{m}{2} v x^{2}(x)=\frac{m}{2} v_{0}^{2}+e \int_{-\infty}^{x} E\left(x^{\prime}\right) d x^{\prime}-\frac{m v_{y}}{2}(x)$. 
Equation (4.13) is a statement of conservation of total energy (non-relativistic) for the ions, which can be more readily seen if put into the form:

$\frac{m v_{0}^{2}}{2}=\frac{m}{2}\left[v_{x}^{2}(x)+v_{y}^{2}(x)\right]+e \Phi(x)$

where we have used:

$\int_{-\infty}^{x} E\left(x^{\prime}\right) d x^{\prime}=-\int_{-\infty}^{x} \frac{d \Phi}{d x^{\prime}} d x^{\prime}=-\Phi(x)$

the electrostatic potential [taking $\Phi(-\infty)=0$ ]. So far no approximations have been made.

We now roughly estimate the differential range of electrons and ions in the boundary layer. Assume the ions penetrate a distance $\delta$ farther than the electrons, producing a positive charge layer of thickness $\delta$. The electrostatic potential developed by the charge separation is given by Poisson's law:

$\frac{d^{2} \Phi(x)}{d x^{2}}=-4 \pi n_{1} e$

which can be integrated over $\delta$ to yield:

$\Delta \Phi=-2 \pi n_{l_{0}} e \delta^{2}$

where for simplicity we have taken the ion ciensity to be the unperturbed density $n_{l_{0}}$

The maximum potential which can be generated is [from Eq. (4.14)]:

$\Phi_{\text {MAX }}=\frac{m_{1} v_{0}^{2}}{2 e}$

i.e., the potential energy of an ion in the field is limited to its original kinetic energy. Substituting this result in Eq. (4.17), we have:

$\delta_{\mathrm{MAX}}=\left|\frac{m_{1} v_{0}^{2}}{4 \pi \mathrm{n}_{\mathrm{j}_{0}} \mathrm{e}^{2}}\right|^{1 / 2}$

which for typical solar wind conditions $\left(v_{0}=300 \mathrm{~km} / \mathrm{s}, n_{\mathrm{l}_{0}}=10 / \mathrm{cm}^{3}\right)$ yields:

$\delta_{\mathrm{MAX}}<130 \mathrm{~m}$.

By the equation of continuity,

$\frac{\partial n_{1}}{\partial t}(x)=-\nabla \cdot\left(n_{1}(x) v\right)=-\frac{d}{d x}\left(n_{1}(x) v_{x_{1}}\right)=0$ in steady state

so $n_{1}(x) v_{x_{1}}(x)=$ constant $=2 n_{I_{0}} v_{0}$, the factor of 2 arising from the reflected flux contribution. Hence as the ions decelerate across the boundary layer the ion density increases, so this estimate $\delta_{\text {MAX }}$ is too large.

More detailed calculations (Davies, 1967) showed that the separation $\delta$ should be of the order of a Debye length:

$\delta \approx\left[\frac{m_{E} v_{0}^{2}}{4 \pi n_{E} e^{2}}\right]^{1 / 2} \approx 1 \mathrm{~m}$. 
Consequently we are justified in assuming charge neutrality throughout the boundary. This implies thi it the $x$-velocities of ions and electrons must be equal.

From Eq. (4.14), we can obtain:

$\left[v_{X}^{2}(x)+v_{Y_{E}}^{2}(x)\right]-\frac{2 e \Phi(x)}{m_{E}}=\left[v_{X}^{2}(x)+v_{Y_{1}}^{2}(x)\right]+\frac{2 e \Phi(x)}{m_{1}}$

which reduces to:

$v_{Y_{E}}^{2}(x)=v_{Y_{i}}^{2}(x)+\frac{2 e \Phi(x)}{m_{E}} ;$

(where a factor of $m_{E} / m_{1}$ has been neglected).

The polarization field does the work of stopping the incident ions. At the maximum penetration (the thickness $L$ of the layer) we have:

$v_{x_{1}}(L)=0$

$\mathrm{v}_{\mathbf{Y}_{1}}(\mathbf{L}) \approx 0$

Therefore from Eq. (4.14), $\mathrm{Q} \Phi(\mathrm{L}) \approx \mathrm{m}_{1} v_{0}^{2} / 2$, and from $\mathrm{Eq} .(4.23)$,

$v_{Y_{E}}^{2}(L) \approx \frac{m_{1} v_{0}^{2}}{m_{E}}$

i.e., the electrons are accelerated by the polarization field to about the initial kinetic energy of the ions. The polarization field acts to transfer kinetic energy from the ions to the electrons entering the boundary. On exit, the energy is transferred back from the electrons to the ions. At the inner edge of the layer, the accelerated electrons carry the bulk of the shielding currents. From Eq. (4.14), we can obtain a pair of equations for ions and electrons:

$v_{0}^{2}=v_{X_{1}}^{2}+v_{Y_{1}}^{2}+2 e \Phi / m_{1}$

$v_{0}^{2}=v_{x_{E}}^{2}+v_{Y_{E}}^{2}-2 \mathrm{e} \Phi / m_{E}$

Substituting from Eq. (4.8) and solving for $\Phi$ yields:

$\Phi(x)=\frac{e}{2 m_{E} c^{2}} \int_{-\infty}^{x} B\left(x^{\prime \prime} d x^{\prime}\right.$.

Substituting Eqs. (4.27) and (4.8) into Eq. (4.26a) and solving for $v_{x_{1}}$ gives:

$$
\begin{aligned}
v_{x_{l}}^{2}(x) & =v_{0}^{2}-\frac{e^{2}}{m_{l} m_{E} c^{2}}\left[\int_{-\infty}^{x} B\left(x^{\prime}\right) d x^{\prime}\right]^{2} \\
& =v_{0}^{2}-\frac{m_{E}}{m_{1}} v_{Y_{E}}^{2} .
\end{aligned}
$$

The overall thickness of the boundary layer can now be estimated.

$\nabla \times B=-\frac{\partial B(x)}{\partial x}=-\frac{4 \pi j}{C} \hat{y}=-\frac{4 \pi e n_{E} v_{Y_{E}}}{C} \hat{y} ;$ 
where we assume that the shielding current is carried predominately by the electrons since $v_{Y_{E}} \gg v_{Y_{1}}$. Substituting in the equation of continuity for the electrons, we have

$$
\begin{aligned}
\frac{\partial B}{\partial x}(x) & =\frac{4 \pi e}{c}\left[\frac{2 n_{0} v_{0}}{v_{X}}\right] v_{Y_{E}} \\
& =\frac{8 \pi e n_{0} v_{0}}{c} \frac{v_{Y_{E}}}{\left[v_{0}^{2}-\frac{m_{E}}{m_{l}} v_{Y_{E}}^{2}\right]^{1 / 2}} .
\end{aligned}
$$

An approximate solution of Eq. (4.30) can be obtained for values of $x$ at a distance from the inner edge $(x=0)$ such that

$$
v_{0}^{2} \gg \frac{m_{E}}{m_{1}} v_{Y_{E}}^{2}(x)
$$

Then

$$
\begin{aligned}
\frac{\partial \mathrm{B}}{\partial x}(x) & \approx \frac{8 \pi e n_{0}}{c} v_{Y_{E}} \\
& \approx \frac{8 \pi e n_{0}}{c}\left[\frac{e}{m_{E} c} \int_{-\infty}^{x} B\left(x^{\prime}\right) d x^{\prime}\right] .
\end{aligned}
$$

Differentiating with respect to $x$ again gives:

$\frac{\partial^{2} \mathrm{~B}}{\partial \mathrm{x}^{2}}(\mathrm{x})=\boldsymbol{a}^{2} \mathrm{~B}(\mathrm{x})$

where $\alpha^{2}=\frac{8 \pi e^{2} n_{0}}{m_{E} c^{2}}$,

which has the solution:

$B=B_{0} \exp (a x), x \leq 0$.

The characteristic scale distance $L$ is therefore

$\mathrm{L}=\frac{1}{\alpha}=\left[\frac{\mathrm{m}_{\mathrm{E}} \mathrm{c}^{2}}{8 \pi \mathrm{e}^{2} \mathrm{n}_{0}}\right]^{1 / 2}=\frac{\delta \mathrm{p}}{\sqrt{2}}$

where $\delta_{\mathrm{p}}=$ plasma skin depth (Jackson, 1962).

From Eqs. (4.29) and (4.27), the current density and the polarization electric field $-\nabla \$$ also show an exponential decrease in the boundary layer. The exponentis. sulution Eq. (4.33) is a good approximation to the true solution of Eq. (4.30), except for the region $-x \ll L$ (Beard, 1964). An interesting feature of the solution is that the scale length $L$ is independent of solar wind kinetic energy. For a typical solar wind density of 10 pairs $/ \mathrm{cm}^{3}, \mathrm{~L} \approx 1 \mathrm{~km}$.

The foregoing model has neglected the magnetospheric plasma, which according to Parker (1967a,b; 1968) would in the steady state act to short out the polarization field. Owing to the high conductivity along the field lines, dense ionospheric plasma could flow to the magnetopause in minutes and neutralize the electrostatic boundary field. Figure 4-3 shows the model obtained for this condition. The boundary is then as thick as an average ion gyroradius in the boundary layer magnetic field, $\approx 100 \mathrm{~km}$, and the 


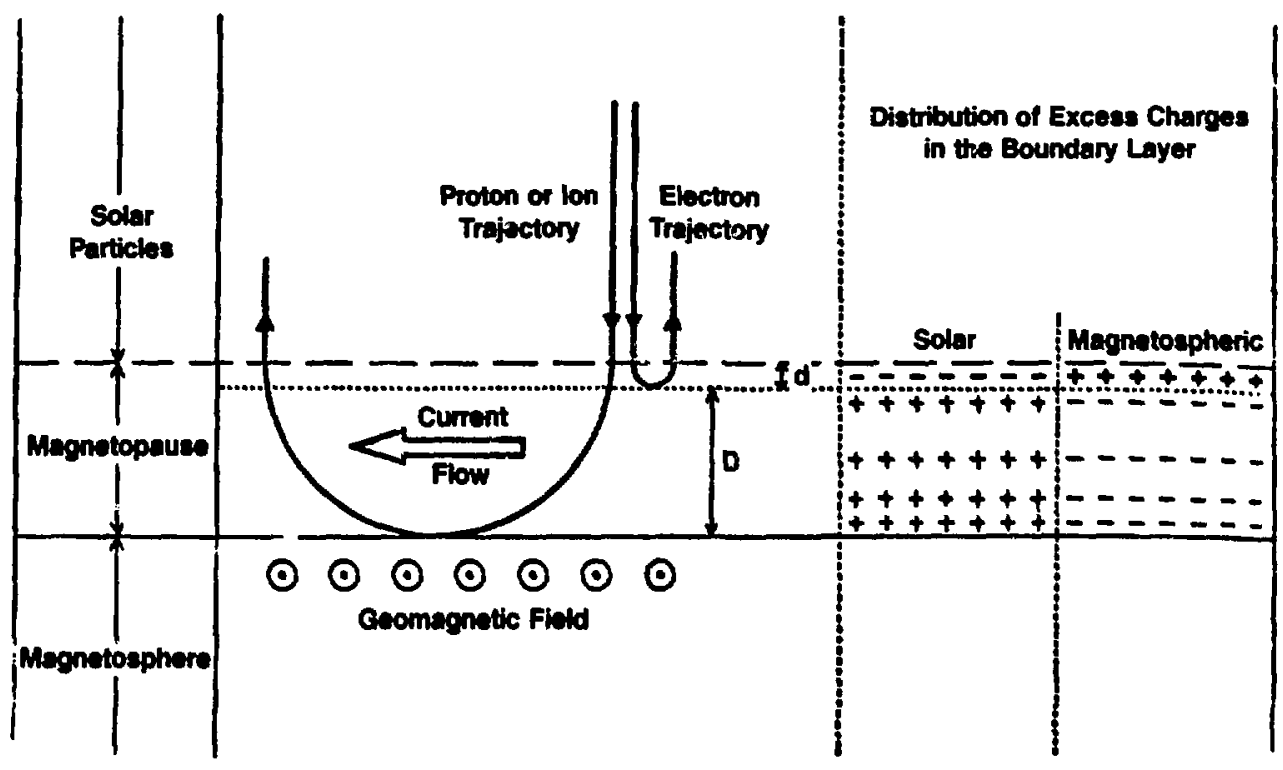

Figure 4-3. A schematic illustration of the trajectories of ions and electrons incident normally on a plane boundary when the polarization electric field is completely neutralized by amivient magnetospheric charged particles; $D \approx 100 \mathrm{~km}, \mathrm{~d} \approx 1 \mathrm{~km}$ (Willis, 1971).

shielding current is carried by the ions with their greater cyclotron radius. Twin-satellite measurements by the ISEE probes (Paschmann et al., 1978) confirmed that the magnetopause structure, at least on some passes, was consistent with Parker's model.

In either case, the boundary layer is thin compared to its radius of curvature. This allows one to treat the interaction between a solar wind particle and the boundary as if the particle were reflected from a plane tangent to the boundary at the point of incidence. That this interaction is a specular reflection is readily demonstrated from the equations of motion.

Consider the trajectory of an incident particle to be divided into an inbound and an outbound section (Fig, 4-4). Let primes denote quantities of the outbound section.

From Eq. (4.5a),

$F_{x}=e E+\frac{e v_{Y} B}{c}$.

$E$ and $B$ are functions of $x$ only, so along the trajectory $F^{\prime}(x)=F(x)$, which implies:

$v_{Y}^{\prime}(x)=v_{Y}(x)$.

Since $E$ is normal to the boundary, the kinetic energy is a function only of $x[E q .(4.14)]$ :

$v_{X}^{\prime 2}(x)+v_{Y}^{\prime 2}(x)=v_{X}^{2}(x)+v_{Y}^{\prime 2}(x) ;$ 


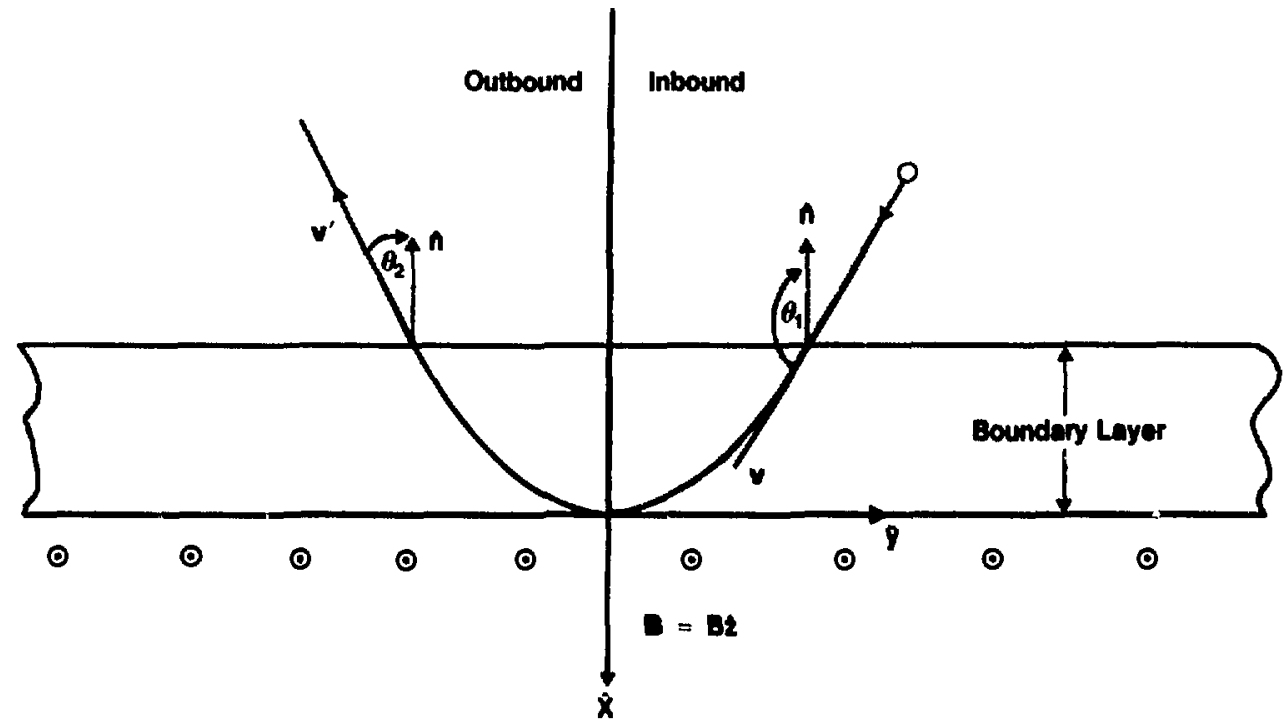

Figure 4-4. Specular reflection of an ion incident on planar magnetopause boundary. Both $E$ and $B$ are functions of $x$ only.

which with Eq. (4.36) implies:

$v_{x}^{\prime 2}(x)=v_{x}^{2}(x) ;$ or

$v_{X}^{\prime}(x)=-v_{x}(x)$ (choosing the minus sign for the outbound trajectory).

Since the inbound and outbound trajectories must span the same range of $x$-values if the particle is to exit, every point in the inbound section is matched to a point in the outbound section where at any $x$,

$v_{x}^{\prime}=-v_{x}$

$v_{Y}^{\prime}=+v_{Y} ;$

$v(x)=v_{x} \hat{\imath}+v_{y} \hat{\jmath}$

$v^{\prime}(x)=-v_{x} \hat{\imath}+v_{y} \hat{\jmath}$.

At the surface, the angles with respect to the outward normai $n$ are:

$\cos \theta_{1}=\frac{\hat{n} \cdot v}{|v|}=-\frac{v_{x}}{|v|}$

$\cos \theta_{2}=\frac{\hat{n} \cdot v^{\prime}}{\left|v^{\prime}\right|}=\frac{v_{x}}{|v|}=-\cos \theta_{1}$

therefore $\theta_{2}=\pi-\theta_{1}$, the condition for specular reflection. 


\section{Self-Consistent Calculation of the Magnetopause}

In the foregoing pages we have argued that the magnetopause can be treated as a thin surface which specularly reflects the incident solar wind ions which stream from the solar direction. We now proceed to sketch the self-consistent method of computing the location of the magnetopause. This method identifies the magnetopause as the locus of a surface in equilibrium between the dynamic pressure of the reflected solar wind (assumed to be unmagnetized and of zero temperature) and the magnetic pressure of the confined magnetospheric field.

Figure 4-5 illustrates the reflective geometry for a section of the boundary surface, where the incident solar wind angle is $\theta_{1}$.

An incident particle undergoing reflection suffers a change in momentum of:

$\delta \mathrm{p}=2 \mathrm{p}_{\mathrm{N}}=2 \mathrm{mv_{ \perp }}=-2 \mathrm{mv} \cos \theta_{1} ;$

where $\mathrm{p}_{\mathrm{N}}=$ component of the incident mumentum in the direction of the local surface normal n (outwarddirected).

The number of particles per unit time incident on the boundary here, per unit area normal to $\hat{n}_{\mathrm{f}}$ is:

$-\Gamma v \cos \theta_{1}$

where $\Gamma$ is the number density of the incident solar wind (assumed uniform). The reflection of this flux produces a dynamic pressure directed normal to the boundary and inwards, of magnitude:

$P_{D}=\left(\Gamma v \cos \theta_{1}\right)(\delta p)=2 \Gamma m_{1} v^{2} \cos ^{2} \theta_{1}$

(The electron contribution to the dynamic pressure is a factor of $m_{E} / m_{I}$ less and can be ignored).

For a current-carrying conductor to be in mechanical equilibrium in a magnetic field, the net body forces must vanish:

$F_{B}=\frac{i \times B}{C}-\nabla \cdot P=0 \quad$ (Krall and Trivelpiece, 1973)

where $\mathrm{P}$ is the pressure tensor. Evaluated normal to the boundary, this condition becomes

$\nabla_{N} P=\frac{(j \times B)_{N}}{c}$

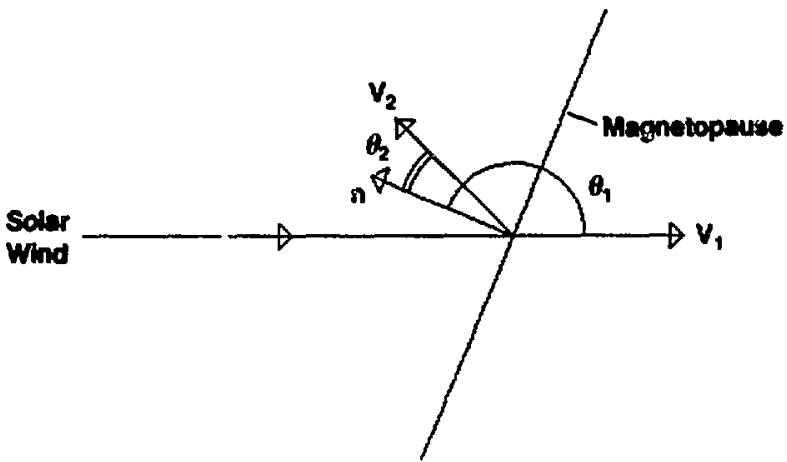

Figure 4-5. Boundary geometry for oblique incidence of solar wind. 
Substituting for $\mathbf{j}$ from Ampere's law:

$\nabla \times B=\frac{4 \pi j}{C} ;$

we have

$\nabla_{N} P=\left[\frac{(\nabla \times B) \times B}{4 \pi}\right]_{N}=\frac{1}{4 \pi}\left[\frac{-1}{2} \nabla_{N} B^{2}\right]=\frac{-\nabla_{N} B^{2}}{8 \pi}$.

Hence

$P+\frac{B^{2}}{8 \pi}=$ constant.

Setting the constant equal to $P_{D}$ we have outside the boundary

$P=P_{0}=2 \Gamma m_{1} v^{2} \cos ^{2} \theta_{1} \quad(B=0)$.

Inside the boundary,

$\frac{B^{2}}{8 \pi}=P_{D}=2 \Gamma m_{1} v^{2} \cos ^{2} \theta_{1} \quad$ (plasma pressure $\left.=0\right) ;$

or

$B= \pm \sqrt{16 \pi \Gamma m_{1} v^{2} \cos ^{2} \partial_{1}}$, the pressure-balance condition.

The cordition that $\nabla \cdot \mathbf{B}=\mathbf{0}$ implies that $\mathrm{B}_{\mathrm{N}}$, the normal component of $\mathbf{B}$, must be cont uous across a boundary. By st: llition $B$ is zero sunward of the boundary, so $B_{N}$ must vanish. There.iore inside the boundary, the toial field $B_{\top}$ is tangential to the surface.

The pressure-balance condition [Eq. (4.52)] can then be rewritten to incorporate the tangential requirement:

$\left|\hat{n} \times B_{T}\right|=-\sqrt{16 \pi \Gamma m_{i} v^{2}} \frac{\hat{n} \cdot \mathbf{v}}{|\mathbf{v}|}$.

The problem of finding the equilibrium location of the magnetopause now reduces to finding $i\left(\mathbf{r}^{\prime}\right)$ and $\mathbf{B}(\boldsymbol{r})$ (for specified $\Gamma, y$ ) such that Eq. (4.53) is satisfied.

Let $\mathrm{R}(\theta, \phi)$ be the geocentric distance to the magnetopause in a polar coordinate system centered on the earth.

$\mathbf{B}_{\mathrm{C}}(\mathbf{r})=$ the field due to the magnetopause currents, evaluated at the center of the boundary at $\mathbf{r}=$ $R(\theta, \phi) \hat{r}(\theta, \phi)$.

$B_{C}(r)$ can be computed from the Biot-Savart law by integrating the shieiding currents over the magnetopause:

$\mathbf{B}_{\mathrm{C}}(\mathbf{r})=\int \frac{\mathbf{J}\left(\mathbf{r}^{\prime}\right) \times \mathbf{r}^{\prime}}{\left|\mathbf{r}-\mathbf{r}^{\prime}\right|^{3}} \mathrm{ds}$

where $J\left(r^{\prime}\right)=$ surface current per unit length at field point $g^{\prime}$.

Note that in the planar approximation (the magnetopause taken as a plane perpendicular to the solar wind) $B_{\mathrm{C}}(\mathbf{r})=\mathbf{0}$ for all $\mathbf{r}$ on the boundary. If evaluated just inside the beundary, the tangential component of the field due to the current sheet is equal and opposite to the component just outside the boundary; if evaluated at the center of the (infinitesimally thick) boundary the tangential component must vanish by 
symmetry. This notion can be extended to the real (curved) magnetopause by imagining that the total field $B_{\mathrm{T}}$ at a point just inside the boundary is the result of a superposition of fields arising from these sources:

1. the geomagnetic dipole (field $\mathbf{B}_{\mathrm{D}}$ );

2. an infinite planar current sheet tangent to the magnetopause at that point (field $B_{p}$ );

3. the curvature of the current sheet (field $\mathbf{B}_{C}$ ).

This decomposition of the field simplifies the magnetic field calculations.

Just inside the surface we have:

$\mathbf{B}_{\mathrm{T}}=\mathbf{B}_{\mathrm{D}}+\mathbf{B}_{\mathrm{P}}+\mathbf{B}_{\mathrm{C}}$

while just outside the surface :

$$
\begin{aligned}
\mathbf{B}_{\mathrm{T}} & =\mathbf{B}_{\mathrm{D}}-\mathbf{B}_{\mathrm{P}}+\mathbf{B}_{\mathrm{C}} \\
& =\mathbf{0} \text { by stipulation. }
\end{aligned}
$$

Therefore $\mathbf{B}_{\mathrm{P}}=\mathbf{B}_{\mathrm{D}}+\mathbf{B}_{\mathrm{C}}$, and

$B_{\mathrm{T}}=2\left(B_{D}+B_{C}\right)$ just inside the boundary.

The surface current J can be expressed in terms of the field:

$\nabla \times B_{T}=\frac{4 \pi J}{c}$.

Integrating over a loop which crosses the boundary yields:

$|J|=\frac{c B_{\mathrm{T}}}{4 \pi}$,

and since $\mathbf{J}, \mathbf{B}_{\mathrm{T}}$, and $\hat{\mathbf{n}}$ are all orthogonal, this can be written:

$J=-\frac{C}{4 \pi} \hat{n} \times B_{T}=-\frac{C}{2 \pi} \hat{n} \times\left(B_{D}+B_{C}\right)$.

This can be substituted into Eq. (4.54) to get an implicit form for $B_{C}$ :

$B_{C}(\mathbf{r})=\frac{-c}{2 \pi} \int \frac{\left[\hat{\mathbf{n}} \times\left(B_{D}+B_{C}\right)\right]}{\left|\mathbf{r}-\mathbf{r}^{\prime}\right|^{3}} \times \mathbf{r}^{\prime} d S$.

Equations (4.53) and (4.61) form the heart of the self-consistent magnetopause calculational method devised by Beard (Beard, 1962; Beard and Jenkins, 1962; Mead and Beard, 1964). Given a first guess at $\mathbf{B}_{7}$ $=B_{D}+B_{C}, E q .(4.53)$ is solved for $R(\theta, \phi)$ and $\hat{n}$, thus determining a first approximation to the surface. This surface and the guess at $B_{T}$ yield an improved estimate of $B_{C}$ from $E q$. (4.61). This iteration process is continued until no further improvement occurs. Beard found that the fourth-iteration surface gave the best results. The field computed from the surface currents cancelled the dipole field at exterior points to within a few tenths of a percent in most locations (Mead and Beard, 1964). A measure of the solution meeting the requirement that $\mathbf{B}_{\mathrm{N}}$ must vanish at the boundary was provided by the ratio $\left|\mathbf{B}_{\text {TANGENTIAL }}\right| / \mathbf{B}_{\mathrm{T}} \mid$; this ratio exceeded .999 everywhere except near the high-latitude neutral points and in the distant tail.

Overall the self-consistent magnetopause method provides an accurate solution to the problem of the reflection of a stream of cold, unmagnetized plasma by the geomagnetic field. We now examine the limitations placed on the model by the simpiifying assumptions. 
The streaming velocity of the solar wind ions is on the order of a factor of $\mathbf{1 0}$ greater than the ion thermal speed, so that the dynamic pressure on the dayside magnetopause is predominately determined by the stream pressure. Hence the neglect of thermal effects does not significantly alter the shape or location of the dayside magnetopause. On the night side however, the thermal pressure and the interplanetary magnetic field must play a critical roie in the closure of the magnetospheric cavity behind the earth. An even more significant omission in this regard is the lack of a tail field current system, which provides about half of the total scalar field in the distant tail. The model assumes the solar wind to be incident from the solar direction, whereas satellite measurements of the plasma flow in the magnetosheath region (between the bow shock and the magnetopause) clearly indicate that piasma flow velocities are nearly tangential to the magneiopause (e.g., Hundhausen et al., $1 \div \delta$ ) as described by gas dynamic models of the bow shock and magnetosphere (Spreiter et al., 1966). This accounts for the slightly compressed appearance of the self-cunsistent model in the "flanks" of the magnetosphere (the region of $x \approx 0$ ) as compared to the empirically-determined magnetopause of Fairfield (1971), Fig. 4-6.

A final issue with respect to model realism is that the model magnetosphere discussed here is closed, i. e., the magnetopause is not penetrated by any terrestrial field line. The theory of the closed magnetosphere was first put forward by Piddington (1960a,b), and elaborated by Axford and Hines (1961). It postulated a viscous-like interaction between the flowing solar wind and the magnetosphere whith transported closed magnetospheric flux tubes, filled with plasma, around from the dayside magnetopause, through the dawn and dusk meridians, to form a stretched-out magnetotail / $/ \mathrm{ig}$. 4-7). In the steady-state, magnetic flux is returned to the dayside again to maintain a dynamic balance. This motion drove the large-scale circulation of plasma known as magnetospheric convection (Fig. 4-8). Solar particle entry is by diffusion across the boundary of the magnetosphere.

A contrary theory of the solar wind-magnetosphere coupling was postulated by Dungey (1961). He

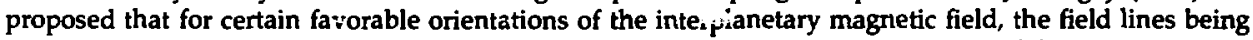
transported by the solar wind could merge or reconnect with the outermost terrestrial field lines, pulling them back over the polar caps (Fig. 4-9). This open model of the magnetosphere allowed solar vind plasma direct access to the magnetosphere. Merging must also occur within the neutral-sheet region of the magnetotail to return flux tubes back to the dayside.

Evidence can be found to support both models. The presence of the mangetosheath-like plasma just inside the low-latitude dayside magnetopause (Eastman et al., 1976) supports diffusive particle entry. Expected consequences of wide-spread, steady-state merging at the dayside-such as plasma heating

Figure 4-6. Intersection of the magnetopause surfaces of various magnetic field models with the equatorial plane. The: in, ChoeBeard-Sullivan, and Mead-Beard models are all "self-consistent magnetopause" models with a nearly identical equatorial trace. The Fairfield trace is an empiricallydetermined houndary for comparisan. (Wilker, 1979).

\section{Projection of Magnetupause Bounderies on Equiforial Plane}

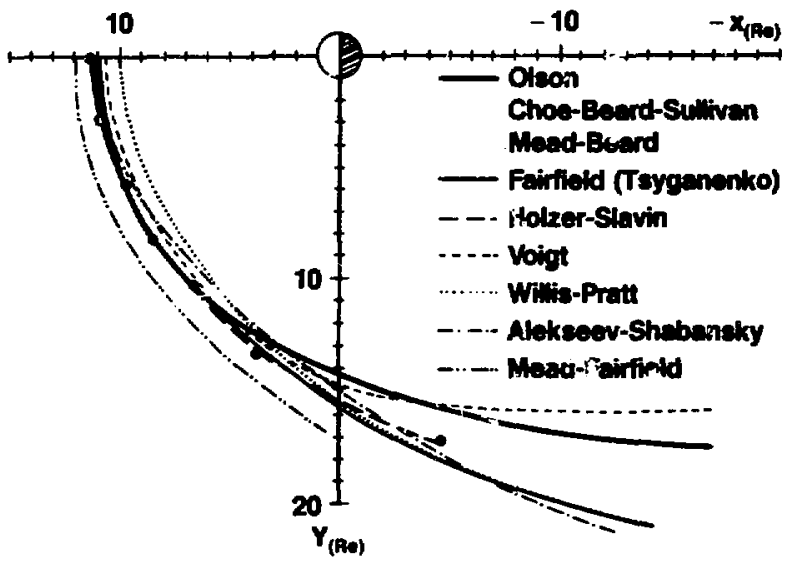


through the conversion of magnetic energy, a normal component of $B$ across the magnetopause, and highvelocity plasma flows-have not been observed (Heikkila, 1975; Mencke Hansen et al., 1976; Haerendel et al., 1978). However a large body of observations does exist whicis favors the open model in explaining such diverse phenomena as rapid solar particle access to the polar caps, the large-scale electric fields distributed over the polar oval, and magnetic substorms [see, e.g., reviews by Fairfield (1979) and Russell and Greenstadt (1983)]. These inconsistencies have led many investigators to conclude that frontside merging is "patchy" (Russell and Elphic, 1979), occurring sporadically in a limited region near the neutral points, and that a large area of the magnetopause is closed (Hill, 1982; Crooker, 1979, 1980). Figure 4-10 illustrates a model combining both merging and viscous transfer. Other researchers maintain that the closed model with some modifications can properly account for the observed phenomena (Johnson, 1978; Piddington, 1979). A variant viewpoint is that neither model is accurate (Alfvén, 1977).

In the present case, the validity of our modeling studies depends only on the configuration of closed field lines on the dayside, not on the details of the solar wind-magnetosphere coupling. We only require that (1) we can discriminate open from closed magnntospheric field lines in our measurements and (2) the topology of the closed field lines in the outer dayside magnetosphere is adequately described by our magnetic field model. On the first point, we note that a terrestrial field line connected to the interplanetary field does not support organized bounce motion; only on closed terrestrial lines do we find the double loss-cone type of pitch angle distribution, and the large fluxes of energetic particles characteristic of magneiospheric radiation. If we restrict our studies to these distributions we will avoid regions of open lines. The question of model topological adequacy will be discussed in the next section, where we describe a particular self-consistent model (the Choe-Beard 1974 model). We will find that in-situ observations are in good agreement with the model field.

\section{The Choe-Beard Model as a Representative Magnetospheric Magnetic Field Model}

In this section we present the Choe-Beard magnetic field model as a generic model for the study of charged particle trajectories in the outer magnetosphere. This model is representative of an important class of field models (including the Olson-Pfitzer and Mead-Williams models) possessing a self-consistently determined magnetopause. By comparison to empirical model fields produced from satellite measurements, this model reproduces the principal features of the actual outer magnetosphe-ic field reasonably well (though perhaps not in detail). In a subsequent section it will be used to illustrate in a qualisatively correct way how the magnetopause current systems greatly distort the dipole field in the outer magnetosphere and give rise to the splitting and branching of adiabatic drift shells.

\section{Definition of the Nominal Choe-Beard Field}

Figure 4-11 depicts the Choe-Beard model field lines traced in the noon-midnight meridian. The field illustrated here is the superposition of fields from several sources: a geocentric dipole (magnetic moment 31100 nanotesla- $R_{E}$ ), the Choe-Beard 1974 self-consistent model of the field due to magnetopause currents (Choe and Beard, 1974; revised by Beard, 1982), and the Beard model of the geomagnetic tail current system (Beard, 1979).

Figure 4-10. A magnetonpheric model illustrating that both forms of solar wind coupling may exist and make sigilificant contributions to the convection system. At the nose, a terrestrial flux tube has reconnected to the interplanetary field, and is being pulled over the polar cap and into the tail. At the ame time, a closed fux tube at the flanks of the magnetosphere is being driven into the tail by a viscous interaction (Cowley, 1982).

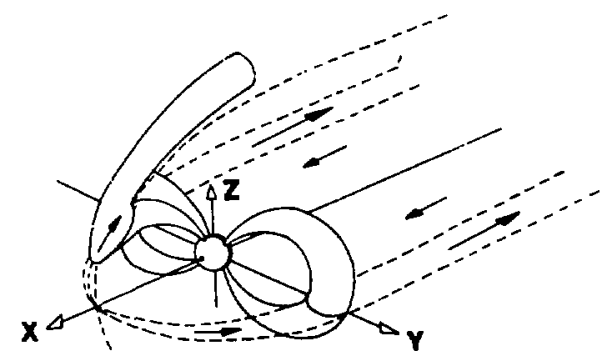




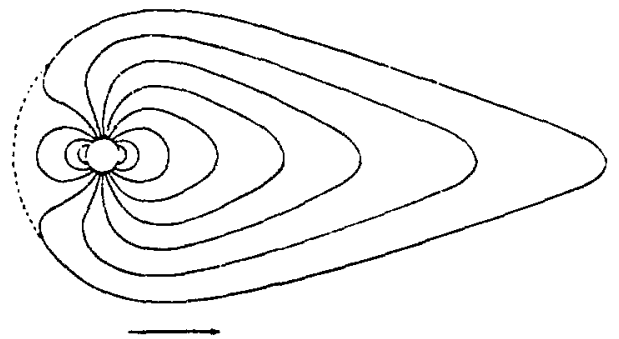

Soler Wind
Figure 4-7. Closed model of the magnetosphere. Solar wind plasma cannot directly penetrate into the magnetoapheric interior. Magnetospheric flux tubes near the magnetopause are dragged in the anti-solar direction by a viscous interaction with the solar wind, forming the magnetotail (Johnson, 1978).

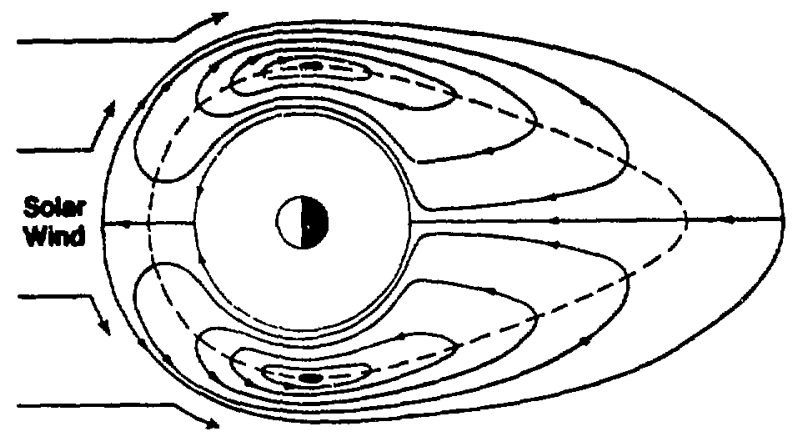

Figure 4-8. Convection of plasma within the closed model. The arrows indicate the direction of the plasma flow caused by the viacous interaction. Solar particles can only enter the magneto. ophere by a diffusive process across the magnetopause (after Axford and Hines, 1961).

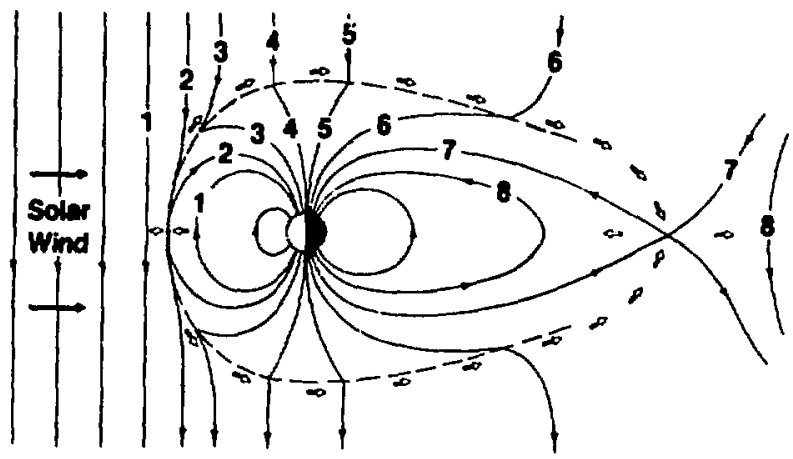

Figure 4-9. The reconnection or merging model of Dungey (1961). As interplanetary field lines embedded in the solar wind plasma are swept by earth, merging of the interplanetary and terrestrial fields can occur. The numbers label the sequential positions of an interplanetary and a terrestrial field line which undergo merging. In (1) an interplanetary line carried by the solar wind arrives at the nose of the magnetosphere, while a terreatrial line carried by the internal convection is brought to the frontside. In (2) the field lines merge to form a line with one foot connected to the earth. In (3)(6) the merged line is carried back over the polar cap, and in (7) reconnection occurs in the vicinity of the neutral sheet. In (8), the newly-reconnected terrestrial line contracts under magnetic tension and moves earthward, driving the convection system which returns flux to the frontside. The newlyreconnected interplanetary field is transported downstream. During the period that the terrestrial line is open to the interplanetary medium, solar wind particles gain direct access to the magnetosphere. Arrows indicate the direction of plasma flow. 


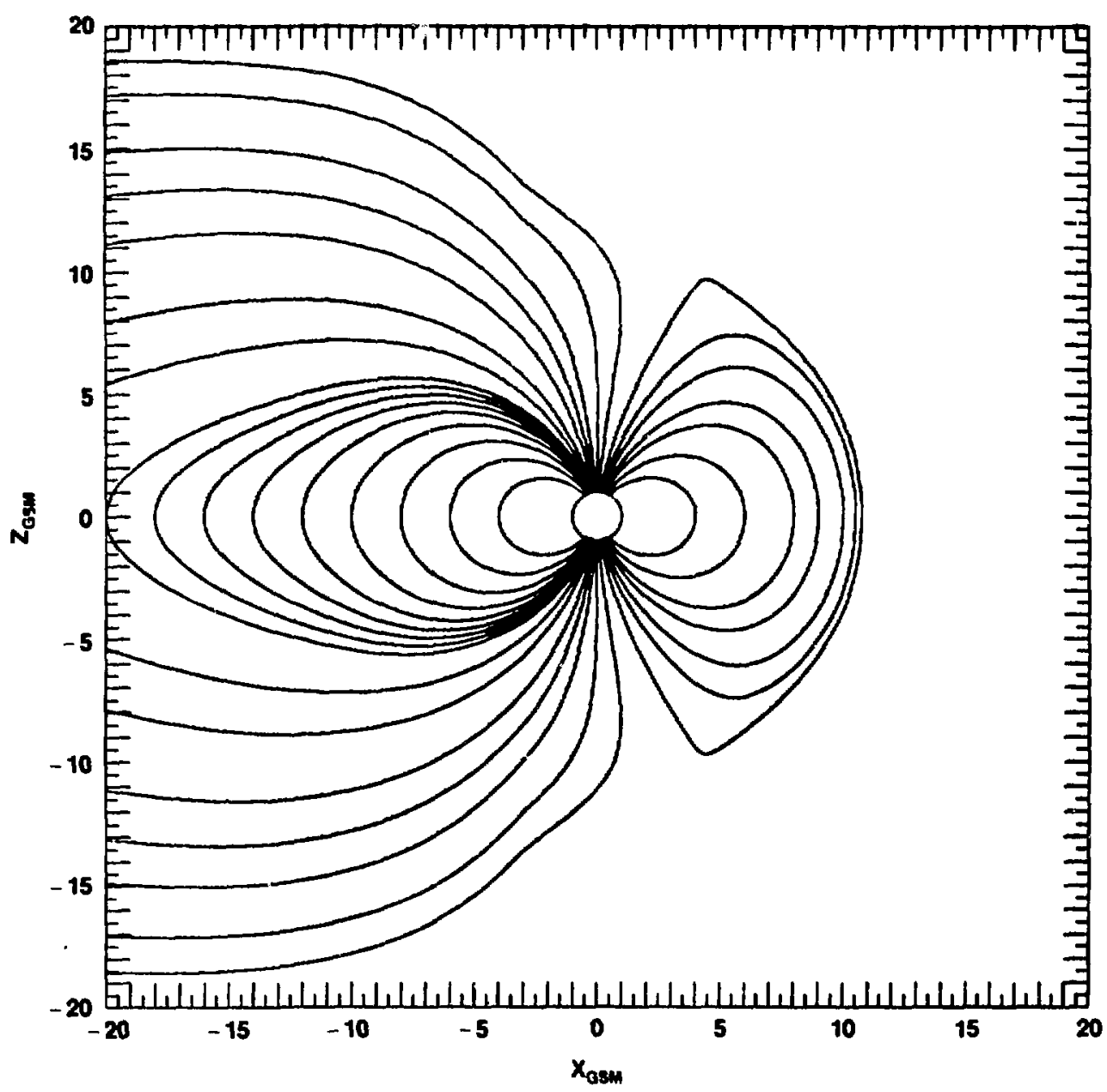

Figure 4-11. Noon-midnight cross-section of the Choe-Beard magnetic field model.

For a magnetcspheric model to be useful for particle trajectory studies it must be expressed in a compact form which can be rapidly evaluated on a computer. Accurately tracing a single field line in the outer magnetosphere (i.e., solving the equations

$\left.d x / B_{X}=d y / B_{Y}=d z / B_{Z}\right)$

can involve $10^{4}$ field evaluations. The computation of the magnetic induction at a field point by integrating the Biot-Savart law over the extended magnetospheric current systems is too costly a process, so field components (or the magnetic scalar or vector potential) are typically expressed as polynomial expansions in the field point coordinates.

Choe and Beard computed the Chapmati-Ferraro currents which flow on the magnetopause and generate a field which nulls the geocentric dipole field in the exterior volume. Inside the magnetosphere 
no currents flow (in the model), so that the field $\mathbf{B}_{M}$ due to the magnetopause currents can be expressed in terms of the magnetic scalar potential $\Phi_{M}$ at any interior point:

$$
\begin{aligned}
& \mathbf{B}_{M}=-\nabla \Phi_{M}, \text { satisfying } \nabla \times B_{M}=\frac{4 \pi J}{c}=0, \text { and: } \\
& \nabla \cdot B_{M}=-\nabla^{2} \Phi_{M}=0 .
\end{aligned}
$$

Equation (4.64) is Laplace's equation, whose solution in three dimnsions can be written in terms of a spherical harmonic expansion:

$\Phi_{M}(r, \theta, \phi)=\sum_{l=1}^{I_{\text {MAX }}} r^{l} \sum_{m=0}^{1} B_{l m} P_{l m}(\cos \theta \cos m \phi ;:$

where the $P_{1 m}(\cos \theta)$ are the associated Legendre functions and the $g_{1 m}$ are the expansion coefficients. $r$ is the radial distance to a field point, $\theta$ is the colatitude measured from the northerm dipole axis, and $\phi$ is the azimuthal coordinate measured from the earth-sun line.

Because this is a solution for the interior, the negative power series in $r$ is omitted. The sinm $\phi$ terms have been omitted since their coefficiunts are zero due to the symmetry of the field about the plane $\phi=0$.

Using the Schmidt-normalized associated Legendre functions, Choe and Beard (1974) expressed the field as:

$B_{i}\langle r, \theta, \phi, \tau)=\sum_{i=1}^{I_{M A X}} r^{1-1} \sum_{m=0}^{l} g_{1 m}(\tau) I_{i l m}(\theta, \phi)$.

The $i$ subscript ranges over $i=1,2,3$, designating respectively the $r, \theta$, and $\phi$ components. The functions $T_{i l m}$ are given by:

$$
\begin{aligned}
& \mathrm{T}_{1 \mathrm{~lm}}(\theta, \phi)=-\mathrm{IP}_{1 \mathrm{~m}}(\cos \theta) \cos m \phi ; \\
& \mathrm{T}_{2 \mathrm{~lm}}(\theta, \phi)=-\left\{m \mathrm{P}_{1 \mathrm{~m}}\left(\cos \theta \cot \theta-\sqrt{(1-\mathrm{m})(1+\mathrm{m}+1)} \mathrm{P}_{1, m+1}(\cos \theta)\right\} \cos m \phi ;\right. \\
& \mathrm{T}_{3 \mathrm{~lm}}(\theta, \phi)=\frac{m \mathrm{P}_{\mathrm{lm}}(\cos \theta) \sin \mathrm{m} \phi}{\sin \theta}
\end{aligned}
$$

(where the sign errors in the referenced work have been corrected). $\boldsymbol{\tau}$ is the dipole tilt angle ( $\tau=0$ for the dipole axis perpendicular to the earth-sun line). The expansion coefficients $g \mathrm{tm}$ for a given value of $\tau$ were determined by least-squares fitting the expansion in Eq. (4.66) to the field at numerous interior points, the field values being computed by a numerical Biot-Savart law integration over the surface cunent distribution. $1_{\mathrm{MAX}}$ in Eq. (4.66) was set to 7. Once the $g_{1 \mathrm{~m}}$ were computed for 8 values of $\tau$, each $\mathrm{g}_{\mathrm{m}}$ could itself be represented by a polynomial expansion in $\tau$ :

$g_{\mid m}(\tau)=\sum_{j=0}^{3} a_{j}(1, m) \tau^{j}$.

This approximation gave good results except near the frontside neutral points and in the region beyond about $10 R_{E}$ into the tail, where the spherical harmonic expansion in Eq. (4.66) converged very slowly and significant errors were made when the expansion was truncated at $1=7$.

Beard (1982) presented an improved model of the field arising from the tailward section of the magnetopause current system. He expanded the scalar magnetic potential in a cylindrical harmonic expansion valid for $: \leq 1.5 R_{E}$ in the tail region. The cylindrical geometry of the tail magnetopause naturally lends itself to a field description in this coordinate system, and the expansion required only 8 terms 
(versus 35 for the Choe-Beari spherical harmonic expansion) to converge to an accurate field representation. For ease in computing, Beard formulated an auxiliary expression for B:

$B_{i}=f_{i}\left(C_{i}, r, \theta, \phi\right) ;$

where the subscript $i=1,2,3$ designates the spherical coordinates $r, \theta$, and $\phi$ respectively. $C_{i}$ is a vector of coefficients determined by least-squares fitting the expression for $B_{i}$ to the field calculated by Biot-Savart integration over the magnetopause current system. The functions $f_{i}$ were chosen to make $B$ divergencefree. (Readers of the referenced work should be alert to misprints in the $B_{\theta}$ and $B_{\phi}$ expressions). In the composite model, the spherical harmonic expression in $\mathrm{Eq} .(4.66)$ is used to represent the magnetopause field on the day side and for $r<1.5 R_{E}$ on the nightside. For $r \geq 1.5 R_{E}$ on the night side the expression (4.69) is used.

The field due to distributed currents flowing inside the tail region of the magnetosphere was also modeled by Beard (1979). He described a tail current system consisting of an equatorial current sheet of finite thickness, flowing from the dawn to dusk sides of the tail. To make this current system divergencefree, he specified return currents that flow from the dusk-side termination of the sheet, around on the cylindrical surface of the magnetopause, to the dawn side. (In a cross-section of the tail, the current pattern resembles the letter $\boldsymbol{\theta}$. See Fig. 1-7, Chapter 1 of the Introduction). The model magnetopause surface was a cylinder of radius $20 R_{E}$, axially terminated by planes at $x=-10 R_{E}$ and $x=-300 R_{E}$. (The $x$-axis lies in the equator and points tailward from the earth). The magnetic field associated with the current system is represented as the vector potential $A(x, y, z)$. $A$ was constrained to produce a field component $B_{x}$ whose $x$-dependence $\left(B_{x} \propto|x|^{-3}\right.$ ) matched satellite observations (Behannon, 1968). Beard generated expressions for $A$ in terms of the sheet thickness parameter $z_{0}$ from which the components of $B$ were obtained by the application of the curl operator (but note the referenced work suffers from a misprint in the formula for $A_{y}$ ). The resultant field is necessarily divergence-free.

With the addition of the tail current system, the magnetopause is no longer completely self-consistent since it was computed from a solar wind-earth dipole pressure balance condition. However the contribution of the tail field in the outer dayside magnetosphere is $\approx 1 \%$ of the total field for $R>8 \mathrm{R}_{\mathrm{E}}$ on the dayside, and therefore does not materially offset the shape or location of the magnetopause. $A$ more significant deficiency in the model is the lack of a model of the diamagnetic "ring" current which is known to flow around the earth in an equatorial sheet (which extends from about $3 R_{E}$ to $8 R_{E}$ ) and produces field depressions within about $6 R_{E}$ on the dayside (Sckopke, 1972).

An advantage of the Choe-Beard model is its flexibility. By varying the dipole tilt and standoff distance parameters one can generate a field which generally matches the field configuration observed during a particular satellite pass through the magnetosphere. This permits the mapping of observed fluxes to other azimuths in a qualitatively realistic manner. The self-consistency of the calculation assures that the field direction and gradients will be realistic near the magnetopause, a crucial requirement for particle flux mapping studi ., in the outer dayside magnetosphere.

\section{Magnetic Field Features of the Choe-Beard Model}

There are several notable features of the model geomagnetic field illustrated in Fig. 4-11. The solar wind is incident along the $x_{C S M}$ axis, in the $-x$ direction (dipole tilt angle $\tau=0$ ). For nominal solar wind conditions the magnetopause subsolar point (the "nose" of the magnetosphere) is approximately $10.5 R_{E}$ distant. The outermost flux tubes on the dayside are highly compressed; the total field just inside the magnetopause at the nose is approximately twice the dipole field (i.e., $B_{\mathrm{T}} \approx 54$ nanotesla). The field lines in this region are nearly circular. Two neutral points are evident, in the northern and southern hemispheres, at which the field magnitude goes to zero and the field reverses direction. Field lines threading the neutral point regions pass just inside the magnetopause. Lines emanating from the earth poleward of the neutral points are swept back to form the extended magnetotail. Currents flowing in the tail beyond $\approx 10 \mathrm{R}_{\mathrm{E}}$ cause the field lines to be drawn out in the antisolar direction.

Figure 4-12 depicts field lines and field magnitude contours in the noon meridian. Note that for the outer field lines (lines with equatorial radius $R_{E Q}>\approx 9 R_{E}$ ) the field magnitude along a line increases slowly with latitude. Along the outermost field lines a reversal in the parallel gradient can be seen.

This is more readily evident in Fig. 4-13, which shows the variation of B with colatitude along a set of outer field lines in the noon meridian. Note that the minimum field magnitude does not occur at the 


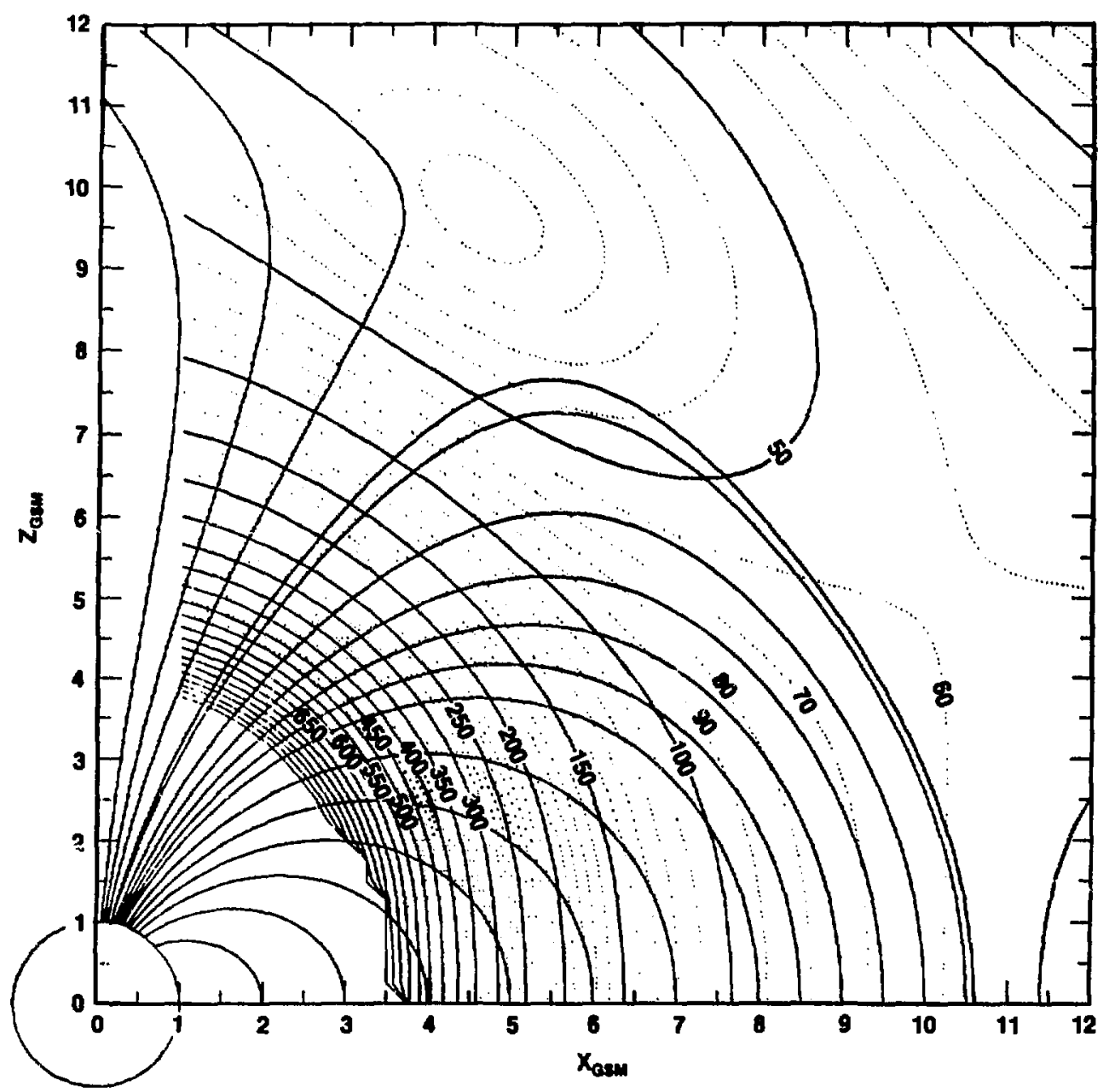

Figure 4-12. Field lines and constant-B contours (in nanotesla) of the Choe-Beard model in the noon meridian. Model parameters are atandoff diatance $R_{S O}=10.0$ (yielding a magnetopause distance of $10.6 R_{E}$ in the noon equator) and dipole tilt $\delta=0$. (Contours beyond the magnetouphere are in a region where the model is invalid, and should be ignored).

magnetic equator $\left(\theta=90^{\circ}\right)$ as it does for a dipole field line. The fields arising from the Chapman-Ferraro currents create high-latitude minimum-B regions termed "pockets" by Roederer (1966). Such pockets are a consistent feature of all mathematical models of the magnetosphere (Antonova and Shabansky, 1974) and were studied in the image dipole model by Shabansky and Antonova (1968) and in the Mead model by Roederer (1966). In the real geomagnetic field these packets can possibly serve to conduct azimuthallydrifting particles away from the noon magnetopause and thus allow closed drift shells for particles which would otherwise encounter the magnetopause in the course of their drift and be lost by scattering. 


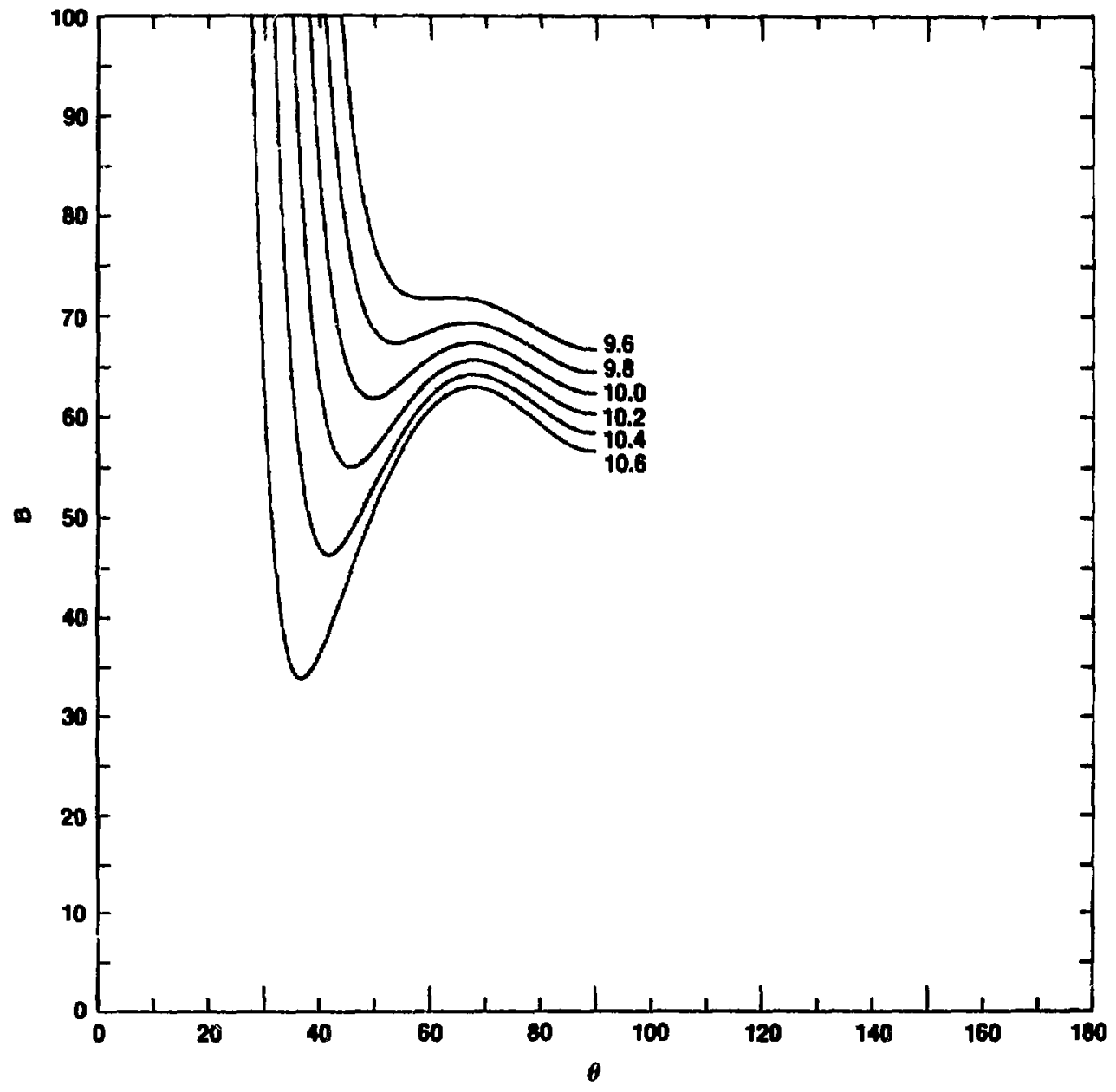

Figure 4-13. B (nanotesla) vs colatitude $\theta_{G S M}$ (degrees) along field lines of the Choe-Beard model in the noon meridian. Model parameters are the sams: as for Fig. 4-12. Lines are labeled at the right with the value of their equatorial-crossing radius $\left(R_{\mathrm{EQ}}\right)$ in $\mathbf{R}_{\mathrm{E}}$. The high-latitude minimum-B pockets are evident in the region $32^{\circ}<\theta<65^{\circ}$, on lines within $1 \mathrm{R}_{\mathrm{E}}$ (in $\mathrm{R}_{\mathrm{EQ}}$ ) of the magnetopause.

\section{Realism of the Choe-Beard Model}

Since we shall be using this model in our drift shell studies, the question of model realism is a substantial concern. Regardless of the theoretical advantages of the self-consistent magnetopause models, realism must be assessed by comparisons to satellite observations of the geomagnetic field. But satellite measurements are not definitive; because field samples are only collected along the orbital path of a spacecraft, a global picture of the geomagnetic field must be built up from a large number of orbits widely separated in time. Such a process unavoidably samples the magnetosphere in many different states (e.g., 
differing dipole tilt, magnetopause standoff distance, level of magnetic activity) and thus produces an average configuration, not a snapshot of a particular configuration.

With this caveat, we show in Fig. 4-14 a map of average scalar $\Delta B$ contours observed by Sugiura and Poros in the noon-midnight meridian. $\Delta B$ as defined by $\left(B_{\text {observed }}-B_{\text {dipole }}\right)$ is a useful quantity for evaluating magnetospheric magnetic field models. This is to be compared with $\Delta B\left(B_{\text {model }}-B_{\text {dipole }}\right)$ contours for the Choe-Beard model shown in Fig. 4-15. Note that for the outer $\left(r>6 R_{E}\right.$ ) dayside region (outside the ring current structure not included in the Choe-Beard model), the dayside contours are reasonably well reproduced, a point substantiated in the 1976 review of models by Walker. The large negative values $(\Delta \mathrm{B} \approx-30 \mathrm{nT})$ of the model in the polar cusp regions are more extreme than those measured by Sugiura and Poros, but are consistent with those measured by the IMP 5 spacecraft (Fairfield and Ness, 1972). Figure 4-16 illustrates their $\Delta B$ measurements on the dayside. The area of the circles is proportional to the field depression. The results of a single IMP 5 pass through the dayside cusp is shown in Fig. 4-17, where depressions as great as $-60 \mathrm{nT}$ were olserved. In general, cusp total field strengths at a distance of $7 R_{E}$ are typically $50-70 \%$ of the dipole field (Fairfield, 1977), indicating $\Delta B$ values of $<-50 \mathrm{nT}$. By this measure, the model's cusp field values are moderate rather than extreme.

The lack of distributed currents in the modei probably accounts for the anomalously high value for the earth intercept of the last closed field line in the noon meridian-about $82^{\circ}$. However several other models (Voigt, 1972; Alekseev and Shabansky, 19\% $\mathrm{i}$; Sugiura and Poros, 1973; among others) have last

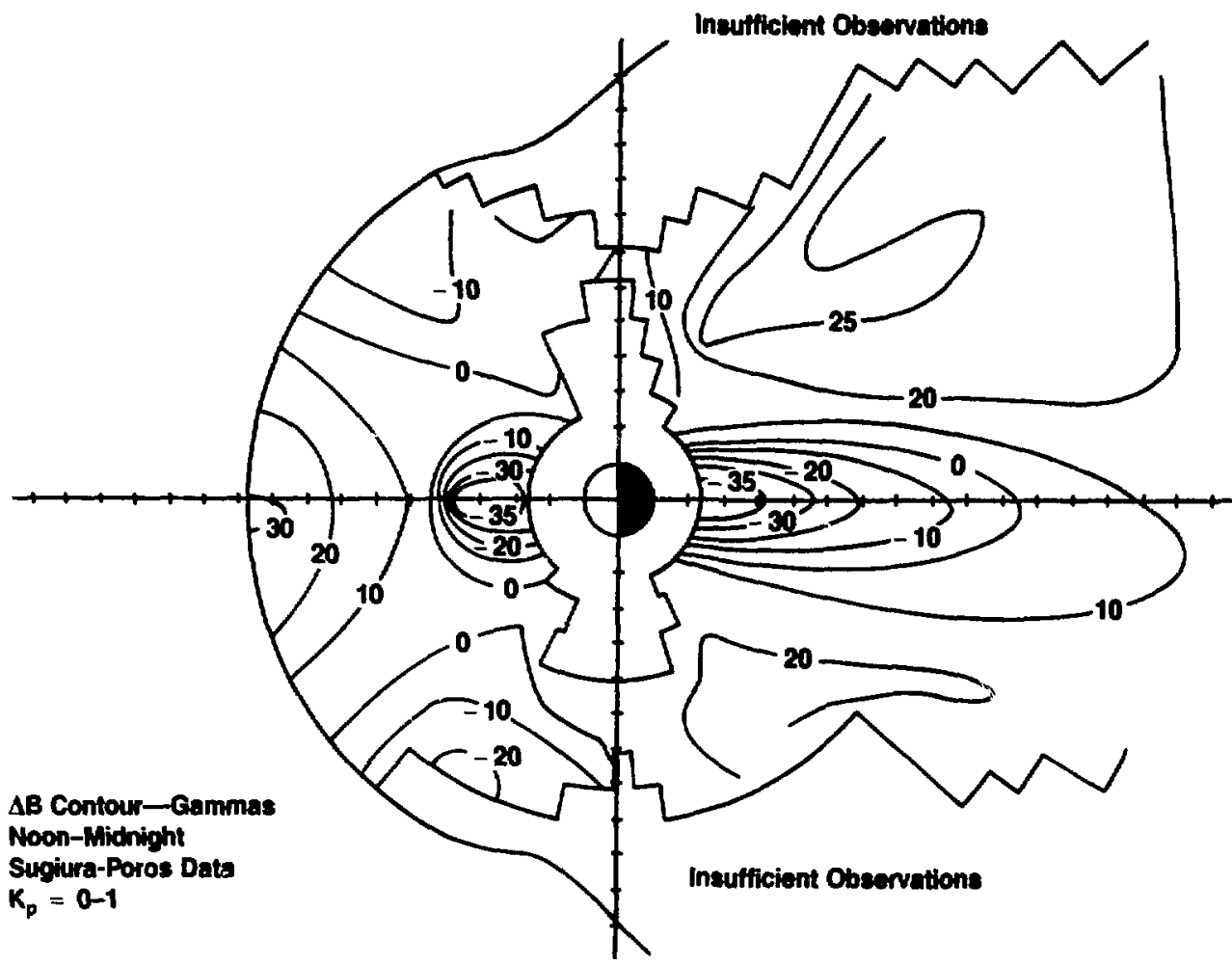

Figure 4-14. Average scalar $\Delta B$ contours in the noon-midnight meridian, as observed by Suguira and Poros, 1973. $\Delta B$ at any point is defined as $B_{\text {obeerved }}-B_{\text {dipole' }}$ 
closed field line intercepts at latitudes above $79^{\circ}$. Satellite measurements (Burch, 1972) indicate that the actual intercept latitude is about $77^{\circ}$.

In the region of particular interest for this study (the outer dayside magnetosphere), we judge the Choe-Beard model to be satisfactory in reproducing the qualitative field features found by satellite measurement. Its ability to produce a realistic field configuration near the magnetopause over a wide range of solar wind pressures and dipole tilt angles makes it a natural choice for interpretation of satellite particle data. Its deficiencies in modeling the ring current or the field-line intercepts do not significantly limit the accuracy of studies of the outer magnetosphere.

\section{Particle Drift Shells in the Outer Magnetosphere}

The Chapman-Ferraro currents flowing on the magnetopause dramatically distort the geomagnetic field in the outer regions. Azimuthal field symmetry disappears and particle drift-shells split, as discussed in the Introduction. At greater distances the distortion increases, producing minimum-B pockets near the high-latitude dayside magnetopause. A fundamental change occurs in the topology of the outer drift shells. Some shells branch into disjoint surface segments which pass through the noon minimum-B regions. In this section we analyze the branching process and determine its effects on the conservation of adiabatic invariants and the directional flux.

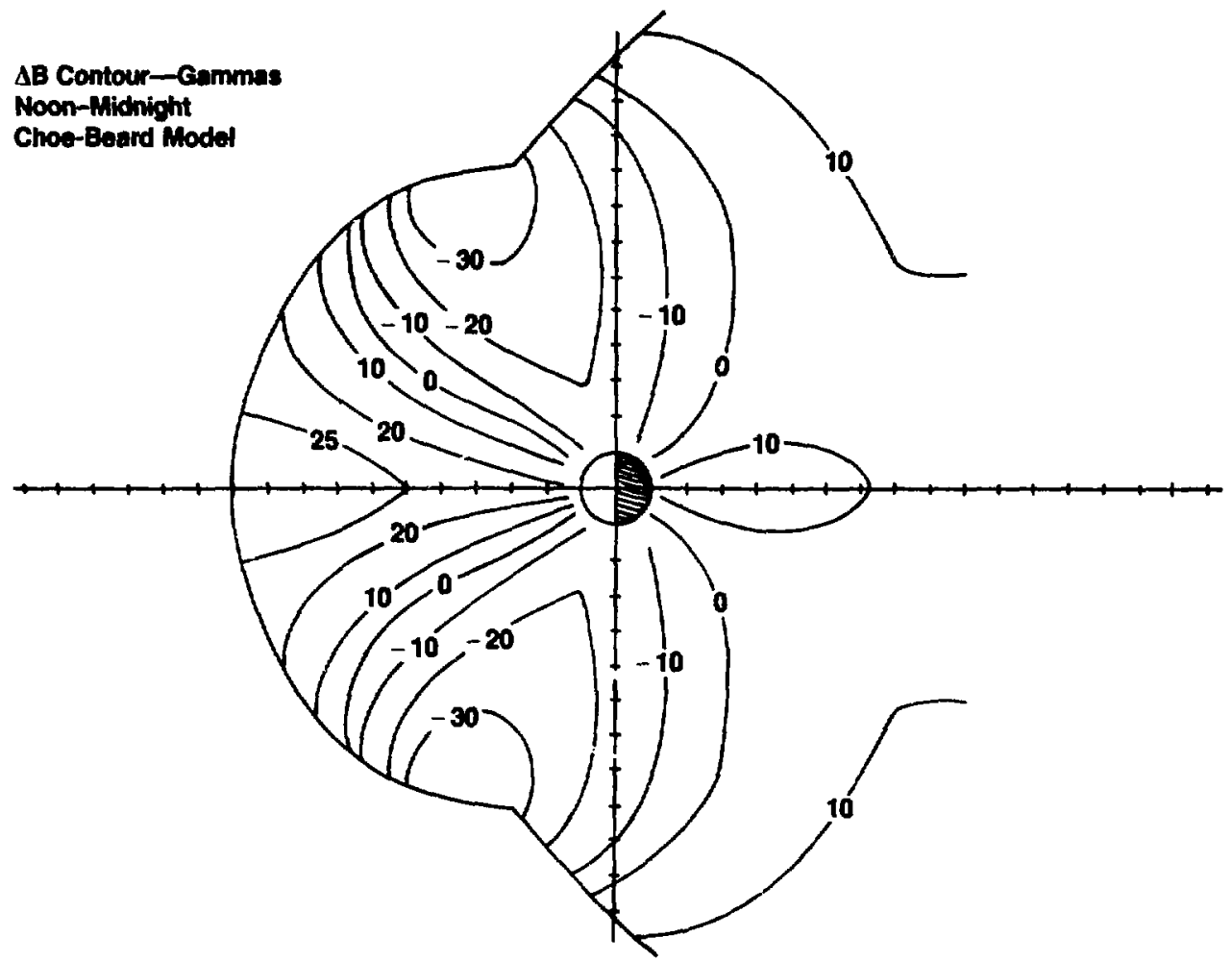

Figure 4-15. Average scalar $\Delta B$ contours in the noon-midnight meridian for the Choe-Beard 1974 model (after Walker, 1976). $\Delta B$ at suy point is defined as $B_{\text {obverved }}-B_{\text {dipole- }}$ 


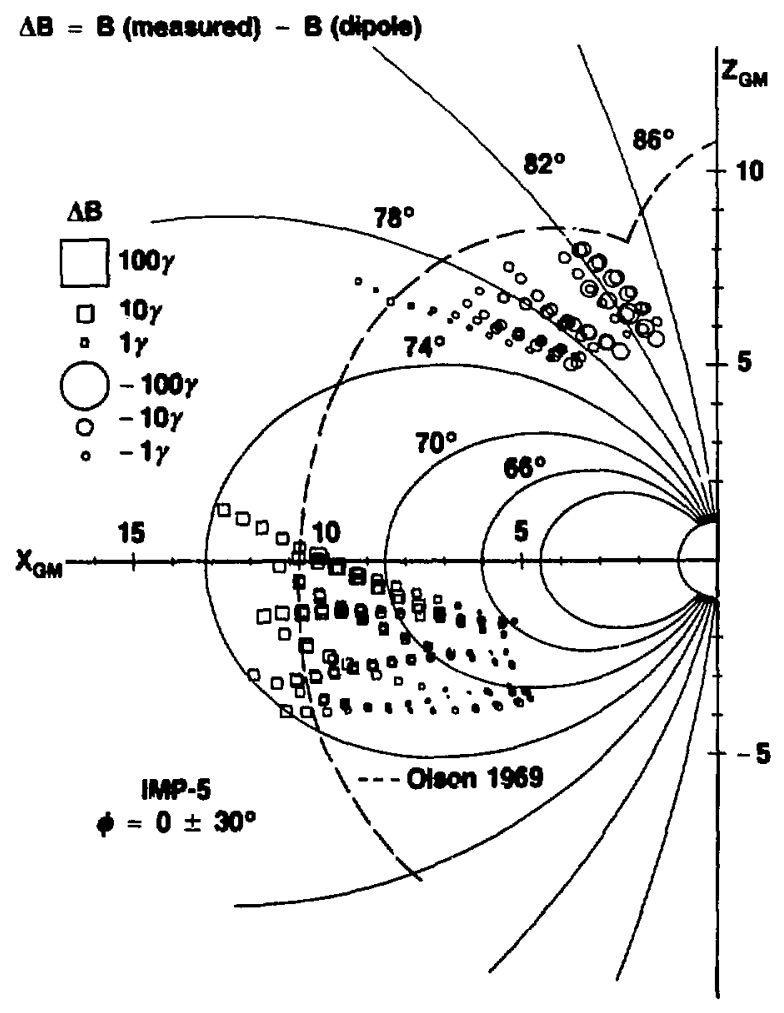

Figure 4-16. Magnetic field observations by the IMP 5 opacecraft in the noon-midnight meridian, during 14 orbits. $\triangle B$ values computed from the field strength measurements are plotted along each trajectory. The area of the plotting symbols indicates the magnitude of $\triangle B$ (Fairfield and Nens, 1972). Field depressions greater than $\mathbf{4 0} \mathrm{nT}$ were observed in the cusp region.

\section{Drift-Shell Branching}

The presence of high-latitude minimum-B pockets in the outer dayside magnetosphere creates the phenomenon of drift-shell branching. As a particle in the outer magnetosphere drifts azimuthally towards noon, it can pass onto lines where minimum-B pockets have formed. For appropriate initial conditions the particle can become trapped in these pockets, se that at noon the particle's bounce trajectory does not cross the equator but is restricted to a limited section of field line at high latitudes. Figure 1-25 of the Introduction depicts the minimum-B pockets at their fullest developmeni in the noon meridian. A particle trapped in the pockets will mirror poleward of the local $B_{\text {Max }}$, found on these lines at a latitude $>30^{\circ}$.

$A$ drift shell will branch if a local $B_{M A X}$ can be found (between the equator and the mirror location) along any line in the shell such that $B_{M A X}=B_{M}$. Figure 4-18.1 illustrates such a branching line. Beyond this line in azimuth (i.e., closer to noon), the local $B_{M A x}$ will exceed $B_{M}$ and the shell will branch and become a multiply-connected surface.

Figure 4-18.2 shows a sequence of lines along the drift shell of a particle initially in the midnight meridian. Parameters are $R_{E Q}=9.5 R_{E}, \alpha_{0}=60^{\circ}, B_{M}=50.5 \mathrm{nT}, I=0.5 R_{E}$, where $R_{E Q}$ is the equatorial distance to the line, $a_{0}$ is the particle's equatorial pitch angle, $B_{M}$ is the mirror field strength, and $I$ is the 


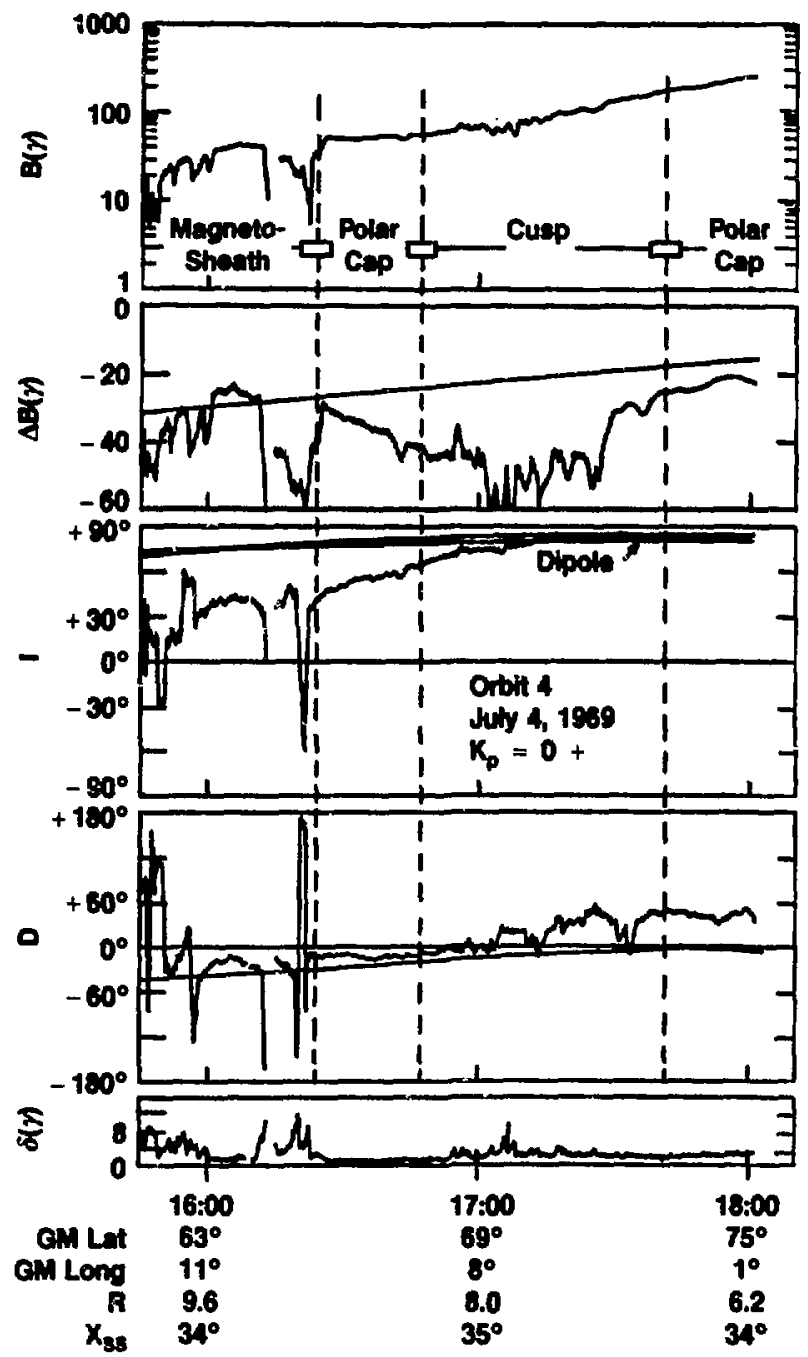

Figure 4-17. Disiant slayside cuop magnetic field as measured by satellite IMP 5 on July 4, 1969. $\triangle B$ depressions as great as, 0 nT were observed (Fairfield and Ness, 1972).

second adiabatic invariant (in the absence of electric fields). Field magnitude $|B|$ in the Choe-Beard model is plotted vs colatitude $\theta$ for each line, which is labeled with the azimuth of its equatorial-plane intercept (midnight $=180^{\circ}$ ). The horizontal line is drawn at the mirror field $B_{M}=50.5 \mathrm{nT}$-the mirror point locations on each field line are determined by the intersection of this line with the $B$ vs $\theta$ trace. Note that as the particle drifts around to the dayside, the field at the equatorial minimum increases (thereby increasing $\alpha_{0}$ ) and the mirror points move to higher latitudes. A local $B_{\text {MAX }}$ develops at about $25^{\circ}$ off the equator. 


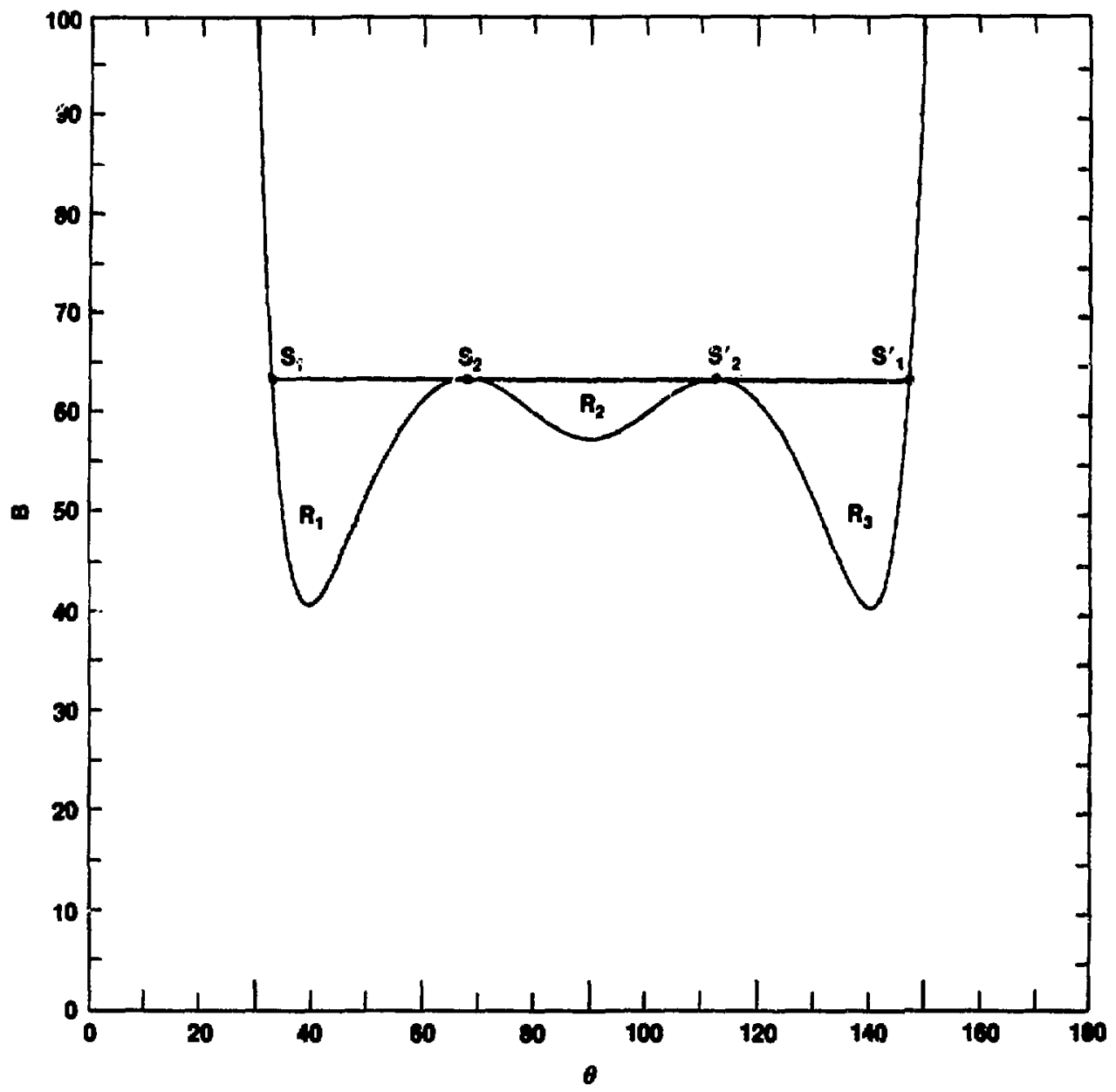

Figure 4-18.1. B (nT) ve colatitude $\theta$ (degrees) along a branching fleld line for a particie drifting from midnight. The field at the local B-maxima (points $S_{2}$ and $S_{2}^{\prime}$ ) now equals the field at the particle's mirror points (points $S_{1}$ and $S_{1}^{\prime}$ ). The thell branches into eczments $S_{1}-S_{2} S_{2}-S_{2}^{\prime}$, and $S_{2}^{\prime}-S_{1}^{\prime}$. 


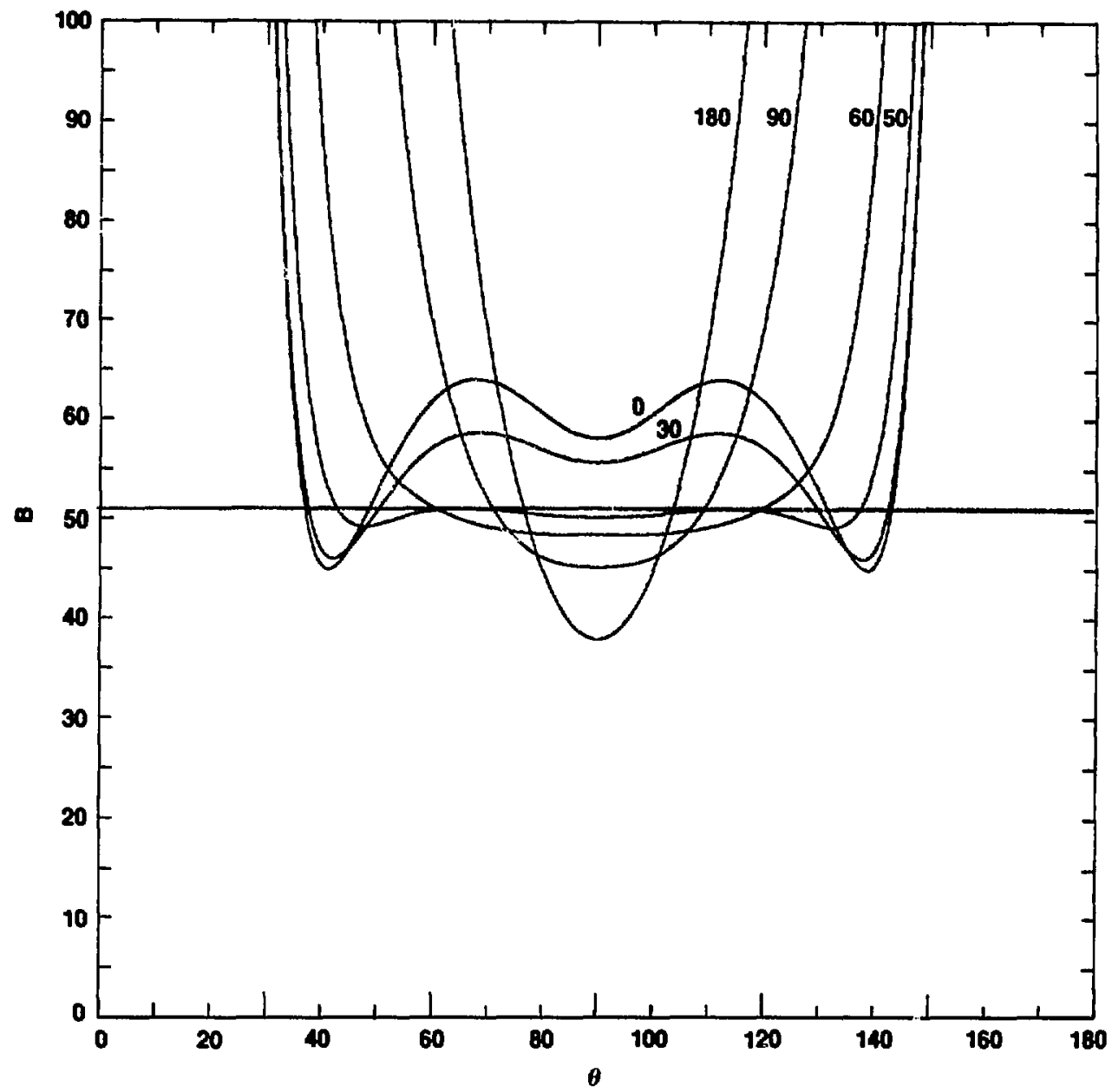

Figure 4-18.2. B (nT) vs colatitude $\theta$ (degrees) along field lines which compose the drift shell of a particle initially mirroring in the midnight meridian, fur the Choe-Beard model. Shell field lines are shown at azimuths of $180^{\circ}$ (midnight), $90^{\circ}, 60^{\circ}, 50^{\circ}, 30^{\circ}$, and $0^{\circ}$ (noon). Drift shell branching occurs at an azimuth of $\approx 50^{\circ}$, and the particle mirrors at noon in a high-latitude minimum-B region. 
At an azimuth of $50^{\circ}, B_{\mathrm{MAX}}=\mathrm{B}_{\mathrm{M}}$ and the drift shell becomes branched, i.e., is no longer a simplyconnected surface. The particle (depending on bounce phase) is either trapped between the two local $B_{\text {MAX }} S$ in an equator-crossing trajectory or else mirrors poleward of one of the $B_{\text {MAX' }} s$ in a B-pocket region. This figure illustrates the case of minimum-B trapping. As the particle drifts beyond the branching azimuth, its bounce motion is confined to the angular range $40^{\circ} \approx<\theta<\approx 50^{\circ}$. (Had the particle taken the equatorial branch it would have encountered the magnetopause before noon and been lost). $A \lambda, \phi$ representation of a branched shell is given in Fig. 4-19, where the latitude and azimuth coordinates of the mirror points along the shell are plotted.

Because the magnetosphere is symmetric about the noon-midnight meridian plane, the branching process is reversed as the particle continues its drift around the dayside magnetosphere and into night. The local $B_{\text {MAX }}$ now decreases with increasing azimuth until the particle once more gains access to the equatorial regions, and the branches rejoin to form a single surface again.

However, a particle pitch-angle distribution (PAD) measured in the post-rejoining region in the afternoon may differ significantly from the corresponding pre-branch PAD measured on the morning side. Suppose we compare a PAD measured at $\left(r_{0}, \lambda=0,-\phi_{0}\right)$ in the outer morning magnetosphere with a PAD measured at $\left(r_{0}, \lambda=0, \phi_{0}\right)$ in the outer afternoon magnetosphere. We find that electrons with pitch angles near $90^{\circ}$ will drift close to the magnetopause before noon, perhaps becoming scattered out of the drift shell (West et al., 1972). Electrons with small pitch angles will move on normal unbranched shells that pass well inside the magnetopause past noon. Electrons in some intermediate range of pitch angles

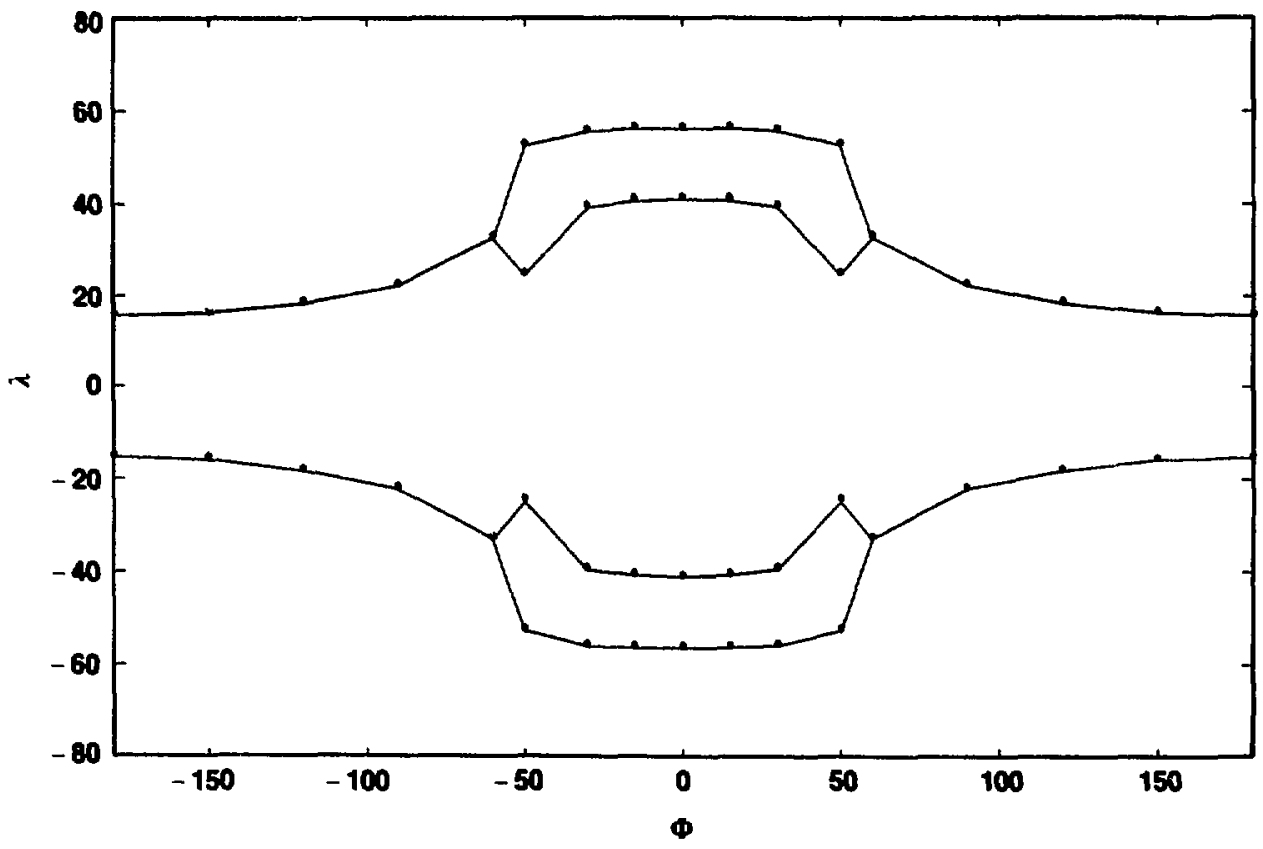

Figure 4-19. Representation of a drift shell in GSM latitude-longitude (2, $\phi)$ space. The traces show the north and south mirror points of a bouncing electron drifting noonwards from midnight $(\phi=$ $-180^{\circ}$ ). At an azimuth prior to $-50^{\circ}$ the shell branches, and the particle moves into the northern or southern shell segment that passes through a high-latitude minimum-B region, depending on its bounce phase at the branching line. At a symmetrical aximuth past noon $\left(\phi=0^{\circ}\right)$ the shell segments rejoin. 
will branch into the high-latitude minimum-B pockets, passing near the polar cusps. When these disparate drift shells rejoin post-noon, we would expect to see changes in flux levels due to the exposure of the particles to varying levels of non-adiabatic effects along their different routes.

\section{Effects of Shell Branching on Adiabatic Invariants $B_{M}$ and $I$}

As a particle drifts in azimuth up to the branching line, its motion is completely adiabatic (i.e., $B_{M}$ and I are adiabatically conserved along the particle's trajectory). We are now interested in the effect of branching on the adiabatic invariants $B_{M}$ and I.

In the static field under consideration, $B_{M}$ will remain constant if the changes in $B$ over a gyroradius remain small. The Alfvén criterion which characterizes adiabatic motion [Chapter 3, Eqs. (3.184a-c)] continues to be satisfied across shell branching; the rate of change of $B$ due to azimuthal drift is much too small to violate conservation of $B_{M}$.

To assess the effect of shell branching on $l$, we consider the following argument. Just prior to branching the particle mirrors between field-line points labeled $S_{1}$ and $S_{1}^{\prime}$ (Fig. 4-18.1). The value of $I$ is given by:

$$
\begin{aligned}
I & =\int_{S_{1}}^{s_{1}^{\prime}} \cos \alpha d s=\int_{S_{1}}^{s_{1}^{\prime}}\left[1-\frac{B(s)}{B_{M}}\right]^{1 / 2} \mathrm{ds}=\int_{s_{1}}^{s_{2}}+\int_{S_{2}}^{s_{2}^{\prime}}+\int_{s_{2}^{\prime}}^{s_{1}^{\prime}} \\
& =I_{1}+I_{2}+I_{3} ; \\
& =I_{0} ;
\end{aligned}
$$

where $S_{2}$ and $S_{2}^{\prime}$ are the locations of the local $B_{M A X} S$ along the line, and $B_{M A X}=B_{M}-\epsilon ; \epsilon$ a small positive number.

Just past the azimuth at which branching occurs, a shell field line can be found for which $\mathrm{B}_{\mathrm{MAX}}=$ $B_{M}+f$ (since $B_{M A X}$ increases monotonically and continuously with azimuth towards noon). The particle is now blocked by the intervening $B_{M A X} s$ from access to all parts of the branched shell line, its bounce motion being confined to one of the regions $R_{1}, R_{2}$, or $R_{3}$, with I-value $I=I_{b 1}, I_{b 2}$, or $I_{b 3}$ respectively.

We can define a total post-branch I-value for the shell as the sum of the I's for the disjoint regions:

$I_{b}=I_{b 1}+I_{b 2}+I_{b 3}$

Now if we move from these pre- and post-branching field lines towards the branching line $|\epsilon|$ decreases, going to zero on the branching line itself. We see that $I_{b j} \rightarrow I_{j}$, and thus

$l_{b} \rightarrow I_{0}$

Following branching, the motion of a particle along any branch of the shell is again completely adiabatic. Therefore $\mathrm{I}_{0}$ continues to characterize the entire drift shell, even though branching has occurred and no particle on a branch has $I=I_{0}$.

We have shown that shell branching can be considered an adiabatic process; the I-value of an individual particle will not be conserved across a branching line, but the sum of the I's for all branched segments will equal the I of the unbranched portion of the shell. In fields which are symmetric about the noonmidnight meridional plane, the original $I$ is restored to every particle when the branched segments rejoin.

\section{Trajectory Calculations}

The foregoing argument for general l-conservation appears sound, however as a check on its validity (and to examine details of particle motion in the vicinity of branching lines) several charged-particle trajectories were computed along branching shells. The details of particle dynamics associated with branching are interesting in themselves, since shell branching was once conjectured not to occur in the geomagnetic field (Northrup and Teller, 1960). For these trials, we numerically integrated the equations of motion [Chapter 3, Eqs. (3.2a-3.2c)] to determine position as a function of time for a $3 \mathrm{MeV}$ electron in the Choe-Beard model magnetic field. This numerical simulation was valid for the general (i.e., non-adiabatic) case of relativistic charged-particle snotion in static electromagnetic fields. Any desired accuracy of the 
computed solution could be achieved (within roundoff error) by controlling the integration time-step. Any non-adiabatic effect associated with the branching process which might have been overlooked in the prior analysis would be apparent by comparing l-values calculated along the particle trajectory with those obtained by adiabatically conserving 1 .

We computed an approximate value for 1 on every bounce of the simulated particle by evaluating the sum

$I_{A P P}=\sum_{i} \frac{P_{\| i} d s_{j}}{P_{\| j}}$,

stepwise along the dynamical path of the particle, where $P_{\| J}$ is the parallel momentum on the $j$-th step, and $d s_{i}=d r_{1} \cdot B_{i} /\left|B_{i}\right|$, the parallel component of the $j$-th step. The summation is taken over all steps between one mirror point and the next (defined as the step on which $v_{\|}$changes sign). We compared this to $\mathrm{I}_{\mathrm{T}}$, the theoretical value of $\mathrm{I}$, as computed by the trapezoidal rule along the particle's drift shell (obtained by assuming general I-conservation at branching lines).

$\mathrm{I}_{\mathrm{T}} \approx \int_{\mathrm{M}}^{\mathrm{M}}\left[1-\frac{\mathrm{B}(\mathrm{s})}{\mathrm{B}_{\mathrm{M}}}\right]^{1 / 2} \mathrm{ds}$

$I_{T}$ is properly defined as an integral along a fixed field line between conjugate mirror points $M$ and $M^{\prime}$. Because the dynamic value $I_{A p p}$ incorporates the effects of the particle's azimuthal drift from line to line between mirror points, detailed agreement between $l_{A P T}$ and $l_{T}$ would not be expected. However as Tables 1 and 2 show, the agreement between $I_{A P r}$ and $I_{T}$ in the pre- and post-branch regions is very good. That is to say, the changes in I due to shell branching as predicted $b /$ our adiabatic theory are reproduced very exactly by our particle trajectory simulations.

We conclude that shell branching is an adiabatic process in the general sense described above. It does not break the first or second adiabatic invariant, and thus is not in itself a mechanism for diffusion of particles. However, a particle population spread over the branched shell segmenis will pass through different spatial regions, and be exposed to non-adiabatic effects in varying amounts, depending on which segments the particles lie on. When the segments rejoin, we may see changes in the particle pitch-angle distribution which arise from these segment-specific effects.

Table 1. Comparison of dynamically-computed adiabatic invariants with analytically-calculated values. The field model employed is the Choe-Beard zero-tilt model, with standoff parameter $R_{a}=10.0$. The dynamic valuen of $B_{M}$ and I were computed by the TIBROE code along the dynamical trajectories of the $3 \mathrm{MeV}$ electrons, for the first bounce. Initial values for the calculation were: $r_{0}=10.5 R_{E} \lambda=0^{\circ}$, $\phi=307^{\circ}$. For comparicon, analytical values of $B_{M}$ were obtained from $\mu$-conservation: $\sin ^{2} \alpha_{0} / B_{0}$ $=1 / B_{M}$. Analytical values of $I$ were obtained by numerically integrating:

$I=\int_{M_{1}}^{M_{2}}\left[1-\frac{B(s)}{n}\right]^{2} d s$

along the initial ficld gine between mirror points.

\begin{tabular}{ccccc}
\hline $\begin{array}{c}\text { Initial } \\
\text { pitch angle }\end{array}$ & $\bar{B}_{M}$ & Dynamic Values & & Analytic Values \\
\hline 60 & 71.0 & 7.19 & 70.9 & \multicolumn{1}{c}{ B } \\
70 & 60.0 & 3.9 & 60.2 & 3.92 \\
80 & 55.0 & 1.06 & 54.8 & 1.06 \\
90 & 53.2 & 0.0 & 53.2 & 0.0 \\
\hline
\end{tabular}

$B_{M}$ in nanotesla.

- $\quad$ in $R_{E}$. 
Table 2. Comparison of dynamically-computed adiabatic invariants with analytically-calculated values, for branching electron trajectories. The field model employed is the Choe-Beard zero-tilt model, with standoff parameter $R_{a}=10.0$. The dynamic values of $B_{M}$ and I were computed by the TIBROE code fing the dynamical trajectories of the $3 \mathrm{MeV}$ electrons, for one complete bounce preand post-branching. Particle trajectories were started at the local $B_{\mathrm{MIN}}$ position on that field line in the noon meridian with $R_{E Q}=10.0 R_{E^{*}}$ For comparison, analytical values of $B_{M}$ were obtained from $\mu$-conservation: $\sin ^{2} \alpha_{0} / B_{0}=1 / B_{M}$. Analytical values of 1 were obtained by numerically integrating:

$I=\int_{M_{1}}^{M_{2}}\left[1-\frac{B(s)}{B_{M}}\right]^{2} d s$

along a representative field line pre- and post-branching.

\begin{tabular}{|c|c|c|c|c|c|c|}
\hline \multirow{2}{*}{$\begin{array}{l}\text { Initial } \\
\text { pitch angle }\end{array}$} & \multicolumn{3}{|c|}{ Dynamic Values } & \multicolumn{3}{|c|}{ Analytic Values } \\
\hline & $\overline{B_{M}}$ & TPRE & Trost & $\overline{B_{M}}$ & tren & Trost \\
\hline 80 & 63.7 & 0.22 & 1.61 & 63.7 & 0.23 & 1.63 \\
\hline 90 & 61.3 & 0.00 & 1.35 & 61.3 & 0.00 & 1.30 \\
\hline
\end{tabular}

$B_{M}$ in nanolesla.

$I$ in $R_{E}$.

\section{Effects of Shell Branching on the Directional Flux}

In the Introduction we pointed out that the directional particle flux $j$ is conserved along the trajectory of a particle, provided there are no collisions or particle sources or sinks. For these conditions we are able to characterize the directional fluxes on an entire drift shell from measurements made on a single line of the shell. If for the shell designated by $B_{M}, I$ we measure the directional flux in the meridian $\phi_{0}$ and obtain at point $\mathbf{P}$ :

$$
\mathrm{i}\left[\boldsymbol{\alpha}(\mathbf{P}), \phi_{0}, \mathrm{E}\right] ; \quad \text { where } \alpha(\mathbf{P})=\sin ^{-1} \sqrt{\left[\mathrm{B}(\mathbf{P}) / \mathrm{B}_{\mathrm{M}}\right]} ;
$$

then go to another meridian $\phi_{1}$ on the same shell and make a mersurement at point $Q$ :

$$
\mathrm{j}\left[\alpha(\mathbf{Q}), \phi_{1}, \mathrm{E}\right] ; \quad \text { where } \alpha(\mathbf{Q})=\sin ^{-1} \sqrt{\mathrm{B}(\mathbf{Q}) / \mathrm{B}_{\mathrm{M}}} ;
$$

we find that:

$\mathrm{j}\left[\alpha(\mathrm{P}), \phi_{0}, \mathrm{E}\right]=\mathrm{j}\left[\alpha(\mathbf{Q}), \phi_{1}, \mathrm{E}\right]$

If in the intervening trajectory of the particle between azimuths $\phi_{0}$ and $\phi_{1}$ non-adiabatic effects orcur (resulting in scattering out of the drift shell), or if particle sinks or sources are operating, then the directional flux may not be conserved. We now investigate the effect of drift shell branching on j-conservation.

For our purposes it $u$ ill be useful to first express $j$ in terms of the particle population in a flux tube. Let $\delta \mathrm{A}(\mathrm{P})=$ normal area of a differential flux tube at point $\mathrm{P}$ (Fig. 4-20) and $\delta \mathrm{N}(\alpha, E)=$ number of particles with energy in the range $\delta \mathrm{E}$ about $\mathrm{E}$ whose mirror points lie in the flux tube and which cross $\delta \mathrm{A}$ in the pitch angle range d $\alpha$ about $\alpha$.

All such particles have a well-defined bounce period $\tau_{B}$ and cross $\delta \mathrm{A}(\mathrm{P})$ in the specified pitch-angle range once per bounce period. Their mirror points are distributed along the tube in the range $\delta B_{M}$ about $\mathrm{B}_{M}$, where $\mathrm{B}_{\mathrm{M}}=\mathrm{B}(\mathrm{P}) / \sin ^{2} \alpha$. We can write $\delta \mathrm{N}$ as

$\delta \mathrm{N}(\alpha, \mathrm{E})=\mathrm{i}[\alpha(\mathrm{P}), \mathrm{E}] \cdot \delta \mathrm{A}_{\perp} \cdot \delta \Omega \cdot \delta \mathrm{E} \cdot \tau_{\mathrm{B}}$.

In terms of the invariant $B_{M}$ we can write this as

$\delta \mathrm{N}\left(\mathrm{B}_{\mathrm{M}}, \mathrm{E}\right)=\mathrm{j}\left(\mathrm{B}_{\mathrm{M}}, \mathrm{E}\right) \cdot \delta \mathrm{A}_{\perp} \cdot \delta \Omega \cdot \delta \mathrm{E} \cdot \tau_{\mathrm{B}}$ 
yielding for $\mathrm{j}$ :

$j\left(B_{M}, E\right)=\frac{\delta N\left(B_{M}, E\right)}{\delta A_{\perp} \cdot \delta \Omega \cdot \delta E \cdot \tau_{B}}$.

We now look at changes in this quantity at a shell branching line. Just prior to branching, we have the situation pictured in Fig. 4-18.1. Particles in their bounce trajectory between mirror points $S_{1}$ and $S_{1}^{\prime}$ pass through three regions of the shell labelled $R_{1}, R_{2}, R_{3}$. Just after branching these regions become disjoint segments of the branched shell. Let $j_{0}\left(B_{M}, E\right)$ be the directional flux just prior to branching;

$\mathrm{j}_{0}\left(\mathrm{~B}_{\mathrm{M}}, \mathrm{E}\right)=\frac{\delta \mathrm{N}_{0}\left(\mathrm{~B}_{M}, \mathrm{E}\right)}{\delta \mathrm{A}_{\perp} \cdot \delta \Omega \cdot \delta \mathrm{E} \cdot \tau_{\mathrm{B}}}$.

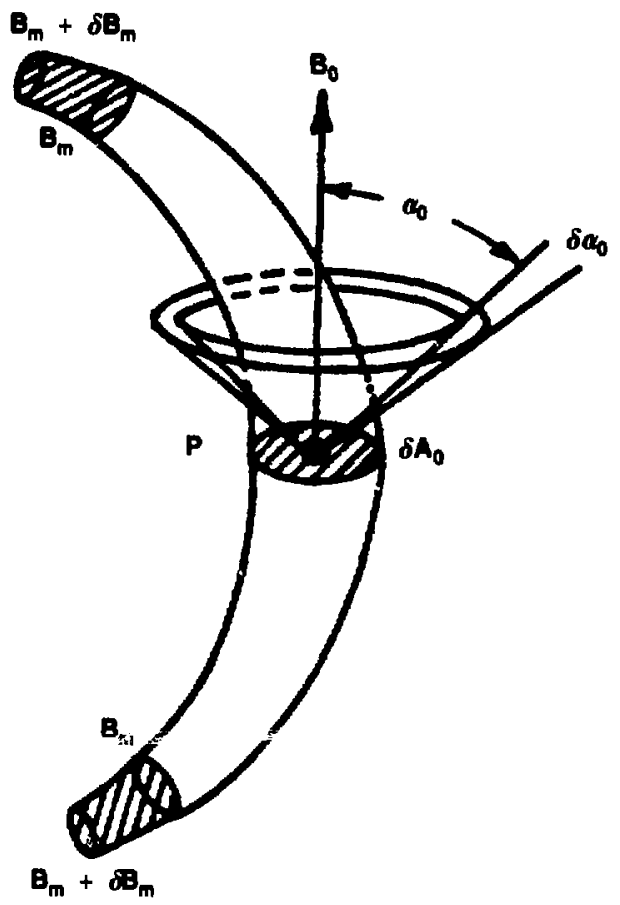

Figure 4-20. Flux-tube relationships. All particles which cross the differential area $\delta A_{0}$ with pitch angles in the range $\delta \alpha_{0}$ about $\alpha_{0}$, have mirror points which are distributed along the flux tube in the range $\delta B_{M}$ about $B_{M}$, where $B_{M}$

$=B(P) / \sin ^{2} \alpha_{0}$ (Roederer, 1970). 
Let $\tau_{i}=$ the time spent in region $R_{j}$ during a pre-branch bounce, so that $\tau_{B}=\tau_{1}+\tau_{2}+\tau_{3}$.

If the bounce phases of the particles which mirror at $S_{1}, S_{1}^{\prime}$ are random [j.e., if the bounce phases of particles in the flux tube between $S_{t}$ and $S_{1}^{\prime}$ are uniformly distributed on $\left.(0,2 \pi)\right]$, then the probability of a particle drifting onto the post-branch segment $R_{i}$ is just

$\operatorname{Pr}\left(R_{\mathrm{i}}\right)=\tau_{\mathrm{j}} / \tau_{\mathrm{B}}$

and the fraction of the $\delta N_{0}$ particles which are trapped upon branching into region $R_{i}$ is

$\delta N_{i} / \delta N_{0}=\operatorname{Pr}\left(R_{i}\right)=\tau_{i} / \tau_{B}$

These particles will oscillate in region $R_{i}$ with bounce period $\tau_{i}$.

Just post-branch, we can write for the directional flux in region $\mathbf{R}_{\mathbf{i}}$ :

$$
\begin{aligned}
j_{i}\left(B_{M}, E\right) & =\frac{\delta N_{i}\left(B_{M}, E\right)}{\delta A_{\perp} \cdot \delta \mathbf{R} \cdot \delta E \cdot \tau_{i}}=\left(\tau_{1} / \tau_{B}\right) \frac{\delta N_{0}}{\delta A_{\perp} \cdot\left(B_{M}, E\right)} \\
& =j_{0}\left(B_{M}, E\right) .
\end{aligned}
$$

We see that directional flux is therefore conserved across shell branching. Although the number of particles mirroring at $B_{M}$ in a post-branch segment is reduced by the ratio $\tau_{i} / \tau_{B}$, the post-branch period is reduced by the same ratio, thus conserving the directional flux.

This phenomenon is analogous to the effect produced by the insertion of a partition into a volume of gas. The number of molecules which can collide with a given end wall is reduced, yet the collision frequency with the wall is compensatingly increased so that the net change in particle flux (pressure) is zero.

When the branched shell segments rejoin post-noon, the combined fluxes reproduce the original directional flux, provided that non-adiabatic processes have not caused the loss (or gain) of particles in any segment. An empirical consequence of adiabatic $j$-conservation is that shell-branching is not in principle detectable as a change in flux across a branch line. However as we will see in the next section, branched shell segments diverge as azimuth increases towards noon, so that any field line with local B-maxima will contain particle populations from different night-side shells. Dayside equatorial PAD measurements on B-maxima field lines exhibit marked discontinuities in flux levels; these changes occur at critical pitch angles which mark the limits of the distinctive populations.

\section{Drift Shell Tracing in the Outer Magnetosphere; the Choe-Beard Model}

We turn now to the computation of energetic particle drift shells in the outer dayside magnetosphere, using a model magnetospheric field. For our field model we choose the Choe-Beard model as discussed in the prior section, chiefly for its self-consistently defined magnetopause and for its variable dipole tilt and standoff parameters. The former property ensures that outer magnetospheric field ines will not cross the magnetopause, and that the field gradients in the magnetopause vicinity will be qualitatively correct. The latter property allows us to adjust the model field to match the magnetospheric conditions observed on a given satellite pass, and thus better compare field modeling results with observed particle fluxes. In this section we will investigate shell splitting and branching in this model, and derive regions of stabletrapping and pseudo-trapping.

\section{Mapping Drift Shells by Means of Adiabatic Invariants}

We have shown that charged particles in a static magnetic field move so as to conserve initial values of $B_{M}$ and $I$ (provided the Alfvén conditions for adiabatic motion are satisfied and that no electric fields exist). We now indicate how the adiabatic invariance of $B_{M}$ and $I$ can be used to map out an entire drift shell in a model field. 
We start our mapping procedure by considering a particle of equatorial pitch angle $\alpha_{0}$, initially mirroring on a field line in the midnight meridian which has equatorial radius $R_{\mathrm{EQ}}$ and equatorial induction $\mathrm{B}_{\mathrm{EQ}}$. We trace this field line in the model from the equator towards the poles to locate the mirror points of the particle, i.e., the points on the line where the induction is

$\mathrm{B}_{\mathrm{M}}=\mathrm{B}_{\mathrm{EQ}} / \sin ^{2} \alpha_{0}$

During the trace we compute the invariant

$I_{0}=\int_{S_{1}}^{S_{2}}\left[1-\frac{B(s)}{B_{M}}\right]^{1 / 2} d s$

where $S_{1}$ and $S_{2}$ are the mirror-point loci. We now move to any other azimuth, say noon, where we wish to locate the field line on which this particle mirrors (assuming that on some intervening azimuth the shell has not branched nor has the particle been lost to the magnetopause or to "open" field lines). Our computer code searches along the equatorial intersection of the noon meridional plane to find a field line containing the point $B=B_{M}, I=I_{0}$. If found, this field line must by definition be part of the desired drift shell. By repeating this process at other azimuths a complete drift shell cai, be mapped out. Roederer employed a similar code to determine the shells in the Mead-Williams model (Figs. 1-22 and 1-24).

\section{The Drift Loss-Cone and Regions of Stable and Pseudo-trapping}

But not all drift shells close within the magnetosphere, or allow completely adiabatic drift. Some drift surfaces generated by particles at midnight intersect the magnetopause before noon. Particles on these shells move to the magnetopause and are lost; the magnetopause acts as a perfect absorber of energetic particles (Williams et al., 1979). If one starts instead in the noon meridian and traces drift shells towards midnight, one finds that some particles drift onto tail field lines which are greatly drawn out in the antisolar direction. Depending on the model employed, some of these lines may be "open," i.e., they are connected to the distant interplanetary field and consequently do not support bounce motion; particles escape from the magnetosphere. Other lines will close through the equatorial "neutral" sheet of the tail but bend sharply as they do so. If the radius of curvature of the line approaches the radius of gyration of the particle, the Alfven criterion for adiabatic motion (Eq. $3.184 \mathrm{~b}$ ) is violated, and the first adiabatic invariant $\mu$ is no longer conserved (Alfvén and Falthammer, 1963). This condition leads to strong pitch angle scattering (Sergeev and Tsyganenko, 1982) which isotropizes PADs (as observed by West et al., $1978 \mathrm{a}, \mathrm{b})$ and precipitates particles into the upper atmosphere.

Particles on such field lines are said to be "pseudo-trapped," in that they can complete but a few drift periods at most before they are lost from the magnetosphere. The distinction between pseudo- and stablytrapped particles is significant in studies of particle distributions. For example, solar wind particles injected into the pseudo-trapped regions cannot contribute to the stable-trapped population, unless some mechanism transports them into the stable trapping regions before loss can occur.

The regions of stable and pseudo-trapping can be delimited in a given meridional plane (for a particular field model) by computing the so-called drift loss cone for a set of field lines in the meridian. At any point in space, the stably-trapped particle population spans a range of pitch angles. Particles with pitch angles outside this range are said to fall into the loss cone. Figure 4-21 depicts the loss cone in the midnight meridian (computed for the Mead-Williams model). In the midnight meridian, electrons of small equatorial pitch angles are lost by Coulomb scattering in the upper atmosphere near their mirror points; these particles are in the bounce loss cone. Electrons of large pitch angles drift into the magnetopause before noon; they are in the drift loss cone. Only in some intermediate range of pitch angles will stable trapping occur. Because of the azimuthal asymmetries of the geomagnetic field, the boundary between stable trapping and pseudo-trapping changes with azimuth.

Equivalent to the loss-cone representation, one can plot for each of a set of field lines, the mirrorpoint locations that separate the regions of stable trapping from pseudo-trapping. The result is a delineation of the mirror-point zones of stable and pseudo-trapping. Roederer was the first investigator to classify adiabatic particle drift trajectories in the geomagnetic field into the categories of stably-trapped and pseudo-trapped drift shells (Roederer, 1967), and to determine the spatial regions which each type occupied. Figure 4-22 displays his analysis of the two drift shell regions for the Mead-Williams model. He 


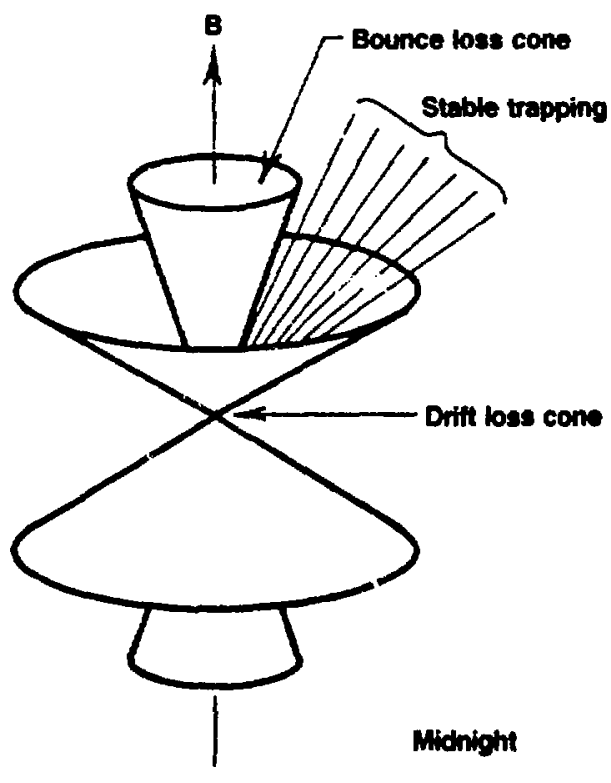

Figure 4-21. Typical loss-cone fo $\leftleftarrows$ an equatorial pitch-angle distribution in the midnight meridian. Particles at small pitch angles are precipitated into the upper atmosphere (i.e., they fall into the bounce loss-cone). Particles at large pitch angles drift to the magnetopause before noon and are lost from the trapping regions (after Roederer, 1970).

found that particles in the outer dayside magnetosphere whose mirror points lay at latitudes $>20^{\circ}$ were pseudo-trapped, moving onto open tail field lines as they drifted into the night hemisphere. The pseudotrapping region in the midnight region consisted of shells $\left(7.2 \approx<R_{E Q}<\approx \nu R_{i E}\right.$ ) which were closed on the night side but intersected the magnetopause before the noon meridian.

\section{Determination of the Stable-trapping Regions of the Choe-Beard Model}

We now wish to determine the stable and pseudo-trapped regions for tre Choe-Beard model. Our analysis is complicated by the fact that some outer drift shells may exhibit branching into the noon highlatitude minimum-B pockets. We begin with the construction of a drift loss cone plot, using our BTRACE code to compute $B_{M}$ and $I$ in the meridian $\phi=-30^{\circ}$ (Fig. 4-23). These are field lines which all pass through the equator at an azimuth of $-30^{\circ}$. Each line is labelled with its $R_{E Q}$ in $R_{E}$. Note that the magnetosphere lies in that part of the plane to the right of the outermost field line $\left(R_{E Q}=10.9\right)$. Let $B_{M}, I_{0}$ be the invariant coordinates of an electron in the midnight meridian. We can locate this particle in the $-30^{\circ}$ meridian by plotting the point corresponding to its invariant coordinates on this figure. If the point falls, for example, at $A$, to the left of the $R_{E Q}=10.9$ line (i.e., outside the magnetosphere), then the particle's drift has taken it into the magnetopause at an earlier azimuth and the particle is lost. If the point falls instead to the right at $B$, the particle's drift shell passes inside the magnetopause at this azimuth and the electron continues its drift towards noon.

By an extension of this process we have determined the regions of stable and pseudo-trapping in the midnight meridian. Figure 4-24 shows a $B_{M}, I$ plot of the outermost field lines of the dayside magnetosphere, for $-45^{\circ} \leq \phi \leq 0^{\circ}$. Superposed are a set of curves that represent equatorial PADs in the midnight meridian, labelled on the right with their midnight $R_{E Q}$. This figure can be used to derive the drift loss cone for a midnight PAD. For instance, for an equatorial PAD at midnight on a line with $R_{E Q}=8.0 R_{E}$, particles of any pitch angle move around noon inside the magnetosphere, and so are stably trapped. By contrast, particles initially on a midnight line with $R_{E Q}=10.0 R_{E}$ with $a_{0}>50^{\circ}$ will drift into the magnetopause before the $-45^{\circ}$ meridian and be lost. These are pseuco-trapped particles, unable to complete a single drift orbit about the earth. As azimuth increases towards noon, the $B_{M}, I$ trace of the outermost field line moves to higher field values as a consequence of the solar-wind compression of the 


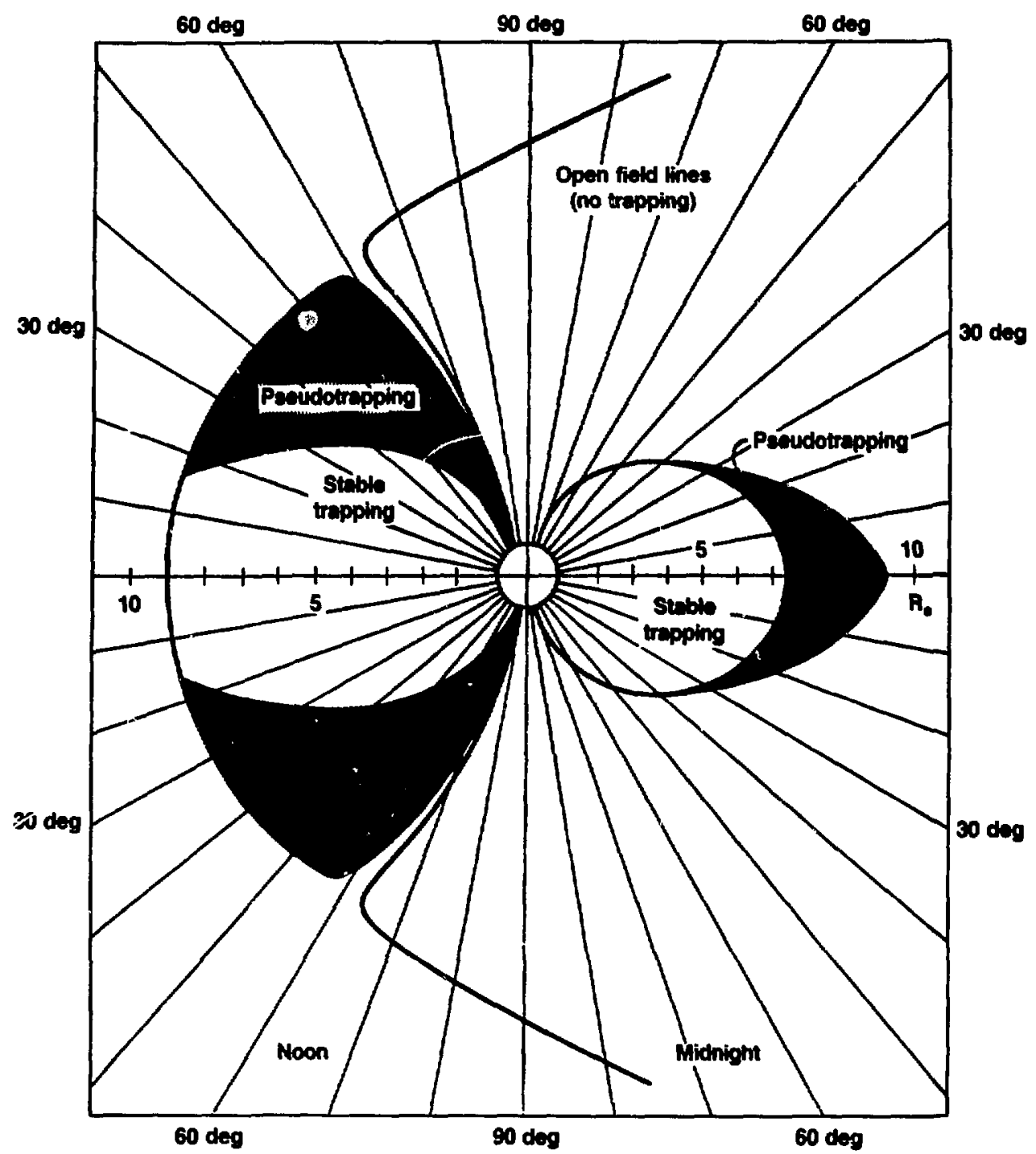

Figure 4-22. Regions of stable-trapping and psuedo-trapping in the noon-midright plane for the Mead-Williams image dipole model, as determined by Roederer (1967).

dayside field. Therefore it is the outermost noon line which serves as the demarcator in the $B_{M}, I$ plane between zones of stable-trapping and pseudo-trapping for particles drifting from midnight around to noon.

If this loss-cone mapping procedure were carried out as indicated, we would obtain stable and pseudo-trapping regions resembling Roederer's for the Mead-Williams model (Fig. 4-22). However this simplified picture must be modified to account for the effects of shell branching. When a shell branches, a 


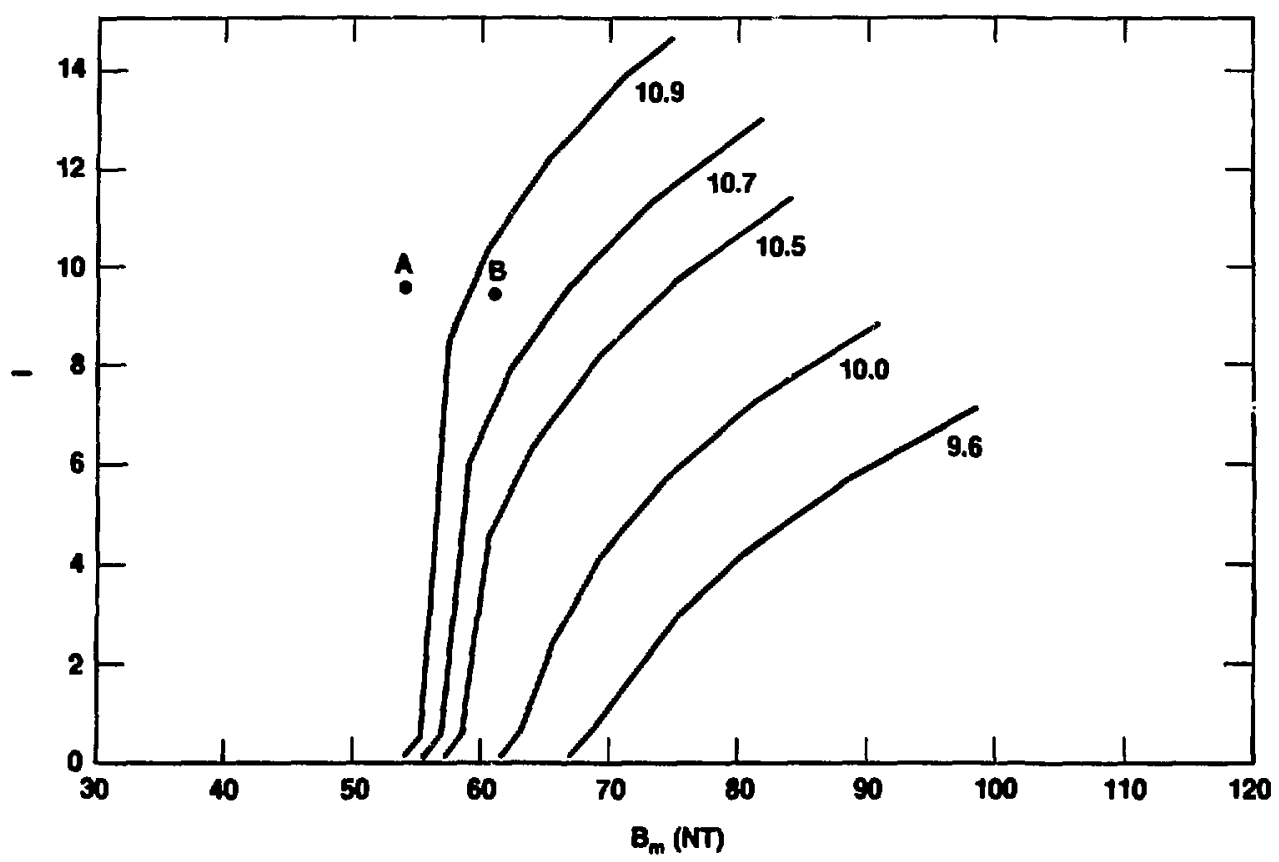

Figure 4-23. I-B ${ }_{M}$ representation of outer magnetospheris field lines in the Choe-Beard model. These are field lines that cross the equator in the azimuth $\phi=-30^{\circ}$, and are labelled with their equatorial crossing distance $\mathbf{R}_{\mathrm{EQ}}$. Electrons dirifting from the midnight meridian with $L_{B} B_{M}$ values that fall in the region of the plane to the left of the outermost field line (e.g., point A) will drift to the magnetopause before this azimuth and be loat. Particles with $1, B_{M}$ values to the right (e.g., point B) are on shells which close within the magnetosphere, up to this azimuth.

particle moves into a high-latitude minimum-B region or onto an equator-crossing branch (if one exists). The B-pocket trajectory is found to always close within the noon magnetopause, forming a stable-trapping circuit. The equatorial branch on the other hand is found to always lie on an open shell. The existence of branching gives rise to the possibility that particles on drift shells that are found from the foregoing analysis to lie in the drift loss cone, could actually lie on branched shells that form part of the stablytrapped region.

Figure 4-25 illustrates the types of drift trajectories that can exist in a branched model. We show constant- $|\mathrm{B}|$ contours in the equatorial plane for the Choe-Beard model (dipole tilt $=0^{\circ}$ ). Also shown are the equatorial intersections of three drift shells generated from an equatorial electron PAD initially in the midnight meridian, at $R_{E Q}=9.5 R_{E}$. The trace labelled 1 follows an electron of initial equatorial pitch angle $\alpha_{0}=60^{\circ}$. At an azimuth of $-50^{\circ}$ the shell branch $\therefore$ and we follow the B-pocket segment around to noon. Note that at noon the shell passes just inside the tragnetopause. As a closed shell, it forms part of the stable-trapping region. The trace labelled 2 follows a stably-trapped electron of $\alpha_{0}=40^{\circ}$. It moves on an unbranched shell passing well inside the magnetopause at noon. The trace labelled 3 follows an electron of $\alpha_{0}=90^{\circ}$. A particle of equatorial pitch angle $\alpha_{0}=90^{\circ}$ is an especially useful case for evaluating drift shells in a model field. This special case particle always mirrors at the equator (maintaining $\mathrm{I}=0$ ) and moves along a $\mathrm{B}$-contour (maintaining $\mathrm{B}=\mathrm{B}_{\mathrm{M}}=38 \mathrm{nT}$ in this irstance). But this contour does not close within the magnetosphere; the particle drifts into the magnetopause at an azimuth of $\approx-65^{\circ}$ and is lost. Thus this is a pseudo-trapping shell, with the immediate consequence that equatorial 


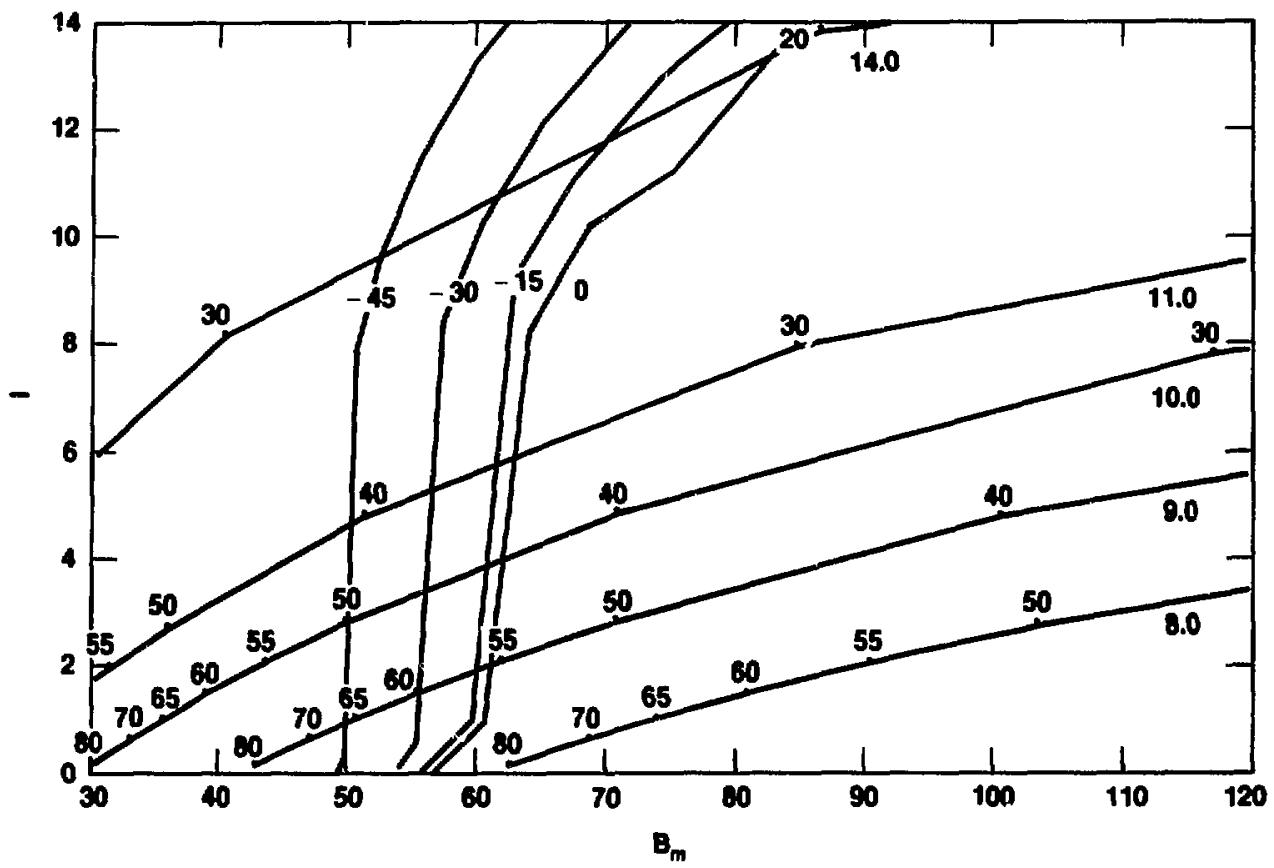

Figure 4-24. Drift loss-cone determination for midnight particles in the Choe-Beard model. The four near-vertical traces are the $1, B_{M}$ representation of the outermost field lines at aximuths of $\phi=$ $-45^{\circ},-30^{\circ},-15^{\circ}, 0^{\circ}$. The more horizontal traces are the representation of PADs in the midnight meridian, on field lines whose $R_{E Q}$ is given at the right. Each point is labeled with the corresponding equatorial pitch angle. Only points to the right of the noon field line $(\phi=0)$ are outside the drift lose cone. The loss cone at $R_{E Q}=9$ is $\alpha_{0}>55^{\circ}$; for $R_{E Q}=10$, it is $\alpha_{0}>45^{\circ}$; for $R_{E Q}=14$, it is $\alpha_{0}>20^{\circ}$.

PAD measurements made in the afternoon on this shell will register low fluxes at $\alpha_{0}=90^{\circ}$ (i.e., only electrons which have scattered onto this drift shell post-noon can contribute to the flux). This phenomenon of "magnetopause shadowing" of directional fluxes due to the cutoff of near-equatoria! drift shells by the magnetopause was reported by West et al. (1972).

The branching of drift shells in a model magnetosphere can be completely determined by reference to a branching map. Figure 4-26 illustrates a branching map we have constructed for the Choe-Beard model. At a given azimuth $\phi$ we have searched along outer field lines to locate branch points (i.e., points where the field strength attains a local maximum). Recalling our exposition of branching in the previous section, a drift shell characterized by $I, B_{M}$ will branch if a local $B_{\mathrm{MAX}}$ can be found on any line of the shell such that $B_{M}=B_{M A X}$. Sunward of the branching line, a particle is constrained to mirror either poleward of the local $B_{\text {MAX }}$ or equatorward of it. In the figure, each branch point $(0)$ is plotted in terms of its $B_{M}, I$ coordinates. By connecting these points with a smooth curve, we have then constructed a graphic representation of a oneparameter family of single-valued functions of $B_{M}$ :

$\mathrm{I}_{0}=f\left(\phi, \mathrm{B}_{M}\right), \mathrm{B}_{\mathrm{M1}}(\phi) \leq \mathrm{B}_{M} \leq \mathrm{B}_{\mathrm{M} 2}(\phi)$,

where $I_{0}$ denotes the unbranched value of $I$. The domain of $B_{M}$ is bounded from below by the branching point values at the magnetopause $B_{M 1}(\phi)$, and from above by the branching point values on the lines where the local $B_{M A X}$ disappears, $B_{M 2}(\phi)$. If $\phi$ is allowed to range over $-180^{\circ} \leq \phi \leq 0^{\circ}$, then Eq. (4.85) 


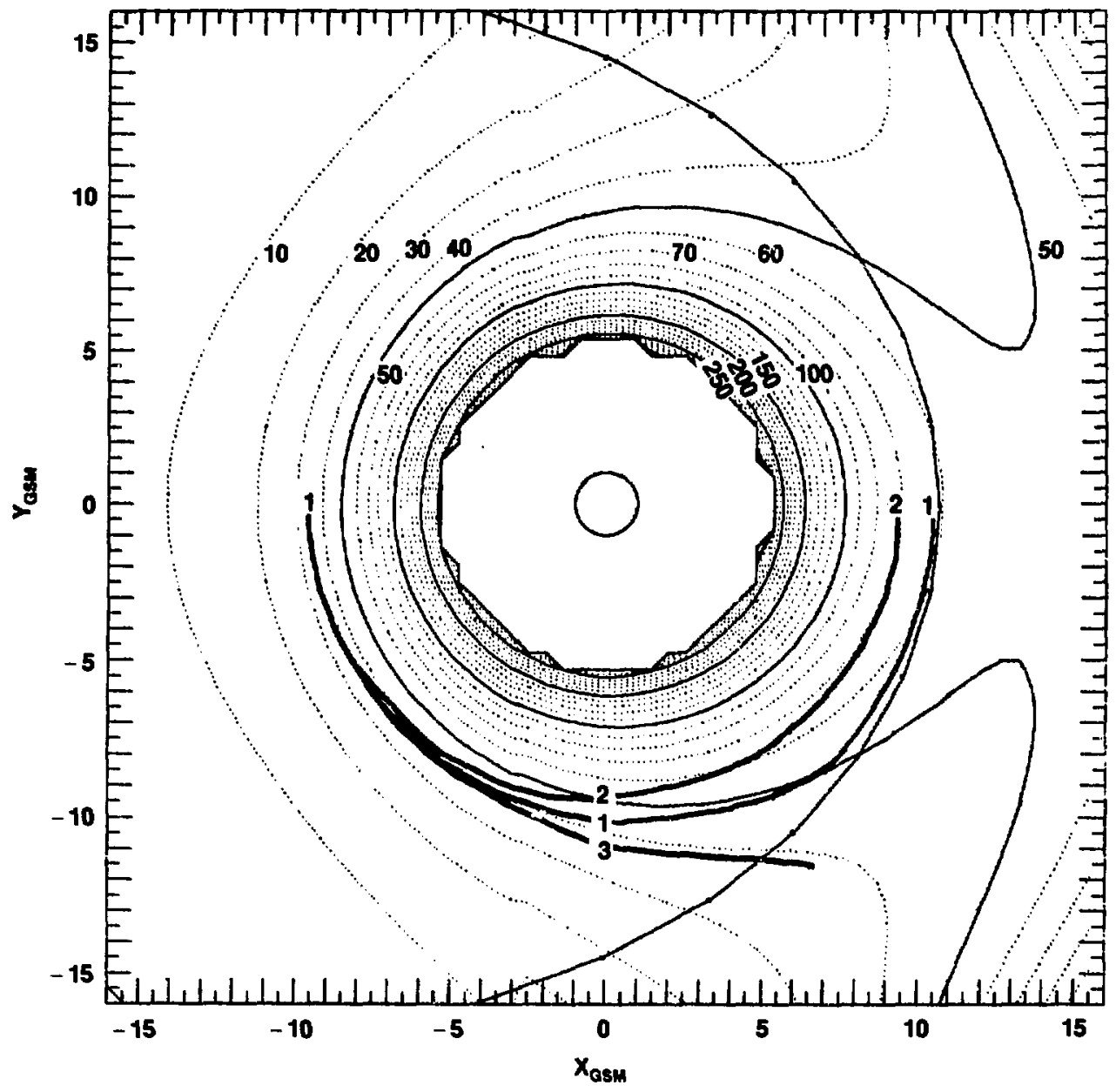

Figure 4-25. |B|-contours (nT) and drift shell traces in the equatorial plane, for the Choe-Beard model. Three drift shell types are illustrated, arising from a single equatorial PAD in the midnight meridian $\left(R_{E Q}=9.5 R_{E}\right)$. The trace labeled 1 is the drift shell of an $\alpha_{0}=60^{\circ}$ electron; at an azimuth of $-50^{\circ}$ the shell branches into high-latitude and equatorial segments. A high-latitude segment is traced from the branching azimuth around to noon. Note that this shell closes just inside the noon magnetopause and therefore forms part of the stably-trapped region. The trace labeled 2 is the drift shell of a stably-trapped $\alpha_{0}=40^{\circ}$ electron; it moves on an unbranched shell that passes well inside the magnetopause at noon. The trace labeled 3 is the drift shell of a pouedo-trapped $\alpha_{0}=90^{\circ}$ electron $(I=0)$; this particle must drift along a constant-B contour $(B=38 \mathrm{nT})$ in the equatorial plane, leading to an encounter with the magnetopause at an azimuth of $\approx-65^{\circ}$.

[N.B.- the spherical harmonic expansion of the model is only valid within the magnetosphere. The contours outside the magnetopause are inphysical.] 


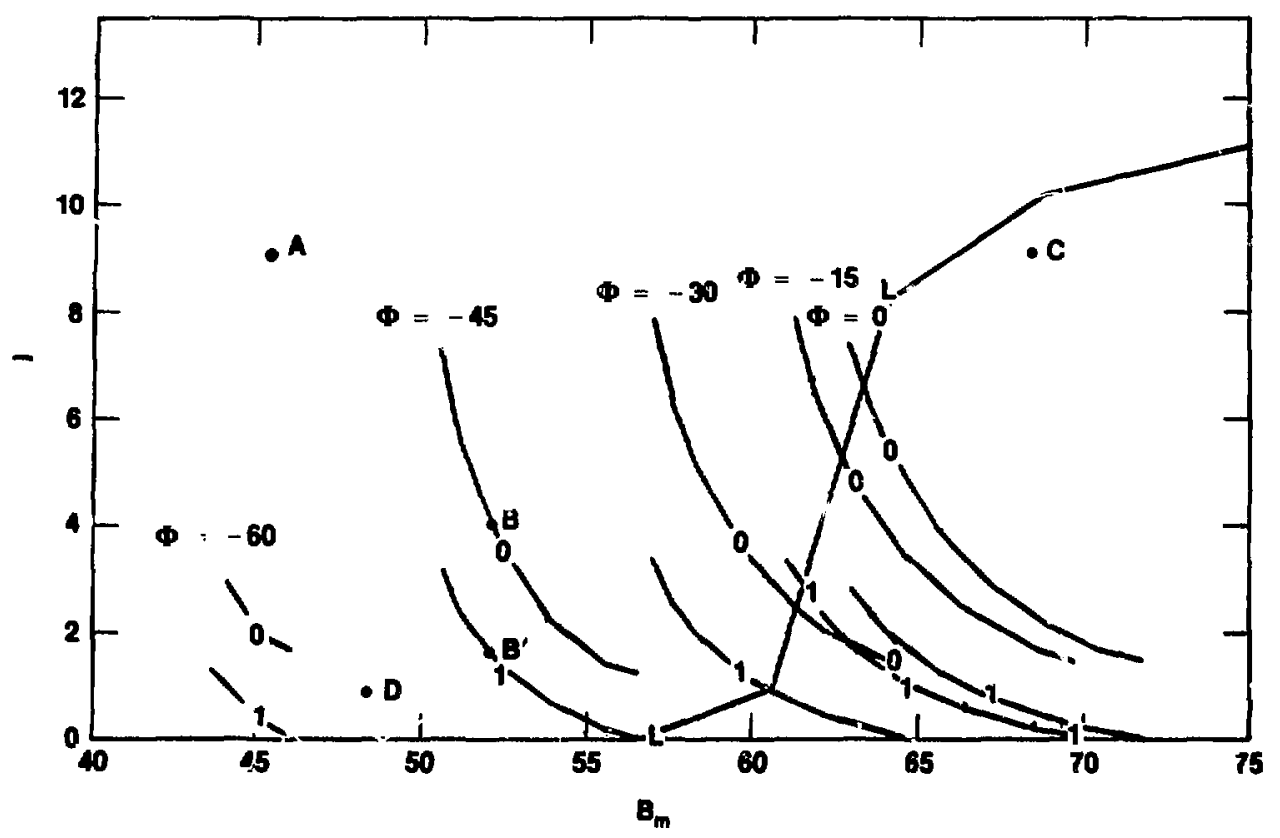

Figure 4-26. "Representation of ahell branching and the drift loar cone in the Choe-Beard model. This figure permits the analysis of the drift trajectory of an electron with afecified invariants $B_{M} I_{0}$ in the midright meridian. Branching curves are given (labeled with $0_{2}$ for a set of azimuths. The lower curves (Labeled with 1) represent the I-valnes of the B-pocket thell sezment emerging from each branch point. The trace at right (labeled with $L$ ) defines the lose-cone for unbranched shells (stable trapping only for points to its righr). Point $A$ represents a particle with an I-value too large ti.; branch and $a \mathrm{~B}_{M}$-value too amall for stable trapping; it lies in the puuedo-trapped region and will exit the magnetosphere befc: ce noon. Point $B$ identifies a particle on a drift shell which branches at the azimuth $\phi=-45^{\circ}$. The B-pocket segment of the shell takes on the reduced I-value given by point B', and the particle continues past noon within the magnetosphere (is part of the arablytrapped region). Point $C$ is outside the branching region but within the stable trapping region in the magnetospheric core. Point $D$ represents a near-equatorial particle which has an Iovalue too s: vall to branch and a $B_{M}$-value too amall for stable trapping; it alas lies in the pauedo-trapping segion.

defines the set of all branching points. The condition for shell branching can then be stated as follows: If for a shell of specified $I_{0}, B_{M}$ in the midnight meridian, there exists a value $\phi$ such that $\left[I_{0}-f\left(\phi, B_{M}\right)=0\right]$ for $\phi$ in the range $-180^{\circ} \leq \phi \leq 0^{\circ}$, then the shell branches at azimuth $\phi$.

$W=$ could equally well view $B_{M}$ as a function of $I_{0}$ and write:

$B_{M}=g\left(\phi, I_{0}\right), I_{1}(\phi) \leq I_{0} \leq I_{2}(\phi)$.

The finite value of $I_{1}(\phi)$ observed in this model $\left(I_{1} \approx 1.2\right)$ regults from the local $B_{M A x}$ point always occuring some (appreviable) distanc: from the equator. Thus particles with large $\alpha_{0}$ (and thereiore small $I_{0}$ ) do rot have access to the high-latitude minimum-B region.s.

Wten branching occurs the original bounce trajectory is broken, in the case of zero dipole tilt, into an equatorial segment and two high-latitude B-pocket segments. (In the general case of non-zero dipole tilt, 
the trajectory is broken into an equatorial segment and one high-latitude B-pock t segment). The particle's motion is subsequently confined to one of these disjoint regions. We have:

$I_{0}=I_{B P N}+I_{B P S}+I_{E Q} ;$

where

$\mathrm{I}_{0} \quad$ the I-value of the original unbranched drift shell;

$I_{B P N}=$ the I-value of the northem B-pocket branch;

$\mathrm{I}_{\mathrm{BPS}}=$ the I-value of the southern B-pocket branch;

$\mathrm{I}_{\mathrm{EQ}}=$ the I-value of the equator-crossing branch.

For the case of zero dipole tilt, $\mathrm{I}_{\mathrm{BPN}}=\mathrm{I}_{\mathrm{BPS}}=\mathrm{l}_{\mathrm{BP}}$.

Below each $l_{0}$ branching curve in Fig. 4-26 we have plotted the $l_{B P}$ values resulting from the branching (curves labeled with 1). Once a shell has branched and the invariant $I_{0}$ has been reduced to $I_{B p \text {, this new }}$ value of $I$ and the unchanged value of $B_{M}$ characterize the branched shell segment. This figure now contains all the information needed to assess the branching process for the Choe-Beard model. We complete the picture by adding the loss-cone information from Fig. 4-24. In the right-hand portion of the figire we plot the loss-cone demarcating noon field line (labeled with $\mathrm{L}$ ).

The fate of any particle drifting adiabatically from any arbitrary initial point in the midnight meridian can now be evaluated. For example, a particle characterized by the $1, B_{M}$ values of point $A$ is in the drift loss cone (i.e., to the left of the $L$-curve) and will strike the magnetopause before reaching noon. Its I-value is above the limit $I_{2}(\phi)$ for shell branching. A particle with the $I, B_{M}$ values of point $B$ would also be lost before noon, except that its drift shell branches at an azimuth of $-45^{\circ}$; if the particle is trapped in the high-latitude B-pocket branch, it will take on the I-value of point B', and pass around the noon meridian within the high-latitude pocket. At an azimuth of $45^{\circ}$ post-noon, the branched segments will rejoin and the particle will resume its normal equator-crossing bounce motion around to midnight (assuming no intervening non-adiabatic effects occur). If the particle instead were trapped into the equatorial branch (mirroring equatorward of the local B-maxima), it would drift to the magnetopause and be lost. Point $\mathrm{C}$ characterizes a particle which moves on a typical closed, unbranched shell within the magnetospheric core. Point D characterizes a particle in the loss-cone whose I-value is below the limit $I_{1}(\phi)$ for branching and will therefore drift to the magnetopause before noon.

In Fig. 4-27 we show how the branching map is employed to determine the branching of an equatorial PAD initially in the midnight meridian. We show here the branching curves from Fig. 4-26, overlaid with traces representing PADs from field lines in the midnight meridian, each labeled on the right with the $\mathbf{R}_{\mathrm{EQ}}$ of its midnight field line. Points on the PAD curves (designated by P) are annotated with the equatorial pitch angle $\alpha_{0}$ which a particle with those $I, B_{M}$ coordinates has at midnight. Where a PAD curve intersects the family of $I_{0}$ curves, shell branching takes place. The post-branch I-value of the particle (assuming it is trapped in the B-pocket segment) is located by noting the I-value which corresponds to its intersection-point $B_{M}$-value on the companion $I_{B P}$ vs $B_{M}$ curve.

A PAD that intersects the branching curves in this diagram contains particles that drift on 3 types of shells. For a PAD on the field line designated by $R_{E Q}=9.0 R_{E}$, for example, we see that particles of $\alpha_{0}>65^{\circ}$ are in the drift loss cone. Particles with pitch angles in the interval $55^{\circ} \leq \alpha_{0} \leq 65^{\circ}$ would also be lost but for shell branching, which allows some of the particles to move into the B-pockets which lie in the stably-trapped region of the magnetosphere. In this case branching acts to decrease the size of the drift loss cone in the midnight meridian. Particles in the range $52^{\circ} \leq \alpha_{0}<55^{\circ}$ are in the stable-trapping region before and after branching into a B-pocket. Particles with $\alpha_{0}<52^{\circ}$ move on unbranched shells in the stably-trapped region of the magnetospheric core.

The regions of stable-trapping, pseudo-trapping, and branching for the Choe-Beard model are shown in Fig. 4-28 as computed by the above process, where we depict field lines and contours of constant equatorial pitch angie $\alpha_{0}$ in the midnight meridian. The boundaries of the different drift shell regions are drawn as heavy lines. Note that the effect of shell branching is to extend the boundary of stable trapping outwards, over a limited range of equatorial pitch angles, $30^{\circ} \leq \alpha_{0} \leq 65^{\circ}$ approximately. Following shell branching, the particles move along a segment of the branched shell towards noon, conserving $B_{M}$ and the post-branch value of $\mathrm{I}$. The mirror points of the B-pocket particles can therefore be located in the noon meridian by searching along the outer noon field lines for points where $B=B_{M}, I=I_{B P}$. 


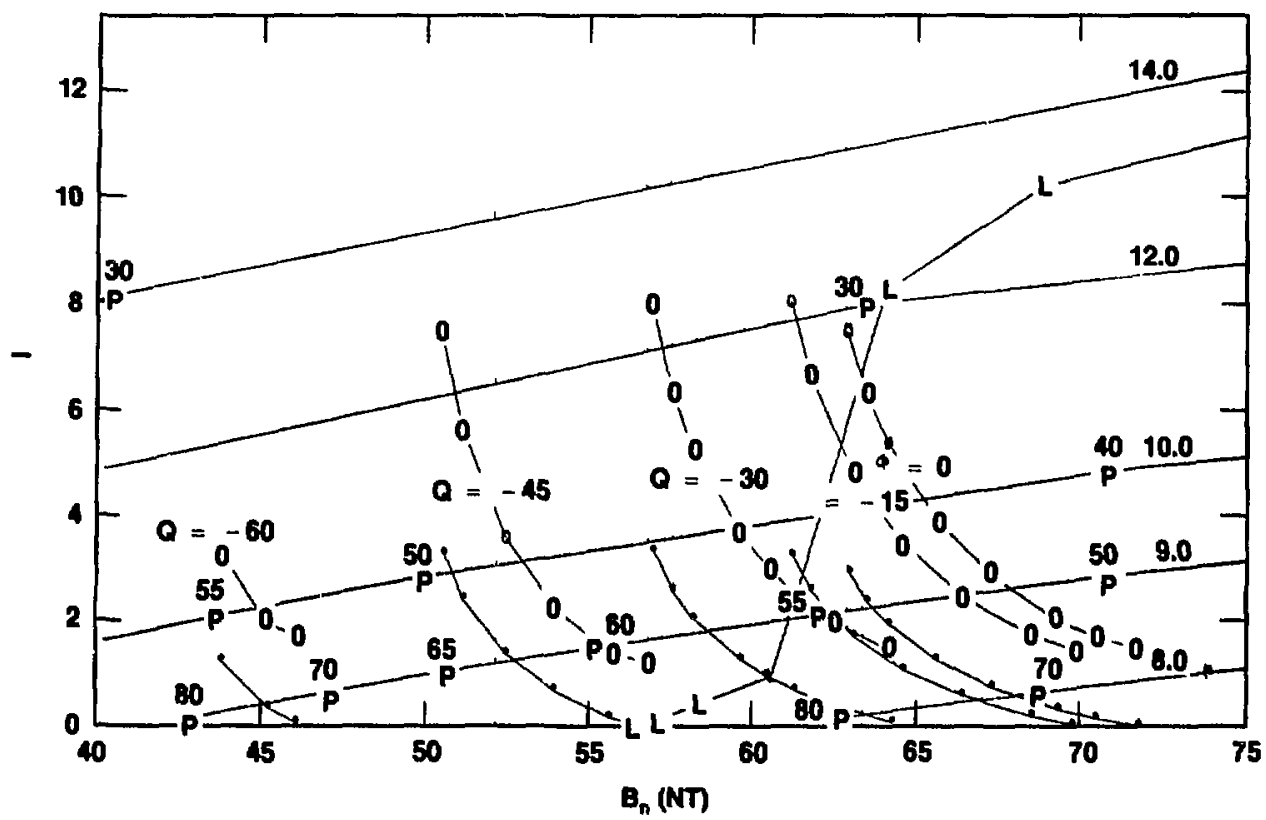

Figure 4-27. Mapping of electron drift shells from midnight to noon in the Choe-Beard model. From this figure one can construct the stable-trapping regions at midnight. Curves labeled with 0,1 , and $L$ are described in the caption of Fig. 4-26. Curves labeled with $P$ represent equatorial PADs obtained in the midnight meridian, along field lines whose $R_{\mathrm{EQ}}$ is given on the right. Each PAD point is annotated with its corresponding equatorial pitch angle $\alpha_{0}$. By inspection, the range of pitch angles on any midnight field line corresponding to drift loss, branching, and stable trapping can be determined.

We have carried out this mapping process for normal and branched shells. Figure 4-29 illustrates the mirror points in the noon meridian for PADs initially on midnight field lines of $R_{E Q}=8$ and $10 R_{E}$. The unbran-hed $R_{E Q}=8$ PAD shows the effects of shell splitting-near equatorial particles mirror on more distant field lines than do particles of small initial $\alpha_{0}$. The $R_{\mathrm{EO}}=10$ PAD shows effects of both shell splitting and shell branching. Particles with $\alpha_{0}$ in the range $43^{\circ} \leq \alpha_{0} \leq 55^{\circ}$ drift on shells that branch before noon; we show here the upper and lower mirror points of the B-pocket segments.

We have performed numerous orbit calculations of drifting electrons to analyze motion in B-pocket regions. Figures 4-30 and 4-31 illustrate the results of numerically integrating the equations of motion to obtain a pointwise trajectory for a drifting particle. Figure 4-30 shows the $Y-Z$ GSM plane projection of the orbit of a $3 \mathrm{MeV}$ electron drifting azimuthally around past the noon meridian $(Y=0)$ on an unbranched shell in the outer magnetosphere. The particle bounces between northern and southern mirror points, at $\mid Z: \approx 7$, and drifts eastward (towards $+Y$ ) under the influence of the gradient- $B$ and centrifugal forces discussed in Chapter 3. Near $|Z|=4$ the orbit shows a local tightening of the helical bounce motion (pitch angle $\alpha$ trending towards $90^{\circ}$ ) as the particle encounters the local B-maxima on the outer field lines. Figure 4-31 shows the orbit of an electron drifting on a branched shell in the same noontime region. The losalized tightening of the turns of the helix is more pronounced. On the fourth bounce the electron becomes trapped in the southern minimum-B pocket near noon; with a different initial bounce phase it 


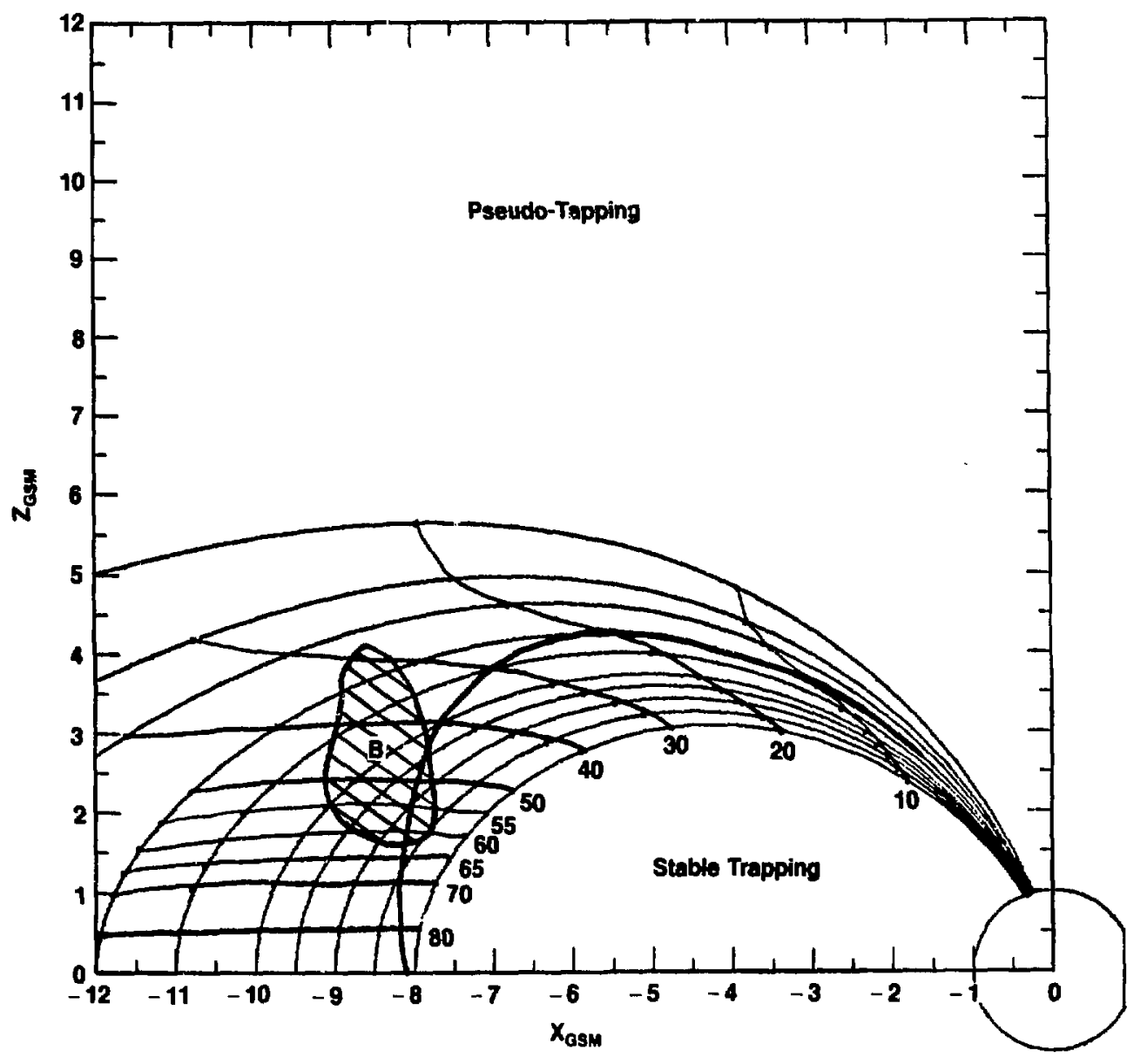

Figure 4-28. Regions of stable trapping (without branching), psuedo-trapping, and branching in the midnight meridian of the Choe-Beard model. Boundaries shown as heavy lines. Field lines and contours of constant equatorial pitch angle $\alpha_{0}$ are also shown. The effect of shell branching is to extend the boundary of stable trapping outwards for a limited range of pitch angles.

would have been trapped in the northern minimum-B pocket or in the equatorial region between the local B-maxima. The shell rejoins post-noon, and the particle regains its initial (pre-branch) l-value.

The stable trapping boundary in the noon meridian is the envelope of all PADs at midnight whose drift trajectories can be mapped around to noon within the magnetosphere. This is shown in Fig. 4-32. By comparison to the Mead-Williams model trapping boundary (Fig. 4-22), we see that shell branching has extended the stable trapping region to significantly higher latitudes at noon. 


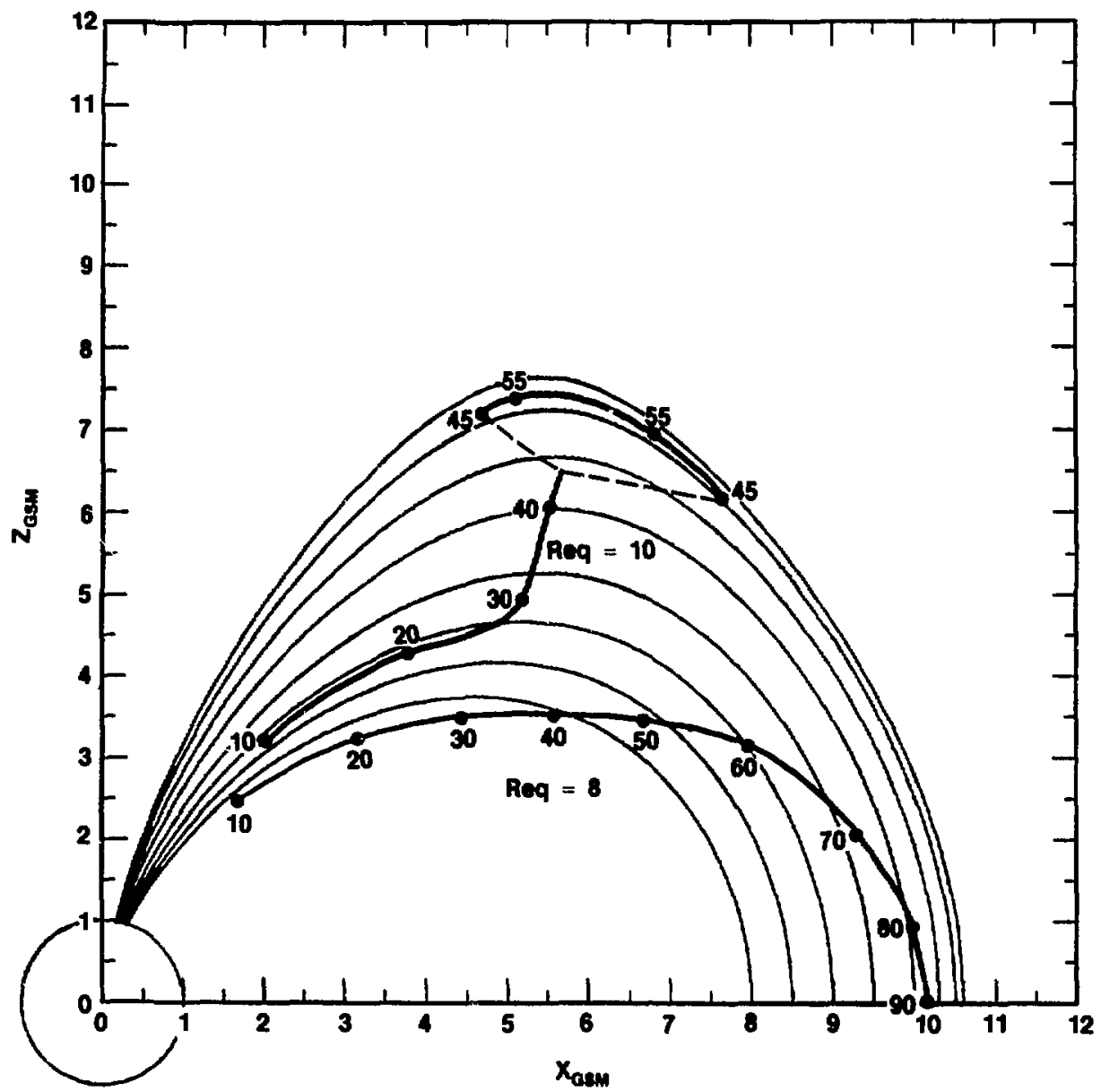

Figure 4-29. Midnight PAD mirror pointo mapped to the noon meridian in the Choe-Beard model. Mirror points are annotated with their $\alpha_{0}$ in the midnight meridian. The PAD from the $R_{\mathrm{EQ}}=8$ line at midnight shows pronounced shell splitting. The PAD from $R_{\mathrm{EQ}}=10$ shows shell branching for $a_{0}$ in the range $43^{\circ} \leq \alpha_{0} \leq 55^{\circ}$; particles with midnight $\alpha_{3}>55^{\circ}$ fall in the drift loas cone. 


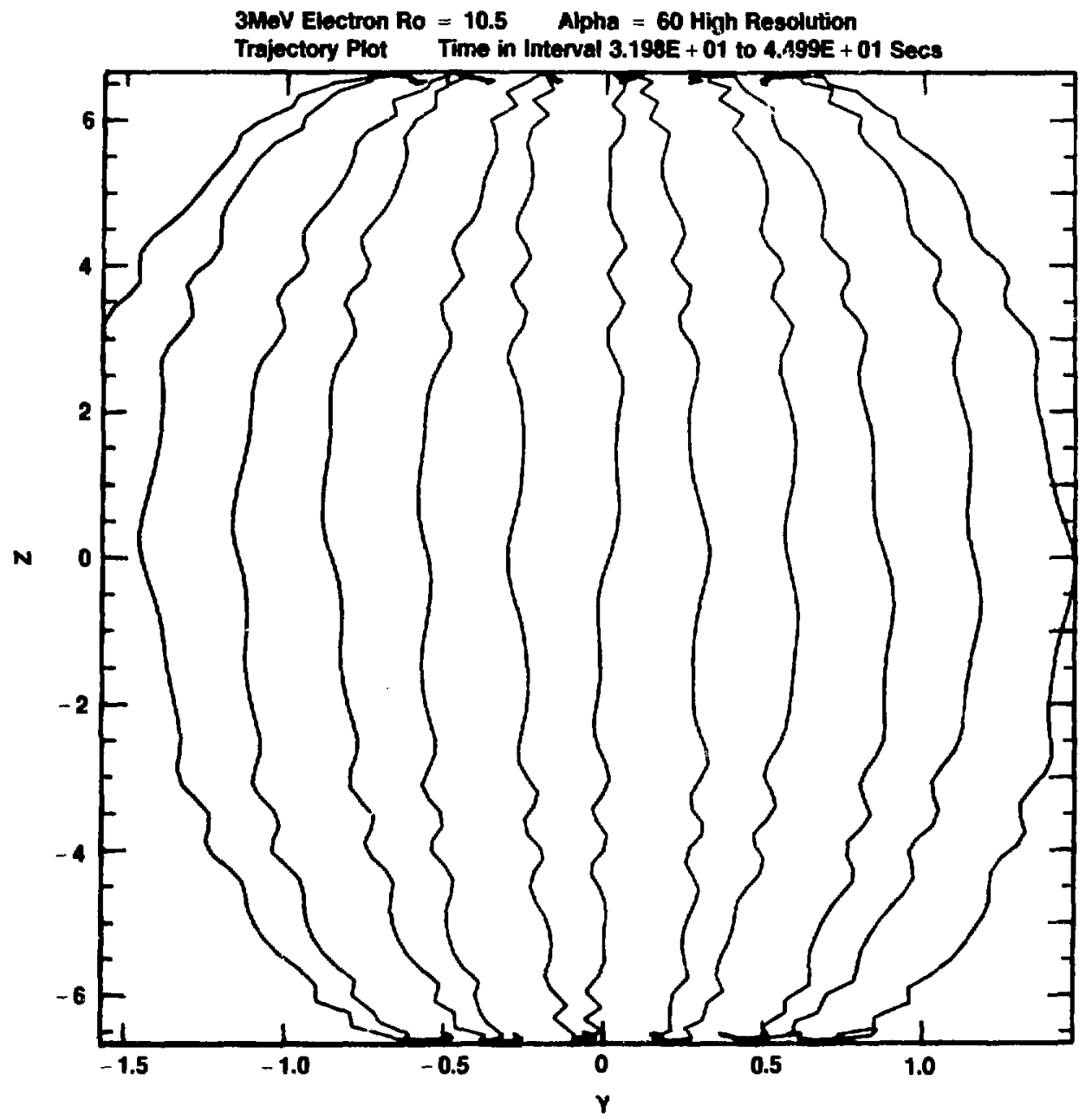

Figure 4-30. Trajectory of a 3 MeV electron on a stably-trapped, unbranched drift shell in the noon sector, computed by numerically integrating the equations of motion. The trajectory is shown projected into the GSM YZ plane. This particle drifts onto field lines near noon which contain local $B$-maxima in the region $|Z| \approx 4$, causing the pitch of the helical orbit to tighten (trend towards $a=$ $90^{\circ}$ ). (Because of sampling, this adiabatic trajectory plot appears irregular.) 


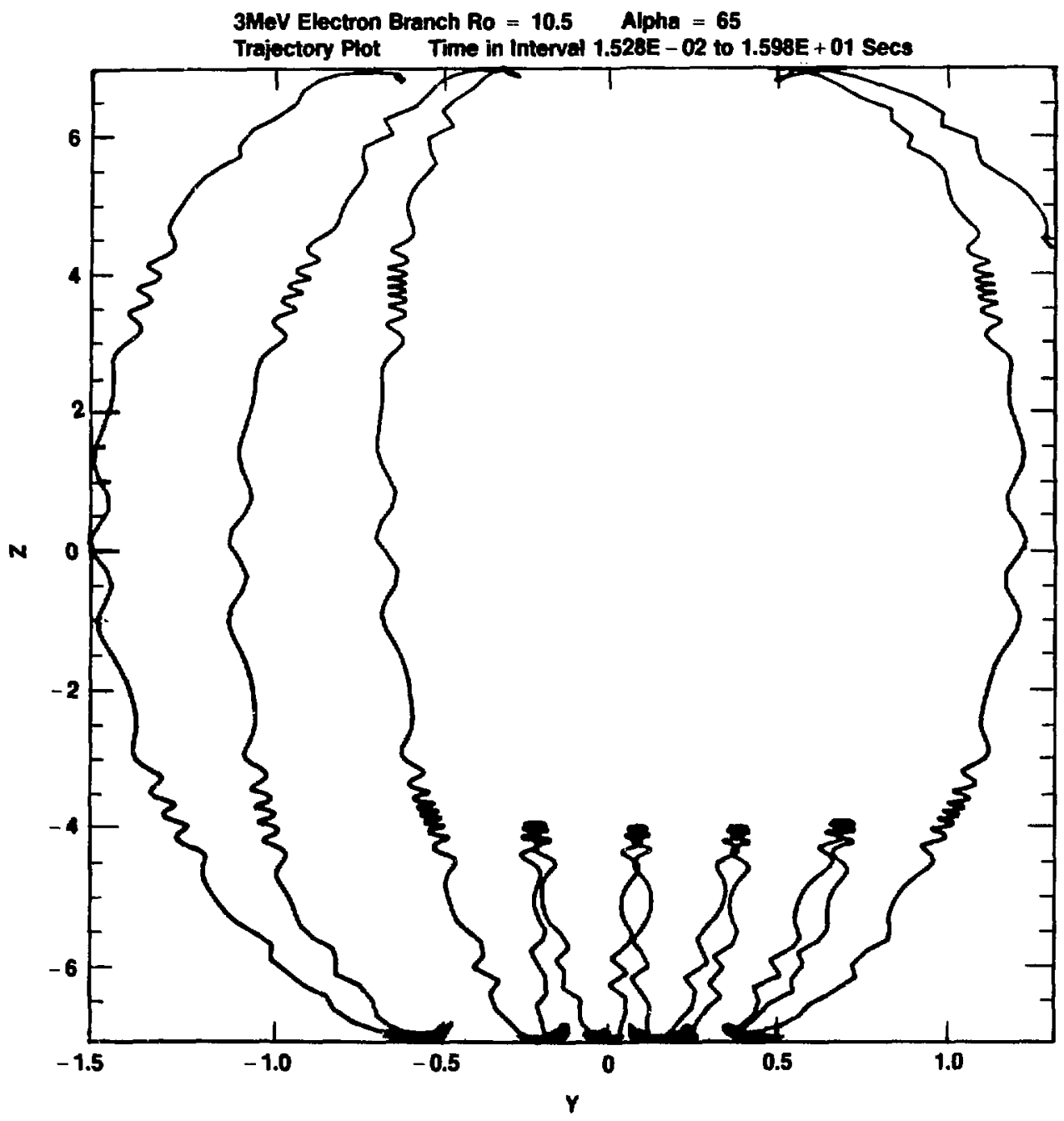

Figure 4-31. Trajectory of a $3 \mathrm{MeV}$ electron on a stably-trapped, branched drift shell in the noon sector, computed by numerically integrating the equations of motion. The trajectory is shown projected into the GSM YZ plane. This particle drifts onto field lines near noon containing local $B$-maxima in the region $|Z| \approx 4$, that exceed the particle's $B_{M}{ }^{-v a l u e . ~ W h e n ~ s h e l l ~ b r a n c h i n g ~ o c c u r s ~ t h e ~}$ particle is trapped in the southern B-pocket, mirroring poleward of the southern B-max. The shell rejoins post-noon, and the particle regains its original I-value. (Because of sampling, this adiabatic trajectory plot appears irregular.) 


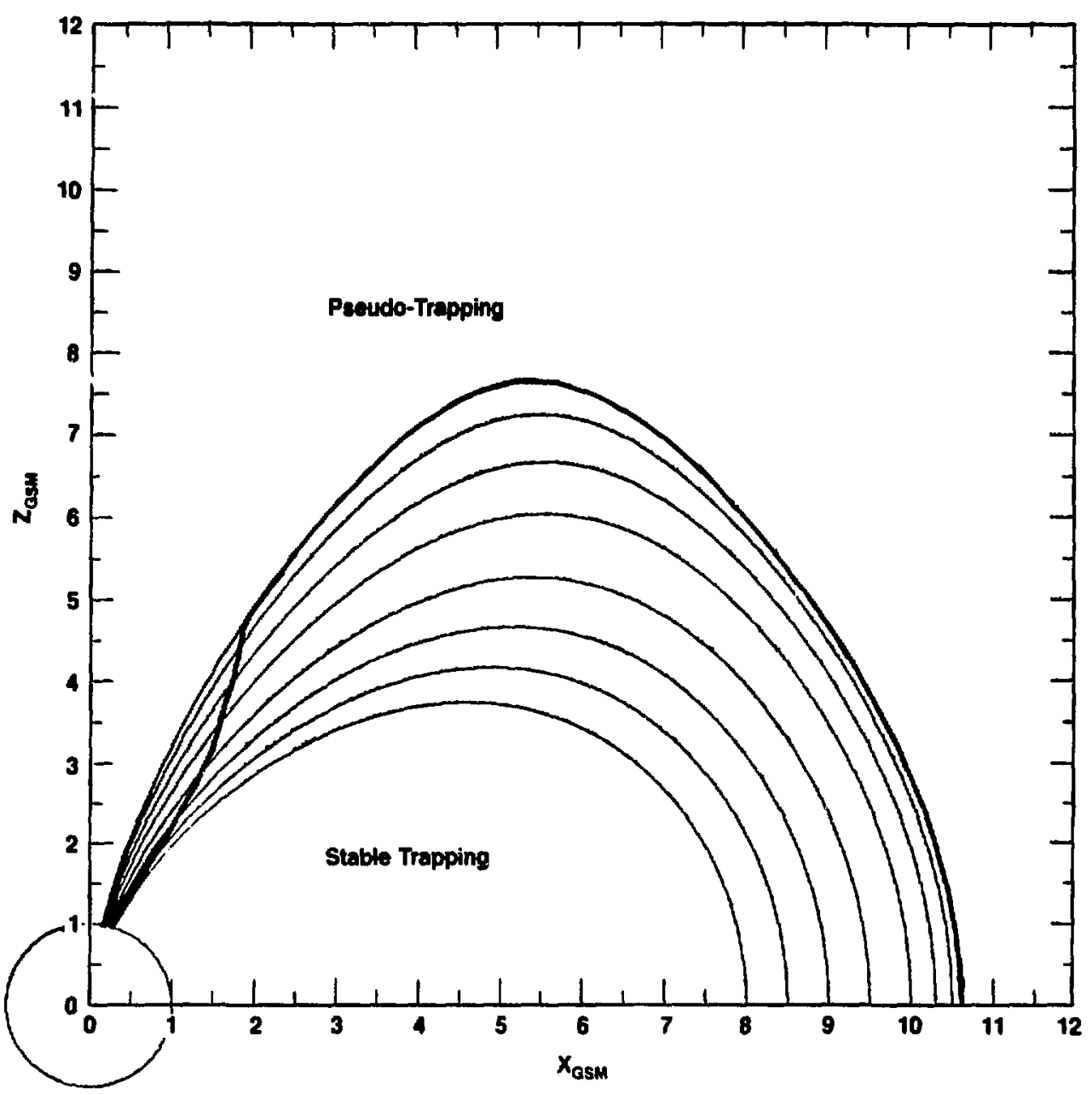

Figure 4-32. Boundary of stable trapping in the noon meridian, for the Choe-Beard model. The presence of high-latitude minimum-B pockets extends the stable-trapping region to high latitudes. 
Shell splitting is a continuous process (although its name would seem to imply a discrete process). We can formulate shell splitting as a transformation which maps a pitch angle distribution measured at a point in the noon meridian, onto a range of field lines in the midnight meridian. Let $R_{\mathrm{EQ}}^{N}$ specify the equatorial radius of a noon-meridian field line, and $\alpha_{0}^{N}$ the equatorial pitch angle of a particle mirroring on that line. For a given field model, this information is sufficient to determine the particle's invariants $B_{M}$ and $I$. The equatorial radius of the midnight field line to which this particle adiabatically maps can be written as:

$R_{\mathrm{EQ}}^{\mathrm{M}}=\mathrm{F}\left(\mathrm{I}, \mathrm{B}_{\mathrm{M}}\right)$

where $F$ is the model-dependent transformation. $F$ is a continuous function of $I$ and $B_{M}$. For a dayside field line of normal geometry (i.e., with one minimum in field strength at the magnetic equator), $B_{M}$ and $l$ vary continuously with equatorial pitch angle $\alpha_{0}$. Therefore $\mathrm{R}_{\mathrm{EQ}}^{\mathrm{M}}$ can be expressed as a continuous function of $\alpha_{0}^{N}$ for a given $R_{E Q}^{N}$

$R_{\mathrm{EQ}}^{M}=f\left(\alpha_{0}^{N}\right)$

As $\alpha_{0}^{N}$ is systematically varied over the range $\alpha_{01}^{N} \leq \alpha_{0}^{N} \leq \alpha_{02}^{N}$ to represent the PAD measured at that point in the noon meridian, the transformations $f$ and $F$ produce mapped $R_{E Q}^{M}$ values in some range $\mathrm{R}_{\mathrm{EQ1}}^{\mathrm{M}} \leq \mathrm{R}_{\mathrm{EQ}}^{\mathrm{M}} \leq \mathrm{R}_{\mathrm{EQ2}}^{\mathrm{M}}$. The condition of no shell splitting can be expressed as:

$\frac{\partial f}{\partial \alpha_{0}^{N}}=0$ for all $a_{0}^{N}$,

which is to say that the mapping is degenerate (all $\alpha_{0}^{N}$ map to a single midnight field line).

Shell branching can introduce discontinuities into the transformation $f . A$ noon field line which threads the B-pocket region can have two high-latitude minima in field strength as well as one at the equator (Fig. 4-33). An equatorial PAD therefore samples paticles mirroring equatorward of the local B-maxima (located at $S_{2}$ and $S_{3}$ ), and also those particles mirroring poleward of the maxima whose $B_{M}$ values exceed $B\left(S_{2}\right)=B\left(S_{3}\right)$. Particles in the magnetic wells can not be observed. At the critical equatorial pitch angle corresponding to mirroring at $\left.S_{2}, \alpha_{0 C}=\sin ^{-1} \sqrt{\mathrm{B}_{\mathrm{EQ}}} / \mathrm{B}\left(\mathrm{S}_{2}\right)\right]$, I changes discontinuously from

$I-=\int_{S_{2}}^{S_{3}}\left[1-\frac{B(s)}{B\left(S_{2}\right)}\right]^{1 / 2} d s$,

$I+=\int_{S_{1}}^{S_{4}}\left[1-\frac{B(s)}{B\left(S_{2}\right)}\right]^{1 / 2} d s$.

Via the transformation $F$, this jump in I produces a jump in $R_{E Q}^{M}$. We illustrate these effects with the ChoeBeard model. Figure 4-34 depicts the distribution of the mirror points of an equatorial PAD for a noon meridian field line $\left(R_{E Q}=10.4 R_{E}\right)$. Particles with $\alpha_{0}$ in the range $72^{\circ} \leq \alpha_{0} \leq 90^{\circ}$ have mirror points which lie equatorward of the local B-maxima $\left(B=64.2 \mathrm{nT}\right.$ ). Particles with $\alpha_{0}<72^{\circ}$ mirror poleward of the local B-maxima. In Fig. 4-35, we indicate the mapped locations of these mirror points in the midnight meridian. Note that particles which mirror equatorward of the local B-maxima on the noon field line, map to field lines at midnight with $R_{E Q}<8.6 R_{E}$. Particles which mirror poleward of the noon local $B$-maxima map to midnight field lines with $R_{E Q}>10.5 R_{E}$. A discontinuity in the mapping is evident at the noon critical equatorial pitch angle $\alpha_{O C}=72^{\circ}$. Since fluxes in the midnight meridian decrease with $R_{E Q}$, discontinuities in $\mathrm{R}_{\mathrm{EQ}}^{\mathrm{M}}$ produce discontinuities in flux levels in the PADs observed at noon.

Figures 4-36 and 4-37 illustrate synthetic equatorial PADs on the noon field line, $R_{E Q}=10.4 R_{E}$ in the Choe-Beard mod $=1$, constructed from models of energetic electron fluxes in the midnight meridian. OGO-5 observations indicate that the prevalent PADs in the midnight sector of the outer magnetosphere are the 


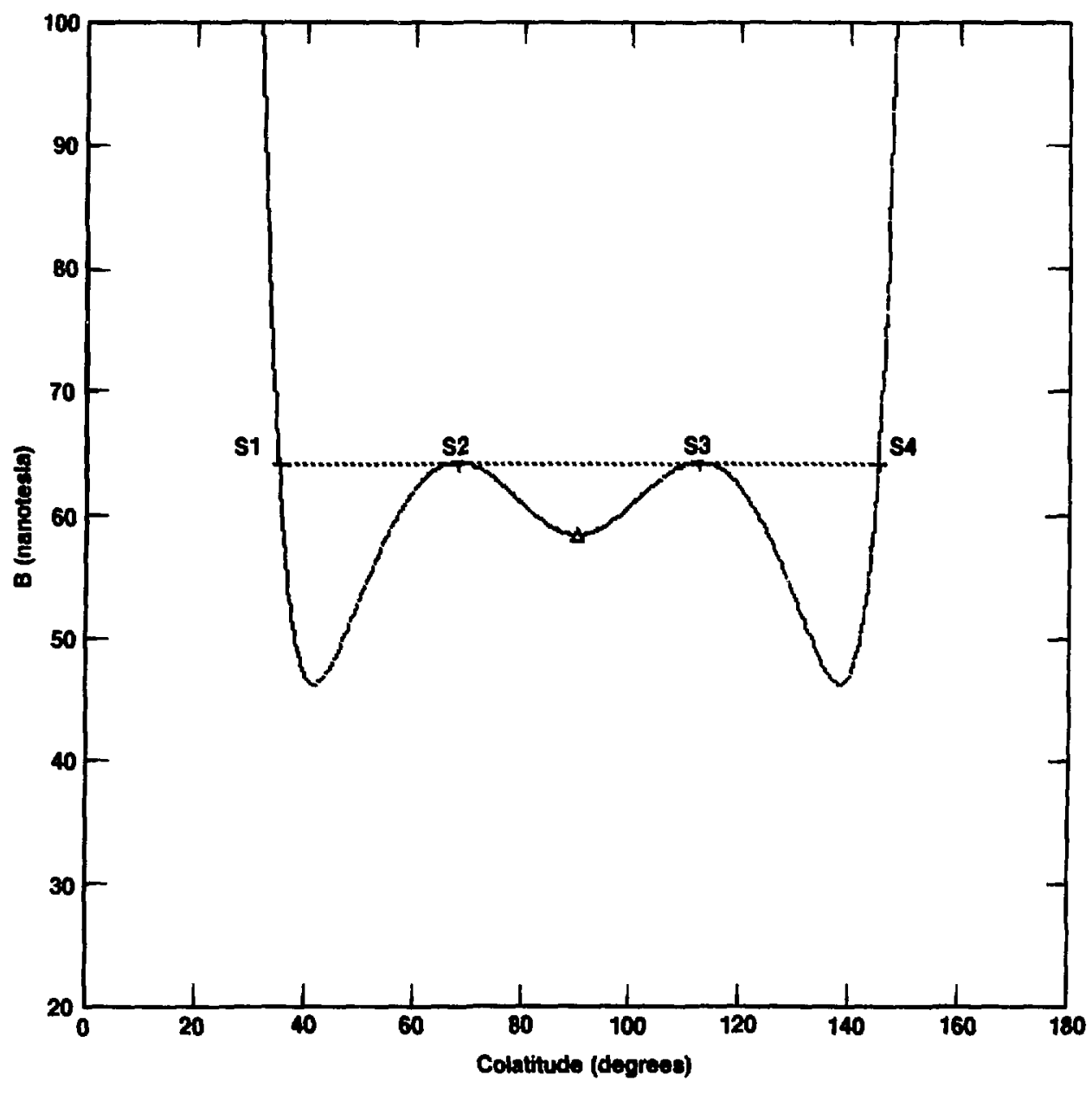

Figure 4-33. An outer magnetospheric field line in the noon meridian, for the zero-tilt Choe-Beard model. Two local B-maxima exist at $S_{2}$ and $S_{3}$ which produce two high-latitude minimum-B pockets, and an equatorial pocket as well. $A$ satellite at the equator (colatitude $90^{\circ}$ ) detects particles whose mirror points lie equatorward of $S_{2}$ and $S_{3}$ and those whose mirror points lie poleward of $S_{1}$ and $S_{4}$. Particles trapped in the high-latitude minima are not observed. 


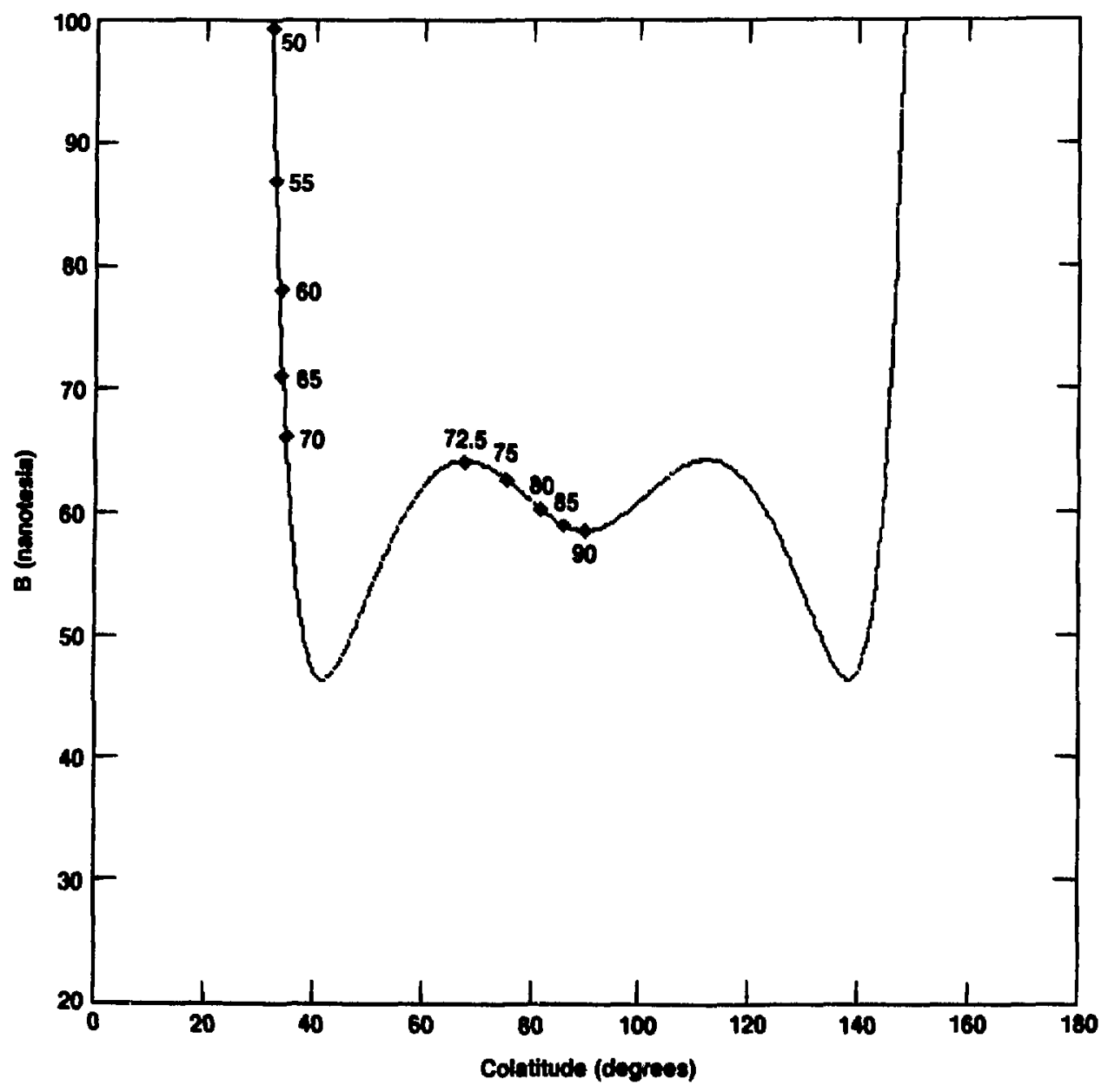

Figure 4-34. Distribution of equatorial PAD mirror points for the field line of Fig. 4-33. Mirror points are labeled with the correaponding equatorial pitch angle. The critical pitch angle of $\alpha \approx 72^{\circ}$ separates the distribution into particles whose mirror points lie poleward of the high-latitude pocket and those whose mirror points lie equatorward of it. 


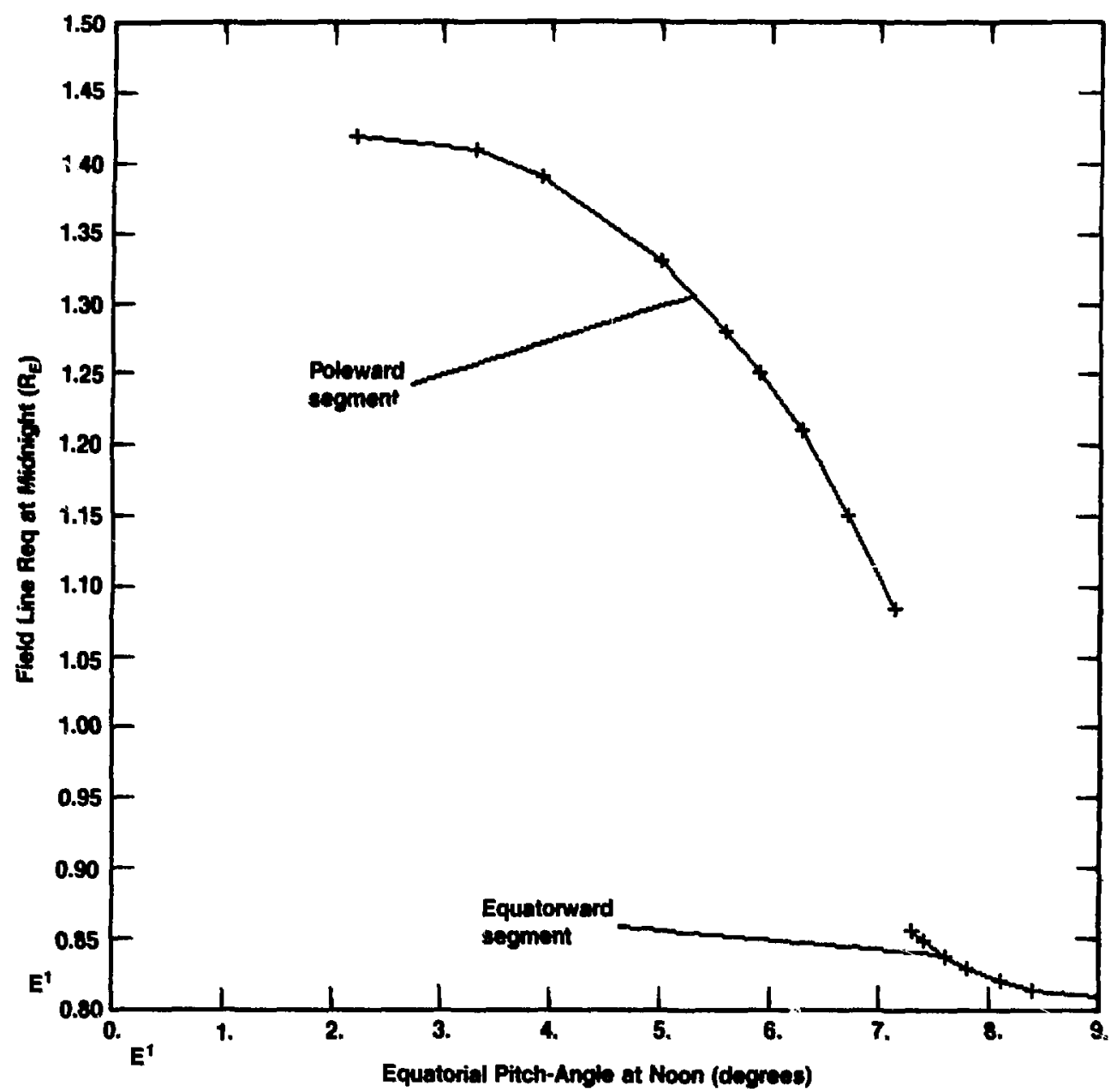

Figure 4-35. Mapping of the mirror points of a noon equatorial PAD into the midnight meridian. Particles ntoving on unbranched shells which mirror equatorward of the noon local B-maxima, map to midnight fieldlines of $R_{\mathrm{EQ}}<9 \mathbf{R}_{\mathrm{E}}$. Particles mirroring poleward of the noon local B-maxima map to distances $>10.5 R_{E}$ at midnight. 


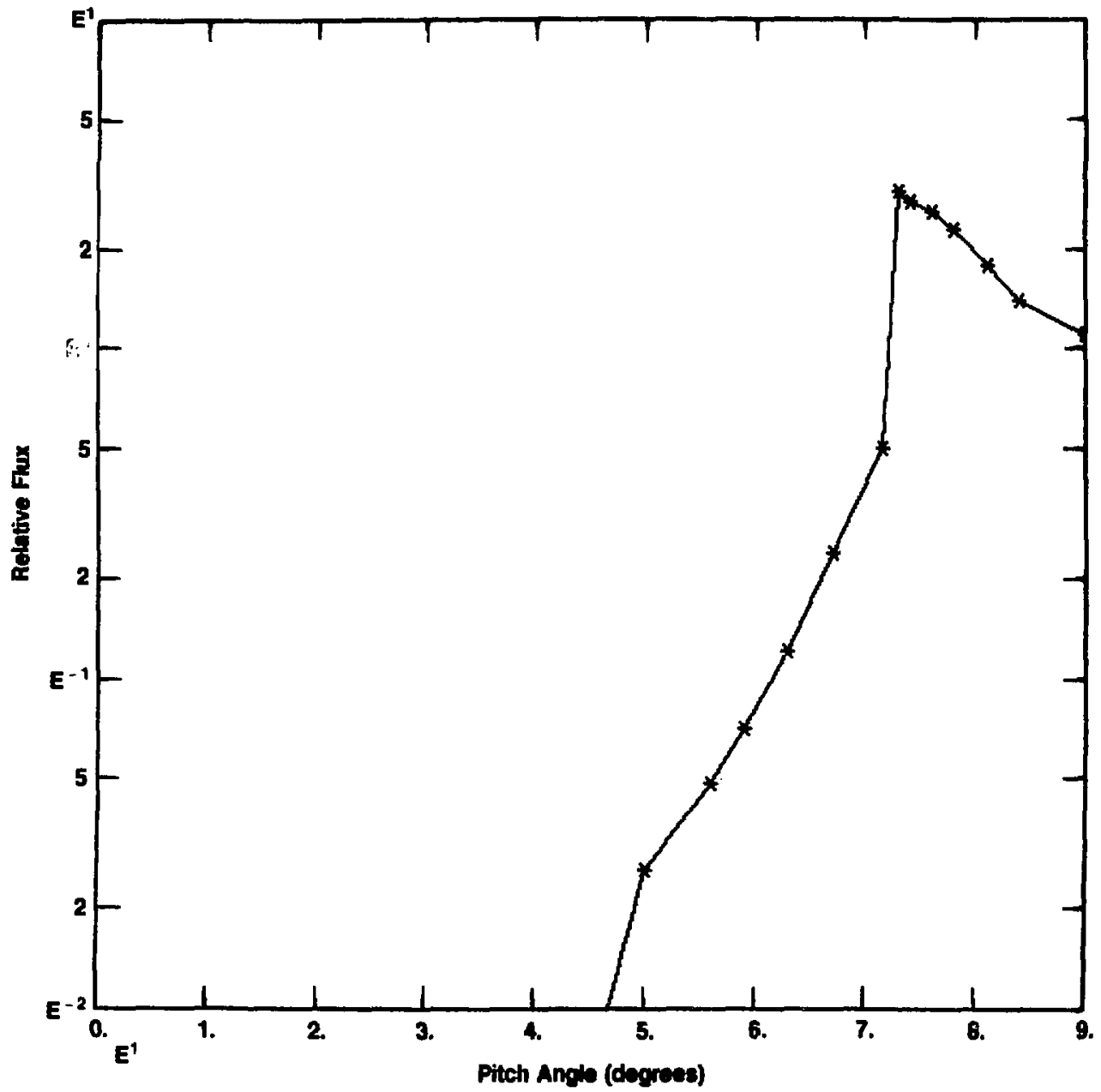

Figure 4-36. Synthetic noon equatorial PAD constructed by mapping fluxes from the midnight meridian to noon, assuming a buticerfly distribution at midnight that decreases exponentially with $R_{E Q}$. The mapping illustrated in Fig, 4-35 wa - wed. 


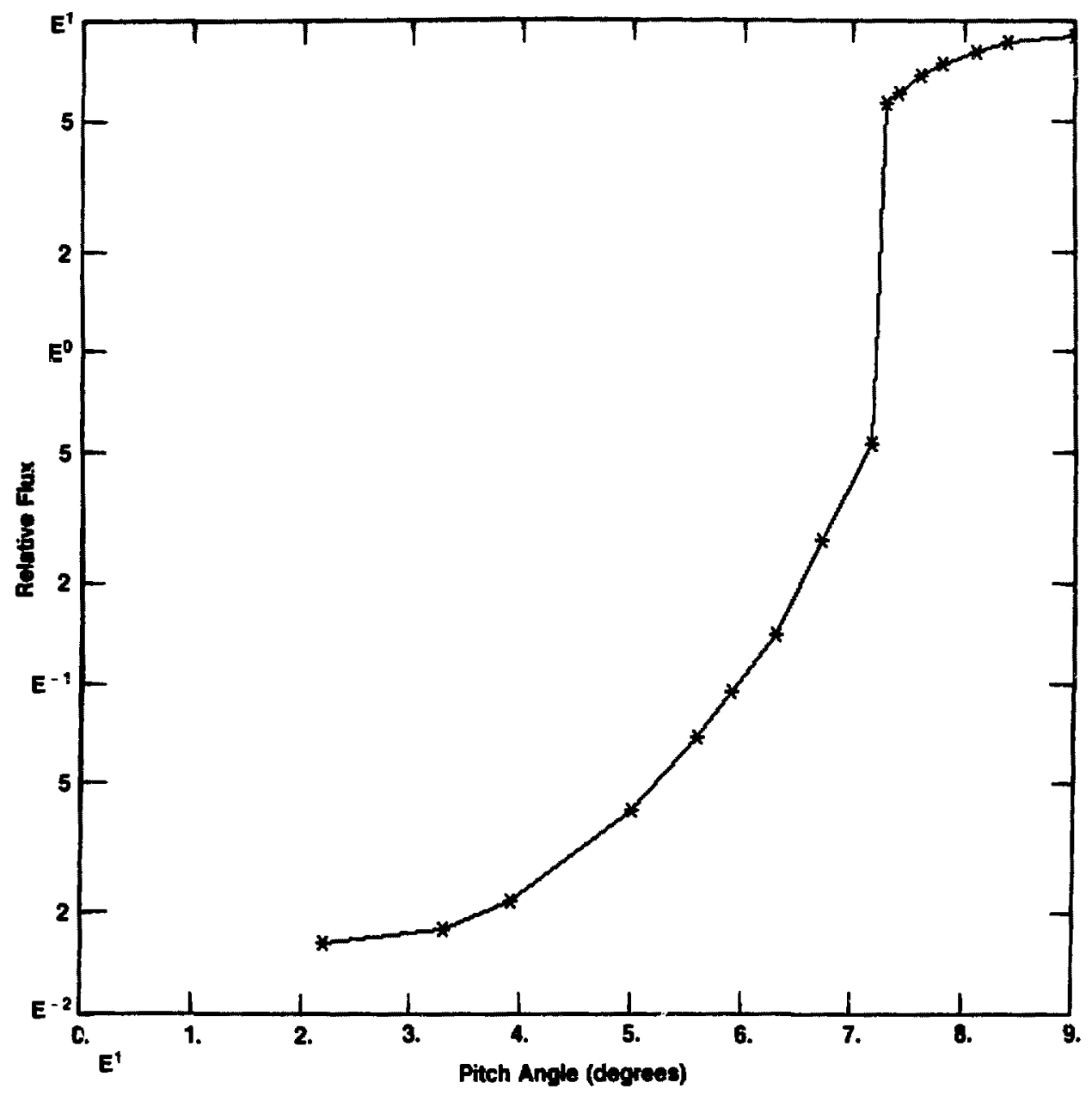

Figure 4-37. Synthetic noon equatorial PAD constructed by mapping fluxes from the midnight meridian to noon, assuming an isotropic distribution at midnight that decreases exponentially with $R_{\mathrm{EQ}}$. The mapping illustrated in Fig. 4-35 was used. 
butterfly distribution (peaking at equatorial pitch angles of $30-40^{\circ}$ ) and the isotropic distribution (constant everywhere except for a small range of pitch angles which falls in the bounce loss cone) (West, 1979). The equatorial directional flux in the midnight meridian was modeled as:

$\mathrm{i}_{M}\left(\mathbf{R}_{\mathrm{EQ}}, \boldsymbol{\alpha}_{0}\right)=j_{0} \cdot 10^{\left[\mathrm{C}\left(\mathrm{R}_{\mathrm{EQ}}-\mathrm{R}_{\mathrm{EDO}}\right)\right]} \cdot \mathrm{G}\left(\alpha_{0}\right) ;$

where $G\left(\alpha_{0}\right)$ gives the dependence of $j_{M}$ on $\alpha_{0} . G\left(\alpha_{0}\right)=1$ for the isotropic distribution, and for the butterfly distribution case, $G\left(\alpha_{0}\right)=G_{8}\left(\alpha_{0}\right)=$ the butierfly distribıtion observed $c_{11}$ Sept. 18, 1968 .

This distribution (illustrated in Fig. 1-23) peaked at about $40^{\circ}$ in ihe $79 \mathrm{keV}$ channel, and was typical of magnetically quiet observations in that region of the near-midnight magnetosphere which maps to the outer dayside (midnight $R_{E C}$ in the range 8-14 $R_{E}$ ). $R_{E Q 0}$ was taken as $8 R_{E}$ and the parameter $C$ (which has units of $\left.\delta \log (j) / \delta \mathrm{R}_{\mathrm{EO}}\right)$ was evaluated from near-equatorial OGO-5 measurements taken in August, 1968. An average value of $C=0.45$ was derised for the radial range $8-\hat{4} 4 R_{E}$.

The noon synthetic PAD based on the isotropic midnight flux model (Fig. 4-36) shows a factor-of-ten jump in flux level at the critica! pitch angle $\alpha_{0 \mathrm{C}}=72^{\circ}$. The synthetic PAD based on the tuiterfly flux model at midnight (Fig. 4-37) shows a lesstr jump at the critical angle. In this latter case, the increase of flux with decreasing pitch angle somewhat offsets the dominant decrease of flux with increasing $R_{\mathrm{EQ}} . A$ final case, not illustrated here because it is uncummon at these distances in the ruldnight region, is that of the "normal" PAD (a distribution peaking at $\alpha_{0}=50^{\circ}$ ). For this case the decrease of tiux with decreasing pitch angle reinforces the dominant decrease of flux with $R_{\mathrm{EQ}}$, leading to a larger flux level jump at the critical noon pitch angle than for the isotropic distribution.

The magnitude of the discontinuity in mapped $R_{E O}$ af midnight, and consequently the magnitude of the observed flux jump in the noon PAD, depends on the depth of the dayside minimum-B puskets. Just inside the dayside masnetopause the pockets are deepest, and the jump $\delta 1$ at the critical pitch angle is therefore the largest, of any field line in that dayside meridian. This implies alsn the greatest jump $\delta R_{E Q}$ in the mapping of fluxes to midnight. As the point of observation on the dayside moves earthward, the B-pocket depth decreases and so does $\delta \mathrm{l}$ and $\delta \mathrm{R}_{\mathrm{E} \Omega}$. Finally a point will be reached at which the local B-maxima vanish, and the maping of fiuxes to midnight becomes a continuous transformation. Ideally, this progression of measurements would show the largest $\mathrm{f}$. . scontinuity just inside the magnetopause, with a progressive decrease in the flux jump until smootnly-varying disirititions are obtained upon transition to normal field line geometry. More realistically, one would expect the motions of the magnetopause to modulate the drifting particle distributions and produce significant time-variations in the observed flux near the magnetopause, complicating the simple adiabatic scenario. Flovever as long as a significant $\delta$ jump occurs in the squatorial PAD, a corresponding flux jump hould 2 . Jserved. This flux jump is in fact a signatur: of field lines which contain local B-maxima, sirce no other at batic process in the magnetosphere can produce it.

\section{Energetic Electron Fluxes in the Outer Dayside Magnetosphere: Experimental Measurements and Adiabatic Theory Interpretation}

Shell sritting and branching phenomena produce significant modifications to the adiabatic trajectories of azimuthally-drifting energetic electron populations. Shell-splitting effects have been seen ir: pitch angle distributions at all azimuths and over an extended radial range in the outer magnetosphere; these effects have been extensively studied and documented. Shell branching is restricted to azimuths within about $60^{\circ}$ of noon, and within a limited radial range in the vicinity of the magnetopause. Only highlyresolved directional flux measuremerts are capable of detecting the characteristic angular flux features which mark a field line that intersects both branched and unbranched drift shells. In this section we discuss the omnidirectional flux observations published to date which bear on the presence of shell branching and minimum-B pockets in the magnetosphere. We then present high resolution PADs gathered by the E6 electron spectrometer experiment on the OGO-5 satellite over a range of azimuths in the outer dayside magnetosphere. These PADs show structure that agrees well with our theoretical predictions of adiabatic particle motion on branched field lines. 


\section{Experimental Evidence for High-Latitude Minimum-B Pockets: Omnidirectional Energetic Electron Flux Measurements}

In the last section, we saw that particle drift shells in the outer magnetosphere can be classified as stable-trapping or psuedo-trapping shells, depending on whether particles on the shells can complete a drift period without being scattered by abrupt changes in the (static) field line configuration encountered along the shell. From an experimental standpoint, stably-trapped regions are characterized by high flux levels of trapped energetic ( $E>40 \mathrm{keV}$ ) electrons, which exhibit small temporal variations. By contrast the fluxes in the psuedo-trapped regions are low and highly-variable, as expected for particles whose lifetimes in the magnetosphere are on the order of minutes.

Early measurements by the Soviet Elektron satellites were used to empirically define regions of stably-trapped and psuedo-trapped particles on the basis of the characteristic variability of $\mathrm{E}>100 \mathrm{keV}$ electrons (Vernov et al., 1968). These observations indicated that the stably-trapped zone extended at noon to significantly higher latitudes than predicted by Roederer's analysis of the Mead-Wi!'iams model (Fig. 4-22). They found that particles with mirror points at latitudes of $30^{\circ}-50^{\circ}$ in the outer dayside magnetosphere were stably trapped. This discovery was also consistent with the stable trapping regions derived for the image dipole model of Shabansky (Shabansky and Antonova, 1968; Antonova and Shabansky, 1968). This model possessed dayside high-latitude minimum-B pockets which were topologically connected to the equatorial regions of the magnetosphere near midnight, forming closed, branched drift shells.

Further trapping-boundary evidence came from the IMP-3 satellite energetic ( $\mathrm{E}>\mathbf{2 5 0} \mathrm{keV}$ ) electron detector D1, provided by the University of Chicago group under J. Simpson. Murayama (1971) used these data to discriminate stably-trapped from psuedo-trapped particles, based on detector counting-rate levels observed on orbits near noon. Comparing his regions so derived to the model of Shabansky (Fig. 4-38), he concluded that the minimum-B regions indeed formed part of the high-flux, stably-trapped radiation zone. Antonova and Shabansky (1974) confirmed that the Elektron and IMP-3 observations were consistent with each other and required a high-latitude extension to the stable-trapping region of Roederer to include the noon minimum-B pockets.

Antonova and Nikolayeva (1979) investigated energetic electron fluxes ( $E>40 \mathrm{keV}$ and $E>300 \mathrm{keV}$ ) measured on the Prognoz 3 spacecraft at mid-to-high latitudes on the dayside. They mapped the observed flux levels into the Mead-Fairfield magnetic field model (Mead and Fairfield, 1975), and found that the regions of enhanced fluxes characteristic of stable trapping extended to the high-latitude minimum-B pockets of the Mead-Fairfield field.

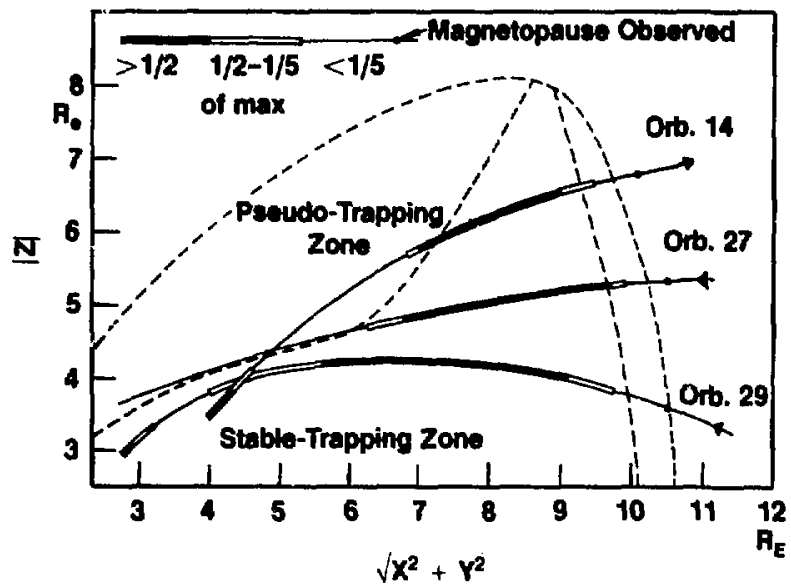

Figure 4-38. Comparison of IMP-3 detector D1 observations of energetic electrons ( $E>250$ keV) along three spacecraft orbits near the noon meridian, with the trapping boundaries derived by Antonova and Shabansky (1974) for the Shabansky image-dipole model (after Murayama, 1971). The highest levels are seen to correlate well with the Shabansky stabletrapping zone. 
Formisano and Domingo (1979) in their study of the polar cusp noted that the trapping-region boundary (as determined by the loss of $\mathrm{E}=0.5-0.8 \mathrm{MeV}$ electrons) fell consistently at a magnetic latitude of $\approx 55^{\circ}$ at noon. This location is poleward of the high-latitude edge of the minimum-B pocket region as computed for the Shabansky image-dipole, Mead-Fairfield, and Choe-Beard models.

Taken together, these observations form convincing evidence for the presence of large fluxes of energetic electrons in regions of the outer dayside magnetosphere which correspond to the location of model minimum-B pockets. It is possible that these regions are segments of closed drift shells in the stable trapping regions, although that has not been conclusively shown. This is all the shell branching information that omnidirectional nux measurements, no matter how extensive, can provide. Because omnidirectional flux measurements are not pitch-angle resolving, they cannot detect the level changes in the directional flux that are associated with B-pocket field lines.

\section{Experimental Evidence for High-Latitude Minimum-B Pockets: Directional Flux Meauurements of Energetic Electrons}

We now show data from several passes of the OGO-5 satellite through the pre- and post-noon sectors of the outer dayside magnetosphere. Features of observed PADs will be compared to model calculations of adiabatic particle drift on branching shells.

These criteria were used in the selection of the passes:

1. Satellite trajectory to be near the magnetic equator, so that nearly all particles mirroring on a field line could be detected;

2. Experiment scan enabled, so that complete PAD information could be obtained;

3. Magnetic activity low, so that dynamic changes in the field configuration, which could cause confounding effects, would be minimized;

4. The set of passes to ccver the azimuthal range of branched shells.

\section{OGO-5 Observation. on Day 66 (March 6), 1968}

We begin with data taken on March 6, 1968 during an inbound pass in the morning sector, at about 10:16 local time (azimuth $\phi_{\mathrm{GSM}}=334^{\circ}$ ), and just above the dipole plane $\left(\lambda_{\mathrm{M}} \approx 5^{\circ}\right)$. The satellite trajectory is depicted in Fig. 4-39 as an equatorial projection in the Choe-Beard model. Figure 4-40 shows the ambient vector magnetic field measured by the UCLA fluxgate magnetometer, Experiment 14, as a function of Universal Time (UT). The three panels give the magnetic field orientation (in dipole inclination and declination coordinates) and magnitude. (Inclination and declination are angles made by the vector magnetic field $\mathbf{B}$ with respect to the magnetic dipole frame of reference. Inclination at a point $\mathbf{P}$ is the angle between $B$ and the plane normal to the geocentric radius (the local horizontal). Declination is the angle between $B$ and the dipole meridional plane at $\mathbf{P})$. The dipole inclination of a dipole line at the spacecraft is plotted as the solid reference line in the top panel for comparison to the observed values. The declination of a dipole line is always zero.

The geophysical coordinates of the spacecraft are given in the columns under the abscissa, for each of the major time annotations. The most important coordinates are $\mathrm{R}$ (geocentric distance), $\lambda_{M}$ (dipole latitude), and the GSM angular coordinates $\phi_{\mathrm{GSM}}$ (azimuth) and $\lambda_{\mathrm{GSM}}$ (latitude). (The GSM system was defined in Fig.4-1.) At the beginning of the interval shown, the spacecraft was in the magnetosheath region of compressed, heated solar wind plasma between the bow shock and the magnetopause. Field magnitude was $\approx 25$ nanotesla (nT) and fluctuating. The declination varied considerably as the spacecraft neared the magnetopause, showing finally a transition to $\approx 0^{\circ}$ when the spacecraft crossed the magnetopause at $\approx 2216 \mathrm{UT}$, at $\mathrm{R}=12.13 \mathrm{R}_{\mathrm{E}}, \phi_{\mathrm{GSM}}=333^{\circ}, \lambda_{\mathrm{CSM}}=11^{\circ}, \lambda_{\mathrm{M}}=7.6^{\circ}$. Dipole tilt was $-5^{\circ}$. Inside the magnetosphere the field declination and inclination were closely dipolar, and the magnitude varied smoothly and slowly along the trajectory.

Figure 4-41 gives the counting rate of the lowest-energy electron channel El (E $=79 \pm 23 \mathrm{keV}$ ). Note that the magnetopause crossing as determined by the magnetometer closely coincided with a large particie flux increase as the satellite moved into the stable particle-trapping regions of the magnetosphere. What appears as a high-frequency oscillation in the counting rate is the result of the constant angular scan of the spectrometer; it generally registers a particle flux maximum at a local magnetic pitch-angle $\alpha$ of $90^{\circ}$ and a minimum near $\alpha=0^{\circ}$ and $180^{\circ}$ (inside the pitch-angle bounce loss cone). The circles plotted two decades 


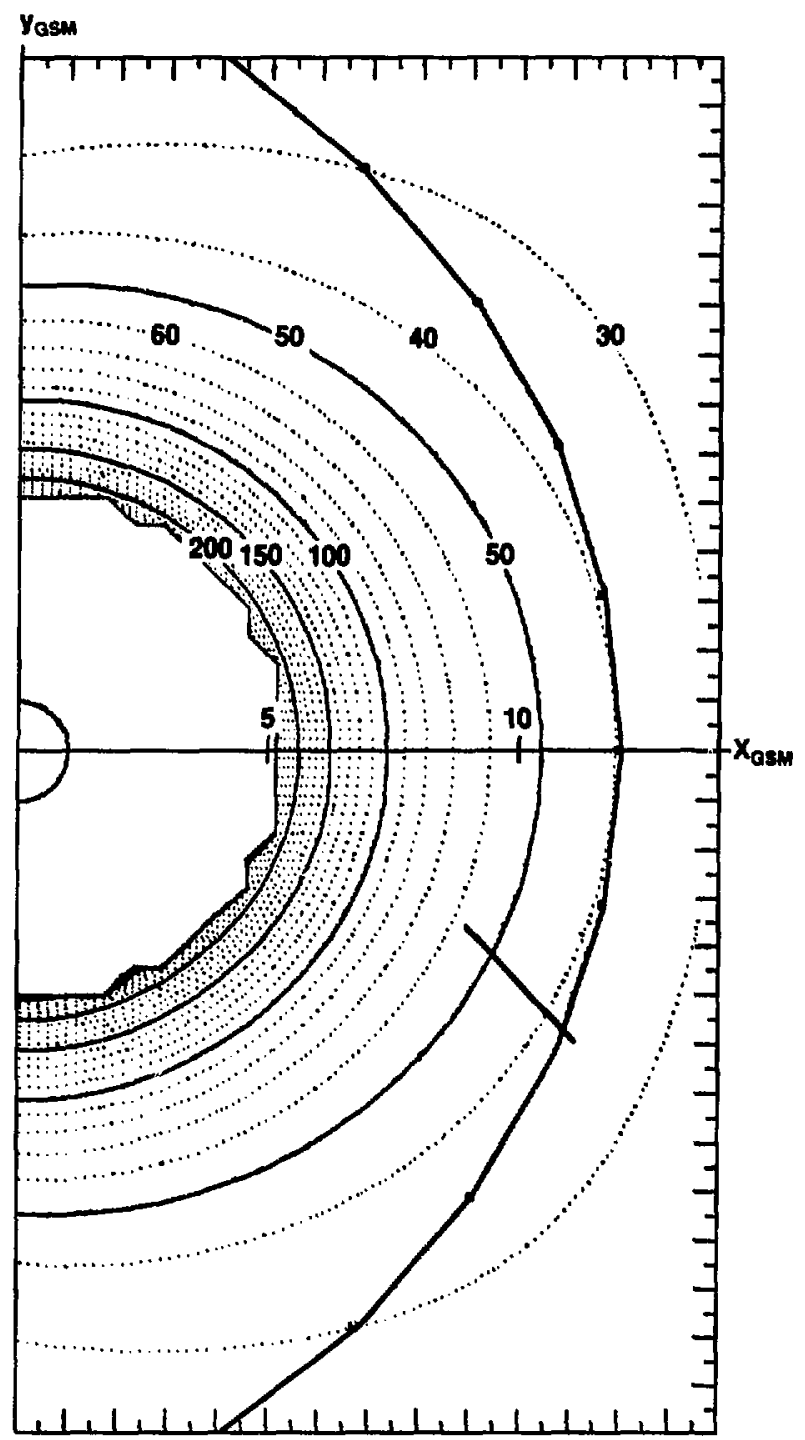

Figure 4-39. Contours of constant magnetic field magnitude (nanoteslas) in the equatorial plane for the Choe-Beard model, appropriate to the OGO-5 spacecraft pass of Day 66, 1968. The spacecraft trajectory appears as a short heavy line at about $27^{\circ}$ before noon $\left(+X_{G S M}\right)$. (Contours outside magnetosphere are not valid).

below the E1 flux represent the counting rate of background detector EB1. By correlating the pointing direction of the instrument with the vector B-field components provided by the UCLA magnetometer, each scan can be transformed into a pitch-angle distribution (PAD) giving directional flux i vs magnetic pitch (aspect) angle $\alpha$. 


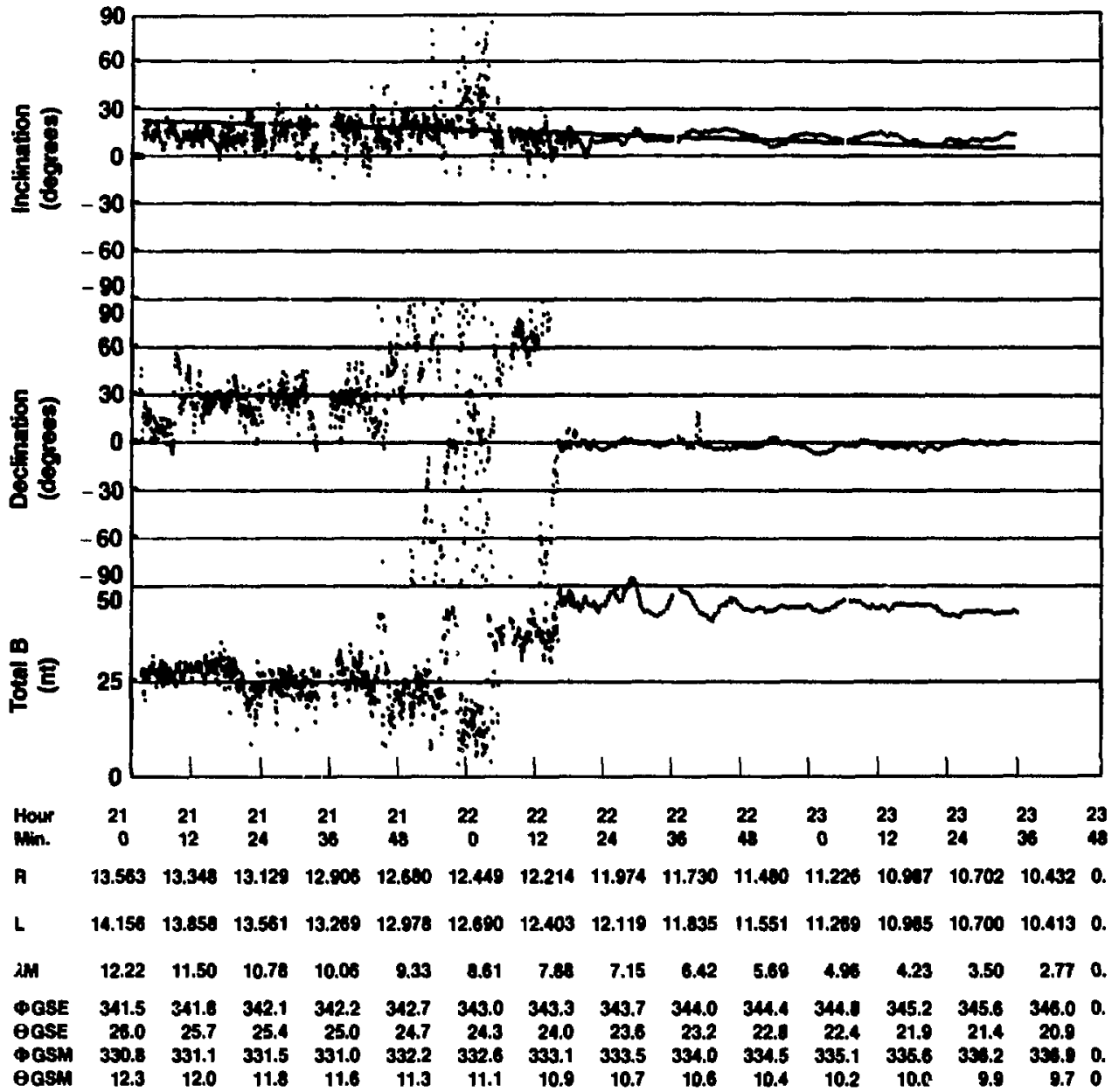

Figure 4-40. Magnetic field observed by the UCLA magnetometer experiment during the OGO-5 inbound pass of Day 66, 1968. X-axis is Universal Time. Upper panela give the inclination and declination of the vector magnetic field, with reference to the dipole coordinate system. Lower panel is field magnitude in nanoteslas. Satellite coordinates are given below each time annotation. Magnetopause crossing occurred at approximately $2216 \mathrm{UT}, \mathbf{R} \approx 12.13 \mathrm{R}_{\mathrm{E}}$. 


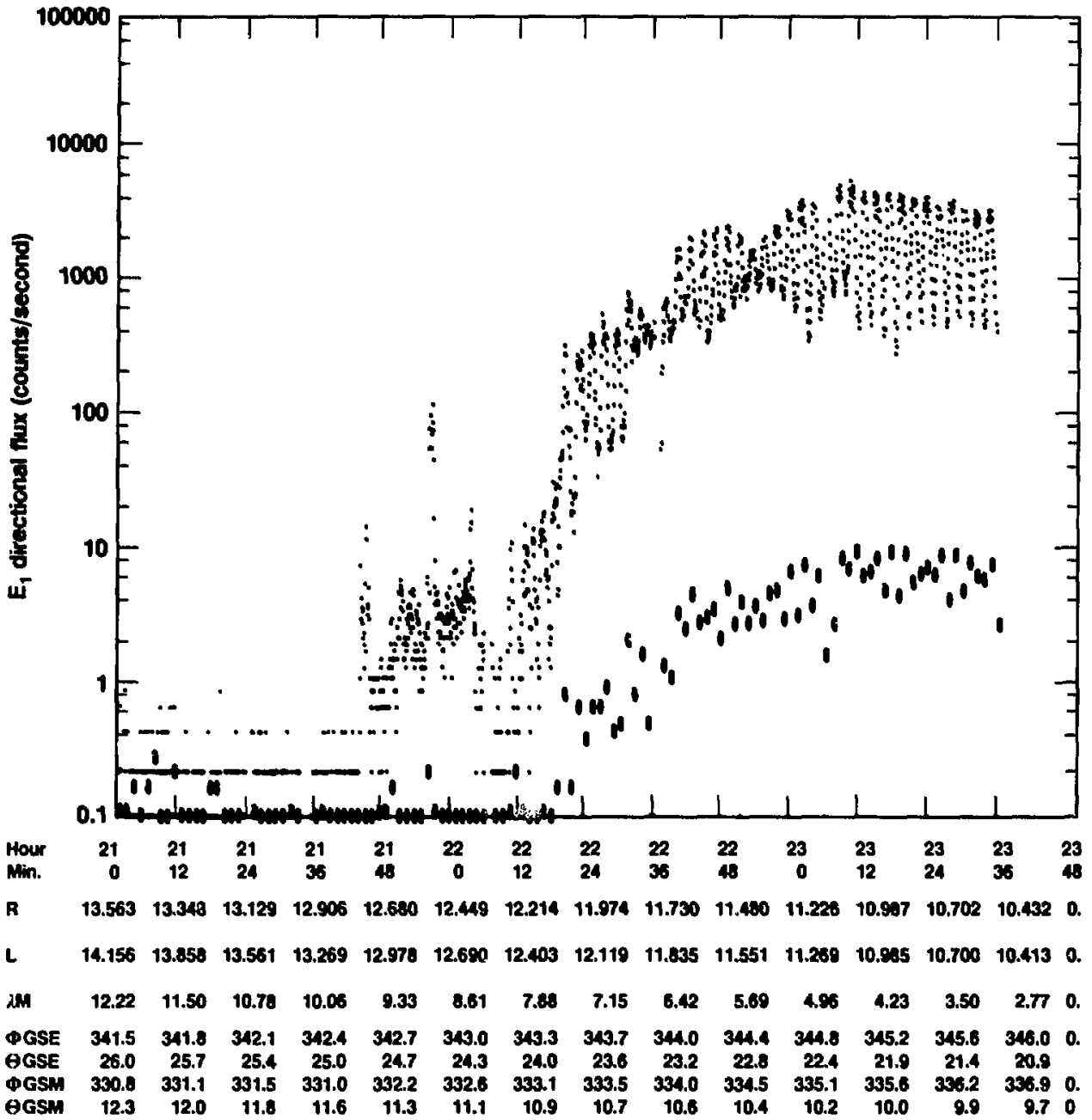

Figure 4-41. Electron counting rate (dots) in the lowest-energy electron channel E1 (79 keV) observed during the OGO-5 inbound pass of Day 66, 1968. The background channel (EB1) counting rate is plotted as zeroes. The oscillation seen in counting rate is due to the scanning of the spectrometer with respect to the spacecraft z-axis (earth-dirested). By correlating the pointing direction of the instrument with the vector B-field components provided by the UCLA magnetometer, each scan can be transformed into a pitch-angle distribution which gives directional flux $\mathbf{j}$ vs magnetic pitch angle a. Abscissa and annotations are the same as in Fig. 4-40. The transition into the magnetosphere occured at about 2220 UT. It is marked by a step increase in flux levels and the appearance of the double-loss-cone pitch-angle distribution. 


\section{Day 66 Field Modeling}

We turn now to modeling the magnetic field configuration appropriate to this interval. Figure 4-42 depicts the outer field lines in the spacecraft meridian $\left(\phi_{\mathrm{CSM}}=334^{\circ}\right)$ for the Choe-Beard model. We have chosen model parameters to match the observed dipole tilt $\left(\delta=-5^{\circ}\right)$ and magnetopause standoff distance $\left(R_{\text {SO }}=11.23 R_{E}\right)$. Analysis of the magnetic field effects on particle PADs measured at the satellite begins with a study of the $B$ vs $\theta$ plot of the lines along the satellite orbit, Fig. 4-43. We note that the outermost lines show high-latitude minimum-B regions and intervening local B-maxima, which owing to dipole tilt are not symmetric about the GSM equator. The minimum-B regions just inside the magnetopause at about $12.0 R_{E}$ have the lowest field values. The depth of these minimum-B pockets decreases as

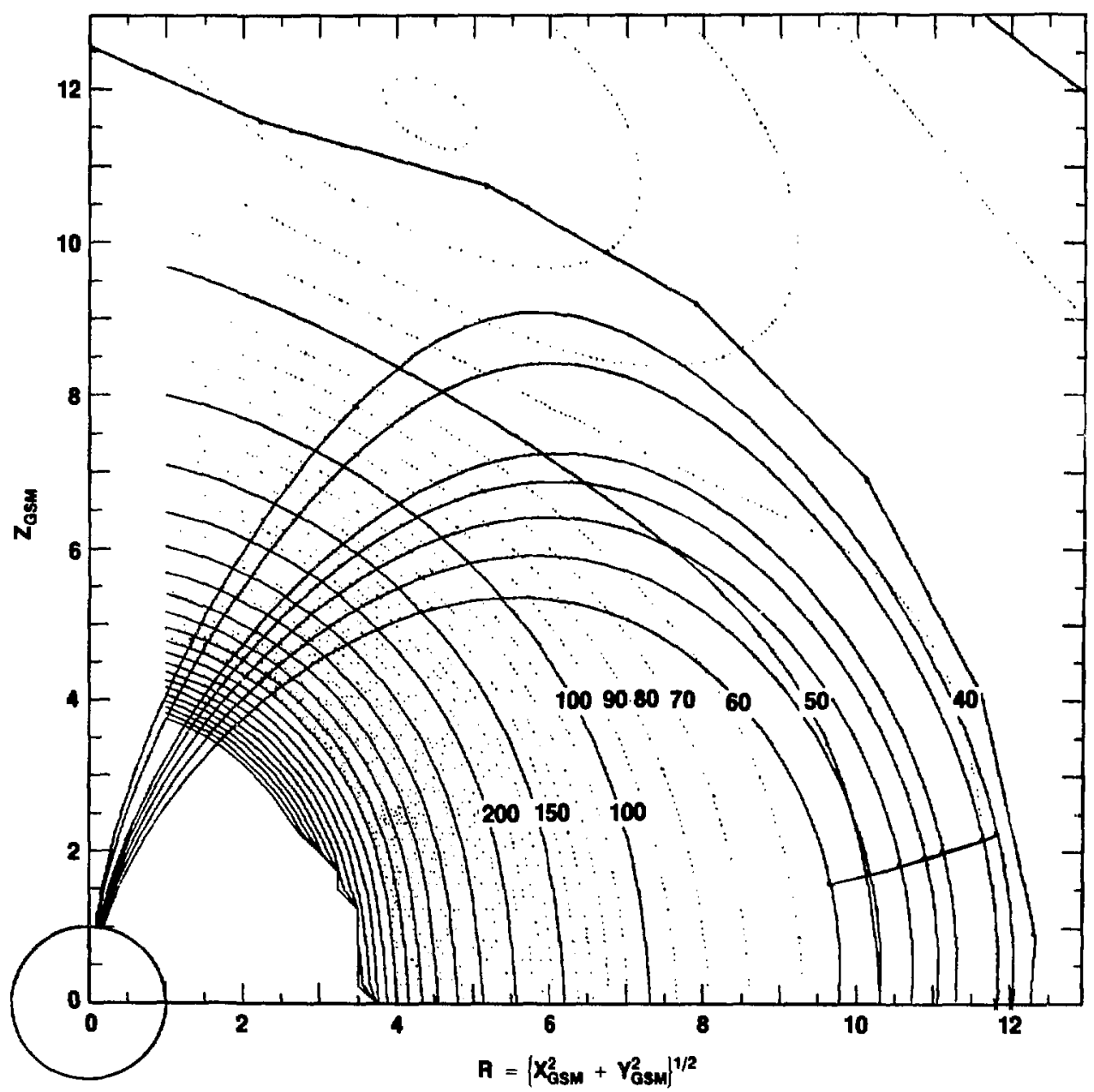

Figure 4-42. Outer magnetospheric field lines and constant-B contours in the azimuth $\phi_{\mathrm{GsM}}=334^{\circ}$ for the Choe-Beard model, appropriate to the inbound pass of Day 66, 1968. The satellite trajectory appears as the short nearly-radial trace. (Contours outside magnetosphere are not valid). 


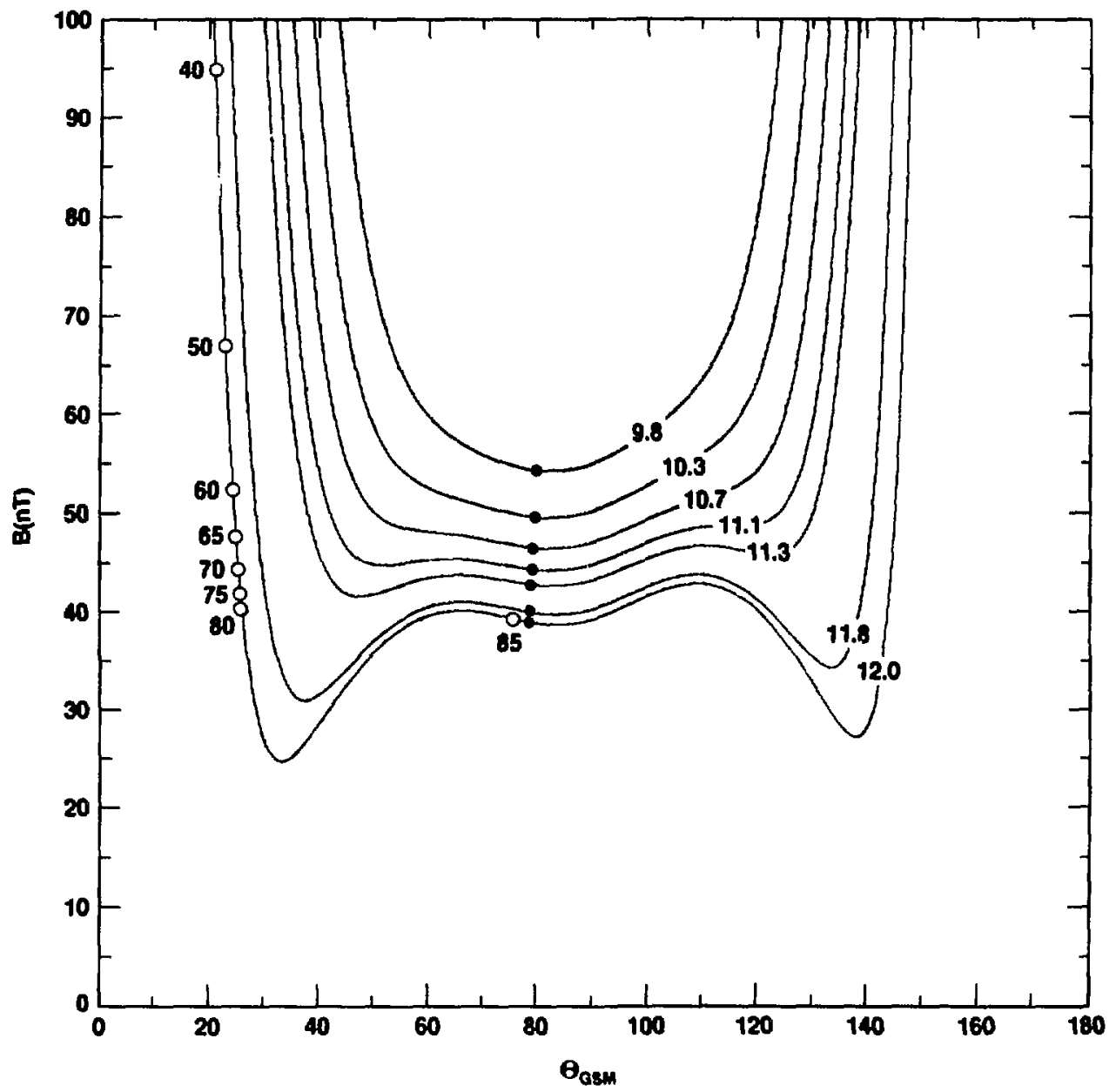

Figure 4-43. Magnetic field magnitude vs GSM colatitude $\theta_{\text {GSM }}$ as computed from the Choe-Beard model along outer magnetospheric lines which intersect the spacecraft trajectory on Day 66, 1963. (The equator is at $\theta_{\mathrm{CSM}}=90 \%$. The spacecraft position is shown as a dot on each line. Lines are labelled on the right with the radial distance of the spacecraft. Numbers along the outermost line denote the spacecraft pitch-angles of particles which mirror at these line locations. High-latitude minimum-B pockets are evident on the outer lines.

the satellite moves earthward, finally vanishing at about $11.0 R_{E}$. Figure 4-44 presents a mirror-point analysis of particles on a B-pocket field line near the magnetopause. It shows several distinct drift shell regions intersecting this single line. We will discuss these regions in terms of the adiabatic drift of particles from the midnight meridian.

Region 0: This region contains particles which move on unbranched shells from midnight. The smallest $B_{M}$ value exceeds the local B-maxima. Both mirror points of every particle lie poleward of the local B-maxima. Their $B_{m}, I$ values map to lines of $R_{E Q}>14.2 R_{E}$ at midnight. At the satellite, their pitch angles lie in the ranges $\alpha_{\mathrm{S}}<73^{\circ}$ and $\alpha_{\mathrm{S}}>107^{\circ}$. 


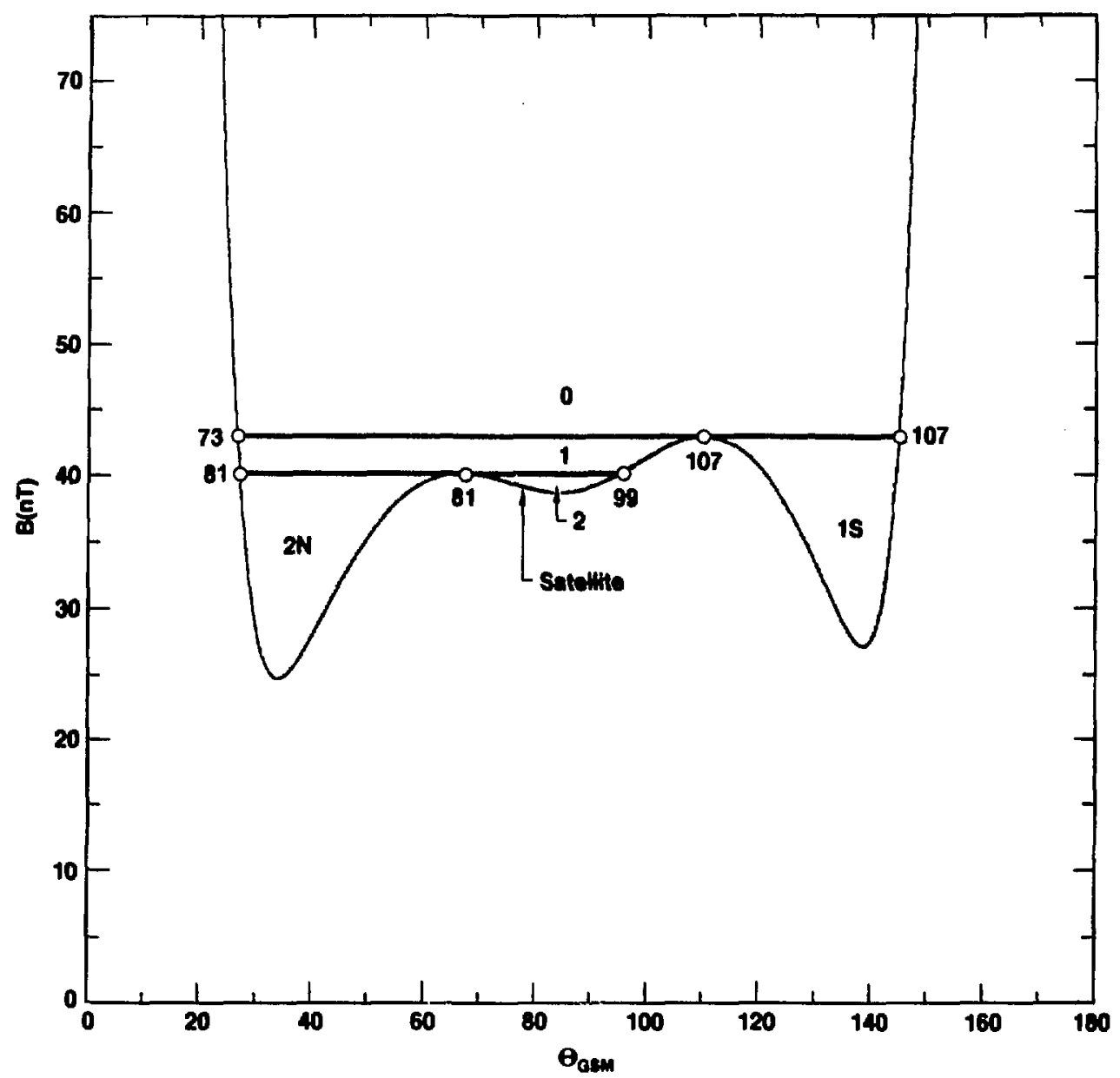

Figure 4-44. Distinct drift shell regions which intersect an outer magnetonpheric field line. Region 0 particles move on unbranched shells from midnight. Region 1 particles move on the equalorial segment of singly-branched shells; Region 1S it the corresponding B-pocket segment. Region 2 contains particles from both unbranched and doubly-branched shell;; Region $2 \mathrm{~N}$ is the corresponding B-pocket segment for doubly-branched shells. Mirror points of particles at the boundaries of the shell regions are labelled with their satellite pitch angles.

Region 1: These particles move on shells which have branched once at some earlier azimuth. One mirror point lies equatorward of the southern $B_{\operatorname{MAX}}$ and one poleward of the (lower) northern $B_{\operatorname{MAX}}$. By use of the branching map of Fig. 4-45 the midnight origins of these particles are found to lie on lines with $11.8 \leq R_{E Q} \leq 14.2 R_{E}$. At the satellite, their pitch angles lie in the ranges $73^{\circ}<\alpha_{\mathrm{S}}<81^{\circ}$ and $99^{\circ}<$ $\boldsymbol{a}_{\mathrm{S}}<107^{\circ}$.

Region 1S: These particles drift on branched shells formed by the same process that produced the Region-1 trajectories. They have taken the southern high-latitude segment of the branched shell into the 


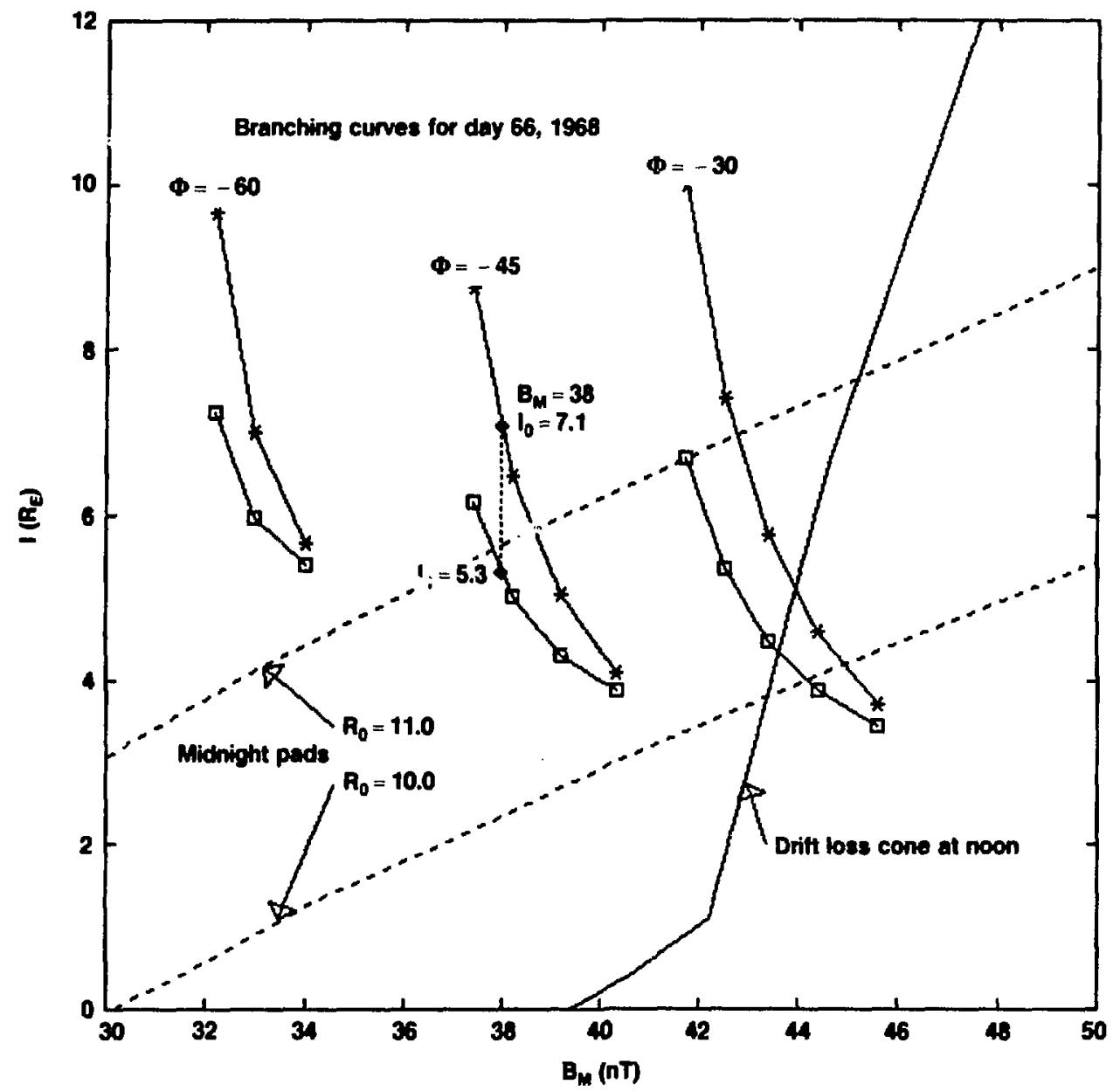

Figure 4-45. Branching map for the Choe-Beard model of the field for Day 66, 1968. At each of three azimuths, values of $B_{M}, 1$ are plotted (symbol ") for which shell branching occurs. The lower curves (symbol $\square$ ) represent the $B_{M}$,I value of the equatorial segment following branching. For example, a shell characterjzed by $B_{M}=38 \mathrm{nT}, I=I_{0}=7.1 R_{E}$ will branch at an azimuth of $-45^{\circ}$. The branched equatorial segment emerging sunwards from this azimuth has $B_{M}=38 \mathrm{nT}, I=I_{1}=5.3 R_{E^{*}}$ Also shown is the drift loss cone defined by the outermost noon line, and the traces of two PADs from the midnight meridian for which branching will occur.

B-pocket region. They pass through noon on trajectories that do not cross the equator (and are not seen by the near-equator satellite).

Region 2: This region contains two particle populations. One moves on twice-branched shells produced when a shell previously branched at a southern $B_{\text {MAX }}$ becomes branched again by a northern $B_{\text {MAX. }}$ That is to say, particles which at some prior azimuth were Region 1 type particles encounter a northern $B_{\text {MAX }}$ such that $B_{\text {MAX }}>B_{M}$ and they branch equatorward. The second population on this line consists of 
particles which in the midnight meridian had small values of 1 and have drifted on unbranched shells to this azimuth. Mirror points of these particles lie equatorward of the local B-maxima.

The first population can be traced back to the midnight meridian by reference to the branching map of Fig. 4-46 constructed to illustrate the more complex calculations needed for the double-branching case. The curves labeled with 0 are branching curves constructed in the azimuths of $-60^{\circ},-45^{\circ}$, and $-30^{\circ}$. A Region 0 drift shell will branch at an azimuth whose branching curve contains the shell's $B_{M}, I$ point. Below each of these curves is plotted the Region 1 I-value for the equatorial segment of the branched Region 1 shell. Also shown is the Region 1 branching surve in the azimuth of $-27^{\circ}$ (the spacecraft Day 66 azimuth), and below that the Region 2 l-value resulting from the Region 1 branching. We can now track a particle from its measured local pitch angle in the azimuth of $-27^{\circ}$ back to its mirror location at midnight.

We choose a particle which has a pitch angle $\alpha_{5} \approx 81^{\circ}$ at the satellite. (This particle has a mirror point at the northern local $B_{M A X}$, moving on a shell that has just branched,) The Choe-Beard model yields invariant values of $B_{M}=40 n T, I \approx .75 R_{E}$. This point is plotted as the dot on the intersection of the line $B_{M}=40 \mathrm{nT}$ with the $\phi=-27^{\circ}$ Region 2 branching curve. We find its pre-branch I-value by following the $B_{M}=40 \mathrm{nT}$ line up to its intersection with the two Region 1 branching curves, one drawn for $\phi=-27^{\circ}$ and one for $\phi=-45^{\circ}$. At this point we read a Region 1 l-value of $\approx 4.0 R_{E}$. Continuing up the $B_{M}=40 \mathrm{nT}$ line to its intersection with the Region 0 branching curve, we read an unbranched value of $\mathrm{I}_{0}=4.4 \mathrm{R}_{\mathrm{E}}$. This is the initial value of the shell in the midnight meridian.

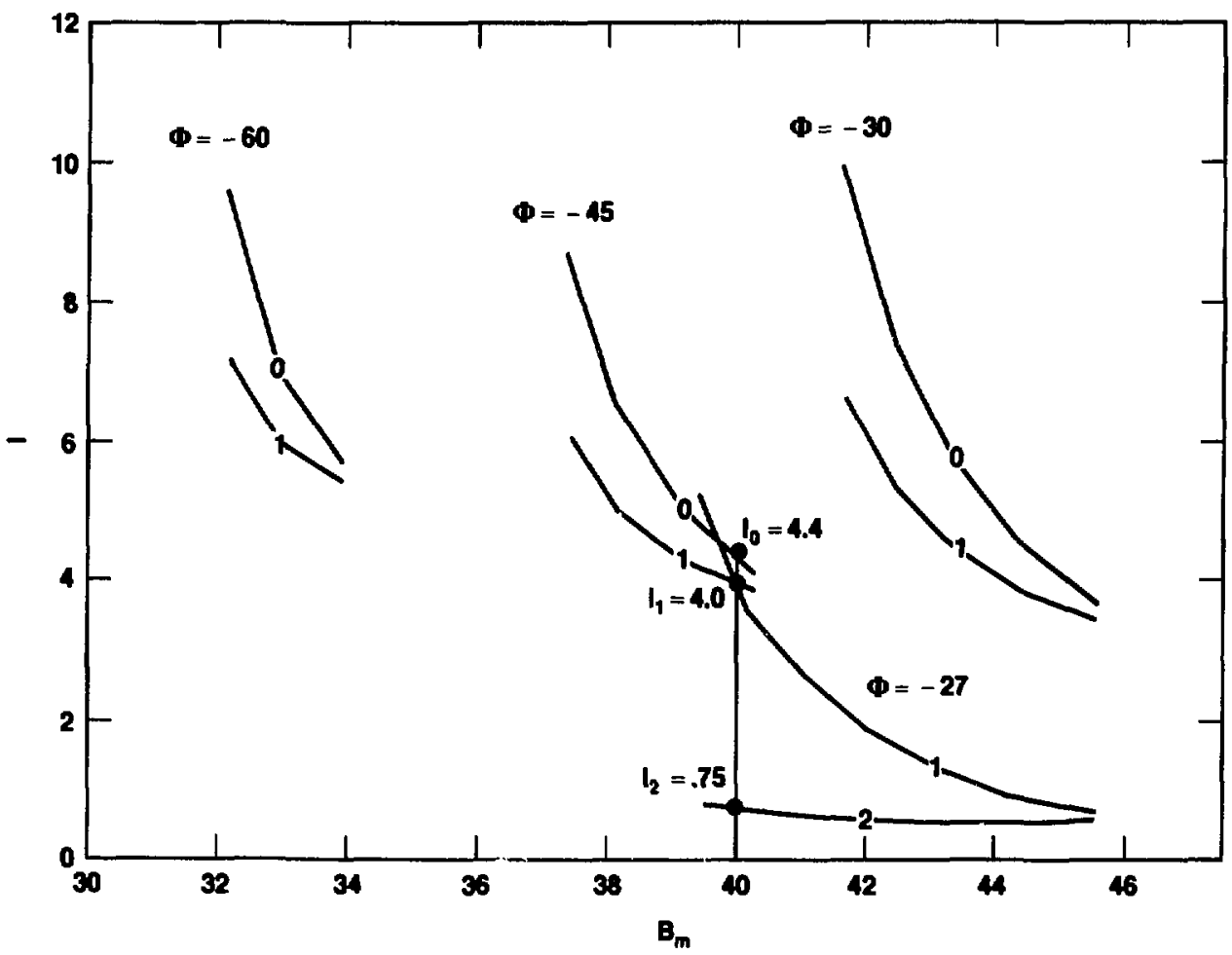

Figure 4-46. Branching map for the Choe-Beard model of the field for Day 66, 1968. The process of double-branching of drift shells is illustrated. See text for explanation. 
This process will seem perhaps clearer if we trace the shell around from midnight. We start in the midnight meridian with a shell of $B_{M}=40 \mathrm{nT}, l_{0}=4.4 R_{E}$. Tracing this shell towards noon, it encounters at $\phi=-45^{\circ}$ a southem local $B_{\text {MAX }}$ which equals the particle's $B_{M}$. The shell splits into a B-pocket segment (Region 1S) and an equatorial segment (Region 1). If the particle becomes trapped into the B-pocket region, it drifts past noon in the (unobserved) Region 1S. If the particle instead branches into the equatorial segment, its I-value is reduced to $I_{1}=4.0 R_{E}$. It continues its drift in azimuth towards noon with one mirror point poleward of the northern local $B_{M A X}$ and one equatorward of the southern local $B_{\text {MAX }}$. until it encounters a line (at $\phi=-27^{\circ}$ ) on which the northern local $B_{M \wedge x}$ equals $B_{M}$. The shell branches again, into a B-pocket segment (Region $2 \mathrm{~N}$ ) and the equatorial segment Region 2, where its I-value is reduced to $\mathrm{I}_{2}=0.75 \mathrm{R}_{\mathrm{E}}$. This particle is observed at the satellite at $\alpha_{S}=81^{\circ}$. By searching in the midnight meridian for the shell $B_{M}=40 n T, I_{0}=4.4 R_{E}$, we locate its equatorial crossing at $R_{E Q} \approx 12 R_{E}$.

The second (unbranched) particle population is found to map back to lines of $R_{E Q} \approx 9.5 R_{E}$ at midnight. Region 2 particles are detected at the satellite in the pitch angle range $81^{\circ}<\alpha_{\mathrm{S}}<99^{\circ}$.

Region 2N: These particles drift on shells which have twice branched, like the first particle population of Region 2. Their bounce motion is confined to the northern high-latitude B-pocket; consequently these particles are not observed at the satellite.

\section{Day 66 Synthetic Pitch Angle Distributions}

Since we have now identified the midnight origins of all shells which contribute to the directional flux measurement at the satellite, we can synthesize an electron PAD at the satellite from a model of electron fluxes at midnight, for comparison to observed dayside distributions. We select as our field point the spacecraft location at $2221 \mathrm{UT}: \mathrm{R}=12.0 \mathrm{R}_{\mathrm{E}}, \lambda_{\mathrm{M}}=7.3^{\circ}, \phi_{\mathrm{GSM}}=333^{\circ}$ (but the magnetic field geometry and the resultant synthetic PAD are nearly constant along the spacecraft trajectory from $2221 \mathrm{UT}$ to 2240). We follow the same procedure used in the previous section to construct the noon PADs of Figs. 4-36 and 4-37. For each of several values of $\alpha_{S}$ we have computed the radial distance $R_{E Q}$ to the equatorial crossing of the field line in the midnight meridian to which a particle of that $\alpha_{S}$ will adiabatically map. This mapping of $\alpha_{s}$ at the satellite into field line $R_{E Q}$ at midnight (using the Choe-Beard model) is shown in Fig. 4-47. The discontinuous nature of the mapping is evident in the $R_{E Q}$ gap between Regions 1 and 2. (For Region 2 we have mapped only the unbranched population; the branched population maps farther into the midnight tail where fluxes are lower and hence this population does not contribute significantly to the Region 2 fluxes). Figures 4-48 and 4-49 show the PADs resulting at the satellite from midnight distributions which are isotropic and butterfly-type, respectively. Both PADs show an abrupt flux decrease at the ends of Region 2.

These synthetic adiabatic PADs do not of course take into account the effects of dynamical variations in the field configuration along the drift path, but these effects can be important for particles drifting in the vicinity of tlue magnetopause. Various studies of the magnetopause structure have determined that the magnetopause is in constant motion, with typical velocities of the order of $10-20 \mathrm{~km} / \mathrm{s}$ (Howe and Siscoe, 1972)-much greater than the spacecraft speed of about $1 \mathrm{~km} / \mathrm{s}$. The magnetopause appears to be KelvinHelmholtz unistable (Fairfield, "2979), and supports surface waves which propagate tailward (Kaufmann and Konradi, 1969).

Magnetopause fluctuations can produce two types of effects on drifting particle distributions. Wavedriven local compressions of the field in the equatorial flanks of the outer magnetosphere can produce magnetopause shadowing effects (Fig. 4-50). If the compression occurs at an azimuth between the midnight meridian and the satellite, contours of B (along which equatorially-mirroring particles drift) which formerly conriected the midnight regions with the satellite, are bent towards the intervening point of compression. Near equaiorially-mirroring particles now drift to the magnetopause before reaching the satellite and are lost. While the perturbation lasts, the directional distributions measured at the satellite will show a flux dropout at pitch angles near $\alpha=90^{\circ}$.

Dynamical variations in the Chapman-Ferraro current systems can also modify PADs in the outer magnetosphere by causing the magnitude of the local B-maxima to change. A drop in magnitude will cause branched shells to temporarily rejoin, allowing particles from the high-latitude B-pockets to have access to the equatorial regions, and vice-versa. The effect on near-equatorial PADs depends on the relative magnitudes of the equatorially-mirroring fluxes with respect to those of the B-pocket. If the B-pocket region is relatively low in particle flux, shell-rejoining will result in a loss of flux from the equatorial region. If however the B-pocket region is relatively high in particle flux, a sudden reduction in 


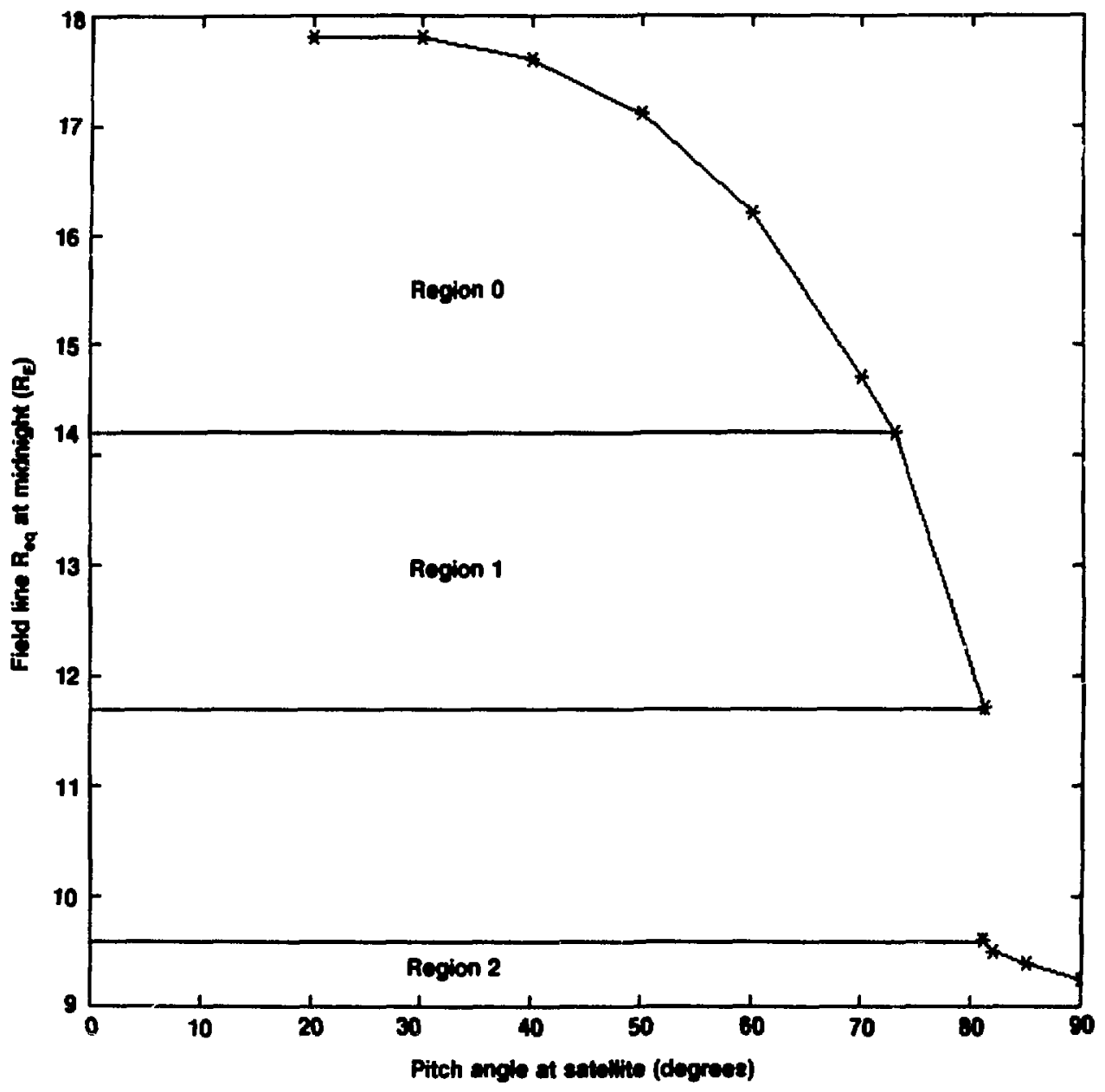

Figure 4-47. Mapping of PAD observed on Day 66, 1968 into the midnight meridian, via the ChoeBeard magnetic field model. The point of PAD observation was $\mathbf{R}=12.06 \mathbf{R}_{\mathrm{E}}, \lambda_{\mathrm{M}}=7.3^{\circ}, \phi_{\mathrm{CSM}}=$ 333․ The presence of local B-maxima on the field line pasing through the point of observation introduces discontinuities into the mapping. 


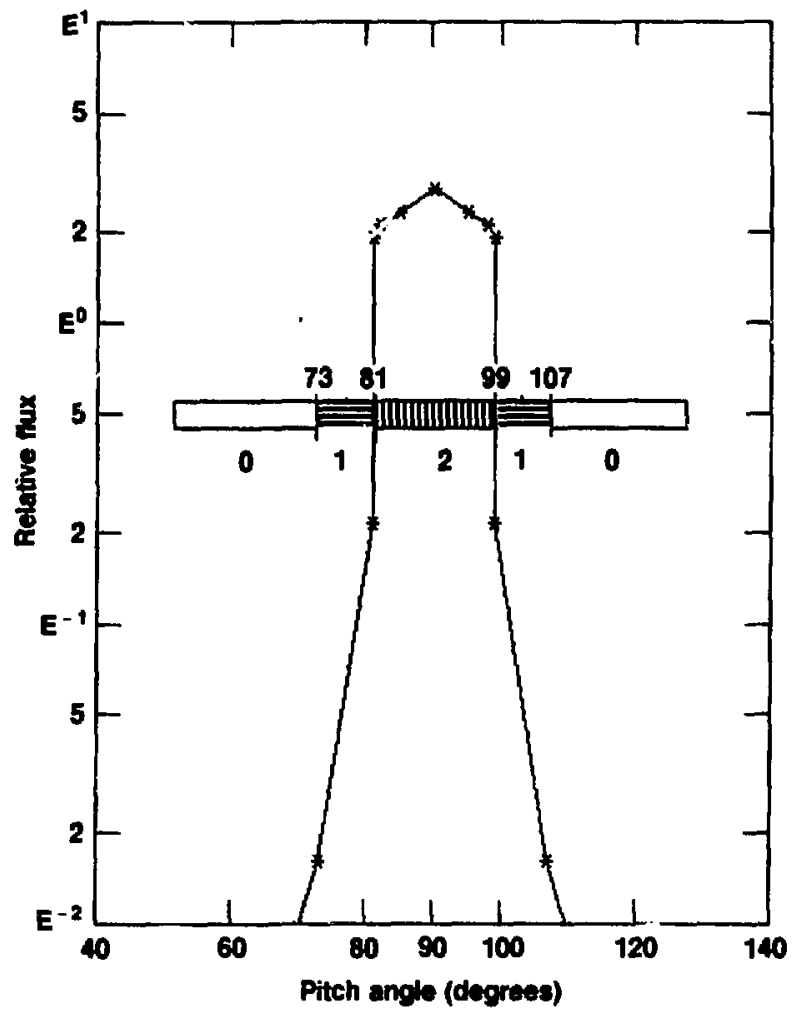

Figure 4-48. Synthetic PAD for Day 66, 1968. It was created by mapping an assumed isotropic distribution at midnight to the satellite point of observation, via the Choe-Beard mode!. It shows abrupt flux transitions at pitch angles corresponding to fluxes which mirror at $B_{M}=$ local B-max, the limits of Regions 0, 1, and 2. 
Figure 4-49. Syntheicic PAD for Day 66, 1968. It was created by mapping an assumed butterfly distribution at midnight to the satellite point of observat s, nn, via the Choe-Beard model. Like the PAD in the previous figure, it shows abrupt flux transitions at pitch angles corresponding to fluxes which mirror at $B_{M}:=10$ cal B-max, the limits of Regions 0,1 , and 2 .

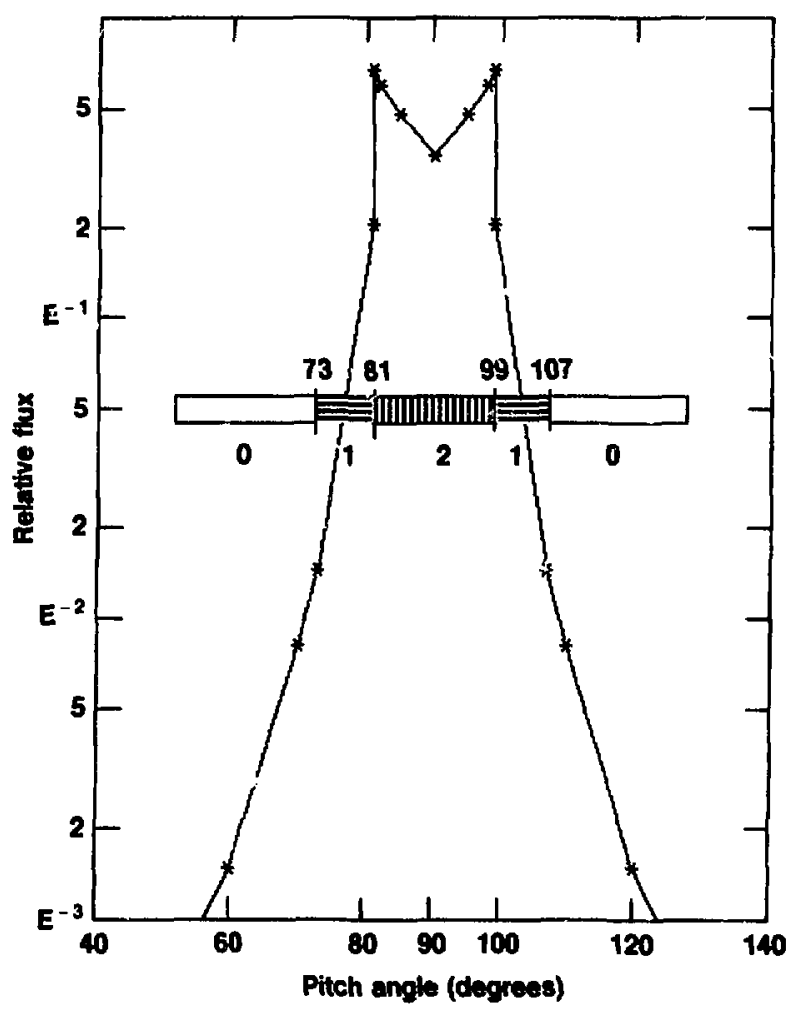




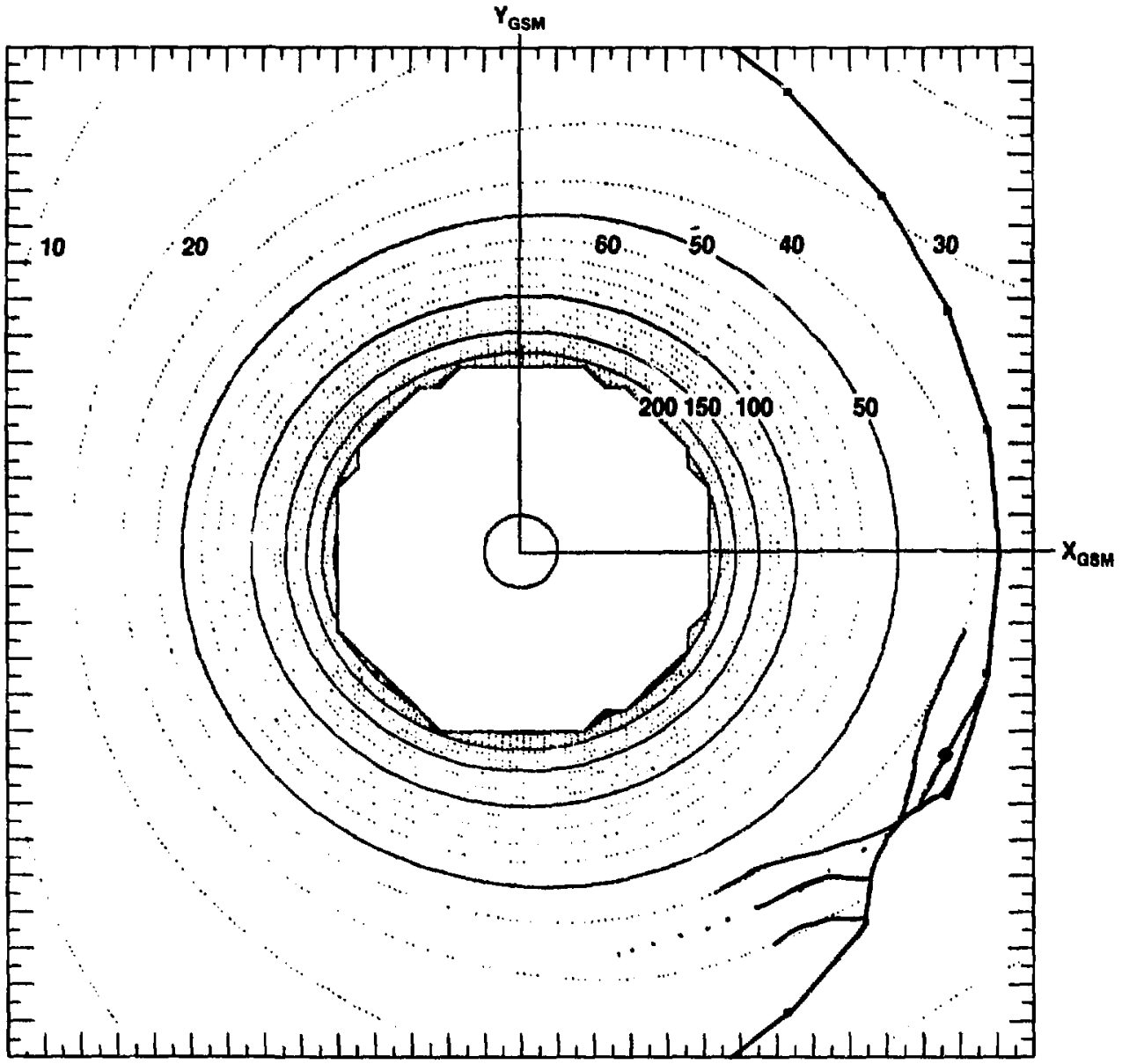

Figure 4-50. Conception of magnetopause shadowing caused by local boundary fluctuations. A section of the magnetopause has moved inwards at an azimuth tailward of the spacecraft (shown as a dot), compressing the outer flux tubes. B-contours which formerly connected the spacecraft to the midnight regions (dotted lines) now terminate at the magnetopause (solid lines), leaving the spacecraft in a temporary particle shadow. 
magnitude of the intervening local $B_{\text {Max }}$ will result in a pulse of particles at the satellite, which will be detected at the local pitch angle corresponding to $B_{M}=B_{M A x}$.

\section{Day 66 Energetic Electron Pitch Angle Distributions}

We now present OGO-5 electron pitch angle distributions measured on Day 66, 1968 in the outer prenoon magnetosphere. Figure 4-51 shows a sequence of pitch angle distributions obtained by the lowest energy electron channel $E 1(E=79 \pm 23 \mathrm{keV})$ as the satellite moved inward from the magnetopause. We have plotted detector counting rate (electrons/sec) vs local magnetic pitch angle $\alpha$. Pitch angles $<70^{\circ}$ were not observed because the oscillating scan mechanism covered a range of only $270^{\circ}$ relative to the spacecraft. The PAD obtained at $R=12.06 R_{E}$ shows an interesting asymmetry in the flux around pitch angle $\alpha=90^{\circ}$. The distribution peaks near $\alpha=99^{\circ}$ at the southern end of Region $2\left(90^{\circ}<\alpha<99^{\circ}\right)$. The distribution decreases rapidly for $\alpha>99^{\circ}$ (Region 1). In Region 2 north of the satellite $\left(81^{\circ}<\alpha<90^{\circ}\right.$ ) the flux decreases rapidly with decreasing pitch angle until the critical pitch angle is reached at the Region end $\left(\alpha=81^{\circ}\right.$ ), where flux levels recover (Region 1). The PAD at $R=12.03 R_{E}$ was acquired a single scan later. The flux gradient in Region 2 has become more strongly positive as Region 2 fluxes mirroring north of the satellite decay further, but flux level transitions still occur near $\alpha=81^{\circ}$ and $99^{\circ}$. By $R=11.95 R_{E}$ the flux levels north of the satellite have recovered, and in fact the flux gradient in Region 2 ras been reversed: the distribution now peaks at $\alpha=81^{\circ}$.

Figure 4-52 displays the channel E2 $(E=158 \pm 36 \mathrm{keV})$ PADs acquired at the same locations as those in Fig. 4-51. The first distribution $\left(R=12.06 R_{E}\right)$ has a maximum at the critical pitch angle $\alpha=91^{\circ}$, and a

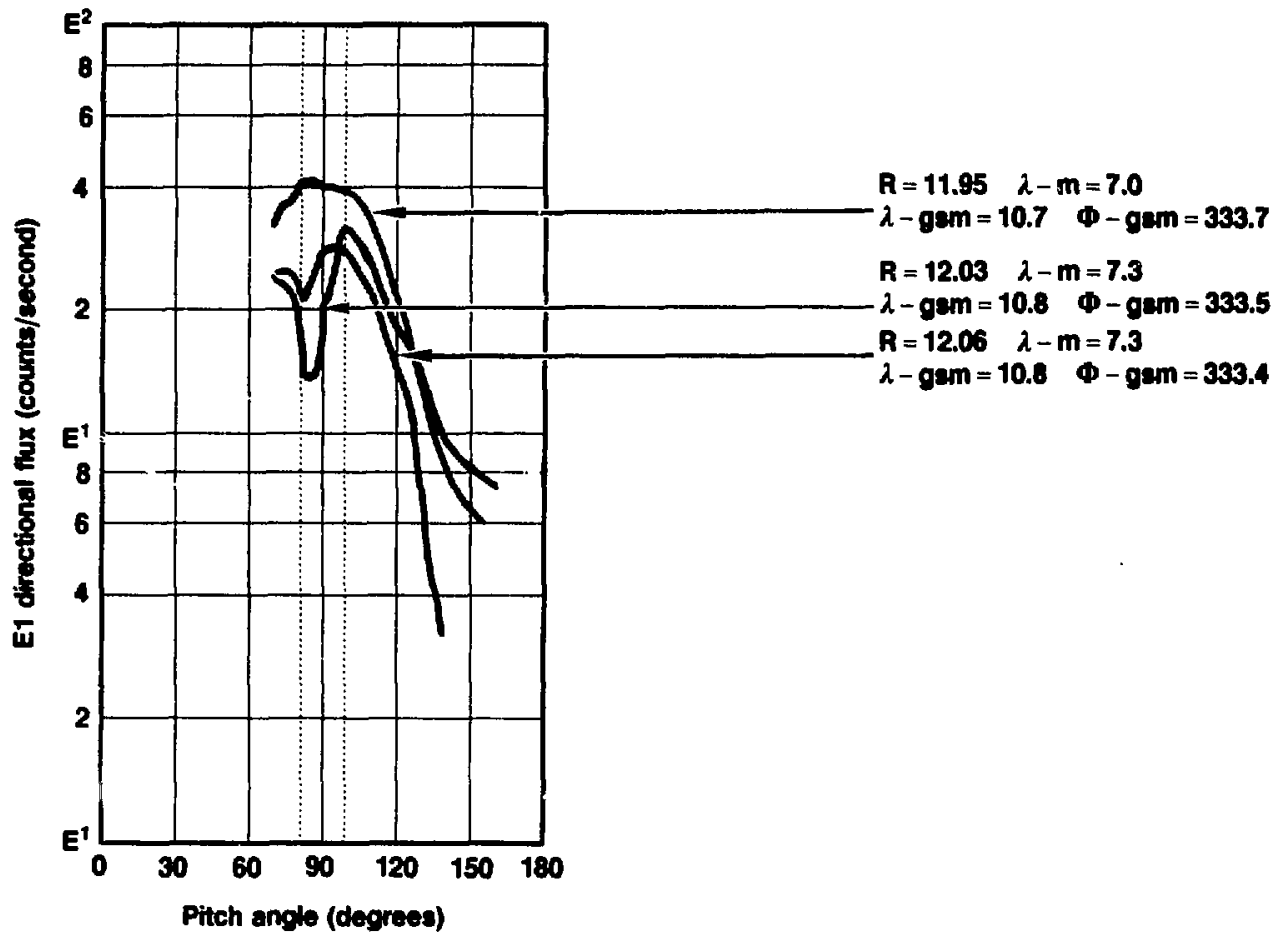

Figure 4-51. Sequence of E1 PADs obtained by OGO-5 on Day 66, 1968, as the satellite moved earthwards from the magnetopause (at $R \approx 12.13 R_{E}$ ). Dotted lines mark the pitch angles $\alpha=81^{\circ}$ and $99^{\circ}$ corresponding to fluxes mirroring at $B_{M}=$ northern local $B$-max. 
positive flux gradient in Region 2. The steepening of the gradient at $R=12.03 R_{E}$ and the flux recovery and gradient reversal at $R=11.95 R_{E}$ mirror the variations seen in channel $E 1$, but the effects are more pronounced here, perhaps because average flux levels are lower.

E1 PADs acquired further along the trajectory are seen in Fig. 4-53. Flux levels increased as the satellite moved deeper into the magnetosphere. The PSD at $R=11.71 R_{E}$ shows a maximum near $\alpha=81^{\circ}$ and a negative flux gradient in Region 2. In the rext sample shown at $R=11.35 R_{E}$ the flux gradient has reversed again; the flux at $\alpha=81^{\circ}$ is now a local minimum and the distribution peaks at $\alpha=99^{\circ}$. The satellite subsequently passed beyond the region of branching drift shells and into the magnetospheric core, where the PADs attained the typical "normal" shape seen at $R=10.99 R_{E}$.

The E2 PADs obtained at the same locations showed similar but more pronounced features (Fig. 4-54). The energy-dependency of fluxes in the outer regions is evident by comparing Figs. 4-53 and 4-54. Whereas E1 fluxes are seen to increase at all pitch angles as the satellite moved earthward, E2 fluxes in Region 2 north of the satellite decreased from $R=11.71 R_{E}$ to $R=11.35 R_{E}$.

During this latter period, fluxes in channels $E 3(E=266 \pm 36 \mathrm{keV})$ and $E 4(E=479 \pm 52 \mathrm{keV})$ rose to significant levels; their PADs are presented in Figs. 4-55 and 4-56 respectively. These distributions show similar transitions at Region edges, with even more pronounced level changes.

\section{OGO-5 Observations on Day 41 (February 10), 1969}

$W_{2}$ next show data acquired on an inbound pass of the satellite through the outer noon equatorial magnetosphere $\left(\lambda_{\mathrm{GSM}} \approx 2^{\circ}, \phi_{\mathrm{CSM}} \approx 7^{\circ}\right)$. Dipole tilt was small $\left(\approx-3^{\circ}\right)$. Figure $4-57$ depicts the satellite trajectory in the equatorial plane of the Choe-Beard model magnetosphere. The magnetometer record for

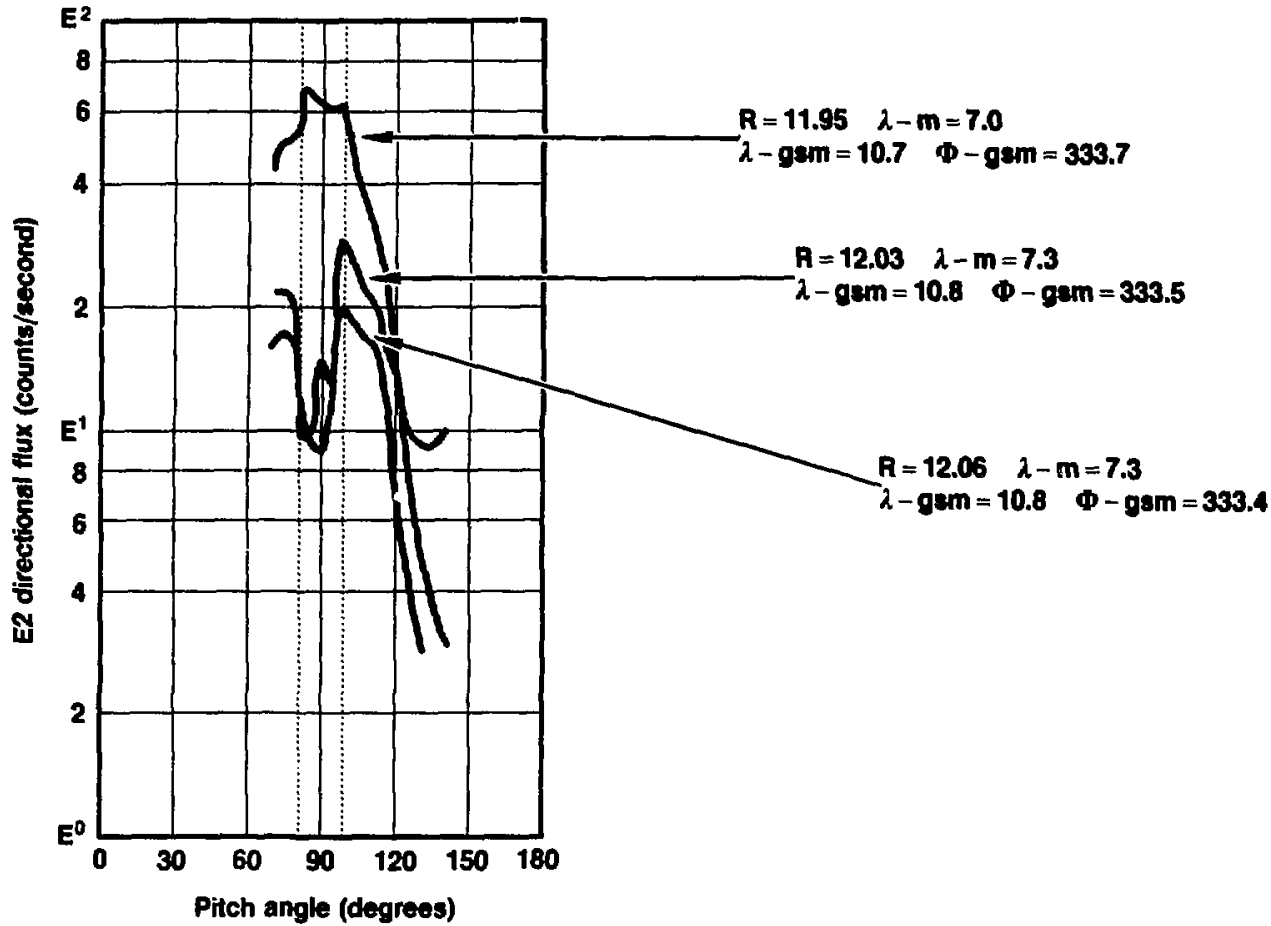

Figure 4-52. Sequence of E2 PADs obtained by OGO-5 on Day 66, 1968, at the same locations as in Fig. 4-51. 


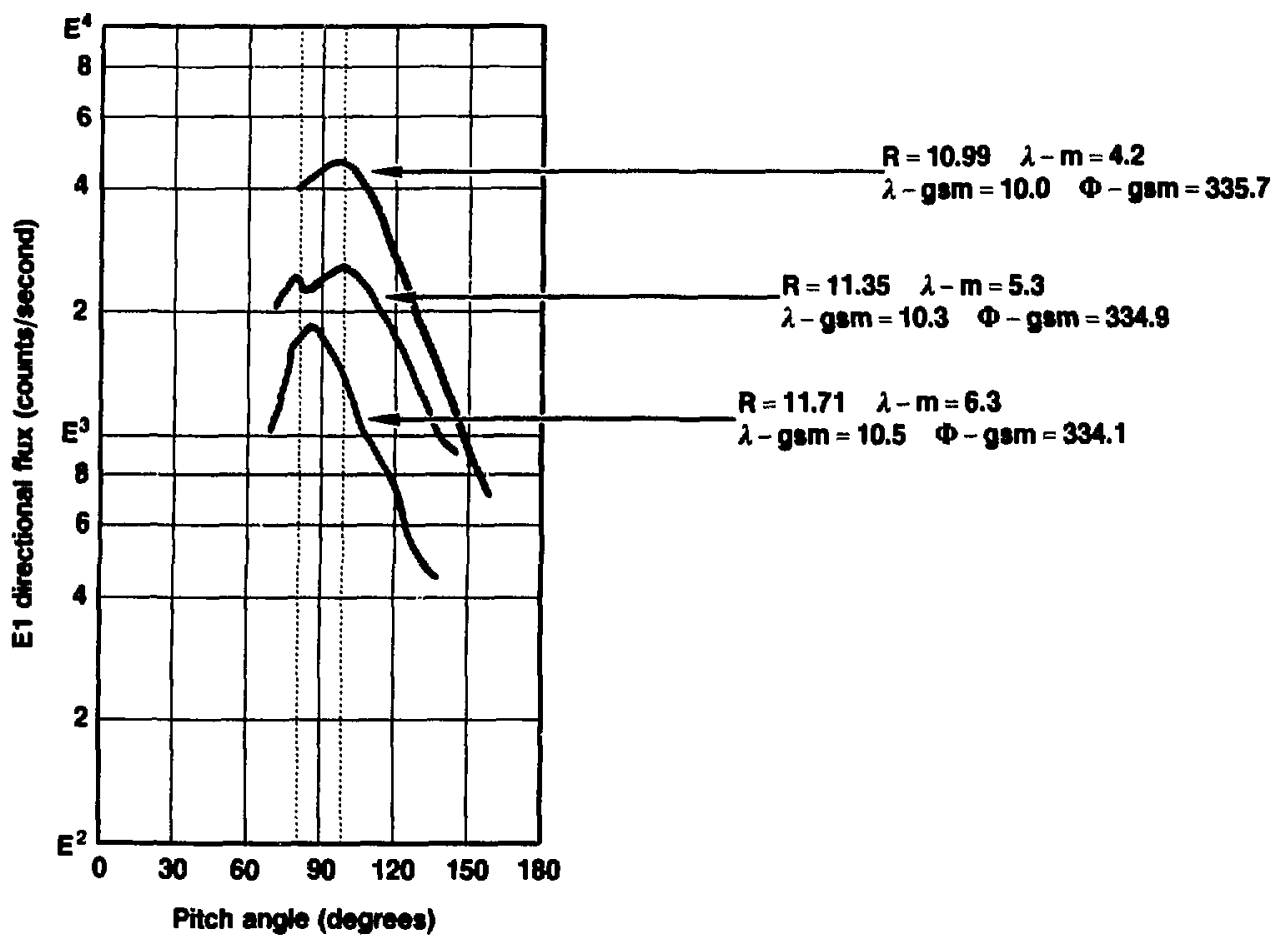

Figure 4-53. Sequence of E1 PADs acquired further along intound orbit, Day 66, 1968. 


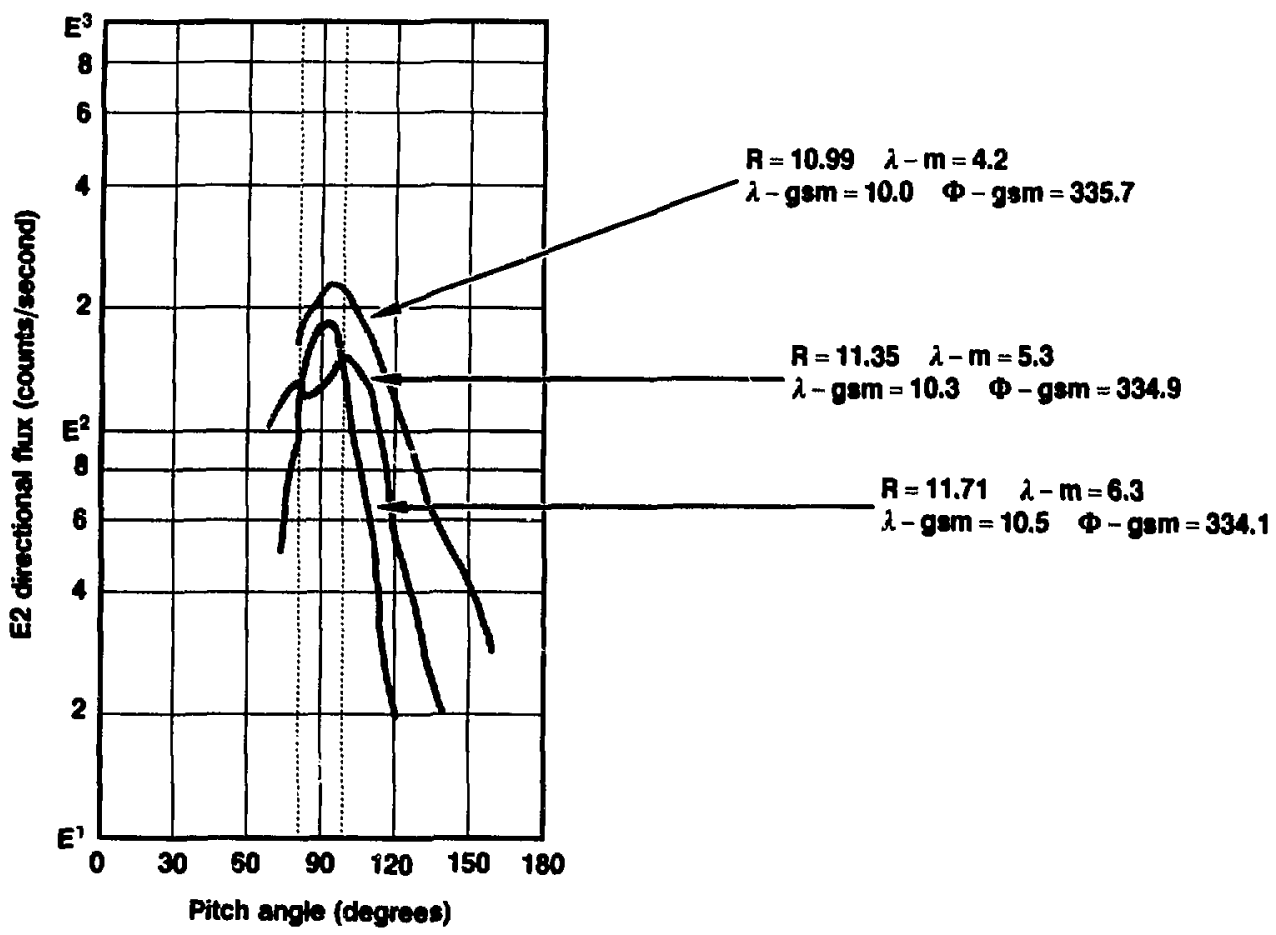

Figure 4-54. Sequence of E2 PADs obtained by OGO-5 on Day 66, 1968, at the same locations as in Fig. 4-53. 


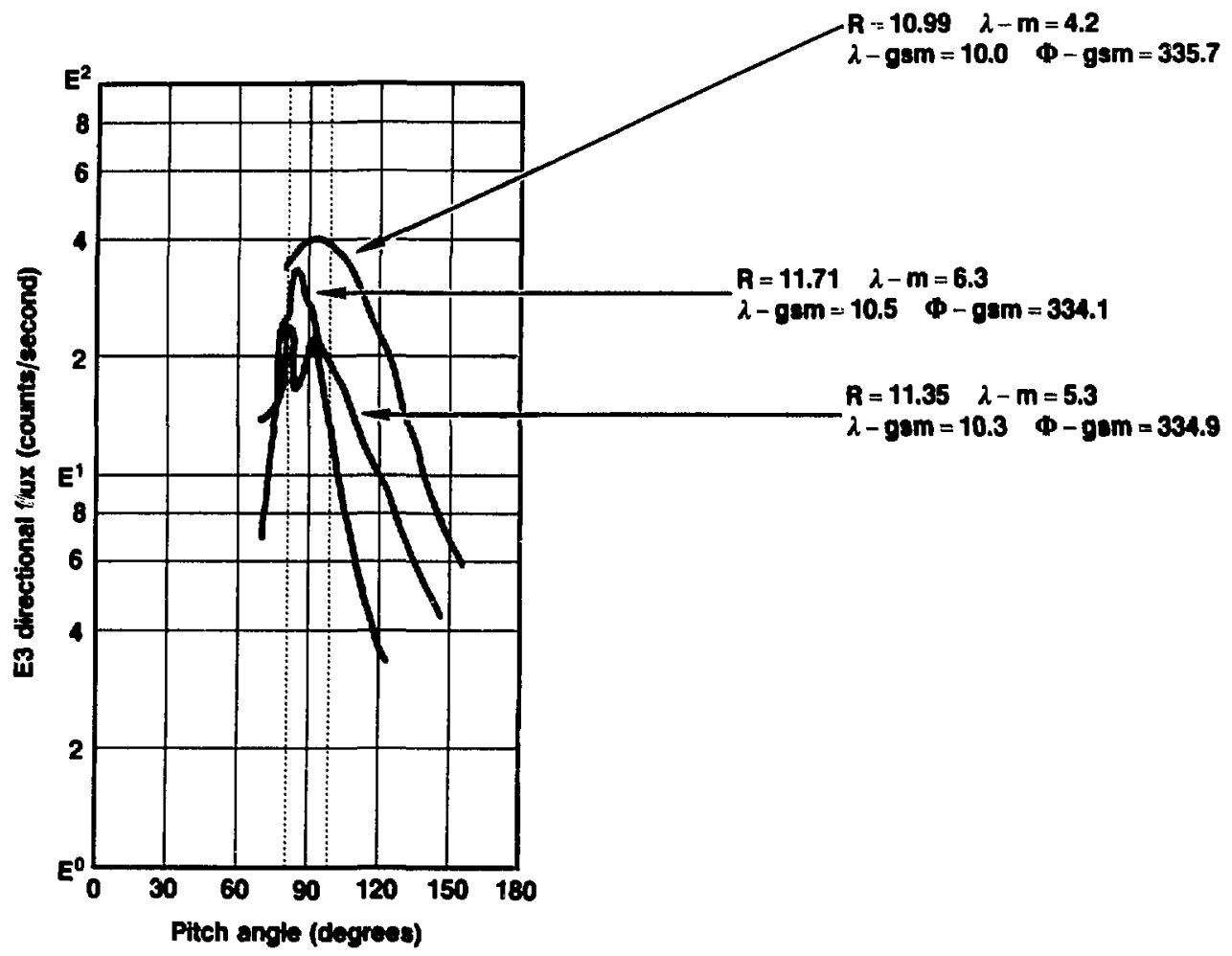

Figure 4-55. Sequence of E3 PADs obtained by OGO-5 on Day 66, 1968, at the same locations as in Fig. 4-53.

148 


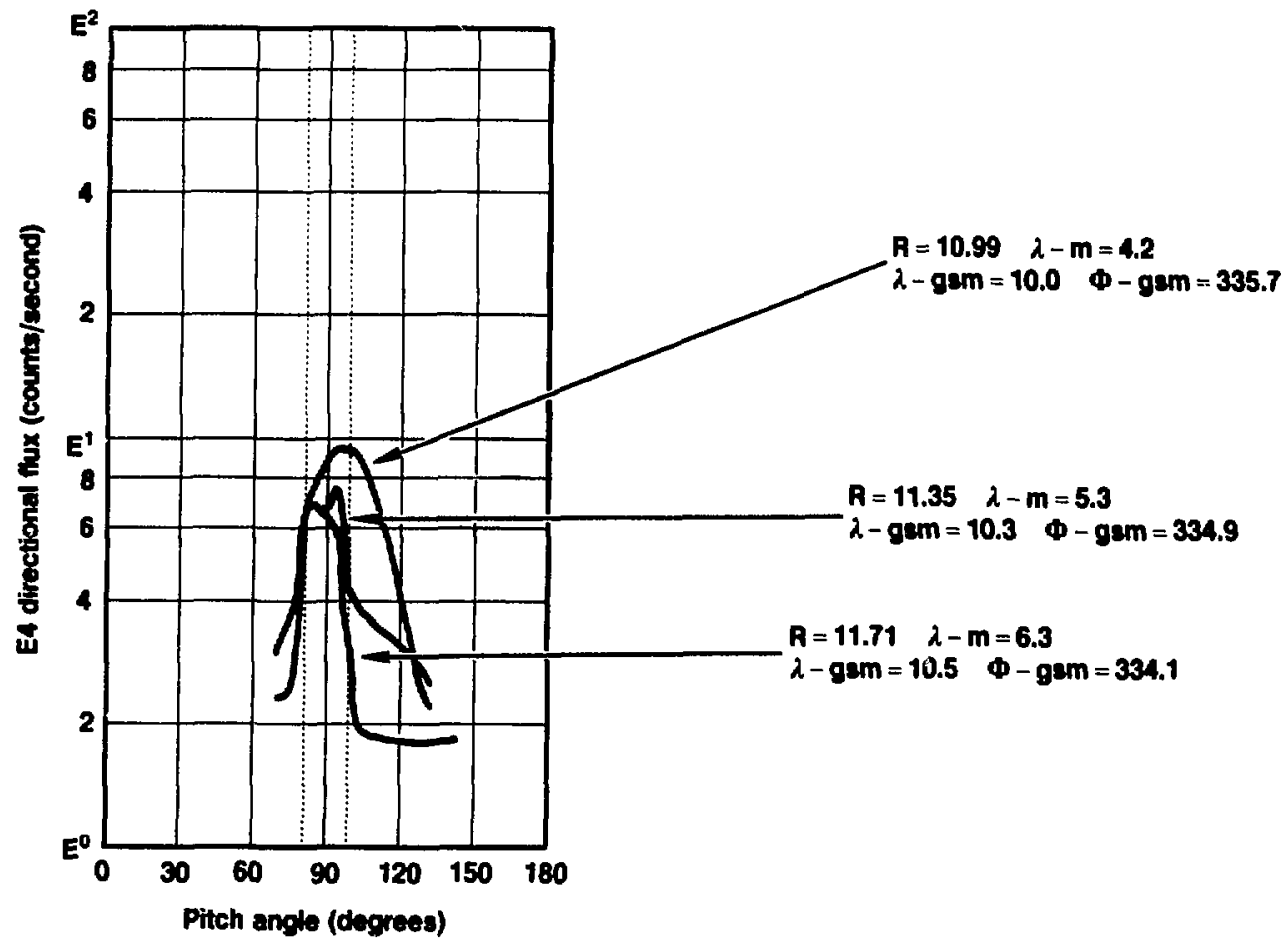

Figure 4-56. Sequence of E4 PADs obtained by OGO-5 on Day 66, 1968, at the same locations as in Fig. 4-53. 


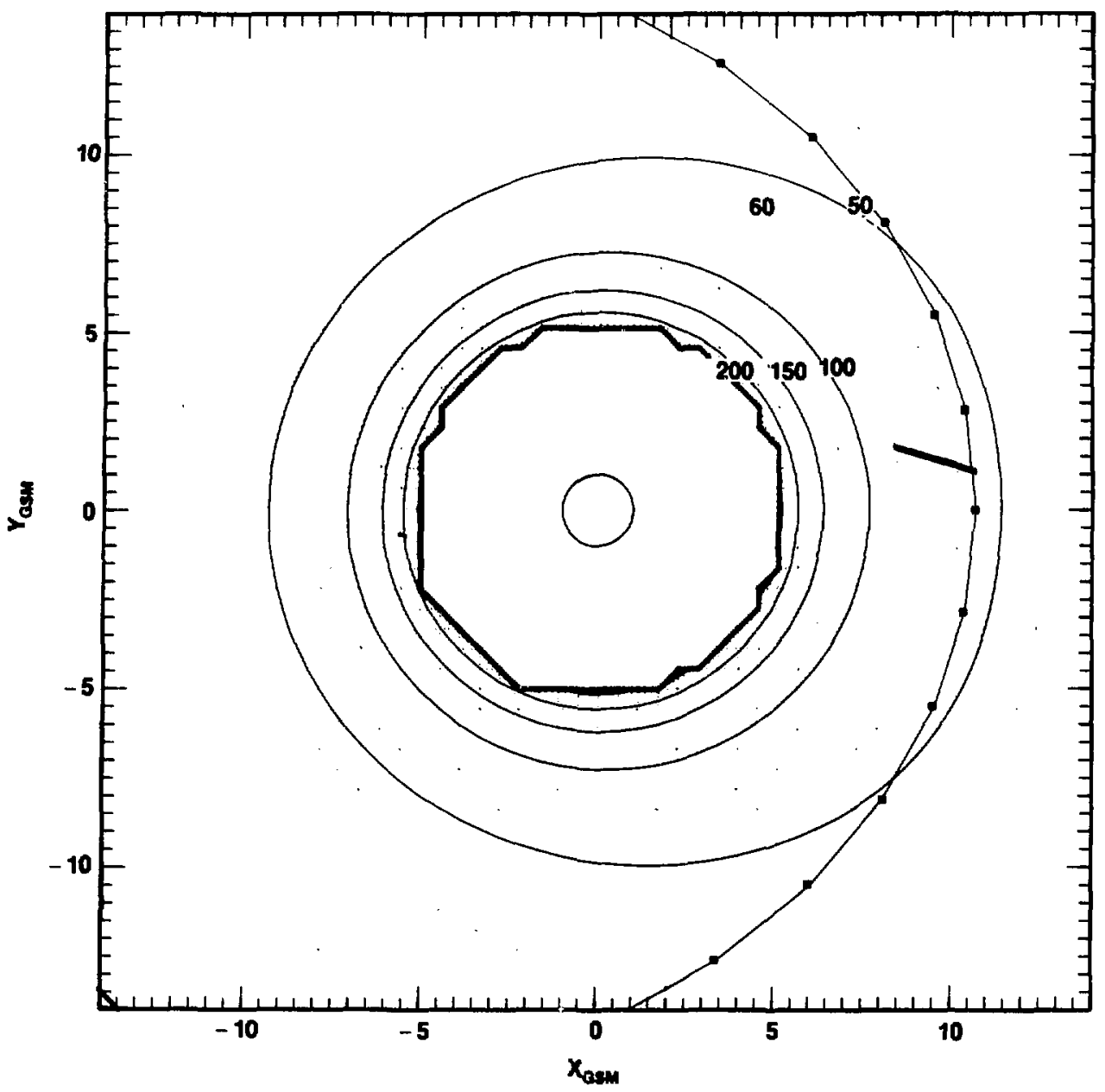

Figure 4-57. Contours of constant magnetic field magnitude (nanoteslas) in the equatorial plane for the Choe-Beard model, appropriate to the OGO-5 apacecraft pass of Day 11, 1969. The opacecraft trajectory appears as a short heavy line at about $7^{\circ}$ past noon. (Contours outside magnetosphere are not vilid). 
this interval is given in Fig. 4-58. The magnetopause was crossed at about $1636 \mathrm{UT}$, at $R=10.7 R_{E}, \phi_{\mathrm{GSM}}=$ $6.1^{\circ}, \lambda_{\mathrm{CSM}}=3.2^{\circ}, \lambda_{\mathrm{M}}=0.5^{\circ}$. The rise in $\mathrm{E} 1$ electron flux to levels characteristic of magnetospheric populations occurred just subsequent to magnetopause crossing (Fig. 4-59).

\section{Day 41 Field Modeling}

Figure 4-60 depicts the out- $r$ field lines in the spacecraft meridian $\left(\phi_{G S M}=7^{\circ}\right)$ for the Choe-Beard model. Model parameters are $a$. ipole tilt angle of $-2.8^{\circ}$ and a magnetopause standoff distance of $10.0 R_{E}$. The variation of the magnetic induction along these lines is given in Fig. 4-61. High-latitude minimum-B pockets are seen on lines that intersect the spacecraft orbit, ranging inwards from the magnetopause to

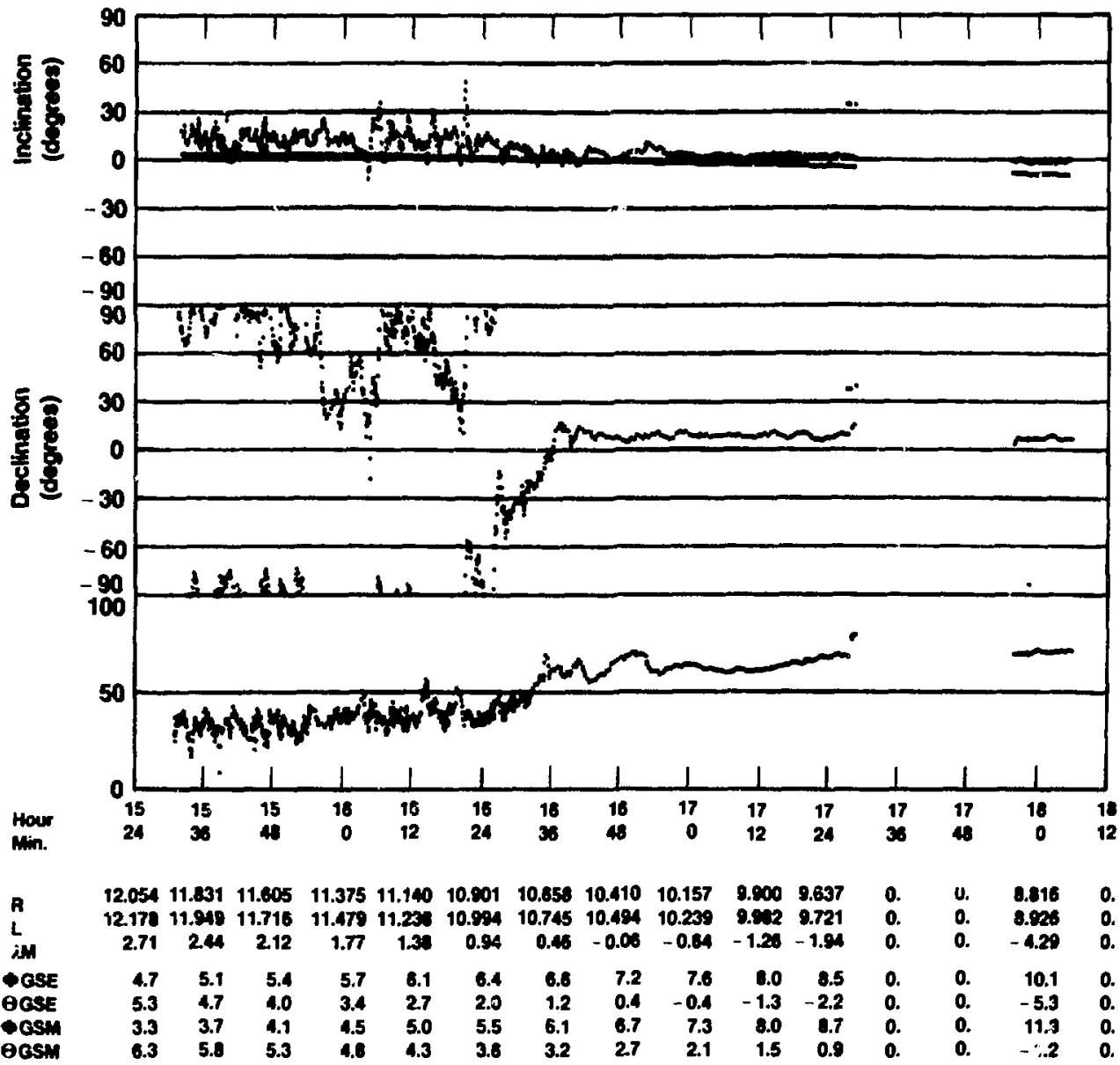

Figure 4-58. Magnetic field observed by the UCLA magnetometer experiment during the OGO-5 inbound pass of Day 41, 1969. X-axis is Universal Time. Upper panels give the inclination and declination of the vector magnetic field, with reference to the dipole coordinate system. Lower panel is field magnitude in nanoteslas. Satellite coordinates are given below each time annotation. Magnetopause crossing occurred at approximately $1636 \mathrm{UT}, R \approx 10.7 \mathrm{R}_{\mathrm{E}}$. 


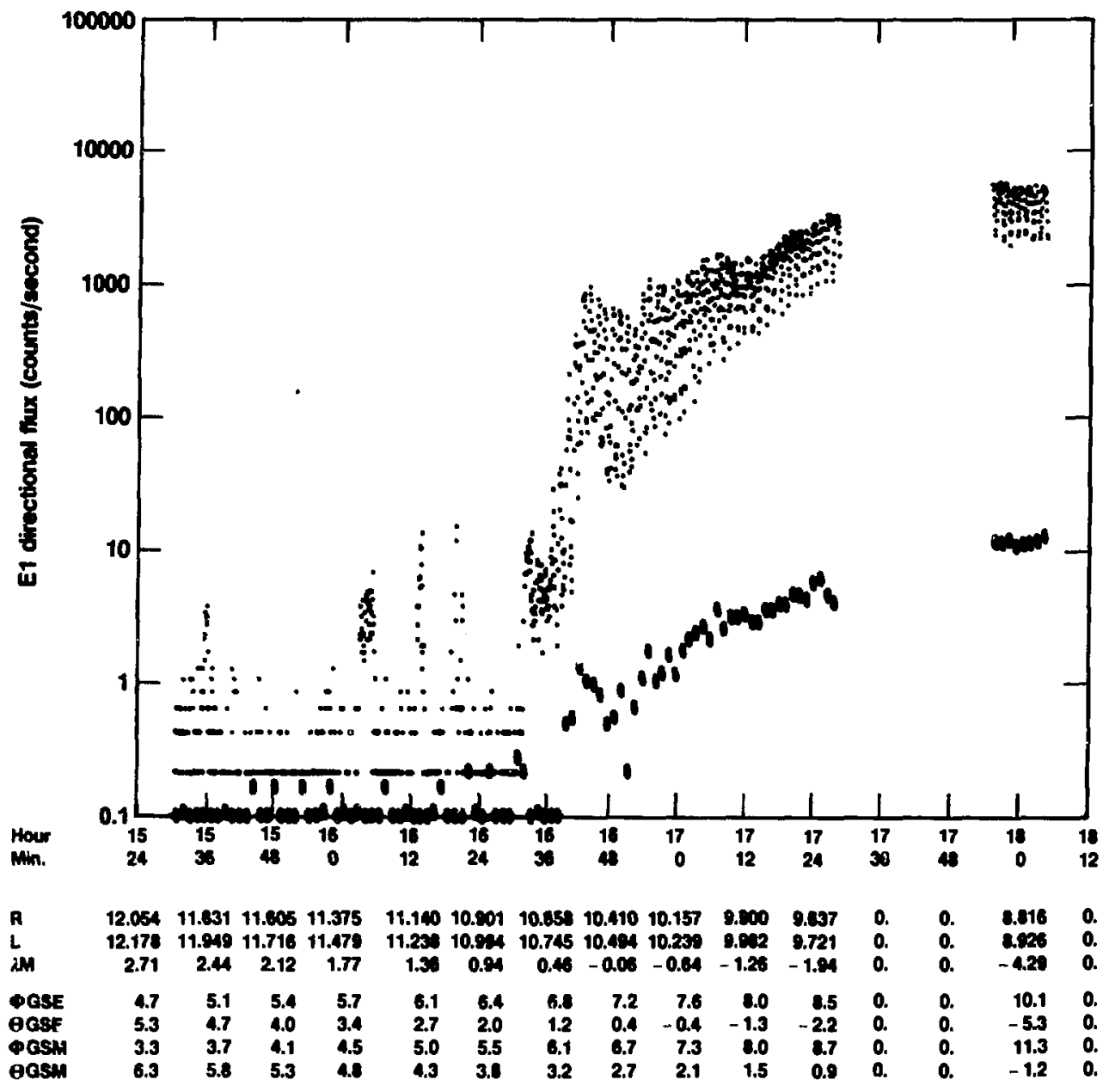

Figure 4-59. Electron counting rate in the loweat-energy electron channel E1 (79 keV) observed during the OGO-5 inbound pass of Day 41, 1969. The transition into the magnetosphere occured at about 1636 UT, when fluxes sharply increased to magnetospheric levels. 


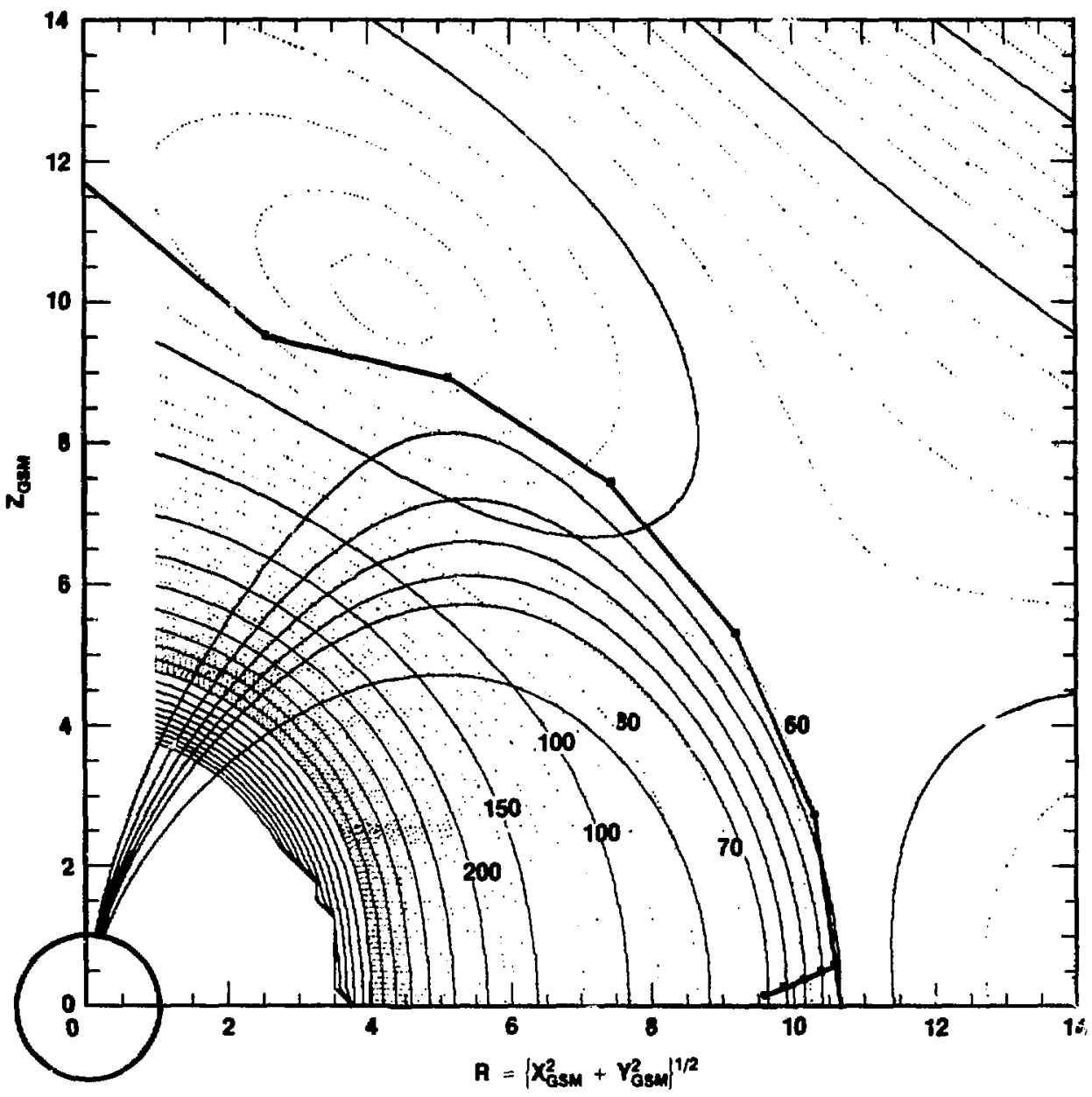

Figure 4-60. Outer magnetospheric field lines and constant-B contours in the aximuth $4 \mathrm{Gsm}=7^{\circ}$ for the Choe-Beard model, appropriate to the inbound pass of Day 41, 1969. The satellite trajectory appears as the short nearly-radial trace. (Contours outside magnetosphere are not valid). 


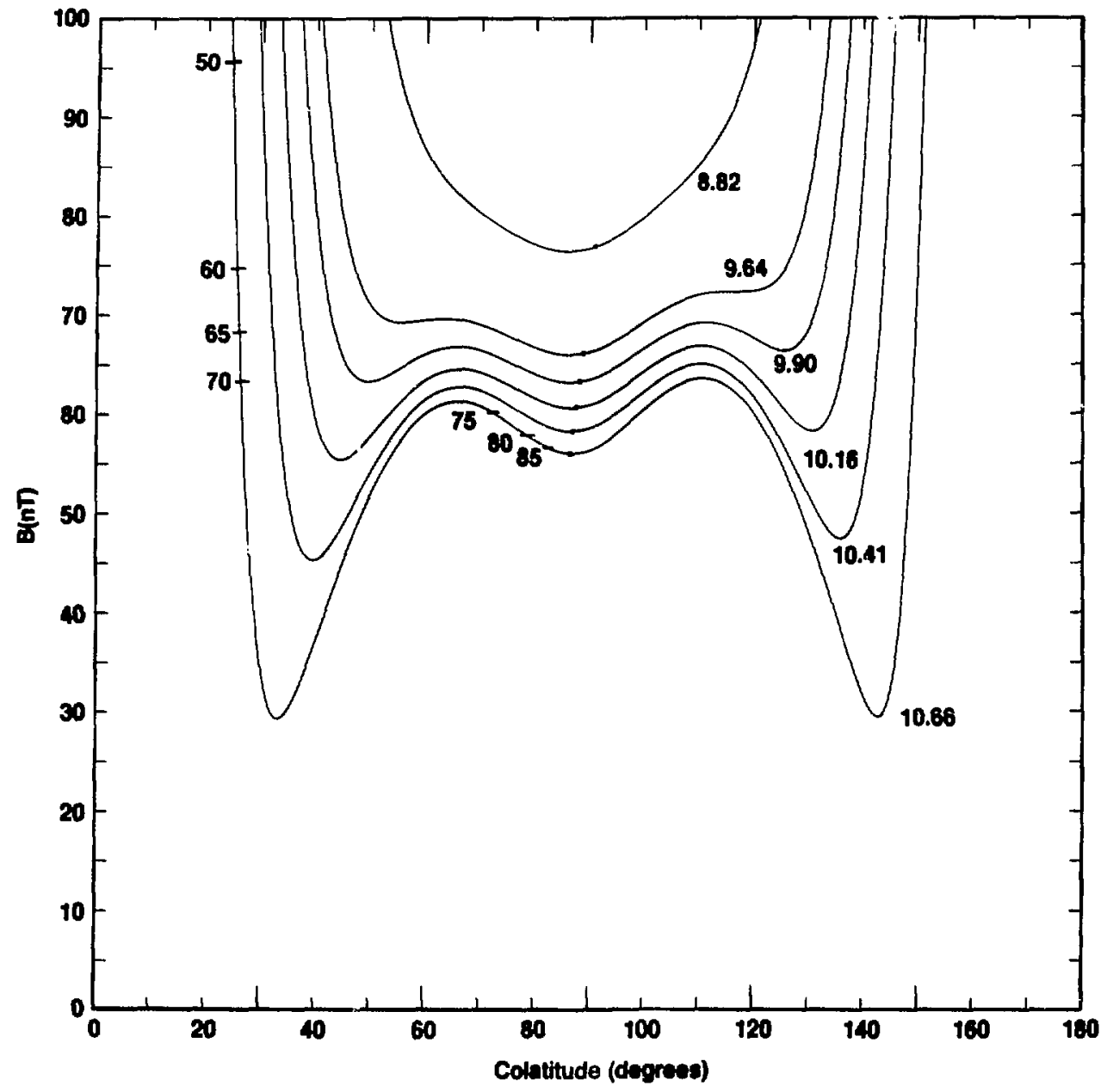

Figure 4-61. Magnetic field magnitude va GSM colatitude $\theta_{\mathrm{GSM}}$ as computed from the Choe-Beard model along outer magnetospheric lines which intersect the spacecraft trajectory on Day 41, 1969. (The equator is at $\theta_{\mathrm{CSM}}=90 \%$. The spacecraft position is shown as a dot on each line. Lines are labelled on the right with the radial distance of the spacecraft in $\mathbf{R}_{\mathrm{E}}$. Numbers along the outermost line denote the spacecraft pitch-angles of particles which mirror at these line locatione. Highlatitude minimum-B pockets are evident on the outer lines. 


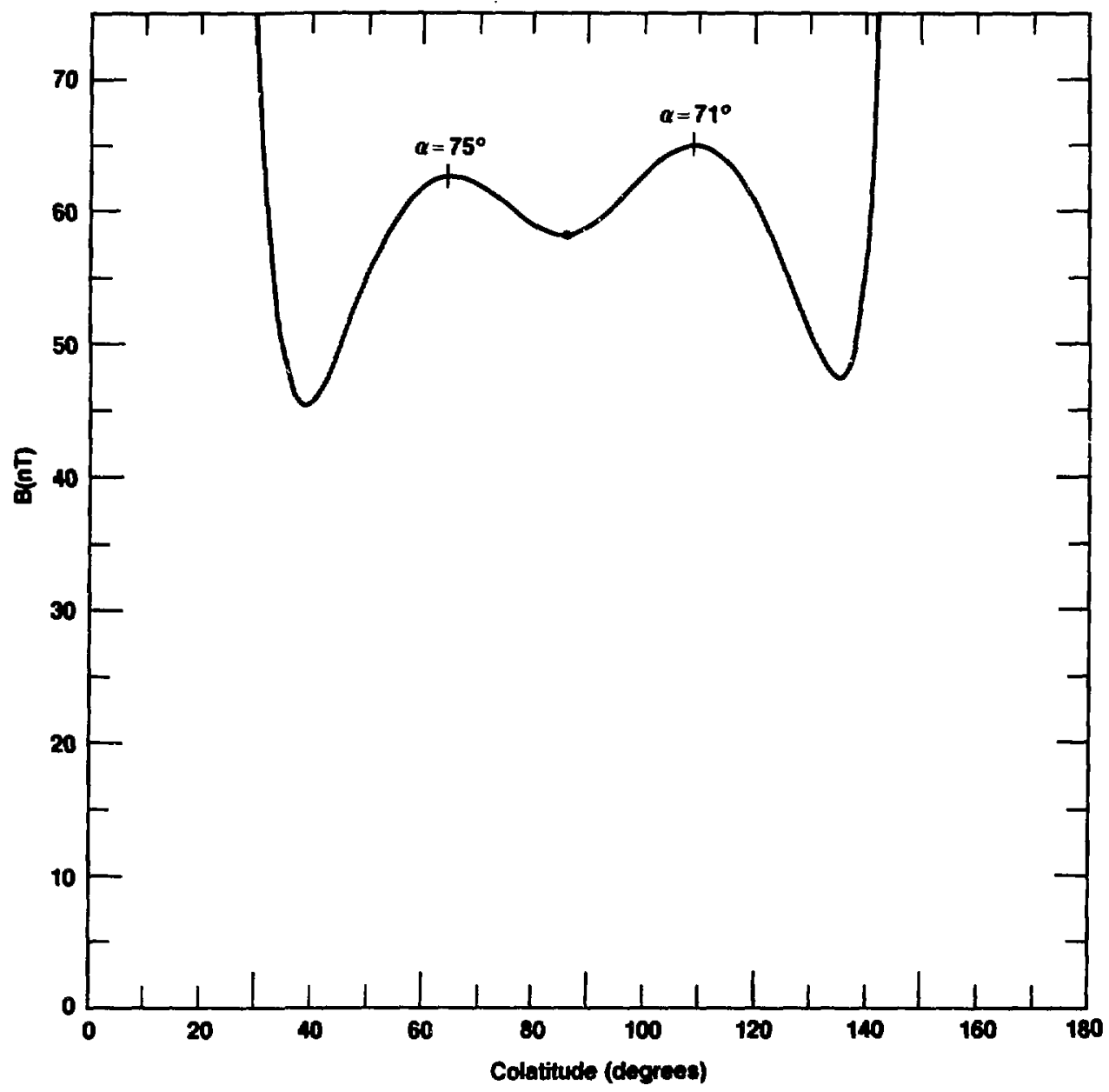

Figure 4-62. Location of local b-maxima on outer line for Day 41, 1969. Fluxes mirroring in the vicinity of the maxima will appear at the satellite at pitch angles of $\alpha=75^{\circ}$ and $71^{\circ}$.

about a radial distance of $9.6 \mathrm{R}_{\mathrm{E}}$. Figure 4-62 illustrates the critical pitch angles at which the local B-maxima occur. Although the dipole tilt causes the northern and southem B-maxima to be represented by slightly different pitch angles at the satellite, they coincide within about $5^{\circ}$ and so we have used average values of $73^{\circ}$ and $107^{\circ}$. Region 0 fluxes $\left(\alpha<73^{\circ}\right.$ and $\alpha>107^{\circ}$ ) mirror poleward of the local B-maxima; Region 1 fluxes $\left(73^{\circ}<\alpha<107^{\circ}\right)$ mirror equatorward of them.

\section{Day 41 Energetic Electron Pitch Angle Distributions}

Figure 4-0. 3 displays a sequence of OGO-5 E1 pitch angle distributions measured on Day 41, 1969 in the outer noon magnetosphere. The PAD obtained just inside the magnetopause $\left(R=10.68 R_{E}\right)$ shows a flux peak near $\alpha=73^{\circ}$, the northern limit of Region 1 fluxes. Region 1 fluxes to the south of the satellite 


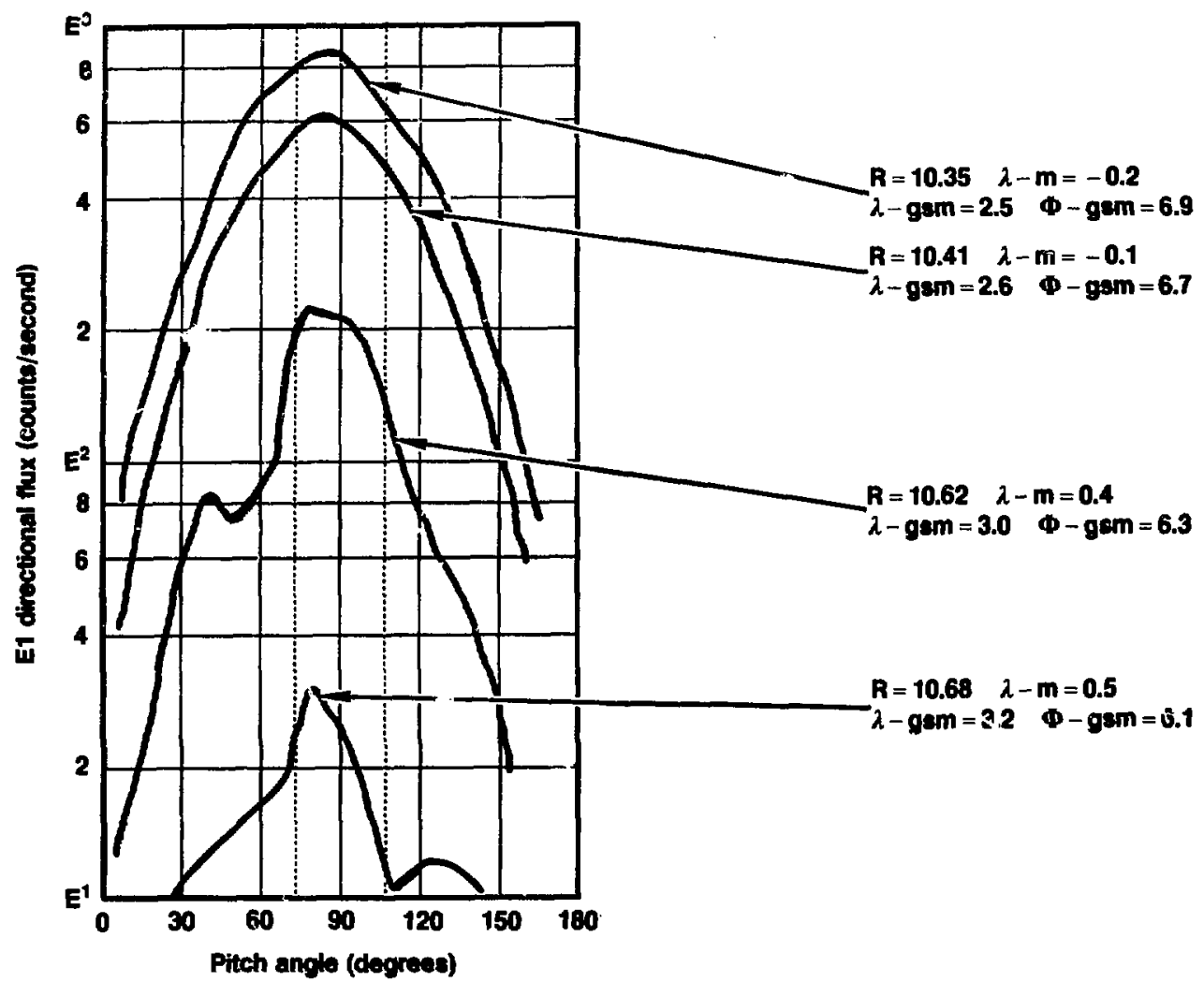

Figure 4-63. Sequence of E1 PADs obtained by OGO-5 on Day 41, 1969, as the satellite moved earthwards from the magnetopause (at $R \approx 10.7 R_{E}$. Dotted lines mark the pitch angles $\alpha=73^{\circ}$ and $107^{\circ}$ corresponding to fluxes mirroring at $B_{M}=$ local $B-m a x$.

are depressed. The PAD obtained at $R=10.62 R_{E}$ shows much increased flux levels, but the transition at $\alpha \approx 73^{\circ}$ is still marked. Further inwards the PADs assume a symmetric, "normal" form; flux transitions are not significant at $R=10.41 R_{E}$ and $10.35 R_{E}$.

Figure 4-64 illustrates the E2 PADs measured at the same locations as for E1 above. The PAD at $R=$ $10.68 R_{E}$ shows an enhancement of Region 1 fluxes mirroring to the north of the satellite. At $R=10.62 R_{E}$ the PAD shows a peak near $\alpha \approx 73^{\circ}$, which persisted as the satellite moved inward to $R=10.41 R_{E}$. At $R=10.35$ the E2 PAD departs from the corresponding E1 measurement. A flux depression near $90^{\circ}$ creates an $M$-shaped distribution with peaks lying close to the Region 1 edges. This distribution closely resembles the PADs obtained in the partial drift shadow region post-noon, which we will present subsequently.

The next-higher energy channel E3 began to register a significant counting rate at the latter two PAD positions (Fig. 4-65). It displays features similar to those of channel E2, showing flux peaks near the pitch angles corresponding to fluxes mirroring at the local B-maxima.

Figures 4-66 and 4-67 depict E1 and E2 PADs further along the inward satellite trajectory. Both citannels showed little deviation from the "normal" distribution as flux levels continued to iricrease. However, channel E3 (Fig. 4-68) continued to show a flux peak at $\alpha=73^{\circ}$ and a negative flux gradient at $R=10.26$ and $9.99 R_{E}$. By $R=9.68 R_{E}$ all distributions showed a broad "normal" configuration. 


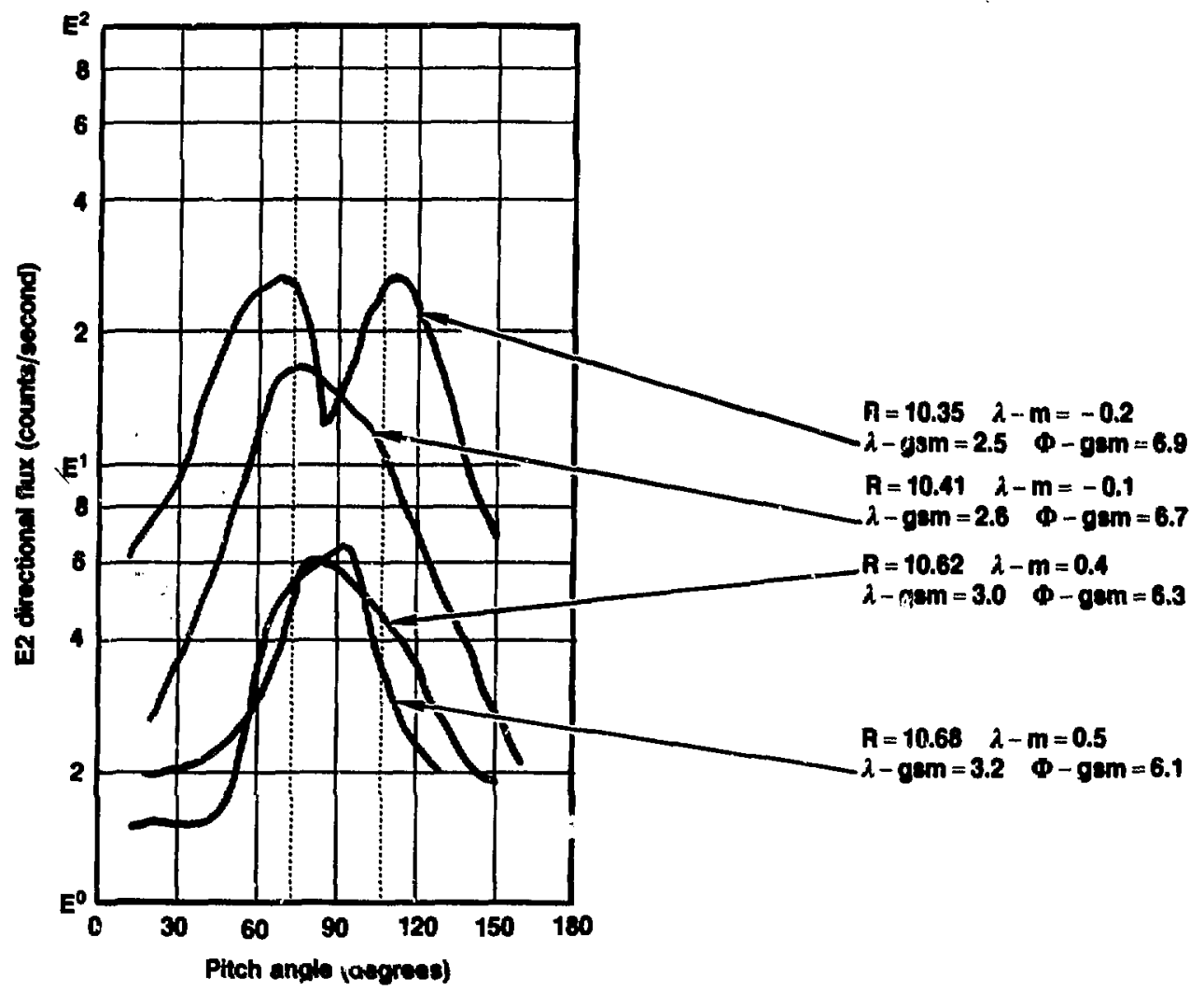

Figure 4-64. Sequence of E2 PADs obtained by OGO-5 on Day 41, 1969, at the same locations as in Fig. 4-63. 


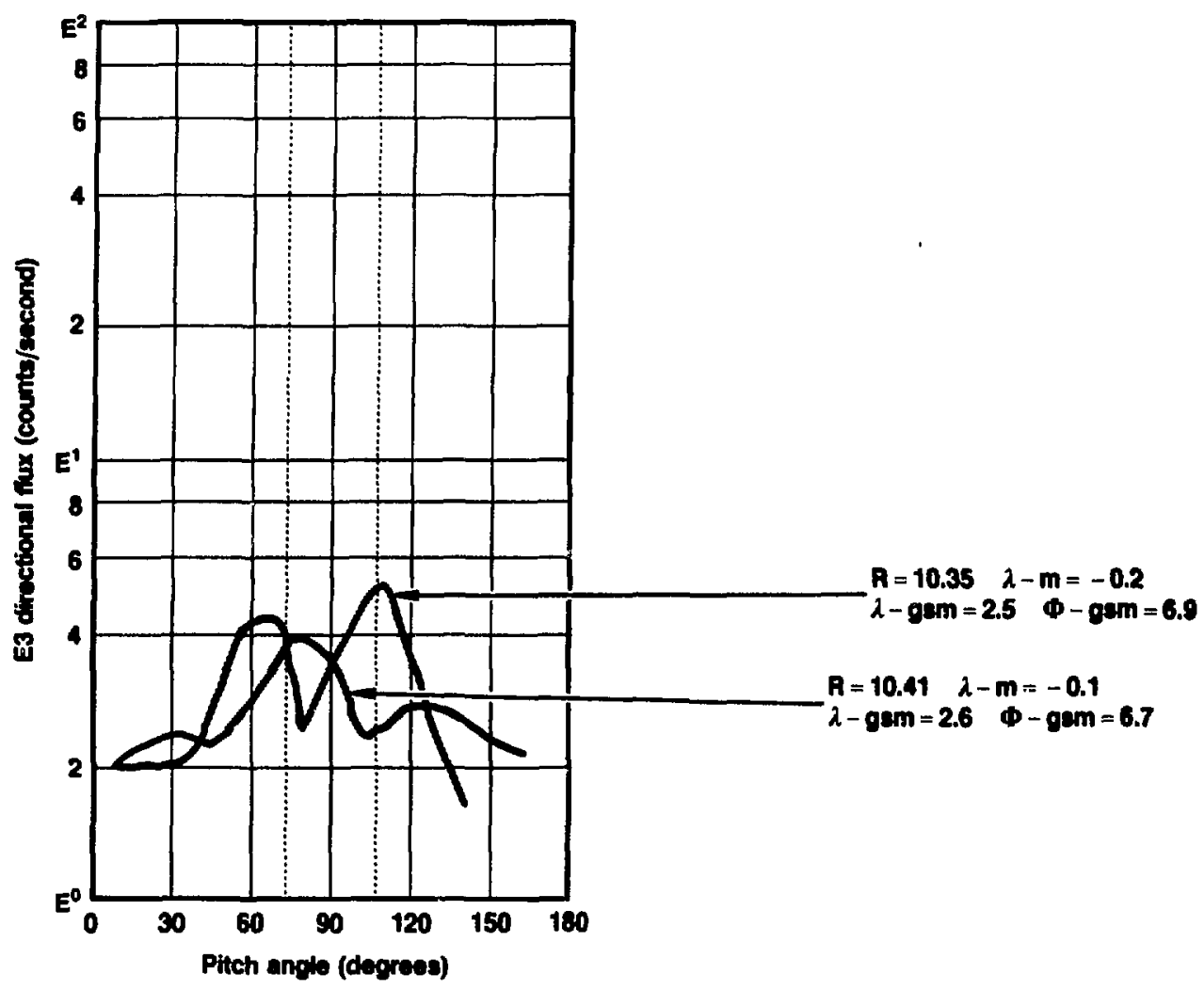

Figure 4-65. Sequence of E3 PADs obtained by OGO-5 on Day 41, 1969, at the latter two locatione of Fig. 4-63. 


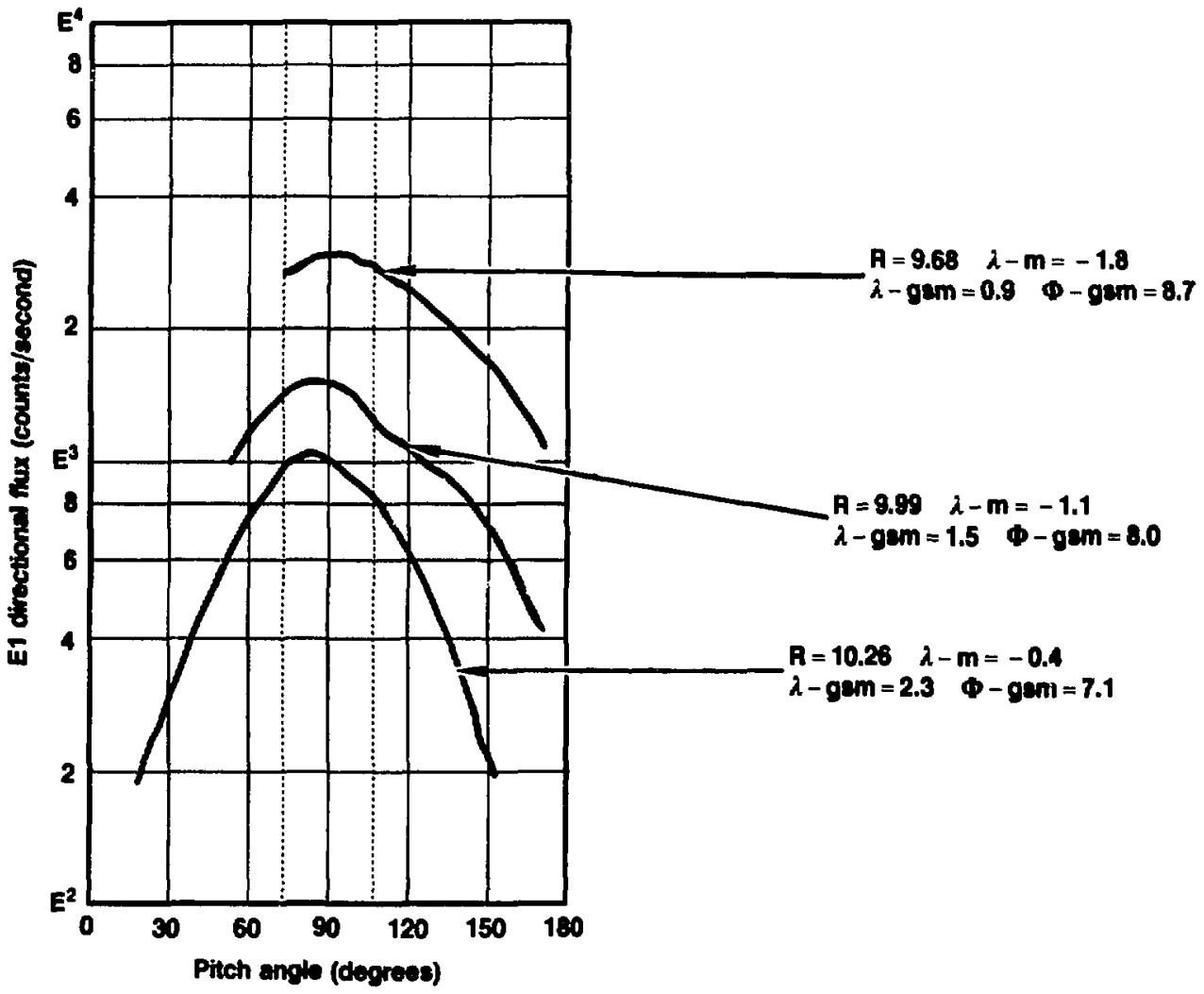

Figure 4-66. Sequence of E1 PADs acquired further along inbound orbit, Day 11, 1969.

159 


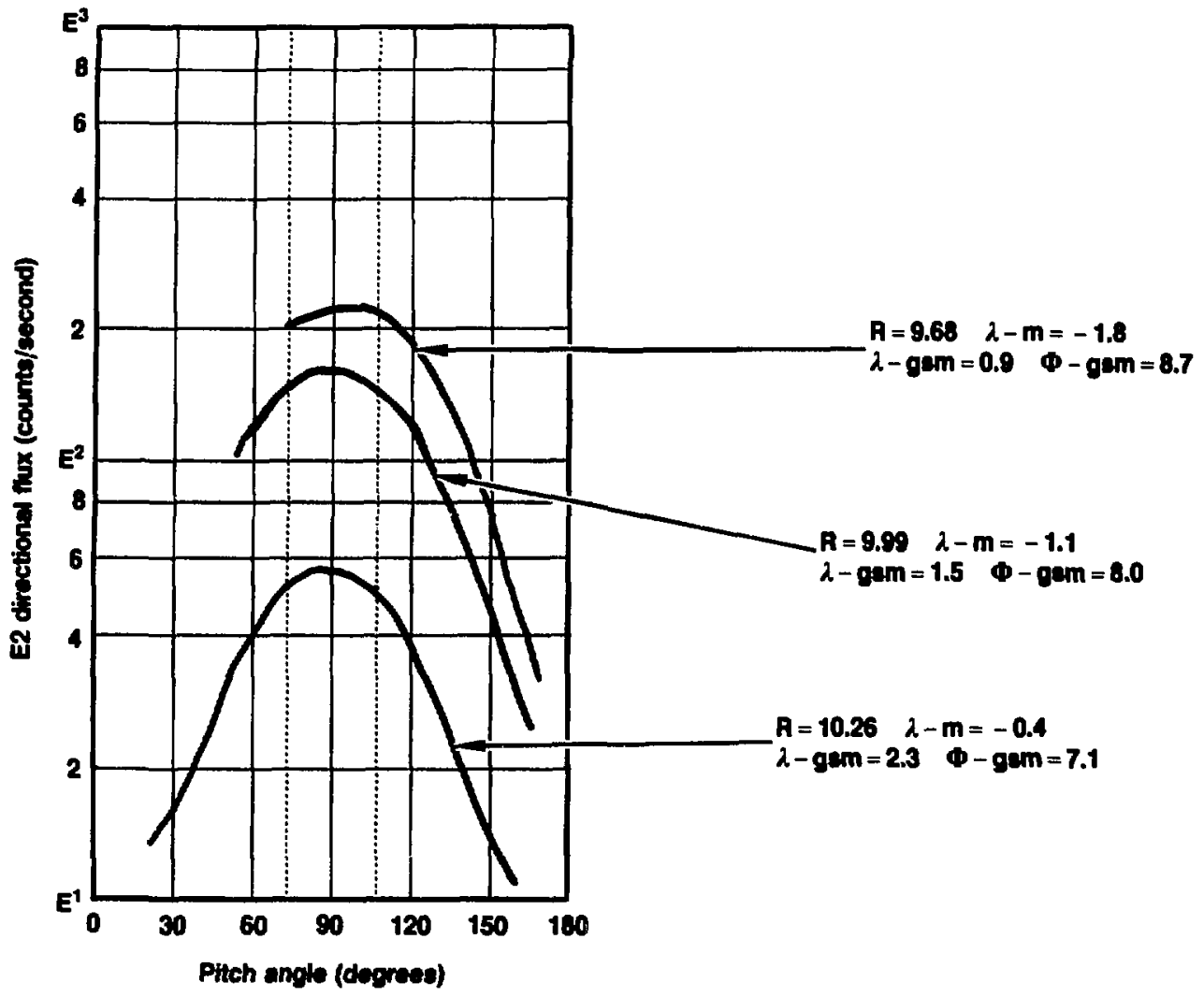

Figure 4-67. Sequence of E2 PADs obtained by OGO-5 on Day 41,1969 , at the aame locations as in Fig. 4-66. 


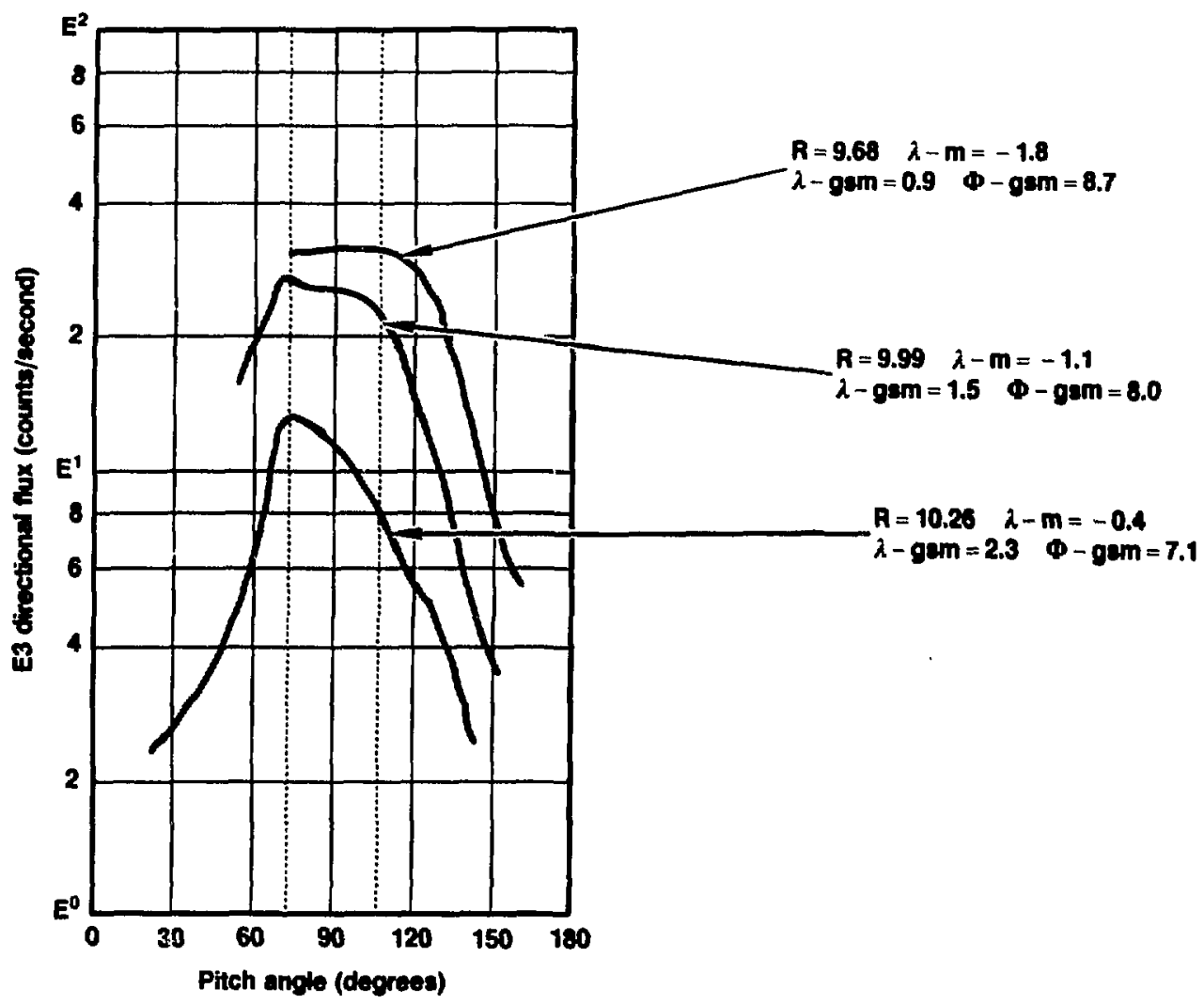

Figure 4-68. Sequence of E3 PADs obtained by OGO-5 on Day 41, 1969, at the same locations as in Fig. 4-66.

\section{OGO-5 Observations on Day 7 (January 7), 1969}

On Day 7, 1969 the satellite passed inbound through the equatorial afternoon magnetosphere, traversing the region where drift shells which had branched in the morning sector were rejoining. Figure 4-69 illustrates the satellite trajectory in the GSM equatorial plane of the Choe-Beard model. The angular coordinates of the satellite during the time of interest were $\lambda_{\mathrm{GSM}} \approx 14^{\circ}, \phi_{\mathrm{CSM}} \approx 39^{\circ}$. Figures 4-70 and 4-71 show the magnetic field and the E1 directional flux recorded along the trajectory. The magnetometer shows that the satellite first crossed the magnetopause at $\approx 2030 \mathrm{UT}$, but was overtaken by the magnetopause moving inwards at $\approx 2036$ UT, leaving the satellite momentarily in the magnetosheath. At $\approx 2045$ UT the satellite passed into the magnetosphere again $\left(R \approx 11 R_{E}\right)$. El fluxes are seen to increase from magnetosheath levels commencing at $\approx 2058 \mathrm{UT}\left(R \approx 10.7 R_{\mathrm{E}}\right)$.

\section{Day 7 Field Modeling}

Preliminary results of modeling the PADs observed on Day 7 were reported by Buck (1975). We are able to improve on the prior analysis by making use of the branching map methodology developed as part of this dissertation. Figure 4-72 depicts the outer field lines in the satellite meridian $\left(\phi_{\mathrm{CSM}}=39^{\circ}\right)$, using 


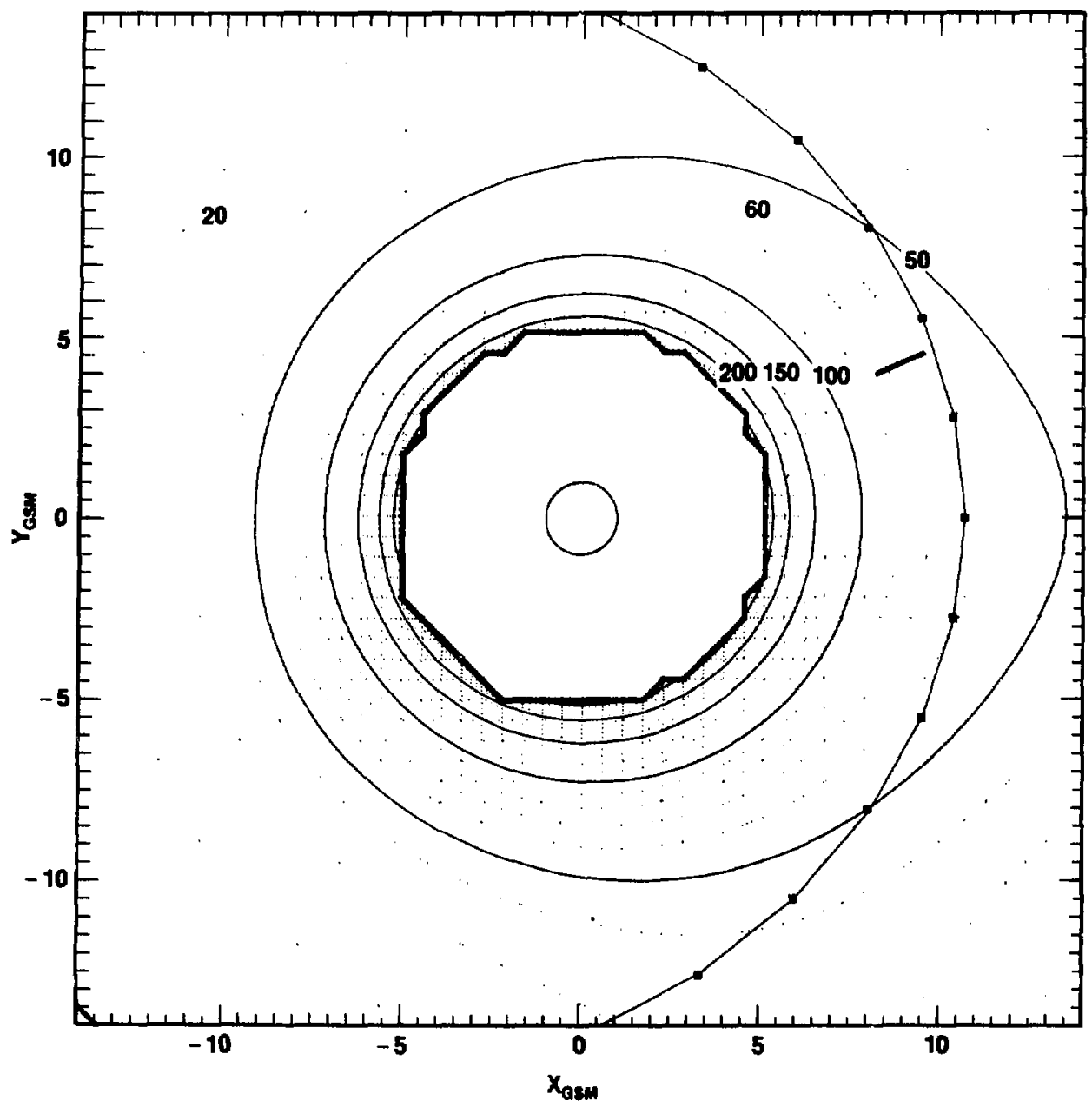

Figure 4-69. Contours of constant magnetic field magnitude (nanoteslas) in the equatorial plane for the Choe-Beard model, appropriate to the OGO-5 spacecraft pass of Day 7, 1969. The spacecraft trajectory appears as a short heavy line at about $39^{\circ}$ past noon. (Contours outside magnetoophere are not valid). 


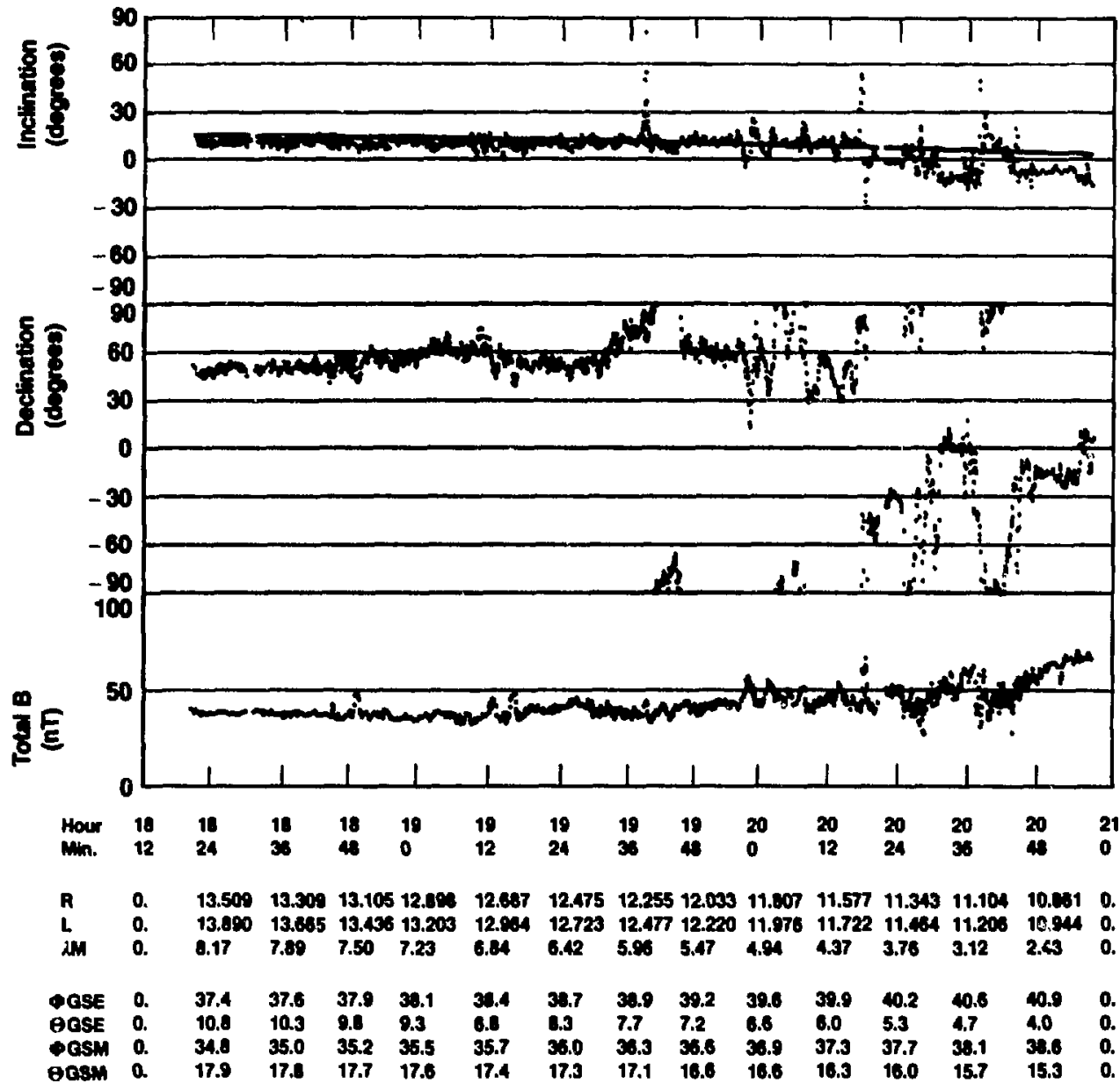

Figure 4-70. Magnetic field observed by the UCLA magnetometer experiment during the OGO-5 inbound pass of Day 7, 1969. X-axis is Universal Time. Upper panels give the inclination and declination of the vector magnetic field, with reference to the dipole coordinate system. Lower panel is field magnitude in nanoteslas. Satellite coordisates are given below each time annotation. Multiple magnetopause crossings are evident at approximately 2030,2036 , and $2045 \mathrm{UT}\left(\mathrm{R} \approx 11.0 \mathrm{R}_{E}\right.$ at this last crossingl. 


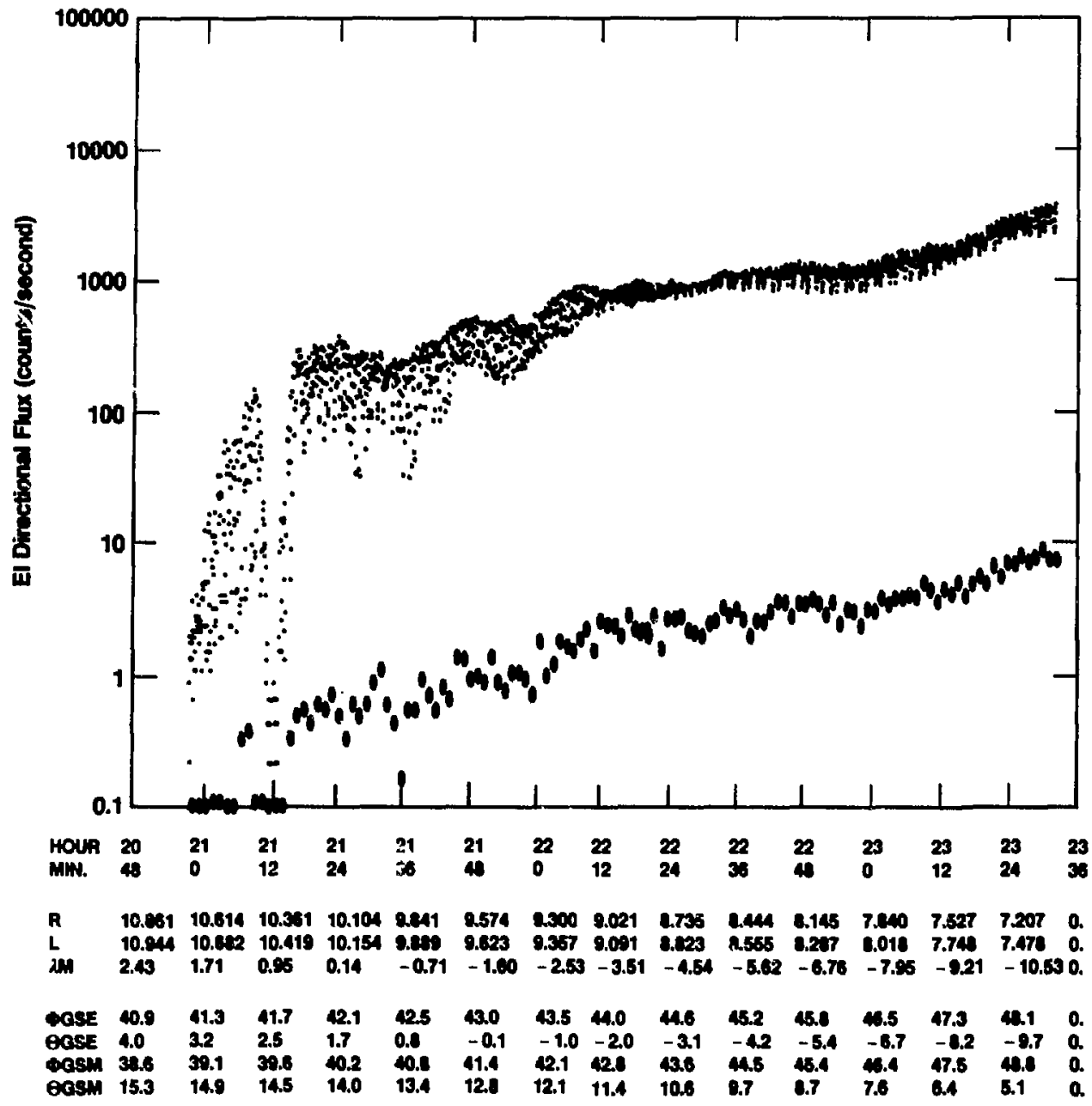

Figure 4-71. Electron counting rate in the lowest-energy electron channel E1 $(79 \mathrm{keV})$ observed during the OGO-5 inbound pass of Day 7,1969 . The counting rate rose to magnetospheric levele at about 2058 UT $\left(R \approx 10.7 R_{E}\right)$. 


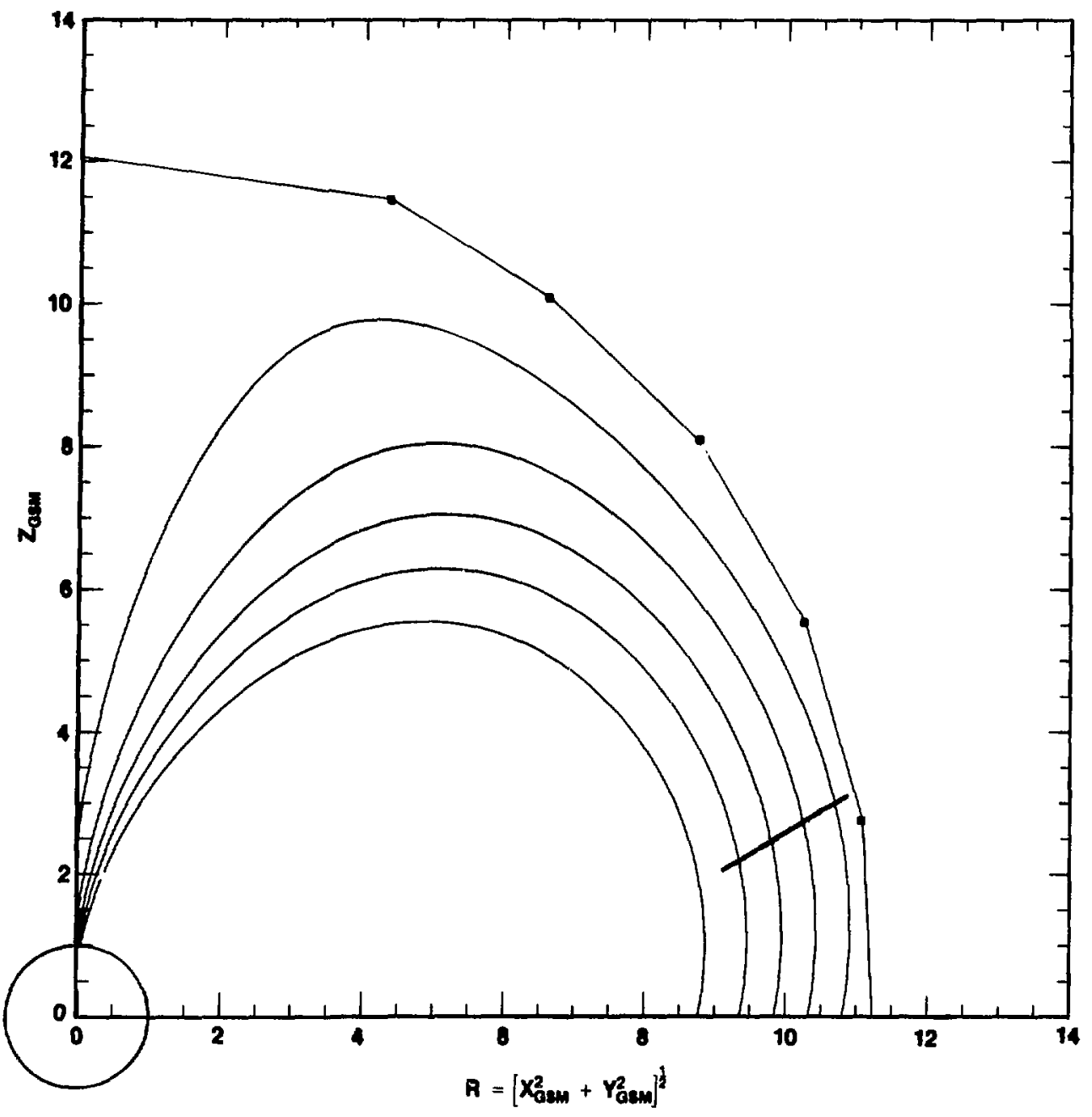

Figure 4-72. Outer magnetospheric field lines in the aximuth $\mathrm{CSM}=39^{\circ}$ for the Choe-Beard model, appropriate to the inbound pass of Day 7, 1969. The satellite trajectory appears as the short nearly-radial trace. 
the Choe-Beard model with standoff distance $R_{S O}=9.93 R_{E}$ and dipole tilt $x-17^{\circ}$. We see from Fig. 4-73 that the outer field lines show high-latitude minimum-B pockets. Owing to dipole tilt, there is a single local B-maxima located south of the satellite at colatitude $\theta \approx 115^{\circ}$.

The principal adiabatic features predicted by the model can be obtained from Fig. 4-74, where the $B_{M}, I$ coordinates of satellite PADs, noon drift loss cone, and branching curves are displayed. This figure illustrates that both magnetopause shadowirig and drift-shell rejoining effects will be present in the PADs measured in the outer regions.

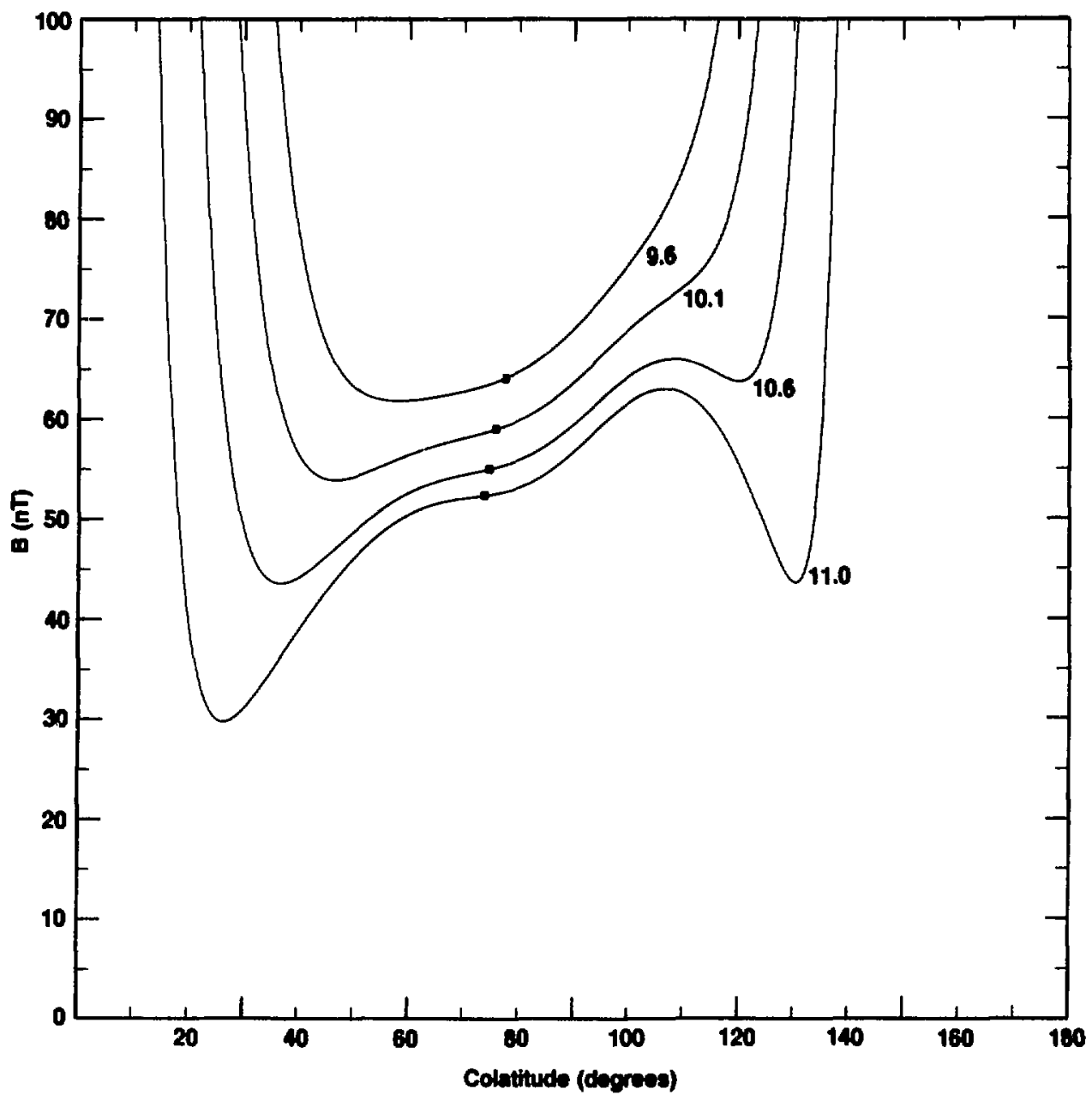

Figure 4-73. Magnetic field magnitude vs GSM colatitude $\theta_{\mathrm{GSM}}$ as computed from the Choe-Beard model along outer magnetonpheric lines which intereect the spacecraft trajectory on Day 7, 1969. (The equator is at $\theta_{\mathrm{CSM}}=90^{\circ}$ ). The spacecraft position is shown as a dot on each line. Lines are labelled on the right with the radial distance of the spacecraft in $R_{E}$. Because of the large dipole tilt $\left(-17^{\circ}\right)$ only a single local B-maximum exists, to the eouth of the satellite. 


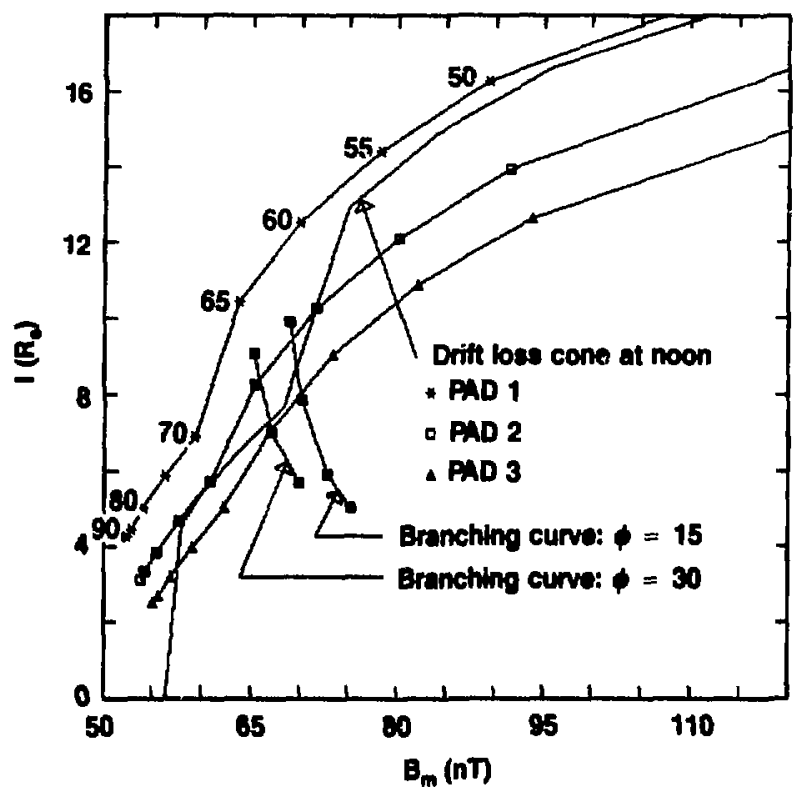

Figure 4-74. Adiabatic drift shell configuration for Day 7, 1969, as computed from the Choe-Beard model. Shown are the $B_{M}, I$ traces of three PADs along the inward-bound spacecraft trajectory, annotated with spacecraft pitch angle. Also depicted is the drift loss cone at noon, delimited by the last closed noon field line. Unbranched drift shells characterized by $B_{M}, 1$ points to the left of this trace do not close within the magnetosphere. Branching curves are drawn at azimuths of $15^{\circ}$ and $30^{\circ}$. Each point on a branching curve designates the $B_{M}, 1$ value of a branched shell which rejoins at that azimuth. The intersection of the branching curves with the PADs indicates the pitch angle range at which the satellite will receive fluxes that drift on rejoined shells, having passed around noon through the high-latitude minimum-B regions. This range is approximately $62^{\circ}<\alpha<66^{\circ}$.

We first note that a PAD obtained by the satellite just inside the magnetosphere (PAD 1) lies entirely in the adiabatic drift shadow. The effective drift loss cone is defined by the trace of the outermost field line at noon; electrons whose $B_{M}, I$ coordinates lie to the left of this trace will drift to the magnetopause prenoon. Consequently the satellite is shielded from electrons drifting adiabatically from the morning sector. A PAD acquired subsequently along the trajectory (PAD 2) displays partial magneiopause shadowing; the satellite is shielded from particles of pitch angles $\alpha>\approx 60^{\circ}$, but begins to register particles at smaller pitch angles. We would expect experimental PADs obtained in the vicinity of PAD 2 to show a flux maximum near $\alpha=60^{\circ}$. Eventually the satellite moves entirely out of the shadow (PAD 3) and records electrons at all pitch angles which have drifted adiabatically around the noon meridian to the satellite.

The effect of the rejoining of branched drift shells can also be determined from this figure. We have plotted branching curves at two azimuths between noon and the satellite meridian (at $\phi_{\mathrm{CSM}}=15^{\circ}$ and $30^{\circ}$ ). We recall from the previous section that a branching curve at a specified azimuth is formed from the loci of all branching points on field lines which intersect the equator at that azimuth. A drift shell branches if any line in the shell contains a local B-maximum such that $B_{M}=B-m a x$. Consequently the drift shell defined by the point of intersection of a PAD with a branching curve, will branch (or rejoin) at the branching curve azimuth. The intersection of these branching curves with PAD 3 indicates that the fluxes observed at the satellite in the pitch angle range $60^{\circ}<\alpha<65^{\circ}$ (and by bounce symmetry in range $115^{\circ}$ $<\alpha<120^{\circ}$ ) include contributions from drift shell segments which have passed through the noon 
minimum-B pockets. Fluxes at greater and lesser pitch angles have moved around noon to the satellite on unbranched shells. If the fraction of particles passing through the B-pockets have undergone scattering, the rejoined fluxes will be depressed relative to those on unbranched shells. We would therefore expect the experimental PADs obtained in the vicinity of PAD 3 to show a local fux minimum at $\alpha \approx 65^{\circ}$ and $115^{\circ}$.

\section{Day 7 Energetic Electron Pitch Angle Distributions}

The next set of figures depict OGO-5 energetic electron pitch angle distributions measured in the outer afternoon magnetosphere on Day 7, 1969. Figure 4-75 shows a sequence of PADs measured by the lowest energy electron channel $E 1(E=79 \pm 23 \mathrm{keV}$ ) as the satellite swept earthward. The PADs at $R=9.99$ and $9.80 R_{E}$ were obtained at trajectory points corresponding approximately to that of PAD 2 in Fig. 4-74, as the satellite lay in the adiabatic drift shadow cast by the noon magnetopause. A deep flux depression is evident in the interval $60^{\circ}<\alpha<120^{\circ}$. indicating that these fluxes were shadowed by the magnetopause. Subsequent PADs (at $R=9.46 R_{E}$ and $9.35 R_{E}$ ) show the flux levels rising in this inverval, indicating that these electrons have drifted around from the morning sector on shells which closed within the magnetosphere. A small local flux minimum is noticeable near $\alpha \approx 70^{\circ}$ and $\alpha \approx 110^{\circ}$, approximately corresponding to the pitch angles in the mudel at which fluxes arrived at the satellite on newly-rejoined drift shells. As the satellite movect earthward the fluxes near $\alpha=90^{\circ}$ increased, until by $R=8.71 R_{E}$ the PADS showed a broad "normal" distribution, peaking at $\alpha=90^{\circ}$. During this interval, th" elative flux minima at the angles corresponding to rejoined shells persisted.

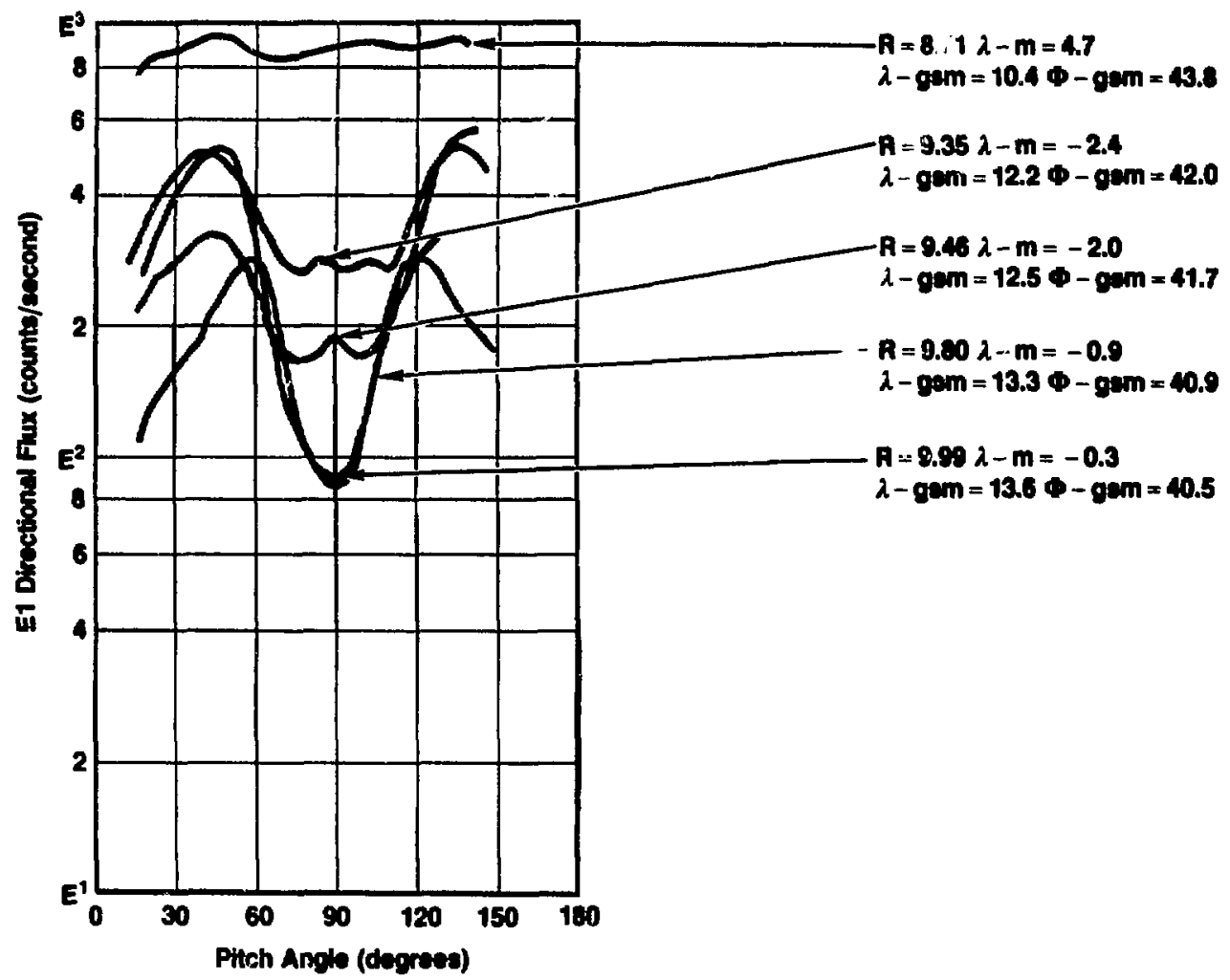

Figure 4-75. Sequence of E1 PADs obtained by OGO-5 on Day 7, 1969, as the eatellite movtd eartinwards from the magnetopause (at $R \approx 11.0 R_{\varepsilon}$ ). 
Figure 4-76 depicts a sequence of PADs for the next higher energy channel E2 (E $=158 \pm 36 \mathrm{keV}$ ) obtained at the same positions as those shown in Fig. 4-75. The same features are observed, namely the initial flux depression in the range $60^{\circ}<\alpha<120^{\circ}$ due to magnetopause shadowing, the gradual filling in of fluxes as the satellite emerged from the drift shadow, and the relative flux minima at $\alpha \approx 70^{\circ}$ and $110^{\circ}$.

The final figure for this day (Fig. 4-77) illustrates the PADs for channel $E 3(E=266 \pm 36 \mathrm{keV}$ ). The distributions are noisier because of the lower counting rates, but features similar to ihose in channels E1 and $\mathrm{E} 2$ can be discerned.

\section{OGO-5 Observations os Day 5 (January 5), 1969}

The Day 5, 1969 observations represent another sample of the afternoon sector of the outer magnetosphere where branched drift shells rejoin. Figure 4-78 illustrates the satellite trajectory in the CSM equatorial plane of the Choe-Beard model. The angular coordinates of the satellite during the time of interest were $\lambda_{\mathrm{GSM}} \approx 8^{\circ}, \phi_{\mathrm{GSM}} \approx 42^{\circ}$. Figures 4-79 and 4-80 show the magnetic field and the E1 directional flux recorded along the trajectory. The magnetometer record shows a transition in field line orientation from magnetosheath-like declination (field vector nearly in the flow direction) to magnetosphere-like declination (field vector nearly in the GSM meridian) at $0434 \mathrm{UT}\left(R \approx 12.9 R_{E}\right)$. However the field remained noisy (characteristic of the magnetosheath) until 0457 UT $\left(R \approx 12.5 R_{E}\right)$. E1 did not observe counts above background until $0520 \mathrm{UT}\left(\mathrm{R} \approx 12.0 \mathrm{R}_{\mathrm{E}}\right)$.

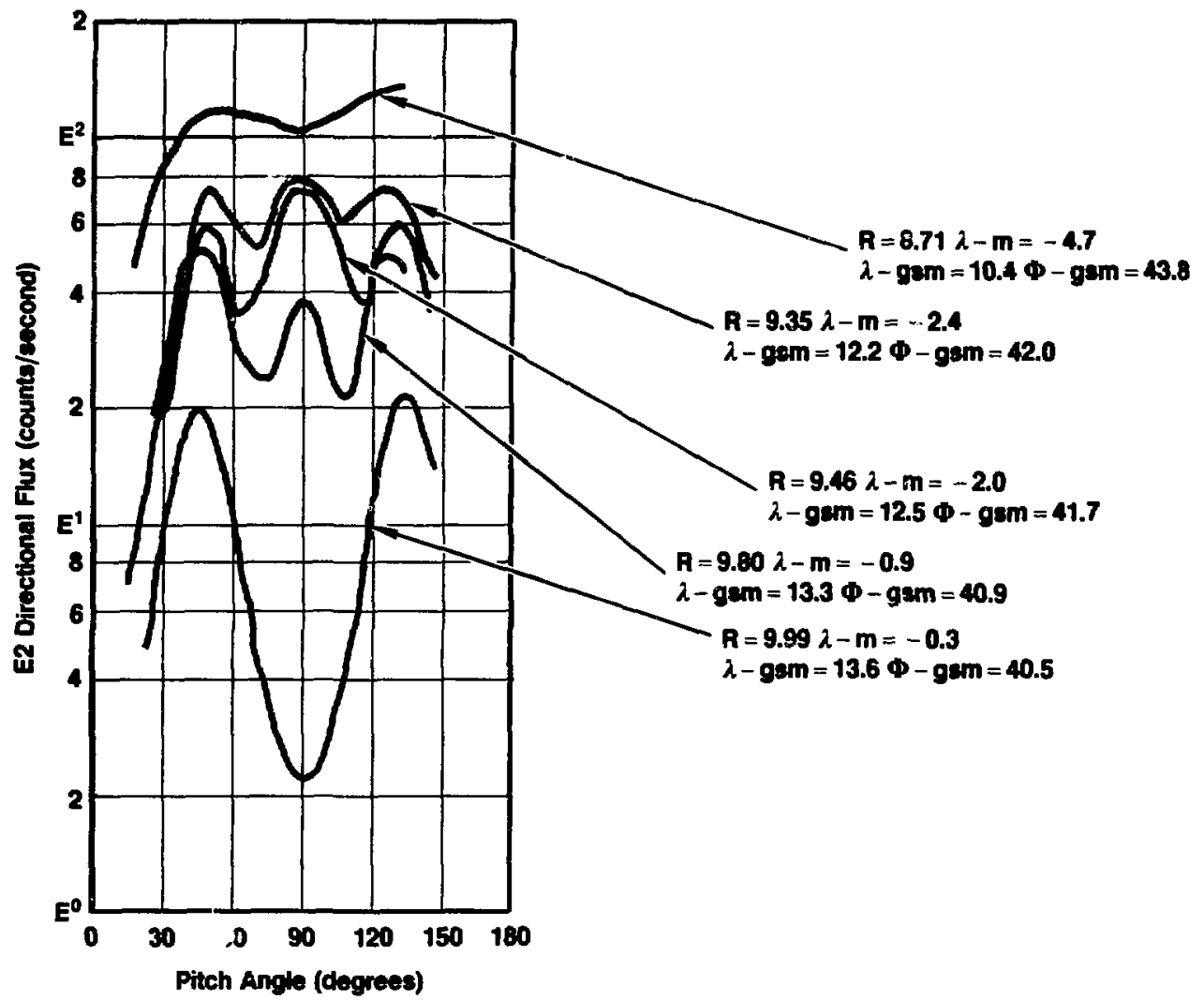

Figure 4-76. Sequence of E2 PADs obtained by OGO-5 on Day 7, 1969, at the same locations as in Fig. 4-75. 


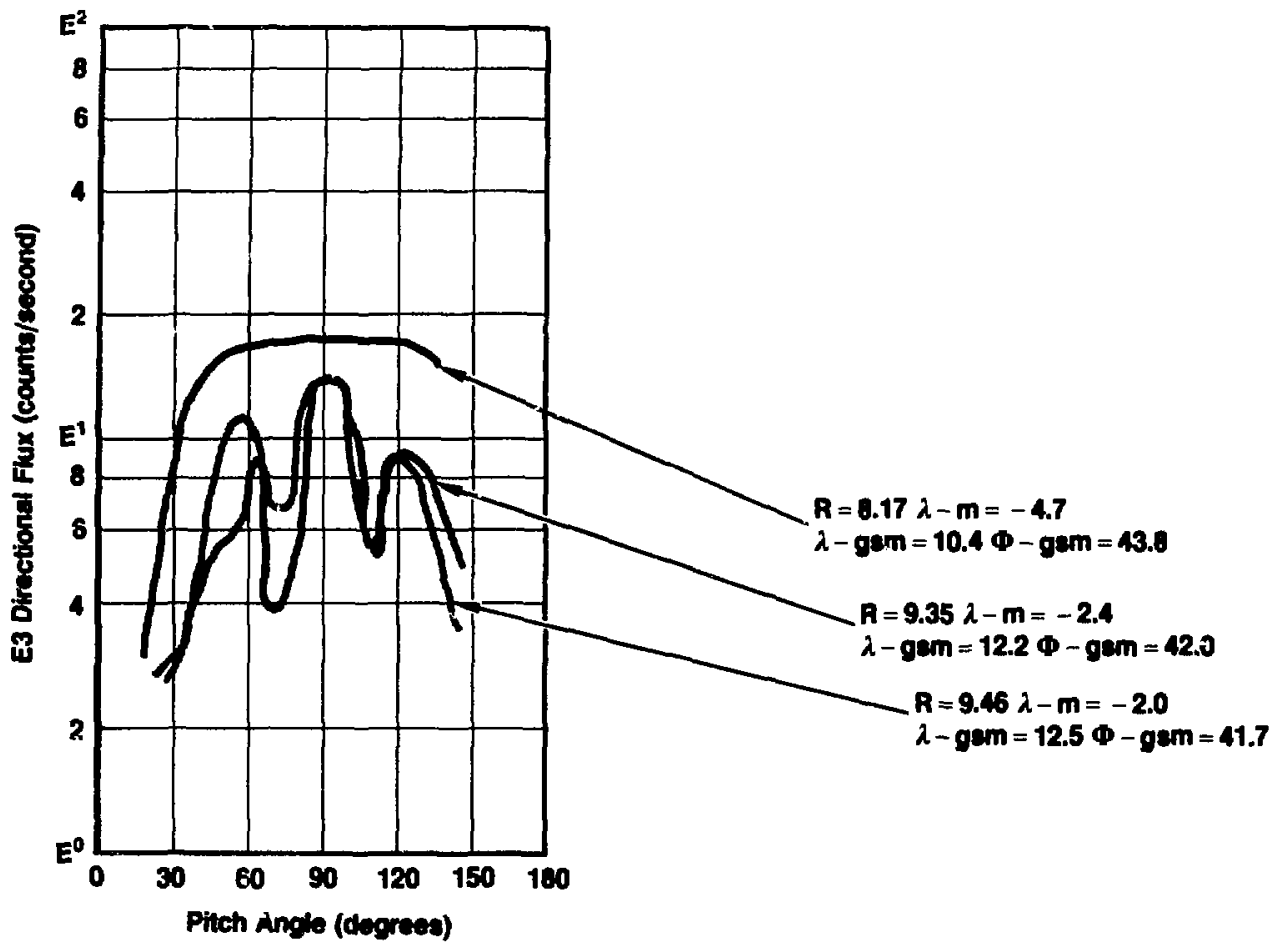

Figure 4-77. Sequence of E3 PADs obtained by OGO-5 on Day 7, 1969, at the same locations as in Fig. 4-75. 


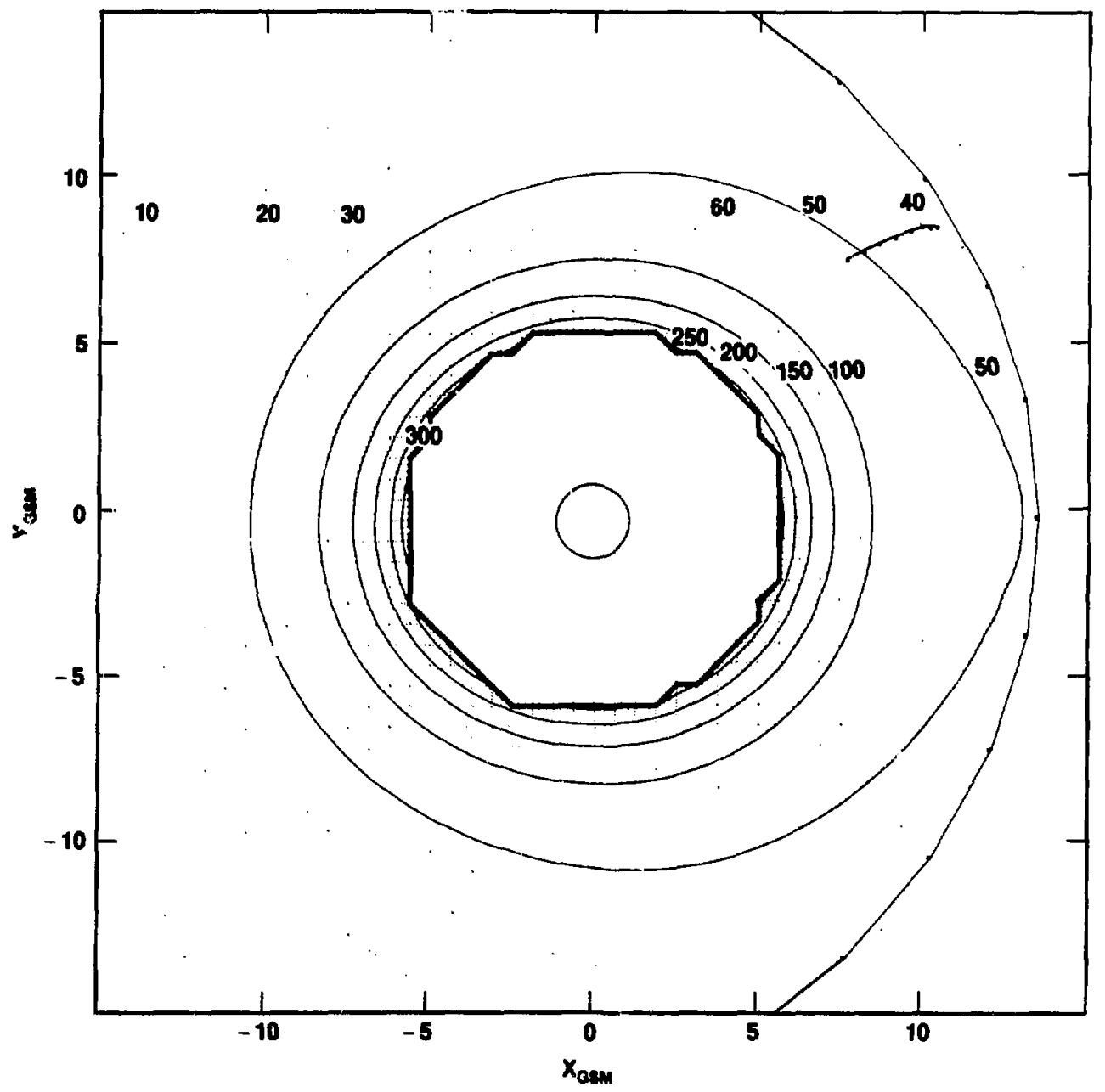

Figure 4-78. Contours of constant magnetic field magnitude (nanoteslau) in the equatorial plane for the Choe-Beard model, appropriate to the OGO-5 spacecraft pass of Day 5, 1969. The apacecraft trajectory appears as a short heavy line at about $42^{\circ}$ past noon. (Contours outside magnetosphere are not valid). 


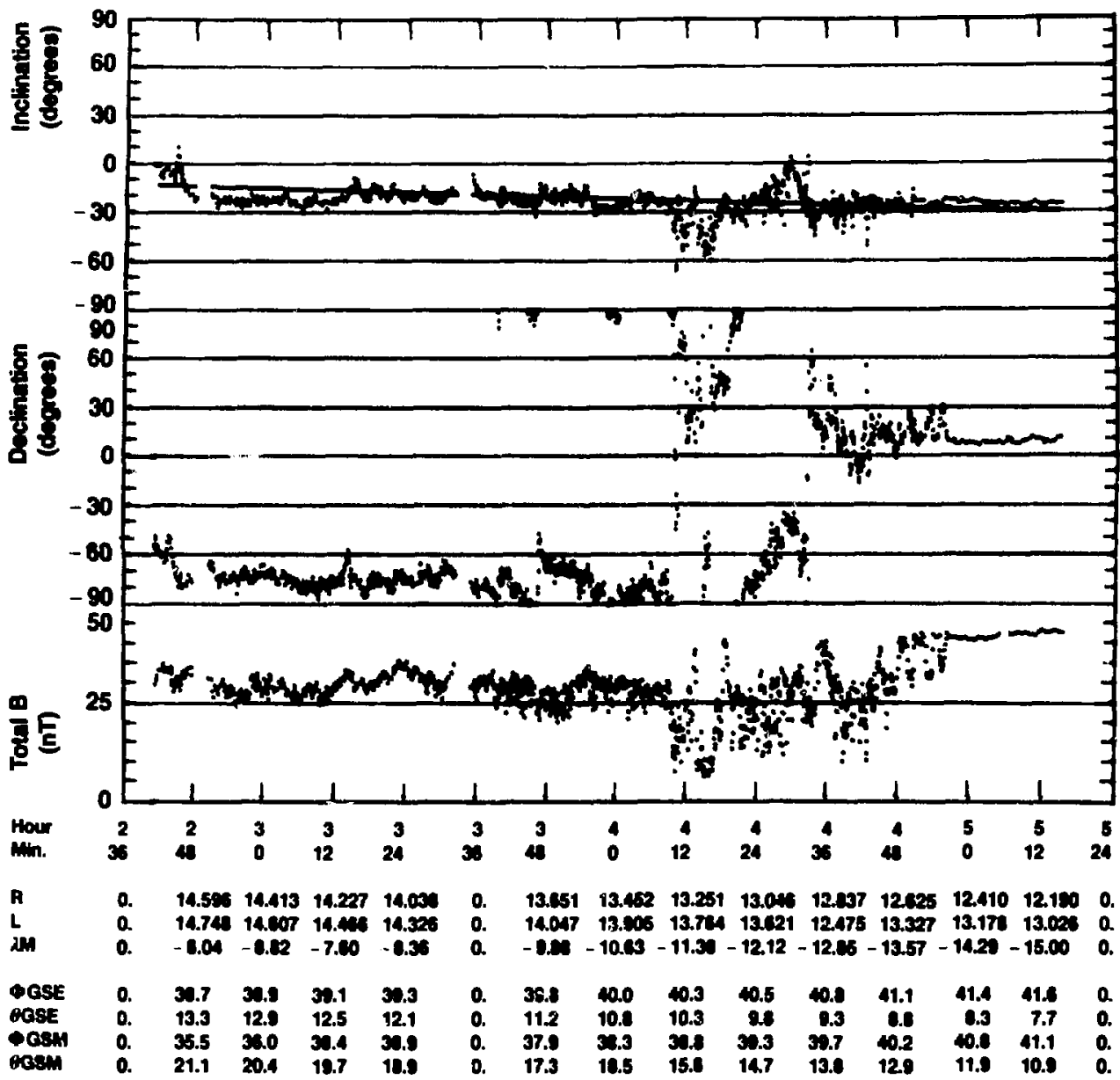

Figure 4-7y Magnetic field observed by the UCLA magnetometer experiment during the OGO-5 inbound pass of Day 5, 1969. X-axis is Univerval Time. Upper panels give the inclination and declination of the vector magnetic field, with reference to the dipole coordinate system. Lower panel is field magnitude in nanoteslas. Satellite coordinates are given below each time annotation. Magnetopause crossing occurred at approximately 0434 UT $\left(R \approx 12.9 R_{E}\right)$. 


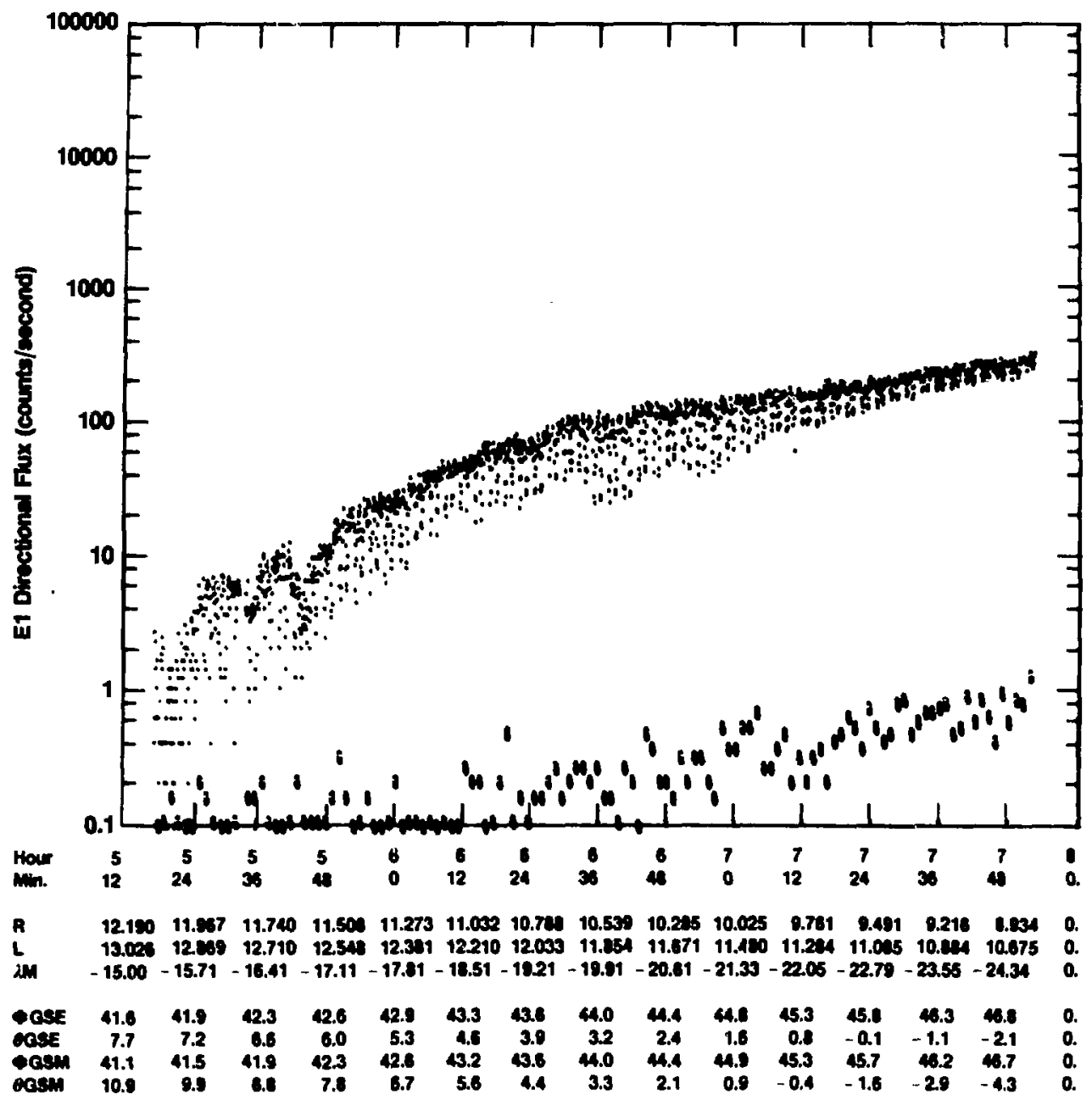

Figure 4-80. Electron counting rate in the lowest-energy electron channel E1 (79 keV) observed during the OGO-5 inbound pass of Day 5, 1969. The counting rate rose to magnetospheric levels at about C:520 UT $\left(R \approx 12.0 R_{E}\right)$. 


\section{Day 5 Field Modeling}

Figure 4-81 depicts the outer field lines in the satelite meridian $\left(\phi_{\mathrm{GSM}}=42^{\circ}\right)$, using the Choe-Beard model with standoff distance $R_{5 O}=11.1 R_{E}$ and dipole tilt $=-33^{\circ}$. Because of the extreme dipole tilt only a single local B-maximum occurs on the outer field lines, to the south of the satellite at colatitude $\theta \approx 100^{\circ}$ (Fig. 4-82).

The predicted flux features for this pass can be deduced from Fig. 4-83, which illustrates the adiabatic drift shell configuration for Day 5. As was the case for Day 7, 1969, the satellite just inside the magnetopause (PAD 1) moves in a particle shadow created by the drift loss cone, which is delimited by the last closed field line at noon. However, particles in a narrow range of pitch angles $55^{\circ}<\alpha<60^{\circ}$ (and by

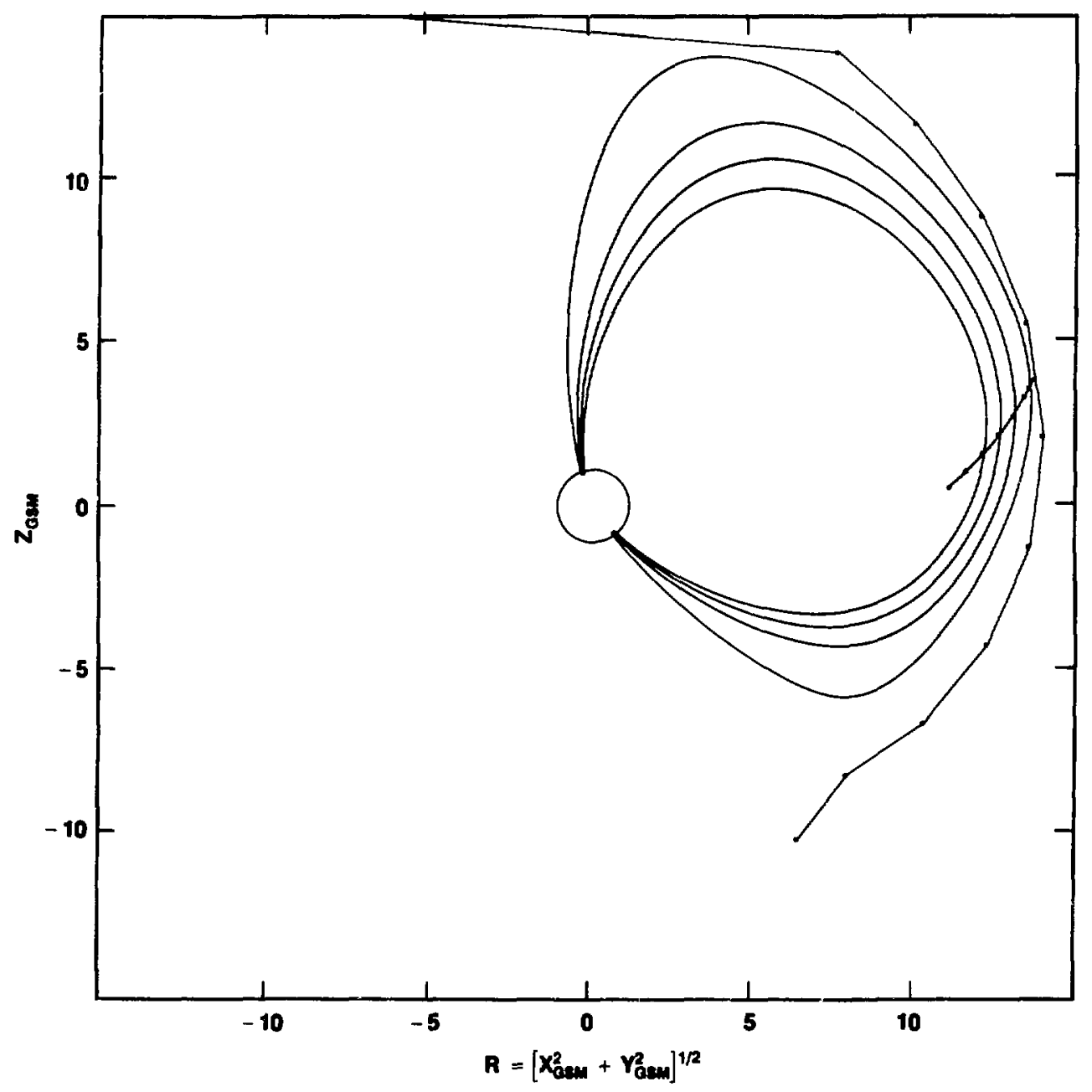

Figure 4-81. Outer magnetoepheric field lines in the azimuth model, appropriate to the inbound pass of Day 5, 1969. Also shown is the satellite trajectory. 
bounce symmetry at $120^{\circ}<\alpha<125^{\circ}$ ) will arrive at the satellite via the rejoining of shells which branched pre-noon, as indicated by the intersection of the PADs with the branching curves constructed for the azimuths $\phi=15^{\circ}$ and $30^{\circ}$. The rejoining of the branched shells in the early afternoon magnetosphere returns particles from the noon minimum-B recions to equator-crossing orbits which can reach the satellite. Particles that have taken the equatorial segment of branched drift shells are lost to the magnetopause pre-noon. We expect that experimental PADs obtained in this region would show a flux maximum in the $55^{\circ}<\alpha<60^{\circ}$ range.

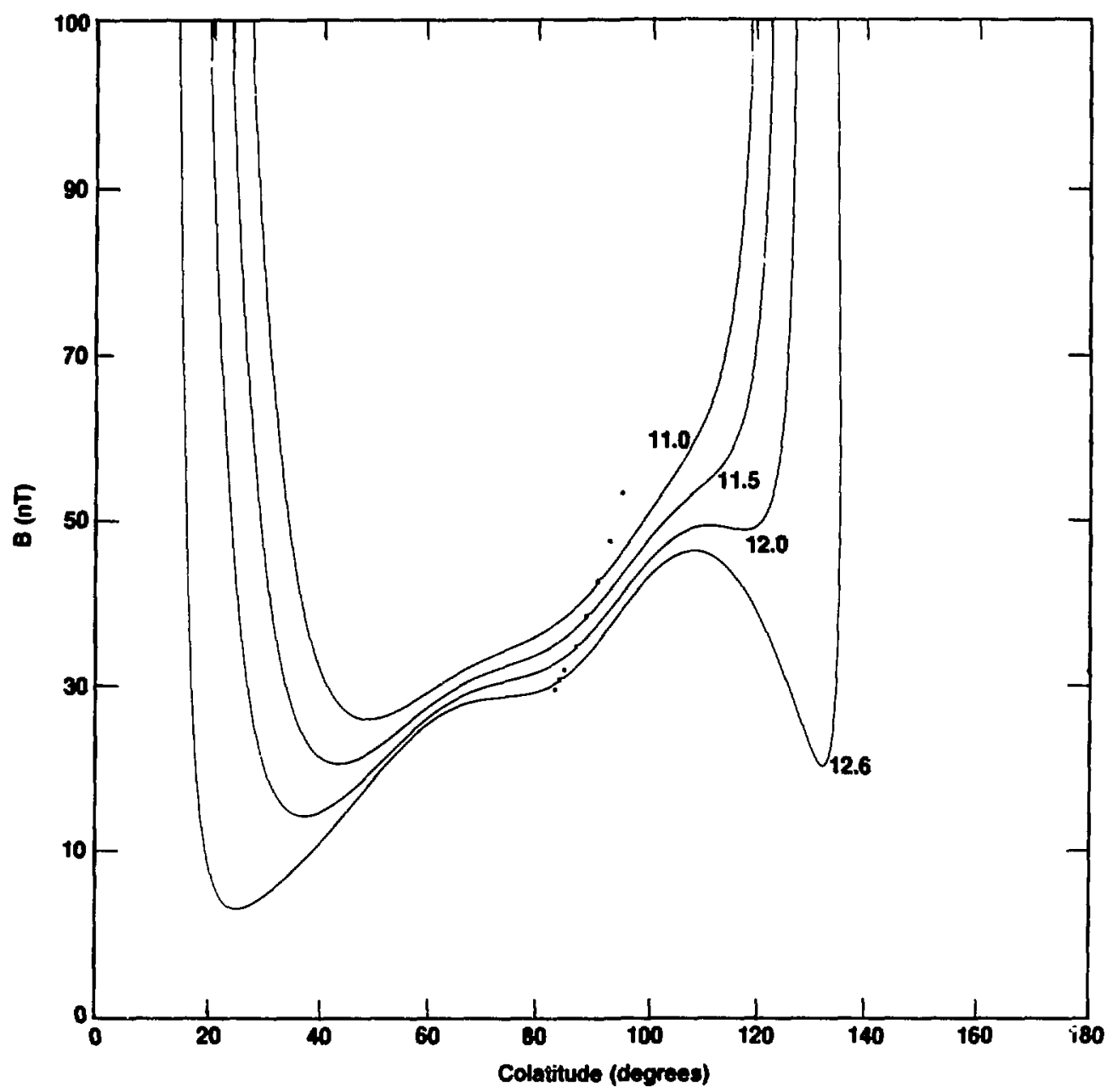

Figure 4-82. Magnetic field magnitude vs GSM colatitude 9 GSM as computed from the Choe-Beard model along outer magnetospheric lines which intersect the spacecraft trajectory on Day 5, 1969. (The equator is at $\theta_{\mathrm{CSM}}=9^{\circ}$ ). The spacecraft position is shown as a dot on each line. Lines are labelled on the right with the radial distance of the spacecraft in $R_{E}$. Because of the extreme dipole tilt $\left(-33^{\circ}\right)$ only a single local B-maximum exists, to the south of the satellite at colatitude $\approx 100^{\circ}$. 


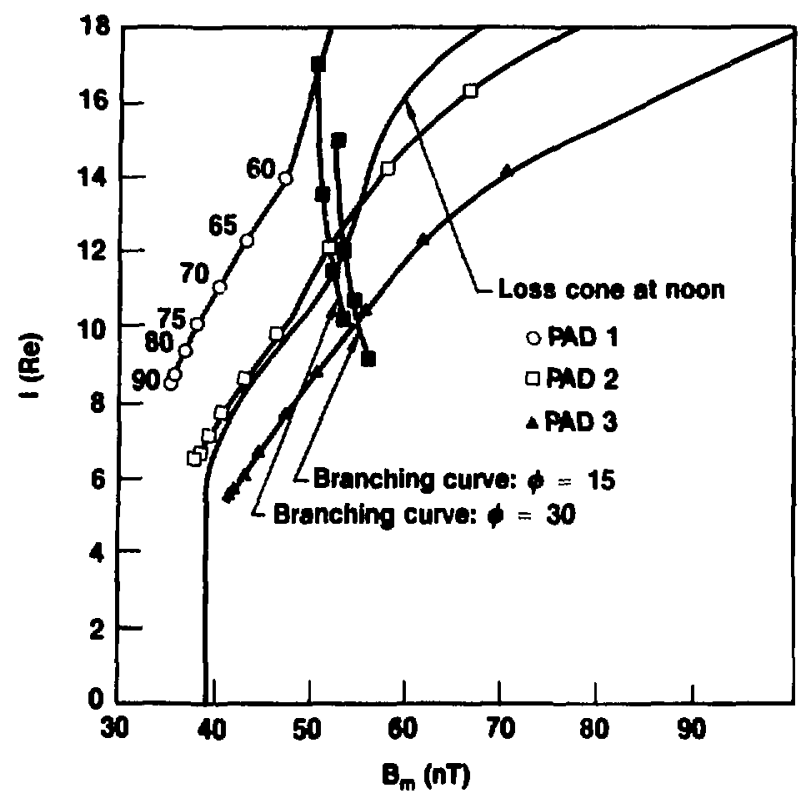

Figure 4-83. Adiabatic drift shell configuration for Day 5, 1969, as computed from the Choe-Beat 1 model. Shown are the $B_{M} I$ traces of three PADs along the inward-bound spacecraft trajectory, annotated with spacecraft pitch angle. Also depicted is the drift loss cone at noon, delimited by the last closed noon field line. Unbranched drift shells characterized by $B_{M} I$ points to the left of this irace do not close within the magnetosphere. Branching curves are drawn at azimuths of $15^{\circ}$ and $30^{\circ}$. Each point on a branching curve designates the $B_{M}, I$ value of a branched shell which rejoins at that azimuth. The intersection of the branching curves with the PADs indicates the pitch angle range at which the satellite will receive fluxes that drift on rejoined ahells, having passed around noon through the high-latitude minimum-B regions. This range is approximately $55^{\circ}<\alpha<65^{\circ}$.

Closer in, the satellite begins to emerge from the drift shadow (PAD 2). Electrons with pitch angles at the satellite $<60^{\circ}$ are now able to drift adiabatically from the moming sector to the satellite on unbranched drift shells. Fluxes which drifted through the noon minimum-B pockets continue to be observed at $\alpha \approx 60^{\circ}$. We expect to see PAD flux levels increase in the range $\alpha<60^{\circ}$.

The satellite moving earthward passes out of the particle shadow altogether (PAD 3), so that particles adiabatically diifting from the morning sector will be observed at all pitch angles. Particles on rejoined shells are observed in the range $60^{\circ}<\alpha<65^{\circ}$ until the satellite passes inward beyond the limit of shell branchisg. PAD: measured in this region should show a flux buildup for $\alpha>60^{\circ}$, leading eventually to the "normal" type of PAD.

\section{Day 5 Energetic Electron Pitch Angle Distributions}

Figure 4-84 displays a sequence of pitch angle distributions obtained by the lowest energy electron channel $E 1$ as the satellite moved inwards from the magnetopause. The PAD at $R=11.99 R_{E}$ is characteristic of the shadowed region, where only electrons which have passed thiough noon in the highlatitude minimum-B pockets can reach the satellite, according to adiabatic theory. This PAD peaks near the predicted pitch angles of $60^{\circ}$ and $120^{\circ}$. PADS in this outer region are highly variable, as exemplified by the PAD at $R=11.61 R_{E}$ which shows a flux dropout at pitch angles $<80^{\circ}$, but maintains a maximum 


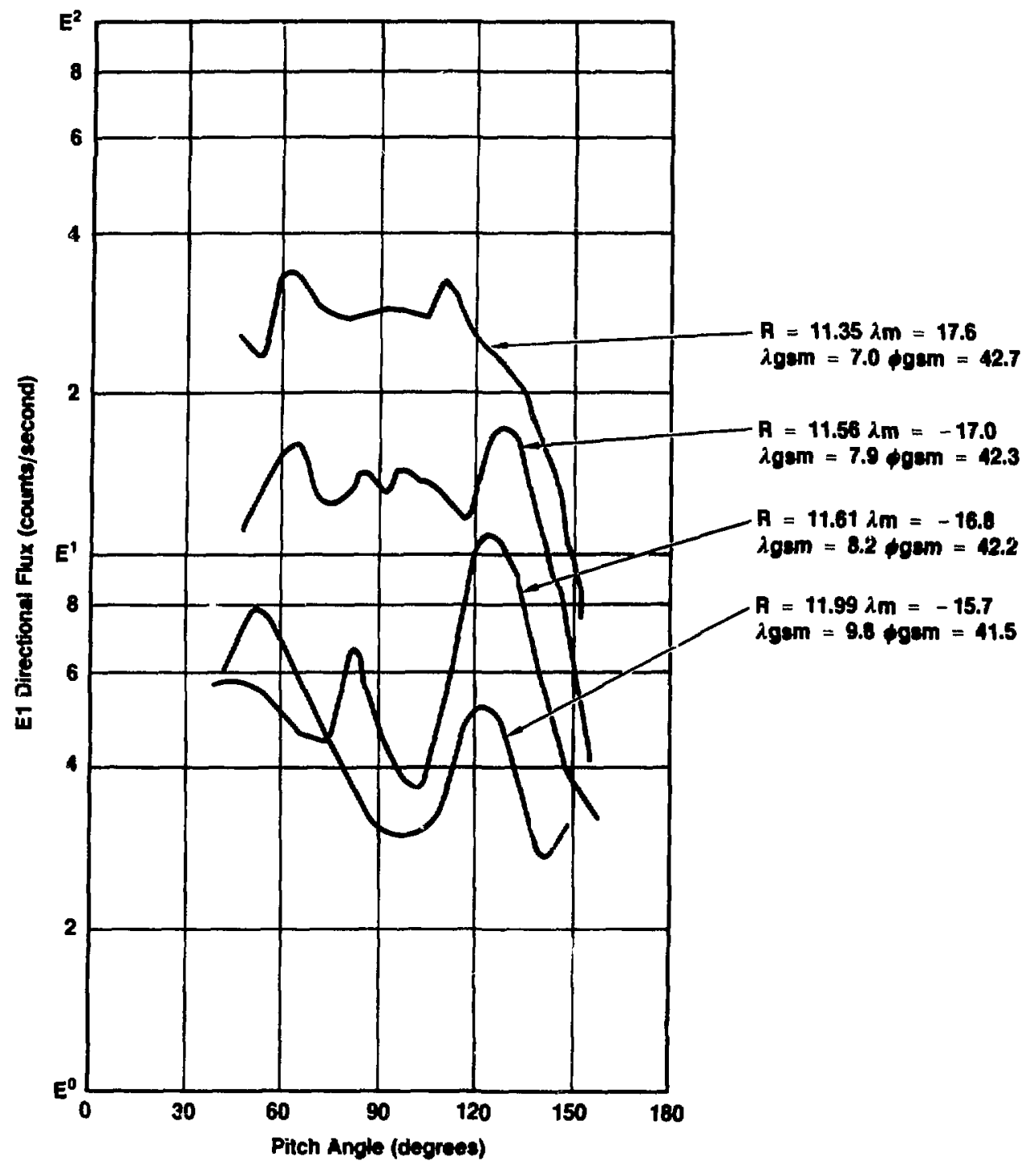

rigure 4-84. Sequence of E1 PADs obtained by OGO-5 on Day 5, 1969, as the satellite moved earthwards from the magnetopause (at $R \approx 12.9 R_{E}$ ).

at $\alpha \approx 120^{\circ}$. PADs measured closer to earth $\left(R=11.56\right.$ and $\left.11.35 R_{E}\right)$ show an increase of flux in the $60^{\circ}<$ $\alpha<120^{\circ}$ range, indicating that the satellite was emarging from the jurift shadow.

Beyond this point, the El PADs (Fig. 4-85) show the flux near $90^{\circ}$ grad ally filling in until the distributions attained the "normal" shape at $R=9.69 R_{E}$. Flux transitions at the pitch angles marking rejoined shells are still evident in the more distant PADs $\left(R=10.95\right.$ and $\left.10.62 R_{E}\right)$, though with decreasing amplitudes. The pitch angles of rejoined shells now correspond to local flux minima in the PADs. 


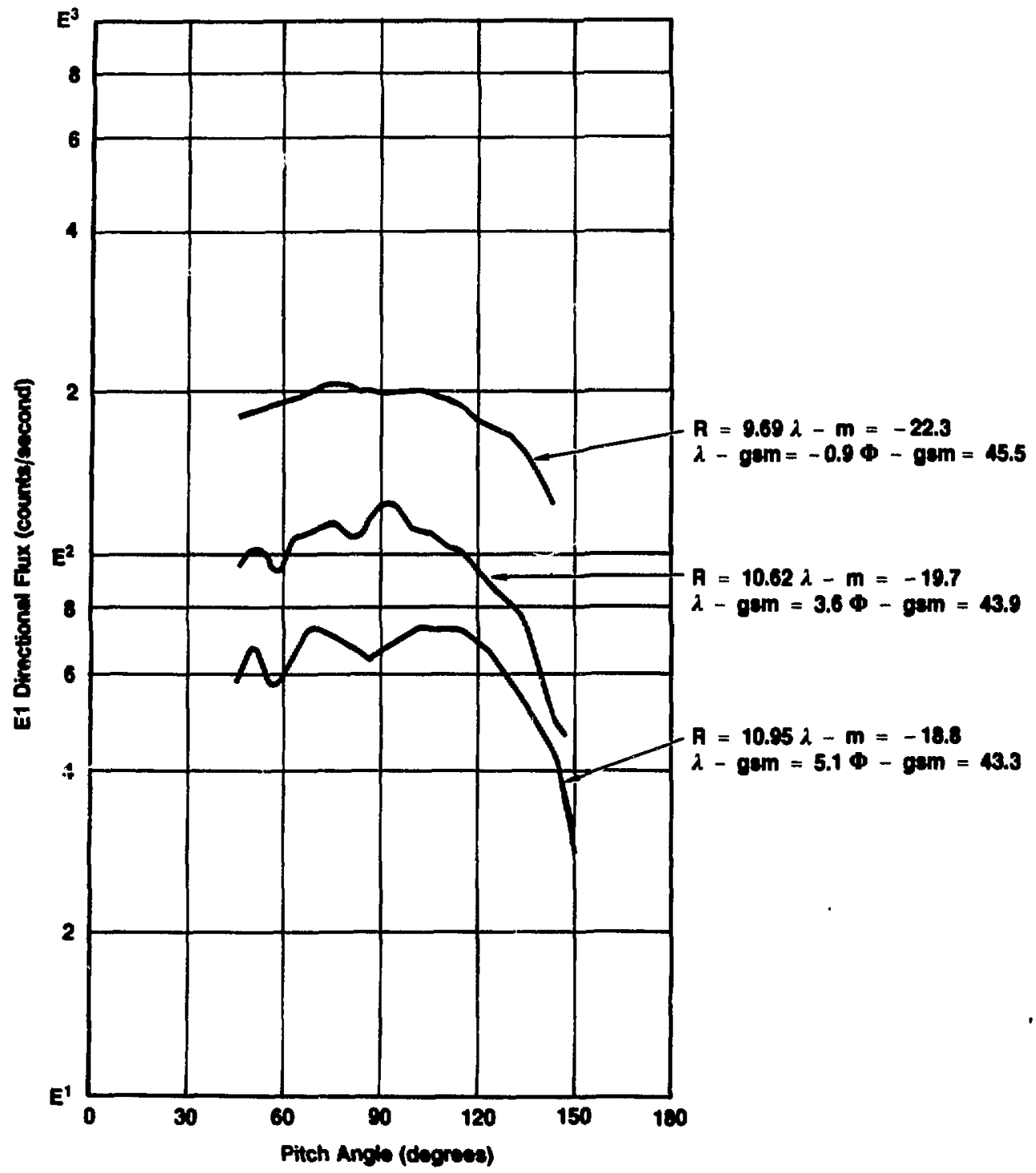

Figure 4-85. Sequence of E1 PADs subsequently obtaint : Sy OGO-5 on Day 5, 1969.

In this region channel E2 began registering significant fluxes (Fig. 4-86). Here the flux transitions at pitch angles corresponding to rejoined drift shells are very apparent in the PADs acquired at $R=10.95$ and $10.62 \mathrm{R}_{\mathrm{E}}$. Although the distributions are somewhat asymmetric, the distributions show a peak near $60^{\circ}$ adjacent to a local minimum near $65^{\circ}-70^{\circ}$. The distributions continue to fill in near $90^{\circ}$ as the satellite moved earthward, until a "normal" type distribution was measured at $R=9.69 \mathbf{R}_{\mathrm{E}}$. 


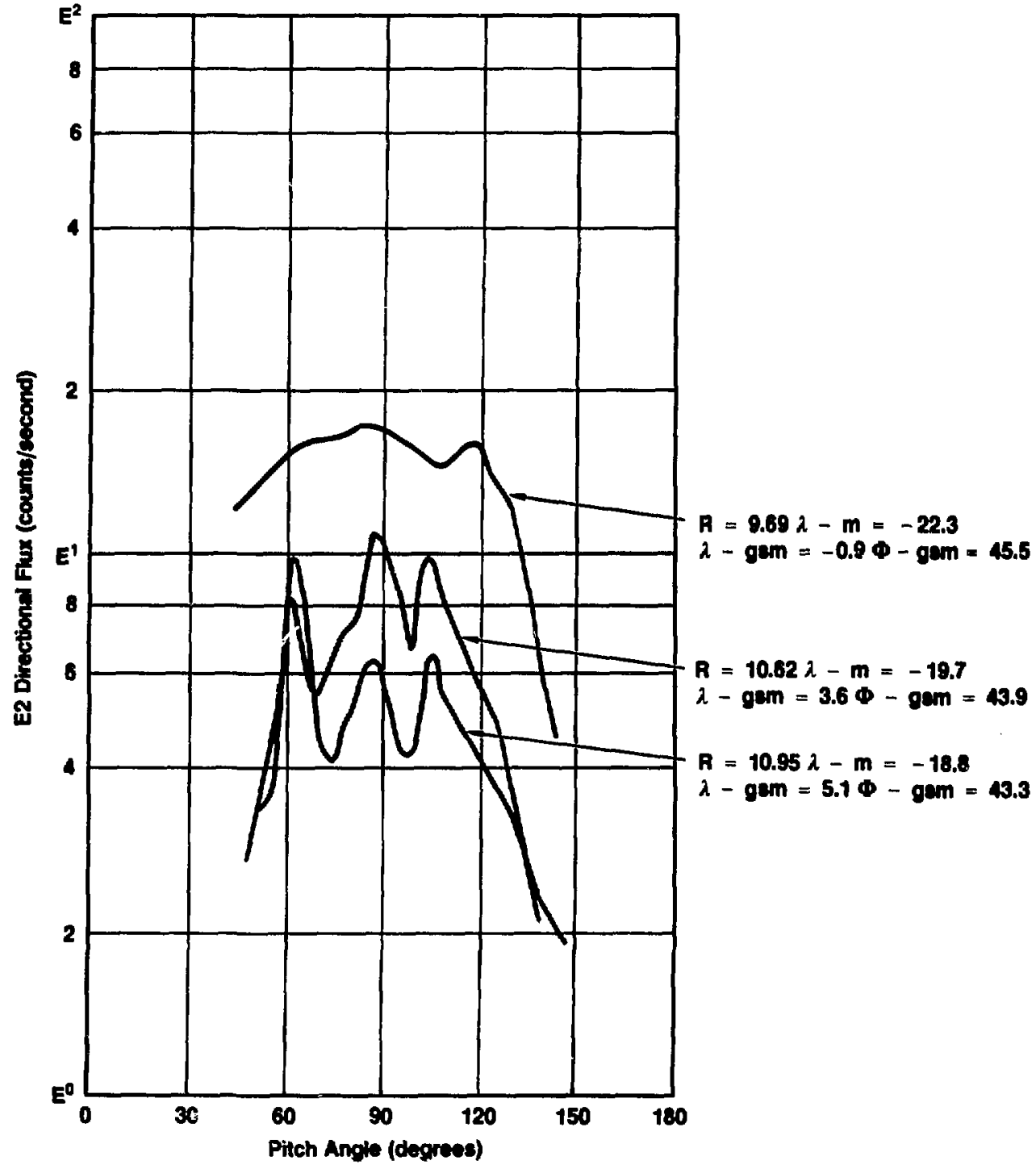

Figure 4-86. Sequence of E2 PADs obtained by OGO-5 on Day 5, 1969, at the same locations as in Fig. 4-85.

\section{CGO-5 Observations on Day 10 (january 10), 1969}

PADs on Day 10, 1969 were acquired during another inbound satellite pass through the outer equatorial magnetosphere in the afternoon sector, in the azimuthal region where we expect branched drift shells to rejoin. The pass occurred at angular coordinates $\phi_{\mathrm{GSM}}=37^{\circ}, \lambda_{\mathrm{GSM}}=4^{\circ}, \lambda_{\mathrm{M}}=-14^{\circ}$. Dipole tilt was very large, at $-26^{\circ}$. Figure $4-87$ depicts the satellite trajectory in the equatorial plane of the Choe-Beard 


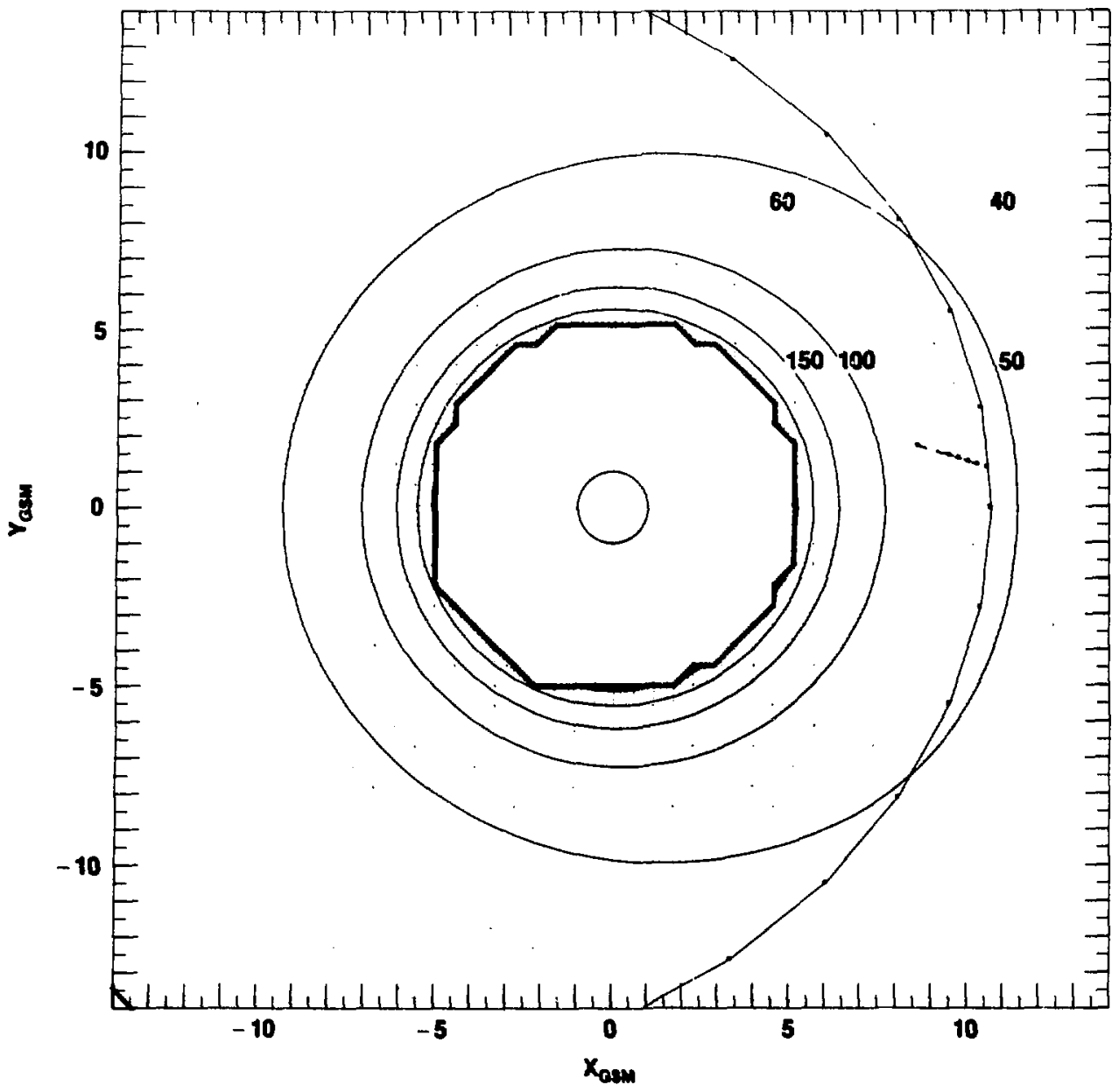

Figure 4-87. Contours of constant magneic field magnitude (nanotealas) in the equatorial plane for the Choe-Beard model, appropriate to the OGO-5 epacecraft pase of Day 10, 1\%69. The epasecraft trajectory appears as a ohort heavy line at about $37^{\circ}$ past noon. (Contours outside magnetosphere are not valid).

model. Figure 4-88 shows the field measured by the UCLA magnetometer. Entry into the magnetosphere first occured at $0920 \mathrm{UT}, \mathrm{R}=13.0 \mathrm{R}_{\mathrm{E}}$, as the observed field declination swung to near-zem. Subsequent episodic changes in declination and magnitude at $\approx 0930$ and 0936 UT may indicate that the fluctuating magnetopause momentarily overtook the sateilite, leaving it for a time in the characteristically noisy magnetosheath. We will present PAD observations following $1000 \mathrm{UT}$, when the field components showed only smooth variations. Figure $\$-89$ gives the E1 directional flux during the interval of interest. The flux increased to magnetospheric levels at $\approx 1004$ UT. The occasional drop-outs in particle flux (at 1012,1025 , and 1036) correlate closely with the $\approx 5 \mathrm{nT}$ increase seen in the Fig. 4-88 magnetometer record, indicating that the field increases resulted from the loss of diamagnetic plasma. 


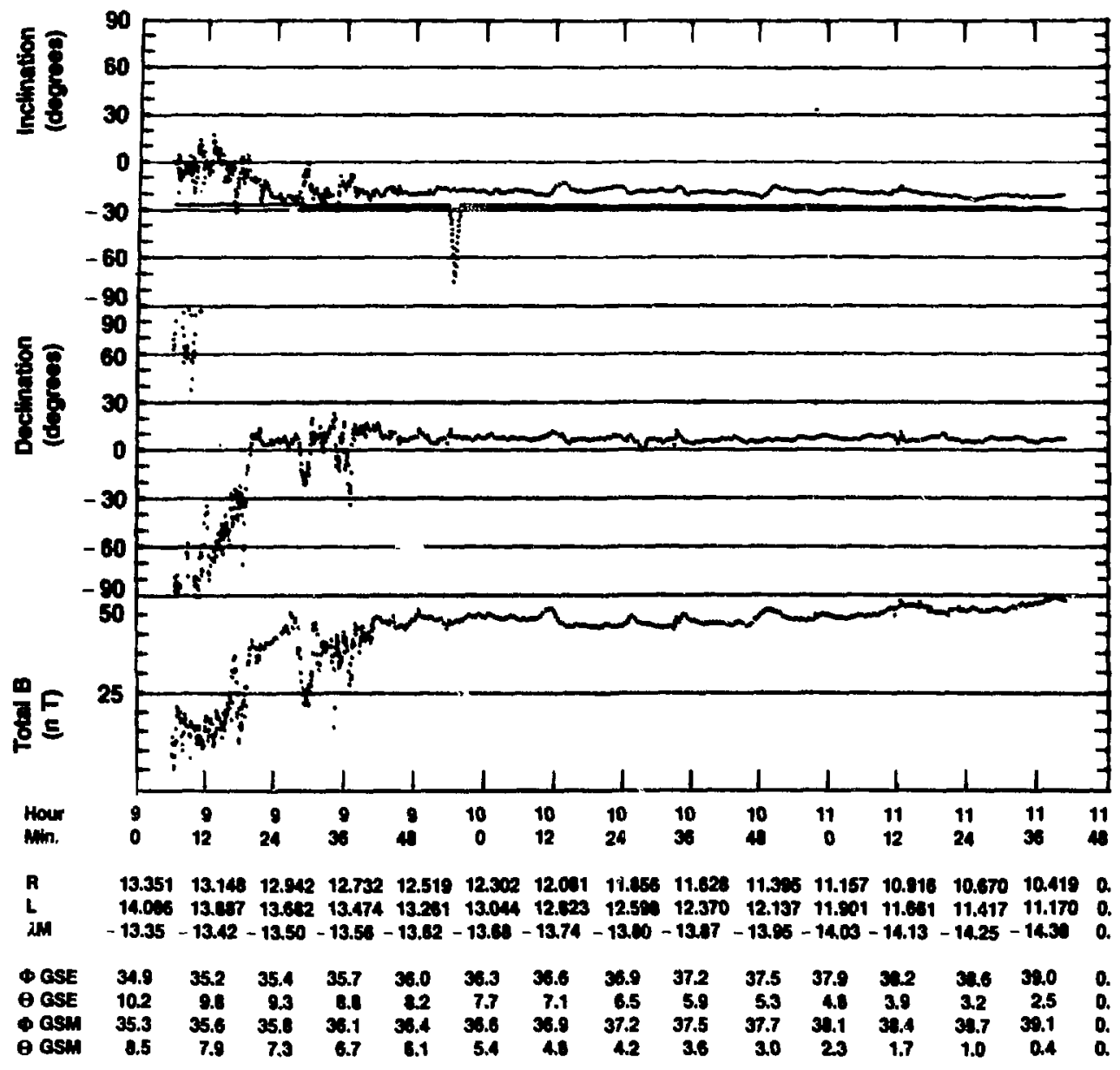

Figure 4-88. Magnetic field observed by the UCLA magnetometer experiment during the OGO-5 inbound pass of Day 10, 1969. X-axis is Universal Time. Upper panels give the inclination and declination of the vector magnetic field, with reference to the dipole coordinate system. Lower panel is field magnitude in nanoteslas. Satellite coordinates are given below each time annotation. Magnetopause crossing occurred at approximately $0920 \mathrm{UT}\left(\mathrm{R} \approx 13.0 \mathrm{R}_{\mathrm{E}}\right)$.

\section{Day 10 Field Mu ding}

The model magnetic field configuration for this pass is portrayed in the next few figures. Figure 4-90 illustrates the geometry of the outermost field lines along the satellite trajectory. Choe-Beard mode! parameters are $R_{S O}=11.84 R_{E}$, tilt $=-25.8^{\circ}$. The magnetic field variation along these lines is given in Fig. 4-91. We see the formation of a single B-pocket on the outermost lines.

Figure 4-92 illustrates the adiabatic drift features on this day. PAD 1, computed for a position just inside the magnetopause along the satellite trajectory, lies wholly in the adiabatic drift shadow cast by the last closed fieldline at noon. The only electrons able to reach this point arrive via branched shell segments 


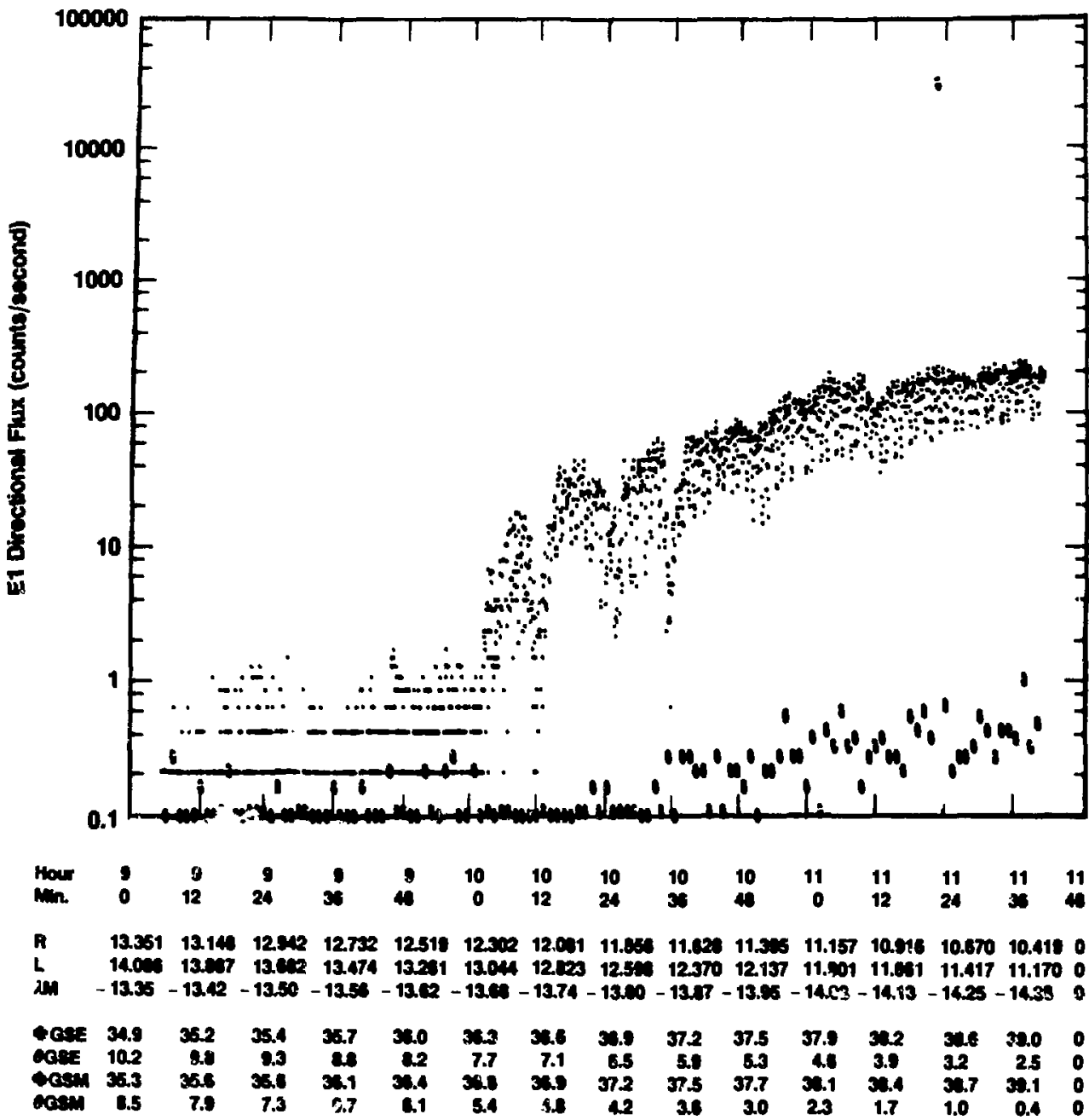

Figure 4-89. Electroa counting sate in the loweat-energy electron channel E1 (79 keV) obwerved during the OGO-5 inbound paw of Day 10, 1969. The counting rate rose to magnetospheric levels at about 1 LSA UT $\left(R \approx 12.2 \Gamma_{\mathrm{rg}}\right)$.

which pass through the noon high-latitude - inimum-B pockets. They are detected in the narrow pitch angle range $60^{\circ}<\alpha<65^{\circ}$ (and by bounce symmetry, in range $115^{\circ}<\alpha<120^{\circ}$ ). As the satellite moves earthward, it passes out of the drift shadow and begins detecting particles at pitch angles away from $90^{\circ}$. PAD 2 illustrates the condition of partial shadowing, where only particles in the range $\alpha<65^{\circ}$ can drift around noon to the satellite on unbranched shells. Further along the trajertory (PAD 3), the sittellite has completely emerged from the drift shadow but still detects electrons which move on newly rejoined drift shells. These appear at the satellite at pitch angles in the range $65^{\circ}<\alpha<70^{\circ}$. 


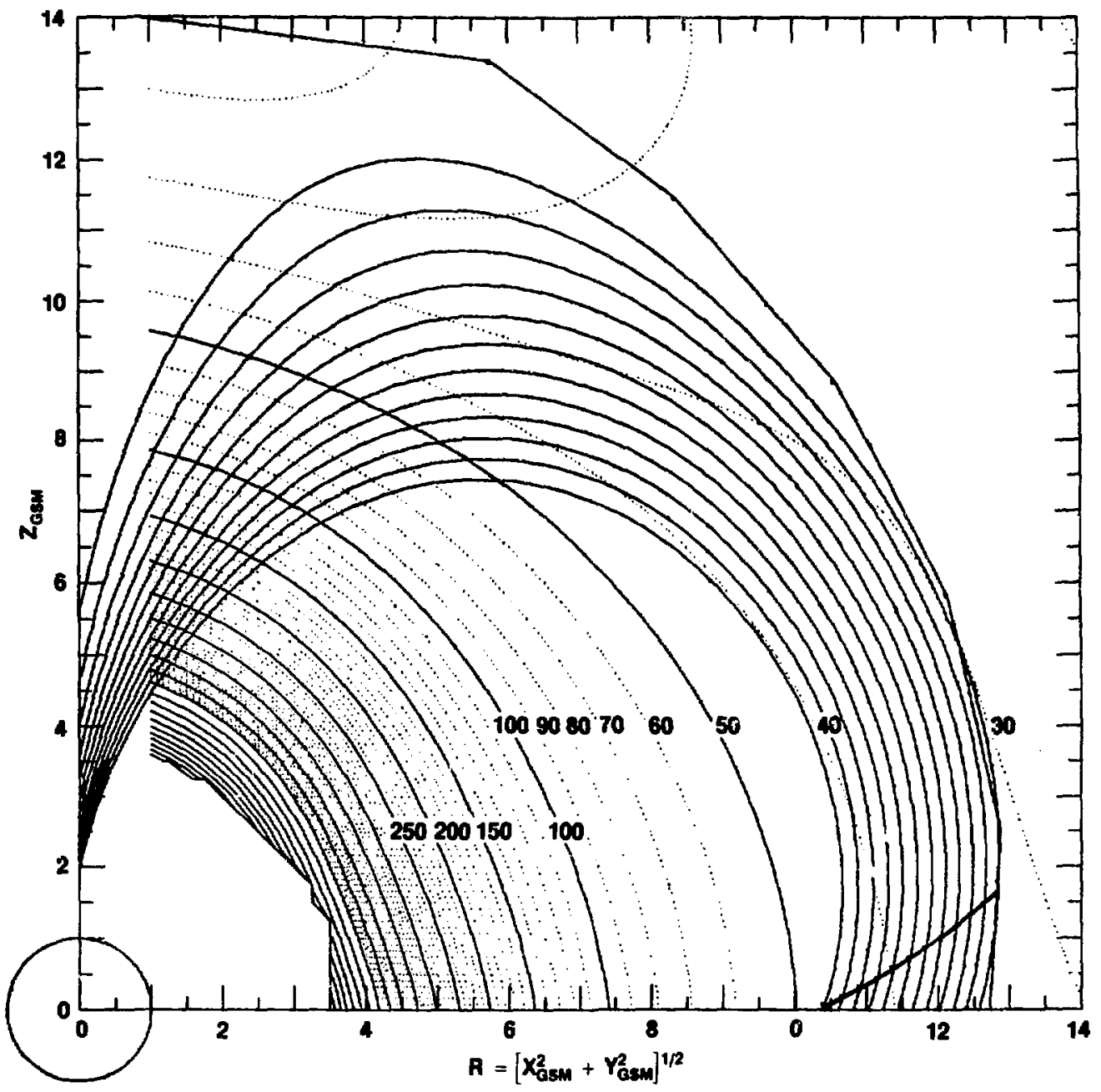

Figure 4-90. Outer magnetospheric field lines and constant-B contours in the azimuth $\phi_{C S M}=37^{\circ}$ for the Choe-Beard model, appropriate to the inbound pass of Day 10, 1969. The satellite trajectory appears as the short heavy trace.

\section{Day 10 Energetic Electron Pitch Angle Distributions}

The PADs acquired by OGO-5 in the region near the magnetopause are shown in the next series of figures. In Fig. 4-93, we see a PAD obtained close to the location where counting rates rose to significant levels, at $R=12.21 \mathrm{R}_{\mathrm{E}}$. It shows peaks at $\alpha=60^{\circ}$ and $120^{\circ}$, consistent with the predictions of the ChoeBeard model that only fluxes on rejoined shells can drift adiabatically through this region. Further earthwards the distribution broadens in the "wings" and begins to fill in near $\alpha=90^{\circ}$, indicating that the satellite began to receive fluxes drifting on unbranched shells from the pre-noon sector. 


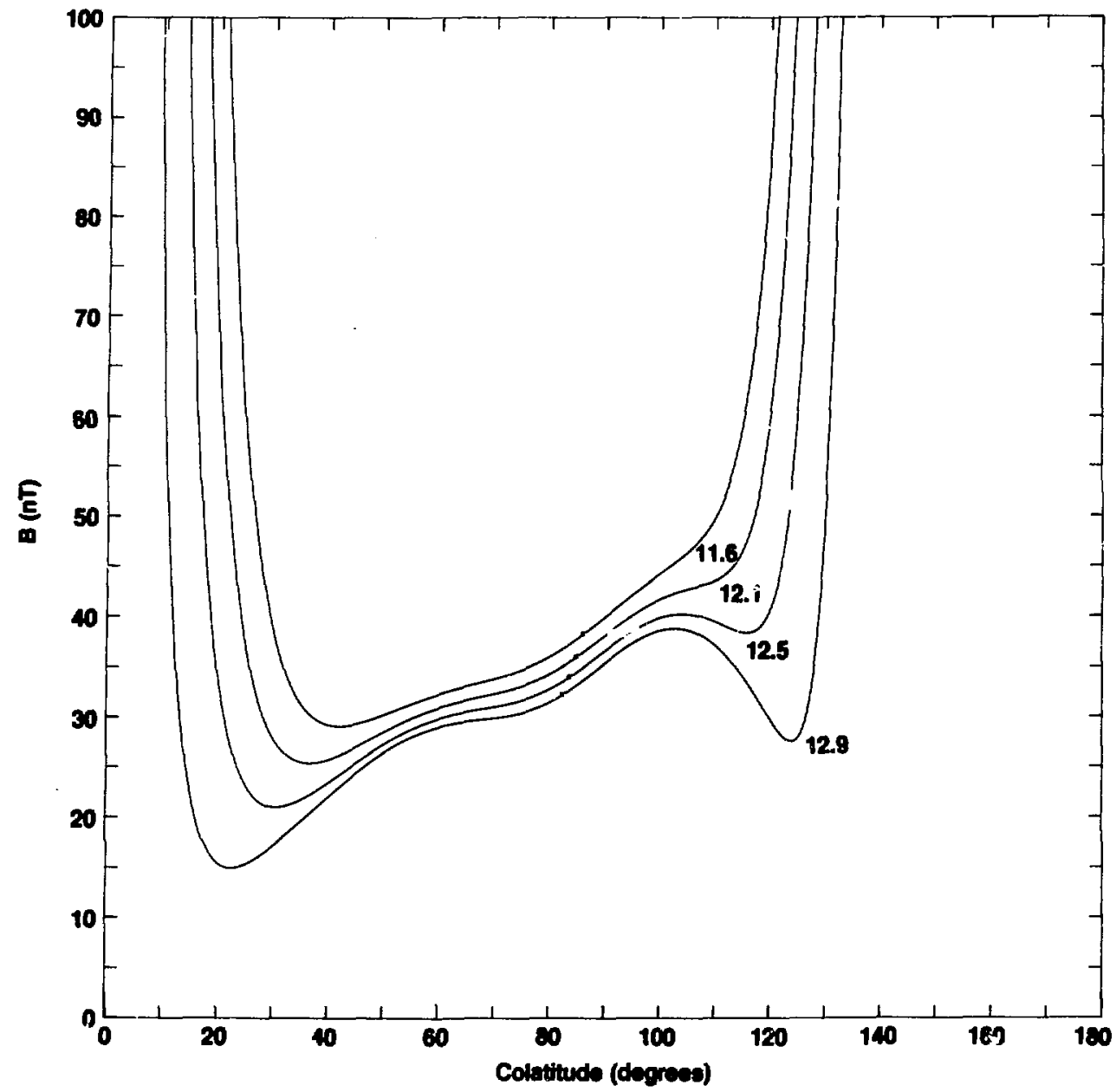

Figure 4-91. Magnetic field magnitude vs GSM colatitude 6 GSM as computed from the Choe-Beard model along suter magnetoopheric lines which internect the ipacecraft trajectory on Day 10, 1969. (The equator is at $\theta_{\mathrm{csm}}=90^{\circ}$ ). The epacecraft position is shown as a dot on each line. Lines are labelled on the right with the radial dintance of the spacecraft in $R_{\mathrm{E}}$. Because of the large dipole tilt $\left(-26^{\circ}\right)$ on! a single local B-maximum exists.

Figure 4-94 continues the sequence of E1 PADs. Flux levels dropped by a factor of two to iour in the interval between the acquisition of the $P A D$ at $R=11.97 R_{E}$ (prior figure) and the one at $R=11.71$, with the largest decrease for $a>60^{\circ}$. A steep flux gradient developed with a flux mirimum near $90^{\circ}$, suggesting the recurrence of magnetopause shadowirg, perhaps caused by an inward fluctuation of the noon magnetopause. By $R=11.61$ flux levels had recovered and local minima appeared in the distributions near $\alpha=65^{\circ}$ and $115^{\circ}$. When the satellite moved inward to $R=11.14 R_{E}$, the E1 channel reported near "normal"-type distributions. 


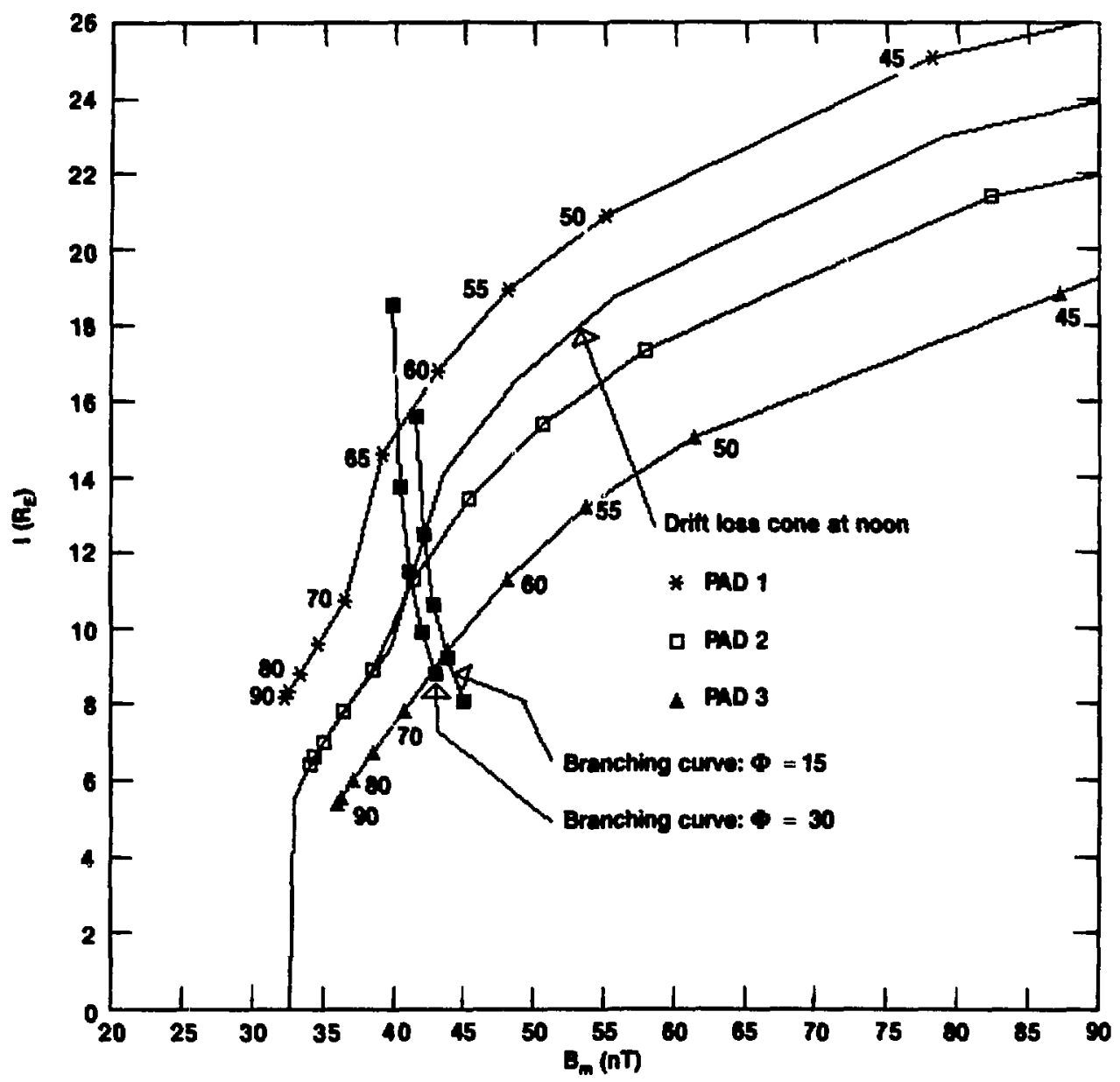

Figure 4-92. Adiabatic drift shell configuration for Day 10, 1969, as computed from the Choe-Beard model. Shown are the $B_{M} I$ traces of three PADs along the inward-bound spacecraft trajectory, annotated with spacecraft pitch angle. Aleo depicted is the drift low cone at noon, delimited by the last closed noon field line. Unbranched drift shells characterized by $B_{M}$ I points to the left of this trace do not close within the magnetosphere. Branching curves are drawn at azimuthe of $15^{\circ}$ and $30^{\circ}$. Each point on a branching curve denignates the $B_{M}$ I value of a branched shell which rejoins at that azimuth. The intersection of the branching curves with the PADs indicates the pitch angle range at which the satellite will receive fluxes that drift on rejoined shells, having paseed around noon through the high-latitude minimum-B regions. This range is approximately $60^{\circ}<\pi<70^{\circ}$.

Figure 4-95 records the PADs obtained by channel E2 during the interval of interest. Although counting rates are quite low, distinct peaks are seen at $\alpha=60^{\circ}$ in PADs at $R=11.88$ and $11.71 R_{E}$ moving to $\alpha \approx 70^{\circ}$ and $110^{\circ}$ at $R=11.61$ and $11.45 R_{E}$. By $R=11.14$ a smoothly-varying normal distribution was observed. 


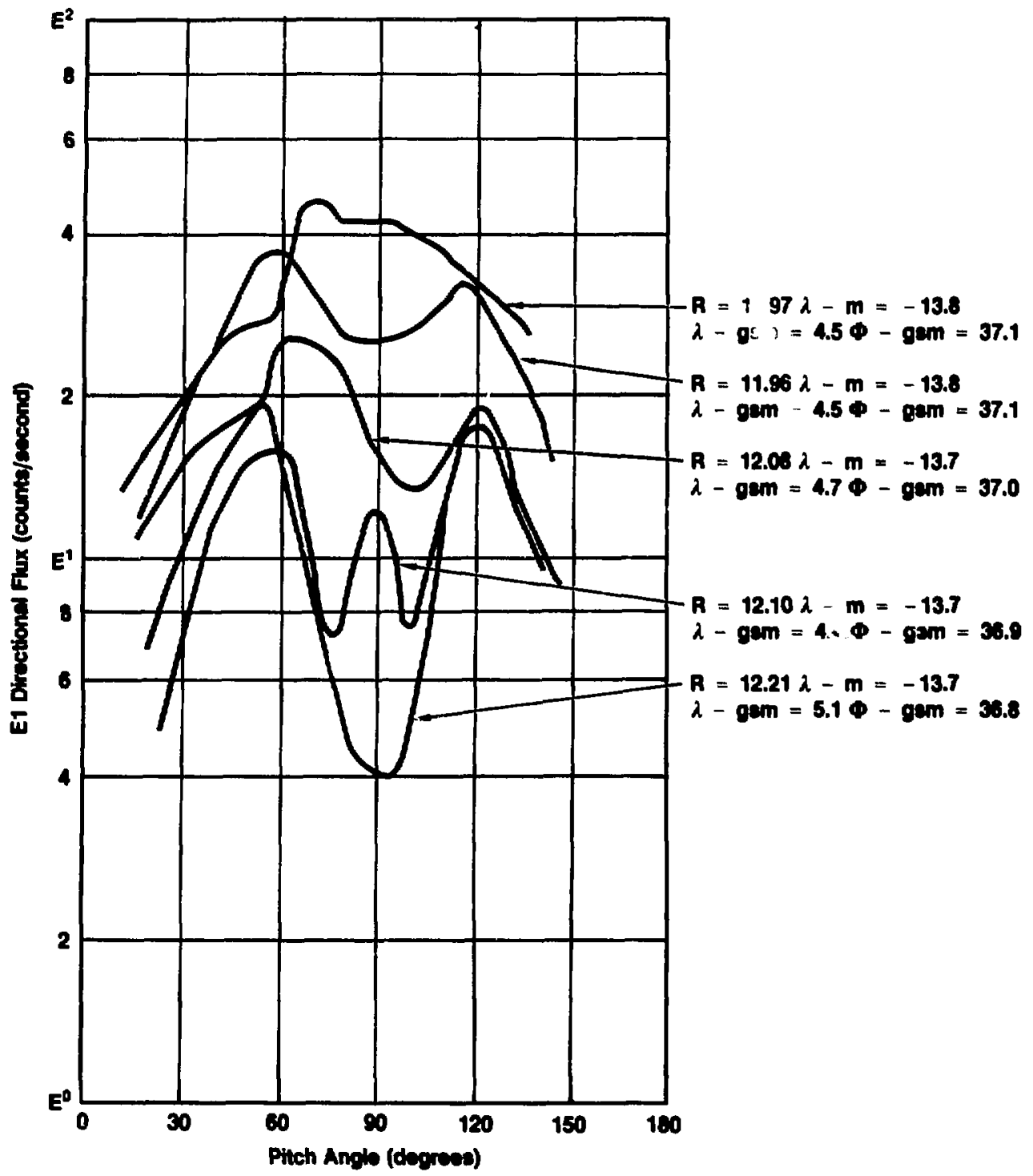

Figure 4-93. Sequence of E1 PADs obtained by OGO-5 on Day 10, 1969, at the satellite moved earthwards from the magnetopause (at $R \approx 13.0 R_{E}$ ). 


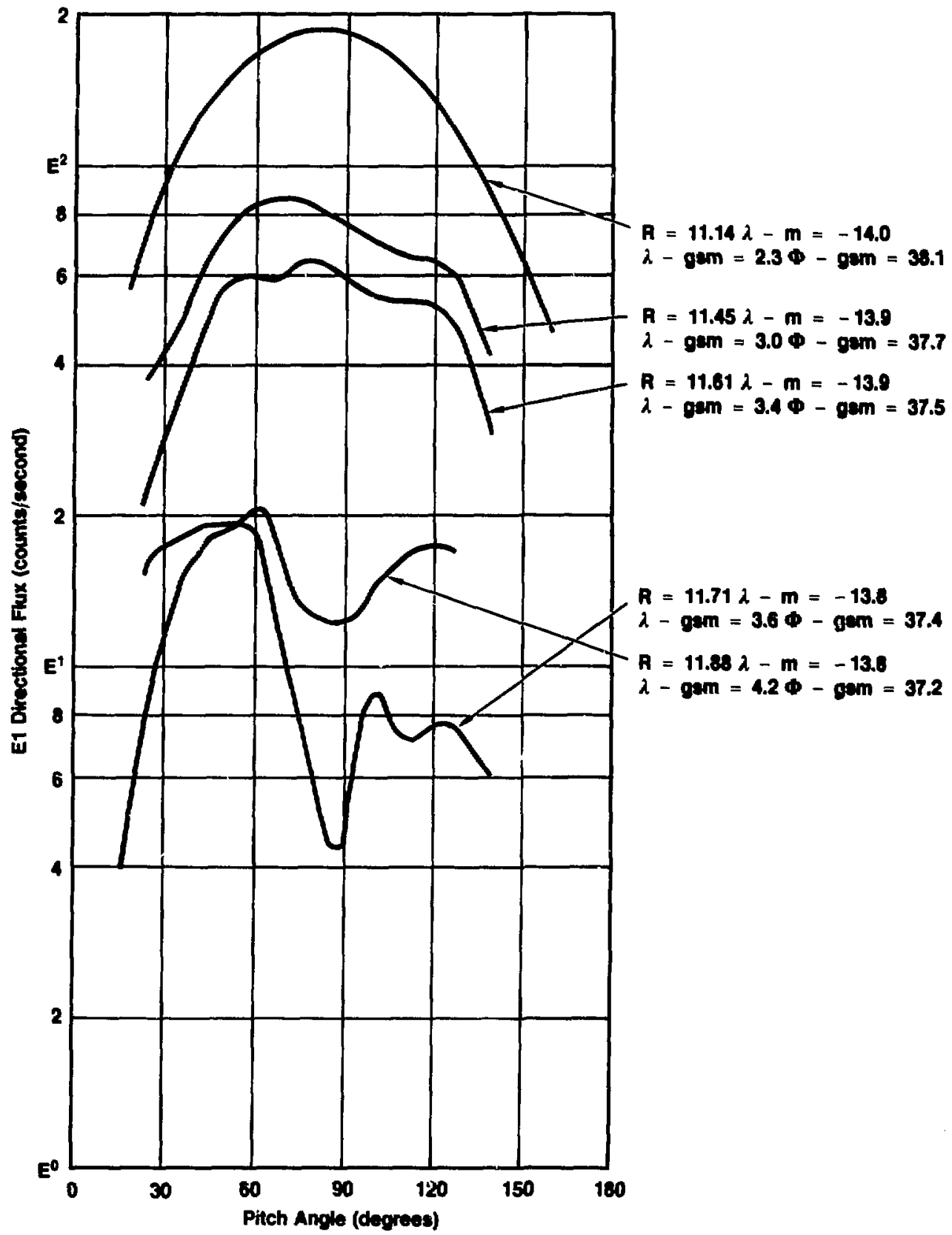

Figure 4-94. Sequence of E1 PADs subsequently obtained by OGO-5 on Day 10, 1969. 


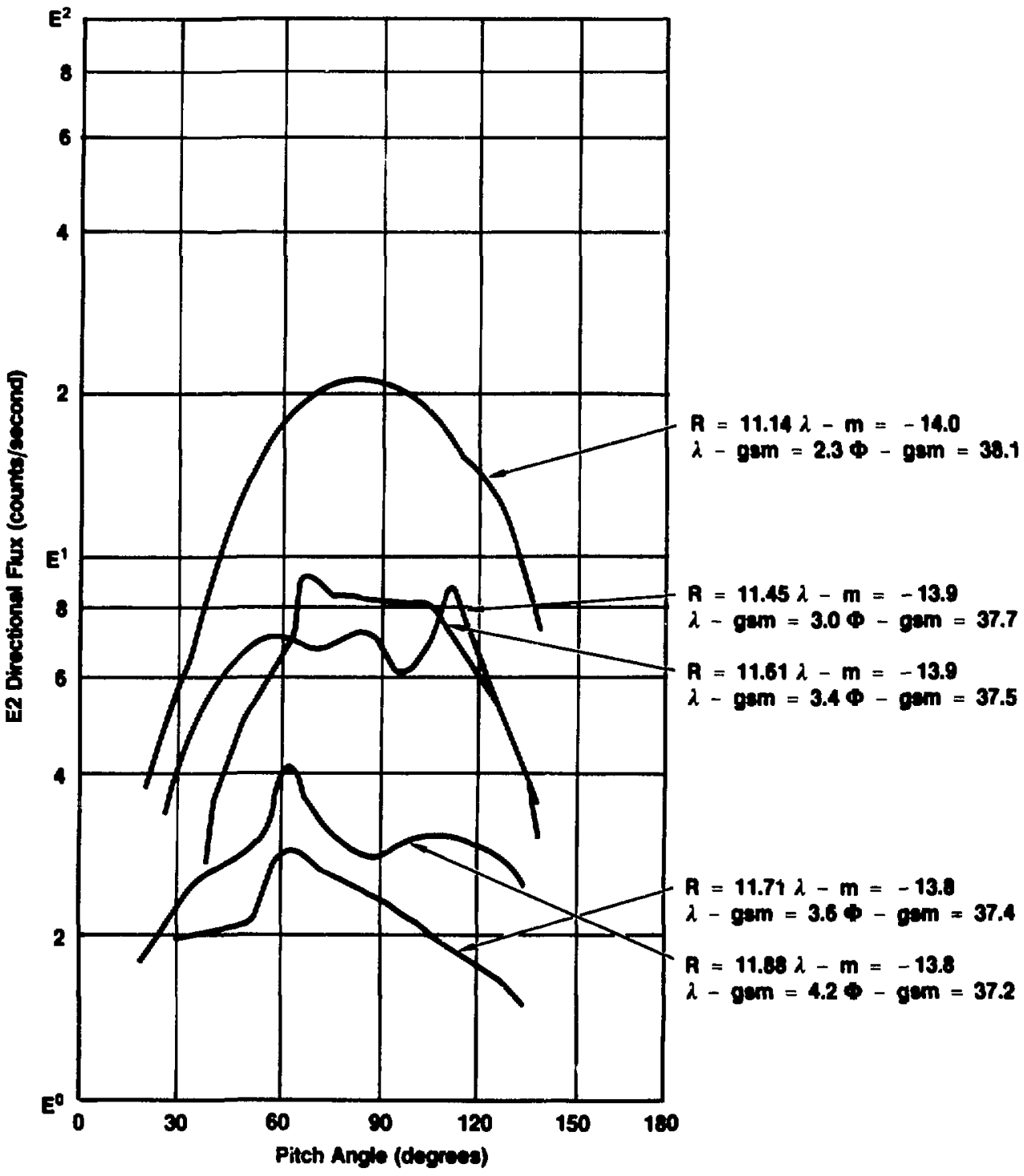

Figure 4-95. Sequence of E2 PADa obtained by OGO-5 on Day 10, 1969, at the same locations as in Fig. 4-94.

\section{Discussion of Experimental Results}

The data presented in the preceding pages were acquired within $\approx 1 R_{E}$ of the magnetopause. These PADs show a volatility of flux level and shape which is characteristic of outer magnetospheric fluxes. Flux dropouts and recoveries can occur, and $\mathrm{dj} / \mathrm{d} \alpha$ gradients can reverse, all on a timescale of minutes. Flux distributions are frequently asymmetric about $\alpha=90^{\circ}$, contrary to those observed in the magnetospheric 
core. Despite these temporal variations, the PADs show two regions that exhibit distinctive flux behaviors. An inner region centered about $\alpha=90^{\circ}$ shows large fluctuations and frequently steep flux gradients. The other region, comprising the "wings" of the PAD beyond the inner region, varies more slowly and exhibits fewer flux features. The boundaries between the two regions are usually marked by large flux transitions. The angular positions of the PAD flux transitions are seen to agree well with the location of branched drift shells, as predicted by the Choe-Beard magnetospheric magnetic field model.

Many of the PAD features evident in these data can be explained in terms of the adiabatic drift of electrons through the distorted and fluctuating magnetic fields of the outer magnetosphere. In the morningto-noon sector (exemplified by the data of Days 66 and 4l) the satellite PADs sampled two particle populations: electrons mirroring equatorward of the local-B maxima (Region 2 of Day 66, Region 1 of Day 41), and electrons mirroring poleward of them. Because the equatorward population has small values of second invariant $I$, the electrons tend to drift along equatorial plane contours of constant-B. The fluctuating magnetopause compresses and expands the equatorial field, and can cause this population to strike the magnetopause and be lost before reaching the satellite. The presence of the local B-maxima on the satellite field line causes the second population seen by the satellite to mirror far off the equator and possess large values of $\mathrm{l}$; these electrons are relatively unaffected by fluctuations in the equatorial field. Therefore shadowing events preferentially remove the equatorward population, producing large flux changes in PADs at pitch angles correspording to the local B-maxima. If these outer field lines had instead a normal geometry (i.e., possessed a single minimum at the equator), the PAD particles' I-values would span a continuous range, and a shadowing event would produce gradual and continuous changes to observed PADs, contrary to our observations on these days.

Flux dropouts in the equatorward regions, accompanied by large flux transitions at the region boundaries, are evident in the PADs for both Day 66 and Day 41 . They occur at $R=12.06,12.03$, and $11.35 R_{E}$ on Day 66 for all detectors, and at $R=10.35 R_{E}$ on Day 41 for detectors E2 and E3.

At other times, usually at positions closer to the earthward limit of shell branching, one sees PADs with a peak near a region boundary, but without the flux dropout in the equatorward region that indicates magnetopause shadowing. Such PADs resemble the synthetic PADs which were constructed for Day 66 by adiabatically mapping fluxes from midnight to the satellite (Figs. 4-48 and 4-49). These PADs are characterized by an equatorward distribution that is flat or increases away from $\alpha=90^{\circ}$, with sharp flux dropoffs at the region boundaries.

The PADs obtained at 11.95 and $11.71 R_{E}$ on Day 66, and those at $R=10.62$ (E1 and E2), 10.41 (E2), 10.26 (E3) and $9.99 R_{E}$ (E3) on Day 41 are of this type, although in no case are the flux transitions as sharp as those of the synthetic distributions. This discrepancy between model and measurement has two probable causes.

The fluxes observed on $\Gamma \cdot 66$ mapped to midnight fieldlines which had $R_{E O}$ values in the range 9.3 $<R_{E Q}<18 R_{E}$, with a ?.capping discontinuity for $9.6<R_{E Q}<11.8 R_{E}$. These ranges overlap the earthward terminus of the model ta:I current sheet, at $10 R_{E}$. Inside this radius the model has no current system, although the actual field (as sinown by the $\Delta B$ contours of Fig. 4-14) indicates large field depressions in this region due to the so-called ring currents circulating about the earth in the equator. One would expect the discontinuous termination of the tail current sheet to produce unrealistic edge artifacts in the model field, producing significant errors in the invariant mappings near the edge.

Even if the mapping were accurate, we have assumed purely adiabatic particle motion in a static field. The fuctuations observed in the outer magnetospheric field will act to diffuse particles in $B_{M}, I$ space, reducing flux gradients in the PADs.

Three of the data sets presented here were obtained on inbound passes which probed the outer equatorial magnetosphere in the afternoon sector, where our model predicted that branched drift shells rejoin. Our graphical analysis in $B_{M}, I$-space of the adiabatic drift trajectories predicted for electron distributions drifting around from the morning sector led us to expect the following PAD features, as the satellite moved inwards from the magnetopause:

1. Just inside the magnetopause, the satellite lies in the drift shadow cast by the noon magnetopause, which shields the satellite from all particles drifting around noon on unbrariched drift shells. Only particles which passed around noon in the high-latitude minimum-B pockets, and subsequently moved onto normal equator-crossing bounce trajectories when the branched shell segments rejoined post-noon, can reach the satellite. Consequently we expect to see flux peaks at pitch angles corresponding to these B-pocket fluxes, and low flux levels elsewhere. 
2. Moving inwards, the satellite begins to pass out of the adiabatic drift shadow. Fluxes increase, first at pitch angles smaller than that of the branched shells, and then at angles closer to $a=90^{\circ}$.

3. Further inwards, the satellite emerges completely from the drift shadow. Flux levels increase most rapidly near $90^{\circ}$, and the PAD eventually attains the normal-type distribution seen at the symmetric prenoon position. But the satellite after emergence still observes fluxes which move on rejoined drift shells, including contributions from both the equatorial and the high-latitude branched shell segments. In the absence of sources or losses along the particles' trajectories from the morning sector, the magnitude of these fluxes at the satellite would not be different from that of fluxes at adjacent pitch angles which travelled to the satellite on unbrancheci shells. We expect the fraction of particles which move through the high-latitude minimum-B pockets to undergo enhanced scattering, mirroring as they do close to the magnetopause and close to the equatorward edge of the noontime cusp, which as we have seen is a region of strong wave activity and pitch-angle scattering. This would produce a local flux minimum in the PAD at angles corresponding to the rejoined shells.

Of the three aftemoon passes, only the one on Day 7 produced distributions with flux features and trends which evolved in a consistent way in all channels, as the satellite moved inwards. The first PADs presented at $R=9.99$ and $9.80 R_{E}$ were acquired in the partial shadow region, where only fluxes with pitch angies in the range $\alpha<60^{\circ}$ and $\alpha>120^{\circ}$ arrived at the satellite. Closer to earth, the PADs began to record fluxes in the region about $90^{\circ}$, at $R=9.46$ and $9.35 R_{E}$. Local flux minima appeared at angles corresponding to fluxes drifting on rejoined shells $\left(\alpha \approx 60^{\circ}-70^{\circ}\right)$. By $R=8.71 R_{\mathrm{E}}$ the distributions had become broad and fairly featureless. These PADs accord qualitatively well with the adiabatic drift model we have advanced here.

The PADs obtained on the other two passes were subject to more variability, both in time (at a fixed energy) and in energy (at a fixed time), and are not so uniformly interpretable as the Day 7 data. However, all of the adiabatic features mentioned above as characteristic of PADs in the region of shell rejoining can be recognized. For instance, the initial members of the sequence of PADs on Days 5 and 10, which were obtained in the shadow region, show flux peaks near the predicted shell-rejoining pitch angle of $\alpha=60^{\circ}$ and $120^{\circ}$, with a subsequent filling in of fluxes near $90^{\circ}$. Some of the PADs acquired inwards show the development of local flux minima at these angles [at $R=11.56(E 1), 10.62$ (E2) on Day 5; $R=11.61$ and 11.45 (E1) on Day 10]. Other PADs did not fit this pattern but showed irregular features, some of them peaking at these angles [e.g., at $R=11.61$ and 11.45 (E2) on Day 10] when overall flux levels were low.

Overall, the post-noon data provide supporting evidence for the notion that a noon-time minimum-B pocket is both a conduit for a branched electron population drifting around the outer noon magnetosphere, and also a scatterer for thoze electrons. When a PAD measurement is made in the post-noon drift shadow region where only electrons on minimum-B trajectories can reach the satellite, a flux peak is observed at the pitch angles corresponding to rejoined drift shelis. When a measurement is made outside the drift shadow, the loss of flux by scattering in the minimum-D regions appears as a local flux minimum.

Given the high variability of PADs in the outer dayside magnetosphere, where significant flux changes occur on the time scale of minutes, and where PAD asymmetries and energy-dependencies are common, it is perhaps surprising that effects predicted from purely adiabatic drift theory are not completely masked by the substantial dynamic effects. This work shows that the phenomenon of drift-shell branching does strongly modify the distributions of drifting particles to produce distinctive flux transitions in observed PADs over a small and stable angular range. We find that the location of these transitions accords well with adiabatic predictions utilizing the Choe-Beard magnetospheric magnetic field model. This is a most significant result.

\section{Shell-branching Studies Using Other Magnetic Field Models}

We turn now to other models of the magnetic field to investigate the model-dependence of shell branching. V. P. Shabansky pioneered branching studies (Shabansky, 1966), using a version of the HonesTaylor image dipole model (Hones, 1963). This model consisted of the geomagnetic dipole plus an auxiliary or image dipole (with a variable moment and distance from earth) placed along the earth-sun line in order to simulate the action of the solar wind on the earth's field (Antonova and Shabansky, 1968). A sheet current situated in the night side equatorial plane and flowing in the GSM Y-direction modeled the effect of the tail current system. Figure 4-96 depicts this model in noon-midnight cross section, with contours of constant-B. In this instarice the sheet current is limited to $-51 R_{E} \leq x \leq-11 R_{E}$. The model contains dayside minimum-B pockets (Fig. 4-97) similar to those of the Choe-Beard model. Shabansky 


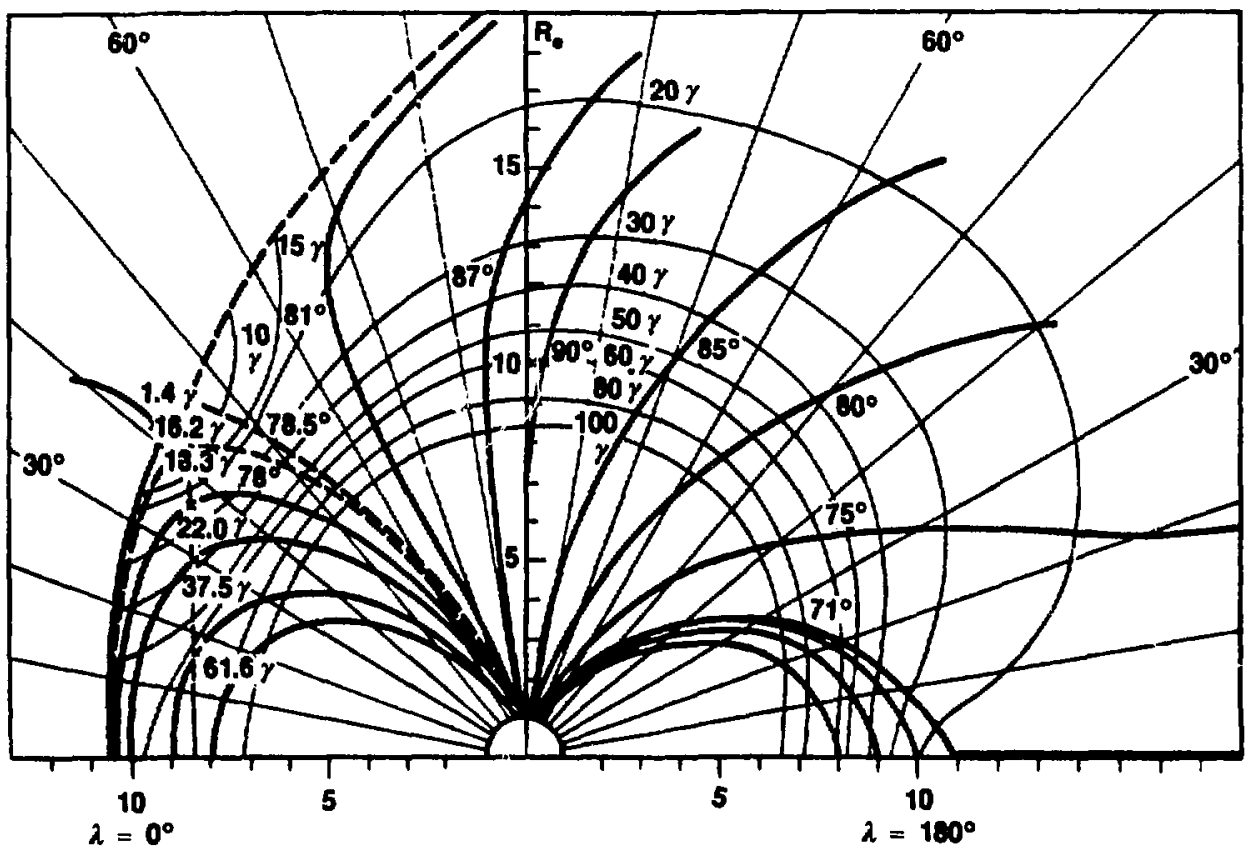

Figure 496. Field lines and constant-B contours in the noon-midnight meridian of the Shabansky image-dipole model (Antonova and Shabansky, 1968). B-pocket regions are evident near the polar cusp.
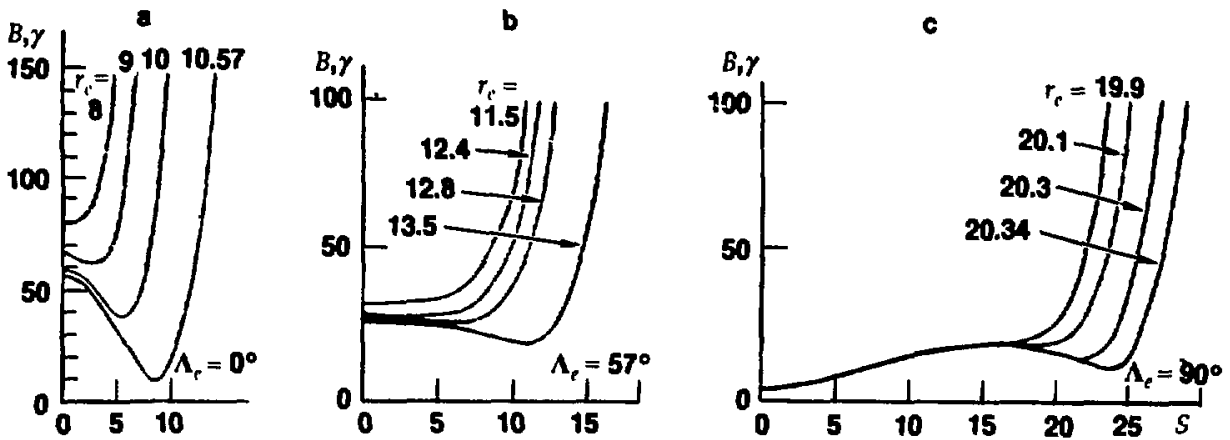

Figure 4-97. Variation of $B$ vs field line arc length $s$ (in $R_{E}$ ) for the Shabansky model (Antonova and Shabansky, 1968). Configuration is shown for azimuths of $0^{\circ}, 57^{\circ}$, and $90^{\circ}$. Lines are labeled with their $\mathbf{R}_{\mathrm{EQ}}\left(\mathbf{R}_{\mathrm{E}}\right)$. B-pockets are seen for all azimuths pictured. 
found (Shabansky and Antonova, 1968) that the B-pockets are topologically connected to the midnight equatorial regions (unlike the Choe-Beard model), so that midnight particles with $I=0$ can pass around noon through the pockets. His zone of stable trapping in the noon meridian (shown in Fig. 4-98) is similar to the one we derived for the Choe-Beard model (Fig. 4-32). The omnidirectional flux measurements noted earlier which support a high-latitude dayside trapping boundary as predicted by the Shabansky model, are consistent with the Choe-Beard model as well. The Shabansky model B-pockets when traced adiabatically back to midnight form a region which includes the equator, whereas the Choe-Beard model branching region at midnight is limited by an equatorial pitch angle $\alpha_{0}<65^{\circ}$ (Fig. 4-28).

The Olson-Pfitzer 1974 model is another analytic representation of the magnetospheric magnetic field which is in general use (Olson, 1974; Olson and Pfitzer, 1974). It forms the total field as the vector sum of the field due to the geomagnetic dipole $\left(B_{G}\right)$, the field due to magnetopause currents $\left(B_{M P}\right)$, and the field due to distributed currents in the magnetosphere $\left(B_{D}\right)$. Figure 4-99 illustrates the Olson-Pfitzer field lines in the noon-midnight rcgion. The presence of $B_{D}$ allows this model to reproduce well the observed field depression caused by the ring curitent for $L<5$ (Suguira and Poros, 1973). However, the magnetopause current distribution was obtained by a self-consistent magnetopause calculation (Olson, 1969) in which the only interior field was $B_{G}$ (and not $B_{G}+B_{D}$ ). As a cci sequence the model exhibits unphysical behavior in the outer magnetosphere where the B-pocket rezions lie. Some of the model field lines penetrate the magnetopause as defined by the self-consistent calculation. Perhaps a more critical problem,

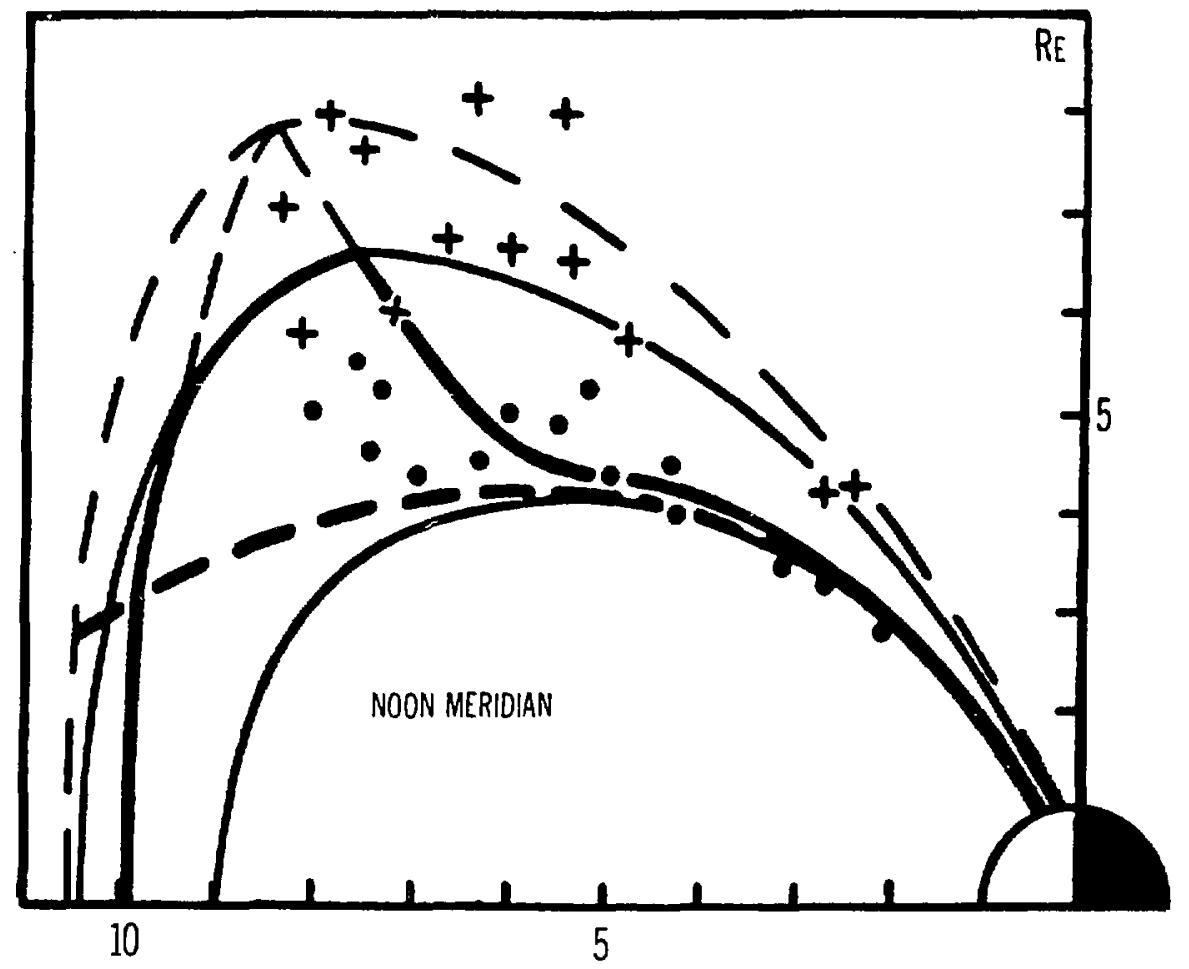

Figure 4-98. Limit of stable trapping in the noon meridian, for the Shabanoky model (thick line). Shown for comparison is the boundary as determined by Roederer (1969) (thick dashed line), and experimental data (crosses and dots) reported by Vernov et al. (1968). The crosess represent the outer boundary, the dots the inner boundary of the zone of unatable radiation (Shabansky, 1971). 
from the standpoint of an investigator who wishes to compute particle trajectories in the outer magnetosphere, is that the radial gradient of the field changes sign. For about $2 \mathbf{R}_{E}$ inwards of the noon magnetopause, the equatorial field decreases earthward (Fig. 4-100). This effect is simply a modeling artifact which is inconsistent with in-situ observations and causes electrons in the model field to drift retrogradely. Even absent these significant difficulties, the lack of an adjustable dipole tilt parameter and magnetopause standoff parameter make it impossible to adjust the model to closely match the ambient conditions on any satellite pass.

The Mead-Fairfield 1975 model (Mead and Fairfield, 1975) is a widely-cited empirical model which is derived from a large number of satellite magnetometer measurements. The total field is represented by a 17-coefficient fit of a polynomial in $x, y$, and $z$ to the vector field observations (Fig. 4-101). This model by

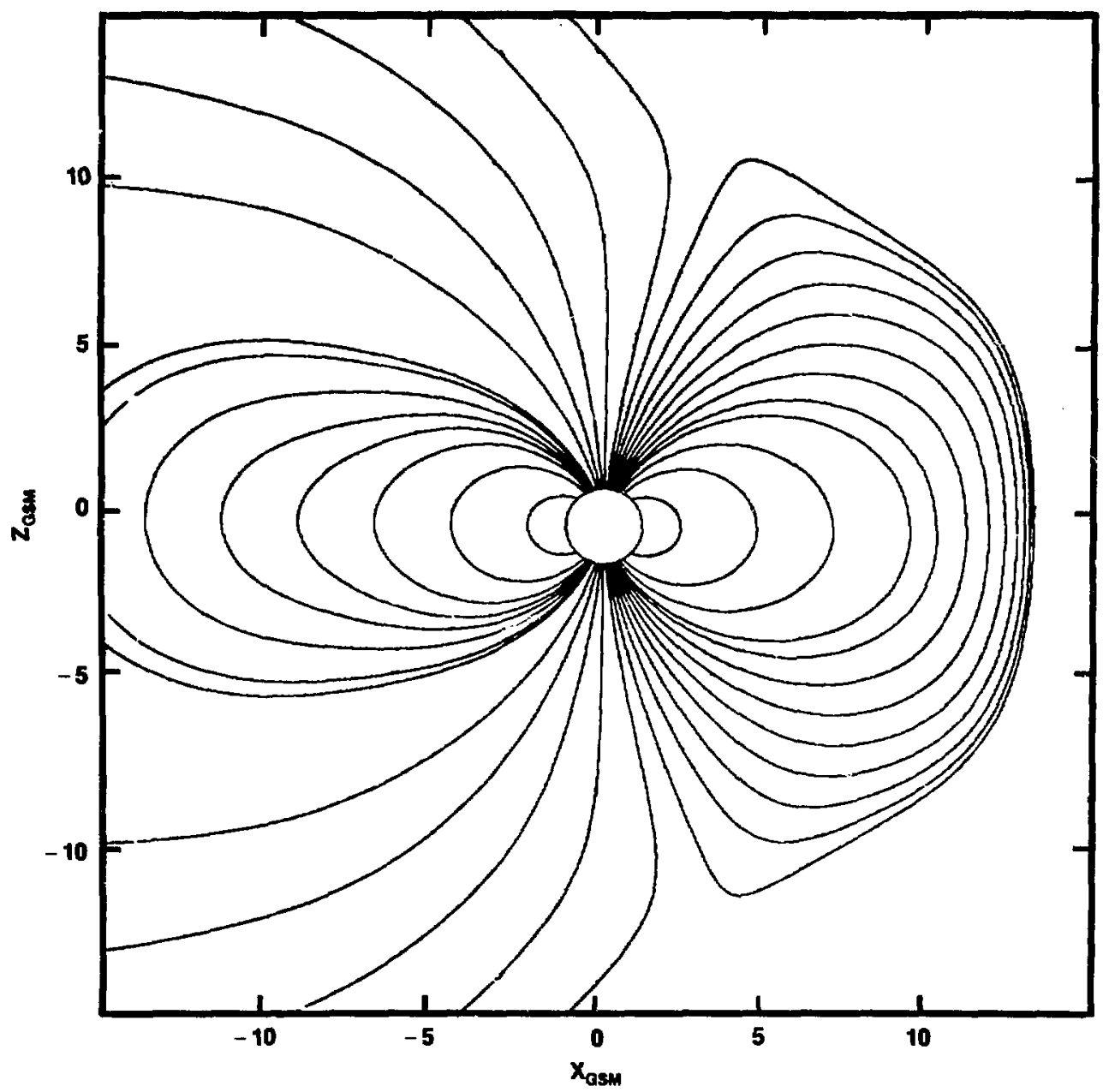

Figure 4-99. Cross section in the noon-midnight plane of the Olson-Pfitzer 1974 magnetospheric magnetic field model. 
Figure 4-100. B vs colatitude $\theta$ along outer noon field lines in the Olson-Pfitzer model. Lines are labeled with their $R_{E Q}\left(R_{E}\right)$. Note the outward gradient of $B$ in the equator, a nonphysical effect arising from inconsistent boundary treatment.

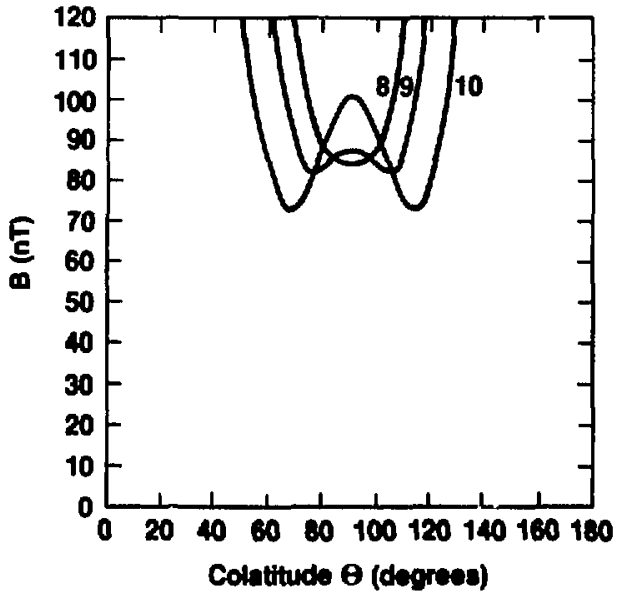

Figure 4-101. Cross section in the noon-midnight plane of the Mead-Fairfield 1975 magnetospheric magnetic field model.

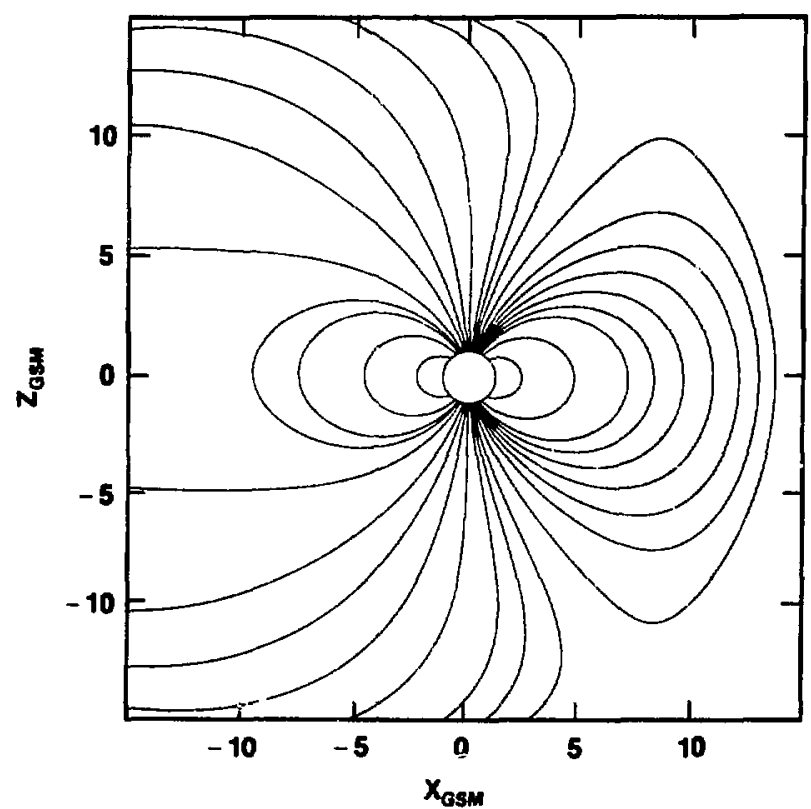

its construction represents the average field configuration quite well and is useful for studies involving time-averaged data sets. Unfortunately for our purposes, the radial field gradient also reverses near the dayside magnetopause as in the Olson-Pfitzer model, as can be seen from the behavior of constant-B contours near the magnetopause (Fig. 4-102). Thus it too is unreliable for trajectory calculations in the outer regions. 


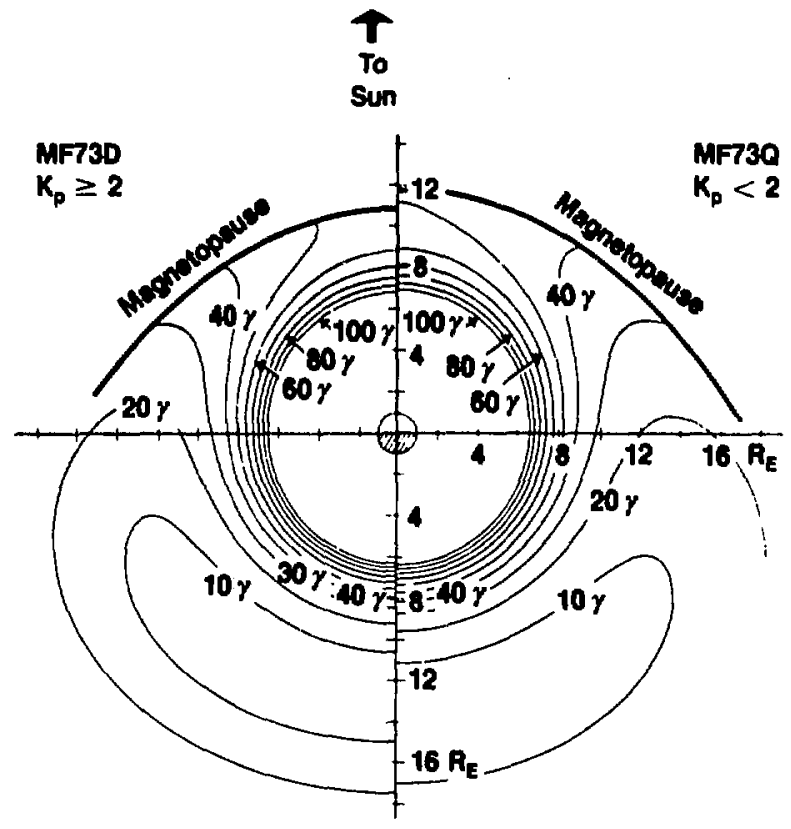

Figure 4-102. Contours of constant-B in the equatorial plane calculated using the Mead-Fairfield model (Walker, 1976). On the right is shown the model generateu for magnetically-quiet conditions (magnetic activity index $K_{P}<2$ ), and on the left is the model appropriate for disturbed conditions $\left(K_{P} \geq 2\right)$. Both show a reversal in the sign of the radial field gradient near the dayside magnetopauce. 


\section{References}

Alekseev, I. I., and V. P. Shabansky, A model of a magnetic field in the geomagnetosphere, Planet. Space Sci., 20, 117, 1972.

Alfvén, H., and C-G Falthammar, Cosmical Electrodynamics, second ed., Clarendon Press, Oxford, 1963.

Alfvén, H., Electric currents in cosmic plasmas, Rev. Geophys. Space Phys., 15, 271, 1977.

Antonova, A. Ye., and V. P. Shabansky, Structure of the geomagnetic field at great distances from the earth, Geomagn. Aeron., 8, 891, 1968.

Antonova, A. Ye., and V. P. Shabansky, Geomagnetic field and trapped particles in the noon sector of the magnetosphere, Geomagn. Aeron., 14, 944, 1974.

Antonova, A. Ye,, and N. S. Nikolayeva, ! ocalization of energetic electrons in the earth's outer magnetosphere, Geomagn. Aeron, 19, 538, $19 / 9$.

Axford, W. I, and C. O. Hines, A unifying theory of high-latitude geophysical phenomena and geomagnetic storms, Can. J. Phys., 39, 1433, 1961.

Beard, D. B., The interaction of the terrestrial magnetic field with the solar corpuscular radiation, J. Geophys. Res., 65, 3559, 1960.

Beard, D. B., The interaction of the terrestral magnetic field with the solar corpuscular radiation, 2, Second order approximation, J. Geophys. Res., 67, 447, 1962.

Beard, D. B., and E. B Jenkins, Correction to the second approximation calculation of the geomagnetic field, solar wind surface, I. Geophys. Res., 67, 4895, 1962.

Beard, D. B., The solar wind geomagnetic field boundary, Rev. Geophys., 2, 335, 1964.

Beard, D. B., The magnetotail magnetic field, J. Geophys. Res., 84, 2118, 1979.

Beard, D. B., The tailward magnetopause field beyond $10 \mathrm{R}_{\mathrm{E}}, J$. Geophys. Res., 87, 2533, 1982.

Behannon, K. W., Mapping of the earth's bow shock and magnetotail by Explorer 33, J. Geophys. Res., 73, 907, 1968.

Buck, R. M., Energetic electron drift motions in the outer dayside magnetosphere - observations and calculations, EOS Trans. Amer. Geophys. Union, 56, 628, 1975.

Burch, J. L., Precipitation of low-energy electrons at high latitudes: Effects of interplanetary magnetic field and dipole tilt angle, J. Geophys. Res., 77, 6696, 1972.

Chapman, W., and V. C. A. Ferraro, A -ew theory of magnetic storms, Terrestrial Magnetism and Atmospheric Electricity, 36, 77, 1931.

Chapman, W., and V. C. A. Ferraro, A new theory of magnetic storms (continued), Terrestrial Magnetism and Atmospheric Electricity, 37, 147, 1932.

Chapman, W., and V. C. A. Ferraro, A new theory of magnetic storms (continued), Terrestrial Magnetism and Atmospheric Electricity, 38, 79, 1933.

Chapman, W., and V. C. A. Ferraro, The theory of the first phase of a geomagnetic storm, Terrestrial Magnetism and Almospheric Electricity, 45, 245, 1940.

Choe, J. Y., and D. B. Beard, The compressed geomagnetic field as a function of dipole tilt, Planet. Space Sci., 22, 595, 1974.

Cowley, S. W. H., The causes of convection in the earth's magnetosphere: A review of the developments during the IMS, Rev. Geophys. Space Phys., 20, 531, 1982.

Crooker, N. U., Dayside merging and cusp geometry, J. Geophys. Res., 84, 951, 1979.

Crooker, N. U., The half-wave rectifier response of the magnetosphere and antiparallel merging, J. Geophys. Res., 85, 575, 1980.

Davies, C. M., Charge separation effects in the Ferraro-Rosenbluth cold plasma sheath model, Phys. Fluids, 10, 391, 1967.

Dungey, J. W., Cosmic Electrodynamics, Cambridge Press, Cambridge, Great Britain, 1958.

Dungey, J. W., Interplanetary magnetic fieid and the auroral zones, Phys. Rev. Lelt., 6, 47, 1961.

Eastman, T. E., E. W. Hones, Jr., S. J. Bame, and J. R. Asbridge, The magnetospheric boundary layer: Site of plasma, momentum and energy transfer from the magnetosheath into the magnetosphere, Geophys. Rrs. Lett., 3, 685, 1976.

Fairfield, D. H., Average and unusual locations of the earth's magnetopause and bow shock, J. Geophys. Res., 76, 6700, 1971.

Fairfield, D. H., Electric and magnetic fields in the high-latitude magnetosphere, Rev. Geophys. Space Phys., 15, 285, 1977. 
Fairfield, D. H., Structure of the Magnetopause: Observations and implications for reconnection, Space Sci. Rev., 23, 427, 1979.

Fairfield, D. H., and N. F. Ness, Configuration of the geomagnetic tail during substorms, ]. Geophys. Res., $75,7032,1970$.

Fairfield, D. H., and N. F. Ness, Imp 5 magnetic-field measurements in the high-latitude outer magnetosphere near the noon meridian, J. Geophys. Res., 77, 611, 1972.

Ferraro, V. C. A., On the theory of the first phase of a geomagnetic storm, J. Geophys. Res., 57, $15,1952$.

Formisano, V., and V. Domingo, Energetic electrons in the cusp and in the high latitude plasma sheet: Evidence for source regions, Planet. Space Sci., 27, 1479, 1979.

Haerendel, G., G. Paschmann, N. Sckopke, H. Rosenbauer, and P. C. Hedgecock, The frontside boundary layer of the magnetosphere and the problem of reconnection, J. Geophys. Res., 83, 3195, 1978.

Halderson, D. W., D. B. Beard, and J. Y. Choe, Corrections to the compressed geomagnetic field as a function of dipole tilt, Planet. Space Sci, 23, 887, 1975.

Heikkila, W. J., Is there an electrostatic field tangential to the dayside magnetopause and neutral line?, Geophys. Res. Lett., 2, 154, 1975.

Hill, T. W., Highlights of theoretical prugress related to the intemational magnetospheric study, Rev. Geophys. Space Phys., 20, 671, 1982.

Hones, E. W., Jr., Motion of charged particles trapped in the earth's magnetosphere, J. Geophys. Res., 68, 1209, 1963.

Howe, H. C., and G. L. Siscoe, Magnetopause Motions at lunar distance determined from the Explorer 35 plasma experiment, J. Geophys. Res., 77, 6071, 1972.

Hundhausen, A. J., J. R. Asbridge, S. J. Bame, H. E. Gilbert, and I. B. Strong, Observations of plasma flow in the earth's magnetosheath, Trans. Am. Geophys. Union, 49, 267, 1968.

Jackson, J. D., Classical Electrodynamics, John Wiley, New York, 1962.

Johnson, F. S., The driving force for magnetospheric convection, Rev. Geophys. Space Phys., 16, 161, 1978.

Kasimov, U., and V. P. Shalansky, Longitudinal transformation of the density of trapped particles in distant regions of the magnetosphere, Geomagn. Aeron., 19, 325, 1975;.

Kaufmann, R. L., and A. Konradi, Explorer 12 magnetopause observations: Large-scale nonuniform motion, J. Geophys. Res., 74, 3609, 1969.

Krall, N. A., and A. W. Trivelpiece, Principles of Plasma Physics, McGraw-Hill, New York, 1973.

Mead, G. D., Deformation of the geomagnetic field by the solar wind, J. Geophys. Res., 69, 1181, 1964.

Mead, G. D., and D. B. Beard, Shape of the geomagnetic field solar wind boundary, J. Geophys. Res., 69, $1169,1964$.

Mead, G. D., and D. H. Fairfield, A quantitative magnetospheric model derived from space craft magnetometer data, J. Geophys. Res., 80, 523, 1975.

Mencke Hansen, A., A. Bahnsen, and N. D'Angelo, The cusp-magnetosheath interface, J. Cesphys. Res., 81, 556, 1976.

Murayama, T., High latitude boundary of the stably-trapped radiation zone on the day-side of the magnetosphere, J. Geomagn. Geoelect., 23, 205, 1971.

Northrop, T. G., and E. Teller, Stability of the adiabatic motion of charged particles in the earth's field, Physical Rev., 11, 215, 1960.

Olson, W. P., The shape of the tilted magnetopause, J. Geophys. Res., 67, 5642, 1969.

Olson, W. P., A model of the distributed magnetospheric currents, J. Geophys. Res., 79, 3731, 1974.

Olson, W. P., and K. A. Pfitzer, A quantitative model of the magnetospheric magnetic field, J. Geophys. $K=., 79,3739,1974$.

Parker, E. N., Confinement of a magnetic field by a beam of ions, J. Geophys. Res., 79, 2315, 1967a.

Parker, E. N., Small-scale nonequilibrium of the magnetopause and its consequences, J. Geophys. Res., 72, $4365,1967 \mathrm{~b}$.

Parker, E. N., Dynamical properties of the magnetosphere, in Physics of the Magnetosphere, edited by R. L. Carovillano, J. F. McClay, and H. F. Radoski, D. Reidel, Dordrecht, Netherlands, 1968.

Paschmann, G., N. Sckopke, G. Haerendel, J. Papamastorakis, S. J. Bame, J. R. Asbridge, J. T. Gosling, E. W. Hones, Jr., and E. R. Tech, ISEE Plesma observations near the subsola. magnetopause, Space Sci. Rev., 22, 717, 1978.

Piddington, J. H., A theory of polar geomagnetic storms, Geophys. J. Royal Astron. Soc., 3, 314, 1960a.

Piddington, J. H., Geomagnetic storm theory, J. Geophys. Res., 65 93, $1960 \mathrm{~b}$. 
Piddington, J. H., The closed model of the earth's magnetosphere, J. Geophys. Res., 84, 93, 1979.

Roederer, J. G., A proposal for trapped particle flux mapping in the outer magnetosphere, NASA-GSFC preprint X-640-66-313, 1966.

Roederer, J. G., On the adiabatic motion of energetic particles in a model magnetosphere, J. Geophys. Res., $72,981,1967$.

Roederer, J. G., Quantitative models of the magnetosphere, Rev. Geophys. Space Phys., 7, 77, 1969.

Roederer, J. G., Dynamics of Geomagnetically Trapped Radiation, Springer-Verlag, New York, 1970.

Russell, C. T., and R. C. Elphic, ISEE observations of flux transfer events at the dayside magnetopause, Geophys. Res. Lett., 6, 33, 1979.

Russell, C. T., and E. W. Gretnstadt, Plasma boundaries and shocks, Rev. Geophys. Space Phys., 21, 449, 1983.

Sckopke, N., A study of self-consistent ring current models, Cosmic Electrodynamics 3, 330, 1972.

Sergeev, V. A., and N. A. Tsyganenko, Energetic particle losses and trapping boundaries as deduced from calculations with a realistic magnetic field model, Planet. Space Sci., 30, 999, 1982.

Shabansky, V. P., Doctoral dissertation, GAISh, MGU, 1966.

Shabansky, V. P., and A. Ye. Antonova, Topology of the drift shells of particles in the magnetosphere, Geomagn. Aeron., 8, 799, 1968.

Shabansky, V. P., Some processes in the magnetosphere, Space Sci. Rev., 12, 299, 1971.

Spreiter, J. R., A. L. Summers, and A. Y. Alksne, Hydromagnetic flow around the magnetosphere, Planet. Space Sci., 14, 223, 1966.

Sugiura, M., and D. J. Poros, A magnetospheric field model incorporating the OGO 3 and 5 magnetic fleld observations, Planel. Space Sci., 21, 1763, 1973.

Taylor, H. E., and E. W. Hones, Jr., Adiabatic motion of auroral particles on a model of the electric and magnetic fields surrounding the earth, J. Geophys. Res., 70, 3605, 1965.

Taylor, H. E., Adiabatic motion of outer-zone particles in a model of the geoelectric and geomagnetic fields, J. Geophys. Kes., 71, 5135, 1966.

Tsyganenko, H. A., A model of the cis-lunar magnetospheric field, Ann. Geophys., 32, 1, 1976.

Vernov, S. N., P. V. Vakulov, S. N. Kuznetsov, Yu. 1. Logachev, E. N. Sosnovets, and V. G. Stolpovskiy, Boundary of the outer radiation belt and zone of unstable radiation, Geomagn. Aeron., 7, 335, 1968.

Voigt, G. H., and K. Fuchs, A macroscopic model for field line interconnection between the magnetosphere and the interplanetary space, in Quantilative Modeling of Magnetospheric Processes, Geophys. Monogr. Ser., Vol. 21, edited by W. P. Olson, AGU, Washington, D.C., 1979.

Voight, G. H., A three dimensional analytical magnetospheric model with defined magnetopause, Z. Geophys., 38, 319, 1972.

Walker, R. J., An evaluation of recent quantitative magnetospheric magnetic field models, Rev. Geophys. Space Phys., 14, 411, 1976.

Walker, R. J., Quantitative modeling of planetary magnetospheric magnetic fields, in Quantitative Modeling of Magnetospheric Processes, Geophys. Monogr. Ser., Vol. 21, edited by W. P. Olson, AGU, Washington, D.C., 1979.

Walker, R. J., Modeling planetary magnetospheres, Rev. Geophys. Space Phys., 21, 495, 1983.

West, H. I., Jr., The signatures of the various regions of the outer magnetosphere in the pitch angle distributions of energetic particles, in Quantitative Modeling of Magnelospheric Process, Geophys. Monogr. Ser., Vol. 21, edited by W. P. Olson, AGU, Washington, D.C., 1979.

West, H. I., Jr., R. M. Buck, and J. R. Walton, Shadowing of electron azimuthal-drift motions near the noon magnetopause, Nature Phys. Sci., 240, 6, 1972.

West, H. I., Jr., R. M. Buck, and M. G. Kivelson, On the configuration of the magnetotail during quiet and weakly disturbed periods: State of the magnetosphere, J. Geophys. Res., 83, 3805, 1978a.

West, H. I., Jr., R. M. Buck, and M. G. Kivelson, On the configuration of the magnetotail during quiet and weakly disturbed periods: Magnetic field modeling, J. Geophys. Res., 83, 3819, 1978b.

Williams, D. J., and G. D. Mead, Night side magnetosphere configuration as obtained from trapped electrons at 1100 kilometers, J. Geophys. Res., 70, 3017, 1965.

Williams, D. J.. T. A. Fritz, B. Wilken, and E. Keppler, An energetic particle perspective of the magnetopause, J. Geophys. Res., 84, 6385, 1979.

Willis, D. M., Structure of the magnetopause, Rev. Geophys. Space Phys., 9, 953, 1971.

Willis, D. M., and R. J. Pratt, A quantitative model of the geomagnetic tail, J. Aimos. Tt Phys., 34, 1955, 1972. 


\section{Chapter 5. Conclusion}

This dissertation has focused on the trajectories of energetic ( $\left.E_{e}>45 \mathrm{keV}\right)$ electroris in the outer dayside magnetosphere. In this region within one $R_{E}$ or so of the magnetopause, the magnetic fields produced by the Chapman-Ferraro currents create high-latitude minimum-B pockets in the total magnetospheric magnetic field which are capable of temporarily trapping azimuthally-drifting electrons. These electrons drift sunward from the midnight meridian, moving on drift shells characterized by initial values of the adiabatic invariants $B_{M}$ and I (for the case of static fields). At some pre-noon azimuth, particles encounter a field line which has a local $B$-maximum of strength $B=B_{M}$. On such a line the drift shell branches into disjoint segments, one lying equatorward of the local maximum and one poleward. Depending on bounce phase, a particle moves onto one of these branched drift shell segments. If it is caught in the high-latitude minimum-B region, it moves around the ncon sector on a bounce trajectory that at noon lies between $40^{\circ}$ and $60^{\circ}$ latitude. At a symmetric location post-noon, the shell segments rejoin and the particle now moves on a normal equator-crossing trajectory around to the midnight meridian.

We examined the details of the shell-branching process. We showed that shell branching conserves $B_{M}, I$, and particle directional fux $j$. I is conserved in the general sense that $I=I_{1}+I_{2}$, where $I$ is the original (unbranched) value and $I_{1}$ and $I_{2}$ are the values associated with the branched segments. Although every particle has its $I$-value reduced to either $I_{1}$ or $I_{2}$ upon branching, it is restored to I when the segments rejoin post-noon. I-conservation on branching shells was argued on a theoretical basis, and confirmed by numerical computation of particle trajectories in a model field. The minimum-B pockets allow azimuthally drifting electrons to pass around noon on closed drift shells, which would otherwise drift to the magnetopause pre-noon and be lost. This effectively extends the boundary of stable trapping on the nightside to greater distances. On the dayside, this process extends the boundary of stable trapping to high latitudes.

Studies of drift trajectories near the magnetopause require an accurate magnetic field model. We surveyed a number of numerical field models in common use for particle studies. We noted that all numericai models which reproduce well the dayside fieldline configuration possess high-latitude minimum-B pockets. However those models which did not model the magnetopause in at least a quasiself-consistent manner produced unphysical effects which limited their utility in trajectory simulations. Fieldlines could be found which penetrated the nominal magnetopause, and field gradients in the outer regions reversed sign. The Choe-Beard 1974 model, however, was found to agree well with satellite measurements and to be free of modeling artifacts, at least near the dayside magnetopause. With its variable dipole tilt and magnetopause standoff parameters, it was easily adjusted to model the actual field conditions encountered on a particular satellite pass.

The previously-reported branching studies used the image dipole model of Antonova and Shabansky, who showed that shell branching occurred for particles which in the midnight meridian mirrored close to the equator $(1=0)$. We found that the Choe-Beard model showed branching for particles at midnight with equatorial pitch angles $\alpha_{0}$ in the range $30^{\circ}<\alpha_{0}<65^{\circ}$, approximately. These differences probably result from the manner in which the tail field is represented. In the image dipole model, the tail field is generated by a current plate on the nightside; in the Choe-Beard model the tail currents are assumed to flow cross-tail in a sheet of finite thickness, returning on the cylindrical tail magnetopause.

For a given model of the magnetospheric magnetic field, we showed that the locations of branching lines and the subsequent adiabatic trajectories of particle on branched shell segments could be completely identified and described by a branching map. This map consists of a $B_{M}, I-$ space representation of midnight meridian PADs, shell branching points at various azimuths, and the drift loss-cone. This construction allowed us to explain the compound branching process found to occur at some angles of dipole tilt. Via this map, it was possible to locate the midnight origins of the particles comprising a noon meridian pitch angle distribution. Under the assumptions of adiabatic motion and conservation of directional flux, the noon PAD could be assigned flux values based on models of the midnight flux distribution. These synthetic noon PADs were seen to have "edges," or abrupt flux transitions, at critical pitch angles corresponding to the location of local B-maxima on the field line passing through the point of observation. Particles mirroring equatorward of the B-maxima mapped to relatively close regions of the midnight tail where flux levels are typically high. Particles mirroring poleward of the B-maxima mapped to relatively 
distant regions of the midnight tail where flux levels are lower. This transition is a characteristic PAD adiabatic signature of a minimum-B fieldline.

The magnetopause is observed to be in continual motion, and to support surface waves. These dynamical effects are expected to modify the trajectories of particles drifting close to the magnetopause. Local compressions of the field due to inward motion of the magnetopause can cause particles to drift into the magnetopause, leaving the satellite in a temporary particle "shadow." Variations in the strength of local B-maxima can cause branched shells to temporarily rejoin, allowing B-pocket fluxes access to the equatorial regions. This latter process would be observed as a sudden flux change at a critical pitch angle.

In the afternoon sector of the outer magnetosphere, our adiabatic drift analysis predicted the following effects. The compressed field of the outer noon sector casts an ndiabatic electron shadow upon the afternoon magnetosphere, producing a region just inside the magnetosphere which is void of particles that drift on unbranched shells. Only particles which pass around noon via the high-latitude minimum-B segments of branched shells, and then move onto normal equator-crossing bounce trajectories when the shell segments rejoin post-noon, can reach a satellite in the shadow region. These particles are observed in a narrow range of pitch angles determined by the magnetic field geometry. As the satellite moves inward, and begins to emerge from the particle shadow, it first observes fluxes bullding up at pitch angles away from $90^{\circ}$, followed by increases in flux about $90^{\circ}$. The flux component from the high-latitude minimum-B regions undergoes scattering in the B-pocket, producing local flux minima at the satellite at angles corresponding to branched shells.

The high-resolution electron directional flux distributions acquired by the $\mathbf{E} 6$ scanning spectrometer experiment on the OGO-5 satellite showed striking evidence of drift shell branching in the outer dayside magnetosphere. Pre-noon PADs showed abrupt flux transitions at pitch angles which matched closely those of the synthetic PADs created by mapping fluxes from the midnight meridian to the satellite. These critical pitch angles corresponded to the locations of local B-maxima along the satellite field line, as derived from the Choe-Beard magnetospheric magnetic field model. The fluxes of electrons mirroring equatorward of the local B-maxima (i.e., appearing at pitch angles between the critical angles) were highly variable, compared to those in the rest of the distribution, and showed effects similar to those observed in magnetopause-shadowed PADs seen in the afternoon magnetosphere. The PADs obtained in the afternoon sector showed flux features consistent with the model predictions of drift shell rejoining noted above, including the magnetopause shadowing of unbranched-shell fluxes, the flux behavior as the satellite emerged from the shadow, and the local flux minima at critical pitch angles.

As a whole, the data set provides strong experimental support for the presence of drift shell branching in the outer dayside magnetosphere. Prominent flux features are seen in the PADs of energetic electrons which correlate well with effects predicted on the basis of adiabatic drift in the Choe-Beard model, over a range of dipole tilt angles, magnetic latitudes, and dayside longitudes.

A possibility arising in this study is that the minirnum-B pockets do more than just permit particles from a relatively restricted spatial region at midnight to drift around the earth; they may also act as conduits for solar wind particles which have entered the magnetosphere in the polar cusp regions adjacent to the minimum-B pockets. Entry of magnetosheath plasma into the polar cusp is well established (e.g., Frank, 1971). The hig' $2 \cdot{ }^{\prime}$ '. $\cdot$ e cusp is a major source of wave activity and a region where wave-particle interactions dominate ‥ . . . asma dynamics (Scarf, 1972). OGO-5 measurements (Russell et al., 1971) show strong, broad specirum activity, with the most intense waves at the cusp boundaries. According to D'Angelo (1977), the fluctuations at the boundary may cause appreciable transverse diffusion of the cusp particles. Cross-field diffusion in the equatorward direction would bring these particles into the B-pocket regions. Particles entering these regions could then be adiabatically transported on stably-trapped drift shells around the earth, and over time could diffuse into the radiation belts. 


\section{Appendix 1: Compute: Cócies}

All tracings of adiabatic drift shells and the production of field model figures were accomplished by the author's BTRACE code on the CDC-7600 computer. This code generates the general magnetospheric magnetic field models examined in this work, including the Choe-Beard, Olson-Pfitzer, Mead-Fairfield, and image dipole models. In addition, several specialized local models are available [e.g., the magnetotail models of Speiser $(1965,1967)$ and West et al. (1978) ]. A major capability of BTRACE is locating the $R_{E O}$ of a field line in a specified azimuth (e.g., noon) which contains points of specified $B_{M}, I$ (e.g., from a PAD originating in the midnight meridian), thus permitting the mapping of stable-trapping regions and shellbranching boundaries. Tracing of field lines was accomplished by integrating the differential equations of a field line:

$\mathrm{dx} / \mathrm{B}_{\mathrm{X}}=\mathrm{dy} / \mathrm{B}_{\mathrm{Y}}=\mathrm{dz} / \mathrm{B}_{\mathbf{Z}} ;$

using the ODE solver from the TIBROE code described below.

Trajectory calculations were performed with the TIBROE code, a derivative of the TIBRO orbit code (Foote et al., 1964). The original code was revised to make the trajectory calculations relativistically correct, to add electric field forces, to incorporate the geomagnetic field models used in BTRACE, to perform first and second adiabatic invariant calculations along a particle trajectory, and to optionally include energy conseivation.

In this latter option (useful for static $B$ and electric field $E=0$ ), the particle velocity is normalized after each timestep to maintain constant particle kinetic energy. The normalization procedure modifies each velocity component by adding a correction term proportional to the component magnitude at the previous timestep. The total correction is such that $|\mathbf{v}|$ remains constant throughout the run. This procedure ensures that particles initially mirroring at the equator, for instance, will not acquire a velocity component parallel to the field line. Tests indicate that the normalization reduces the trajectory error per step by about a factor of two, compared to the standard unnormalized calculation.

TIBROE integrates the equations of motion using a third-difference Adams predictor-corrector method, with a Runge-Kutta starter. Accuracy of results reported in this work was checked by reversing the orbits. Under a change in sign of particle charge and a reversal of final velocity direction, a particle will ideally retrace its trajectory from the final point at time $\mathrm{T}$ to the initial point of the calculation, arriving at time 2T. The errors in the calculation are cumulative, before and after the orbit reversal, so that the difference between the particle positions at $t=0$ and $t=2 T$ is a measure of the computational accuracy. Accuracies achieved in this work were of the order of $0.2 R_{E}$ over times of $T=20$ seconds-more than enough accuracy to validate the changes in I that accompany shell-branching and shell-rejoining. TIBROE runs also verified that the motions of $3 \mathrm{MeV}$ electrons in the B-pocket regions were completely adiabatic (maintaining constant $B_{M}$ and I). Therefore the mappings deduced in this work from adiabatic drift theory are valid for electrons up to this energy.

\section{References}

D'Angelo, N., Plasma waves and instabilities in the polar cusp: A review, Rev. Geophys. Space Phys., 15, $299,1977$.

Foote, J. H., R. P. Freis, J. Killeen, R. W. Moir, and J. A. Staples, Computer code for calculating particle trajectories in a confining magnetic field, in Controlled Thermonuclear Research Semi-Annual Report-july-December 1964, Lawrence Livermore National Laboratory, Livermore, CA, UCRL-12303, p128, 1965.

Frank, L. A., and K. L. Ackerson, Observations of charged particle precipitation into the auroral zone, J. Geophys. Res., 76, 3612, 1971.

Russell, C. T., C. R. Chappell, M. D. Montgomery, M. Neugebauer, and F. L. Scarf, OGO 5 observations of the polar cusp on November 1, 1968, J. Geophys. Res., 76, 6743, 1971.

Scarf, F. L., R. W. Fredricks, I. M. Green, and C. T. Russell, Plasma waves in the dayside polar cusp, J. Geophys. Res., 77, 2274, 1972. 
Speiser, T. W., Particle trajectories in model current sheets, 1, Analyticaì Solutions, J. Geophys. Res., 70, 4219, 1965.

Speiser, T. W., Particle trajectories in model current sheets, 2, Applications to auroras using a geomagnetic tail model, J. Geophys. Res., 72, 3919, 1967.

West, H. I., Jr., R. M. Buck, and M. G. Kivelson, On the configuration of the magnetotail during quiet and weakly disturbed periods: Magnetic field modeling, J. Geophys. Res., 83, 3819, 1978. 